\title{
1999 Report on Hanford Site Land Disposal Restrictions for Mixed Waste
}

D. G. Black

Date Published

April 1999

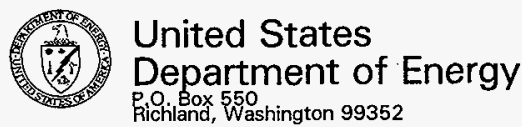

Richland, Washington 99352

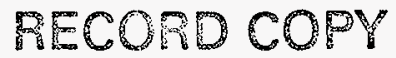

Approved for Public Release 
LEGAL DISCLAIMER

This report was prepared as an account of work sponsored by an agency of the United States Government. Neither the United States Government nor any agency thereof, nor any of their employees, nor any of their contractors, subcontractors or their employees, makes any warranty, express or implied, or assumes any legal liability or responsibility for the accuracy, completeness, or any third party's use or the results of such use of any information, apparatus, product, or process disclosed, or represents that its use would not infringe privately owned rights. Reference herein to any specific commercial product, process, or service by trade name, trademark, manufacturer, or otherwise, does not necessarily constitute or imply its endorsement, recommendation, or favoring by the United States Government or any agency thereof or its contractors or subcontractors. The views and opinions of authors expressed herein do not necessarily state or reflect those of the United States Government or any agency thereof.

This report has been reproduced from the best available copy.

Printed in the United States of Americe

DISCLM-2.CHP $(1-91)$ 


\section{EXECUTIVE SUMMARY}

This report was submitted to meet the requirements of Hanford Federal Facility Agreement and Consent Order ${ }^{1}$ (Tri-Party Agreement) Milestone M-26-01I. This milestone requires the preparation of an annual report that covers characterization, treatment, storage, minimization, and other aspects of managing land-disposal-restricted mixed waste at the Hanford Facility.

The U.S. Department of Energy, its predecessors, and contractors on the Hanford Facility were involved in the production and purification of nuclear defense materials from the early 1940 s to the late 1980s. These production activities have generated large quantities of liquid and solid mixed waste. This waste is regulated under authority of both the Resource Conservation and Recovery Act of $1976^{2}$ and the Atomic Energy Act of $1954^{3}$. This report covers only mixed waste.

The Washington State Department of Ecology, U.S. Environmental Protection Agency, and U.S. Department of Energy have entered into the Tri-Party Agreement' to bring the Hanford Facility operations into compliance with dangerous waste regulations. The Tri-Party Agreement required development of the original land disposal restrictions (LDR) plan and its annual updates to comply with LDR requirements for mixed waste. This report is the ninth update of the plan first issued in 1990.

The Tri-Party Agreement requires and the baseline plan and annual update reports provide the following information.

Waste Characterization Information. Provides information about characterizing each LDR mixed waste stream. The sampling and analysis methods and protocols, past characterization results, and, where available, a schedule for providing the characterization information are discussed.

Storage Data. Identifies and describes the mixed waste on the Hanford Facility. Storage data include the Resource Conservation and Recovery Act of $1976^{2}$ dangerous waste codes, generator process knowledge needed to identify the waste and to make LDR determinations, quantities stored, generation rates, location and method of storage, an assessment of storage-unit compliance status, storage capacity, and the bases and assumptions used in making the estimates.

\footnotetext{
${ }^{1}$ Ecology, EPA, and DOE, 1989, Hanford Federal Facility Agreement and Consent Order, Vol. 1 and 2, as amended, Washington State Department of Ecology, U.S. Environmental Protection Agency, and U.S. Department of Energy, Olympia, Washington.

${ }^{2}$ Resource Conservation and Recovery Act of 1976, as amended, 42 U.S.C. 6901, et seq.

${ }^{3}$ Atomic Energy Act of 1954, as amended, 42 U.S.C. 2011.
} 
Treatment Information. Identifies the current treatment processes, plans, and schedules for developing treatment technologies that meet LDR treatment standards. Includes all applicable milestones and associated schedules for the development and implementation of treatment or technologies to achieve compliance with the LDR requirements. Also includes discussions of treatment alternatives and accelerated treatment.

Waste Reduction Information. Identifies methods for reducing the generation of LDR waste. Includes treatment methods and process changes made or planned to reduce the generation of LDR waste, methods to minimize the volume of LDR waste, and methods to minimize the toxicity of newly generated waste.

Schedule. Provides schedules depicting the events necessary to achieve compliance with LDR requirements, including planned or completed variances or treatment equivalencies needed to achieve LDR compliance.

Progress. Identifies progress made in achieving compliance since the last LDR report.

Waste generated on the Hanford Facility resulted from primarily defense materials production. Usable defense materials were separated from fission-product waste using precipitation and solvent extraction processes. The large quantities of liquid waste that resulted from these separation processes were stored in underground single-shell tanks (SST) and doubleshell tanks (DST). Additional waste resulted from the following:

- Nuclear fuel fabrication

- Process laboratory activities

- Equipment and structure cleaning and decontamination

- Process- and storage-unit closure

- Research and development activities, such as Fast Flux Test Facility operation.

After waste reduction, the total projected annual generation rates for the streams covered in this report range from 7533 cubic meters in 2003 to 12,084 cubic meters in 2001.

The following types of mixed waste (i.e., hazardous waste that contains radionuclides) are addressed in this report:

- Waste designated as characteristic dangerous waste and as toxic or persistent by the Washington State criteria

- Listed mixed waste because it contains small amounts of spent solvents and discarded pure chemical products.

The waste consists of liquid, sludge, hard crystalline material (salt cake), and such materials as contaminated equipment, paper, and rags. Much is already known about the waste 
characteristics from process knowledge and sampling and analysis programs. Action schedules have been developed to further characterize the waste.

The waste currently is stored in underground SSTs and DSTs, in containers placed in storage units such as the Central Waste Complex (CWC), caissons, and other facilities units. The waste is removed from these storage units, treated to meet LDR standards, and sent for final disposal in accordance with permits and, where applicable, Tri-Party Agreement milestones.

Approximately 216,937 cubic meters of mixed LDR waste are currently in storage. The DSTs are expected to have adequate storage capacity through 2004 under the current planning baseline. To.alleviate any future space shortages, DST contents are concentrated through the 242-A Evaporator. This has allowed DOE to indefinitely postpone building new DSTs. The storage space at the CWC is currently 60 to 65 percent full. Storage projections show that the CWC's capacity will not be exceeded during the waste management life cycle (through 2032) based on the fiscal year (FY) 1999 treatment and disposal planned funding levels and forecast waste volumes.

The waste treatment processes for these waste streams include the current treatment processes to reduce corrosion of storage tanks and planned treatment processes to reduce waste toxicity and immobilize waste constituents in DSTs. Current waste treatment consists of adjusting $\mathrm{pH}$ and adding corrosion inhibitors in DSTs and using sorbents and solidifying agents (requirements placed on generators before the CWC will accept the waste). Surface decontamination and waste segregation, separation, and repackaging are performed at the T Plant complex. Planned waste treatment processes include developing neutralization and toxic constituent destruction processes; developing waste separation, pretreatment, and stabilization processes (commercial facilities); and separating tank waste (pretreatment) into low-activity and high-level waste fractions, both of which will be vitrified. The low-level fraction will be disposed of on site. The high-level fraction will be sent to an offsite geologic repository for disposal.

The Hanford Facility developed a sitewide waste minimization plan that sets minimization goals and establishes processes for measuring progress toward these goals. Each plant or process has a program in place to implement the sitewide goals.

The Hanford Facility has a preexisting agreement (Tri-Party Agreement) that meets the legal requirements specified under the Federal Facilities Compliance Act of 1992. This agreement exempts the Hanford Facility from having to develop a site treatment plan, although other activities under the Act are still required. Both the Washington State Department of Ecology and the U.S. Environmental Protection Agency determined that this Hanford Site Land Disposal Restrictions Report for Mixed Waste, required by the Tri-Party Agreement, meets the requirements of a site treatment plan. In accordance with the Act, DOE submitted an LDR plan and an order requiring compliance with the plan (the Tri-Party Agreement). Accordingly, various Tri-Party Agreement milestones have been set to fulfill LDR storage, characterization, and/or treatment requirements identified in the LDR report. Failure to meet any of these 
milestones would constitute a deviation from the LDR plan and a violation of the Tri-Party Agreement.

The continued storage of land-disposal-restricted waste until sufficient treatment and disposal capacity is available was negotiated as part of the Tri-Party Agreement. Schedule to implement the dangerous waste management compliance activities until treatment capacity is available are described in the Tri-Party Agreement and this report. Any newly identified compliance actions will be scheduled in accordance with procedures established in the agreement. 


\section{ACKNOWLEDGMENTS}

The efforts of many people across the Hanford Facility were instrumental in preparing and issuing this report. Direct major support was provided by the following:

Report Lead Author and Coordinator:
D. G. Black
Waste Management Federal Services Hanford Inc.

Technical Editing and Document Services:

M. P. Curry Lockheed Martin Services, Inc.

Report Input and Contractor Review:

F. M. Coony Waste Management Federal Services of Hanford, Inc.

C. R. Haas Waste Management Federal Services of Hanford, Inc.

G.D. Hendricks

B\&W Hanford Company

O. S. Kramer

Fluor Daniel Hanford, Inc.

G. J. LeBaron

M. W. McCoy

B\&W Hanford Company

A. G. Miskho Fluor Daniel Hanford, Inc.

D. E. Nester

Waste Management Federal Services of Hanford, Inc.

D. H. Nichols

Waste Management Federal Services of Hanford, Inc.

P. A. Powell

Lockheed Martin Hanford Corporation

G. S. Robinson

Bechtel Hanford Inc.

M. M. Serkowski

F. M. Simmons

$B \& W$ Hanford Company

J. N. Strode

B\&W Hanford Company

Lockheed Martin Hanford Corporation

\section{U.S. Department of Energy, Richland Operations Office} review and approval coordination:
R. N. Krekel
G. L. Sinton 
DOE/RL-99-01

This page intentionally left blank. 
DOE/RL-99-01.

\section{CONTENTS}

1.0 INTRODUCTION

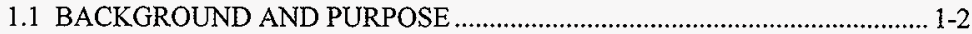

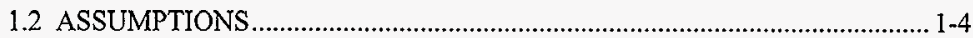

1.3 SCHEDULE AND MECHANICS OF PLAN UPDATE ...............................1-10

1.4 MILESTONE PLANNING PROCESS ....................................................... 1-11

1.5 ACTIVITIES AND ACHIEVEMENTS ..................................................... 1-11

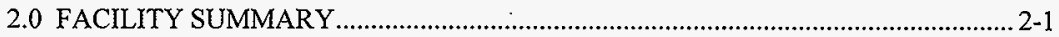

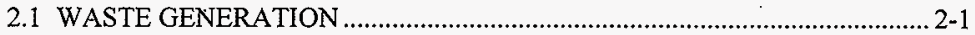

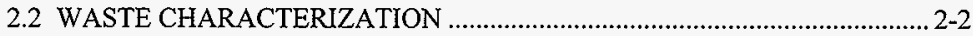

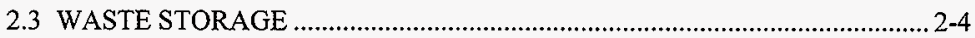

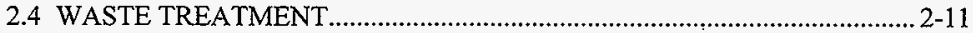

2.4.1 Double-Shell Tank System Waste ................................................... 2-12

2.4.2 Single-Shell Tank System Waste.................................................... 2-12

2.4.3 Central Waste Complex Stored Waste................................................. 2-29

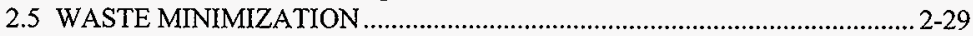

2.5.1 Waste Minimization Program Elements ........................................... 2-29

2.5.2 Program Objectives..................................................................... 2-30

2.5.3 Location-Specific Waste Minimization ............................................. 2-31

2.6 TREATABILITY VARIANCES, EQUIVALENCY PETITIONS, RULEMAKING PETITIONS, AND CASE-BY-CASE EXEMPTIONS........ 2-32

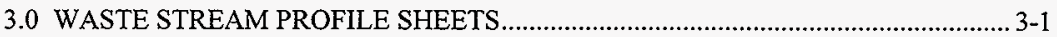

LDR-Compliant Solids, MLLW-01-A through MLLW-01-D ….............................. 3-5

Inorganic Solids, MLLW-02-A and MLLW-02-B ............................................. 3-25

Organic Solids, MLLW-03-A and MLLW-03-B ……....................................... 3-37

Debris, MLLW-04-A and MLLW-04-B ........................................................ 3-51

Elemental Lead, MLLW-05-A and MLLW-05-B ……….................................... 3-63

Elemental Mercury, MLLW-06-A and MLLW-06-B........................................ 3-73

M91 MLLW, MLLW-07-A and MLLW-07-B .............................................. 3-83

Greater-Than-Class 3, MLLW-08 .................................................................... 3-93

Contact-Handled TRU, TRUM-01A through TRUM-01K .................................... 3-97

Remote-Handled TRU, TRUM-02A and TRUM-02B ..................................... 3-153

TRU Hydraulic Fluids w/PCBs, TRUM-03 ……............................................ 3-165

300 Area CH-TRUM, TRUM-04A through TRUM-04C.................................... 3-171

TRUM Long-Length Equipment, TRUM-05A and TRUM-05B ....................... 3-187

Miscellaneous RH-TRUM, TRUM-06 ........................................................ 3-197

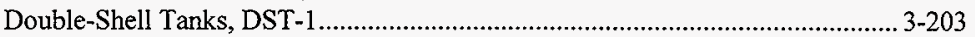

Single-Shell Tanks, SST-1 ........................................................................ 3-211

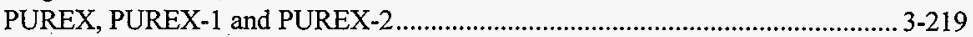

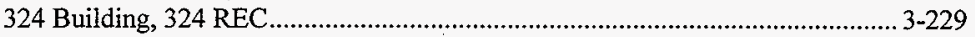




\section{CONTENTS (cont)}

B Plant Complex, B PLANT-1 AND B PLANT-2 …………................................ 3-233

T Plant Complex, T-Drag-off Tank, Condenser …................................................ 3-241

Cesium and Strontium Capsules, WESF ......................................................... 3-253

4.0 ADDITIONAL WASTE STREAM INFORMATION ................................................... 4-1

4.1 THE HANFORD FACILITY'S SOLID LOW-LEVEL MIXED WASTE ........ 4-1

4.1.1 MLLW Summary .......................................................................... 4-1

4.1.2 MLLW Generation.......................................................................... 4-2

4.1.3 MLLW Characterization ....................................................................... 4-9

4.1.4 MLLW Storage ............................................................................. 4-11

4.1.5 MLLW Treatment ........................................................................... 4-15

4.1.6 MLLW Strategic Plan Objective ................................................... 4-25

4.1.7 MLLW Waste Reduction.................................................................. 4-25

4.1.8 MLLW Treatability Variances, Equivalency Petitions, Rulemaking Petitions, and Case-by-Case Exemptions ....................... 4-25

4.2 TRUM WASTE SUMMARY.

4.2.1 Mixed TRU Waste Generation ...................................................... 4-28

4.2.2 TRUM Characterization................................................................. 4-30

4.2.3 TRUM Storage .............................................................................. 4-32

4.2.4 TRUM Treatment and WIPP Certification ..................................... 4-33

4.2.5 TRUM Waste Reduction.............................................................. 4-34

4.2.6 TRUM Treatability Variances, Equivalency Petitions, Rulemaking Petitions, and Case-by-Case Exemptions ....................... 4-35

4.3 DOUBLE-SHELL TANK SYSTEM WASTE ............................................ 4-35

4.3.1 DST System Generation .................................................................. 4-35

4.3.2 DST System Storage .................................................................... 4-39

4.3.3 DST System Characterization........................................................ 4-40

4.3.4 DST System Treatment ...................................................................... 4-42

4.3.5 DST System Treatability Variances, Equivalency Petitions, Rulemaking Petitions, and Case-by-Case Exemptions ....................... 4-44

4.4 SINGLE-SHELL TANK SYSTEM WASTE .............................................. 4-44

4.4.1 SST System Generation ................................................................. 4-44

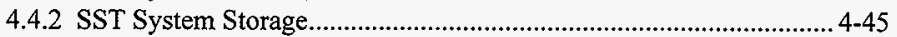

4.4.3 SST System Characterization .......................................................... 4-46

4.4.4 SST System Treatment ..................................................................... 4-47

4.4.5 SST System Treatability Variances, Equivalency Petitions,

Rulemaking Petitions, and Case-by-Case Exemptions...................... 4-47

4.5 PUREX CONTAINMENT BUILDING WASTE ........................................... 4-47

4.5.1 PUREX Containment Building Generation......................................... 4-47

4.5.2 PUREX Containment Building Storage ............................................ 4-48

4.5.3 PUREX Containment Building Characterization ............................... 4-48

4.5.4 PUREX Containment Building Treatment ........................................ 4-48 


\section{CONTENTS (cont)}

4.6 PUREX STORAGE TUNNELS WASTE …..............................................48

4.6.1 PUREX Storage Tunnels Generation.................................................. 4-48

4.6.2 PUREX Storage Tunnels Storage ………………............................... 4-50

4.6.3 PUREX Storage Tunnels Characterization ....................................... 4-51

4.6.4 PUREX Storage Tunnels Treatment................................................. 4-52

4.7324 RADIOCHEMICAL ENGINEERING CELLS WASTE ............................ 4-53

4.7.1 324 REC Generation .................................................................... 4-53

4.7.2 324 REC Storage ............................................................................. 4-54

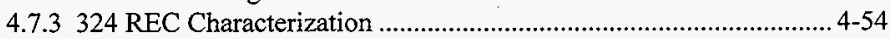

4.7.4 324 REC Treatment ................................................................. 4-54

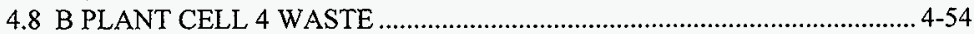

4.8.1 B Plant Cell 4 Generation .................................................................. 4-55

4.8.2 B Plant Cell 4 Storage ..................................................................... 4-55

4.8.3 B Plant Cell 4 Characterization.............................................................. 4-55

4.8.4 B Plant Cell 4 Treatment.................................................................... 4-55

4.9 B PLANT CONTAINMENT BUILDING WASTE....................................... 4-56

4.9.1 B Plant Containment Building Generation ........................................... 4-56

4.9.2 B Plant Containment Building Storage.............................................. 4-56

4.9.3 B Plant Containment Building Characterization............................... 4-56

4.9.4 B Plant Containment Building Treatment …………............................ 4-57

4.10 T PLANT COMPLEX DRAG-OFF BOX …………................................ 4-57

4.10.1 Drag-Off Box Generation .............................................................. 4-57

4.10.2 Drag-Off Box Storage ................................................................. 4-57

4.10.3 Drag-Off Box Characterization...................................................... 4-57

4.10.4 Drag-Off Box Treatment............................................................... 4-58

4.11 T PLANT COMPLEX TANK TRAILER WASTE ........................................ 4-58

4.11.1 Tank Trailer Waste Generation............................................................ 4-58

4.11.2 Tank Trailer Waste Storage ............................................................... 4-58

4.11.3 Tank Trailer Waste Characterization ............................................... 4-58

4.11.4 Tank Trailer Waste Treatment ......................................................... 4-59

4.12 T PLANT EC-1 CONDENSER ………................................................. 4-59

4.12.1 EC-1 Condenser Generation ....................................................... 4-59

4.12.2 EC-1 Condenser Storage ................................................................ 4-59

4.12.3 EC-1 Condenser Characterization.................................................. 4-59

4.12.4 EC-1 Condenser Treatment................................................................... 4-59 


\section{CONTENTS (cont)}

4.13 CESIUM AND STRONTIUM CAPSULES, WESF ....................................... 4-60

4.13.1 Cesium and Strontium Capsule Waste Generation........................... 4-60

4.13.2 Cesium and Strontium Capsule Waste Storage .................................. 4-60

4.13.3 Cesium and Strontium Capsule Waste Characterization .................. 4-60

4.13.4 Cesium and Strontium Capsule Waste Treatment ............................. 4-60

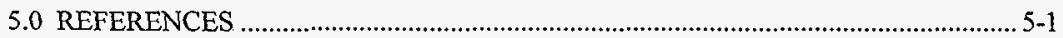

5.1 APPLICABLE DOCUMENTS .............................................................. 5-1

5.2 CODE OF FEDERAL REGULATIONS AND FEDERAL REGISTER ............ 5-2

5.3 FEDERAL AND STATE ACTS ……....................................................... 5-4

5.4 WASHINGTON STATE REGULATIONS ……........................................... 5-4 


\section{LIST OF FIGURES}

2-1 Tri-Party Agreement Action Plan Work Schedule ...................................................... 2-13

4-1 Hanford MLLW Baseline Disposition Map .............................................................. 4-17

4-2 Hanford Facility TRU Waste Baseline Disposition Map ………….............................. 4-36

4-3 DST Space Summary ......................................................................................... 4-41

4-4 Hanford Tank Waste Remediation System................................................................ 4-43

\section{LIST OF TABLES}

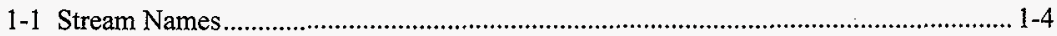

1-2 Streams No Longer Applicable to Report ……....................................................... 1-7

2-1 Waste Inventory and Generation Summary .......................................................... 2-1

2-2 LDR Summary Table.................................................................................................... 2-5

3-1 Estimated Mass of Nonradioactive Chemical Components of Single-Shell Tank and Double-Shell Tank Systems Waste ........................................................................ 3-206

3-2 Hanford Facility Single-Shell Tank System Releases ............................................ 3-215

4-1 Waste Stream Summaries ..................................................................................... 4-2

4-2 Past-Practice Waste Characterization Verification Schedule ......................................... 4-12

4-3 FY98 Characterization Verification Activity ........................................................ 4-13

4-4 Waste Treatment Schedule and Volume................................................................. 4-20

4-5 Mixed TRU Waste Streams ......................................................................................... 4-27

4-6 Mixed TRU Waste Generation ................................................................................ 4-29

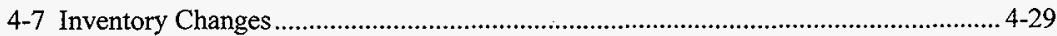

4-8 TRUM Disposition Schedule and Waste Volume ...................................................... 4-35

4-9 Waste Generation for Various Locations and Programs ............................................... 4-39

4-10 DST System Summary ......................................................................................... 4-39

4-11 SST System Summary ……............................................................................... 4-46

4-12 Plutonium Uranium Extraction Plant Storage Tunnels Inventories .......................... 4-49 


\section{ACRONYMS AND ABBREVIATIONS}

\begin{tabular}{|c|c|}
\hline ALARA & as low as reasonably achievable \\
\hline ATG & Allied Technology Group \\
\hline CERCLA & $\begin{array}{l}\text { Comprehensive Environmental Response, Compensation, } \\
\text { and Liability Act of } 1980\end{array}$ \\
\hline CFR & Code of Federal Regulations \\
\hline $\mathrm{CH}$ & contact handled \\
\hline CWC & Central Waste Complex \\
\hline DOE & U.S. Department of Energy \\
\hline DOE-HQ & U.S. Department of Energy-Headquarters \\
\hline DSSF & double-shell slurry feed \\
\hline DSSI & Diversified Scientific Services, Inc. \\
\hline DST & double-shell tank \\
\hline Ecology & Washington State Department of Ecology \\
\hline EM & U.S. Department of Energy, Office of Environmental Management \\
\hline EPA & U.S. Environmental Protection Agency \\
\hline FFCAct & Federal Facilities Compliance Act of 1992 \\
\hline FR & Federal Register \\
\hline FY & fiscal year \\
\hline HEPA & high-efficiency particulate air (filter) \\
\hline HLW & high-level waste \\
\hline LDR & land disposal restriction \\
\hline LLBG & low-level burial grounds \\
\hline LLCE & long-length contaminated equipment \\
\hline LLMW & low-level mixed waste \\
\hline MLLW & mixed low-level waste \\
\hline MWDF & mixed waste disposal facility \\
\hline NA & not applicable \\
\hline National Report & National Report on Prohibited Wastes and Treatment Options \\
\hline NCAW & neutralized current acid waste \\
\hline NDA & nondestructive analysis \\
\hline NDE & nondestructive examination \\
\hline PCB & polychlorinated biphenyl \\
\hline PFP & Plutonium Finishing Plant \\
\hline PHMC & Project Hanford Management Contract \\
\hline PUREX & Plutonium-Uranium Extraction (Plant) \\
\hline RCRA & Resource Conservation and Recovery Act of 1976 \\
\hline REC & Radiochemical Engineering Cells \\
\hline RH & remote handled \\
\hline $\mathrm{RL}$ & U.S. Department of Energy, Richland Operations Office \\
\hline SDAR & Storage Disposal Approval Record \\
\hline
\end{tabular}

as low as reasonably achievable

Allied Technology Group

Comprehensive Environmental Response, Compensation

and Liability Act of 1980

contact handled

Central Waste Complex

U.S. Department of Energy

double-shell slurry feed

double-shell tank

Washington State Department of Ecology

U.S. Environmental Protection Agency

Federal Facilities Compliance Act of 1992

fiscal year

high-efficiency particulate air (filter)

and disposal restriction

low-level burial grounds

long-length contaminated equipmen

low-level mixed waste

mixed low-level waste

not applicable

National Report on Prohibited Wastes and Treatment Options

nondestructive analysis

nondestructive examination

Plutonium Finishing Plant

Project Hanford Management Contract

Resource Conservation and Recovery Act of 1976

remote handled

U.S. Department of Energy, Richland Operations Office

age Disposal Approval Record 


\section{ACRONYMS AND ABBREVIATIONS (cont)}

SST

STP

SWITS

TBD

Tri-Party Agreement

TRU

TRUM

TRUSAF

TSCA

TSD

TWRS

UHC

WAC

WERF

WESF

WIPP

WMH

WRAP

WSRd single-shell tank

Site treatment plan

Solid Waste Information and Tracking System (database)

to be determined

Hanford Federal Facility Agreement and Consent Order transuranic

transuranic mixed

Transuranic Waste Storage and Assay Facility

Toxic Substances Control Act of 1976

treatment; storage, and disposal

Tank Waste Remediation System

underlying hazardous constituents

Washington Administrative Code

Waste Experimental Reduction Facility

Waste Encapsulation and Storage Facility

Waste Isolation Pilot Plant

Waste Management Federal Services of Hanford, Inc.

Waste Receiving and Processing (Facility) (2336-W)

Waste Specification Records 
DOE/RL-99-01

This page intentionally left blank. 


\subsection{INTRODUCTION}

The baseline land disposal restrictions (LDR) plan was prepared in 1990 in accordance with Hanford Federal Facility Agreement and Consent Order (Tri-Party Agreement) (Ecology et al. 1989) Milestone M-26-00. The text of this milestone is as follows.

"LDR requirements include limitations on storage of specified hazardous wastes (including mixed wastes). In accordance with approved plans and schedules, the U.S. Department of Energy (DOE) shall develop and implement technologies necessary to achieve full compliance with LDR requirements for mixed wastes at the Hanford Facility. LDR plans and schedules shall be developed with consideration of other action plan milestones and will not become effective until approved by the U.S. Environmental Protection Agency (EPA) (or Washington State Department of Ecology [Ecology]) upon authorization to administer LDRs pursuant to Section 3006 of the Resource Conservation and Recovery Act of 1976 (RCRA). Disposal of LDR wastes at any time is prohibited except in accordance with applicable LDR requirements for nonradioactive wastes at all times. The plan will include, but not be limited to, the following:

Waste characterization plan

Storage report

Treatment report

Treatment plan

Waste minimization plan

A schedule depicting the events necessary to achieve full compliance with LDR requirements

A process for establishing interim milestones."

The original plan was published in October 1990. This is the ninth of a series of annual updates required by Tri-Party Agreement Milestone M-26-01. A Tri-Party Agreement change request approved in March 1992 changed the annual due date from October to April and consolidated this report with a treatment alternatives report prepared under Milestone M-25-00. The reporting period for this report is from April 1, 1998, to March 31, 1999.

The 1990 baseline plan was a follow-on document to the National Report on Prohibited Wastes and Treatment Options (National Report) (DOE 1990), which identified all solvents (40 Code of Federal Regulations [CFR] 268.30) and California List (40 CFR 268.32) waste 
restricted from land disposal, and a subsequent effort by DOE (WHC 1990a) to identify any additional waste restricted from land disposal as a result of First-, Second-, and Third-Third LDRs promulgation (55 Federal Register [FR] 22520).

This report is used as the Hanford Facility's equivalent to the final site treatment plan (STP) submitted to the U.S. Department of Energy-Headquarters (DOE-HQ) as required by the Federal Facility Compliance Act of 1992 (FFCAct). Washington State and the EPA concurred that the U.S. Department of Energy, Richland Operations Office (RL) was not obligated to complete a site treatment plan. However, RL and the Hanford Facility contractors have been participating in the FFCAct process by providing data and cost information to support a complex-wide effort to set priorities for treatment projects. Because the Tri-Party Agreement addresses compliance with RCRA, Section 3021(b)(5), the requirements of RCRA, Section 021(b), do not apply to mixed waste stored on the Hanford Facility.

In response to Ecology comments on the 1997 LDR report, the report's format was changed in 1998 . Where past reports were broken down primarily by storage locations, the new format separates the information by waste stream. Chapter 3 includes a waste stream profile sheet for each stream. The profile sheets present most of the generation; characterization; minimization; and treatment, storage, and disposal (TSD) information for the streams.

\subsection{BACKGROUND AND PURPOSE}

On September 19, 1989, DOE entered into a federal facilities compliance agreement with the EPA and the Colorado Department of Health regarding the storage of certain radioactive mixed waste at the Rocky Flats Plant. The agreement required the DOE to prepare and submit the National Report (DOE 1990) to the EPA. This report was submitted to the EPA in January 1990. It included information on all DOE sites that store radioactive mixed waste subject to the LDRs in effect when the report was prepared.

The EPA has promulgated various new LDR rules since the Rocky Flats compliance agreement. (Major LDR rulemakings are listed in Chapter 5, "References.") The LDRs apply to the hazardous component of mixed waste. The most recent rulemakings include the LDR Phase IV (63 FR 28556) and Hazardous Waste Identification Rule for Environmental Media (63 FR 65874).

Washington State regulations [Washington Administrative Code (WAC) 173-303-070(3) and 173-303-070(5)] effective November 20,1995, significantly affected the designation of waste generated and waste in storage. First, federal waste codes (e.g., D001) are assigned to a waste stream, then state codes (e.g., WT01) are assigned. Based on these amendments, the state code is not assigned in many cases. (The new regulations have not been applied to the complete inventory of containerized waste in storage that was generated before the effective date. The new regulations will be applied to stored waste as funding is available to evaluate the characterization 
of containerized waste. In most cases, the waste characterization will be evaluated when the mixed waste is moved to a new storage location or removed from storage for treatment.)

This report describes the generation and management of LDR mixed waste generated, treated, and stored at the Hanford Facility. Discussions focus on the hazardous aspects of mixed waste, although treatment, storage, and disposal are frequently complicated by the radioactive components. This report discusses the LDR mixed waste managed at the Hanford Facility by waste stream. The waste is separated into streams based primarily on physical characteristics and future treatment before disposal. This grouping results in 47 streams of LDR waste being defined. Table 1-1 shows the 47 stream names used for this report. Most of the information on each waste stream is presented in a waste stream profile sheet. These sheets make up Chapter 3; the narrative that supports and augments the sheets is in Chapter 4.

To allow this report to be relatively concise, some LDR mixed waste streams have been combined where treatment and disposal pathways are the same. The Central Waste Complex (CWC) and double-shell tank (DST) waste streams, in particular, include in their waste generation projections waste generated at other locations, such as the Plutonium Finishing Plant and laboratories. For example, if a generated waste stream is routinely sent to the $\mathrm{CWC}$, the waste inventory and future generation will be reported only as future projected receipts (waste generation) for specific CWC waste streams. The specific locations that generate the waste typically will be mentioned in the description of the stream in this report, but a separate generation projection for that location will not be included. However a stored LDR mixed-waste inventory that is not routinely transferred to the CWC, and, therefore, is not included in another waste generation projection, is listed as a separate waste stream in this report. An example of this situation is the waste in the Plutonium-Uranium Extraction (PUREX) tunnels. In this way, this report covers the future treatment and disposal of all LDR mixed waste using the fewest separate waste streams.

Decommissioning and remediation activities for RCRA past-practice units are anticipated to generate large volumes of contaminated soil and debris (e.g., contaminated structures, drums, tanks, piping, equipment, and cleanup debris). Some of this soil and debris may be subject to regulation under the LDR Program. Volumes from these and other onsite waste management activities governed by the Comprehensive Environmental Response, Compensation, and Liability Act of 1980 (CERCLA) are typically documented in records of decision, action memoranda and/or decontamination and decommissioning work plans. Waste generated from CERCLA activities that meets the Environmental Restoration Disposal Facility waste acceptance criteria will be disposed of in the Environmental Restoration Disposal Facility. This waste is not included in this report.

Some CERCLA waste is included in this report. This waste consists of waste that does not meet the Environmental Restoration Disposal Facility's waste acceptance criteria and is sent to the CWC for storage. This waste is reported as part of the waste receipt projections for the CWC. 
A significant number of waste streams that were included in previous LDR reports no longer are included as separate waste streams in this report. Table 1-2 lists the names of these streams, identifies the sources of the waste, and summarizes the reasons the reporting mechanism was changed. Streams that are broken down differently from before, but that are still within the same overall category (e.g., transuranic mixed [TRUM] waste or mixed LLW [MLLW]) are not included in the table. Waste streams are deleted from the report if the waste is being stored on Site solely to facilitate its proper recovery, treatment, and disposal in accordance with 40 CFR 268.50, "Prohibitions on Storage of Restricted Wastes."

\subsection{ASSUMPTIONS}

This section lists key milestones and assumptions used to prepare this report.

RL has negotiated changes to the Tri-Party Agreement to allow the pretreatment and immobilization of MLLW from single-shell tanks (SST) and DSTs to be turned over to private contractors. The private companies will treat the tank waste and return the treated waste products to DOE. To integrate the privatization process, the LLW pretreatment process will be included with the LLW immobilization process.

Table 1-1. Stream Names. (4 sheets)

\begin{tabular}{|l|l|l|}
\hline \multicolumn{1}{|c|}{ Stream ID } & \multicolumn{1}{|c|}{ Stream Name } & \multicolumn{1}{|c|}{ Waste Source } \\
\hline MLLW-01 & $\begin{array}{l}\text { LDR Compliant Solids (overall name } \\
\text { for next four streams) }\end{array}$ & $\begin{array}{l}\text { Inorganic salt waste, excavated soil, and } \\
\text { contaminated equipment expected to be suitable for } \\
\text { direct disposal. }\end{array}$ \\
\hline MLLW-01A & Backlog Soils (Past-Practice) & $\begin{array}{l}\text { Soils excavated from the various tank farms, } \\
\text { incidentally contaminated with tank waste. }\end{array}$ \\
\hline MLLW-01B & $\begin{array}{l}\text { General LDR-Compliant Waste at } \\
\text { CWC (Past-Practice) }\end{array}$ & As previous, past practice. \\
\hline MLLW-01C & $\begin{array}{l}\text { General LDR-Compliant Waste at } \\
\text { CWC (Waste Specification System) }\end{array}$ & As previous, waste specification system. \\
\hline MLLW-01D & $\begin{array}{l}\text { Trench 34 Stored Inventory (Waste } \\
\text { Specification System) }\end{array}$ & Trench 34 stored waste. \\
\hline MLLW-02 & $\begin{array}{l}\text { Inorganic Solids (overall name for } \\
\text { next two streams) }\end{array}$ & $\begin{array}{l}\text { Inorganic particulates, absorbed liquids and sludge, } \\
\text { paint waste, salt waste, and aqueous labpacks from } \\
\text { numerous onsite generators. }\end{array}$ \\
\hline MLLW-02A & $\begin{array}{l}\text { General Inorganic Solids and } \\
\text { Labpacks (Past-Practice) }\end{array}$ & As previous, past practice. \\
\hline MLLW-02B & $\begin{array}{l}\text { General Inorganic Solids and } \\
\text { Labpacks (Waste Specification } \\
\text { System) }\end{array}$ & As previous, waste specification system. \\
\hline MLLW-03 & $\begin{array}{l}\text { Organic Solids (overall name for next } \\
\text { two streams) }\end{array}$ & $\begin{array}{l}\text { Organic waste similar to RL-MLLW-02. } \\
\text { (Past-Practice) }\end{array}$ \\
\hline MLLW-03A & $\begin{array}{l}\text { General Organic Solids and Labpacks } \\
\text { (Waste Specification System) }\end{array}$ & As previous, waste specification system. \\
\hline
\end{tabular}


Table 1-1. Stream Names. (4 sheets)

\begin{tabular}{|c|c|c|}
\hline Stream ID & Stream Name & Waste Source \\
\hline MLLW-04 & $\begin{array}{l}\text { Debris (overall name for next two } \\
\text { streams) }\end{array}$ & $\begin{array}{l}\text { Inorganic and organic debris including plastic and } \\
\text { rubber, metal, and heterogeneous debris. }\end{array}$ \\
\hline MLLW-04A & Past-Practice Hazardous Debris & As previous, past practice. \\
\hline MLLW-04B & $\begin{array}{l}\text { Waste Specification System } \\
\text { Hazardous Debris }\end{array}$ & As previous, waste specification system. \\
\hline MLLW-05 & $\begin{array}{l}\text { Elemental Lead (overall name for } \\
\text { next } 2 \text { streams) }\end{array}$ & Elemental lead and lead shielding. \\
\hline MLLW-05A & Elemental Lead (Past-Practice) & As previous, past practice. \\
\hline MLLW-05B & $\begin{array}{l}\text { Elemental Lead (Waste Specification } \\
\text { System) }\end{array}$ & As previous, waste specification system. \\
\hline MLLW-06 & $\begin{array}{l}\text { Elemental Mercury (overall name for } \\
\text { next two streams) }\end{array}$ & Elemental mercury from various sources. \\
\hline MLLW-06A & Elemental Mercury (Past-Practice) & As previous, past practice. \\
\hline MLLW-06B & $\begin{array}{l}\text { Elemental Mercury (Waste } \\
\text { Specification System) }\end{array}$ & As previous, waste specification system. \\
\hline MLLW-07 & $\begin{array}{l}\text { M-91 Remote-Handled Mixed Low- } \\
\text { Level Waste (overall name for next } \\
\text { two streams) }\end{array}$ & Remote-handled waste generated on and off Site. \\
\hline MLLW-07A & $\begin{array}{l}\text { M-9.1 Remote-Handled Mixed Low- } \\
\text { Level Waste (Past-Practice) }\end{array}$ & As previous, past practice. \\
\hline MLLW-07B & $\begin{array}{l}\text { M-91 Remote-Handled Mixed Low- } \\
\text { Level Waste (Waste Specification } \\
\text { System) }\end{array}$ & As previous, waste specification system. \\
\hline MLLW-08 & $\begin{array}{l}\text { Greater Than Class } 3 \text { Waste (Past- } \\
\text { Practice) }\end{array}$ & $\begin{array}{l}\text { Greater Than Class } 3 \text { waste. Consists of } \\
\text { radioisotope thermoelectric generators received in } \\
1980 \text { and } 1983 \text {. }\end{array}$ \\
\hline TRUM-01 & $\begin{array}{l}\text { Contact-Handled TRU Mixed Waste } \\
\text { (overall name for next } 10 \text { streams) }\end{array}$ & \\
\hline TRUM-01A & Contact-Handled Combustible Debris & $\begin{array}{l}\text { PFP, PUREX Plant, Critical Mass Laboratory, } \\
\text { Materials Engineering Laboratory, Kerr-McGee, } \\
\text { and REDOX facility }\end{array}$ \\
\hline TRUM-01B & Contact-Handled Composite Filters & PFP \\
\hline TRUM-01C & $\begin{array}{l}\text { Contact-Handled Heterogeneous } \\
\text { Debris }\end{array}$ & $\begin{array}{l}\text { PFP, PUREX Plant, Kerr-McGee, Radiochemistry } \\
\text { Building, and the Strontium Semi-works D\&D }\end{array}$ \\
\hline TRUM-01D & Contact-Handled Metal Debris & $\begin{array}{l}\text { PUREX Plant, PFP, Kerr-McGee, and } \\
\text { Radiochemistry Building }\end{array}$ \\
\hline TRUM-01E & $\begin{array}{l}\text { Contact-Handled Non-metal } \\
\text { Inorganic Particulates }\end{array}$ & $\begin{array}{l}\text { Radiological Calibrations Laboratory, Kerr-McGee, } \\
\text { and the PFP }\end{array}$ \\
\hline TRUM-0IF & Contact-Handled Lead Waste & $\begin{array}{l}\text { PFP, Radiochemistry Building, research } \\
\text { laboratories, and Fuels Development Laboratory }\end{array}$ \\
\hline TRUM-01G & Contact-Handled Solidified Organics & PFP \\
\hline TRUM-0IH & $\begin{array}{l}\text { Contact-Handled Solidified } \\
\text { Inorganics }\end{array}$ & PFP, Chemical Development Laboratory \\
\hline TRUM-01J & Contact-Handled Soils & Semi-works D\&D Project, and PFP \\
\hline TRUM-01K & $\begin{array}{l}\text { Contact-Handled Unknown or } \\
\text { Mixture }\end{array}$ & $\begin{array}{l}\text { Radiochemistry Building, PFP, and the PUREX } \\
\text { Plant }\end{array}$ \\
\hline TRUM-02 & $\begin{array}{l}\text { Remote-Handled Transuranic Mixed } \\
\text { Waste (overall name for next two } \\
\text { streams) }\end{array}$ & $\begin{array}{l}\text { Specific sources listed under TRUM-02A and } \\
\text { TRUM-02B }\end{array}$ \\
\hline
\end{tabular}


Table 1-1. Stream Names. (4 sheets)

\begin{tabular}{|c|c|c|}
\hline Stream ID & Stream Name & Waste Source \\
\hline TRUM-02A & Shielded Heterogeneous Debris & $\begin{array}{l}\text { Radiochemistry Building, and Post-Irradiation Test } \\
\text { Laboratory }\end{array}$ \\
\hline TRUM-02B & Shielded Lead Waste & $\begin{array}{l}\text { Argonne National Laboratories-East, Chemical } \\
\text { Engineering Building, Battelle Columbus } \\
\text { Laboratories, and Post-Irradiation Test Laboratory }\end{array}$ \\
\hline TRUM-03 & $\begin{array}{l}\text { PFP Hydraulic Fluids Contaminated } \\
\text { With PCBs }\end{array}$ & PFP \\
\hline TRUM-04 & $\begin{array}{l}300 \text { Area Revitalization Contact- } \\
\text { Handled Mixed TRU (overall name } \\
\text { for next three streams) }\end{array}$ & $\begin{array}{l}300 \text { Area facilities in which the process missions } \\
\text { have terminated }\end{array}$ \\
\hline TRUM-04A & $\begin{array}{l}300 \text { Area Revitalization Contact- } \\
\text { Handled Mixed TRU Heterogeneous } \\
\text { Debris }\end{array}$ & $\begin{array}{l}300 \text { Area facilities in which the process missions } \\
\text { have terminated }\end{array}$ \\
\hline TRUM-04B & $\begin{array}{l}300 \text { Area Revitalization Contact- } \\
\text { Handled Mixed TRU Inorganic } \\
\text { Nonmetal Debris }\end{array}$ & $\begin{array}{l}300 \text { Area facilities in which the process } \\
\text { missions have terminated }\end{array}$ \\
\hline TRUM-04C & $\begin{array}{l}300 \text { Area Revitalization Contact- } \\
\text { Handled Mixed TRU Metal Debriss }\end{array}$ & $\begin{array}{l}300 \text { Area facilities in which the process } \\
\text { missions have terminated }\end{array}$ \\
\hline TRUM-05 & $\begin{array}{l}\text { TRU Single-Shell Tank Long-Length } \\
\text { Equipment (overall.name for next } \\
\text { two streams) }\end{array}$ & 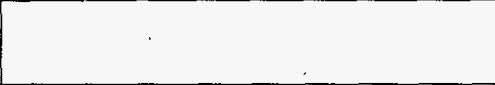 \\
\hline TRUM-05A & $\begin{array}{l}\text { Contact-Handled TRU Single-Shell } \\
\text { Tank Long-Length Equipment }\end{array}$ & $\begin{array}{l}\text { Future maintenance of underground waste storage } \\
\text { tanks at Ḣanford }\end{array}$ \\
\hline TRUM-05B & $\begin{array}{l}\text { Remote-Handled TRU Single-Shell } \\
\text { Tank Long-Length Equipment }\end{array}$ & $\begin{array}{l}\text { Future maintenance of underground waste storage } \\
\text { tanks at Hanford }\end{array}$ \\
\hline TRUM -06 & $\begin{array}{l}\text { Miscellaneous Remote-Handled } \\
\text { Mixed TRU Waste }\end{array}$ & National Laboratories and T-Plant Operations \\
\hline DST-1 & Double-shell tank waste & $\begin{array}{l}\text { Widely varying waste from chemical separations } \\
\text { processes (e.g., PUREX, PFP, and cesium and } \\
\text { strontium separations) and related support facilities } \\
\text { used from } 1970 \text { to date. }\end{array}$ \\
\hline SST-1 & Single-shell tank waste & $\begin{array}{l}\text { Waste from spent nuclear fuel processing and } \\
\text { related support facilities between } 1944 \text { and } 1980 \text {. }\end{array}$ \\
\hline PUREX-1 & PUREX Containment Building & $\begin{array}{l}\text { Chromium-contaminated debris from the E-Cell } \\
\text { floor stored in F-Cell. }\end{array}$ \\
\hline PUREX-2 & PUREX Storage Tunnels & $\begin{array}{l}\text { Discarded equipment and waste from PUREX and } \\
\text { other facilities containing mercury, lead, silver, } \\
\text { cadmium, chromium, barium, and mineral oil. }\end{array}$ \\
\hline 324REC & $\begin{array}{l}324 \text { Radiochemical Engineering Cell } \\
\text { waste }\end{array}$ & $\begin{array}{l}\text { Variety of high-activity radioactive waste, } \\
\text { containing toxic heavy metals, generated during } \\
\text { research and development activities since the mid- } \\
1960 \text { 's and the processing of } 324 \text { Building high- } \\
\text { level vault waste. }\end{array}$ \\
\hline B Plant-1 & B Plant Cell-4 Waste & $\begin{array}{l}\text { Containers of mixed and/or highly radioactive solid } \\
\text { waste generated in the Waste Encapsulation and } \\
\text { Storage Facility hot cells since } 1988 \text {. }\end{array}$ \\
\hline B Plant-2 & $\begin{array}{l}\text { B Plant Containment Building } \\
\text { Storage }\end{array}$ & $\begin{array}{l}\text { Discarded and failed process jumpers and } \\
\text { equipment from B Plant operational processes } \\
\text { stored in the B Plant canyon deck and process cells. }\end{array}$ \\
\hline
\end{tabular}


Table 1-1. Stream Names. (4 sheets)

\begin{tabular}{|c|c|c|}
\hline Stream ID & Stream Name & Waste Source \\
\hline T-Drag-off & T Plant Complex Drag-off Box & $\begin{array}{l}\text { Miscellaneous pieces of equipment accumulated } \\
\text { over a period of years stored in a box in the } \\
221 \text {-T canyon. }\end{array}$ \\
\hline T-Condenser & T Plant Complex EC-1 Condenser & $\begin{array}{l}\text { A condenser from the 242-A Evaporator now } \\
\text { stored next to the } 2706-\mathrm{T} \text { complex. }\end{array}$ \\
\hline T-Tank & T Plant Complex Tank Trailer Waste & $\begin{array}{l}\text { Liquid waste from analytical laboratories and } \\
\text { B Plant. Stored in a tank trailer within the T Plant } \\
\text { complex. }\end{array}$ \\
\hline WESF & Cesium and Strontium Capsules & $\mathrm{CsCl}_{\mathrm{Salt}} \mathrm{SrF}_{2}$ salt reclaimed from DSTs and SSTs \\
\hline$\overline{D S T}$ & tank $\quad$ SST & Single-shell tank \\
\hline PCB & ed biphenyl & Transuranic \\
\hline Plutoniu & aishing Plant & Waste Encapsulation and Storage Facility \\
\hline
\end{tabular}

PUREX Plutonium-Uranium Extraction Plant

Table 1-2. Streams No Longer Applicable to Report. (2 sheets)

\begin{tabular}{|l|l|l|}
\hline \multicolumn{1}{|c|}{ Stream name } & \multicolumn{1}{|c|}{ Waste source } & \multicolumn{1}{c|}{ Reason } \\
\hline PUREX Plant Aging Waste & $\begin{array}{l}\text { First extraction-column fission } \\
\text { products from PUREX plant. }\end{array}$ & $\begin{array}{l}\text { Waste no longer generated. } \\
\text { Inventory is in DSTs. }\end{array}$ \\
\hline $\begin{array}{l}\text { W433 Sodium Storage Facility } \\
\text { Waste }\end{array}$ & $\begin{array}{l}\text { Waste sodium from FFTF } \\
\text { operations. }\end{array}$ & $\begin{array}{l}\text { Significant amounts of alkali metal } \\
\text { waste are no longer generated. } \\
\text { Inventory is being stored at the } \\
\text { CWC and the TSD unit has } \\
\text { undergone RCRA closure } \\
\text { (April 14, 1997). }\end{array}$ \\
\hline $\begin{array}{l}\text { PUREX Ammonia Scrubber } \\
\text { Waste }\end{array}$ & $\begin{array}{l}\text { Waste generated from adsorption } \\
\text { of gaseous ammonia from fuel } \\
\text { processing operations at the } \\
\text { PUREX Plant. }\end{array}$ & $\begin{array}{l}\text { Waste no longer generated. } \\
\text { Inventory in DSTs. }\end{array}$ \\
\hline PUREX Process Condensate & $\begin{array}{l}\text { Condensed vapors from PUREX } \\
\text { Plant operations. }\end{array}$ & $\begin{array}{l}\text { Waste no longer generated. } \\
\text { Inventory in DSTs. }\end{array}$ \\
\hline Hexone Waste & $\begin{array}{l}\text { Hexone that had been planned } \\
\text { for use in the 202-S solvent } \\
\text { extraction process. }\end{array}$ & $\begin{array}{l}\text { Hexone has been incinerated off } \\
\text { site. }\end{array}$ \\
\hline $\begin{array}{l}\text { 183-H Solar Evaporation } \\
\text { Basins Waste }\end{array}$ & $\begin{array}{l}\text { Containerized solids retrieved } \\
\text { from 183-H Area Solar } \\
\text { Evaporations Basins, generated } \\
\text { from 300 Area fuel fabrication } \\
\text { waste from 1973 to 1985. }\end{array}$ & $\begin{array}{l}\text { Closure plan activities for the TSD } \\
\text { unit are complete. Unit is in } \\
\text { postclosure care. Process waste } \\
\text { inventory is now in storage at the } \\
\text { CWC. }\end{array}$ \\
\hline 303-K Stored Waste & $\begin{array}{l}\text { Temporary storage of 300 Area } \\
\text { fuel fabrication solid and liquid } \\
\text { waste. }\end{array}$ & $\begin{array}{l}\text { TSD unit is undergoing closure. } \\
\text { Waste treated and disposed of or } \\
\text { now in storage at the CWC. }\end{array}$ \\
\hline
\end{tabular}


Table 1-2. Streams No Longer Applicable to Report. (2 sheets)

\begin{tabular}{|c|c|c|c|c|}
\hline \multicolumn{2}{|c|}{ Stream name } & \multicolumn{2}{|c|}{ Waste source } & Reason \\
\hline \multicolumn{2}{|c|}{$\begin{array}{l}\text { 224-T Transuranic Storage } \\
\text { and Assay Facility Stored } \\
\text { Waste }\end{array}$} & \multicolumn{2}{|c|}{$\begin{array}{l}\text { TRU waste from on Site and off } \\
\text { Site packaged for eventual WIPP } \\
\text { disposal. }\end{array}$} & $\begin{array}{l}\text { Inventory moved to the CWC. } \\
\text { TSD will be closed. }\end{array}$ \\
\hline \multicolumn{2}{|c|}{ B Plant Organic Waste } & \multicolumn{2}{|c|}{$\begin{array}{l}\text { Organic solvent waste from the } \\
\text { strontium solvent extraction } \\
\text { process that operated in B Plant } \\
\text { from } 1968 \text { to } 1979 .\end{array}$} & $\begin{array}{l}\text { Waste has been shipped to a } \\
\text { permitted disposal site for } \\
\text { destruction. }\end{array}$ \\
\hline \multicolumn{2}{|c|}{$\begin{array}{l}\text { 242-A Evaporator Process } \\
\text { Condensate }\end{array}$} & \multicolumn{2}{|c|}{$\begin{array}{l}\text { 242-A Evaporator (evaporation } \\
\text { of water from DST and SST } \\
\text { system waste). }\end{array}$} & $\begin{array}{l}\text { Waste stream is being treated and } \\
\text { disposed of as generated. }\end{array}$ \\
\hline CWC & \multirow{2}{*}{\multicolumn{3}{|c|}{$\begin{array}{l}\text { Central Waste Com } \\
\text { Double-shell tank }\end{array}$}} & \multirow{2}{*}{ of 1976} \\
\hline DST & & & & \\
\hline FFTF & \multicolumn{2}{|c|}{ Fast Flux Text Facility } & SST & Single-shell tank \\
\hline PCB & \multicolumn{2}{|c|}{ Polychlorinated biphenyl } & TRU & Transuranic \\
\hline PFP & \multicolumn{2}{|c|}{ Plutonium Finishing Plant } & TSD & \multirow{2}{*}{$\begin{array}{l}\text { Treatment, storage, and/or d } \\
\text { Waste Isolation Pilot Plant }\end{array}$} \\
\hline PUREX & \multicolumn{2}{|c|}{ Plutonium-Uranium Extraction } & WIPP & \\
\hline
\end{tabular}

The following other Tri-Party Agreement milestones related to the management of LDR waste, including approved change requests, are some of the most significant.

- Complete pretreatment and immobilization of Hanford Facility low-activity waste by December 2024 (M-60-00).

- Start hot operations of two low-activity waste pretreatment and immobilization facilities by December 2002 (M-60-12).

- Complete SST interim stabilization by September 2000 (M-41-00). Complete closure of all SST farms by September 2024 (M-45-00). This milestone includes a requirement to initiate tank waste retrieval from one SST by December 2003 (M-45-05T1).

- Develop and submit the Waste Identification and Requirements Document to Ecology, issue tank characterizations consistent with the requirements and enter the information into the database (M-44-00).

- Complete construction and initiate operations of expanded laboratory hot cells for highlevel mixed waste by June 1994 (M-11-00). This milestone is complete.

- Initiate treatment of contact-handled mixed low-level waste (MLLW) by September 1999 (M-19-01), and complete treatment and/or direct disposal of at least 1,644 cubic meters of contact-handled low-level mixed waste by September 2002 (M-19-00). The M-19 change request that establishes these alternatives to the construction and operation of the Waste Retrieval and Processing (WRAP) 2A facility received final approval in October 1996. 
- Complete closure of the non-permitted mixed waste units in the 324 Building radiochemical engineering cells (REC) B-Cell, REC D-Cell, and the high-level vault (HIV) (M-89-00) by October 31, 2005.

- Complete removal of 324 Building REC B-Cell mixed waste and equipment by November 30, 2000 (M-89-02).

- Complete B Plant facility transition phase and initiate the surveillance and maintenance phase by September 1999 (M-82-00). This milestone includes removing organic waste from the B Plant canyon by June 1997 (M-82-03), documenting any hazardous substances or dangerous waste remaining in B Plant by June 1998 (M-82-07), and completing disposition of the organic waste by September 1998 (M-82-08). All but the major milestone (M-82-00) have been accomplished.

- Complete acquisition of new facilities and modification of existing and planned facilities needed for storage, treatment, if necessary, and disposal of all Hanford Facility transuranic (TRU) and TRUM waste, MLLW, and greater than category 3 (GTC3) waste (M-91-00 series of milestones; see details in Chapter 2).

The following are key assumptions that have been used to develop the treatment plans and schedules for DST waste and assumptions related to the use of tank space.

- The pretreatment methods to be developed will include acceptable technology to separate the waste into LLW and high-level waste (HLW)' streams so that the bulk of chemical waste is in the low-activity stream and the bulk of radionuclides are in the high-activity stream.

- Pretreated waste from all DSTs and SSTs will be provided to the LLW and HLW vitrification facilities, using selective blending if necessary.

- The level of cyanides and organics in DST and SST waste received from pretreatment will be treatable by vitrification, and the glass waste forms will comply fully with leachability requirements or appropriate variances will be obtained.

- Space in DSTs will be available to support DST and SST waste management activities.

- Liquid waste from SSTs will continue to be transferred to DSTs as part of the stabilization program for the SSTs.

\footnotetext{
${ }^{1}$ The terms LLW, HLW, and TRU are used in this report to be consistent with Section 8 of the third-third LDR regulations ( 55 FR 22520). These terms are not intended to match the definitions in the contracts for privatization activities.
} 
- Process condensate from the 242-A Evaporator and hazardous wastewater from other sources, including liquid effluents from tank waste pretreatment and vitrification will continue to be treated in the 200 Area Effluent Treatment Facility (ETF).

For waste generated by the Hanford Facility Environmental Restoration Program, only the quantities of waste that are sent to the CWC are considered part of the scope of this report. Waste disposed of to the Environmental Restoration Disposal Facility is not included in this report.

The PUREX tunnels, canyon facilities, and other inoperative Site facilities will not be dispositioned, operate, or otherwise generate LDR waste during the next 5 years. The waste generation projections in this report are based on this assumption.

\subsection{SCHEDULE AND MECHANICS OF PLAN UPDATE}

Information in the baseline plan will be updated in future annual reports in accordance with Tri-Party Agreement Milestone M-26-01. In addition to or as part of content required by the milestone, the annual report revisions include the following:

- Revision of the stream generation rates to reflect current operating plans and schedules

- Revision of treatment plans and schedules to reflect further-defined waste treatments and treatment schedules

- Revision of the stream characterizations to reflect additional sample analyses or process changes

- Revision of the compliance status of the units to reflect future compliance assessments and permitting activities

- Reevaluation of the adequacy of the capacity of current units for storing LDR waste

- Addition of new or proposed milestones, as applicable

- Changes in the configuration of the mixed waste complex required under the FFCAct

- Addition of new LDR mixed waste streams as they are identified or declared to be waste. These may include streams such as spent nuclear fuel basin sludge, bulk sodium waste, and 300 Area special-case waste. Tri-Party Agreement milestones for some of this waste have been established in the M-92 milestone series. 


\subsection{MILESTONE PLANNING PROCESS}

Milestones and work schedules for activities related to the management of LDR mixed waste will be consistent with the work schedules contained in Appendix D of the Tri-Party Agreement (Ecology et al. 1989) and the annual update to the work schedule. The scope of these schedules includes interim milestones and additional target dates to accomplish the major milestones contained in Section 2.0 of the Tri-Party Agreement. Summary milestone schedules for activities related to the management of LDR mixed waste are discussed in Section 3.0 of the Tri-Party Agreement. Any new LDR milestones and changes to approved LDR milestone schedules will be implemented via the Change Control System process defined in Section 12.0 of the Tri-Party Agreement.

Tri-Party Agreement Milestone M-26-01 also requires that appropriate new milestones be proposed through this annual report. No new milestones are proposed for this reporting period.

The LDR milestone planning process used by DOE and its contractors also must consider the DOE and federal budget processes; integration with concurrent Hanford Facility operations, including waste management and environmental restoration activities; and overall Sitewide regulatory compliance and coordination with other milestone initiatives described in the Tri-Party Agreement. Because these planning elements are numerous and complex, issues will be coordinated and reconciled through the ongoing project managers and the Inter-Agency Management Integration Team meetings within the broader framework provided by Section 8.0 of the Tri-Party Agreement. Also, LDR waste management activities will be included, as appropriate, in Tri-Party Agreement milestone review meetings.

\subsection{ACTIVITIES AND ACHIEVEMENTS}

This section summarizes the major activities and accomplishments related to compliance with LDRs from April 1, 1998, through March 31, 1999.

- The 324 Facility special-case waste assessment in support of 324 closure was issued (milestone M-89-05).

- The closure plan for the 324 Building REC HLV, low-level vault (LLV), and associated areas has been developed and issued.

- The DST Dangerous Waste Part B Permit application, DOE/RL-90-39 (1991a) is being revised. In December 1997, Chapter 3, "Waste Analysis," and Appendix 3A, "DoubleShell Tank System Waste Analysis Plan,"were transmitted to Ecology for review. Workshops will be scheduled to address remaining issues.

- All outstanding safety issues pertaining to the safety status of waste in the tanks have been or are being resolved. The organic safety issue has been resolved and 18 SSTs will 
be removed from the Organic Waste Tank Watch List. (Eight of the SSTs removed from the Organic Waste Watch List will remain on the Flammable Gas Watch List.)

- In September 1998, deactivation of the B Plant Canyon Facility, 221B, was completed. The canyon facility was closed except for quarterly surveillance activities. No LDR waste is generated at B Plant and LDR waste stored at B Plant is documented in the report.

- Custody of the PUREX Containment Building is expected to transfer from B\&W Hanford Company to Bechtel Hanford, Inc., in the first half of calendar year 1999. This is a step in the process toward decontamination and decommissioning of the facility, or other final action.

- CWC and WRAP Dangerous Waste Part B Permit applications were certified in May 1998 for incorporation into the Hanford Facility RCRA permit. Modification is ongoing.

- The Transuranic Waste Storage and Assay Facility (TRUSAF) has been administratively transferred to DOE, Office of Assistant Manager for Facilities and Laboratory Management. This changes the facility's classification from operations to decontamination and decommissioning.

- During September 1998, 96 drums (approximately 20 cubic meters) of MLLW containing organic/carbonaceous debris were shipped to the Waste Experimental Reduction Facility (WERF) for incineration. Incineration of this waste is scheduled to be performed during FY 1999 with treatment residues to be received back the same year. This is considered to be a demonstration activity because the economics and effectiveness of the process will be evaluated once treatment has been completed (incineration at the WERF followed by stabilization at $\mathrm{T}$ Plant). If the treatment approach proves economical, additional waste will be scheduled for treatment at the WERF beginning in FY 2001.

- Three containers (approximately 1 cubic meter) of ash from incineration of Bettis Atomic Power Laboratory waste at the WERF were stabilized at the T Plant Facility during FY 1998. The waste originally was incinerated at the WERF, but the treatment residue required secondary treatment for RCRA-regulated heavy metals (cadmium, chromium, and lead). The waste was successfully treated and returned to Bettis on August 28, 1998. This activity was associated with Bettis's obligations under authority of the FFCAct as implemented at their site.

- During September 1998, the environmental assessment associated with the Allied Technologies Group (ATG) nonthermal treatment contract was completed for the nonthermal treatment activity with a finding of no significant impact. Completion of this environmental assessment met Tri-Party Agreement compliance agreement milestone M-19-01-T03. 
- The WRAP facility successfully completed the contractor and RL operational readiness reviews for the LLW and TRU processing lines. These lines were then declared operational and began processing waste during September 1998.

- The Waste Isolation Pilot Plant (WIPP) received its first shipment of TRU waste from the Los Alamos National Laboratory in March 1999, paving the way for future shipments from DOE sites across the complex, including the Hanford Site.

- Low-level burial grounds final status permitting action was deferred because State Environmental Policy Act documentation was not complete.

- The State-only LDR on organic/carbonaceous waste prohibition does not apply to Hanford Facility waste, effective December 1, 1998. The prohibition will not apply until Ecology determines that a recycling, treatment, or incineration facility is available within 1600 kilometers (1,000 miles) of Washington State borders and DOE is unable to demonstrate otherwise. Practically, the state-only LDR prohibition will become effective again when treatment capacity becomes available at ATG in Richland, Washington.

- Fourteen capsules of cesium chloride were overpacked at the 324 Building and transferred into WESF for storage. Five of these capsules include pencil-type sources created at the Oak Ridge Site. The remaining nine capsules, which were placed in Type-W overpacks, were from WESF.

- Waste minimization accomplishments in reducing LDR waste include the following activities.

- Numerous pieces of process tanks, machinery, associated equipment, and piping at a 300 Area facility were identified for disposal. The equipment was chemically decontaminated to low-level waste, thus saving mixed waste storage or disposal costs for 169 cubic meters of waste.

- Approximately 18,900 liters (5,000 gallons) of runoff (rainwater and snowmelt) annually flood the $2706 \mathrm{~T}$ Building by accumulating along several exterior doors, eventually passing beneath the doors at grade level, and entering the building. Once it enters the building and contacts the floor areas and collection pits, the runin (otherwise clean water) must be treated as F-listed waste by association. The run-in collected in the $2706 \mathrm{~T}$ railroad sump is eventually pumped to the canyon tank system and into the double-shelled railcars for disposal. The modification entails raising the exterior doors several inches and placing concrete landings on the inside and outside of the doors. This effectively raises the grade level and prevents the run-in from entering the building. This was a return-on-investmentfunded project that eliminated more than 40 cubic meters of mixed waste during 1998. 
- An application was submitted in 1998 to remove the restrictions on the type of multisource leachate (F039 Waste) that can be treated at the 200 Area Effluent Treatment Facility. This would expand the variety of mixed waste that could be treated. 


\subsection{FACILITY SUMMARY}

This section summarizes the generation, characterization, storage, treatment, and reduction of LDR waste at the Hanford Facility that is subject to this report.

\subsection{WASTE GENERATION}

The projected volumes of mixed waste to be generated are presented in Table 2-1 and the Waste Stream Profile Sheets in Chapter 3. Assumptions governing these generation rates are discussed in Section 1.2.

The waste generation projections represent the current best estimates of future waste generation for each LDR mixed waste stream or the quantity of mixed waste that will be added to TSD units. These estimates are based on a detailed evaluation of plant operating schedules, past operating history, and projections of future waste generation. The projected generation volumes may be higher or lower than the actual generation rates because of changes in waste treatment or production schedules or waste minimization activities.

Table 2-1. Waste Inventory and Generation Summary.

\begin{tabular}{|c|c|c|c|c|c|c|c|}
\hline \multirow[t]{2}{*}{ Waste stream ID } & \multirow[t]{2}{*}{ Waste stream name } & \multirow{2}{*}{$\begin{array}{l}\text { Current } \\
\text { inventory } \\
\left(\mathrm{m}^{3}\right)\end{array}$} & \multicolumn{5}{|c|}{ Generation projection (fiscal year) $\left(\mathrm{m}^{3}\right)$} \\
\hline & & & 1999 & 2000 & 2001 & 2002 & 2003 \\
\hline MLLW-01 & $\begin{array}{l}\text { LDR Compliant } \\
\text { Solids }\end{array}$ & 1538 & 0 & 1132 & 706 & 704 & 142 \\
\hline MLLW-02 & Inorganic Solids & 3035 & 23 & 25 & 42 & 30 & 45 \\
\hline MLLW-03 & Organic Solids & 546 & 49 & 63 & 56 & 54 & 56 \\
\hline MLLW-04 & Debris & 3639 & 349 & 433 & 412 & 556 & 514 \\
\hline MLLW-05 & Elemental Lead & 342 & 17 & 22 & 10 & 14 & 10 \\
\hline MLLW-06 & Elemental Mercury & 9 & 0.4 & 0.4 & 0.4 & 0.4 & 0.4 \\
\hline MLLW-07 & M91 RH-MLLW & 61 & 47 & 160 & 177 & 176 & 136 \\
\hline MLLW-08 & GTC3 & 1 & 0 & 0 & 0 & 0 & 0 \\
\hline TRUM-01 & $\begin{array}{l}\text { Generalized } \\
\text { CH-TRUM }\end{array}$ & 255 & 7 & 34 & 51 & 35 & 80 \\
\hline TRUM-02 & $\begin{array}{l}\text { Generalized Shielded } \\
\text { TRUM }\end{array}$ & 8 & 0.4 & 0.4 & 0.4 & 11 & 11 \\
\hline TRUM-03 & $\begin{array}{l}\text { PFP Hydraulic Fluid } \\
\text { with PCBs }\end{array}$ & 73 & 0 & 0 & 0 & 0 & 0 \\
\hline TRUM-04 & $\begin{array}{l}00 \text { Area } \\
\text { Revitalization } \\
\text { CH-TRUM }\end{array}$ & 0 & 0 & 100 & 100 & 100 & 100 \\
\hline TRUM-05 & $\begin{array}{l}\text { TRU SST long-length } \\
\text { equipment }\end{array}$ & 0 & $\overline{0}$ & 0 & 328 & 328 & 328 \\
\hline
\end{tabular}


Table 2-1. Waste Inventory and Generation Summary.

\begin{tabular}{|c|c|c|c|c|c|c|c|}
\hline \multirow[t]{2}{*}{ Waste stream ID } & \multirow[t]{2}{*}{ Waste stream name } & \multirow{2}{*}{$\begin{array}{c}\text { Current } \\
\text { inventory } \\
\left(\mathrm{m}^{3}\right)\end{array}$} & \multicolumn{5}{|c|}{ Generation projection (fiscal year) $\left(\mathrm{m}^{3}\right)$} \\
\hline & & & 1999 & 2000 & 2001 & 2002 & 2003 \\
\hline TRUM-06 & $\begin{array}{l}\text { Miscellaneous } \\
\text { RH-TRUM }\end{array}$ & 0 & 1 & 1 & 1 & 1 & 1 \\
\hline$\overline{\mathrm{DST}}$ & $\begin{array}{l}\text { Double-Shell Tank } \\
\text { System }\end{array}$ & 70,976 & 6890 & 7420 & 10,200 & 9600 & 6110 \\
\hline SST & $\begin{array}{l}\text { Single-Shell Tank } \\
\text { System }\end{array}$ & 133,600 & .0 & $\overline{0}$ & 0 & 0 & 0 \\
\hline PUREX-1 & $\begin{array}{l}\text { PUREX Containment } \\
\text { Building }\end{array}$ & 1 & 0 & 0 & 0 & 0 & 0 \\
\hline PUREX-2 & $\begin{array}{l}\text { PUREX Storage } \\
\text { Tunnels }\end{array}$ & 2800 & 400 & 0 & 0 & 0 & 0 \\
\hline 324REC & $\begin{array}{l}324 \text { Building } \\
\text { Radiochemical } \\
\text { Engineering Cells }\end{array}$ & 2.66 & 0 & 0 & 0 & 0 & 0 \\
\hline B PLANT-1 & B Plant Cell 4 Waste & 1.4 & 0 & 0 & 0 & 0 & 0 \\
\hline B PLANT-2 & $\begin{array}{l}\text { B Plant Containment } \\
\text { Building Storage }\end{array}$ & (a) & 0 & 0 & 0 & 0 & 0 \\
\hline T-Dragoff & $\begin{array}{l}\text { T Plant Complex } \\
\text { Drag-Off Box }\end{array}$ & 10 & $\overline{0}$ & 0 & 0 & $\overline{0}$ & 0 \\
\hline T-Tank & $\begin{array}{l}\text { T Plant Complex } \\
\text { Tank Trailer Waste }\end{array}$ & 4.54 & 0 & 0 & 0 & 0 & $\overline{0}$ \\
\hline T-Condenser & $\begin{array}{l}\text { T Plant Complex } \\
\text { EC-1 Condenser }\end{array}$ & 32.11 & 0 & 0 & 0 & 0 & 0 \\
\hline WESF & $\begin{array}{l}\text { Cesium and } \\
\text { Strontium Capsules }\end{array}$ & 2 & 0 & 0 & 0 & 0 & 0 \\
\hline & Totals & 216,937 & 7784 & 9391 & 12,084 & 11.609 & 7533 \\
\hline
\end{tabular}

(a) No volume estimate available. Estimated at $500 \mathrm{~kg}$ generation for 1998 . Current inventory is $294,000 \mathrm{~kg}$.

$\mathrm{CH}$ Contact handled

DST Double-shell tank

GTC3 Greater than class 3

LDR Land disposal restrictions

MLLW Mixed low-level waste

PCB Polychlorinated biphenyl

RH Remote handied

TRUM Transuranic mixed (waste)

\subsection{WASTE CHARACTERIZATION}

Characterization of mixed waste stored at the Hanford Facility has been based on current process knowledge and, where available, waste sample analyses. Waste will be sampled and characterized as necessary until the waste is disposed of. Future characterization plans for the waste streams and their status are summarized in Table 2-2. Characterization of individual waste streams is further described in Chapters 3 and 4. 
The dangerous waste designations for the waste streams are summarized in the waste stream profile sheets in Chapter 3. In some cases, additional waste codes are included for waste that may be received in the future. Dangerous waste, as defined by Washington State regulations, encompasses more waste types than are covered under the federal hazardous waste program. In some cases, the waste designations will include federal and state-only waste codes because waste from many sources will be managed under the waste stream profile sheet. Future waste characterization and complete implementation of the WAC 173-303 amendments may show that more or fewer waste codes apply to a waste stream managed under a given profile sheet. Any changes resulting from future characterization will be included in updates to this report. Washington State-only information is included in this report for completeness only; state-only LDRs are not subject to the RCRA statutory requirements.

The F001 through F005 waste codes (spent halogenated and nonhalogenated solvents) have been assigned to the SST- and DST-system waste because of the mixture rule from the introduction of spent solvents. Also, these waste codes apply to all downstream waste derived from DST system waste, such as process condensate from the 242-A Evaporator. The past discharges of spent solvents to SST and DST systems, and the resultant tank-to-tank transfers have mixed essentially all the waste in the tanks. The tank waste does not contain large quantities of organic solvents, as is typically the case for waste designated F001 through F005. The SST and DST system waste is primarily inorganic with trace contamination by F001 through F005 solvents.

In November 1994, the F039 waste code was added to the Part A Form 3 Permit Applications for the DST System, Low-Level Burial Grounds, CWC, WRAP Module 1, and other TSD units. This was done to allow for management of F039 waste generated during onsite mixed waste disposal operations. The 200 Area ETF can delist F039 waste streams if they are derived from just F001 through F005 sources. Currently, no federally regulated F039 waste is being generated or stored at the Hanford Facility.

Waste codes and required treatments for underlying hazardous constituents (UHC) are within the scope of this report. The waste characterization section of the waste stream profile sheets in Chapter 3 contains lists of constituents where UHCs are to be identified. The Hanford Facility TSD unit contractors are identifying applicable UHCs and determining waste treatment impacts. As UHC information becomes available, it will be included in this report.

The schedule and means for reporting waste characterization data are outlined in the Tri-Party Agreement (Ecology et al: 1989) as amended by Section 9.6, "Data Access and Delivery Requirements." This section states that DOE will make available to Ecology and EPA all relevant electronic data and databases. Unvalidated data will available within 7 days after receipt from the laboratories. All validated data will be entered into the selected database in accordance with the data delivery schedule in Section 9.6.6. The time limits are 216 days for SST analyses, 176 days for hot cell analyses (136-day annual average), and 126 days for low-level and mixed waste (111-day annual average). (All time limits are computed from the 
date of sampling.) Waste characterization data for CWC waste are maintained in the TSD unit records and currently are available only in hard copy.

\subsection{WASTE STORAGE}

The storage unit capacity for solid mixed waste at the Hanford Facility is projected to be adequate for all generated mixed waste throughout the waste management life cycle (FY 2032), assuming direct disposal and treatment programs are funded to their current levels. This projection is based on the individual projections of waste streams shipped to the CWC and the availability of planned treatment facilities. The projection of waste generation rates is reevaluated annually.

Using current space projections, the currently available DST system will have space available through at least 2004 . The baseline plans are to consolidate and evaporate DST system waste, which will eliminate the need to construct additional tanks. This will allow for their continued use for storage of new waste. Tri-Party Agreement change request M-42-95-01, which was approved December 1, 1995, postponed the construction of new DSTs indefinitely.

Except for the SST system, the storage units for mixed waste described in this report have not been known to release any dangerous constituents to the environment. This has been determined by analyzing all available information such as monitoring data, inspections, and operational history. The SST system is estimated to have released between 2862 cubic meters and 4022 cubic meters of liquid waste to the ground (FDH 1999). This estimate excludes any cooling water added to tanks after they were known to be leaking. To minimize further releases from this storage unit, the pumpable liquid portion of the waste stored in the SST system is being transferred to the DST system.

Releases to the environment before waste was stored or packaged in substantially its current configuration are not included in this report. For example, some of the waste now stored at the CWC came from the 183-H Solar Basins. While the 183-H Solar Basins leaked waste to the ground, the leaks occurred before the waste was packaged into drums, so the leakage estimate is not included in this report. For the purposes of this report, emissions allowed by permits and spills that are promptly cleaned up are not considered releases to the environment and are not included in the waste stream profile sheets in Chapter 3. 
Table 2-2A. LDR Summary Table, MLIW. (2 sheets)

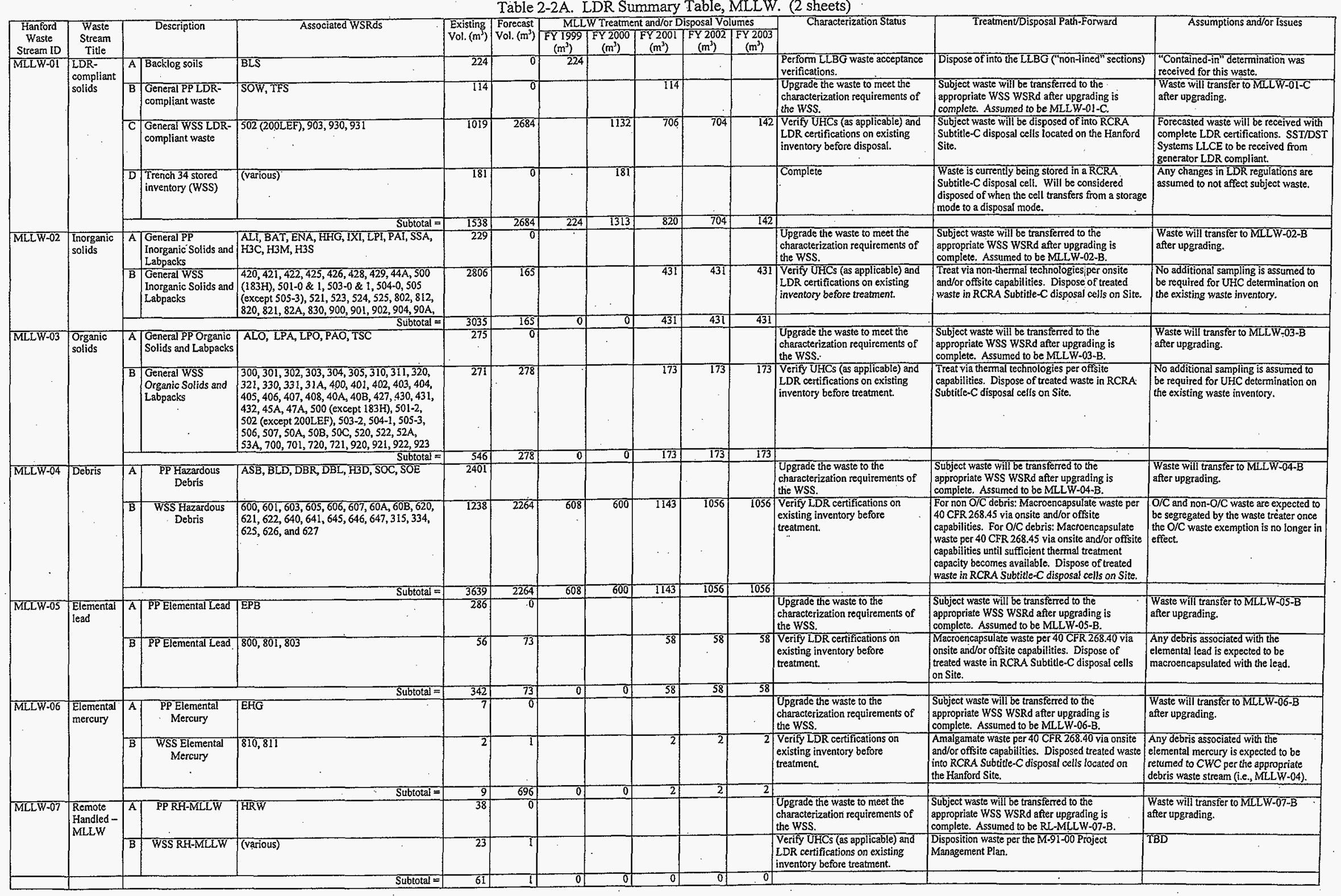


Table 2-2A. LDR Summary Table, MLLW. (2 sheets)

\begin{tabular}{|c|c|c|c|c|c|c|c|c|c|c|c|c|c|}
\hline $\begin{array}{l}\text { Hanford } \\
\text { Waste }\end{array}$ & $\begin{array}{l}\text { Waste } \\
\text { Stream }\end{array}$ & Description & Associated WSRds & \begin{tabular}{|l|}
$\begin{array}{l}\text { Existings } \\
\text { Vol. }\left(\mathbf{m}^{\prime}\right)\end{array}$ \\
(a)
\end{tabular} & $\begin{array}{l}\text { Forecast } \\
\text { Vol. }\left(\mathrm{m}^{\prime}\right)\end{array}$ & $\begin{array}{r}\text { MLL } \\
\text { FY } 19999\end{array}$ & $\begin{array}{l}\text { W Treatm } \\
\text { FY } 2000\end{array}$ & ent and/ort & $\frac{D i s p o s a l V C}{1 F Y 2002}$ & $\begin{array}{l}\frac{1}{\text { liumes }} \\
\text { FY } 2003\end{array}$ & Characterization Status & TreatmentDisposal Path-Forward & Assumptions and/or Issues \\
\hline \begin{tabular}{|l|} 
Stream ID \\
MLLW-08
\end{tabular} & & & & & & & & & & & & & \\
\hline MLLW-08 & $\begin{array}{l}\text { GTC3 } \\
\text { (PP) }\end{array}$ & & DBR (RTG's only) & & & & & & & & TBD & TBD & TBD \\
\hline
\end{tabular}


Table 2-2B. LDR Summary Table, TRUM Waste. (2 sheets)

\begin{tabular}{|c|c|c|c|c|c|c|c|c|c|c|c|c|c|}
\hline \multirow[t]{2}{*}{ Waste Stream ID } & \multirow[t]{2}{*}{ Waste Stream Title } & \multirow{2}{*}{$\begin{array}{c}\text { Associated } \\
\text { WSRds }\end{array}$} & \multirow{2}{*}{$\mid$\begin{tabular}{|l|}
$\begin{array}{l}\text { End of } \mathrm{FY} \\
\text { inventory }\left(\mathrm{m}^{3}\right)\end{array}$ \\
ing
\end{tabular}} & \multirow{2}{*}{$\begin{array}{c}\text { FY 99.03 } \\
\text { Forecast }\left(\mathrm{m}^{3}\right)\end{array}$} & \multirow{2}{*}{ Package Type } & \multicolumn{5}{|c|}{ TRUM Treatment and/or Disposal Volume } & \multirow{2}{*}{ Characterization Status } & \multirow{2}{*}{$\begin{array}{l}\text { TreatmentDisposal Path } \\
\text { Forward } \\
\end{array}$} & \multirow{2}{*}{ Treatment'Disposal Assumptions and/or Issues } \\
\hline & & & & & & FY [999] & FY 2000 & FY 2001 & FY2002 & FY 2003 & & & \\
\hline TRUM-OIA & $\begin{array}{l}\text { Contact-handled mixed } \\
\text { TRU combustible debris }\end{array}$ & CBDTC & 73. & 35 & $\begin{array}{l}\text { Drums and } \\
\text { boxes }\end{array}$ & (a) & (a) & (a) & (a) & (a) & $\begin{array}{l}\text { Waste will be characterized as } \\
\text { necessary to meet the waste acceptance } \\
\text { criteria at WIPP. }\end{array}$ & $\begin{array}{l}\text { TRU waste is exempt from } \\
\text { LDRs. }\end{array}$ & 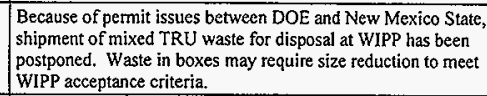 \\
\hline TRUM-01B & $\begin{array}{l}\text { Contact-handled mixed } \\
\text { TRU composite filters }\end{array}$ & CMFTC & 8 & 0 & Drums & (a) & (a). & (a) & (a) & (a) & $\begin{array}{l}\text { Waste will be characterized as } \\
\text { necessary to meet the waste acceptance } \\
\text { criteria at WIPP. }\end{array}$ & \begin{tabular}{|l|} 
TRU waste is exempt from \\
LDRs. \\
\end{tabular} & $\begin{array}{l}\text { Because of permit issues between DOE and New Mexicos State, } \\
\text { shipments of mixed TRU waste for disposal at WIPP has been } \\
\text { postponed. }\end{array}$ \\
\hline TRUM-01C & $\begin{array}{l}\text { Contact-handled mixed } \\
\text { TRU heteregenous debris }\end{array}$ & HTDTC & 131 & 88 & $\begin{array}{l}\text { Drums and } \\
\text { boxes }\end{array}$ & (a) & (a) & (a) & (a) & (a) & $\begin{array}{l}\text { Waste will be characterized as } \\
\text { neccssary to meet the waste acceptance } \\
\text { criteria at WIPP. }\end{array}$ & $\begin{array}{l}\text { TRU waste is exempt from } \\
\text { LDRs. }\end{array}$ & $\begin{array}{l}\text { Because of permit issues between DOE and New Mexico State, } \\
\text { shipment of mixed TRU waste for disposal at WIPP has been } \\
\text { postponed. Waste in boxes may require size reduction to meet } \\
\text { WTPP acceptance criteria. }\end{array}$ \\
\hline TRUM-01D & $\begin{array}{l}\text { Contact-handled mixed } \\
\text { TRU metal debris }\end{array}$ & MTDTC & 16 & 16 & $\begin{array}{l}\begin{array}{l}\text { Drums and } \\
\text { boxes }\end{array} \\
\text { b }\end{array}$ & (a) & (a) & (a) & (a) & (a) & $\begin{array}{l}\text { Waste will be characterized as } \\
\text { necessary to meet the waste acceptance } \\
\text { criteria at WIPP. }\end{array}$ & $\begin{array}{l}\text { TRU waste is exempt from } \\
\text { LDRs. }\end{array}$ & $\begin{array}{l}\text { Because of permit issues between DOE and New Mexico State, } \\
\text { shipment of mixed TRU waste for dissposal at WIPP has been } \\
\text { postponed. Waste in boxes may require size reduction to meet } \\
\text { WIPP acceptance criteria. }\end{array}$ \\
\hline TRUM-01E & $\begin{array}{l}\text { Contact-handled mixed } \\
\text { TRU non-metal inorganic } \\
\text { particulates }\end{array}$ & $\begin{array}{l}\text { NMTTC } \\
\end{array}$ & 1 & 45 & Drums & (a) & (a) & (a) & (a) & (a) & $\begin{array}{l}\text { Waste will be characterized as } \\
\text { necessary to meet the waste acceptance } \\
\text { criteria at WIPP. }\end{array}$ & $\begin{array}{l}\text { TRU waste is exempt from } \\
\text { LDRs. }\end{array}$ & $\begin{array}{l}\text { Because of permit issues between DOE and New Mexico State, } \\
\text { shipment of mixed TRU waste for disposal at WIPP has been } \\
\text { postponed. }\end{array}$ \\
\hline TRUM-01F & $\begin{array}{l}\text { Contact-handled mixed } \\
\text { TRU lead waste }\end{array}$ & $\begin{array}{l}\text { PBMTC } \\
\end{array}$ & 5 & 15 & Drums & (a) & (a) & (a) & (a) & (a) & $\begin{array}{l}\text { Waste will be characterized as } \\
\text { necessary to meet the waste acceptance } \\
\text { criteria at wIPP. }\end{array}$ & $\begin{array}{l}\text { TRU waste is exempt from } \\
\text { LDRs. }\end{array}$ & $\begin{array}{l}\text { Because of permit issues between DOE and New Mexico State, } \\
\text { shipment of mixed TRU waste for disposal at WIPP has been } \\
\text { postponed. }\end{array}$ \\
\hline TRUM-01G & $\begin{array}{l}\text { Contact-handled mixed } \\
\text { TRU solidified inorganics }\end{array}$ & SLITC & 1 & 6 & Drums & (a) & (a) & (a) & (a) & (a) & $\begin{array}{l}\text { Waste will be characterized as } \\
\text { necessary to meet the waste acceptance } \\
\text { criteria at WIPP. }\end{array}$ & $\begin{array}{l}\text { TRU waste is exempt from } \\
\text { LDRs. }\end{array}$ & $\begin{array}{l}\text { Because of permit issues between DOE and New Mexico State, } \\
\text { shipment of mixed TRU waste for disposal at WIPP has been } \\
\text { postponed. }\end{array}$ \\
\hline TRUM-01H & $\begin{array}{l}\text { Contact-handided mixed } \\
\text { TRU solidifited organics }\end{array}$ & SLOTC & 3 & 2 & Drums & (a) & (a) & (a) & (a) & (a) & $\begin{array}{l}\text { Waste will be characterized as } \\
\text { necessary to meet the waste acceptance } \\
\text { criteria at WIPP. }\end{array}$ & \begin{tabular}{|l} 
TRU waste is exempt from \\
LDRs.
\end{tabular} & $\begin{array}{l}\text { Because of permit issues between DOE and New Mexico State, } \\
\text { shipment of mixed TRU waste for disposal at WIPP has been } \\
\text { postponed. }\end{array}$ \\
\hline TRUM-01J & $\begin{array}{l}\text { Contact-handied mixed } \\
\text { TRU soils }\end{array}$ & SOITC & 10 & 0 & Drums & (a) & (a) & (a) & (a) & (a) & \begin{tabular}{|l|} 
Waste will be characterized as \\
necessary to meet the waste acceptance \\
criteria at WIPP.
\end{tabular} & $\begin{array}{l}\text { TRU waste is exempt from } \\
\text { LDRs. }\end{array}$ & $\begin{array}{l}\begin{array}{l}\text { Because of permit issues between DOE and New Mexico State, } \\
\text { shipment of mixed TRU waste for disposal at WIPP has been } \\
\text { postponed. }\end{array}\end{array}$ \\
\hline TRUM-01K & $\begin{array}{l}\text { Contact-handled mixed } \\
\text { TRU unknown or mixture }\end{array}$ & UNKTC & 7 & 0 & Drums & (a) & (a) & (a) & (a) & (a) & $\begin{array}{l}\text { Waste will be characterized as } \\
\text { necessary to meet the waste acceptance } \\
\text { criteria at WIPP. }\end{array}$ & $\begin{array}{l}\text { TRU waste is exempt from- } \\
\text { LDRs. }\end{array}$ & $\begin{array}{l}\text { Because of permit issues between DOE and New Mexico State, } \\
\text { shipment of mixed TRU waste for disposal at WIPP has been } \\
\text { postponed. }\end{array}$ \\
\hline TRUM-02A & $\begin{array}{l}\text { Shielded mixed TRU } \\
\text { heterogeneous debris }\end{array}$ & HTDTS & 5 & 22 & Shielded drums & (a) & (a) & (a) & (a) & (a) & $\begin{array}{l}\text { Waste will be characterized as } \\
\text { necessary to meet the waste acceptance } \\
\text { criteria at WIPP. }\end{array}$ & $\begin{array}{l}\text { TRU waste is exempt from } \\
\text { LDRs. }\end{array}$ & $\begin{array}{l}\text { Because of permiti issues between DOE and New Mexico State, } \\
\text { shipment of mixed TRU waste for disposal at WIPP has been } \\
\text { postponed. }\end{array}$ \\
\hline TRUM-02B & $\begin{array}{l}\text { Shielded mixed TRU lead } \\
\text { waste }\end{array}$ & PBMTS & 3 & & \begin{tabular}{|l|}
$\begin{array}{l}\text { Shielded } \\
\text { drums, boxes, } \\
\text { and casks }\end{array}$ \\
\end{tabular} & (a) & (a) & (a) & (a) & (a) & \begin{tabular}{|l|}
$\begin{array}{l}\text { Waste will be characterized as } \\
\text { necessary to meet the waste acceptance } \\
\text { criteria at wilPP. }\end{array}$ \\
\end{tabular} & $\begin{array}{l}\text { TRU waste is exempt from } \\
\text { LDRs. }\end{array}$ & $\begin{array}{l}\text { Because of permiti issues between DOE and New Mexico State, } \\
\text { shipment of mixed TRU waste for disposal at WIPP has been } \\
\text { posiponed. }\end{array}$ \\
\hline TRUM-03 & $\begin{array}{l}\text { PFP hydraulic fluids } \\
\text { contaminated with PCBs }\end{array}$ & PCBTC & 73 & 0 & $\begin{array}{l}\begin{array}{l}\text { Boxes and } \\
\text { drums }\end{array} \\
\end{array}$ & (a) & (a) & (a) & (a) & (a) & $\begin{array}{l}\begin{array}{l}\text { Because a forward path has yet to } \\
\text { exist, characterization activities are } \\
\text { unknown. }\end{array} \\
\end{array}$ & $\begin{array}{l}\text { TRU waste is exempt from } \\
\text { LDRs. }\end{array}$ & $\begin{array}{l}\text { Waste contaminated with PCBs cannot be accepted by WIPP. } \\
\text { A path forward for this waste needs to be determined. } \\
\text { Evaluation of options that will lead to disposal is planned. } \\
\end{array}$ \\
\hline TRUM $=04 \mathrm{~A}$ & \begin{tabular}{|l|}
300 Area Revitalization \\
contact-handled mixed \\
TRU heterogeneous debris
\end{tabular} & $\mathrm{NA}$ & 0 & 216 & $\begin{array}{l}\text { Drums and } \\
\text { boxes }\end{array}$ & (a) & (a) & (a) & (a) & (a) & $\begin{array}{l}\text { Waste will be characterized as } \\
\text { necessary to meet the waste acceptance } \\
\text { criteria at WIPP. }\end{array}$ & $\begin{array}{l}\text { TRU waste is exempt from } \\
\text { LDRs. }\end{array}$ & $\begin{array}{l}\text { Because of permit issues berween DOE and New Mexico State, } \\
\text { shipment of mixed TRU waste for disposal at WIPP has been } \\
\text { postponed. }\end{array}$ \\
\hline TRUM-04B & $\begin{array}{l}300 \text { Area Revitalization } \\
\text { contact-handled mixed } \\
\text { rRU inorganic nonmetal } \\
\text { debris }\end{array}$ & $\mathrm{NA}_{\mathrm{A}}$ & 0 & 16 & $\begin{array}{l}\text { Drums and } \\
\text { boxes }\end{array}$ & (a) & (a) & (a) & (a) & (a) & $\begin{array}{l}\text { Waste will be characterized as } \\
\text { necessary to meet the waste acceptance } \\
\text { criteria at WIPP. }\end{array}$ & $\begin{array}{l}\text { TRU waste is exempt from } \\
\text { LDRs. }\end{array}$ & $\begin{array}{l}\text { Because of permit issues between DOE and New Mexico State, } \\
\text { shipment of mixed TRU waste for disposal at WIPP has been } \\
\text { postponed. }\end{array}$ \\
\hline TRUM-04C & $\begin{array}{l}00 \text { Area Revitalization } \\
\text { contact-handled mixed } \\
\text { TRU metal debris }\end{array}$ & $\mathrm{NA}$ & 0 & 168 & $\begin{array}{l}\text { Drums and } \\
\text { boxes }\end{array}$ & (a) & (a) & (a) & (a) & (a) & $\begin{array}{l}\text { Waste will be characterized as } \\
\text { necessary to mect the waste acceptance } \\
\text { criteria at WIPP. }\end{array}$ & $\begin{array}{l}\text { TRU waste is exempt from } \\
\text { LDRs. }\end{array}$ & $\begin{array}{l}\text { Recause of permiti issues between DOE and New Mexico State, } \\
\text { shipment of mixed TRU waste for disposal at WIPP has been } \\
\text { possponed. }\end{array}$ \\
\hline TRUM-05A & $\begin{array}{l}\text { Contact-handled TRU } \\
\text { single-shell tank long- } \\
\text { length equipment }\end{array}$ & $\mathrm{NA}$ & & 492 & Special cask & (a) & (a) & (a) & (a) & (a) & $\begin{array}{l}\text { Waste will be characterized as } \\
\text { necessary to meet the waste acceptance } \\
\text { criteria at WIPP. }\end{array}$ & $\begin{array}{l}\text { TRU waste is exempt from } \\
\text { LDRs. }\end{array}$ & $\begin{array}{l}\text { Because of permiti issues between DOE and New Mexico State, } \\
\text { shipment of mixed TRU waste for disposal at WIPP has been } \\
\text { postponed. Waste will need to be sized reduced to meet the } \\
\text { WWPP requirements. }\end{array}$ \\
\hline
\end{tabular}


Table 2-2B. LDR Summary Table, TRUM Waste. (2 sheets)

\begin{tabular}{|c|c|c|c|c|c|c|c|c|c|c|c|c|c|}
\hline Waste Stream ID & Waste Stream Title & $\begin{array}{c}\text { Associated } \\
\text { WSRds }\end{array}$ & $\begin{array}{l}\text { End of FY } 1998 \\
\text { inventory }\left(\mathrm{m}^{3}\right)\end{array}$ & $\begin{array}{c}\text { FY } 99-03 \\
\text { Forecast }\left(\mathrm{m}^{3}\right)\end{array}$ & Package Type & \multicolumn{5}{|c|}{$\begin{array}{l}\text { TRUM Treatment and/or Disposal Volume } \\
\end{array}$} & Characterization Status & $\begin{array}{c}\text { Treatmentolisposal Path } \\
\text { Forward } \\
\end{array}$ & TreatmentDisposal Assumptions and/or Issues \\
\hline TRUM-05B & $\begin{array}{l}\text { Remote-handled TRU } \\
\text { single-shell tank long- } \\
\text { length equipment }\end{array}$ & $\mathrm{NA}$ & 0 & 492 & Special cask & (a) & (a) & (a) & (a) & (a) & $\begin{array}{l}\text { Waste will be characterized as } \\
\text { necessary to meet the waste acceptance } \\
\text { criteria at WIPP. }\end{array}$ & $\begin{array}{l}\text { TRU waste is exempt from } \\
\text { LDRs. }\end{array}$ & $\begin{array}{l}\text { Because of permit issues between DOE and the State of New } \\
\text { Mexico, shipments of mixed TRU waste for disposal at WIPP } \\
\text { has been postponed. Waste package size will need to be } \\
\text { reduced to meet WIPP requirements. }\end{array}$ \\
\hline TRUM-06 & $\begin{array}{l}\text { Miscellaneous remote- } \\
\text { handled mixed TRU waste }\end{array}$ & $\mathrm{NA}$ & 0 & 5 & Shielded drums & (a) & (a) & (a) & (a) & (a) & $\begin{array}{l}\text { Waste will be characterized as } \\
\text { necessary to meet the waste acceptance } \\
\text { criteria at WIPP. }\end{array}$ & \begin{tabular}{|l} 
TRU waste is exempt from \\
LDRs.
\end{tabular} & $\begin{array}{l}\text { Because of permit issues between DOE and New Mexico State, } \\
\text { shipment of mixed TRU waste for disposal at WIPP has been } \\
\text { postponed. }\end{array}$ \\
\hline & & TRU Total & 336 & 1618 & & & & & & & & & \\
\hline
\end{tabular}

NOTE: NA (not applicable) means that no WSRd is associated with this stream because this stream does not exist yet.
(a) TRUM is exempt for the LDR treatment requirements. Because of permit issues between DOE and the State of New Mexico, shipments of mixed TRU waste have been postponed, and a shipment schedule is not available. 
Table 2-2C. LDR Summary Table, Non-CWC Stored Waste. (2 sheets)

\begin{tabular}{|c|c|c|c|c|c|c|}
\hline Waste stream name & $\begin{array}{c}\text { FY } 1998 \text { inventory } \\
\left(\mathrm{m}^{3}\right)\end{array}$ & $\begin{array}{l}\text { Number of } \\
\text { packages }\end{array}$ & Package type & Characterization status & Treatment/disposal path forward & $\begin{array}{c}\text { Treatment/disposal } \\
\text { capabilities/capacities/issucs }\end{array}$ \\
\hline $\begin{array}{l}\text { Double-Shell Tank } \\
\text { System }\end{array}$ & 70.976 & 28 & $\begin{array}{l}\text { Underground } \\
\text { tanks }\end{array}$ & In progress per M-44-00. & $\begin{array}{l}\text { Retricve the waste, pretreat into } \\
\text { LLW and HLW streams, and vitrify. }\end{array}$ & $\begin{array}{l}\text { Dispose of LLW waste on site } \\
\text { in a retrievable mode. Store } \\
\text { HLW onsite until offsite } \\
\text { geologic repository is open. } \\
\text { Onsite TSD vaults for LL,W } \\
\text { and ILW must be constructed. }\end{array}$ \\
\hline $\begin{array}{l}\text { Single-Shell Tank } \\
\text { System }\end{array}$ & 133,600 & 149 & $\begin{array}{l}\text { Underground } \\
\text { tanks }\end{array}$ & In progess per M-44-00. & Retrieve into DST. Same as DST. & Same as DST. \\
\hline $\begin{array}{l}\text { PUREX } \\
\text { Containment } \\
\text { Building }\end{array}$ & 1 & 1 & Metal box & Fully characterized. & $\begin{array}{l}\text { No path forward developed. Will } \\
\text { remain at PUREX facility until plans } \\
\text { are developed by Environmental } \\
\text { Restoration Contractor. }\end{array}$ & $\begin{array}{l}\text { Transfer of the PUREX facility } \\
\text { to the Environmental } \\
\text { Restoration Contractor must } \\
\text { still occur. }\end{array}$ \\
\hline $\begin{array}{l}\text { PUREX Storage } \\
\text { Tunnels }\end{array}$ & 2800 & 36 & $\begin{array}{l}\text { Various, on rail } \\
\text { cars }\end{array}$ & $\begin{array}{l}\text { Partially characterized. May } \\
\text { have to be segregated and } \\
\text { more fully characterized. }\end{array}$ & $\begin{array}{l}\text { No path forward developed. Will } \\
\text { remain in place until the PUREX } \\
\text { facility is dispositioned. }\end{array}$ & $\begin{array}{l}\text { No path forward developed. } \\
\text { The waste is highly radioactive } \\
\text { and requires remote handling. }\end{array}$ \\
\hline $\begin{array}{l}324 \text { Building } \\
\text { Radiochemical } \\
\text { Enginecring Cells }\end{array}$ & 2.66 & $\mathrm{~N} / \mathrm{A}$ & $\begin{array}{l}\text { Not yet } \\
\text { packaged }\end{array}$ & Fully characterized. & $\begin{array}{l}\text { Will be transported to the PUREX } \\
\text { Tumnels and CWC for storage and } \\
\text { possible future treatment. }\end{array}$ & $\begin{array}{l}324 \mathrm{REC} \text { is not a permitted } \\
\text { storage unit. Will be } \\
\text { transferred to permitted TSDS } \\
\text { by } 5 / 31 / 99 \text { per } \mathrm{M}-89-02 \text {. }\end{array}$ \\
\hline $\begin{array}{l}\text { B Plant Cell } 4 \\
\text { Wastc }\end{array}$ & 1.4 & 43 & $0.21 \mathrm{~m}^{3}$ drums & $\begin{array}{l}\text { Information based on process } \\
\text { knowledge. No additional } \\
\text { characterization planned. }\end{array}$ & $\begin{array}{l}\text { No path forward developed. Will } \\
\text { remain at the B Plant Complex until } \\
\text { facility is dispositioned by the } \\
\text { Environmental Restoration } \\
\text { Contractor. }\end{array}$ & $\begin{array}{l}\text { Waste is highly radioactive and } \\
\text { requires remote handling. }\end{array}$ \\
\hline $\begin{array}{l}\text { B Plant } \\
\text { Containment } \\
\text { Building Storage }\end{array}$ & Unknown' & Unknown & Unknown & $\begin{array}{l}\text { Information based on process } \\
\text { knowledge. No additional } \\
\text { characterization planned. }\end{array}$ & $\begin{array}{l}\text { No path forward developed. Will } \\
\text { remain at the B Plant Complex until } \\
\text { facility is dispositioned by the } \\
\text { Environmental Restoration } \\
\text { Contractor. }\end{array}$ & $\begin{array}{l}\text { Waste is highly radioactive and } \\
\text { requires remote handling. }\end{array}$ \\
\hline $\begin{array}{l}\text { T Plant Complex } \\
\text { Drag-off Box }\end{array}$ & 10 & 1 & Storage box & $\begin{array}{l}\text { Further characterization to be } \\
\text { done. Currently not } \\
\text { scheduled. }\end{array}$ & $\begin{array}{l}\text { Planned disposal location is } \\
\text { Subtitle-C or } L L W \text { portion of } \\
\text { LLBG. }\end{array}$ & Not currently tunded. \\
\hline $\begin{array}{l}\text { TPlant Complex } \\
\text { Tank Trailer Waste }\end{array}$ & 4.54 & 1 & $\begin{array}{l}5000 \text { gal tank } \\
\text { trailer }\end{array}$ & Fully characterized. & $\begin{array}{l}\text { DST system likely to accept waste. } \\
\text { Plans are to remove contents of tank } \\
\text { trailers to meet requirements for } \\
\text { empty containers.- }\end{array}$ & $\begin{array}{l}\text { If waste cannot be accepted } \\
\text { into the DST system, an } \\
\text { alternative pathway will be } \\
\text { developed. }\end{array}$ \\
\hline
\end{tabular}


Table 2-2C. LDR Summary Table, Non-CWC Stored Waste. (2 sheets)

\begin{tabular}{|c|c|c|c|c|c|c|}
\hline Waste stream name & $\begin{array}{l}\text { FY } 1998 \text { inventory } \\
\left(\mathrm{m}^{3}\right)\end{array}$ & $\begin{array}{c}\text { Number of } \\
\text { packages }\end{array}$ & Package type & Characterization status & Treatment/disposal path forward & $\begin{array}{c}\text { Treatment/disposal } \\
\text { capabilitics/capacities/issues }\end{array}$ \\
\hline $\begin{array}{l}\text { F Plant Complex } \\
\text { EC-1 Condenser }\end{array}$ & 32.11 & 1 & Condenser & $\begin{array}{l}\text { Whether additional } \\
\text { characterization will be } \\
\text { performed is not known. } \\
\text { Whether this equipment } \\
\text { contains regulated } \\
\text { constituents is not known at } \\
\text { this time. }\end{array}$ & $\begin{array}{l}\text { Planned disposal location is } \\
\text { Subtitle-C or LLW portion of } \\
\text { LLBG. }\end{array}$ & $\begin{array}{l}\text { Dependent on FY } 2000 \\
\text { funding. Treatment required, } \\
\text { if any, will depend on } \\
\text { characterization results. }\end{array}$ \\
\hline $\begin{array}{l}\text { Cesium and } \\
\text { Strontium Capsules }\end{array}$ & 2 & $\begin{array}{r}1335 \mathrm{Cs} \\
601 \mathrm{St}\end{array}$ & Capsules & $\begin{array}{l}\text { Fully characterized based on } \\
\text { process history and flow } \\
\text { sheets. }\end{array}$ & $\begin{array}{l}\text { Capsules are scheduled to be } \\
\text { blended into the tank waste for } \\
\text { vitrification beginning in } 2013\end{array}$ & $\begin{array}{l}\text { Capsules will be cut up and } \\
\text { salts dissolved either at WESF } \\
\text { or the vitrification plant. }\end{array}$ \\
\hline $\begin{array}{l}\text { Total waste volume } \\
\text { and package count, } \\
\text { not including CWC } \\
\text { ("N/A "entries not } \\
\text { counted) }\end{array}$ & 207,430 & 2196 & & - & & \\
\hline $\begin{array}{l}\text { Total waste } \\
\text { inventory including } \\
\text { CWC }\end{array}$ & 216,937 & 25,641 & & & & \\
\hline
\end{tabular}

\section{ALARA as low as reasonably achievable}

CI) contact-handled

CWC Central Waste Complex

DOE U.S. Department of Energy

DST double-shell tank

ETF Effluent Treatment Facility

IDPE high-density polyethylene

HEPA high-efficiency particulate air

IILW high-level waste

LDR L Land Disposal Restriction

LLBG low-level burial ground

L.LCE long-length contaminated equipment

LLW low-level waste

N/A not applicable

$\mathrm{O} / \mathrm{C} \quad$ organic/carbonaceous

PCB polychlorinated biphenyl

PFP Plutonium Finishing Plant
PP

RCRA

REC

RTR

SST

STABL

Tri-Party Agreement Hanford Federal Facility Agreement and Consent Order

TRU

TRUM

TSCA

TSD

TWRS

UHC

WIPP

WRAP

WSRd

WSS

past practice

Resource Conservation and Recovery Act of 1976

radiochemical engineering ccll

Real-time radiography

single-shell tank

stabilization

\section{transuranic}

transuranic mixed

Toxic Substances Control Act of 1976

treatment, storage, and/or disposal

Tank Waste Remediation System

underlying hazardous constituen

Wastc Isolation Pilot Plant

Waste Receiving and Processing (facility)

Waste Specification Record (specific WSRds are not defined)

Waste Specification System 
The volume and types of the mixed waste currently in storage at the Hanford Facility are summarized in Table 2-1. The table shows that, as of 1998, the Hanford Facility stored approximately 216,937 cubic meters of land disposal restricted radioactive mixed waste. All this waste is considered to be a compliant system except SST waste. SST waste is stored in tanks that do not have secondary containment and do not have an integrity assessment. As such, SSTs will be closed rather than upgraded.

\subsection{WASTE TREATMENT}

The federal LDRs apply to each type of hazardous waste that has been restricted from land disposal. Treatment standards are identified as either concentration based or technology based. Concentration-based standards have been developed based on "best demonstrated available technology." Except for cyanides, which must be destroyed, treatment to meet concentrationbased standards can be pursued via any technology other than dilution, which is not permissible. The only requirement is that the waste be treated to reduce the concentration of the constituents of concern. However, waste types to which technology-based standards apply must be treated by that specified technology. Hazardous waste designated with multiple RCRA codes must be treated in accordance with the standards for each waste code and subcategory, when applicable, unless 40 CFR 268.9(a) and (b) apply. In situations where overlap occurs, the more stringent standard must be applied. EPA's LDR rulemaking efforts, 59 FR 47982, resulted in the universal treatment standards, which contain numerical limits for underlying hazardous constituents (UHC). Based on LDR Phase IV requirements, the UHC requirements are applicable to most characteristic waste types destined for land disposal. The UHC requirements become applicable when using treatment standards contained in 40 CFR 268.40. If mixed waste that was placed into storage now requires a UHC determination before treatment and/or disposal, additional characterization may be required.

Applicable planned treatment methods and treatment alternatives are described in the waste stream profile sheets in Chapter 3 and summarized in Table 2-2. The Hanford Site Technology Coordination Group science and technology needs for FY 1998 are available on the Internet at http://www.pnl.gov/stcg. These science and technology needs can be reviewed for applicability to the waste stream in Section 4, "Waste Stream Treatment," of the waste stream profile sheet. Another relevant Internet site, "Accelerating Cleanup: Paths to Closure," is at http://www.env.doe.gov/closure.

The use of offsite commercial treatment technologies currently is being considered for some waste streams. The use of onsite commercial technologies also is possible. The DOE is considering using nontraditional contracting approaches (i.e., "privatization") for site remediation work. The use of commercial technologies is likely to play a role in site remediation work (primarily under CERCLA regulations). Certain waste treatment operations for waste stored at the CWC, such as stabilization and thermal treatment and vitrification of SST and DST waste, are planned to be provided by either Hanford Facility or private contractors.

The Tri-Party Agreement specifies the required dates for construction and startup of, and waste treatment in, the major treatment units. No requirements have been established for accelerated treatment beyond these dates. (Figure 2-1 shows the Tri-Party Agreement work 
schedule.) Further details on accelerated treatment are located in the discussions of individual waste streams, found in Chapter 4.

The following paragraphs summarize the three largest volume LDR.mixed-waste streams at the Hanford Facility. Subsequent chapters provide more detail about these and other streams.

\subsubsection{Double-Shell Tank System Waste}

The DST system waste consists of LLW, TRU waste, and HLW. In the interim storage mode, however, the waste is managed as HLW and some of the liquid fraction is reduced in volume by processing in the 242-A Evaporator. To meet disposal requirements, present plans are to separate the waste into a LLW stream and a HLW/TRU stream before treatment. The HLW/TRU stream may undergo additional treatment as necessary to reduce its volume and concentrate its radionuclide loading.

Before ultimate disposal, the DST waste will be treated to meet applicable LDR requirements. The present plans are for a private company to build and operate a vitrification facility where the DST system waste will be formed into glass logs. Vitrification is the specified LDR treatment technology for $\mathrm{HLW}$ that exhibits the corrosive and heavy metal characteristics at the point of generation. At this time, it is unclear if vitrification is also the specified treatment for LLW and TRU waste. If it is not the specified treatment, the LLW and TRU waste will have to be treated to meet appropriate concentration-based standards.

Once vitrification is comple: $;$ present plans are to send the waste for storage or disposal. LLW will be stored and disposed of on Site. TRU waste will be stored on Site and disposed of at the WIPP facility. HLW will be stored on Site and disposed of at a national geologic repository. Because the repository cannot accept hazardous waste, additional permitting, legislation, or some other action might be required before actual shipment of the vitrified HLW to the national repository.

\subsubsection{Single-Shell Tank System Waste}

The SST system waste consists of LLW, TRU waste, and HLW; however, while in storage, it is managed as HLW. The physical forms of SST system waste are sludge, salt cake, and liquid. Liquid waste, which includes supernatant and interstitial liquid in the salt cake, is being transferred to the DST system for later treatment as long as the safety status of the SST system does not worsen after pumping. The planning base for the SST system is to retrieve the waste, as specified by the Tri-Party Agreement, and transfer it to the DST system. The SST system waste will then be disposed of as DST system waste. No additional waste will be generated and stored in the SST system; waste has not been introduced into the SST system since 1980. 
DOE/RL-99-01

Figure 2-1. Tri-Party Agreement Action Plan Work Schedule. (16 sheets)

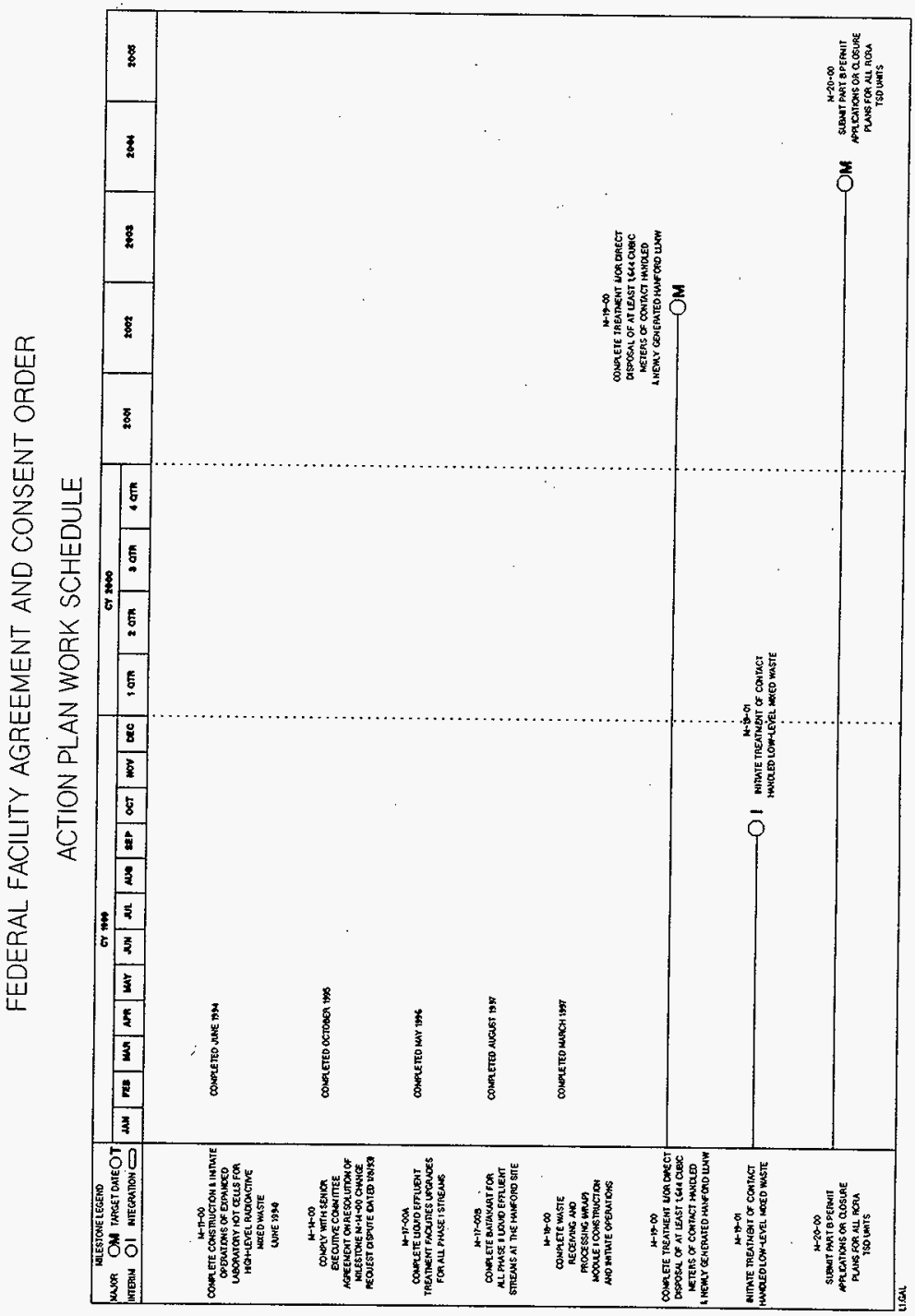


DOE/RL-99-01

Figure 2-1. Tri-Party Agreement Action Plan Work Schedule. (16 sheets)

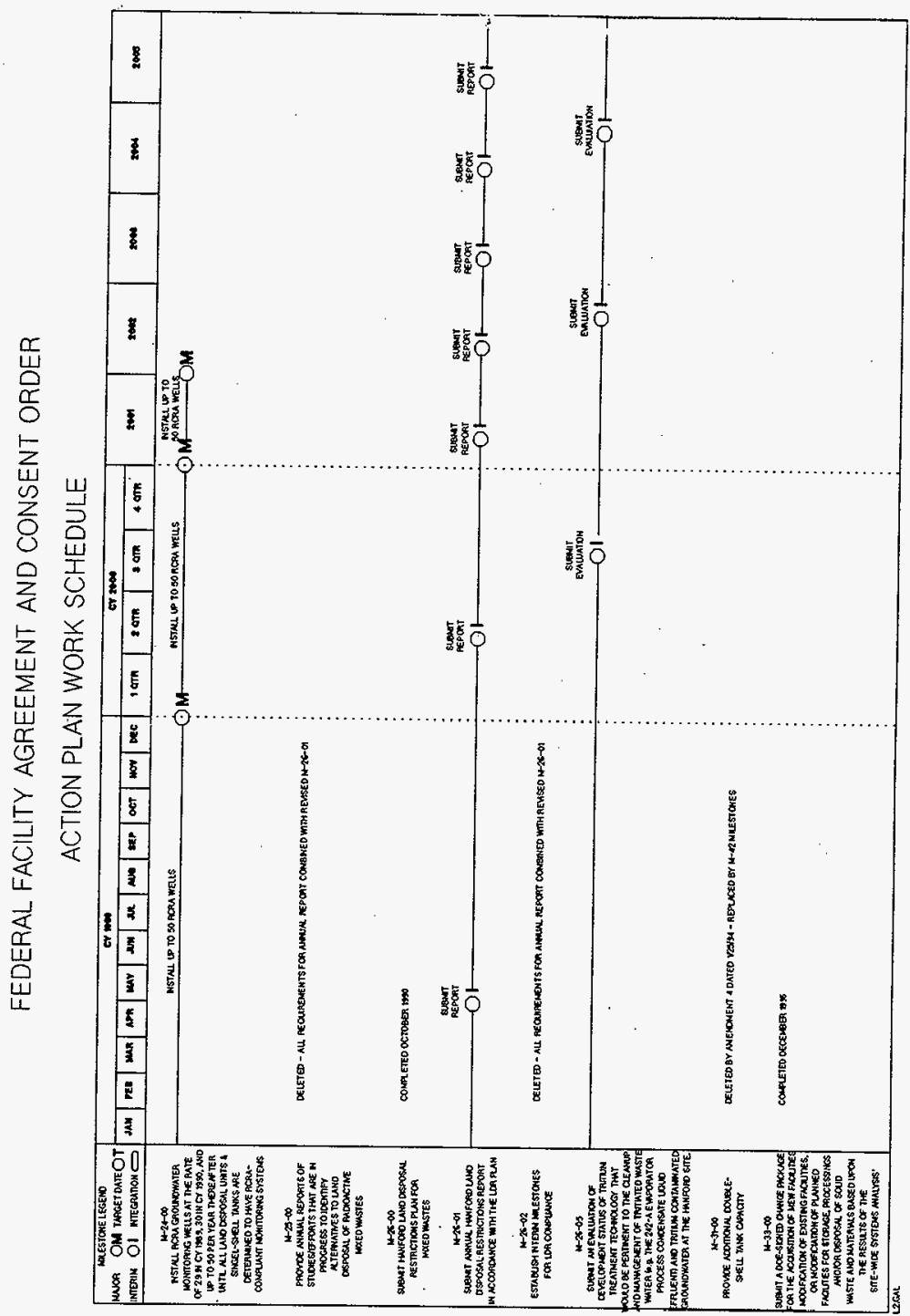


DOE/RL-99-01

Figure 2-1. Tri-Party Agreement Action Plan Work Schedule. (16 sheets)

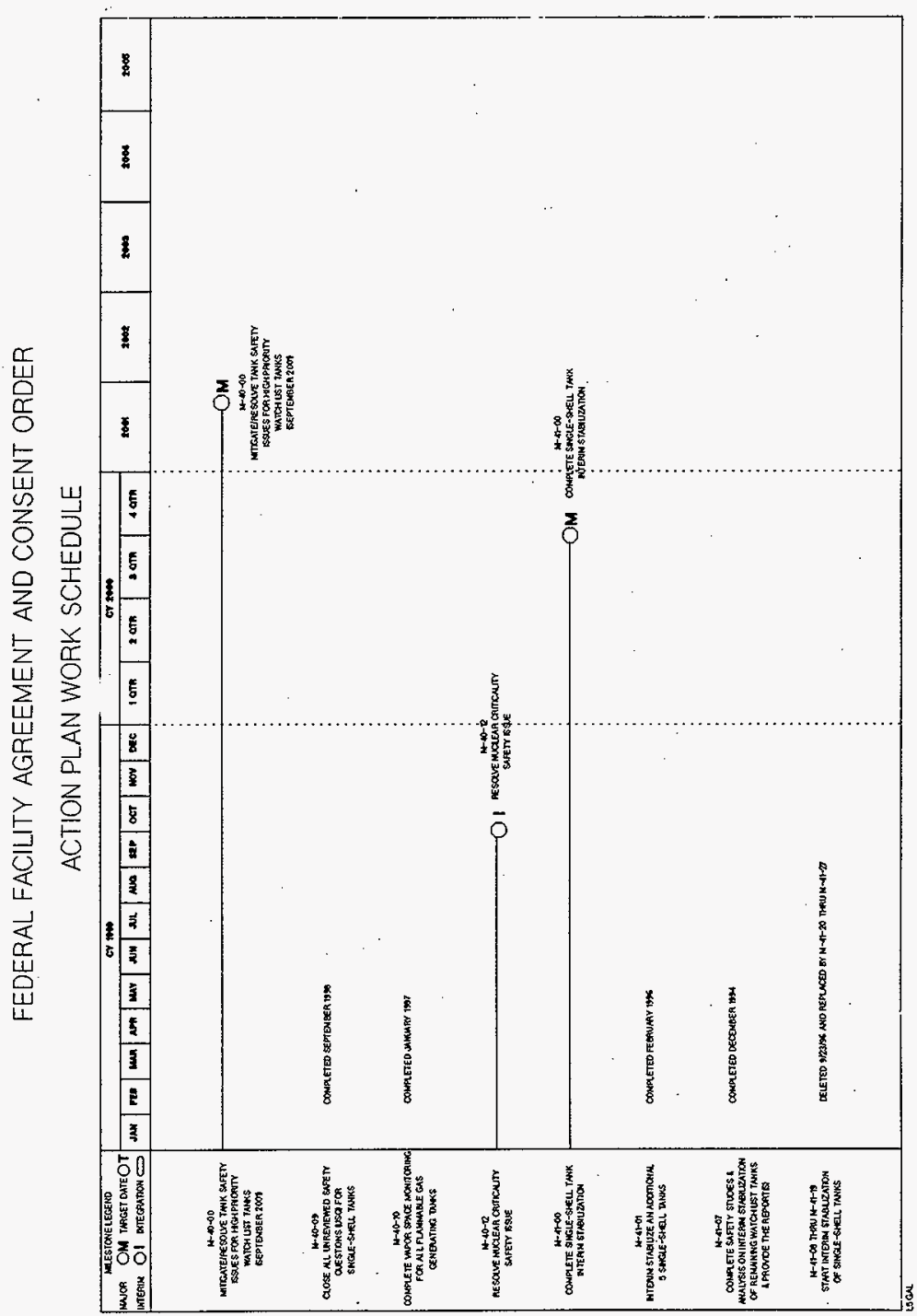


FEDERAL FACILITY AGREEMENT AND CONSENT ORDEH

ACTION PLAN WORK SCHEDULE

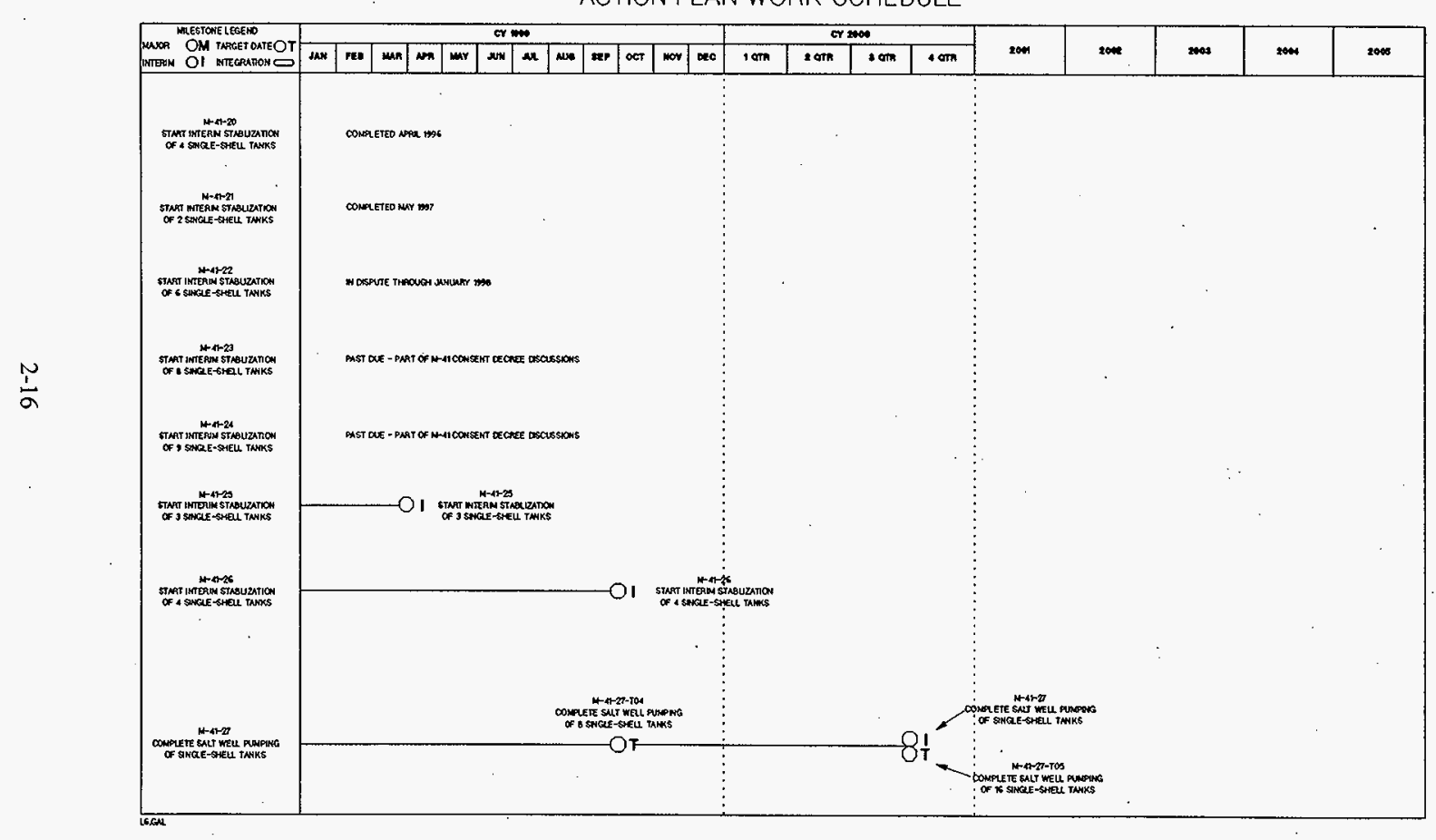

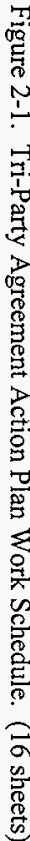


DOE/RL-99-01

Figure 2-1. Tri-Party Agreement Action Plan Work Schedule. (16 sheets)

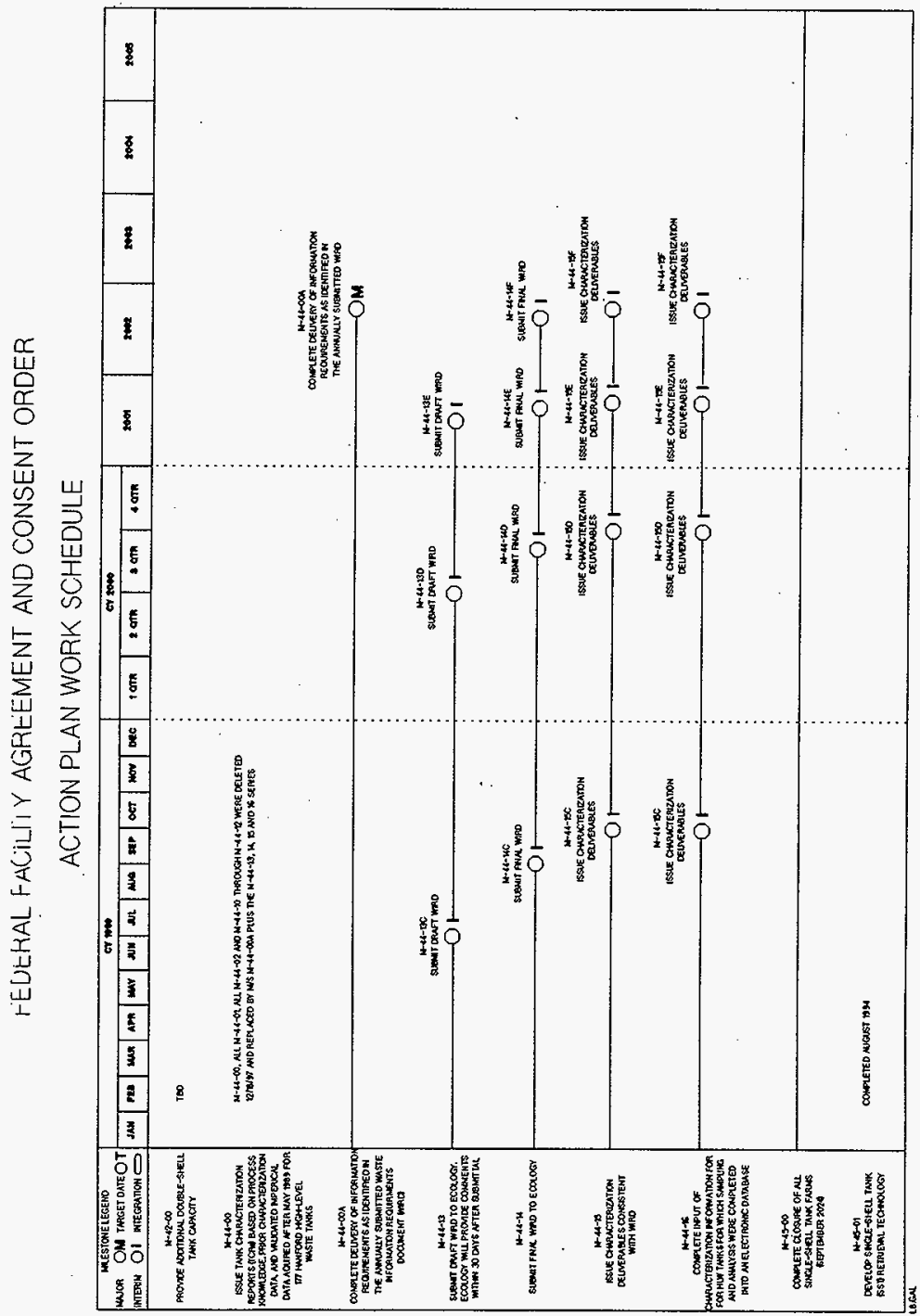


FEDERAL FACILITY AGREEMENT AND CONSENT ORDER

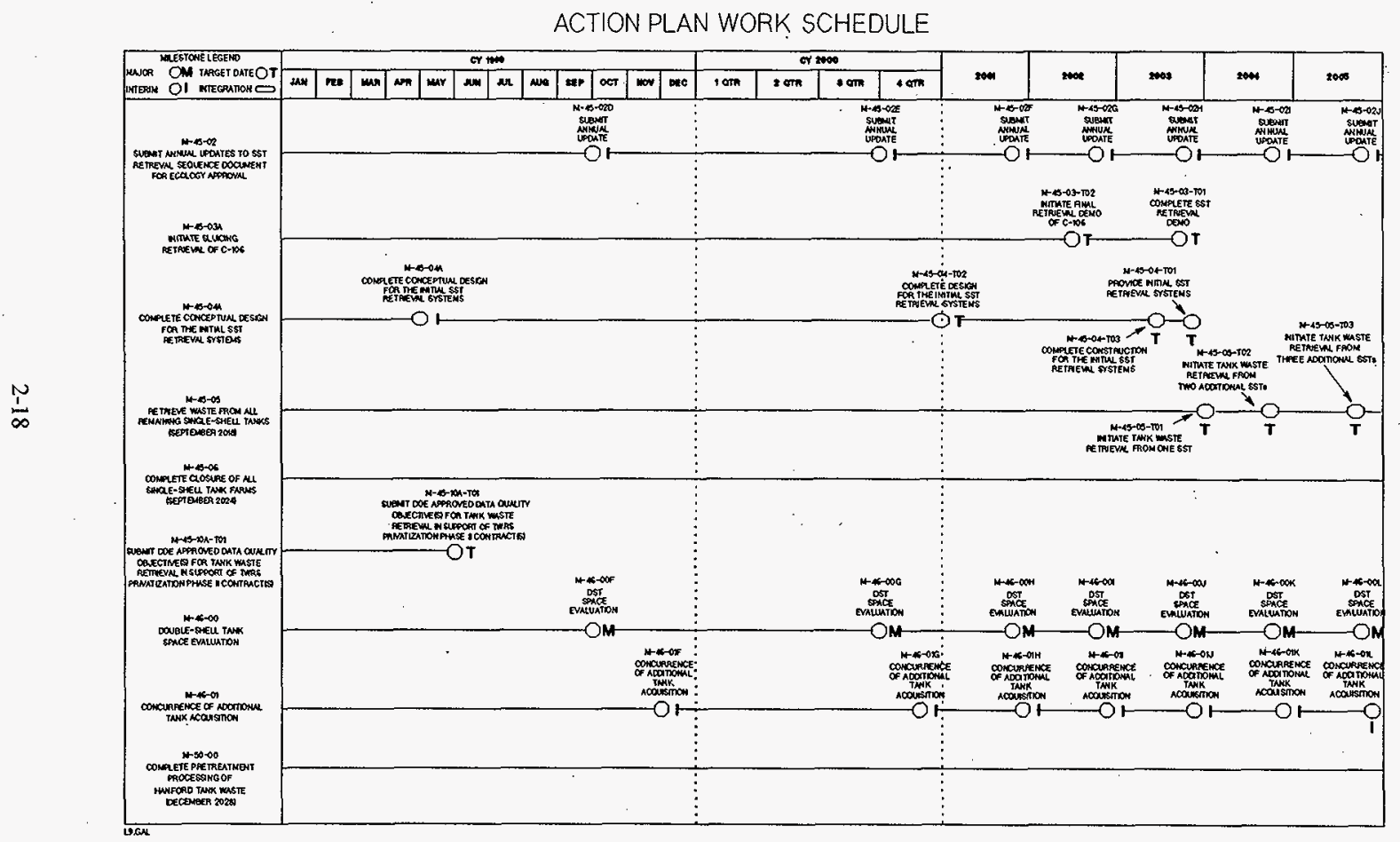


FEDERAL FACILITY AGREEMENT AND CONSENT ORDER

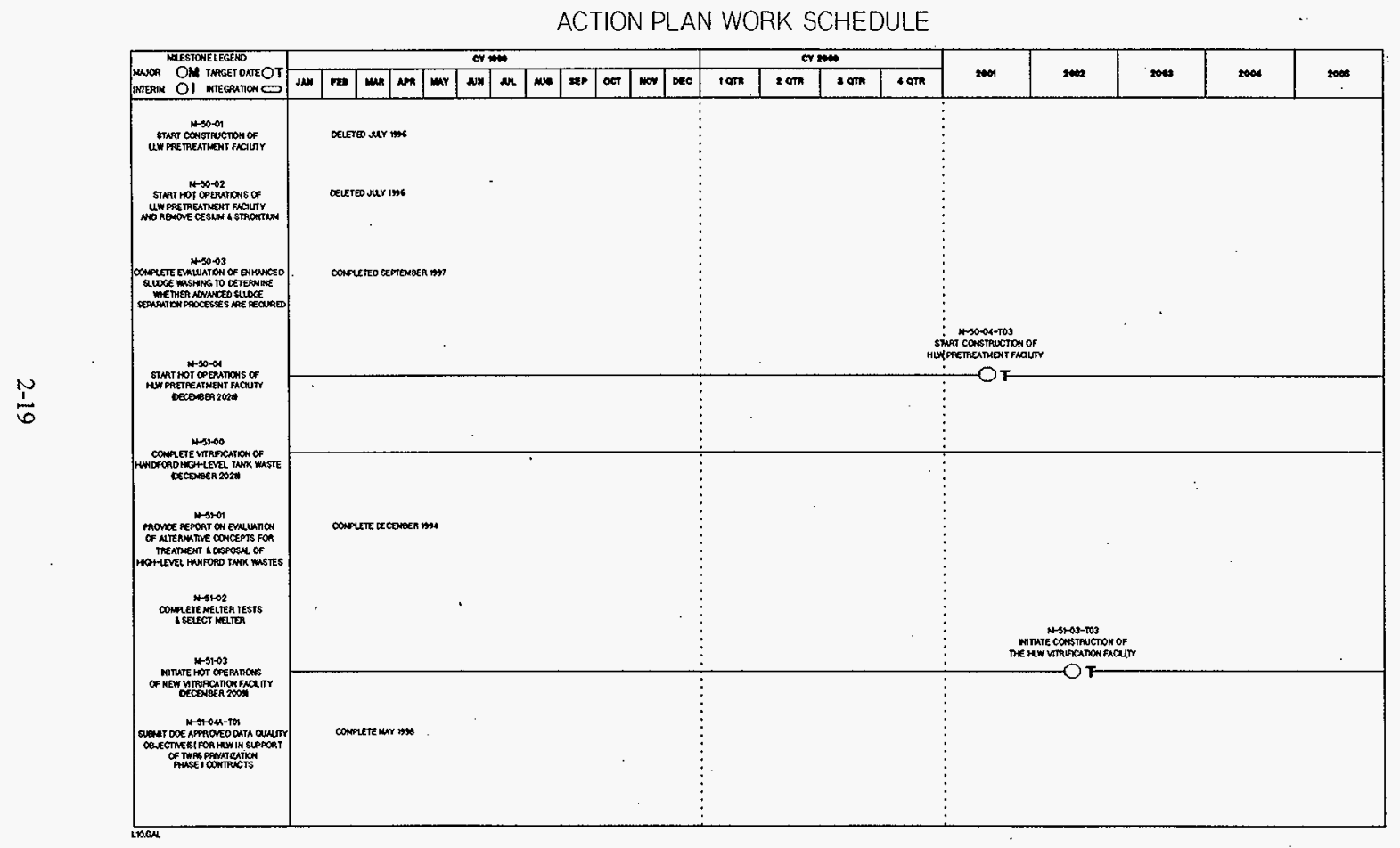


DOE/RL-99-01

Figure 2-1. Tri-Party Agreement Action Plan Work Schedule. (16 sheets)

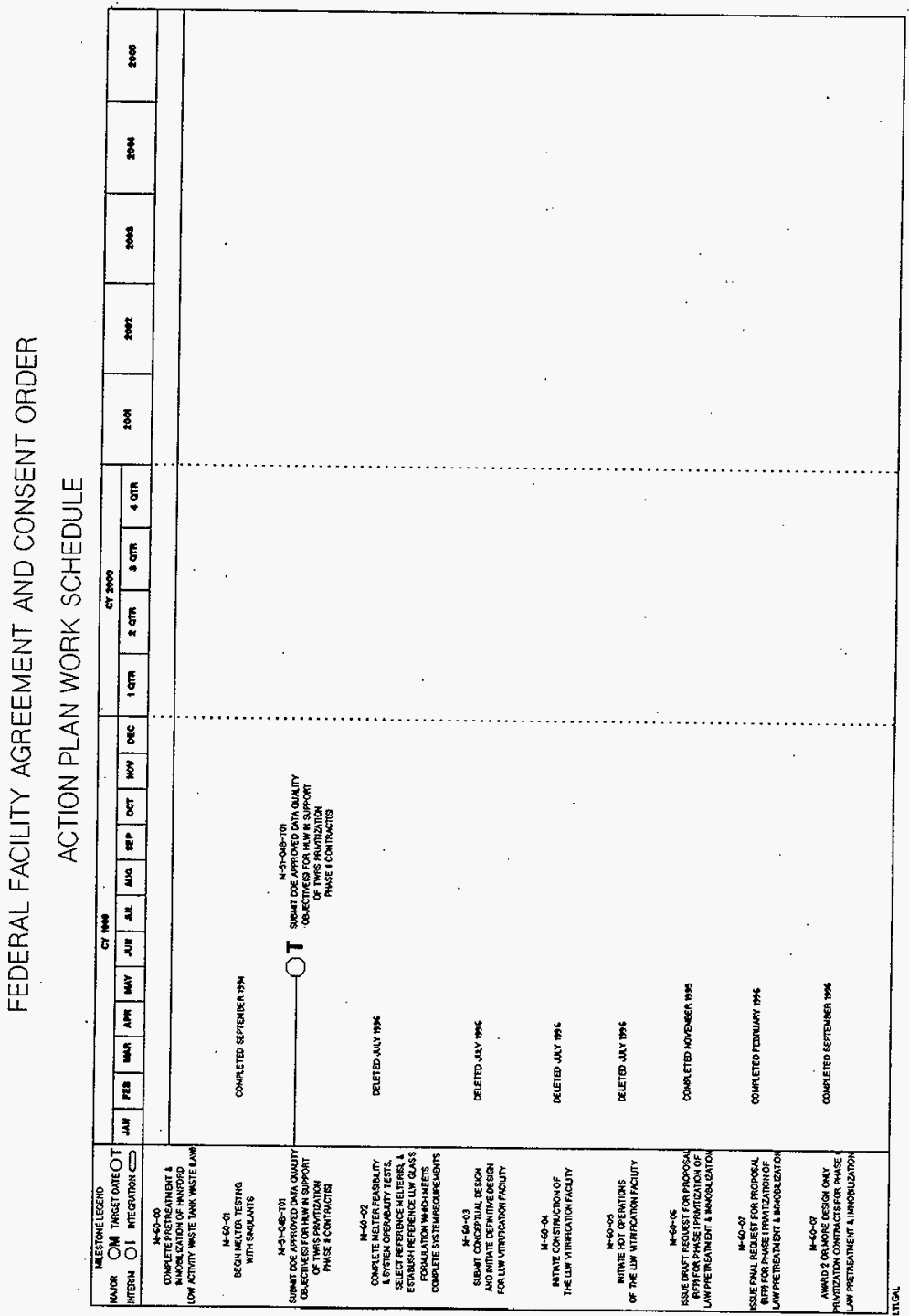


FEDERAL FACILITY AGREEMENT AND CONSENT ORDER

ACTION PLAN WORK SCHEDULE

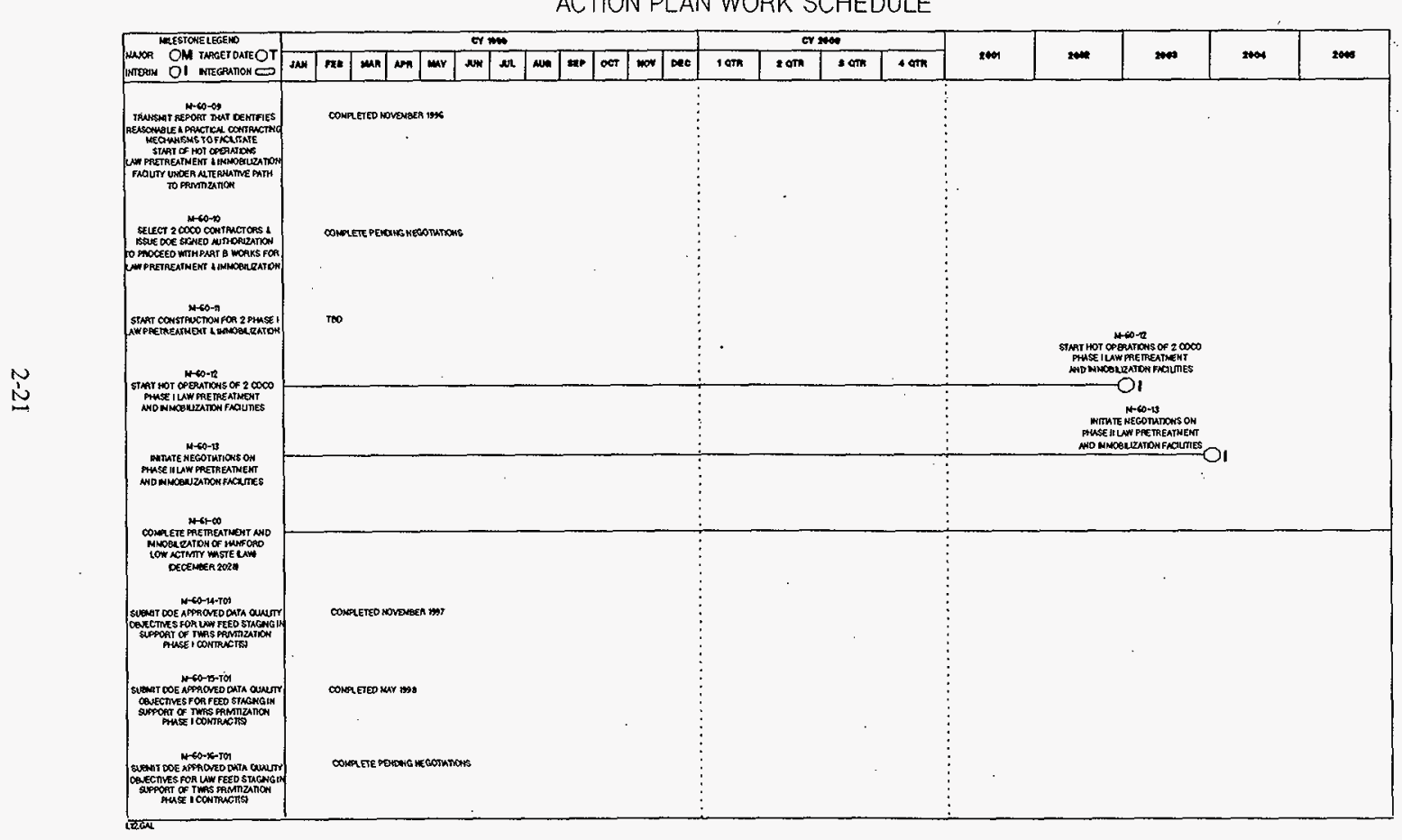


FEDERAL FACILITY AGREEMENT AND CONSENT ORDER

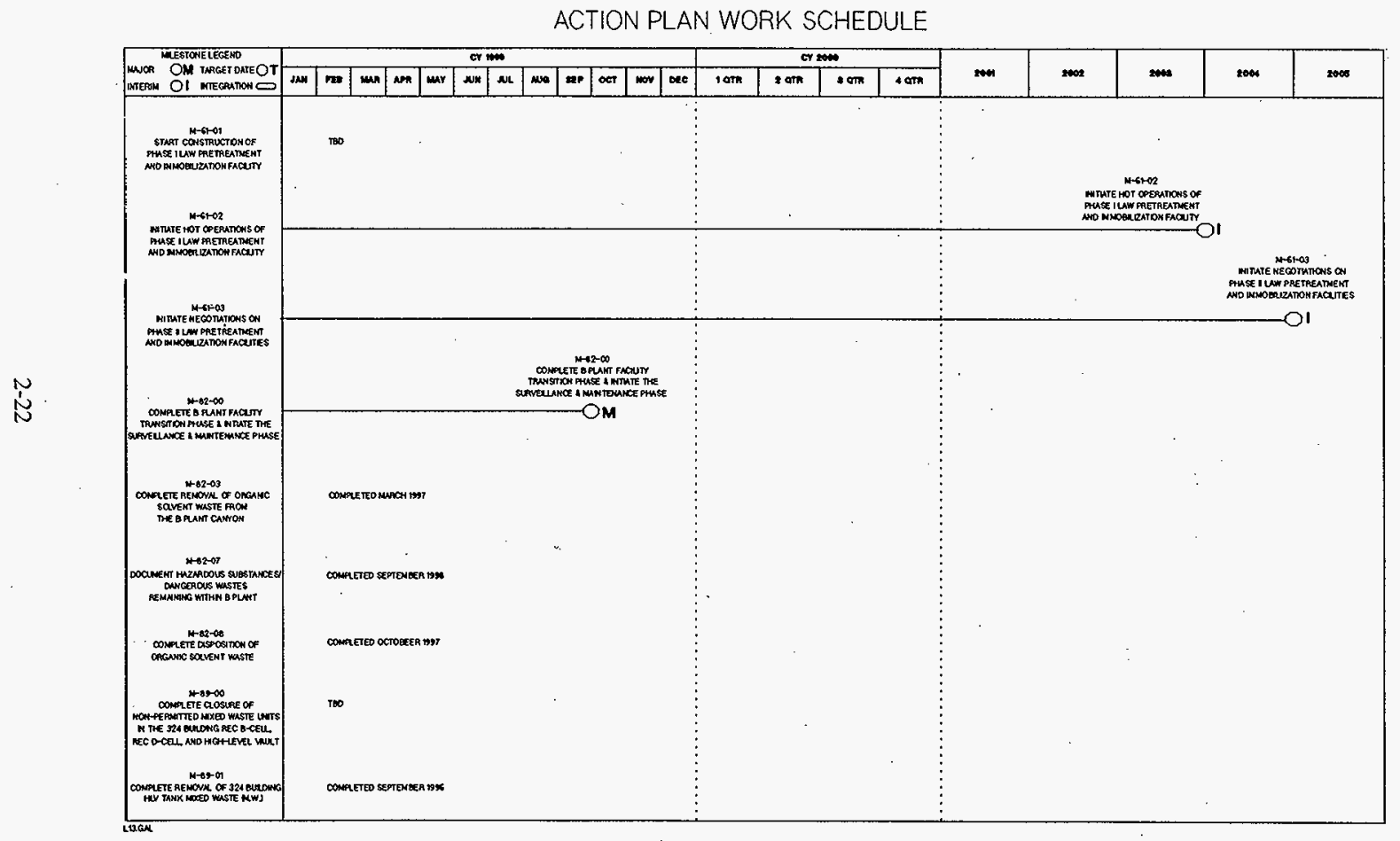

$\sum$

早

号

를

a

5
$\frac{2}{0}$
$\frac{0}{0}$

has 
FEDERAL FACILITY AGREEMENT AND CONSENT ORDER

ACTION PLAN WORK SCHEDULE

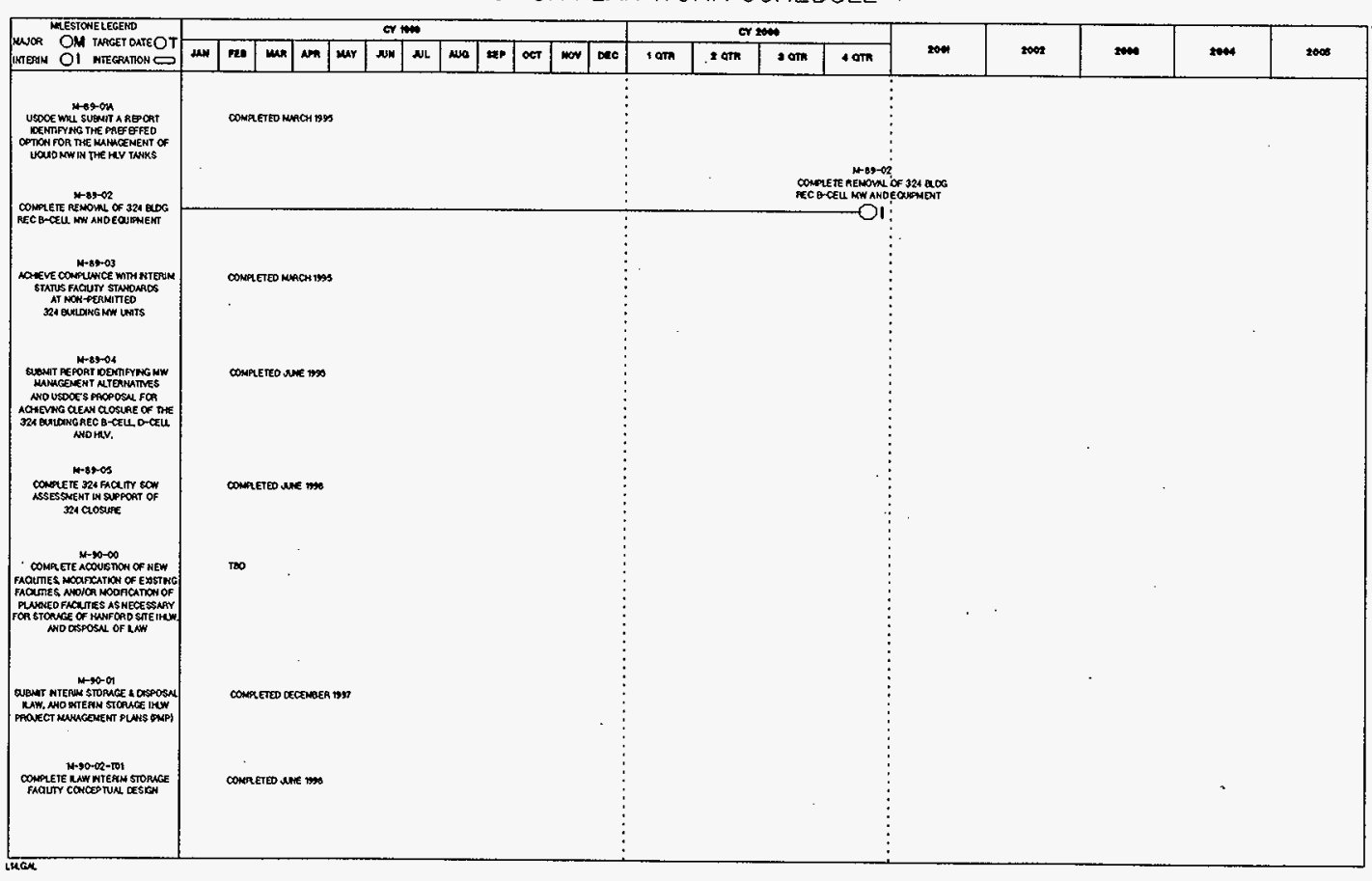


FEDERAL FACILITY AGREEMENT AND CONSENT ORDER

ACTION PLAN WORK SCHEDULE

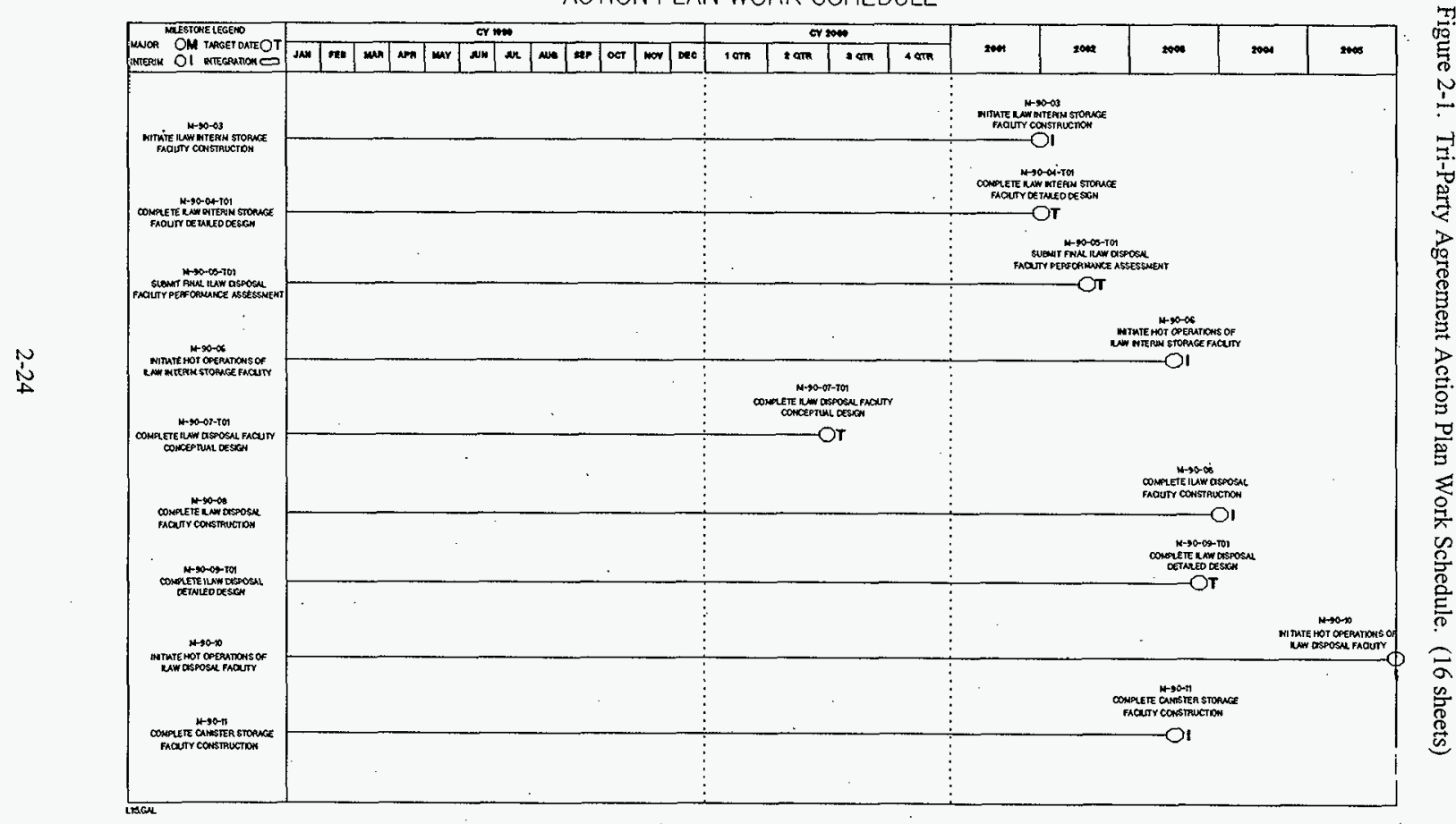


DOE/RL-99-01

Figure 2-1. Tri-Party Agreement Action Plan Work Schedule. (16 sheets)

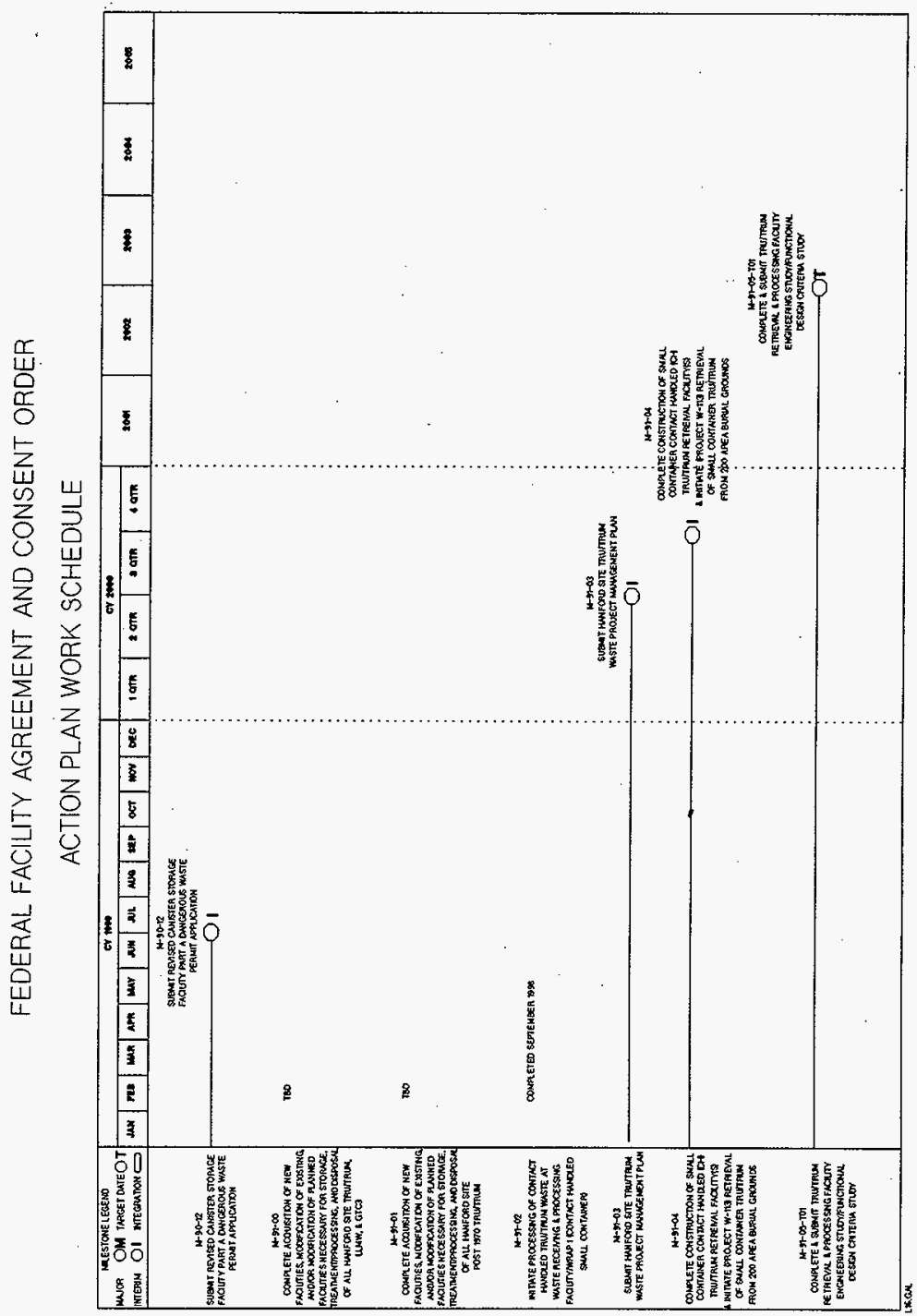


FEDERAL FACILITY AGREEMENT AND CONSENT ORDER

ACTION PLAN WORK SCHEDULE

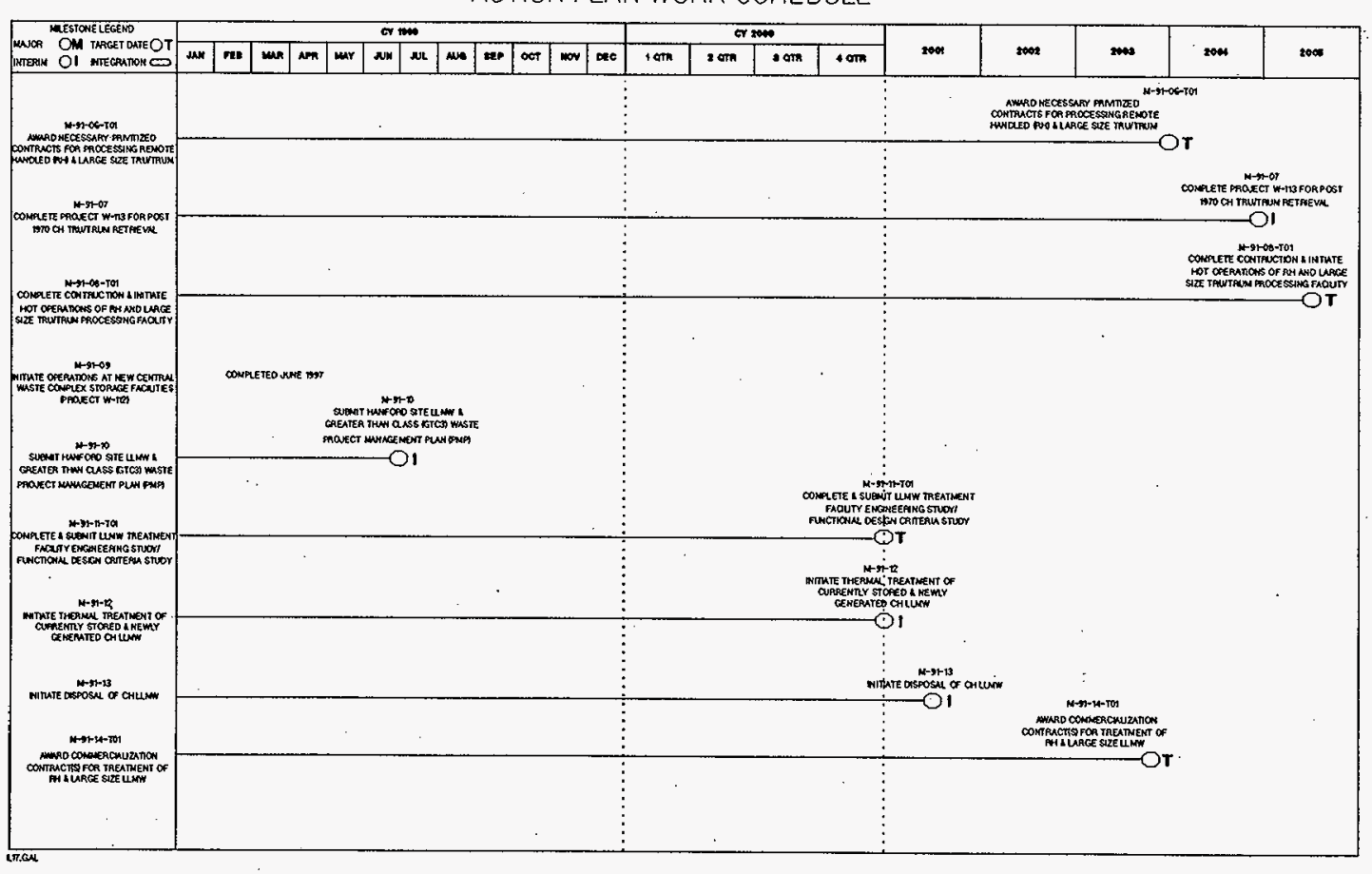


FEDERAL FACILITY AGREEMENT AND CONSENT ORDER

ACTION PLAN WORK SCHEDULE

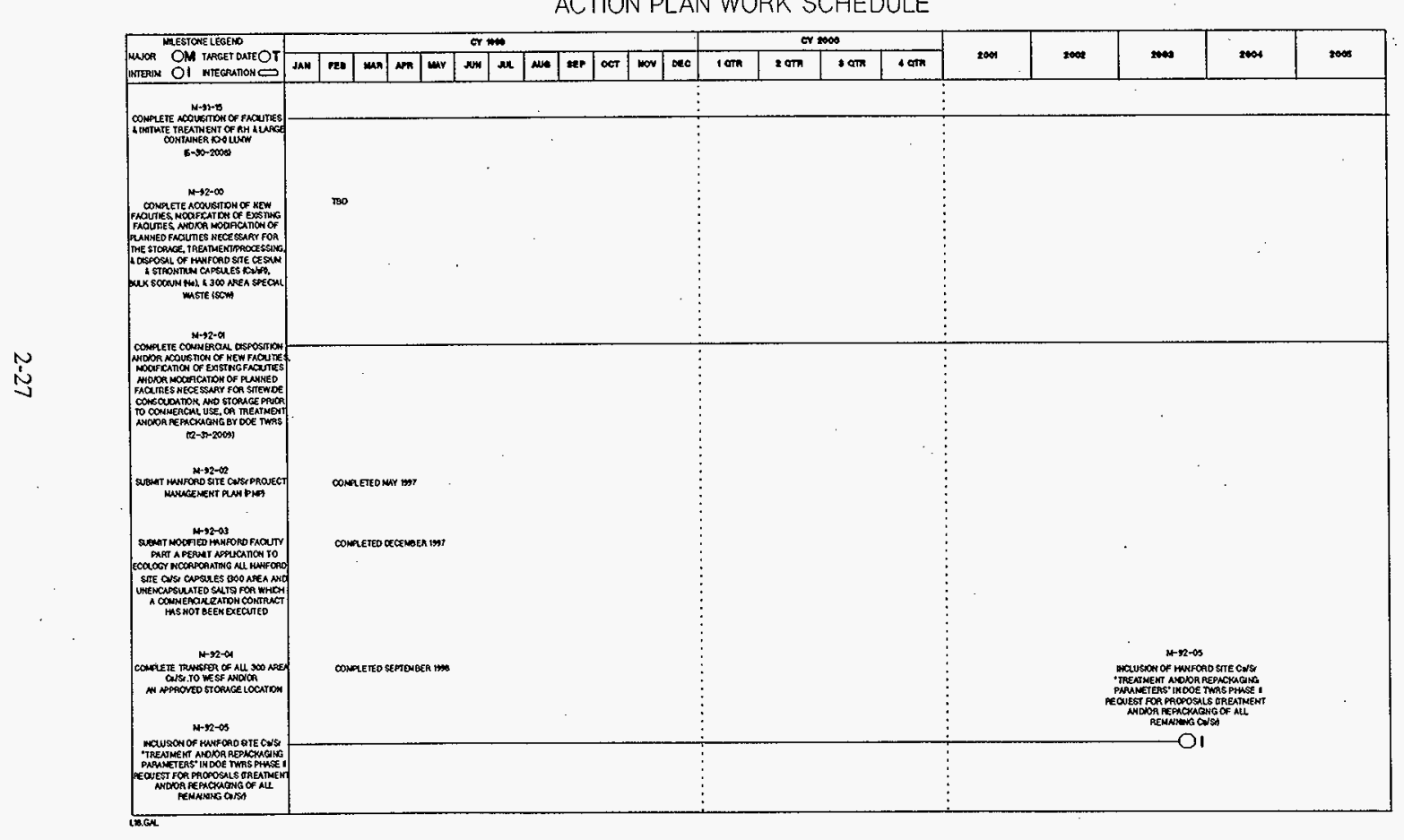


FEDERAL FACILITY AGREEMENT AND CONSENT ORDER

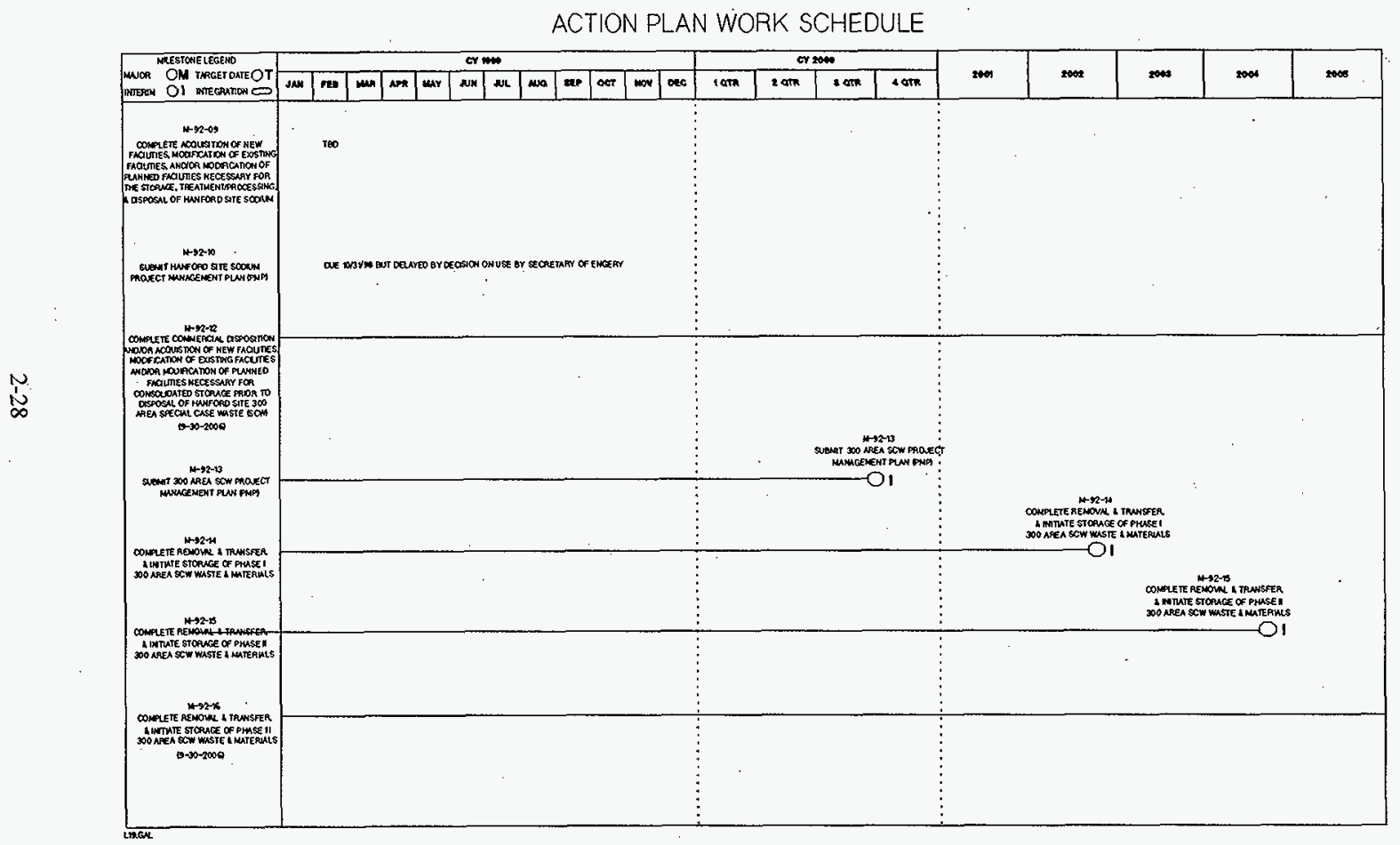




\subsubsection{Central Waste Complex Stored Waste}

The Hanford Facility's CWC is a RCRA interim-status storage unit for MLLW and TRU and TRUM waste. Some of the waste also is contaminated with polychlorinated biphenyls (PCB) and is regulated under the Toxic Substances Control Act of 1976 (TSCA).

The CWC has functioned as the Hanford Facility's main mixed waste storage location for containerized waste since 1987. During its first 10 years of operation, waste inventories at the CWC increased significantly. The lack of characterization, treatment, and disposal capabilities for handling MLLW and TRUM waste contributed to this increase.

In 1997, significantly more treatment and direct disposal activities began, which is reducing the yearly increase in the MLLW storage inventory. Additional onsite and offsite treatment activities will prepare significant volumes of MLLW for disposal. The Hanford Facility's LLMW Subtitle-C disposal site, the Subtitle-C portion of the LLBG, is expected to open for disposal operations during FY 2000; this should cause the CWC's waste inventory to decline. RCRA permit issues between DOE-Carlsbad and New Mexico State have caused shipments of mixed TRU waste for disposal at the Waste Isolation Pilot Plant (WIPP) to be postponed. A shipment schedule for mixed TRU waste is not available. The Hanford Facility contractors will continue to achieve certification status for shipment of non-mixed TRU waste to the WIPP.

\subsection{WASTE MINIMIZATION}

Annual goals have been established by both RL and contractor management for all types of waste generated at the Hanford Facility. Through the performance of waste minimization assessments and the selection of economically practicable options, the overall Site goals are translated into specific goals for each waste stream.

\subsubsection{Waste Minimization Program Elements}

Six basic elements make up the waste minimization program: top management support, characterization of waste generated and the process that generates it, waste minimization assessments, cost allocation, technology transfer, and program evaluation.

2.5.1.1 Statement of Management Support/Commitment. The RL manager and contractor management are committed to minimizing the generation of waste by giving preference to source reduction, material substitution, and environmentally sound recycling over treatment, storage, and disposal of such waste. A written policy, signed by the RL manager, covering this commitment can be found in Hanford Site Waste Minimization and Pollution Prevention Awareness Program Plan, DOE/RL-91-31 (RL 1998). Management takes appropriate action to provide adequate personnel, budget, training, and resources on a continuing basis to ensure that the objectives of the waste minimization program are met. 
Contractor management further demonstrates its support by including waste minimization training in the.Hanford general employee training program, sponsoring incentive programs that reward individual and group contributions, and including waste minimization in job performance evaluations of persons having waste minimization responsibilities.

2.5.1.2 Characterization of Waste Generation. Waste that is generated is characterized to obtain information on the quantity generated and its hazardous constituents and their concentration.

2.5.1.3 Cost Allocation System. A cost accounting system that accounts for the "true cost" of waste generated includes short- and long-term costs arising from all aspects of waste generation. These include under-use of raw materials found in the waste stream, management of the waste generated, and third-party liabilities if the waste is improperly disposed of. Associated costs will include those for personnel, record keeping, transportation, pollution control, equipment, treatment, storage, and disposal. Costs for all waste types generated at the Hanford Facility are updated annually and published in DOE/RL-97-12, Return on Investment (ROI) Proposal Preparation Guide (RL 1998).

2.5.1.4 Technology Transfer. The transfer of federally developed technology between laboratories and potential users is a contractual responsibility of DOE facilities and laboratories. Transfer of technologies specific to waste minimization may develop from information exchange systems, workshops, or topical conferences.

2.5.1.5 Program Success Criteria. The following success criteria are available to aid in demonstrating effective waste-minimization efforts:

- Reduced amount of all waste types

- Reduced waste management costs

- Improved regulatory compliance

- Reduced health risks

- Increased production efficiency

- Reduced accident risk

- Improved public relations.

\subsubsection{Program Objectives}

The objectives of the waste minimization program are as follows:

- Foster a philosophy to conserve resources and minimize waste and poliution, while achieving Hanford Facility strategic objectives.

- Promote the use of nonhazardous materials in operations to minimize the potential risks to human health and the environment. 
- Reduce or eliminate the generation of waste through input substitution, process modification, improved housekeeping, and closed-loop recycling to achieve minimal adverse effects to the air, water, and land.

- Comply with applicable federal and state regulations and DOE requirements for waste minimization, waste reduction, and pollution prevention.

- Characterize waste streams and develop a baseline of waste-generation data.

- Identify and implement methods and technologies for waste minimization.

- Target policies, procedures, or practices that may be barriers to waste minimization.

- Promote integration and coordination of waste generators and waste managers on waste minimization matters.

- Develop specific goals for waste minimization activities and schedules for achieving .those goals.

- Enhance communication of waste minimization objectives, goals, and ideas.

- Create incentives for waste minimization.

- Collect and exchange waste minimization information through technology transfer, outreach, and educational networks.

- Develop mechanisms for fully disseminating current technical information to Hanford Facility users.

\subsubsection{Location-Specific Waste Minimization}

All permittees that generate hazardous, mixed, and/or radioactive waste are required to have a waste minimization program in place. The managers of waste-generating activities on the Hanford Facility are required to certify, in writing, that a waste minimization program exists. The following are key components of the program.

- To the extent practical, all mixed waste is segregated and packaged separately from LLW or TRU waste that contains no hazardous or dangerous constituents.

- To minimize the generation of mixed waste, the generator actively seeks nondangerous alternatives for the dangerous constituents in their processes.

- Waste is characterized and the potential for minimization is investigated.

- Minimization goals are set annually and tracked quarterly. 
- Where allowed by regulation, mixed waste is treated to remove the dangerous constituents.

- Where allowed by regulation, corrosive materials are neutralized to remove their corrosive characteristics or are packaged in a manner that ensures the integrity of the containment barriers.

- Waste handling, segregation, and certification will be performed following detailed procedures when the disposal criteria are promulgated.

\subsection{TREATABILITY VARIANCES, EQUIVALENCY PETITIONS, RULEMAKING PETITIONS, AND CASE-BY-CASE EXEMPTIONS}

If variances, equivalency petitions, rulemaking petitions, or case-by-case exemptions are required because the treatment standard is not appropriate for the mixed waste or because of delays in the development of treatment, storage, or disposal capacity, they will be applied for in accordance with the procedures detailed in the Tri-Party Agreement (Ecology et al. 1989) and/or applicable regulations. Specific variances, exemptions, etc., that have been granted or applied for or are known to be needed in the future are covered in the applicable waste stream profile sheets (Chapter 3) and/or narrative (Chapter 4).

The Tri-Party Agreement provides for extending a schedule or deadline on receipt of a timely request for extension and when good cause exists for the requested extension. Any request for an extension must be submitted in writing and specify the following:

- The timetable and deadline or schedule for which the extension is sought

- The length of the extension sought

- The good cause for the extension

- Any related deadline or schedule that would be affected if the extension were granted.

Good causes for an extension include the following:

- An event of force majeure as defined in Article XLVII of the Tri-Party Agreement, subject to Ecology's reservation in Paragraph 147

- A delay caused by another party's failure to meet any requirement of the Tri-Party Agreement

- A delay caused by invocation of dispute resolution to the extent provided by Paragraph 30(f) and Paragraph 59(i) or judicial order

- A delay caused or likely to be caused by an extension granted to another deadline or schedule 
- Any other event or series of events mutually agreed to by the parties as constituting good cause. 
DOE/RL-99-01

This page intentionally left blank. 
DOE/RL-99-01

\subsection{WASTE STREAM PROFILE SHEETS}

A profile sheet has been prepared for each waste stream. These sheets make up this chapter. The following paragraphs describe the data fields of each sheet. Information augmenting the data on the profile sheets is presented in Chapter 4 . The numbers at the beginning of each of the following paragraphs refer to the section numbers on the waste stream profile sheets.

1.1 Waste stream ID. Uniquely identifies the stream.

1.2 Waste stream name. A short, descriptive name for the waste stream.

1.3.1 Stream source and history description. Describes where the waste came from, why it was generated, when it was placed into storage, and how it was managed before being stored in the current location. The generator's name is included if the waste was not generated at the Hanford Facility.

1.3.2 Waste category(s). Lists categories in a multiple-choice format. The box is checked that best describes the function or type of process that generated the waste.

1.3.3 Additional notes. Provides information on waste generation that cannot be supplied in the multiple-choice format.

2.1 Waste stream description. Briefly describes the physical contents of the stream.

2.2.1 Waste type. Lists options in a multiple-choice format. One box is checked for radiological content; another is checked to differentiate between contact- and remote-handled waste types.

2.2.2 Comments on radiological characteristics. Provides space for information on radiological characteristics of the waste that cannot be supplied in a multiple-choice format.

2.3.1 Matrix characteristics table. Amplifies the waste stream description in field 2.1. The "Matrix Parameter Category Code" is the treatability group code (e.g., S5320) from $D O E$ Treatability Group Guidance, DOE/LLW-217 (DOE 1995). The "matrix code description" is the name that applies to the code (e.g., wood debris). For some streams, one entry makes up 100 percent of the waste. "Typical" or "range (\%)" lists the estimated percentage of the waste that fits this category. The overall matrix parameter category code is the overall code from the table that describes the greatest percentage of the waste. "Overall matrix description" is the name associated with this overall code.

2.3.2 Confidence level for matrix characteristics data. Provides a subjective judgement of how accurately the physical contents of the waste are known (i.e., the data discussed in item 2.3.1). For example, a drum that has not been inspected might be ranked "low." A low or medium ranking could imply that this stream needs further characterization. 
2.3.3 Comments on matrix characteristics and/or confidence level. Provides space for information on matrix characteristics of the waste and the confidence level that cannot be supplied in the multiple-choice format.

2.4.1 Wastewater/non-wastewater under RCRA. Lists options in a multiple-choice format. The appropriate box is checked for whether, under federal LDR requirements, the waste is considered wastewater or nonwastewater. This does not apply for state-only dangerous waste.

2.4.2 Regulated contaminant table. Provides the following information in a tabular format. The "EPA/State codes" are the listed or characteristic waste codes such as D001, F005, etc. "Waste description" is the characteristic or contaminant of concern (e.g., ignitable or methyl ethyl ketone). The "LDR Subcategory" is any applicable subcategory of the waste code, e.g., Corrosive Characteristic waste or Radioactive High Level Waste for D002. The LDR subcategory only applies to D001 though D011. Some profile sheets may add the constituent of concern in this field for "F-coded" waste. Concentration of the constituent, if known, is included in the table. In many cases, the concentration is not known, so this may be left blank or "TBD." "Basis" explains how the concentration information was determined (e.g., process knowledge, laboratory analysis, etc.). The final column is for the regulatory-required method for treating the waste or the required concentration, as obtained from the applicable regulations. UHC information will be entered into this table when it applies to the waste.

\subsubsection{List of any waste codes from the table for which the stream already meets established LDR treatment standards. Self-explanatory.}

2.4.3 Does this waste stream contain PCBs? Lists options in a multiple-choice format. The appropriate box is checked for polychlorinated biphenyl content and the concentration range is indicated. The basis for the determination can be process knowledge or waste characterization.

2.4.4 What is the confidence level for the regulated contaminant characteristic data? Lists options in a multiple-choice format. This assigns a subjective rating to the accuracy of the information presented on contaminants, waste codes, etc. A low or medium rating implies that more needs to be done in this area.

\subsubsection{Comments on regulated contaminant characteristics and/or confidence level.} Provides space for comments on regulated contaminant characteristics of the waste and confidence in the accuracy of the information.

2.4.6 Will further characterization be performed? Lists options in a multiple-choice format. The appropriate box is checked and details, schedule information, and milestone numbers are given.

3.1 Is this waste stream currently stored? Lists options in a multiple-choice format. All or most streams will have "yes" checked. If the waste currently is being generated and none is stored, "no" is checked.

3.2 Current storage method. Indicates the type of storage unit. 
3.3 TSD unit and building number and (if available/applicable) number of containers/tanks in each. Provides space for listing this information in a fill-in-the-blanks format.

3.4 Is storage of waste in compliance with applicable State and Federal standards? "Yes" or "No" is checked. If waste storage is not in compliance, details are provided.

3.5 Applicable Tri-Party Agreement milestones related to storage. Includes milestone numbers related to waste storage. These include such items as construction of storage facilities, unit upgrades, and completion of compliance assessments, permitting, and environmental impact statements.

3.6 Current inventory for this stream. Gives the inventory for the stream in cubic meters. Typically the inventory listed is as of September 30,1998 . The volume is the total container volume (if boxes, drums, etc.), the internal filled tank volume, or the volume of waste that will have to be treated (if contaminated equipment, etc.). If only some other volume measurement (e.g., a gross volume) is available, this is explained in the comments section of field 3.6.

3.7 Is this waste stream currently generated; if not, will this waste stream be generated in the next 5 years? Lists options in a multiple-choice format. "Yes" is checked for streams that were being generated from April 1998 through March 1999, or will be generated in the period ending in September 2002.

3.8 An indication of whether the generation of this waste is best described as routine, or one-time/sporadic. Lists options in a multiple-choice format.

3.9 Estimated generation projection table by fiscal year. Lists options in a multiple-choice format. The table is completed for waste projected to be generated for the next 5 fiscal years.

3.10 Last year's fiscal year 1998 generation projection. Compares the actual waste quantity generated in 1998 to the quantity that was projected in last year's report. (Some quantities may be figured by calendar year instead of fiscal year, but the comparisons are consistent).

3.11 Has there ever been any unusual release of this stream to the environment? Lists options in a multiple-choice format. Provides information on releases. For the purposes of this report, emissions allowed by permits and spills that are promptly cleaned up are not considered releases to the environment and are not included in the profile sheets. Releases that must be noted include leaks that have not been cleaned up and long-term releases not permitted under RCRA.

4.1 Is this stream currently being treated? Lists options in a multiple-choice format. The appropriate treatment box is checked and details are provided if treatment is currently under way.

4.2 Planned treatment. Lists options in a multiple-choice format. The appropriate box is checked indicating plans for treating the waste stream to meet applicable regulations. 
4.3 Planned treatment method, facility, extent of treatment capacity available. Gives details of planned treatment, for both onsite TSD units and offsite facilities, as well as details of how much of the required capacity is or is not available.

4.4 Treatment schedule information. Provides space to include such information as when treatment starts and ends and how much waste will be treated each year.

4.5 Applicable Tri-Party Agreement milestone numbers, including permitting. Provides space to list appropriate milestone numbers.

4.6 If treating or planning to treat on site, was or will waste minimization be addressed in developing and/or selecting the treatment method? Provides space to describe how waste minimization will be considered in developing the treatment method.

4.7 Treatability variances, equivalency petitions, rulemaking petitions, and case-by-case exemptions. Gives details of any existing or future treatability variances (40 CFR 268.44), equivalency petitions (40 CFR 268.42(b)), rulemaking petitions (WAC 173-303-910, 40 CFR 260.20), and case-by-case exemptions (WAC 173-303-140(6)).

5.1 After treatment, how will the waste stream be disposed of? Provides space to describe methods, locations, variances required, etc., as applicable.

5.2 Ápplicable Tri-Party Agreement milestone numbers for disposal. Self-explanatory.

5.3 Planned disposal dates/time-frames. Lists any established or estimated schedules for waste disposal.

6.1 Has a waste minimization assessment been completed for the stream? Lists options in a multiple-choice format. The appropriate box is checked.

6.2 Explanation of any waste minimization activities (e.g., process changes) under way or to be implemented for generation of this stream. Provides space to write explanation.

6.3 Schedule for implementing waste minimization methods. Provides space to include waste minimization schedule.

6.4 Waste reduction achieved during the calendar year and projected future reductions. Provides space to list any assumptions used in preparing the estimates. 


\title{
WASTE STREAM PROFILE SHEET
}

\subsection{WASTE STREAM IDENTIFICATION AND SOURCE}

\author{
1.1 Waste stream ID: MLLW-01-A \\ 1.2 Waste stream name: Backlog Soils \\ 1.3 Waste stream source information
}

1.3.1 Stream source and history description. Include how the waste was managed prior to storage, timeframe when waste was placed into storage. Subject waste was generated in the early $1990 \mathrm{~s}$ through various operational activities at the 200 East and 200 West DST and SST systems. During FY 1994 and FY 1995, a significant effort (Tank Farms Backlog Waste Program) was undertaken to fully characterize this waste to the CWC storage requirements. This activity was completed in FY 1995, and all waste was put into compliant storage at the CWC. This waste was assigned the BLS (Backlog Soils) WSRd to maintain its link with the Tank Farms Backlog Waste Program.
1.3.2 Source category(s)
$\square$ Pollution control or waste treatment process $\quad \square$ Materials production/recovery effluents
Facility or equipment operation and maintenance waste
Discarded excess or expired materials $R \& D / R \& D$ laboratory waste
Other (explain):
Spill clean-ups or emergency response actions

1.3.3 Additional notes: Subject waste was selected as a Direct Disposal waste stream per DOE/RL95-35, Direct Disposal Team Report (RL 1995a).

\subsection{WASTE STREAM CHARACTERIZATION}

2.1 Waste stream description (content): This waste consists of soils (dirt, sand, gravel, rocks, etc.) that were excavated from the various waste tank farms. The waste was incidentally contaminated with tank waste; therefore, the waste is designated with F001 through F005 based on the "containedin" policy. The waste is packaged in drums and boxes. The WSRd making up this waste stream is BLS.

\subsection{Radiological characteristics}

2.2.1 Mixed Waste type $\square$ High-level $\square$ Transuranic \Low-level 囚Contact-handled $\square$ Remote-handled

2.2.2 Comments on radiological characteristics (e.g., more specific content, treatment concerns caused by radiation, confidence level): Since the waste was only incidentally contaminated with tank waste, the radiological characteristics are minimal. No shielding was used in the packaging of the waste.

\subsection{Matrix characteristics (physical content)}

2.3.1 Matrix constituent table (comprising at least $1 \%$ of the total volume or mass)

Matrix Parameter

Matrix Constituent

Typical

Category Code

Description or Range (\%) 
Overall matrix parameter category code: $\mathbf{S 4 0 0 0}$

Overall matrix description: Soil/Gravel

2.3.2 Confidence level for matrix characteristics data in 2.3.1

$\square$ Low $\square$ Medium $\bigotimes$ High

2.3.3 Comments on matrix characteristics and/or confidence level: Waste has been verified through the Backlog Waste Program per the Backlog Waste Analysis Plan (BWAP). A contained-in determination was approved for the subject waste by Ecology. The waste is acceptable for disposal into the LLW portion of Hanford's LLBGs. Note: Even though this waste carries the "BLS" PastPractice WSRd, it meets the requirements of the Waste Specification System and is ready for disposal.

\subsection{Regulated contaminant characteristics}

\subsubsection{Wastewater/non-wastewater under RCRA}

$\square$ Wastewater $\bigotimes$ Non-wastewater $\square$ Unknown

2.4.2 Regulated contaminant table including treatment requirements, and UHCs if applicable

\begin{tabular}{|c|c|c|c|c|c|}
\hline $\begin{array}{l}\text { EPA/ } \\
\text { State } \\
\text { Code }\end{array}$ & Waste Description & $\begin{array}{c}\text { LDR } \\
\text { Sub- } \\
\text { category }\end{array}$ & $\begin{array}{c}\text { Concentration } \\
\text { (Typical or } \\
\text { Range) }\end{array}$ & Basis & $\begin{array}{c}\text { LDR } \\
\text { Concentration Limit or } \\
\text { Technology Code }\end{array}$ \\
\hline F001 & $\begin{array}{c}\text { 1,1,1-Trichloro- } \\
\text { ethane }\end{array}$ & Spent sc & ent $<1 \mathrm{mg} / \mathrm{kg}$ & Analysis & $6.0 \mathrm{mg} / \mathrm{kg}$ \\
\hline F002 & Methylene Chloride & Spent sc & ent $<1 \mathrm{mg} / \mathrm{kg}$ & Analysis & $30 \mathrm{mg} / \mathrm{kg}$ \\
\hline F003 & Acetone \& Hexone & Spent so & ent $<1 \mathrm{mg} / \mathrm{kg}$ & Analysis & $160 \mathrm{mg} / \mathrm{kg}$ \\
\hline F004 & o-Cresol \& p-Cresol & Spent s & ent $<1 \mathrm{mg} / \mathrm{kg}$ & Analysis & $5.6 \mathrm{mg} / \mathrm{kg}$ \\
\hline F005 & Methyl Ethyl Ketone & Spent $\mathbf{s}$ & ent $<1 \mathrm{mg} / \mathrm{kg}$ & Analysis & $36 \mathrm{mg} / \mathrm{kg}$ \\
\hline WP02 & Persistant, DW & NA & $\mathbf{N A}$ & NA & None \\
\hline
\end{tabular}

* If waste isn't consistent in concentration, this may not apply. Describe in 2.4.5.

2.4.2.1 List any waste codes from 2.4.2 for which the stream already meets established LDR treatment standards: All hazardous constituents are below the LDR limits. Furthermore, a "contained-in" determination was granted by Ecology to allow disposal of the subject waste into the $L L W$ portion of Hanford's LLBGs.

\subsubsection{Does this waste stream contain PCBs?}

$$
\square \text { Yes } \bigotimes \text { No } \square \text { Unknown. }
$$

If no, skip to 2.4.4.

2.4.3.1 Is waste stream subject to TSCA regulations for PCBs?
$\square$ Yes $\square$ No $\square$ Unknown

2.4.3.2 Indicate the $\mathrm{PCB}$ concentration range.

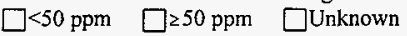

2.4.4 What is the confidence level for the regulated contaminant characteristic data?

$\square$ Low $\square$ Medium $\bigotimes$ High


2.4.5 Comments on regulated contaminant characteristics and/or confidence level: All hazardous constituents are below the LDR limits. Furthermore, a "contained-in" determination was granted by Ecology (March 1997) to allow disposal of the subject waste into the LLW portion of Hanford's LLBGs.

2.4.6 Will further characterization be performed? $\square$ Yes \No $\square$ Unknown

2.4.6.1 If yes, provide details and schedule:

2.4.6.2 If yes, provide Tri-Party Agreement milestone numbers:

\subsection{WASTE STREAM STORAGE, INVENTORY, AND GENERATION}

3.1 Is this waste stream currently stored? $\quad$ Yyes $\square$ No If no, skip to 3.7.

3.2 Current storage method

$\square$ Container (pad)
$\square$ Tank
$\square$ Other (explain)

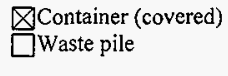

$\square$ Container (retrievably.buried)

Surface impoundment

3.3 TSD unit name and building number and (if applicable/available) number of containers/tanks in each: Central Waste Complex. Approximately 600 packages (224 cubic meters).

3.4 Is storage of waste in compliance with applicable State and Federal standards?

$\triangle$ Yes $\square$ No

If no, provide details:

3.5 Applicable Tri-Party Agreement milestones related to storage: None

3.6 Current inventory for this stream

Total LDR volume (cubic meters): 224

Date of inventory values: $9 / 30 / 98$

Comments on waste inventory: Based on inventory residing at the CWC reported in the

Solid Waste Information Tracking System (SWITS) under the "BLS" WSRd.

3.7 Is this waste stream currently generated; if not, will this waste stream be generated in the next 5 years?

$\square$ Yes $\bigotimes$ No. If no, skip to 3.11.

3.8 The current or future generation of this waste is best described as:

$\square$ Routine $\square$ One-time or sporadic

3.9 Estimated generation projection by fiscal year

$\begin{array}{lllll}1999 & \mathrm{~m}^{3} \quad \text { (and/or) } & \mathrm{kg} & \\ 2000 & & & & \\ 2001 & & & & \\ 2002 & & & & \\ 2003 & & & & \end{array}$

3.10 Last year's fiscal year 1998 generation projection: NA

Actual quantity generated in 1998: 0 (both in $\mathrm{m}^{3}$ unless otherwise noted)

3.11 Has there ever been any unusual release of this stream to the environment? 
$\square$ Yes 区No

If yes, summarize releases and quantities:

\subsection{WASTE STREAM TREATMENT}

4.1 Is this stream currently being treated?

पYes 囚No If yes, provide details:

4.2 Planned treatment: Check the appropriate box indicating future plans for treating this waste stream to meet applicable regulations, including LDR treatment standards.

冈 No treatment required (skip to 5.0)

$\square$ Treating or plan to treat onsite

Treating or plan to treat offsite

Treatment options still being assessed

4.3 Planned treatment method, facility, extent of treatment capacity available:

4.4 Treatment schedule information:

4.5 Applicable treatment Tri-Party Agreement milestone numbers (including permitting):

4.6 If treating or planning to treat on site, was or will waste minimization be addressed in developing and/or selecting the treatment method? $\square$ Yes $\square$ No $\square$ Unknown

If yes, please describe:

4.7 Treatability equivalency petitions, rulemaking petitions, and case-by-case exemptions needed for treatment:

\subsection{WASTE STREAM DISPOSAL}

5.1 After treatment, how will the waste stream be disposed of (include description, locations, variances required, etc., as applicable): Hanford LLBG (LLW portion) is planned to receive the subject waste.

5.2 Applicable Tri-Party Agreement milestone number(s) for disposal: Counts toward M-19-00 volume commitments (direct disposal).

5.3 Planned disposal dates/time-frame: FY $1999-2000$

\subsection{WASTE MINIMIZATION}

6.1 Has a waste minimization assessment been completed for this stream?

$$
\square \text { Yes } \bigotimes \text { No } \square \text { NA }
$$

6.2 Explain any waste minimization activities (e.g., process changes) under way or to be implemented for generation of this stream: . No new waste was generated during 1998. Therefore a waste assessment for this stream is not necessary and was not conducted during the calendar year.

6.3 Schedule for implementing waste minimization methods: Since subject waste is currently generated and being disposed, no additional waste minimization activities are planned.

6.4 Waste reduction achieved during the calendar year and projected future reductions (volume or mass): NA 
DOE/RL-99-01

6.4.1 Assumptions used in above estimates: NA 


\section{WASTE STREAM PROFILE SHEET}

\subsection{WASTE STREAM IDENTIFICATION AND SOURCE}

1.1 Waste stream ID: MLLW-01B

1.2 Waste stream name: General Past-Practice LDR Compliant Waste

1.3 Waste stream source information

1.3.1 Stream source and history description. Include how the waste was managed prior to storage, timeframe when waste was placed into storage. Subject waste was generated and put into CWC storage prior to the implementation of the Waste Specification System (WSS); therefore, it is considered as a past-practice waste. The waste was generated by onsite organizations and offsite generators.

\subsubsection{Source category(s)}

$\bigotimes$ Pollution control or waste treatment process $\square$ Materials production/recovery effluents

Facility or equipment operation and maintenance waste

$\triangle$ Discarded excess or expired materials

R\&D/R\&D laboratory waste

Spill clean-ups or emergency response actions

$\bigotimes$ Analytical laboratory waste

冈 Remediation/D\&D waste

Other (explain):

1.3.3 Additional notes: The waste is anticipated not to be restricted by LDRs; however, the waste will remain under dangerous waste regulation and be directly disposed into a RCRA Subtitle-C disposal cell located on the Hanford Site.

\subsection{WASTE STREAM CHARACTERIZATION}

2.1 Waste stream description (content): This waste consists of soils (dirt, sand, gravel, rocks, etc.), debris, other particulates, and solidified liquids,. All waste forms are anticipated to contain LDR compliant levels of dangerous waste constituents. The waste is packaged in drums and boxes. The current WSRds making up this waste stream are SOW and TFS.

\subsection{Radiological characteristics}

\subsubsection{Mixed Waste type $\square$ High-level $\square$ Transuranic \Low-level} $\bigotimes$ Contact-handled $\square$ Remote-handled

2.2.2 Comments on radiological characteristics (e.g., more specific content, treatment concerns caused by radiation, confidence level): Since this waste is a general category based on dangerous waste characteristics, the radiological characteristics are expected to vary greatly. However, there is high confidence that the waste is CH-MLLW.

2.3 Matrix characteristics (physical content)

2.3.1 Matrix constituent table (comprising at least $1 \%$ of the total volume or mass)

\begin{tabular}{ccc}
$\begin{array}{c}\text { Matrix Parameter } \\
\text { Category Code }\end{array}$ & $\begin{array}{c}\text { Matrix Constituent } \\
\text { Description }\end{array}$ & $\begin{array}{c}\text { Typical } \\
\text { or Range (\%) }\end{array}$ \\
\hline $\mathbf{S 4 0 0 0}$ & Soil/Gravel & $\mathbf{4 5 \%}$
\end{tabular}


Overall matrix parameter category code: $\mathbf{S}$

Overall matrix description: Solids

\subsubsection{Confidence level for matrix characteristics data in 2.3.1}

Low $\bigotimes$ Medium $\square$ High

2.3.3 Comments on matrix characteristics and/or confidence level: Subject waste will undergo matrix characterization verifications as part of the past-practice "upgrading" program. Once the waste meets all the upgrading requirements, it will be assigned to the proper WSS WSRd associated with waste stream MLLW-01C. If some of the waste does not meet direct disposal criteria (i.e., does not meet all LDRs), it will be reassigned into the appropriate waste stream that requires treatment (e.g., MLLW-02 through 06).

\subsection{Regulated contaminant characteristics}

\subsubsection{Wastewater/non-wastewater under RCRA}
$\square$ Wastewater
$\bigotimes$ Non-wastewater
Unknown

2.4.2 Regulated contaminant table including treatment requirements, and UHCs if applicable

\begin{tabular}{|c|c|c|c|c|c|}
\hline $\begin{array}{l}\text { EPA/ } \\
\text { State } \\
\text { Code }\end{array}$ & Waste Description & $\begin{array}{c}\text { LDR } \\
\text { Sub- } \\
\text { category }\end{array}$ & $\begin{array}{l}\text { Concentration } \\
\text { (Typical or } \\
\text { Range)* }\end{array}$ & Basis & $\begin{array}{c}\text { LDR } \\
\text { Concentration Limit or } \\
\text { Technology Code }\end{array}$ \\
\hline F001 & $\begin{array}{c}\text { 1,1,1-Trichloro- } \\
\text { ethane }\end{array}$ & Spent solvent & $<1 \mathrm{mg} / \mathrm{kg}$ & Process Knowledge & $6.0 \mathrm{mg} / \mathrm{kg}$ \\
\hline $\mathrm{FO02}$ & Methylene Chloride & Spent solvent & $<\mathbf{I m g} / \mathbf{k g}$ & Process Knowledge & $30 \mathrm{mg} / \mathrm{kg}$ \\
\hline F003 & Acetone \& Hexone & Spent solvent & $<$ lmg/kg & Process Knowledge & $160 \mathrm{mg} / \mathrm{kg}$ \\
\hline FoO4 & o-Cresol \& p-Cresol & Spent solvent & $<1 \mathrm{mg} / \mathrm{kg}$ & Process Knowledge & $5.6 \mathrm{mg} / \mathrm{kg}$ \\
\hline F005 & Methyl Ethyl Ketone & Spent solvent & $<1 \mathrm{mg} / \mathrm{kg}$ & Process Knowledge & $36 \mathrm{mg} / \mathrm{kg}$ \\
\hline WT02 & Toxic, DW & NA & 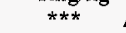 & Analysis or Knowledge & None \\
\hline WSC2 & Solid Corrosive & NA & $\mathrm{pH}>12.5$ & " & None \\
\hline W001 & State Only PCBs & NA & $1<=[P C B s]<5$ & $50 \mathrm{ppm}$ & $<50$ ppm \\
\hline UHCs & NA & & & & \\
\hline
\end{tabular}

* If waste isn't consistent in concentration, this may not apply. Describe in 2.4.5.

2.4.2.1 List any waste codes from 2.4.2 for which the stream already meets established LDR treatment standards: Initially, all dangerous waste constituents are assumed to meet LDR limits; however, subject waste will undergo characterization verifications as part of the past-practice upgrading program. Once the waste meets all the upgrading requirements, it will be assigned to the proper WSS WSRd associated with waste stream MLLW-01C. If some of the waste does not meet direct disposal criteria (i.e., does not meet all LDRs), it will be reassigned in to the appropriate waste stream that requires treatment (e.g., MLLW-02 through 06).

\subsubsection{Does this waste stream contain PCBs?}

\Yes $\square$ No $\square$ Unknown.

If no, skip to 2.4 .4 .

2.4.3.1 Is waste stream subject to TSCA regulations for PCBs?

$\square$ Yes $\triangle$ No $\square$ Unknown

2.4.3.2 Indicate the PCB concentration range. 


\section{$\bigotimes<50$ ppm $\square \geq 50$ ppm $\square$ Unknown}

2.4.4 What is.the confidence level for the regulated contaminant characteristic data?

$\square$ Low $\bigotimes$ Medium $\square$ High

2.4.5 Comments on regulated contaminant characteristics and/or confidence level: Subject waste will undergo characterization verifications as part of the past-practice upgrading program. Once the waste meets all the upgrading requirements, it will be assigned to the proper WSS WSRd associated with waste stream MLLW-01C. If some of the waste does not meet direct disposal criteria (i.e., does not meet all $L D R s$ ), it will be reassigned into the appropriate waste stream that requires treatment (e.g., MLLW-02 through 06).

\subsubsection{Will further characterization be performed? $\quad$ Yes $\square$ No $\square$ Unknown}

2.4.6.1 If yes, provide details and schedule: Currently scheduled to go through characterization upgrading in FY2001

2.4.6.2 If yes, provide Tri-Party Agreement milestone numbers: No TPA milestone is directly associated with characterization of this waste stream.

\subsection{WASTE STREAM STORAGE, INVENTORY, AND GENERATION}

3.1 Is this waste stream currently stored? $\bigotimes$ Yes $\quad \square$ No $\quad$ If no, skip to 3.7.

3.2 Current storage method

$\square$ Container (pad)
$\square$ Tank
$\square$ Other (explain):

$$
\begin{array}{ll}
\bigotimes \text { Container (covered) } & \square \text { Container (retrievably buried) } \\
\square \text { Waste pile } & \square \text { Surface impoundment }
\end{array}
$$

3.3 TSD unit name and building number and (if applicable/available) number of containers/tanks in each: There are approximately 400 packages residing in the $\mathrm{CWC}$.

3.4 Is storage of waste in compliance with applicable State and Federal standards?

$\triangle$ Yes $\square$ No

If no, provide details:

3.5 Applicable Tri-Party Agreement milestones related to storage: None

3.6 Current inventory for this stream

Total $L D R$ volume (cubic meters):

Date of inventory values: $\quad 9 / 30 / 98$

114

Comments on waste inventory: Based on inventory residing at the $\mathrm{CWC}$ as reported in the Solid Waste Information Tracking System (SWITS) for WSRds TFS and SOW.

3.7 Is this waste stream currently generated; if not, will this waste stream be generated in the next 5 years? $\square$ Yes $\bigotimes$ No. If no, skip to 3.11 .

3.8 The current or future generation of this waste is best described as:

$\square$ Routine $\square$ One-time or sporadic

3.9 Estimated generation projection by fiscal year

$\mathrm{m}^{3} \quad$ (and/or) $\mathrm{kg}$

NA

2000

$\bar{X}$ 
3.10 Last year's fiscal year 1998 generation projection: NA

Actual quantity generated in 1998: 0 (both in $\mathrm{m}^{3}$ unless otherwise noted)

3.11 Has there ever been any unusual release of this stream to the environment?

$\square$ Yes 区No

If yes, summarize releases and quantities:

\subsection{WASTE STREAM TREATMENT}

4.1 Is this stream currently being treated?

$\square$ Yes $\bigotimes$ No If yes, provide details:

4.2 Planned treatment: Check the appropriate box indicating future plans for treating this waste stream to meet applicable regulations, including LDR treatment standards.

$\bigotimes$ No treatment required (skip to 5.0 )

Treating or plan to treat onsite

Treating or plan to treat offsite

$\square$ Treatment options still being assessed

4.3 Planned treatment method, facility, extent of treatment capacity available:

4.4 Treatment schedule information:

4.5 Applicable treatment Tri-Party Agreement milestone numbers (including permitting):

4.6 If treating or planning to treat on site, was or will waste minimization be addressed in developing and/or selecting the treatment method? $\square$ Yes $\square$ No $\square$ Unknown

If yes, please describe:

4.7 Treatability equivalency petitions, rulemaking petitions, and case-by-case exemptions needed for treatment:

\subsection{WASTE STREAM DISPOSAL}

5.1 After treatment, how will the waste stream be disposed of (include description, locations, variances required, etc., as applicable): Subject waste will be disposed into a RCRA Subtitle-C disposal cell located on the Hanford Site

5.2 Applicable Tri-Party Agreement milestone number(s) for disposal: Counts toward the M-19-00 volume commitments.

5.3 Planned disposal dates/time-frame: FY2001

\subsection{WASTE MINIMIZATION}

6.1 Has a waste minimization assessment been completed for this stream?

$\square$ Yes $\bigotimes$ No $\square$ NA




\section{DOE/RL-99-01}

6.2 Explain any waste minimization activities (e.g., process changes) under way or to be implemented for generation of this stream: No new waste was generated during 1998. Therefore a waste assessment for this stream is not necessary and was not conducted during the calendar year.

6.3 Schedule for implementing waste minimization methods: Since subject waste is currently generated and being directly disposed, no additional waste minimization activities are planned.

6.4 Waste reduction achieved during the calendar year and projected future reductions (volume or mass): NA

6.4.1 Assumptions used in above estimates: NA 


\section{WASTE STREAM PROFHE SHEET}

\subsection{WASTE STREAM IDENTIFICATION AND SOURCE}

1.1 Waste stream ID: MLLW-01C

\subsection{Waste stream name: General Waste Specification System LDR-Compliant Waste}

\subsection{Waste stream source information}

1.3.1 Stream source and history description. Include how the waste was managed prior to storage, timeframe when waste was placed into storage. Subject waste was generated and put into CWC storage either after the implementation of the Waste Specification System (WSS) or has been "upgraded" from various past-practice waste streams. The waste was generated by onsite organizations.

\subsubsection{Source category(s)}

$\bigotimes$ Pollution control or waste treatment process

$\bigotimes$ Facility or equipment operation and maintenance waste

$\triangle$ Discarded excess or expired materials

$\triangle$ R\&D/R\&D laboratory waste

Spill clean-ups or emergency response actions

Other (explain):

1.3.3 Additional notes: The waste is not restricted by LDRs; however, the waste will remain under dangerous waste regulation and be directly disposed into a RCRA Subtitle-C disposal cell located on the Hanford Site.

\subsection{WASTE STREAM CHARACTERIZATION}

2.1 Waste stream description (content): This waste consists of soils (dirt, sand, gravel, rocks, etc.), debris, particulates, and solidified liquids. All waste forms contain LDR compliant levels of dangerous waste constituents. The waste is packaged in drums and boxes. Subject waste also includes the long-length contaiminated equipment (LLCE) items forecasted to be received from SST/DST systems. The current WSRds making up this waste stream are 500 (200LEF only), 903, 930 and 931.

\subsection{Radiological characteristics}

\subsubsection{Mixed Waste type $\square$ High-level $\square$ Transuranic QLow-level}

$\square$ Contact-handled $\square$ Remote-handled

2.2.2 Comments on radiological characteristics (e.g., more specific content, treatment concerns caused by radiation, confidence level): Since this waste is a general catigory based on dangerous waste characteristics, the radiological characteristics are expected to vary greatly. However, there is high confidence that the waste is CH-MLLW.

2.3 Matrix characteristics (physical content)

2.3.1 Matrix constituent table (comprising at least $1 \%$ of the total volume or mass)

$$
\text { Matrix Parameter Matrix Constituent Typical }
$$




\begin{tabular}{ccc} 
Category Code & Description & or Range (\%) \\
\hline S3150 & Soilidified Homogeneous Solids & $90 \%$ \\
S3121 & Waste Water Treatment Sludges & $\mathbf{7 \%}$ \\
S3100 & Inorganic Homogeneous Solids & $\mathbf{3 \%}$
\end{tabular}

Overall matrix parameter category code: $\mathbf{S}$

Overall matrix description: Solids

\subsubsection{Confidence level for matrix characteristics data in 2.3.1}

$\square$ Low $\square$ Medium QHigh

2.3.3 Comments on matrix characteristics and/or confidence level: This waste stream can consist of many different physical matrix characteristic types since it based on LDR requirements for disposal of a dangerious waste. There is a very high confidence that subject waste stream will not contain any physical matrix characteristics that do not meet the LDRs (i.e., free liquids).

\subsection{Regulated contaminant characteristics}

\subsubsection{Wastewater/non-wastewater under RCRA}
$\square$ Wastewater
囚Non-wastewater
Uunknown

2.4.2 Regulated contaminant table including treatment requirements, and UHCs if applicable

\begin{tabular}{|c|c|c|c|c|c|}
\hline $\begin{array}{l}\text { EPA/ } \\
\text { State } \\
\text { Code }\end{array}$ & Waste Description & $\begin{array}{c}\text { LDR } \\
\text { Sub- } \\
\text { category } \\
\end{array}$ & $\begin{array}{c}\text { Concentration } \\
\text { (Typical or } \\
\text { Range) }\end{array}$ & Basis & $\begin{array}{c}\text { LDR } \\
\text { Concentration Limit or } \\
\text { Technology Code }\end{array}$ \\
\hline D001 & Ignitable Ign & nitable Char & $* * *$ & $* * *$ & EACT \& meet 268.48 \\
\hline D002 & Corrosive & orrosive Char & $* * \star$ & *** & EACT \& meet 268.48 \\
\hline D009 & TC-Mercury & w Mercury & $<.20 \mathrm{mg} / \mathrm{I} \mathrm{TCLP}$ & $0.20 \mathrm{mg} / \mathrm{l}$ & 1 TCLP\& meet 268.48 \\
\hline 001 & $\begin{array}{c}\text { 1,1,1-Trichloro- } \\
\text { ethane }\end{array}$ & Spent solvent & $<6 \mathrm{mg} / \mathrm{kg} \quad$ Pro & cess Knowledge & $6.0 \mathrm{mg} / \mathrm{kg}$ \\
\hline 002 & Methylene Chloride $\mathrm{Sp}$ & pent solvent & $<30 \mathrm{mg} / \mathrm{kg}$ & ocess Knowledge & $30 \mathrm{mg} / \mathrm{kg}$ \\
\hline 003 & Acetone \& Hexone & Spent solvent & $<160 \mathrm{mg} / \mathrm{kg}$ & rocess Knor & $160 \mathrm{mg} / \mathrm{kg}$ \\
\hline 004 & o-Cresol \& p-Cresol S & Spent solvent & $<5.6 \mathrm{mg} / \mathrm{kg} \quad \mathrm{Pr}$ & rocess Knowledge & $5.6 \mathrm{mg} / \mathrm{kg}$ \\
\hline 005 & ethyl Ethyl Ketone, $\mathrm{Sp}$ & ent solvent & $<36 \mathrm{mg} / \mathrm{kg}$ Pro & cess Knowledge & $36 \mathrm{mg} / \mathrm{kg}$ \\
\hline 029 & cyanide & NA & $10 / 0.32 \mathrm{mg} / \mathrm{kg}$ & Anaylsis & $590 / 30 \mathrm{mg} / \mathrm{kg}$ \\
\hline 030 & Cyan & NA & $10 / 0.32 \mathrm{mg} / \mathrm{kg}$ & Anaylsis & $590 / 30 \mathrm{mg} / \mathrm{kg}$ \\
\hline 098 & Potas & NA & $10 / 0.32 \mathrm{mg} / \mathrm{kg}$ & Anaylsis & $590 / 30 \mathrm{mg} / \mathrm{kg}$ \\
\hline P106 & Sodiu & NA & $10 / 0.32 \mathrm{mg} / \mathrm{kg}$ & Anaylsis & $590 / 30 \mathrm{mg} / \mathrm{kg}$ \\
\hline P120 & ntoxide & NA & $32.3 \mathrm{mg} / \mathrm{kg}(\max )$ & Analysis & STABL \\
\hline U123 & Formic Acid (Formate) & NA & $366 \mathrm{mg} / \mathrm{kg}(\max )$ & Analysis & STABL(Equivalency) \\
\hline WT02 & Toxic, DW & NA & $* * *$ Anal & lysis or Knowledg & None \\
\hline $\begin{array}{l}\text { WSC2 } \\
\text { UHCS }\end{array}$ & $\begin{array}{l}\text { Solid Corrosive } \\
\text { see } 2.4 .2 .1\end{array}$ & NA & $\mathrm{pH}>12.5$ & & None \\
\hline
\end{tabular}

* If waste isn't consistent in concentration, this may not apply. Describe in 2.4.5.

2.4.2.1 List any waste codes from 2.4.2 for which the stream already meets established LDR treatment standards: Currently, subject waste meets all applicable LDRs including any applicable UHCs. Specific UHCs for a given waste have been treated to meet Universal Treatment Standards.

2.4.3 Does this waste stream contain PCBs?

$\square$ Yes $\square$ No $\square$ Unknown.

If no, skip to 2.4.4. 
2.4.3.1 Is waste stream subject to TSCA regulations for PCBs?
$\square$ Yes
No
$\square$ Unknown

2.4.3.2 Indicate the $\mathrm{PCB}$ concentration range.

$\square<50 \mathrm{ppm} \quad \square \geq 50 \mathrm{ppm} \quad \square$ Unknown

2.4.4 What is the confidence level for the regulated contaminant characteristic data?

$\square$ Low $\square$ Medium $\square$ High

2.4.5 Comments on regulated contaminant characteristics and/or confidence level: Subject waste is ready for disposal.

2.4.6 Will further characterization be performed? $\square$ Yes $\quad$ No $\square$ Unknown

2.4.6.1 If yes, provide details and schedule:

2.4.6.2 If yes, provide Tri-Party Agreement milestone numbers:

\subsection{WASTE STREAM STORAGE, INVENTORY, AND GENERATION}

3.1 Is this waste stream currently stored? $\square$ Yes $\square$ No If no, skip to 3.7.

3.2 Current storage method

$\square$ Container (pad)
$\square$ Tank
$\square$ Other (explain):
QContainer (covered)
$\square$ Waste pile Container (retrievably buried)
Surface impoundment

3.3 TSD unit name and building number and (if applicable/available) number of containers/tanks in each: There are approximately $\mathbf{3 1 0 0}$ packages residing in the CWC.

3.4 Is storage of waste in compliance with applicable State and Federal standards?

QYes $\square$ No

If no, provide details:

3.5 Applicable Tri-Party Agreement milestones related to storage: No TPA milestone is directly associated with storage of this waste stream.

3.6 Current inventory for this stream

Total LDR volume (cubic meters): 1019

Date of inventory values: $\quad 9 / 30 / 98$

Comments on waste inventory: Based on inventory residing at the $\mathrm{CWC}$ as reported in the Solid Waste Information Tracking System (SWITS) for WSRds 502 (200LEF only), 903, 930, and 931.

3.7 Is this waste stream currently generated; if not, will this waste stream be generated in the next 5 years? QYes $\square$ No. If no, skip to 3.11 .

3.8 The current or future generation of this waste is best described as:

$\square$ Routine $\square$ One-time or sporadic

3.9 Estimated generation projection by fiscal year

$\begin{array}{rrrrr} & \mathrm{m}^{3} & \text { (and/or) } & \mathrm{kg} & \mathrm{NA} \\ 1999 & \mathbf{0} & & & \square \\ 2000 & 1132 & & & \square\end{array}$




$\begin{array}{ll}2001 & 706 \\ 2002 & 704 \\ 2003 & 142\end{array}$

3.10 Last year's fiscal year 1998 generation projection: $\quad 76$

Actual quantity generated in 1998: 105 (both in $\mathrm{m}^{3}$ unless otherwise noted)

3.11 Has there ever been any unusual release of this stream to the environment?

$\square$ Yes 区No

If yes, summarize releases and quantities:

\subsection{WASTE STREAM TREATMENT}

4.I Is this stream currentily being treated?

$\square$ Yes $\triangle$ No If yes, provide details:

4.2 Planned treatment: Check the appropriate box indicating future plans for treating this waste stream to meet applicable regulations, including LDR treatment standards.

$\bigotimes$ No treatment required (skip to 5.0)

$\square$ Treating or plan to treat onsite

Treating or plan to treat offsite

Treatment options still being assessed

4.3 Planned treatment method, facility, extent of treatment capacity available:

4.4 Treatment schedule information:

4.5 Applicable treatment Tri-Party Agreement milestone numbers (including permitting):

4.6 If treating or planning to treat on site, was or will waste minimization be addressed in developing and/or selecting the treatment method? $\square$ Yes $\square$ No $\square$ Unknown

If yes, please describe:

4.7 Treatability equivalency petitions, rulemaking petitions, and case-by-case exemptions needed for treatment:

\subsection{WASTE STREAM DISPOSAL}

5.1 After treatment, how will the waste stream be disposed of (include description, locations, variances required, etc., as applicable): Subject waste will be disposed into a RCRA Subtitle-C disposal cell located on the Hanford Site

5.2 Applicable Tri-Party Agreement milestone number(s) for disposal: Counts toward the M-19-00 volume commitments.

5.3 Planned disposal dates/time-frame: Beginning in FY2000

\subsection{WASTE MINIMIZATION}

6.1 Has a waste minimization assessment been completed for this stream?

$\square$ Yes $\square$ No $\square$ NA 
6.2 Explain any waste minimization activities (e.g., process changes) under way or to be implemented for generation of this stream: No new waste was generated during 1998. Therefore a waste assessment for this stream is not necessary and was not conducted during the calendar year.

To the extent practical, all mixed waste is segregated and packaged separately from LLW or TRU wastes. The volume of mixed waste is reduced by in-drum compaction when possible, and where it does not interfere with future treatment activities. To minimize the generation of mixed waste, generators actively seek nondangerous alternatives for the dangerous constituents in their processes. Minimization goals are set annually and tracked quarterly, and waste treatment is used to destroy the hazardous constituents, as allowable.

6.3 Schedule for implementing waste minimization methods: Since subject waste is currently generated and being directly disposed, no additional waste minimization activities are planned.

6.4. Waste reduction achieved during the calendar year and projected future reductions (volume or mass): NA

6.4.1 Assumptions used in above estimates: NA 


\section{WASTE STREAM PROFILE SHEET}

\subsection{WASTE STREAM IDENTIFICATION AND SOURCE}

1.1 Waste stream ID: MLLW-01D

1.2 Waste stream name: Trench 34 Stored Inventory

\subsection{Waste stream source information}

1.3.1 Stream source and history description. Include how the waste was managed prior to storage, timeframe when waste was placed into storage. Subject waste originated from onsite generating organizations and offsite generators between 1987 and 1997. This waste was stored in the CWC prior to treatment, and after treatment it was put into Trench $\mathbf{3 4}$ for storage to open up more space in the CWC.

\subsubsection{Source category(s)}

$\square$ Pollution control or waste treatment process $\square$ Materials production/recovery effluents

Facility or equipment operation and maintenance waste

Discarded excess or expired materials

$\mathrm{R} \& \mathrm{D} / \mathrm{R} \& \mathrm{D}$ laboratory waste

$\square$ Spill clean-ups or emergency response actions $\square$ Source unknown

$\square$ Analytical laboratory waste

Other (explain): Nuclear reactor dismantled components

1.3.3 Additional notes: All waste residing in Trench 34 storage is LDR compliant and will be disposed in place once Trench 34 transfers from a storage mode to a disposal mode.

\subsection{WASTE STREAM CHARACTERIZATION}

2.1 Waste stream description (content): This waste consists of ten (10) macroencapsulated LongLength Contaminated Equipment (LLCE) items from the SST/DST system, twenty-two (22) macroencapsulated packages containing compacted debris waste from SST/DST system operations and maintenance, and one (1) macroencapsulated reactor core basket from the US Navy.

\subsection{Radiological characteristics}

\subsubsection{Mixed Waste type $\square$ High-level $\square$ Transuranic QLow-level \Contact-handled $\square$ Remote-handled}

2.2.2 Comments on radiological characteristics (e.g., more specific content, treatment concerns caused by radiation, confidence level): All waste is LDR compliant and packaged for disposal. Currently there are a few radiological "hot-spots" (based on dose-rate) on the LLCE items that are externally shielded by lead blankets. These blankets are not part of the waste and will be removed prior to disposal.

\subsection{Matrix characteristics (physical content)}

2.3.1 Matrix constituent table (comprising at least $1 \%$ of the total volume or mass)

\begin{tabular}{ccc}
$\begin{array}{c}\text { Matrix Parameter } \\
\text { Category Code }\end{array}$ & $\begin{array}{c}\text { Matrix Constituent } \\
\text { Description }\end{array}$ & $\begin{array}{c}\text { Typical } \\
\text { or Range (\%) }\end{array}$ \\
\hline Z1200 & Macroencapsulated Forms & $\mathbf{1 0 0 \%}$
\end{tabular}


Overall matrix parameter category code: $\quad \mathbf{Z 1 2 0 0}$

Overall matrix description: Macroencapsulated Forms

2.3.2 Confidence level for matrix characteristics data in 2.3 .1

$\square$ Low $\square$ Medium $\otimes$ High

2.3.3 Comments on matrix characteristics and/or confidence level: All of the subject waste is macroencapsulated and has been verified through the Waste Specification System. Paperwork associated with disposal is complete and is awaiting the initiation of disposal operations of Hanford's LLBG (Subtitle-C portion).

\subsection{Regulated contaminant characteristics}

2.4.1 Wastewater/non-wastewater under RCRA

$\square$ Wastewater छNon-wastewater $\square$ Unknown

2.4.2 Regulated contaminant table including treatment requirements, and UHCs if applicable

\begin{tabular}{|c|c|c|c|c|c|}
\hline $\begin{array}{l}\text { EPA/ } \\
\text { State } \\
\text { Code }\end{array}$ & Waste Description & $\begin{array}{c}\text { LDR } \\
\text { Sub- } \\
\text { category }\end{array}$ & $\begin{array}{l}\text { Concentration } \\
\text { (Typical or } \\
\text { Range) }\end{array}$ & $\begin{array}{cr} & \text { Conc } \\
\text { Basis } & \mathrm{T} \\
\end{array}$ & $\begin{array}{l}\text { LDR } \\
\text { centration Limit or } \\
\text { Technology Code }\end{array}$ \\
\hline F001 & $\begin{array}{c}\text { 1,1,1-Trichloro- } \\
\text { ethane }\end{array}$ & Spent solvent & $<100 \mathrm{mg} / \mathrm{kg}$ & Treatment Technology & (1) \\
\hline F002 & Methylene Chloride & Spent solvent & $<100 \mathrm{mg} / \mathrm{kg}$ & $"$ & (1) \\
\hline F003 & Acetone \& Hexone & Spent solvent & $<100 \mathrm{mg} / \mathrm{kg}$ & " & (1) \\
\hline F004 & o-Cresol \& p-Cresol & Spent solvent & $<100 \mathrm{mg} / \mathrm{kg}$ & $"$ & (1) \\
\hline F005 & Methyl Ethyl Ketone & Spent solvent & $<100 \mathrm{mg} / \mathrm{kg}$ & $"$ & (1) \\
\hline D007 & Chromium & NA & Unknown & $"$ & (1) \\
\hline D008 & Lead & TC-Lead & Unknown & $"$ & (1) \\
\hline WT01 & Toxic, EHW & N.A & Unknown & $"$ & None (2) \\
\hline WT02 & Toxic, DW & NA & Unknown & $"$ & None \\
\hline WP02 & Persistent, DW & NA & Unknown & $"$ & None \\
\hline
\end{tabular}

(1) Debris standards in 40 CFR 268.45

(2) Mixed extremely hazardous wastes may be land-disposed in Washington State in DOE facilities in accordance with $\mathrm{RCW} 70.105 .050(2)$.

The combination of waste codes varies on a per-package basis in accordance with WAC 173-303$070(3)$ and (5).

* If waste isn't consistent in concentration, this may not apply. Describe in 2.4.5.

2.4.2.1 List any waste codes from 2.4 .2 for which the stream already meets established LDR treatment standards: Waste has been macroencapsulated and meets the Alternative Treatment Standards for Hazardous Debris LDRs.

2.4.3 Does this waste stream contain PCBs?

$$
\square \text { Yes } \mathrm{N} \text { No } \square \text { Unknown. }
$$

If no, skip to 2.4.4. 
2.4.3.1 Is waste stream subject to TSCA regulations for PCBs?

$\square$ Yes $\square$ No $\square$ Unknown

2.4.3.2 Indicate the PCB concentration range.

$\square<50 \mathrm{ppm} \quad \square \geq 50 \mathrm{ppm} \quad \square$ Unknown

2.4.4 What is the confidence level for the regulated contaminant characteristic data?

$\square$ Low $\square$ Medium $\triangle$ High

2.4.5 Comments on regulated contaminant characteristics and/or confidence level: Subject waste has met the rigors of the Waste Specification System for waste that is ready to be disposed.

2.4.6 Will further characterization be performed? $\square$ Yes $\quad$ No $\square$ Unknown

2.4.6.1 If yes, provide details and schedule:

2.4.6.2 If yes, provide Tri-Party Agreement milestone numbers:

\subsection{WASTE STREAM STORAGE, INVENTORY, AND GENERATION}

3.1 Is this waste stream currently stored? $\bigotimes$ Yes $\square$ No If no, skip to 3.7.

3.2 Current storage method

\begin{tabular}{|c|c|c|}
\hline $\begin{array}{l}\text { 冈Container (pad) } \\
\text { Trank }\end{array}$ & $\begin{array}{l}\square \text { Container (covered) } \\
\square \text { Waste pile }\end{array}$ & $\begin{array}{l}\square \text { Container (retrievably buried) } \\
\text { Surface impoundment }\end{array}$ \\
\hline
\end{tabular}

3.3 TSD unit name and building number and (if applicable/available) number of containers/tanks in each: There are approximately 33 packages residing in the 218 W5 LLBG, Trench 34 (RCRA Subtitle-C disposal cell that is permitted for storage).

3.4 Is storage of waste in compliance with applicable State and Federal standards?

区Yes $\square$ No

If no, provide details:

3.5 Applicable Tri-Party Agreement milestones related to storage: No TPA milestone is directly associated with storage of this waste stream.

3.6 Current inventory for this stream

Total LDR volume (cubic meters):

Date of inventory values: $9 / 30 / 98$

181

Comments on waste inventory: Based on inventory residing in the 218W5 LLBG, Trench 34 as reported in the Solid Waste Information Tracking System (SWITS).

3.7 Is this waste stream currently generated; if not, will this waste stream be generated in the next 5 years?

囚Yes $\square$ No. If no, skip to 3.11 .

3.8 The current or future generation of this waste is best described as:

$\square$ Routine 叉One-time or sporadic

3.9 Estimated generation projection by fiscal year

$\begin{array}{lllll}1999 & 0^{m^{3}} & \text { (and/or) } & \mathrm{kg} & \mathrm{NA} \\ 2000 & 0 & & & \square\end{array}$




$\begin{array}{ll}2001 & 0 \\ 2002 & 0 \\ 2003 & 0\end{array}$

3.10 Last year's fiscal year 1998 generation projection: 32 Actual quantity generated in 1998: 0 (both in $\mathrm{m}^{3}$ unless otherwise noted)

3.11 Has there ever been any unusual release of this stream to the environment?

$\square$ Yes $\bigotimes$ No

If yes, summarize releases and quantities:

\subsection{WASTE STREAM TREATMENT}

4.1 Is this stream currently being treated?
$\square$ Yes \No If yes, provide details:

4.2 Planned treatment: Check the appropriate box indicating future plans for treating this waste stream to meet applicable regulations, including LDR treatment standards.

No treatment required (skip to 5.0)

Treating or plan to treat onsite

Treating or plan to treat offsite

Treatment options still being assessed

4.3 Planned treatment method, facility, extent of treatment capacity available:

4.4 Treatment schedule information:

4.5 Applicable treatment Tri-Party Agreement milestone numbers (including permitting):

4.6 If treating or planning to treat on site, was or will waste minimization be addressed in developing and/or selecting the treatment method? $\square$ Yes $\square$ No $\square$ Unknown

If yes, please describe:

4.7 Treatability equivalency petitions, rutemaking petitions, and case-by-case exemptions needed for treatment:

\subsection{WASTE STREAM DISPOSAL}

5.1 After treatment, how will the waste stream be disposed of (include description, locations, variances required, etc., as applicable): Subject waste will be disposed in place when Trench 34 transfers from a storage mode to a disposal mode.

5.2 Applicable Tri-Party Agreement milestone number(s) for disposal: M-91-13

5.3 Planned disposal dates/time-frame: Beginning in FY2000

\subsection{WASTE MINIMIZATION}

6.1 Has a waste minimization assessment been completed for this stream?
$\square$ Yes $\square$ No $\square$ NA 
6.2 Explain any waste minimization activities (e.g., process changes) under way or to be implemented for generation of this stream: No new waste was generated during 1998. Therefore a waste assessment for this stream is not necessary and was not conducted during the calendar year.

6.3 Schedule for implementing waste minimization methods: Since subject waste is currently generated and being directly disposed, no additional waste minimization activities are planned.

6.4 Waste reduction achieved during the calendar year and projected future reductions (volume or mass): NA

6.4.1 Assumptions used in above estimates: NA 


\section{WASTE STREAM PROFILE SHEET}

\subsection{WASTE STREAM IDENTIFICATION AND SOURCE}

1.1 Waste stream ID: MLLW-02A

1.2 Waste stream name: General Past-Practice Inorganic Solids \& Labpacks

\subsection{Waste stream source information}

1.3.1 Stream source and history description. Include how the waste was managed prior to storage, timeframe when waste was placed into storage. Subject waste was generated and placed into CWC storage prior to the implementation of the Waste Specification System (WSS); therefore, it is considered a past-practice waste. The waste was generated by many onsite generating organizations and offsite generators.

\subsubsection{Source category(s)}

$\bigotimes$ Pollution control or waste treatment process $\square$ Materials production/recovery effluents
$\square$ Facility or equipment operation and maintenance waste

$\square$ Discarded excess or expired materials $\square$ Analytical laboratory waste

R\&D/R\&D laboratory waste

$\triangle$ Spill clean-ups or emergency response actions

Remediation/D\&D waste

Other (explain):

1.3.3 Additional notes: This is a general waste category based on physical, chemical, radiological and regulatory factors.

\subsection{WASTE STREAM CHARACTERIZATION}

2.1 Waste stream description (content): This waste stream consists of many different inorganic solids including particulates, absorbed liquids, sladges, labpacks, paint waste, salt waste, regulated batteries, alkali metals, etc. This waste stream does not include hazardous debris other than incidential debris material comingled with the non-debris. The current WSRds making up this waste stream are ALI, BAT, ENA, HHG, IXI, LPI, PAI, SSA, H3C, H3M, and H3S.

\subsection{Radiological characteristics}

\subsubsection{Mixed Waste type $\square$ High-level $\square$ Transuranic QLow-level} 冈Contact-handled $\square$ Remote-handled

2.2.2 Comments on radiological characteristics (e.g., more specific content, treatment concerns caused by radiation, confidence level): Since this waste is a general category based on dangerous waste characteristics, the radiological characteristics are expected to vary greatly. However, there is high confidence that the waste is CH-MLLW.

2.3 Matrix characteristics (physical content)

2.3.1 Matrix constituent table (comprising at least $1 \%$ of the total volume or mass)

\begin{tabular}{ccc}
$\begin{array}{c}\text { Matrix Parameter } \\
\text { Category Code }\end{array}$ & $\begin{array}{c}\text { Matrix Constituent } \\
\text { Description }\end{array}$ & $\begin{array}{c}\text { Typical } \\
\text { or Range (\%) }\end{array}$ \\
\hline S3110 & Inorganic Particulates & $\mathbf{4 0 - 5 0 \%}$
\end{tabular}




$\begin{array}{lll}\text { X6000 } & \text { LapPacks (Inorganic only) } & \mathbf{2 5 - 3 5 \%} \\ \text { S3121 } & \text { Wastewater Treatment Sludges } & \mathbf{5 - 1 0 \%} \\ \text { X7500 } & \text { Reactive Metals } & \mathbf{5 - 1 0 \%} \\ \text { S5300 } & \text { Heterogeneous Debris } & \mathbf{5 - 1 0 \%} \\ \text { X7400 } & \text { Batteries } & <5 \%\end{array}$

Overall matrix parameter category code: $\mathbf{S 3 1 0 0}$

Overall matrix description: Inorganic Solids

2.3.2 Confidence level for matrix characteristics data in 2.3.1

$\square$ Low $\bigotimes$ Medium $\square$ High

2.3.3 Comments on matrix characteristics and/or confidence level: Subject waste will undergo matrix characterization verifications as part of the past-practice "upgrading" program. Once the waste meets all the upgrading requirements, it will be assigned to the proper WSS WSRd associated with waste stream MLLW-02B. If, during the upgrading process, it is determined that some of the waste does not meet one of the MLLW-02B waste stream WSRds, then the appropriate WSRd will be assigned to the waste, and then the waste will be transferred to the correct waste stream.

2.4 Regulated contaminant characteristics

2.4.1 Wastewater/non-wastewater under RCRA

$\square$ Wastewater $\bigotimes$ Non-wastewater $\square$ Unknown

2.4.2 Regulated contaminant table including treatment requirements, and UHCs if applicable

\begin{tabular}{|c|c|c|c|c|c|c|}
\hline $\begin{array}{l}\text { EPA/ } \\
\text { State } \\
\text { Code }\end{array}$ & Waste Descriotion & & $\begin{array}{c}\text { LDR } \\
\text { Sub- } \\
\text { category }\end{array}$ & $\begin{array}{c}\text { Concentration } \\
\text { (Typical or } \\
\text { Range) }\end{array}$ & Basis & $\begin{array}{c}\text { LDR } \\
\text { Concentration Limit or } \\
\text { Technology Code }\end{array}$ \\
\hline D001 & Ignitable & & nitable Char & $\pi * *$ & $* * *$ & DEACT \& meet 268.48 \\
\hline D002 & Corrosive & & rrosive Char & $\star * *$ & $* * *$ & DEACT \& meet 268.48 \\
\hline D003 & Reactive & & active Cyanide & $* * *$ & $* * *$ & $590 / 30 \mathrm{mg} / \mathrm{kg}$ \\
\hline D004 & TC-Arsenic & NA & & $* * *$ & $* * *$ & $5.0 \mathrm{mg} / \mathrm{TCLP}$ \\
\hline D005 & TC-Barium & NA & & $* * *$ & $* * *$ & $100 \mathrm{mg} / \mathrm{l} \mathrm{TCLP}$ \\
\hline D006 & TC-Cadmium & & dmium Char & $* * *$ & $* * *$ & $1.0 \mathrm{mg} / 1$ TCLP \\
\hline D007 & TC-Chromium & NA & & $k * *$ & $* \star \star$ & $5.0 \mathrm{mg} / 1$ TCLP \\
\hline D008 & TC-Lead & & ad Char & $* * *$ & $* * *$ & $5.0 \mathrm{mg} / \mathrm{ICLP}$ \\
\hline D009 & TC-Mercury & & wercury & $<260 \mathrm{mg} / \mathrm{kg}$ & $* * *$ & $0.20 \mathrm{mg} / 1 \mathrm{TCLP}$ \\
\hline D010 & TC-Selenium & NA & & $* * *$ & *** & $5.7 \mathrm{mg} / 1 \mathrm{TCLP}$ \\
\hline D011 & TC-Silver & NA & & $* * *$ & $* * *$ & $5.0 \mathrm{mg} / \mathrm{l}$ TCLP \\
\hline F001 & \multicolumn{3}{|c|}{ 1,1,1-trichloroethane Spent solvent } & $<6.0 \mathrm{mg} / \mathrm{kg}$ & $* * *$ & $6.0 \mathrm{mg} / \mathrm{kg}$ \\
\hline F002 & \multicolumn{2}{|c|}{ Methylene Chloride S } & Spent solvent & $<30 \mathrm{mg} / \mathrm{kg}$ & $* * *$ & $30 \mathrm{mg} / \mathrm{kg}$ \\
\hline F003 & \multicolumn{2}{|l|}{ Acetone \& Hexone } & Spent solvent & $<160 \mathrm{mg} / \mathrm{kg}$ & $* * *$ & $160 \mathrm{mg} / \mathrm{kg}$ \\
\hline F004 & \multicolumn{2}{|l|}{ o-Cresol \& p-Cresol } & Spent solvent & $<5.6 \mathrm{mg} / \mathrm{kg}$ & $* * *$ & $5.6 \mathrm{mg} / \mathrm{kg}$ \\
\hline F005 & & Spent solvent & $<36 \mathrm{mg} / \mathrm{kg}$ & $* * *$ & $36 \mathrm{mg} / \mathrm{kg}$ \\
\hline P029 & \multicolumn{2}{|c|}{ Copper cyanide } & NA 1 & $10 / 0.32 \mathrm{mg} / \mathrm{kg}$ & Anaylsis & $590 / 30 \mathrm{mg} / \mathrm{kg}$ \\
\hline P030 & \multicolumn{2}{|l|}{ Cyanides } & NA & $10 / 0.32 \mathrm{mg} / \mathrm{kg}$ & Anaylsis & $590 / 30 \mathrm{mg} / \mathrm{kg}$ \\
\hline P098 & \multicolumn{2}{|l|}{ Potassium cyanide } & NA & $10 / 0.32 \mathrm{mg} / \mathrm{kg}$ & Anaylsis & $590 / 30 \mathrm{mg} / \mathrm{kg}$ \\
\hline P106 & \multicolumn{2}{|c|}{ Sodium cyanide } & NA & $10 / 0.32 \mathrm{mg} / \mathrm{kg}$ & Anaylsis & $590 / 30 \mathrm{mg} / \mathrm{kg}$ \\
\hline $\mathbf{P} 120$ & & NA & $32.3 \mathrm{mg} / \mathrm{kg}(\max )$ & Analysis & STABL \\
\hline U123 & & & Formic Acid (Formate) & $366 \mathrm{mg} / \mathrm{kg}(\max )$ & Analysis & STABL(Equivalency) \\
\hline WT01 & Toxic, EHW & NA & & $* * *$ & $* * *$ & None (1) \\
\hline WT02 & Toxic, DW & NA & & $* * *$ & $* * *$ & None \\
\hline WP02 & Persistant, DW & NA & & $* * *$ & **** & None \\
\hline WSC2 & Solid Corrosive & NA & & $<=2.5 \mathrm{pH}$ & & ove Solid-Acid Char \\
\hline
\end{tabular}

UHCs TBD on a per-package basis during waste receipt or from characterization activities. 
(1) Mixed extremely hazardous wastes may be land-disposed in Washington State in DOE facilities in accordance with RCW 70.105.050(2).

* If waste isn't consistent in concentration, this may not apply. Describe in 2.4.5.

2.4.2.1 List any waste codes from 2.4 .2 for which the stream aiready meets established LDR treatment standards: It is assumed that all waste in this waste stream will require various levels of treatment prior to disposal

2.4.3 Does this waste stream contain PCBs?

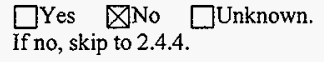

2.4.3.1 Is waste stream subject to TSCA regulations for PCBs?

$\square$ Yes $\square$ No $\square$ Unknown

2.4.3.2 Indicate the $\mathrm{PCB}$ concentration range.
$\square<50 \mathrm{ppm}$
$\square \geq 50 \mathrm{ppm}$
$\square$ Unknown

2.4.4 What is the confidence level for the regulated contaminant characteristic data?

$\square$ Low $\bigotimes$ Medium $\square$ High

2.4.5 Comments on regulated contaminant characteristics and/or confidence level: Subject waste will undergo characterization verifications as part of the past-practice upgrading program. Once the waste meets all the upgrading requirements, it will be assigned to the proper WSS WSRd associated with waste stream MLLW-02B. If, during the upgrading process, it is determined that some of the waste does not meet one of the MLLW-02B waste stream WSRds, then the appropriate WSRd will be assigned to the waste, and then the waste will be transferred to the correct waste stream.

\subsubsection{Will further characterization be performed? 叉Yes $\square$ No $\square$ Unknown}

2.4.6.1 If yes, provide details and schedule: Currently scheduled to go through characterization upgrading beginning in FY2000; however, this is contingent on other Hanford Site priorities and budget constraints.

2.4.6.2 If yes, provide Tri-Party Agreement milestone numbers: 'No TPA milestone is directly associated with characterization of this waste stream.

\subsection{WASTE STREAM STORAGE, INVENTORY, AND GENERATION}

3.1 is this waste stream currently stored? $\square Y$ es $\square$ No If no, skip to 3.7.

3.2 Current storage method

$\square$ Container (pad)
$\square$ Tank
$\square$ Other (explain):

$\begin{array}{ll}\bigotimes \text { Container (covered) } & \square \text { Container (retrievably buried) } \\ \square \text { Waste pile } & \square \text { Surface impoundment }\end{array}$

3.3 TSD unit name and building number and (if applicable/available) number of containers/tanks in each: There are approximately 1,025 packages residing in the $\mathrm{CWC}$.

3.4 Is storage of waste in compliance with applicable State and Federal standards?

$\triangle$ Yes $\square$ No 
If no, provide details:

3.5 Applicable Tri-Party Agreement milestones related to storage: No TPA milestone is directly associated with storage of this waste stream.

3.6 Current inventory for this stream

Total LDR volume (cubic meters): 229

Date of inventory values: $\quad 9 / 30 / 98$

Comments on waste inventory: Based on inventory residing at the $\mathrm{CWC}$ as reported in the Solid Waste Information Tracking System (SWITS) for WSRds: ALI, BAT, ENA, HHG, IXI, LPI, PAI, SSA, H3C, H3M, and H3S.

3.7 Is this waste stream currently generated; if not, will this waste stream be generated in the next 5 years? $\square$ Yes $\bigotimes$ No. If no, skip to 3.i1.

3.8 The current or future generation of this waste is best described as:

$\square$ Routine $\square$ One-time or sporadic

3.9 Estimated generation projection by fiscal year

$\begin{array}{lllll}1 & \mathrm{~m}^{3} \quad \text { (and/or) } & \mathrm{kg} & \\ 1999 & & & & \\ 2000 & & & & \\ 2001 & & & & \\ 2002 & & & & \end{array}$

3.10 Last year's fiscal year 1998 generation projection:

Actual quantity generated in 1998:

(both in $\mathrm{m}^{3}$ unless otherwise noted)

3.I I Has there ever been any unusual release of this stream to the environment?

$\square$ Yes QNo

If yes, summarize releases and quantities:

\subsection{WASTE STREAM TREATMENT}

4.1 Is this stream currently being treated?

$\square$ Yes $\square$ No If yes, provide details:

4.2 Planned treatment: Check the appropriate box indicating future plans for treating this waste stream to meet applicable regulations, including LDR treatment standards.

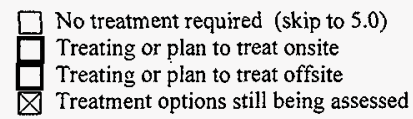

4.3 Planned treatment method, facility, extent of treatment capacity available: Because this waste is a past-practice waste stream and must undergo characterization upgrading before treatment, the specific treatment methods, facilities and schedules will not be determined until the appropriate WSS WSRd is assigned.

\subsection{Treatment schedule information: FY 2001 - FY 2008}

4.5 Applicable treatment Tri-Party Agreement milestone numbers (including permitting): Counts toward M-19-00 volume commitments (treatment). 
4.6 If treating or planning to treat on site, was or will waste minimization be addressed in developing and/or selecting the treatment method? $\square$ Yes $\square$ No $\square$ Unknown

If yes, please describe:

4.7 Treatability equivalency petitions, rulemaking petitions, and case-by-case exemptions needed for treatment:

\subsection{WASTE STREAM DISPOSAL}

5.1 After treatment, how will the waste stream be disposed of (include description, locations, variances required, etc., as applicable): Subject waste ultimately will be disposed into a RCRA Subtitle-C disposal cell located on the Hanford Site; however, the waste must under go upgrading and treatment prior to disposal.

\subsection{Applicable Tri-Party Agreement milestone number(s) for disposal: $\quad$ M-91-13}

\subsection{Planned disposal dates/time-frame: TBD}

\subsection{WASTE MINIMIZATION}

6.1 Has a waste minimization assessment been completed for this stream?
$\square$ Yes $\otimes$ No $\square$ NA

6.2 Explain any waste minimization activities (e.g., process changes) under way or to be implemented for generation of this stream: No new waste was generated during 1998. Therefore a waste assessment for this stream is not necessary and was not conducted during the calendar year.

To the extent practicaI, all mixed waste is segregated and packaged separately from LLW or TRU wastes. The volume of mixed waste is reduced by in-drum compaction when possible, and where it does not interfere with future treatment activities. To minimize the generation of mixed waste, generators actively seek nondangerous alternatives for the dangerous constituents in their processes. Minimization goals are set annually and tracked quarterly, and waste treatment is used to destroy the hazardous constituents, as allowable.

6.3 Schedule for implementing waste minimization methods: Waste minimization activities are an ongoing activity.

6.4 Waste reduction achieved during the calendar year and projected future reductions (volume or mass): None

\subsubsection{Assumptions used in above estimates: NA}




\section{WASTE STREAM PROFILE SHEET}

\subsection{WASTE STREAM IDENTIFICATION AND SOURCE}

\subsection{Waste stream ID: MLLW-02B}

\subsection{Waste stream name: General Waste Specification System Inorganic Solids and Labpacks}

\subsection{Waste stream source information}

1.3.1 Stream source and history description. Include how the waste was managed prior to storage, timeframe when waste was placed into storage. Subject waste was generated and put into CWC storage either after the implementation of the Waste Specification System (WSS) or has been "upgraded" from various past-practice waste streams. The waste was generated by many onsite generating locations and offsite generators. The primary generator for the existing waste was the closure of the 183-H Solar Basins.

\subsubsection{Source category(s)}

$\bigotimes$ Pollution control or waste treatment process

Materials production/recovery effluents

冈 Facility or equipment operation and maintenance waste

Discarded excess or expired materials $\bigotimes$ Analytical laboratory waste

\ R\&D/R\&D laboratory waste

Spill clean-ups or emergency response actions Other (explain):

1.3.3 Additional notes: This is a general waste category based on physical, chemical, radiological and regulatory factors.

\subsection{WASTE STREAM CHARACTERIZATION}

2. I Waste stream description (content): This waste stream consists of many different inorganic solicis including particulates, absorbed liquids, sludges, labpacks, paint waste, salt waste, regulated batteries, alkali metals, etc. This waste strean does not include hazardous debris other than incidental debris material comingled with the non-debris. The current WSRds making up this waste stream are 420, 421, 422, 425, 426, 428, 429, 44A, 500 (183H only), 501-0 \& 1, 503-0 \& 1, 504-0, 505 (except 505-3), 521, 523, 524, 525, 802, 812, 820, 821, 82A, 830, 900, 901, 902, 904, and 90A.

\subsection{Radiological characteristics}

\subsubsection{Mixed Waste type $\square$ High-level $\square$ Transuranic \Low-level \Contact-handled $\square$ Remote-handled}

2.2.2 Comments on radiological characteristics (e.g., more specific content, treatment concerns caused by radiation, confidence level): Since this waste is a general category based on dangerous waste characteristics, the radiological characteristics are expected to vary greatly. However, there is high confidence that the waste is CH-MLLW.

2.3 Matrix characteristics (physical content)

2.3.1 Matrix constituent table (comprising at least $1 \%$ of the total volume or mass)

Matrix Parameter

Matrix Constituent
Typical 
DOE/RL-99-01

\begin{tabular}{ccc} 
Category Code & Description & or Range (\%) \\
\hline S3121 & Wastewater Treatment Sludges & $\mathbf{9 0 - 9 5 \%}$ \\
S3120 & Misc. Inorganic Particulates & $<\mathbf{5 \%}$ \\
S5300 & Heterogeneous Debris & $<\mathbf{5 \%}$ \\
X6000 & Labpacks (Inorganic only) & $<1 \%$ \\
X7500 & Reactive Metals & $<1 \%$ \\
X7400 & Batteries & $<1 \%$
\end{tabular}

Overall matrix parameter category code: $\mathbf{S 3 1 0 0}$

Overall matrix description: Inorganic Homogeneous. Solids

2.3.2 Confidence level for matrix characteristics data in 2.3.1

$\square$ Low $\square$ Medium $\bigotimes$ High

2.3.3 Comments on matrix characteristics and/or confidence level: This waste stream can consist of many different physical matrix characteristic types since it based on LDR requirements for treatment and disposal of a dangerous waste. There is a high confidence that the subject waste stream will not contain physical matrix characteristics that do not meet the waste stream description.

\subsection{Regulated contaminant characteristics}

\subsubsection{Wastewater/non-wastewater under RCRA}

$\square$ Wastewater Non-wastewater $\square$ Unknown

2.4.2 Regulated contaminant table including treatment requirements, and UHCs if applicable

\begin{tabular}{|c|c|c|c|c|c|c|}
\hline $\begin{array}{l}\text { EPA/ } \\
\text { State } \\
\text { Code } \\
\end{array}$ & Waste Description & & $\begin{array}{l}\text { LDR } \\
\text { Sub- } \\
\text { category } \\
\end{array}$ & $\begin{array}{l}\text { Concentration } \\
\text { (Typical or } \\
\text { Range) } \\
\end{array}$ & Basis & $\begin{array}{c}\text { LDR } \\
\text { Concentration Limit or } \\
\text { Technology Code } \\
\end{array}$ \\
\hline D001 & Ignitable & & nitable Char & $k x t$ & $* * * \hbar$ & DEACT \& meet 268.48 \\
\hline D002 & Corrosive & & rrosive Char & **** & $\star \star \star \star$ & DEACT \& meet 268.48 \\
\hline D003 & Reactive & & eactive Cyanide & $* * *$ & $* * *$ & $590 / 30 \mathrm{mg} / \mathrm{kg}$ \\
\hline D004 & TC-Arsenic & NA & & $* * *$ & $* * *$ & $5.0 \mathrm{mg} / \mathrm{l} \mathrm{TCLP}$ \\
\hline D005 & TC-Barium & NA & & $* * *$ & $* * *$ & $100 \mathrm{mg} / \mathrm{I} \mathrm{TCLP}$ \\
\hline D006 & TC-Cadmium & & dmium Char & $* * *$ & $\star * *$ & $1.0 \mathrm{mg} / \mathrm{I}$ TCLP \\
\hline D007 & TC-Chromium & NA & & $* * *$ & $* * *$ & $5.0 \mathrm{mg} / \mathrm{l}$ TCLP \\
\hline D008 & TC-Lead & & ad Char & $* * *$ & $* * *$ & $5.0 \mathrm{mg} / 1 \mathrm{TCLP}$ \\
\hline D009 & TC-Mercury & & w Mercury & $<260 \mathrm{mg} / \mathrm{kg}$ & $\star * * *$ & $0.20 \mathrm{mg} / 1 \mathrm{TCLP}$ \\
\hline D010 & TC-Selenium & NA & & $* * *$ & $* * *$ & $5.7 \mathrm{mg} / \mathrm{l} \mathrm{TCLP}$ \\
\hline D011 & TC-Silver & NA & & $* * *$ & $* * *$ & $5.0 \mathrm{mg} / 1$ TCLP \\
\hline F001 & 1,1,1-trichloroetha & ne $S_{l}$ & Spent solvent & $<6.0 \mathrm{mg} / \mathrm{kg}$ & $* * *$ & $6.0 \mathrm{mg} / \mathrm{kg}$ \\
\hline F002 & Methylene Chlorid & & Spent solvent & $<30 \mathrm{mg} / \mathrm{kg}$ & $\star \star *$ & $30 \mathrm{mg} / \mathrm{kg}$ \\
\hline F003 & Acetone \& Hexone & & Spent solvent & $<160 \mathrm{mg} / \mathrm{kg}$ & $* * *$ & $160 \mathrm{mg} / \mathrm{kg}$ \\
\hline F004 & o-Cresol \& p-Cres & & Spent solvent & $<5.6 \mathrm{mg} / \mathrm{kg}$ & *** & $5.6 \mathrm{mg} / \mathrm{kg}$ \\
\hline F005 & Methyl Ethyl Keto & & Spent solvent & $<36 \mathrm{mg} / \mathrm{kg}$ & $* * *$ & $36 \mathrm{mg} / \mathrm{kg}$ \\
\hline P029 & Copper cyanide & & NA 1 & $10 / 0.32 \mathrm{mg} / \mathrm{kg}$ & Anaylsis & $590 / 30 \mathrm{mg} / \mathrm{kg}$ \\
\hline P030 & Cyanides & & NA & $10 / 0.32 \mathrm{mg} / \mathrm{kg}$ & Anaylsis & $590 / 30 \mathrm{mg} / \mathrm{kg}$ \\
\hline P098 & Potassium cyanide & & NA & $10 / 0.32 \mathrm{mg} / \mathrm{kg}$ & Anaylsis & $590 / 30 \mathrm{mg} / \mathrm{kg}$ \\
\hline P106 & Sodium cyanide & & NA & $10 / 0.32 \mathrm{mg} / \mathrm{kg}$ & Anaylsis & $590 / 30 \mathrm{mg} / \mathrm{kg}$ \\
\hline P120 & Vanadium pentoxi & & NA & $32.3 \mathrm{mg} / \mathrm{kg}(\max )$ & Analysis & STABL \\
\hline 0123 & Formic Acid (Forn & nate) & ) NA & $366 \mathrm{mg} / \mathrm{kg}(\max )$ & Analysis & STABL(Equivalency) \\
\hline WT01 & Toxic, EHW & NA & & . $\quad * * *$ & $* * *$ & None (1) \\
\hline WT02 & Toxic, DW & NA & & $* * * *$ & *** & None \\
\hline WP02 & Persistant, DW & NA & & $\star \dot{x} *$ & $\star * \star *$ & None \\
\hline WSC2 & Solid Corrosive & NA & & $<=2.5 \mathrm{pH}$ & & Remove Solid-Acid Char \\
\hline
\end{tabular}


UHCs TBD on a per-package basis during waste receipt and/or when the waste is sent for treatment (see Section 2.4.5).

(1) Mixed extremely hazardous wastes may be land-disposed in Washington State in DOE facilities in accordance with RCW 70.105.050(2).

* If waste isn't consistent in concentration, this may not apply. Describe in 2.4.5.

2.4.2.1 List any waste codes from 2.4.2 for which the stream already meets established LDR treatment standards: It is assumed that all waste in this waste stream will require various levels of treatment prior to disposal.

2.4.3 Does this waste stream contain PCBs?

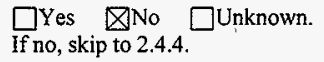

2.4.3.1 Is waste stream subject to TSCA regulations for PCBs?

$\square$ Yes $\square$ No $\square$ Unknown

2.4.3.2 Indicate the PCB concentration range.

$\square<50 \mathrm{ppm} \quad \square \geq 50 \mathrm{ppm} \quad \square$ Unknown

2.4.4 What is the confidence level for the regulated contaminant characteristic data?

$\square$ Low $\square$ Medium $\otimes$ High

2.4.5 Comments on regulated contaminant characteristics and/or confidence level: Subject waste has met the rigors of the Waste Specification System for waste storage and treatment. However, the WSS came into effect in 1995 and was based on the dangerous waste regulations imposed at that time. There have been several changes to the dangerous waste regulations since then that impose additional characterization requirements onto the generator, namely identifcation of UHCs for all waste designated with a characteristic waste code (i.e., D001 through D043).

2.4.6 Will further characterization be performed? $\quad$ Yes $\square$ No $\square$ Unknown

2.4.6.1 If yes, provide details and schedule: UHCs will be determined and identified on the corresponding LDR notification/certification at the time subject waste is sent to treatment.

2.4.6.2 If yes, provide Tri-Party Agreement milestone numbers: No TPA milestones are directly associated with characterization of this waste stream.

\subsection{WASTE STREAM STORAGE, INVENTORY, AND GENERATION}

3.1 Is this waste stream currently stored? $\quad$ Yes $\square$ No If no, skip to 3.7.

3.2 Current storage method

$\square$ Container (pad)
$\square$ Tank
$\square$ Other (explain):

$\bigotimes$ Container (covered) $\square$ Container (retrievably buried)

$\square$ Waste pile $\square$ Surface impoundment

3.3 TSD unit name and building number and (if applicable/available) number of containers/tanks in each: There are approximately $\mathbf{9 , 5 3 0}$ packages of this waste stream residing in the $\mathrm{CWC}$.

3.4 Is storage of waste in compliance with applicable State and Federal standards? 
$\triangle$ Yes $\square$ No

If no, provide details:

3.5 Applicable Tri-Party Agreement milestones related to storage: No TPA milestone is directly associated with storage of this waste stream.

3.6 Current inventory for this stream

Total LDR volume (cubic meters): $\quad \mathbf{2 8 0 7}$

Date of inventory values: $9 / 30 / 98$

Comments on waste inventory: Based on inventory residing at the $\mathrm{CWC}$ as reported in the Solid Waste Information Tracking System (SWITS) for WSRds: 420, 421, 422, 425, 426, 428, 429, 44A, 500 (183H only), 501-0 \& 1, 503-0 \& 1, 504-0, 505 (except 505-3), 521, 523, 524, 525, 802, 812, $820,821,82 \mathrm{~A}, 830,900,901,902,904$, and $90 \mathrm{~A}$.

3.7 Is this waste stream currently generated; if not, will this waste stream be generated in the next 5 years? $\square$ Yes $\square$ No. If no, skip to 3.11 .

3.8 The current or future generation of this waste is best described as:

囚Routine $\square$ One-time or sporadic

3.9 Estimated generation projection by fiscal year

$\begin{array}{lllll} & \mathrm{m}^{3} & \text { (and/or) } & \mathrm{kg} & \\ 1999 & \mathbf{2 3} & & \\ 2000 & \mathbf{2 5} & & & \\ 2001 & \mathbf{4 2} & & \\ 2002 & \mathbf{3 0} & & \\ 2003 & \mathbf{4 5} & & \square\end{array}$

3.10 Last year's fiscal year 1998 generation projection: 117

Actual quantity generated in 1998: 21 (both in $\mathrm{m}^{3}$ unless otherwise noted)

3.11 Has there ever been any unusual release of this stream to the environment?

$\square$ Yes QNo

If yes, summarize releases and quantities:

\subsection{WASTE STREAM TREATMENT}

4.1 Is this stream currently being treated?

$\square$ Yes $\bigotimes$ No If yes, provide details:

4.2 Planned treatment: Check the appropriate box indicating future plans for treating this waste stream to meet applicable regulations, including LDR treatment standards.

$\square$ No treatment required (skip to 5.0)
Treating or plan to treat onsite
Treating or plan to treat offsite
Treatment options still being assessed

4.3 Planned treatment method, facility, extent of treatment capacity available: Stabilization is targeted as the primary treatment technology for subject waste. Treatment will be performed by means of onsite and offsite commercial treatment contracts, and/or by onsite treatment units (mainly Hanford's T-Plant complex). Currently, offsite treatment would be up to 100 cubic meters in FY 2000; however, this is an optional volume per the ATG Non-Thermal Treatment contract and is not currently being planned to be exercised. The onsite treatment capacity for this type of waste is very 
limited at this time (i.e., less than 10 cubic meters per year). Additional offsite/onsite treatment capacity will need to be obtained to meet Hanford's treatment needs.

\begin{tabular}{|c|c|}
\hline 4 Treatment schedule information: & 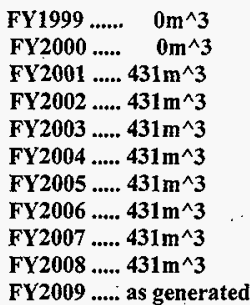 \\
\hline
\end{tabular}

4.5 Applicable treatment Tri-Party Agreement milestone numbers (including permitting): Counts toward M-19-00 volume commitments (treatment).

4.6 If treating or planning to treat on site, was or will waste minimization be addressed in developing and/or selecting the treatment method? $\bigotimes$ Yes $\square$ No $\square$ Unknown

If yes, please describe: TBD

4.7 Treatability equivalency petitions, rulemaking petitions, and case-by-case exemptions needed for treatment: Obtained a treatability equivalency from Ecology to allow stabilization in lieu of combustion treatment for formic acid (U123) for waste originating from the closure of the 183-H Solar Evaporation Basins. Hanford will utilize its organic/carbonaceous waste exemption on an asneeded basis until thermal treatment capacity becomes available for organic/carbonaceous waste.

\subsection{WASTE STREAM DISPOSAL}

5.1 After treatment, how will the waste stream be disposed of (include description, locations, variances required, etc., as applicable): - Subject waste will be disposed into a RCRA Subtitle-C disposal cell located on the Hanford Site.

5.2 Applicable Tri-Party Agreement milestone number(s) for disposal: M-91-13

5.3 Planned disposal dates/time-frame: Beginning in FY2000

\subsection{WASTE MINIMIZATION}

6.1 Has a waste minimization assessment been completed for this stream?

$$
\square \text { Yes } \bigotimes \text { No } \square \text { NA }
$$

6.2 Explain any waste minimization activities (e.g., process changes) under way or to be implemented for generation of this stream: To the extent practical, all mixed waste is segregated and packaged separately from LLW or TRU wastes. The volume of mixed waste is reduced by in-drum compaction when possible, and where it does not interfere with future treatment activities. To minimize the generation of mixed waste, generators actively seek nondangerous alternatives for the dangerous constituents in their processes. Minimization goals are set annually and tracked quarterly, and waste treatment is used to destroy the hazardous constituents, as allowable.

6.3 Schedule for implementing waste minimization methods: Waste minimization activities are an ongoing activity. 
6.4 Waste reduction achieved during the calendar year and projected future reductions (volume or mass): None

6.4.1 Assumptions used in above estimates: NA 
DOE/RL-99-01

This page intentionally left blank. 


\section{WASTE STREAM PROFILE SHEET}

\subsection{WASTE STREAM IDENTIFICATION AND SOURCE}

1.1 Waste stream ID: MLLW-03A

1.2 Waste stream name: General Past-Practice Organic Solids \& Labpacks

1.3 Waste stream source information

1.3.1. Stream source and history description. Include how the waste was managed prior to storage, timeframe when waste was placed into storage. Subject waste was generated and put into CWC storage prior to the implementation of the Waste Specification System (WSS); therefore, it is considered as a past-practice waste. The waste was generated by many onsite generating organizations and offsite generators.

\subsubsection{Source category(s)}

$\bigotimes$ Pollution control or waste treatment process $\quad \square$ Materials production/recovery effluents

$\bigotimes$ Facility or equipment operation and maintenance waste

Discarded excess or expired materials

Q R\&D/R\&D laboratory waste

Spill clean-ups or emergency response actions

$\bigotimes$ Analytical laboratory waste

\. Remediation/D\&D waste

Other (explain):

1.3.3 Additional notes: This is a general waste category based on physical, chemical, radiological and regulatory factors.

\subsection{WASTE STREAM CHARACTERIZATION}

2.1 Waste stream description (content): This waste stream consists of many different inorganic and organic solids (e.g., particulates, absorbed liquids, sludges, resins, soils) and labpacks that are contaminated with organic regulated dangerous waste constituents, including PCBs. This waste stream does not include hazardous debris other than incidental debris material comingled with the non-debris. The current WSRds making up this waste stream are ALO, LPA, LPO, PAO and TSC.

2.2 Radiological characteristics

\subsubsection{Mixed Waste type $\square$ High-level $\square$ Transuranic $\bigotimes$ Low-level} $\bigotimes$ Contact-handled $\square$ Remote-handled

2.2.2 Comments on radiological characteristics (e.g., more specific content, treatment concerns caused by radiation, confidence level): Since this waste is a general category based on dangerous waste characteristics, the radiological characteristics are expected to vary greatly. However, there is high confidence that the waste is CH-MLLW.

2.3 Matrix characteristics (physical content)

2.3.1 Matrix constituent table (comprising at least $1 \%$ of the total volume or mass)

\begin{tabular}{ccc}
$\begin{array}{c}\text { Matrix Parameter } \\
\text { Category Code }\end{array}$ & $\begin{array}{c}\text { Matrix Constituent } \\
\text { Description }\end{array}$ & $\begin{array}{c}\text { Typical } \\
\text { or Range (\%) }\end{array}$ \\
\hline X6000 & Labpacks & $\mathbf{3 5 - 4 0 \%}$
\end{tabular}


DOE/RL-99-01

$\$ 3200$

$\mathbf{S} 3210$

S3114
Misc Solids with PCBs

Organic Particulates

Absorbed Organic Liquids
$25-30 \%$

$15-20 \%$

$15-20 \%$

Overall matrix parameter category code: $\mathrm{S}$

Overall matrix description: Soljds

\subsubsection{Confidence level for matrix characteristics data in 2.3.1}

$\square$ Low $\quad$ Medium $\square$ High

2.3.3 Comments on matrix characteristics and/or confidence level: Subject waste will undergo matrix characterization verifications as part of the past-practice upgrading program. Once the waste meets all the upgrading requirements, it will be assigned to the proper WSS WSRd associated with waste stream MLLW-03B. If during the upgrading process it is determined that some of the waste does not meet one of the MLLW-03B waste stream WSRds, then the appropriate WSRd will be assigned to the waste, and then the waste will be transferred to the correct waste stream.

\subsection{Regulated contaminant characteristics}

\subsubsection{Wastewater/non-wastewater under RCRA}

$\square$ Wastewater $\bigotimes$ Non-wastewater $\square$ Unknown

\subsubsection{Regulated contaminant table including treatment requirements, and UHCs if applicable}

\begin{tabular}{|c|c|c|c|c|c|}
\hline $\begin{array}{l}\text { EPA/ } \\
\text { State } \\
\text { Code }\end{array}$ & Waste Description & $\begin{array}{c}\text { LDR } \\
\text { Sub- } \\
\text { category } \\
\end{array}$ & $\begin{array}{c}\text { Concentration } \\
\text { (Typical or } \\
\text { Range) }\end{array}$ & Basis & $\begin{array}{c}\text { LDR } \\
\text { Concentration Limit or } \\
\text { Technology Code }\end{array}$ \\
\hline D001 & Ignitable & Low TOC & $* * *$ & $* * \dot{*}$ & DEACT \& meet 268.48 \\
\hline D002 & Corrosive & Corrosive Char & $* * *$ & $* * *$ & DEACT \& meet 268.48 \\
\hline D004 & TC-Arsenic & NA & $* * *$ & $* * *$ & $5.0 \mathrm{mg} / 1 \mathrm{TCLP}$ \\
\hline D005 & TC-Barium & NA & $* * *$ & $* * *$ & $100 \mathrm{mg} / \mathrm{T} \mathrm{TCLP}$ \\
\hline D006 & TC-Cadmium & Cadmium Char & $* * *$ & $* * *$ & 1.0mg/l TCLP \\
\hline D007 & TC-Chromium & NA & $* * *$ & $* * *$ & $5.0 \mathrm{mg} / \mathrm{TCLP}$ \\
\hline D008 & TC-Lead & Lead Char & $* * *$ & $* \star \star$ & $5.0 \mathrm{mg} / 1 \mathrm{TCLP}$ \\
\hline D009 & TC-Mercury & Low Mercury & $<260 \mathrm{mg} / \mathrm{kg}$ & $* * *$ & $0.20 \mathrm{mg} / \mathrm{I}$ TCLP \\
\hline D010 & TC-Selenium & NA & $* * * 0$ & $* * *$ & $5.7 \mathrm{mg} / \mathrm{I}$ TCLP \\
\hline D011 & TC-Silver & NA & $* * *$ & $* * *$ & $5.0 \mathrm{mg} / 1 \mathrm{TCLP}$ \\
\hline D012 & Endrin & NA & $* * *$ & $* \dot{*} *$ & $0.13 \mathrm{mg} / \mathrm{kg}$ \& meet 268.48 \\
\hline D016 & 2,4-D & NA & $\star * *$ & $* * *$ & $10 \mathrm{mg} / \mathrm{kg} \&$ meet 268.48 \\
\hline D018 & Benzene & NA & $* * *$ & $* * *$ & $10 \mathrm{mg} / \mathrm{kg} \&$ meet 268.48 \\
\hline D019 & Carbon Tetrachlorid & ide $\mathbf{N A}$ & $* * *$ & $* * *$ & $6.0 \mathrm{mg} / \mathrm{kg} \&$ meet 268.48 \\
\hline D020 & Chlordane & NA & $* * *$ & $* * *$ & $0.26 \mathrm{mg} / \mathrm{kg} \&$ meet 268.48 \\
\hline D021 & Chlorobenzene & NA & $* * *$ & $* * *$ & $6.0 \mathrm{mg} / \mathrm{kg} \mathrm{\&} \mathrm{meet} 268.48$ \\
\hline D022 & Chloroform & NA & $\star \star \star \star *$ & $* * *$ & $6.0 \mathrm{mg} / \mathrm{kg} \&$ meet 268.48 \\
\hline D023 & o-Cresol & NA & $* * *$ & $* * *$ & $5.6 \mathrm{mg} / \mathrm{kg} \&$ meet 268.48 \\
\hline D026 & Cresol & NA & $* * *$ & $* * * *$ & $11.2 \mathrm{mg} / \mathrm{kg} \&$ meet 268.48 \\
\hline D027 & p-Dichlorobenzene I & NA & $\star \star * *$ & $* * *$ & $6.0 \mathrm{mg} / \mathrm{kg} \&$ meet 268.48 \\
\hline D028 & 1,2-Dichloroethane & NA & $* * *$ & $* * *$ & $6.0 \mathrm{mg} / \mathrm{kg} \&$ meet 268.48 \\
\hline D029 & 1,1-Dichlorethylene & $\mathbf{N A}$ & $* * *$ & $* * *$ & $6.0 \mathrm{mg} / \mathrm{kg} \&$ meet 268.48 \\
\hline D030 & 2,4-Dinitrotoluene & NA & $* * *$ & $* * *$ & $140 \mathrm{mg} / \mathrm{kg} \&$ meet 268.48 \\
\hline D031 & Heptachlor & NA & $* * *$ & $* * *$ & $0.066 \mathrm{mg} / \mathrm{kg} \&$ meet 268.48 \\
\hline D033 & Hexachlorobutadien & ne NA & $* * *$ & $* * * *$ & $5.6 \mathrm{mg} / \mathrm{kg} \&$ meet 268.48 \\
\hline D034 & Hexachloroethane & NA & $* * *$ & $* * *$ & $30 \mathrm{mg} / \mathrm{kg} \&$ meet 268.48 \\
\hline D035 & Methyl Ethyl Ketone & NA & $* * *$ & $* * *$ & $36 \mathrm{mg} / \mathrm{kg} \mathrm{\&} \mathrm{meet} 268.48$ \\
\hline D036 & Nitrobenzene & NA & *** & $* * *$ & $14 \mathrm{mg} / \mathrm{kg} \&$ meet 268.48 \\
\hline D037 & Pentachlorophenol & NA & $\star * \star *$ & $* * *$ & $7.4 \mathrm{mg} / \mathrm{kg} \&$ meet 268.48 \\
\hline
\end{tabular}




\begin{tabular}{|c|c|c|c|c|c|}
\hline D038 & Pyridine & NA & $* * *$ & $* * *$ & $16 \mathrm{mg} / \mathrm{kg} \&$ meet 268.48 \\
\hline D039 & Tetracholorethylene & $\mathbf{N A}$ & $* * *$ & $* * *$ & $6.0 \mathrm{mg} / \mathrm{kg} \&$ meet 268.48 \\
\hline D040 & Trichloroethylene & NA & $\star * * *$ & $* * *$ & $6.0 \mathrm{mg} / \mathrm{kg} \mathrm{\&} \mathrm{meet} 268.48$ \\
\hline D043 & Vinyl Chloride & NA & $* * *$ & $* * *$ & $6.0 \mathrm{mg} / \mathrm{kg} \&$ meet 268.48 \\
\hline F001 & 1,1,1-Trichloroethane $S$ & Spent solvent & $\star \star \star$ & $* * *$ & $6.0 \mathrm{mg} / \mathrm{kg}$ \\
\hline $\mathrm{F} 002$ & Methylene Chloride & Spent solvent & $* * *$ & $* * *$ & $30 \mathrm{mg} / \mathrm{kg}$ \\
\hline F003 & Acetone \& Hexone & Spent solvent & $* * *$ & $* * *$ & $160 \mathrm{mg} / \mathrm{kg}$ \\
\hline F004 & o-Cresol \& p-Cresol & Spent solvent & $* * *$ & $* * *$ & $5.6 \mathrm{mg} / \mathrm{kg}$ \\
\hline F005 & Methyl Ethyl Ketone & Spent solvent & $* * *$ & $* * *$ & $36 \mathrm{mg} / \mathrm{kg}$ \\
\hline $\mathrm{F} 022$ & Process Waste Tetra-, & , penta-, or hexa & hloro-benzenes & $* * *$ & Various \\
\hline P012 & Arsenic Acid & NA & $* * *$ & $* * *$ & $5.0 \mathrm{mg} / \mathrm{l}$ \\
\hline P022 & Carbon Disulfide & NA & $\star * *$ & $* * *$ & CMBST \\
\hline P023 & Chloroacetaldehyde & NA & $* * *$ & $\star \star \star$ & CMBST \\
\hline $\mathbf{P} 030$ & Cyanide & NA & $* * *$ & $* * *$ & $590 / 30 \mathrm{mg} / \mathrm{kg}$ \\
\hline P102 & Propargyl Alcohol & NA & $* * *$ & $* * *$ & CMBST \\
\hline U001 & Acetaldehyde & NA & $* * *$ & $* * *$ & CMBST \\
\hline $\mathrm{UeO2}$ & Acetone & NA & $* * *$ & $* * *$ & $160 \mathrm{mg} / \mathrm{kg}$ \\
\hline U003 & Acetonitrile & NA & $* * *$ & $\star * *$ & CMBST \\
\hline U004 & Acetophenone & NA & $* * *$ & $* * *$ & $9.7 \mathrm{mg} / \mathrm{kg}$ \\
\hline U006 & Acetyl Chloride & NA & $* * *$ & $* * *$ & CMBST \\
\hline U019 & Benzene & NA & $* * *$ & $* * *$ & $10 \mathrm{mg} / \mathrm{kg}$ \\
\hline U025 & Bis(2-Chloroethyl)ether & NA & $* * *$ & $* * *$ & $6.0 \mathrm{mg} / \mathrm{kg}$ \\
\hline U031 & n-Butyl Alcohol & $\mathbf{N A}$ & $* * *$ & $\approx * *$ & $2.6 \mathrm{mg} / \mathrm{kg}$ \\
\hline U044 & Chloroform & NA & $* * *$ & $* * *$ & $6.0 \mathrm{mg} / \mathrm{kg}$ \\
\hline U056 & Cyclohexane & NA & $\star \star *$ & $* * *$ & CMBST \\
\hline U057 & Cyclohexanone & NA & ** & $* * \dot{\pi}$ & CMBST \\
\hline U080 & Methylene Chloride & NA & $* * *$ & $* * *$ & $30 \mathrm{mg} / \mathrm{kg}$ \\
\hline U103 & Dimethyl Sulfate & NA & $\star * *$ & $* * *$ & CMBST \\
\hline $\mathrm{U} 108$ & 1,4-Dioaxane & NA & $* * *$ & $* * *$ & CMBST \\
\hline U112 & Ethyl Acetate & NA & $* * *$ & $* * *$ & $33 \mathrm{mg} / \mathrm{kg}$ \\
\hline U117 & Ethyl Ether & NA & $\star * * \dot{\pi}$ & $* * *$ & $160 \mathrm{mg} / \mathrm{kg}$ \\
\hline U121 & Trichloromonofluorome & ethane NA & $* * *$ & $* * *$ & $30 \mathrm{mg} / \mathrm{kg}$ \\
\hline $\mathrm{U} 123$ & Formic Acid & $\mathbf{N A}$ & $\star \star * x$ & $* * *$ & CMBST \\
\hline U133 & Hydrazine & NA & $* * *$ & $* * *$ & CMBST \\
\hline U134 & Hydrogen Fluoride & NA & $* * *$ & $* * *$ & NEUTR \\
\hline $\mathrm{U} 144$ & Lead Acetate & $\mathbf{N A}$ & $* * *$ & $* * *$ & $0.37 \mathrm{mg} / \mathrm{kg}$ \\
\hline U154 & Methanol & NA & $* \star *$ & $\star * *$ & CMBST \\
\hline U159 & Methyl Ethyl Ketone & NA & $* * *$ & $* * *$ & $36 \mathrm{mg} / \mathrm{kg}$ \\
\hline U160 & Methyl Ethyl Ketone Pe & eroxide NA & $* * *$ & $* * *$ & CMBST \\
\hline U161 & Methyl Isobutyl Ketone & $\mathbf{N A}$ & $* * *$ & $* * *$ & $33 \mathrm{mg} / \mathrm{kg}$ \\
\hline U162 & Methyl Methacrylate & NA & $\star \star \star$ & $* * *$ & $160 \mathrm{mg} / \mathrm{kg}$ \\
\hline $\mathrm{U} 165$ & Naphthalene & NA & $* \star *$ & $\star * *$ & $5.6 \mathrm{mg} / \mathrm{kg}$ \\
\hline U169 & Nitrobenzene & $\mathbf{N A}$ & $* * *$ & $* * *$ & $14 \mathrm{mg} / \mathrm{kg}$ \\
\hline U170 & p-Nitropropane & NA & $* * *$ & $* * *$ & $29 \mathrm{mg} / \mathrm{kg}$ \\
\hline U187 & Phenacetin & NA & $* * *$ & $* * *$ & $16 \mathrm{mg} / \mathrm{kg}$ \\
\hline U188 & Phenol & NA & $* * *$ & $* * *$ & $6.2 \mathrm{mg} / \mathrm{kg}$ \\
\hline U189 & Phosphorus Sulfide & NA & $* * *$ & $\star \star *$ & CMBST \\
\hline U196 & Pyridine & NA & $* *$ & $* * *$ & $16 \mathrm{mg} / \mathrm{kg}$ \\
\hline $\mathrm{U} 203$ & Safrole & NA & $* * *$ & $* * *$ & $22 \mathrm{mg} / \mathrm{kg}$ \\
\hline $\mathrm{U} 210$ & Tetracholorethylene & NA & $* * *$ & $\star * *$ & $6.0 \mathrm{mg} / \mathrm{kg}$ \\
\hline U211 & Carbon Tetrachloride & $\mathbf{N A}$ & $* * *$ & $* * *$ & $6.0 \mathrm{mg} / \mathrm{kg}$ \\
\hline $\mathrm{U} 213$ & Tetrahydrofuran & NA & $* * *$ & $* * *$ & CMBST \\
\hline U218 & Thioacetamide & $\mathbf{N A}$ & $* \star \star \star$ & $\star * *$ & CMBST \\
\hline $\mathrm{U} 220$ & Toluene & NA & $* * *$ & $* * *$ & $10 \mathrm{mg} / \mathrm{kg}$ \\
\hline $\mathrm{U} 226$ & 1,1,1-Trichloroethane & NA & $* * *$ & $* * *$ & $6.0 \mathrm{mg} / \mathrm{kg}$ \\
\hline $\mathrm{U} 228$ & Trichloroethylene & NA & $* * *$ & $* * *$ & $6.0 \mathrm{mg} / \mathrm{kg}$ \\
\hline U239 & Xylenes & NA & $* * *$ & $* * *$ & $30 \mathrm{mg} / \mathrm{kg}$ \\
\hline
\end{tabular}




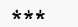

WT01 Toxic, EHW

WT02 Toxic, DW

WP01 Persistant, EHW

WP02 Persistant, DW

WSC2 Solid Corrosive

$\begin{array}{ll}\text { NA } & * * * \\ \text { NA } & * * * \\ \text { NA } & * * * \\ \text { NA } & * * * \\ \text { NA } & * * * \\ \text { NA } & \end{array}$

$* * *$

$* * *$

$* * *$

$* * *$

$\star \star * *$

$\star \star \star *$
CMBST

None (1)

None

None (1)

None

Remove Solid-Acid Char

UHCs TBD on a per-package basis during waste receipt or from characterization activities.

(1) Mixed extremely hazardous wastes may be land-disposed in Washington State in DOE facilities in accordance with RCW 70.105.050(2).

* If waste isn't consistent in concentration, this may not apply. Describe in 2.4.5.

2.4.2.1 List any waste codes from 2.4 .2 for which the stream already meets established LDR treatment standards: It is assumed that all waste in this waste stream will require various levels of treatment prior to disposal

2.4.3 Does this waste stream contain PCBs?

\Yes $\square$ No $\square$ Unknown.

If no, skip to 2.4 .4 .

2.4.3.1 Is waste stream subject to TSCA regulations for PCBs?

囚Yes $\square$ No $\square$ Unknown

2.4.3.2 Indicate the $\mathrm{PCB}$ concentration range.

$\bigotimes<50 \mathrm{ppm} \quad \bigotimes \geq 50 \mathrm{ppm} \square$ Unknown

2.4.4 What is the confidence level for the regulated contaminant characteristic data?

$\square$ Low 囚Medium $\square$ High

2.4.5 Comments on regulated contaminant characteristics and/or confidence level: Subject waste will undergo characterization verifications as part of the past-practice upgrading program. Once the waste meets all the upgrading requirements, it will be assigned to the proper WSS WSRd associated with waste stream MLLW-03B. If during the upgrading process it is determined that some of the waste does not meet one of the MLLW-03B waste stream WSRds, then the appropriate WSRd will be assigned to the waste, and then the waste will be transferred to the correct waste stream.

Some of the waste in this waste stream does contain PCBs subject to TSCA regulation. If a waste package is regulated by TSCA, it is identified as such on storage records and tracked in SWITS.

2.4.6 Will further characterization be performed? $\quad$ Yes $\square$ No $\square$ Unknown

2.4.6.1 If yes, provide details and schedule: Currently scheduled to go through characterization upgrading beginning in FY2000; however, this is contingent on other Hanford Site priorities and budget constrants.

2.4.6.2 If yes, provide Tri-Party Agreement milestone numbers: No TPA milestone is directly associated with characterization of this waste stream.

\subsection{WASTE STREAM STORAGE, INVENTORY, AND GENERATION}

3.1 Is this waste stream currently stored? ?Yes $\square$ No If no, skip to 3.7. 
3.2 Current storage method

$\square$ Container (pad)
$\square$ Tank
$\square$ Other (explain):

冈Container (covered) $\square$ Waste pile $\square$ Container (retrievably buried)

$\square$ Surface impoundment

3.3 TSD unit name and building number and (if applicable/available) number of containers/tanks in each: There are approximately 1,185 packages residing in the $\mathrm{CWC}$.

3.4 Is storage of waste in compliance with applicable State and Federal standards?

$\triangle$ Yes $\square$ No

If no, provide details:

3.5 Applicable Tri-Party Agreement milestones related to storage: No TPA milestone is directly associated with storage of this waste stream.

3.6 Current inventory for this stream

Total LDR volume (cubic meters): 275

Date of inventory values: $9 / 30 / 98$

Comments on waste inventory: Based on inventory residing at the $\mathrm{CWC}$ as reported in the Solid Waste Information Tracking System (SWITS) for WSRds: ALO, LPA, LPO, PAO and TSC.

3.7 Is this waste stream currently generated; if not, will this waste stream be generated in the next 5 years? $\square$ Yes $\bigotimes$ No. If no, skip to 3.11.

3.8 The current or future generation of this waste is best described as:

$\square$ Routine $\square$ One-time or sporadic

3.9 Estimated generation projection by fiscal year

$\begin{array}{lllll} & \mathrm{m}^{3} & \text { (and/or) } & \mathrm{kg} & \\ 1999 & & & & \text { МA } \\ 2000 & & & & \\ 2001 & & & & \\ 2002 & & & & \\ 2003 & & & \end{array}$

3.10 Last year's fiscal year 1998 generation projection:

Actual quantity generated in 1998:

(both in $\mathrm{m}^{3}$ unless otherwise noted)

3.11 Has there ever been any unusual release of this stream to the environment?

$\square$ Yes QNo

If yes, summarize releases and quantities:

\subsection{WASTE STREAM TREATMENT}

4.1 Is this stream currently being treated?

$\square$ Yes $\square$ No If yes, provide details:

4.2 Planned treatment: Check the appropriate box indicating future plans for treating this waste stream to meet applicable regulations, including. LDR treatment standards.
No treatment required (skip to 5.0)
Treating or plan to treat onsite
$\triangle$ Treating or plan to treat of fsite
Treatment options still being assessed 
4.3 Planned treatment method, facility, extent of treatment capacity available: Because this waste is a past-practice.waste stream, it must undergo characterization upgrading before treatment. Once upgraded, majority of this waste will be treated by thermal treatment by an offsite commercial waste treatment unit.

\subsection{Treatment schedule information: FY2001 through FY2008}

4.5 Applicable treatment Tri-Party Agreement milestone numbers (including permitting): Counts toward M-91-12 volume commitments (treatment).

4.6 If treating or planning to treat on site, was or will waste minimization be addressed in developing and/or selecting the treatment method? $\square$ Yes $\square$ No $\square$ Unknown

If yes, please describe:

4.7 Treatability equivalency petitions, rulemaking petitions, and case-by-case exemptions needed for treatment:

\subsection{WASTE STREAM DISPOSAL}

5.1 After treatment, how will the waste stream be disposed of (include description, locations, variances required, etc., as applicable): Subject waste uitimately will be disposed into a RCRA Subtitle-C disposal cell located on the Hanford Site; however, the waste must undergo upgrading and treatment prior to disposal.

5.2 Applicable Tri-Party Agreement milestone number(s) for disposal: $\quad$ M-91-13

5.3 Planned disposal dates/time-frame: TBD

\subsection{WASTE MINIMIZATION}

6.1 Has a waste minimization assessment been completed for this stream?

$$
\square \text { Yes } \square \text { No } \square \text { NA }
$$

6.2 Explain any waste minimization activities (e.g., process changes) under way or to be implemented for generation of this stream: No new waste was generated during 1998. Therefore a waste assessment for this stream is not necessary and was not conducted during the calendar year.

To the extent practical, all mixed waste is segregated and packaged separately from LLW or TRU wastes. The volume of mixed waste is reduced by in-drum compaction when possible, and where it does not interfere with future treatment activities. To minimize the generation of mixed waste, generators actively seek nondangerous alternatives for the dangerous constituents in their processes. Minimization goals are set annually and tracked quarterly, and waste treatment is used to destroy the hazardous constituents, as allowable.

6.3 Schedule for implementing waste minimization methods: Waste minimization activities are an ongoing activity.

6.4 Waste reduction achieved during the calendar year and projected future reductions (volume or mass): None

\subsubsection{Assumptions used in above estimates: NA}




\title{
WASTE STREAM PROFILE SHEET
}

\subsection{WASTE STREAM IDENTIFICATION AND SOURCE}

\author{
1.1 Waste stream ID: MLLW-03B
}

1.2 Waste stream name: General Waste Specification System Organic Solids and Labpacks

1.3 Waste stream source information

1.3.1 Stream source and history description. Include how the waste was managed prior to storage, timeframe when waste was placed into storage. Subject waste was generated and put into CWC storage either after the implementation of the Waste Specification System (WSS) or it has been "upgraded" from various past-practice waste streams. The waste has been generated by many onsite generating organizations and offsite generators.

\subsubsection{Source category(s)}

$\bigotimes$ Pollution control or waste treatment process $\quad \square$ Materials production/recovery effluents

Facility or equipment operation and maintenance waste

Discarded excess or expired materials

$\triangle \mathrm{R} \& D / R \& D$ laboratory waste

Spill clean-ups or emergency response actions

$\bigotimes$ Analytical laboratory waste

$\square$ Other (explain):

1.3.3 Additional notes: This is a general waste category based on physical, chemical, radiological and regulatory factors.

\subsection{WASTE STREAM CHARACTERIZATION}

2.1 Waste stream description (content): This waste stream consists of many different inorganic and organic solids (e.g., particulates, absorbed liquids, sludges, resins, soils) and labpacks that are contaminated with organic regulated dangerous waste constituents, including PCBs. This waste stream does not include hazardous debris other then incidental debris material comingled with the non-debris. The current WSRds making up this waste stream are $300,301,302,303,304,305,310$, $311,320,321,330,331,31 \mathrm{~A}, 400,401,402,403,404,405,406,407,408,40 \mathrm{~A}, 40 \mathrm{~B}, 427,430,431,432$, 45A, 47A, 500 (except 183H), 501-2, 502 (except 200LEF), 503-2, 504-1, 505-3, 506, 507, 50A, 50B, $50 \mathrm{C}, 520,522,52 \mathrm{~A}, 53 \mathrm{~A}, 700,701,720,721,920,921,922$, and 923.

2.2 Radiological characteristics

2.2.1 Mixed Waste type $\square$ High-level $\square$ Transuranic $\bigotimes$ Low-level \Contact-handled $\square$ Remote-handled

2.2.2 Comments on radiological characteristics (e.g., more specific content, treatment concerns caused by radiation, confidence level): Since this waste is a general cafegory based on dangerous waste characteristics, the radiological characteristics are expected to vary greatly. However, there is high confidence that the waste is CH-MLLW.

2.3 Matrix characteristics (physical content)

2.3.1 Matrix constituent table (comprising at least $1 \%$ of the total volume or mass) 
DOE/RL-99-01

\begin{tabular}{ccc}
$\begin{array}{c}\text { Matrix Parameter } \\
\text { Category Code }\end{array}$ & $\begin{array}{c}\text { Matrix Constituent } \\
\text { Description }\end{array}$ & $\begin{array}{c}\text { Typical } \\
\text { or Range ( } \%)\end{array}$ \\
\hline S4100 & Soils/Gravel (Org. contaminants) & $35-40 \%$ \\
X6000 & Labpacks & $25-30 \%$ \\
S & Misc. Solids with PCBs & $15-20 \%$ \\
S3210 & Organic Particulates & $15-20 \%$
\end{tabular}

Overall matrix parameter category code: $\mathrm{S}$

Overall matrix description: Solids

2.3.2 Confidence level for matrix characteristics data in 2.3.1 $\quad \square$ Low $\square$ Medium $\quad$ High

2.3.3 Comments on matrix characteristics and/or confidence level: This waste stream can consist of many different physical matrix characteristic types since it based on LDR requirements for treatment and disposal of a dangerous waste. There is a high confidence that subject waste stream will not contain physical matrix characteristics that do not meet the waste stream description.

\subsection{Regulated contaminant characteristics}

\subsubsection{Wastewater/non-wastewater under RCRA}

$\square$ Wastewater \Non-wastewater $\square$ Unknown

2.4.2 Regulated contaminant table including treatment requirements, and UHCs if applicable

\begin{tabular}{|c|c|c|c|c|c|}
\hline $\begin{array}{l}\text { EPA/ } \\
\text { State } \\
\text { Code } \\
\end{array}$ & Waste Description & $\begin{array}{c}\text { LDR } \\
\text { Sub- } \\
\text { category } \\
\end{array}$ & $\begin{array}{c}\text { Concentration } \\
\text { (Typical or } \\
\text { Range)* }\end{array}$ & Basis & $\begin{array}{c}\text { LDR } \\
\text { Concentration Limit or } \\
\text { Technology Code }\end{array}$ \\
\hline D001 & Ignitable & Low TOC & $\star * *$ & $\star \star \star \star *$ & DEACT \& meet 268.48 \\
\hline D002 & Corrosive & Corrosive Char & $* * *$ & $* * *$ & DEACT \& meet 268.48 \\
\hline D004 & TC-Arsenic & NA & $* * \dot{x}$ & $* * *$ & $5.0 \mathrm{mg} / \mathrm{l}$ TCLP \\
\hline D005 & TC-Barium & NA & $* * *$ & $* * *$ & $100 \mathrm{mg} / \mathrm{l} \mathrm{TCLP}$ \\
\hline D006 & TC-Cadmium & Cadmium Char & $* * *$ & $* * *$ & $1.0 \mathrm{mg} / \mathrm{I} \mathrm{TCLP}$ \\
\hline D007 & TC-Chromium & NA & $\star * *$ & $* * *$ & $5.0 \mathrm{mg} / \mathrm{l} \mathrm{TCLP}$ \\
\hline D008 & TC-Lead & Lead Char & $* * *$ & $* * *$ & $5.0 \mathrm{mg} / \mathrm{I}$ TCLP \\
\hline D009 & TC-Mercury & Low Mercury & $<260 \mathrm{mg} / \mathrm{kg}$ & $* * *$ & $0.20 \mathrm{mg} / \mathrm{l} \mathrm{TCLP}$ \\
\hline D010 & TC-Selenium & NA & $* * *$ & $* * *$ & $5.7 \mathrm{mg} / \mathrm{TCLP}$ \\
\hline D011 & TC-Silver & NA & *** & $* * *$ & $5.0 \mathrm{mg} / \mathrm{T} \mathrm{TCLP}$ \\
\hline D012 & Endrin & NA & **** & $\star * * *$ & $0.13 \mathrm{mg} / \mathrm{kg} \&$ meet 268.48 \\
\hline D016 & 2,4-D & NA & $\star \star *$ & $* * *$ & $10 \mathrm{mg} / \mathrm{kg} \&$ meet 268.48 \\
\hline D018 & Benzene & NA & $* * *$ & $* * *$ & $10 \mathrm{mg} / \mathrm{kg} \&$ meet 268.48 \\
\hline D019 & Carbon Tetrachlorid & ide NA & $* * *$ & $* * *$ & $6.0 \mathrm{mg} / \mathrm{kg} \&$ meet 268.48 \\
\hline D020 & Chlordane & NA & *** & $\star * *$ & $0.26 \mathrm{mg} / \mathrm{kg} \&$ meet 268.48 \\
\hline D021 & Chlorobenzene & NA & $* * *$ & $* * *$ & $6.0 \mathrm{mg} / \mathrm{kg} \&$ meet 268.48 \\
\hline D022 & Chioroform & NA & $* * *$ & $* * *$ & $6.0 \mathrm{mg} / \mathrm{kg} \&$ meet 268.48 \\
\hline D023 & 0 -Cresol & NA & *** & $\star * \star$ & $5.6 \mathrm{mg} / \mathrm{kg} \&$ meet 268.48 \\
\hline D026 & Cresol & NA & $* * *$ & $* * *$ & $11.2 \mathrm{mg} / \mathrm{kg} \&$ meet 268.48 \\
\hline D027 & p-Dichlorobenzene & NA & $* * *$ & $* * *$ & $6.0 \mathrm{mg} / \mathrm{kg} \&$ meet 268.48 \\
\hline D028 & 1,2-Dichloroethane & NA & $* * *$ & $* * *$ & $6.0 \mathrm{mg} / \mathrm{kg} \&$ meet 268.48 \\
\hline D029 & 1,1-Dichlorethylene & NA & *** & *** & $6.0 \mathrm{mg} / \mathrm{kg} \&$ meet 268.48 \\
\hline D030 & 2,4-Dinitrotoluene & NA & $* * *$ & $* * *$ & $140 \mathrm{mg} / \mathrm{kg} \&$ meet 268.48 \\
\hline D031 & Heptachlor & NA & $* * *$ & $* * *$ & $0.066 \mathrm{mg} / \mathrm{kg} \&$ meet 268.48 \\
\hline D033 & Hexachlorobutadien & ne NA & $* * *$ & $* * *$ & $5.6 \mathrm{mg} / \mathrm{kg} \&$ meet 268.48 \\
\hline D034 & Hexachloroethane & NA & $\div * * *$ & $* * *$ & $30 \mathrm{mg} / \mathrm{kg} \mathrm{\&} \mathrm{meet} 268.48$ \\
\hline D035 & Methyl Ethyl Ketone & NA & $* * *$ & *** & $36 \mathrm{mg} / \mathrm{kg}$ \& meet 268.48 \\
\hline D036 & Nitrobenzene & NA & $* * *$ & $* * *$ & $14 \mathrm{mg} / \mathrm{kg} \&$ meet 268.48 \\
\hline
\end{tabular}




\begin{tabular}{|c|c|c|c|c|c|}
\hline D037 & Pentachlorophenol & NA & $* * *$ & $* * *$ & $7.4 \mathrm{mg} / \mathrm{kg} \&$ meet 268.48 \\
\hline D038 & Pyridine & NA & $* * *$ & $* * *$ & $16 \mathrm{mg} / \mathrm{kg} \&$ meet 268.48 \\
\hline D039 & Tetracholorethylene & NA & $* * *$ & $* * *$ & $6.0 \mathrm{mg} / \mathrm{kg} \&$ meet 268.48 \\
\hline D040 & Trichloroethylene & NA & $* * * *$ & $* \star *$ & $6.0 \mathrm{mg} / \mathrm{kg} \&$ meet 268.48 \\
\hline D043 & Vinyl Chloride & NA & $\star * *$ & $* \star * *$ & $6.0 \mathrm{mg} / \mathrm{kg} \&$ meet 268.48 \\
\hline F001 & 1,1,1-Trichloroethane $S_{p}$ & Spent solvent & $* * *$ & $* * *$ & $6.0 \mathrm{mg} / \mathrm{kg}$ \\
\hline F002 & Methylene Chloride S & Spent solvent & $* * *$ & $* * *$ & $30 \mathrm{mg} / \mathrm{kg}$ \\
\hline F003 & Acetone \& Hexone & Spent solvent & $\star * \star$ & $* * *$ & $160 \mathrm{mg} / \mathrm{kg}$ \\
\hline F004 & o-Cresol \& p-Cresol & Spent solvent & $* * *$ & $* * *$ & $5.6 \mathrm{mg} / \mathrm{kg}$ \\
\hline F005 & Methyl Ethyl Ketone & Spent solvent & $\star * *$ & $* * *$ & $36 \mathrm{mg} / \mathrm{kg}$ \\
\hline F022 & Process Waste Tetra-, & penta-, or hexach & hloro-benzenes & $* * *$ & Various \\
\hline P012 & Arsenic Acid & NA & $* * *$ & $* * *$ & $5.0 \mathrm{mg} / \mathrm{l}$ \\
\hline P022 & Carbon Disulfide & NA & $* * *$ & $\star * *$ & CMBST \\
\hline P023 & Chloroacetaldehyde & NA & $* * *$ & $* * *$ & CMBST \\
\hline P030 & Cyanide & NA & $* * *$ & $* * *$ & $590 / 30 \mathrm{mg} / \mathrm{kg}$ \\
\hline P102 & Propargyl Alcohol & NA & $\star * *$ & $* * *$ & CMBST \\
\hline U001 & Acetaldehyde & NA & $* * *$ & $* * *$ & CMBST \\
\hline U002 & Acetone & NA & $* * *$ & $* * *$ & $160 \mathrm{mg} / \mathrm{kg}$ \\
\hline U003 & Acetonitrile & NA & $* * *$ & $* * *$ & CMBST \\
\hline U004 & Acetophenone & NA & $* * *$ & $* * *$ & $9.7 \mathrm{mg} / \mathrm{kg}$ \\
\hline U006 & Acetyl Chloride & NA & $* * *$ & $* * *$ & CMBST \\
\hline U019 & Benzene & NA & $* * *$ & $* \dot{*} *$ & $10 \mathrm{mg} / \mathrm{kg}$ \\
\hline U025 & Bis(2-Chloroethyl)ether & $\mathrm{NA}$ & $* * *$ & $* * *$ & $6.0 \mathrm{mg} / \mathrm{kg}$ \\
\hline U031 & n-Butyl Alcohol & NA & $\star \star \star *$ & $* * *$ & $2.6 \mathrm{mg} / \mathrm{kg}$ \\
\hline U044 & Chloroform & NA & $* * *$ & $* * *$ & $6.0 \mathrm{mg} / \mathrm{kg}$ \\
\hline U056 & Cyclohexane & NA & $* * *$. & $* * *$ & CMBST \\
\hline U057 & Cyclohexanone & $\mathbf{N A}$ & $* * *$ & $* * *$ & CMBST \\
\hline U080 & Methylene Chloride & NA & $* * *$ & $* * *$ & $30 \mathrm{mg} / \mathrm{kg}$ \\
\hline U103 & Dimethyl Sulfate & NA & $* * *$ & $* * * *$ & CMBST \\
\hline U108 & 1,4-Dioaxane & NA & $\star \star \star \star$ & $\star * * *$ & CMBST \\
\hline U112 & Ethyl Acetate & NA & $* * *$ & $* * *$ & 33 mg/kg \\
\hline U117 & Ethyl Ether & NA & $* * *$ & $* * *$ & $160 \mathrm{mg} / \mathrm{kg}$ \\
\hline U121 & Trichloromonofiuoromet & ethane NA & $* \star *$ & $* * *$ & $30 \mathrm{mg} / \mathrm{kg}$ \\
\hline U123 & Formic Acid & NA & $* * *$ & $* * *$ & CMBST \\
\hline U133 & Hydrazine & NA & $* * *$ & $* * *$ & CMBST \\
\hline U134 & Hydrogen Fluoride & NA & $\star \star \star \star$ & $\star * *$ & NEUTR \\
\hline U144 & Lead Acetate & NA & $* * *$ & $\star \star * *$ & $0.37 \mathrm{mg} / \mathrm{kg}$ \\
\hline U154 & Methanol & NA & $* * *$ & $* * *$ & CMBST \\
\hline U159 & Methyl Ethyl Ketone & $\mathbf{N A}$ & $k * k$ & $* * *$ & $36 \mathrm{mg} / \mathrm{kg}$ \\
\hline U160 & Methyl Ethyl Ketone Per & roxide NA & $* * *$ & $\star \star \star *$ & CMBST \\
\hline U161 & Methyl Isobutyl Ketone & NA & $* * *$ & $* * *$ & $33 \mathrm{mg} / \mathrm{kg}$ \\
\hline U162 & Methyl Methacrylate & NA & $* * *$ & $* * * *$ & $160 \mathrm{mg} / \mathrm{kg}$ \\
\hline U165 & Naphthalene & NA & $\dot{* * *}$ & $* * *$ & $5.6 \mathrm{mg} / \mathrm{kg}$ \\
\hline U169 & Nitrobenzene & NA & $* * *$ & $* * \star$ & $14 \mathrm{mg} / \mathrm{kg}$ \\
\hline U170 & p-Nitropropane & NA & $* * *$ & $* \star * *$ & $29 \mathrm{mg} / \mathrm{kg}$ \\
\hline U187 & Phenacetin & NA & $* * *$ & $* \star *$ & $16 \mathrm{mg} / \mathrm{kg}$ \\
\hline U188 & Phenol & $\mathbf{N A}$ & $* * *$ & $* * *$ & $6.2 \mathrm{mg} / \mathrm{kg}$ \\
\hline U189 & Phosphorus Sulfide & NA & $* * *$ & $* * *$ & CMBST \\
\hline U196 & Pyridine & NA & $* * *$ & $* * *$ & $16 \mathrm{mg} / \mathrm{kg}$ \\
\hline $\mathrm{U} 203$ & Safrole & NA & $* * *$ & $* * * *$ & $22 \mathrm{mg} / \mathrm{kg}$ \\
\hline $\mathbf{U} 210$ & Tetracholorethylene & NA & $* * *$ & $* * \dot{*}$ & $6.0 \mathrm{mg} / \mathrm{kg}$ \\
\hline U211 & Carbon Tetrachloride & NA & $* * *$ & $* * *$ & $6.0 \mathrm{mg} / \mathrm{kg}$ \\
\hline U213 & Tetrahydrofuran & NA & $* * *$ & $* * *$ & CMBST \\
\hline U218 & Thioacetamide. & NA & $* * *$ & $* \star *$ & CMBST \\
\hline U220 & Toluene & NA & $* * *$ & $\star \star *$ & $10 \mathrm{mg} / \mathrm{kg}$ \\
\hline U226 & 1,1,1-Trichloroethane & NA & $* * *$ & $* * *$ & $6.0 \mathrm{mg} / \mathrm{kg}$ \\
\hline U228 & Trichloroethylene & NA & $* * *$ & $* * *$ & $6.0 \mathrm{mg} / \mathrm{kg}$ \\
\hline
\end{tabular}




$\begin{array}{llllll}\text { U239 } & \text { Xylenes } & \text { NA } & * * * & * * * & 30 \mathrm{mg} / \mathrm{kg} \\ \text { U359 } & \text { 2-Ethoxyethanol } & \text { NA } & * * * & * * * & \text { CMBST } \\ \text { WT01 } & \text { Toxic, EHW } & \text { NA } & * * * & * * * & \text { None (1) } \\ \text { WT02 } & \text { Toxic, DW } & \text { NA } & * * * & * * * & \text { None } \\ \text { WP01 } & \text { Persistant, EHW } & \text { NA } & * * * & * * * & \text { None (1) } \\ \text { WP02 } & \text { Persistant, DW } & \text { NA } & * * * & * * * & \text { None } \\ \text { WSC2 } & \text { Solid Corrosive } & \text { NA } & * * * & * * * & \text { Remove Solid-Acid Char }\end{array}$

UHCs TBD on a per-package basis during waste receipt and/or when the waste is sent for treatment (see Section 2.4.5).

(1) Mixed extremely hazardous wastes may be land-disposed in Washington State in DOE facilities in accordance with RCW 70.105.050(2).

* If waste isn't consistent in concentration, this may not apply. Describe in 2.4.5.

2.4.2.1 List any waste codes from 2.4 .2 for which the stream already meets established LDR treatment standards: It is assumed that all waste in this waste stream will require various levels of treatment prior to disposal.

2.4.3 Does this waste stream contain PCBs?

$\triangle$ Yes $\square$ No $\square$ Unknown.

If no, skip to 2.4.4.

2.4.3.1 Is waste stream subject to TSCA regulations for $P C B$ ?

$\triangle$ Yes $\square$ No $\square$ Unknown

2.4.3.2 Indicate the $\mathrm{PCB}$ concentration range.

$\bigotimes<50 \mathrm{ppm} \quad \square \geq 50 \mathrm{ppm} \quad \square$ Unknown

2.4.4 What is the confidence level for the regulated contaminant characteristic data?

$\square$ Low $\square$ Medium $\bigotimes$ High

2.4.5 Comments on regulated contaminant characteristics and/or confidence level: Subject waste has met the rigors of the Waste Specification System for waste storage and treatment. However, the WSS came into effect in 1995 and was based on the dangerous waste regulations imposed at that time. There have been several changes to the dangerous waste regulations since then that impose additional characterization requirements onto the generator, namely identifcation of UHCs for all waste designated with a characteristic waste code (i.e., D001 through D043).

Some of the waste in this waste stream does contain PCBs subject to TSCA regulation. If a waste package is regulated by TSCA, it is identified as such on storage records and tracked in SWITS

2.4.6 Will further characterization be performed? 囚Yes $\square$ No $\square$ Unknown

2.4.6.1 If yes, provide details and schedule: UHCs will be determined and identified on the corresponding LDR notification/certification at the time subject waste is sent to treatment.

2.4.6.2 If yes, provide Tri-Party Agreement milestone numbers: No TPA milestones are directly associated with characterization of this waste stream.

\subsection{WASTE STREAM STORAGE, INVENTORY, AND GENERATION}

3.1 Is this waste stream currently stored? $\quad$ Yes $\square$ No If no, skip to 3.7. 
DOE/RL-99-01

3.2 Current storage method

$\square$ Container (pad)
$\square$ Tank
$\square$ Other (explain)
区Container (covered)
Waste pile

Container (retrievably buried)

Surface impoundment

3.3 TSD unit name and building number and (if applicable/available) number of containers/tanks in each: There are approximately 1,165 waste packages that make up this stream residing in the CWC.

3.4 Is storage of waste in compliance with applicable State and Federal standards?

$\triangle$ Yes $\square$ No

If no, provide details:

3.5 Applicable Tri-Party Agreement milestones related to storage: No TPA milestone is directly associated with storage of this waste stream.

3.6 Current inventory for this stream

Total LDR volume (cubic meters): 271

Date of inventory values: $\quad 9 / 30 / 98$

Comments on waste inventory: Based on inventory residing at the $\mathrm{CWC}$ as reported in the Solid Waste Information Tracking System (SWITS) for WSRds: 300, 301, 302, 303, 304, 305, 310, $311,320,321,330,331,31 \mathrm{~A}, 400,401,402,403,404,405,406,407,408,40 \mathrm{~A}, 40 \mathrm{~B}, 427,430,431,432$, 45A, 47A, 500 (except 183H), 501-2, 502 (except 200LEF), 503-2, 504-1, 505-3, 506, 507, 50A, 50B, $50 \mathrm{C}, 520,522,52 \mathrm{~A}, 53 \mathrm{~A}, 700,701,720,721,920,921,922$, and 923.

3.7 Is this waste stream currently generated; if not, will this waste stream be generated in the next 5 years? QYes $\square$ No. If no, skip to 3.11 .

3.8 The current or future generation of this waste is best described as:

$\triangle$ Routine $\square$ One-time or sporadic

3.9 Estimated generation projection by fiscal year

$\begin{array}{lllll} & \mathrm{m}^{3} & \text { (and/or) } & \mathrm{kg} & \\ 1999 & \mathbf{4 9} & & & \\ 2000 & \mathbf{6 3} & & & \\ 2001 & \mathbf{5 6} & & & \\ 2002 & \mathbf{5 4} & & & \\ 2003 & \mathbf{5 6} & & \end{array}$

3.10 Last year's fiscal year 1998 generation projection: 79 Actual quantity generated in 1998: 69 (both in $\mathrm{m}^{3}$ unless otherwise noted)

3.11 Has there ever been any unusual release of this stream to the environment?

$\square$ Yes $\mathrm{XNo}$

If yes, summarize releases and quantities:

\subsection{WASTE STREAM TREATMENT}

4.1 Is this stream currently being treated?

$\square$ Yes $\bigotimes N_{0}$ If yes, provide details:

4.2 Planned treatment: Check the appropriate box indicating future plans for treating this waste stream to meet applicable regulations, including LDR treatment standards. 
$\square$ No treatment required (skip to 5.0)

$\square$ Treating or plan to treat onsite

Treating or plan to treat offsite

Treatment options still being assessed

4.3 Planned treatment method, facility, extent of treatment capacity available: Thermal treatment is targeted as the primary treatment technology for subject waste. Treatment will be performed by means of an offsite commercial treatment contract with ATG (contract currently in place). ATG has stated that the treatment volumes specified in the contract are approximately $20 \%$ of their anticipated thermal treatment capacity. The ATG contract has a required 5-year base performance period and 5 optional individual years that could extend treatment out through FY2010.

4.4 Treatment schedule information: $\quad$ FY1999 ...... $0 \mathrm{~m}^{\wedge} 3$

FY $2000 \ldots . . .0 \mathrm{~m}^{\wedge} 3$

FY2001 ..... 173m^3

FY2002 .....173m^3

FY2003 ..... 173m^3

FY2004 …. 120m^3

FY2005 .....120 $\mathrm{m}^{\wedge} 3$

FY2006 ..... 120m^3

FY2007 .... 120 $\mathrm{m}^{\wedge} 3$

FY2008 ..... 120 $\mathrm{m}^{\wedge} 3$

FY2009 ..... as generated

4.5 Applicable treatment Tri-Party Agreement milestone numbers (including permitting): Counts toward M-91-12 volume commitments (treatment).

4.6 If treating or planning to treat on site, was or will waste minimization be addressed in developing and/or selecting the treatment method? $\square$ Yes $\square$ No $\square$ Unknown

If yes, please describe:

4.7 Treatability equivalency petitions, rulemaking petitions, and case-by-case exemptions needed for treatment: TBD

\subsection{WASTE STREAM DISPOSAL}

5.1 After treatment, how will the waste stream be disposed of (include description, locations, variances required, etc., as applicable): Subject waste will be disposed into a RCRA Subtitle-C disposal cell located on the Hanford Site.

5.2 Applicable Tri-Party Agreement milestone number(s) for disposal: $\quad$ M-91-13

5.3 Planned disposal dates/time-frame: Beginning in FY2001

\subsection{WASTE MINIMIZATION}

6.1 Has a waste minimization assessment been completed for this stream?

$\square$ Yes $\bigotimes$ No $\square$ NA

6.2 Explain any waste minimization activities (e.g., process changes) under way or to be implemented for generation of this stream: No new waste was generated during 1998. Therefore a waste assessment for this stream is not necessary and was not conducted during the calendar year.

To the extent practical, all mixed waste is segregated and packaged separately from LLW or TRU wastes. The volume of mixed waste is reduced by in-drum compaction when possible, and where it 
does not interfere with future treatment activities. To minimize the generation of mixed waste, generators actively seek nondangerous alternatives for the dangerous constituents in their processes. Minimization goals are set annually and tracked quarterly, and waste treatment is used to destroy the hazardous constituents, as allowable.

6.3 Schedule for implementing waste minimization methods: Waste minimization activities are an ongoing activity.

6.4 Waste reduction achieved during the calendar year and projected future reductions (volume or mass): None

6.4.1 Assumptions used in above estimates: NA 
DOE/RL-99-01

This page intentionally left blank. 


\title{
WASTE STREAM PROFILE SHEET
}

\subsection{WASTE STREAM IDENTIFICATION AND SOURCE}

\author{
1.1 Waste stream ID: $\quad$ MLLW-04A
}

\subsection{Waste stream name: Past-Practice Hazardous Debris}

\subsection{Waste stream source information}

1.3.1 Stream source and history description. Include how the waste was managed prior to storage, timeframe when waste was placed into storage. Subject waste was generated and put into CWC storage prior to the implementation of the Waste Specification System (WSS); therefore, it is considered as a past-practice waste. The waste was generated by many onsite generating organizations and offsite generators.

\subsubsection{Source category(s)}

$\square$ Pollution control or waste treatment process
Facility or equipment operation and maintenance waste
$\square$ Discarded excess or expired materials
R\&D/R\&D laboratory waste
Spill clean-ups or emergency response actions
Other (explain):

1.3.3 Additional notes: This is a general waste category based on physical, chemical, radiological and regulatory factors.

\subsection{WASTE STREAM CHARACTERIZATION}

2.1 Waste stream description (content): This waste stream includes past-practice waste meeting the definition of hazardous debris. This waste was received into the $C W C$ prior to the implementation of the WSS, and the waste was generated from many different onsite generating organizations and offsite generators. The primary onsite generating organizations for this waste stream have been TWRS ( 1700 cubic meters) and PFP (130 cubic meters).

From evaluations performed on the hazardous debris inventory in the CWC, the waste contains approximately $90 \%$ organic/carbonaceous $(\mathrm{O} / \mathrm{C})$ constituents (e.g., plastics, rubber, wood, paper, rags, etc.) and $10 \%$ non-O/C constituents (e.g., metals, concrete, asbestos). Since Hanford has not segregated $\mathrm{O} / \mathrm{C}$ constituents from non- $\mathrm{O} / \mathrm{C}$ constituents, the entire debris waste inventory is essentially $\mathrm{O} / \mathrm{C}$ waste as defined in WAC 173-303-040. If the debris is contaminated with PCBs at concentrations greater than $50 \mathrm{ppm}$ it is not included in this waste stream and is considered organic solids. Since this waste requires upgrading to the WSS requirements prior to treatment, the actual treatment and disposal of this waste would take place under waste stream MLLW-04B. The current WSRds making up this waste stream are ASB, BLD, DBR, DBL, H3D, SOC and SOE.

2.2 Radiological characteristics

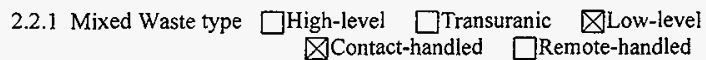

2.2.2 Comments on radiological characteristics (e.g., more specific content, treatment concerns caused by radiation, confidence level): Since this waste is a general category based on dangerous waste 
characteristics, the radiological characteristics are expected to vary greatly. However, there is high confidence that the waste is CH-MLLW.

2.3 Matrix characteristics (physical content)

2.3.1 Matrix constituent table (comprising at least $1 \%$ of the total volume or mass)

\begin{tabular}{ccc}
$\begin{array}{c}\text { Matrix Parameter } \\
\text { Category Code }\end{array}$ & $\begin{array}{c}\text { Matrix Constituent } \\
\text { Description }\end{array}$ & $\begin{array}{c}\text { Typical } \\
\text { or Range (\%) }\end{array}$ \\
\hline S5300 & Organic/Carbonaceous Debris & $\mathbf{8 0 - 9 0 \%}$ \\
S5110 & Metal Debris & $\mathbf{5 - 1 0 \%}$ \\
S5120 & Inorganic Non-metal Debris & $<5 \%$ \\
S3000 & Homogeneous Solids & $<\mathbf{5 \%}$ \\
S4000 & Soll/Gravel & $<\mathbf{5 \%}$
\end{tabular}

Overall matrix parameter category code: $\quad \mathbf{\$ 5 4 0 0}$

Overall matrix description: Hetergeneous Debris

\subsubsection{Confidence level for matrix characteristics data in 2.3.1}

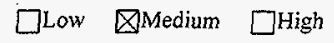

2.3.3 Comments on matrix characteristics and/or confidence level: Subject waste will undergo matrix characterization verifications as part of the past-practice "upgrading" program. Once the waste meets all the upgrading requirements, it will be assigned to the proper WSS WSRd associated with waste stream MLLW-04B. If during the upgrading process it is determined that some of the waste does not meet one of the MLLW-04B waste stream WSRds, then the appropriate WSRd will be assigned to the waste, and then the waste will be transferred to the correct waste stream.

2.4 Regulated contaminant characteristics

2.4.1 Wastewater/non-wastewater under RCRA

$\square$ Wastewater $\bigotimes$ Non-wastewater $\square$ Unknown

2.4.2 Regulated contaminant table including treatment requirements, and UHCs if applicable

\begin{tabular}{|c|c|c|c|c|c|c|}
\hline $\begin{array}{l}\text { EPA/ } \\
\text { State } \\
\text { Code }\end{array}$ & Waste Description & & $\begin{array}{c}\text { LDR } \\
\text { Sub- } \\
\text { category }\end{array}$ & $\begin{array}{c}\text { Concentration } \\
\text { (Typical or } \\
\text { Range)* }\end{array}$ & Basis & $\begin{array}{c}\text { LDR } \\
\text { Concentration Limit or } \\
\text { Technology Code }\end{array}$ \\
\hline$\overline{\mathrm{D} 001}$ & Ignitable & Ignita & able Char & $* * *$ & $\begin{array}{l}\text { Treatment } \\
\text { Technology }\end{array}$ & $\begin{array}{l}\text { Alternative Treatment } \\
\text { Stds. For Haz. Debris } \\
\text { (40 CFR 268.45) }\end{array}$ \\
\hline D002 & Corrosive & Corro & osive Char & $* * * *$ & $"$ & " \\
\hline D003 & Reactive & React & ctive Cyanides & $* * *$ & $"$ & $"$ \\
\hline D004 & TC-Arsenic & & NA & $* * * *$ & $"$ & $"$ \\
\hline D005 & TC-Barium & & $\mathbf{N A}$ & $* * *$ & $"$ & $"$ \\
\hline D006 & TC-Cadmium & Cadm & mium Char & $* * *$ & $"$ & $"$ \\
\hline D007 & TC-Chromium & & NA & $* * *$ & $"$ & $"$ \\
\hline D008 & TC-Lead & Lead & d Char & $* * *$ & $"$ & $"$ \\
\hline D009 & TC-Mercury & Low 1 & Mercury & $<260 \mathrm{mg} / \mathrm{kg}$ & $"$ & $"$ \\
\hline D010 & TC-Selenium & & NA & $* * *$ & $"$ & $"$ \\
\hline D011 & TC-Silver & & NA & $* * *$ & $"$ & $"$ \\
\hline D012 & Endrin & & NA & $* * *$ & $"$ & " \\
\hline D018 & Benzene & & $\mathbf{N A}$ & $* * *$ & $"$ & $"$ \\
\hline D019 & Carbon Tetrachl & loride & NA & $* * * \pi$ & $"$ & $"$ \\
\hline D022 & Chioroform & & NA & $* * * *$ & $"$ & $"$ \\
\hline D026 & Cresol & & NA & $* * * *$ & 1 & $"$ \\
\hline D027 & p-Dichlorobenzer & & NA & $* * *$ & " & $"$ \\
\hline
\end{tabular}




\begin{tabular}{|c|c|c|c|c|c|}
\hline D028 & 1,2-Dichloroethane & NA & $* * *$ & $"$ & $"$ \\
\hline D029 & 1,1-Dichlorethylene & NA & $* * *$ & $"$ & $"$ \\
\hline D030 & 2,4-Dinitrotoluene & NA & $* * *$ & $"$ & $"$ \\
\hline D031 & Heptachlor & NA & $* * *$ & $"$ & $"$ \\
\hline D033 & Hexachlorobutadiene & NA & $* * *$ & $"$ & $"$ \\
\hline D034 & Hexachloroethane & NA & $* * *$ & $"$ & $"$ \\
\hline D035 & Methyl Ethyl Ketone & NA & $* * *$ & $"$ & $"$ \\
\hline D036 & Nitrobenzene & NA & $* \star *$ & $"$ & $"$ \\
\hline D037 & Pentachlorophenol & NA & $* * *$ & $"$ & $"$ \\
\hline D038 & Pyridine & NA & $* * *$ & $"$ & $"$ \\
\hline D039 & Tetracholorethylene & NA & $* * *$ & $"$ & $"$ \\
\hline D040 & Trichloroethylene & NA & $* * *$ & $"$ & $"$ \\
\hline D043 & Vinyl Chloride & NA & $* * \hbar$ & $"$ & $"$ \\
\hline F001 & 1,1,1-Trichloroethane $s$ & Spent solvent & $* * *$ & $"$ & $"$ \\
\hline F002 & Methylene Chloride & Spent solvent & $* * *$ & $"$ & $"$ \\
\hline F003 & Acetone \& Hexone & Spent solvent & $* * *$ & $"$ & $"$ \\
\hline F004 & o-Cresol \& p-Cresol & Spent solvent & $* * *$ & $"$ & $"$ \\
\hline F005 & Methyl Ethyl Ketone & Spent solvnet & $* * *$ & $"$ & $"$ \\
\hline P029 & Copper Cyanide & NA & $k * *$ & $"$ & $"$ \\
\hline P030 & Cyanides & NA & $* * *$ & $\because$ & $"$ \\
\hline P098 & Potassium Cyanide & NA & $* * *$ & $"$ & $"$ \\
\hline $\mathbf{P 1 0 2}$ & Propargyl Alcohol & NA & $* * *$ & $"$ & $"$ \\
\hline P106 & Sodium Cyanide & NA & $* * *$ & $"$ & $"$ \\
\hline P120 & Vanadium Pentoxide & NA & $* * *$ & $"$ & $"$ \\
\hline U002 & Acetone & NA & $* * *$ & $"$ & $"$ \\
\hline U006 & Acetyl Chloride & NA & $* * *$ & $"$ & $"$ \\
\hline U031 & n-Butyl Alcohol & $\mathbf{N A}$ & $* * *$ & $"$ & $"$ \\
\hline U057 & Cyclohexanone & NA & $\star \star * *$ & $"$ & $"$ \\
\hline U080 & Methylene Chloride & NA & $* * *$ & $"$ & $"$ \\
\hline$U 123$ & Formic Acid & NA & $* * *$ & $"$ & $"$ \\
\hline U151 & Mercury Low & Nercury & $<260 \mathrm{mg} / \mathrm{kg} \mathrm{Hg}$ & $"$ & $\because$ \\
\hline U159 & Methyl Ethyl Ketone & NA & $* * *=$ & $"$ & $"$ \\
\hline U161 & Methyl Isobutyl Ketone & NA & $* * *$ & $"$ & $"$ \\
\hline U196 & Pyridine & NA & $* * *$ & $"$ & $"$ \\
\hline U220 & Toluene & $\mathbf{N A}$ & $* * *$ & $"$ & $"$ \\
\hline U226 & 1,1,1-Trichlorethane & NA & $* * *$ & $"$ & $"$ \\
\hline U239 & Xylenes & NA & $* * *$ & $"$ & $"$ \\
\hline WT01 & Toxic, EHW & NA & $\star \star \star *$ & $* * *$ & None (1) \\
\hline WT02 & Toxic, DW & NA & $* * *$ & $* * *$ & None \\
\hline WP01 & Persistant, EHW & NA & $* * *$ & $* * *$ & None (1) \\
\hline WP02 & Persistant, DW. & NA & $* * *$ & $* * *$ & None \\
\hline WSC2 & Solid Corrosive & NA & $* * *$ & $* * *$ & $\begin{array}{l}\text { Remove Solid-Acid } \\
\text { Characteristic }\end{array}$ \\
\hline
\end{tabular}

UHCs Identification not required when using the alternative treatment standards for hazardous debris.

1) Mixed extremely hazardous wastes may be land-disposed in Washington State in DOE facilities in accordance with RCW 70.105.050(2).

2) The combination of waste codes varies on a per-package basis in accordance with WAC 173-303$070(3)$ and (5).

* If waste isn't consistent in concentration, this may not apply. Describe in 2.4.5. 
2.4.2.1 List any waste codes from 2.4.2 for which the stream aiready meets established LDR treatment standards: It is assumed that all waste in this waste stream will require various levels of treatment prior to disposal.

2.4.3 Does this waste stream contain PCBs?

\Yes $\square$ No $\square$ Unknown.

If no, skip to 2.4.4.

2.4.3.1 Is waste stream subject to TSCA regulations for $\mathrm{PCBs}$ ?

$\square$ Yes QNo $\square$ Unknown

2.4.3.2 Indicate the $\mathrm{PCB}$ concentration range.

$\square<50 \mathrm{ppm} \square>50 \mathrm{ppm} \square$ Unknown

2.4.4 What is the confidence level for the regulated contaminant characteristic data?

$\square$ Low $\square$ Medium $\square$ High

2.4.5 Comments on regulated contaminant characteristics and/or confidence level: Subject waste will undergo characterization verifications as part of the past-practice "upgrading" program. Once the waste meets all the upgrading requirements, it will be assigned to the proper WSS WSRd associated with waste stream MLLW-04B. If during the upgrading process it is determined that some of the waste does not meet one of the MLLW-04B waste stream WSRds, then the appropriate WSRd will be assigned to the waste, and then the waste will be transferred to the correct waste stream.

2.4.6 Will further characterization be performed? $\quad$ Yes $\square$ No $\square$ Unknown

2.4.6.1. If yes, provide details and schedule: Currently scheduled to go through characterization upgrading beginning in FY2000; however, this is contingent on other Hanford Site priorities and budget constrants.

2.4.6.2 If yes, provide Tri-Party Agreement milestone numbers: No TPA milestone is directly associated with characterization of this waste stream.

\subsection{WASTE STREAM STORAGE, INVENTORY, AND GENERATION}

3.1 Is this waste stream currently stored? $\bigotimes$ Yes $\square$ No If no, skip to 3.7.

3.2 Current storage method

$\square$ Container (pad)
$\square$ Tank
$\square$ Other (explain):
\Container (covered) $\square$ Container (retrievably buried)
$\square$ Waste pile $\square$ Surface impoundment

3.3 TSD unit name and building number and (if applicable/available) number of containers/tanks in each:

There are approximately 2,518 packages residing in the $C W C$.

3.4 Is storage of waste in compliance with applicable State and Federal standards?

\Yes $\square$ No

If no, provide details:

3.5 Applicable Tri-Party Agreement milestones related to storage: No TPA milestone is directly associated with storage of this waste stream.

3.6 Current inventory for this stream

Total $L D R$ volume (cubic meters):

Date of inventory values: $\quad 9 / 30 / 98$ 
Comments on waste inventory: Based on inventory residing at the $C W C$ as reported in the Solid Waste Information Tracking System (SWITS) for WSRds: ASB, BLD, DBR, DBL, H3D, SOC and SOE.

3.7 Is this waste stream currently generated; if not, will this waste stream be generated in the next 5 years? $\square$ Yes $\square$ No. If no, skip to 3.11.

3.8 The current or future generation of this waste is best described as:

$\square$ Routine $\square$ One-time or sporadic

3.9 Estimated generation projection by fiscal year

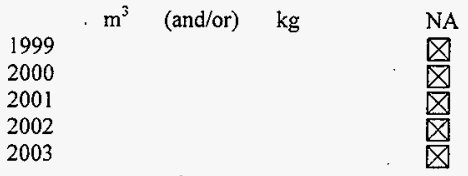

3.10 Last year's fiscal year 1998 generation projection:

Actual quantity generated in 1998: - (both in $\mathrm{m}^{3}$ unless otherwise noted)

3.11 Has there ever been any unusual release of this stream to the environment?

$\square$ Yes $\square \mathrm{No}$

If yes, summarize releases and quantities:

\subsection{WASTE STREAM TREATMENT}

4.1 Is this stream currently being treated?

$\square$ Yes $\square$ No If yes, provide details:

4.2 Planned treatment: Check the appropriate box indicating future plans for treating this waste stream to meet applicable regulations, including LDR treatment standards.

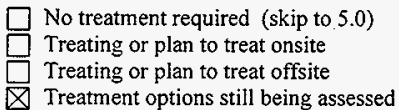

4.3 Planned treatment method, facility, extent of treatment capacity available: Since this waste is a past-practice waste stream and must undergo characterization upgrading before treatment, the specific treatment methods, facilities and schedules will not be determined until the appropriate WSS WSRd is assigned.

4.4 Treatment schedule information: TBD

4.5 Applicable treatment Tri-Party Agreement milestone numbers (including permitting): Counts toward M-19-00 volume commitments (treatment).

4.6 If treating or planning to treat on site, was or will waste minimization be addressed in developing and/or selecting the treatment method? $\square$ Yes $\square$ No $\square$ Unknown

If yes, please describe:

4.7 Treatability equivalency petitions, rulemaking petitions, and case-by-case exemptions needed for treatment: 


\subsection{WASTE STREAM DISPOSAL}

5.1 After treatment, how will the waste stream be disposed of (include description, locations, variances required, etc., as applicable): Subject waste ultimately will be disposed into a RCRA Subtitle-C disposal cell located on the Hanford Site; however, the waste must under go upgrading and treatment prior to disposal.

5.2 Applicable Tri-Party Agreement milestone number(s) for disposal: $\quad$ M-91-13

5.3 Planned disposal dates/time-frame: Beginning in FY 2000 and continuing until all the waste is dispositioned.

\subsection{WASTE MINIMIZATION}

6.1 Has a waste minimization assessment been completed for this stream?
$\square$ Yes $\bigotimes$ No $\square$ NA

6.2 Explain any waste minimization activities (e.g., process changes) under way or to be implemented for generation of this stream: No new waste was generated during 1998. Therefore a waste assessment for this stream is not necessary and was not conducted during the calendar year.

6.3 Schedule for implementing waste minimization methods: Waste minimization activities are an ongoing activity.

6.4 Waste reduction achieved during the calendar year and projected future reductions (volume or mass): None

6.4.1 Assumptions used in above estimates: NA 


\subsection{WASTE STREAM IDENTIFICATION AND SOURCE}

\subsection{Waste stream ID: MLLW-04B}

1.2 Waste stream name: Waste Specification System Hazardous Debris

1.3 Waste stream source information

1.3.1 Stream source and history description. Include how the waste was managed prior to storage, timeframe when waste was placed into storage. Subject waste was generated and put into CWC storage either after the implementation of the Waste Specification System (WSS) or has been "upgraded" from various past-practice waste streams. The waste was generated by many onsite generating organizations and offsite generators. The primary onsite generating organization for the existing waste was SST/DST Systems operations.

1.3.2 Source category(s)
$\square$ Pollution control or waste treatment process
Materials production/recovery effluents
Facility or equipment operation and main
Discarded excess or expired materials
Discarded excess or expired
Spill clean-ups or emergency response actions
ce waste
Analytical laboratory waste
\ Remediation/D\&D waste
\ Source unknown
Other (explain):

1.3.3 Additional notes: This is a general waste category based on physical, chemical, radiological and regulatory factors.

\subsection{WASTE STREAM CHARACTERIZATION}

2.1 Waste stream description (content): This waste stream consists of hazardous debris containing primarily organic debris material (e.g., plastic, rubber, wood, paper, cloth, tumbleweeds, etc.). Some inorganic debris material (e.g., building rubble, metals, asbestos, etc.) would be present in the waste; however, the debris would be considered as organic/carbonaceous waste (as defined per WAC 173303-040) on a per-container basis. If the debris is contaminated with PCBs at concentrations greater than $50 \mathrm{ppm}$ it is not included in this waste stream and is considered organic solids. The current WSRds making up this waste stream are $600,601,603,605,606,607,60 \mathrm{~A}, 60 \mathrm{~B}, 620,621,622,640$, $641,645,646,647,315,334,625,626$, and 627 .

\subsection{Radiological characteristics}

\subsubsection{Mixed Waste type $\square$ High-level $\square$ Transuranic 叉Low-level 区Contact-handled $\square$ Remote-handled}

2.2.2 Comments on radiological characteristics (e.g., more specific content, treatment concerns caused by radiation, confidence level): Since this waste is a general category based on dangerous waste physical characteristics, the radiological characteristics are expected to vary greatly. However, there is high confidence that the waste is CH-MLLW.

2.3 Matrix characteristics (physical content)

2.3.1 Matrix constituent table (comprising at least $1 \%$ of the total volume or mass) 
DOE/RL-99-01

\begin{tabular}{ccc}
$\begin{array}{c}\text { Matrix Parameter } \\
\text { Category Code }\end{array}$ & $\begin{array}{c}\text { Matrix Constituent } \\
\text { Description }\end{array}$ & $\begin{array}{c}\text { Typical } \\
\text { or Range }(\%)\end{array}$ \\
\hline $\mathbf{S 5 3 0 0}$ & Organic/Carbonaceous Debris & $\mathbf{8 0 - 9 0 \%}$ \\
$\mathbf{S 5 1 1 0}$ & Metal Debris & $\mathbf{5 - 1 0 \%}$ \\
$\mathbf{S 5 1 2 0}$ & Inorganic Non-metal Debris & $<5 \%$ \\
$\mathbf{S 3 0 0 0}$ & Homogeneous Solids & $<\mathbf{5 \%}$ \\
$\mathbf{S 4 0 0 0}$ & Soil/Gravel & $<5 \%$
\end{tabular}

Overall matrix parameter category code: $\mathbf{S 5 4 0 0}$

Overall matrix description: Hetergeneous Debris

2.3.2 Confidence level for matrix characteristics data in 2.3.1 $\square$ Low $\square$ Medium $\square$ High

2.3.3 Comments on matrix characteristics and/or confidence level: This waste stream can consist of many different physical matrix characteristic types since it based on LDR requirements for treatment and disposal of a dangerous waste. There is a high confidence that subject waste stream will not contain physical matrix characteristics that do not meet the waste stream description.

2.4 Regulated contaminant characteristics

2.4.1 Wastewater/non-wastewater under RCRA

$\square$ Wastewater \Non-wastewater $\square$ Unknown

2.4.2 Regulated contaminant table including treatment requirements, and UHCs if applicable

\begin{tabular}{|c|c|c|c|c|c|c|}
\hline $\begin{array}{l}\text { EPA/ } \\
\text { State } \\
\text { Code }\end{array}$ & Waste Description & & $\begin{array}{l}\text { LDR } \\
\text { Sub- } \\
\text { category } \\
\end{array}$ & $\begin{array}{l}\text { Concentration } \\
\text { (Typical or } \\
\text { Range)* }\end{array}$ & Basis & $\begin{array}{c}\text { LDR } \\
\text { Concentration Limit or } \\
\text { Technology Code }\end{array}$ \\
\hline D001 & Ignitable & Ignital & able Char & $* * *$ & $\begin{array}{l}\text { Treatment } \\
\text { Technology }\end{array}$ & $\begin{array}{l}\text { Alternative Treatment } \\
\text { Stds. For Haz. Debris } \\
\text { (40 CFR 268.45) }\end{array}$ \\
\hline D002 & Corrosive & Corro: & osive Char & $* * *$ & $"$ & " \\
\hline D003 & Reactive & React & tive Cyanides & $* * *$ & $"$ & $"$ \\
\hline D004 & TC-Arsenic & & NA & $* * *$ & $"$ & $"$ \\
\hline D005 & TC-Barium & & NA & $* * *$ & " & $"$ \\
\hline D006 & TC-Cadmium & Cadm & mium Char & $* * *$ & $"$ & $"$ \\
\hline D007 & TC-Chromium & & NA & *** & $"$ & " \\
\hline D008 & TC-Lead & Lead & Char & $\star * \dot{\pi} *$ & $"$ & $"$ \\
\hline D009 & TC-Mercury & Low i & Mercury & $<260 \mathrm{mg} / \mathrm{kg}$ & $"$ & $"$ \\
\hline D010 & TC-Selenium & & NA & $* * *$ & $"$ & $"$ \\
\hline D011 & TC-Silver & & NA & $* * *$ & $"$ & $"$ \\
\hline D018 & Benzene & & NA & $* * *$ & $"$ & $"$ \\
\hline D019 & Carbon Tetrachl & loride & NA & $* * *$ & $"$ & $"$ \\
\hline D022 & Chloroform & & NA & $* * *$ & $"$ & $"$ \\
\hline D026 & Cresol & & NA & $* * *$ & $"$ & $"$ \\
\hline D027 & p-Dichlorobenze & & NA & $* * *$ & $"$ & $"$ \\
\hline D028 & 1,2-Dichloroetha & & NA & $* * *$ & $"$ & $"$ \\
\hline D029 & 1,1-Dichlorethyle & ene & NA & $* * *$ & $"$ & $"$ \\
\hline D030 & 2,4-Dinitrotoluen & & NA & $* * *$ & $"$ & $"$ \\
\hline D033 & Hexachlorobutad & diene & NA & *** & $"$ & $n$ \\
\hline D034 & Hexachloroethan & & NA & $* * *$ & $"$ & $"$ \\
\hline D035 & Methyl Ethyl Ket & tone & NA & $* * *$ & $"$ & $"$ \\
\hline D036 & Nitrobenzene & & NA & $* * *$ & $"$ & $"$ \\
\hline D039 & Tetracholorethyle & & NA & $* * *$ & $"$ & $"$ \\
\hline D040 & Trichloroethylene & & NA & $* * *$ & $"$ & $"$ \\
\hline
\end{tabular}




\begin{tabular}{|c|c|c|c|c|c|}
\hline D043 & Vinyl Chloride & NA & $\star \star \star * *$ & $"$ & $"$ \\
\hline F001 & 1,1,1-Trichloroethane & Spent solvent & $* * *$ &. & $"$ \\
\hline F002 & Methylene Chloride & Spent solvent & $* * *$ & " & $"$ \\
\hline F003 & Acetone \& Hexone & Spent solvent & $* * *$ & $"$ & $"$ \\
\hline F004 & o-Cresol \& p-Cresol & Spent solvent & **क & $"$ & $"$ \\
\hline F005 & Methyl Ethyl Ketone & Spent solvnet & $* * *$ & $"$ & $"$ \\
\hline U006 & Acetyl chloride & NA & $* * *$ & $"$ & $"$ \\
\hline U043 & Vinyl chloride & NA & $* * *$ & $"$ & $"$ \\
\hline U080 & Methylene Chloride & NA & $* * *$ & $"$ & $"$ \\
\hline WT01 & Toxic, EHW & NA & $* * *$ & $* * *$ & None (1) \\
\hline WT02 & Toxic, DW & NA & $* * *$ & $* * *$ & None \\
\hline WP01 & Persistant, EHW & NA & $* * *$ & $* * *$ & None (1) \\
\hline WP02 & Persistant, DW & NA & $* * *$ & $* * *$ & None \\
\hline WSC2 & Solid Corrosive & NA & $\star \star \star *$ & $* * *$ & $\begin{array}{c}\text { Remove Solid-Acid } \\
\text { Characteristic }\end{array}$ \\
\hline
\end{tabular}

UHCs Identification not required when using the alternative treatment standards for hazardous debris.

1) Mixed extremely hazardous wastes may be land-disposed in Washington State in DOE facilities in accordance with RCW 70.105.050(2).

2) The combination of waste codes varies on a per-package basis in accordance with WAC 173-303$070(3)$ and (5).

* If waste isn't consistent in concentration, this may not apply. Describe in 2.4.5.

2.4.2.1 List any waste codes from 2.4 .2 for which the stream already meets established LDR treatment standards: It is assumed that all waste in this waste stream will require various levels of treatment prior to disposal

2.4.3 Does this waste stream contain PCBs?

区Yes $\square$ No $\square$ Unknown.

If no, skip to 2.4.4.

2.4.3.1 Is waste stream subject to TSCA regulations for PCBs?

$\square$ Yes 区No $\square$ Unknown

2.4.3.2 Indicate the $\mathrm{PCB}$ concentration range.

Q<50 ppm $\square \geq 50 \mathrm{ppm} \square$ Unknown

2.4.4 What is the confidence level for the regulated contaminant characteristic data?

$\square$ Low 区Medium $\square$ High

2.4.5 Comments on regulated contaminant characteristics and/or confidence level: Subject waste has met the rigors of the Waste Specification System for waste storage and treatment.

2.4.6 Will further characterization be performed? $\square$ Yes $\quad$ No $\square$ Unknown

2.4.6.1 If yes, provide details and schedule:

2.4.6.2 If yes, provide Tri-Party Agreement milestone numbers:

\subsection{WASTE STREAM STORAGE, INVENTORY, AND GENERATION}

3.1 Is this waste stream currently stored? YYes $\square$ No If no, skip to 3.7. 
3.2 Current storage method

$\begin{array}{lll}\square \text { Container (pad) } & \text { QContainer (covered) } & \square \text { Container (retrievably buried) } \\ \square \text { Tank } & \square \text { Waste pile } & \square \text { Surface impoundment } \\ \square \text { Other (explain): } & \end{array}$

3.3 TSD unit name and building number and (if applicable/available) number of containers/tanks in each: There are approximately 2,081 packages residing in the CWC.

3.4 Is storage of waste in compliance with applicable State and Federal standards?

QYes $\square$ No

If no, provide details:

3.5 Applicable Tri-Party Agreement milestones related to storage: No TPA milestone is directly associated with storage of this waste stream.

3.6 Current inventory for this stream

Total LDR volume (cubic meters): 1218

Date of inventory values: $\quad 9 / 30 / 98$

Comments on waste inventory: Based on inventory residing at the $\mathrm{CWC}$ as reported in the Solid Waste Information Tracking System (SWITS) for WSRds: 600, 601, 603, 605, 606, 607, 60A, $60 \mathrm{~B}, 620,621,622,640,641,645,646,647,315,334,625,626$, and 627 .

3.7 Is this waste stream currently generated; if not, will this waste stream be generated in the next 5 years? 区Yes $\square$ No. If no, skip to.3.11.

3.8 The current or future generation of this waste is best described as:

QRoutine $\square$ One-time or sporadic

3.9 Estimated generation projection by fiscal year

$\begin{array}{lllll} & \mathbf{m}^{3} & \text { (and/or) } & \mathrm{kg} & \mathrm{NA} \\ 1999 & \mathbf{3 4 9} & & & \\ 2000 & \mathbf{4 3 3} & & & \\ 2001 & \mathbf{4 1 2} & & & \\ 2002 & \mathbf{5 5 6} & & & \\ 2003 & \mathbf{5 1 4} & & & \end{array}$

3.10 Last year's fiscal year 1998 generation projection: 308

Actual quantity generated in 1998: 265 (both in $\mathrm{m}^{3}$ unless otherwise noted)

3.11 Has there ever been any unusual release of this stream to the environment?

$\square$ Yes $\square$ No

If yes, summarize releases and quantities:

\subsection{WASTE STREAM TREATMENT}

4.1 Is this stream currently being treated?

$\square$ Yes $\square$ No If yes, provide details:

4.2 Planned treatment: Check the appropriate box indicating future plans for treating this waste stream to meet applicable regulations, including LDR treatment standards.

No treatment required (skip to 5.0 )

Treating or plan to treat onsite 
$\square$ Treating or plan to treat offsite

Treatment options still being assessed

4.3 Planned treatment method, facility, extent of treatment capacity available: Macroencapsulation per the Alternative Debris Rules (40 CFR 268.45) is targeted as the primary treatment technology for this waste stream. Treatment via macroencapsulation will occur as long as the state-only LDR for organic/carbonaceous waste continues to apply.

Macroencapsulation will take place offsite at the ATG facility located in north Richland (per the nonthermal treatment contract currently in place with ATG), and onsite at the T-Plant and/or WRAP. Thermal destruction is currently planned to take place offsite.at the Waste Experimental Reduction Facility (WERF) and the ATG facility.

\begin{tabular}{|c|c|}
\hline Treatment schedule information: & 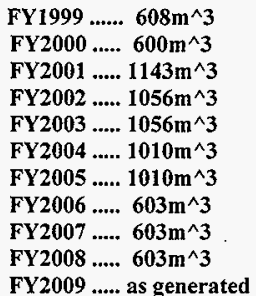 \\
\hline
\end{tabular}

4.5 Applicable treatment Tri-Party Agreement milestone numbers (including permitting): Counts toward M-19-00 volume commitments (treatment).

4.6 If treating or planning to treat on site, was or will waste minimization be addressed in developing and/or selecting the treatment method? $\square$ Yes $\square$ No Wunknown

If yes, please describe:

4.7 Treatability equivalency petitions, rulemaking petitions, and case-by-case exemptions needed for treatment: Exemption for the state-only LDR on the disposal of $O / C$ waste.

\subsection{WASTE STREAM DISPOSAL}

5.1 After treatment, how will the waste stream be disposed of (include description, locations, variances required, etc., as applicable): Subject waste will be disposed into a RCRA Subtitle-C disposal cell located on the Hanford Site.

5.2 Applicable Tri-Party Agreement milestone number(s) for disposal: $\quad$ M-91-13

5.3 Planned disposal dates/time-frame: Following treatment

\subsection{WASTE MINIMIZATION}

6.1 Has a waste minimization assessment been completed for this stream?

$\square$ Yes $\bigotimes$ No $\square$ NA

6.2 Explain any waste minimization activities (e.g., process changes) under way or to be implemented for generation of this stream: No new waste was generated during 1998 and generation of this waste stream is expected to continue. A waste assessment was not conducted during the calendar year nor was any waste reduction achieved. This waste stream will be identified to the on-site generating facility management as a candidate for a waste minimization assessment. 
To the extent practical, all mixed waste is segregated and packaged separately from LLW or TRU wastes. The volume of mixed waste is reduced by in-drum compaction when possible, and where it does not interfere with future treatment activities. To minimize the generation of mixed waste, generators actively seek nondangerous alternatives for the dangerous constituents in their processes. Minimization goais are set annually and tracked quarterly, and waste treatment is used to destroy the hazardous constituents, as allowable.

6.3 Schedule for implementing waste minimization methods: Waste minimization activities are an ongoing activity.

6.4 Waste reduction achieved during the calendar year and projected future reductions (volume or mass): None

6.4.1 Assumptions used in above estimates: NA 


\section{WASTE STREAM PROFILE SHEET}

\subsection{WASTE STREAM IDENTIFICATION AND SOURCE}

\subsection{Waste stream ID: MLLW-05A}

\section{- 1.2 Waste stream name: Past-Practice Elemental Lead}

\subsection{Waste stream source information}

1.3.1 Stream source and history description. Include how the waste was managed prior to storage, timeframe when waste was placed into storage. Subject waste was generated and put into CWC storage prior to the implementation of the Waste Specification System (WSS); therefore, it is considered as a past-practice waste. The waste was generated by many onsite generating organizations and offsite generators. The waste stream is primarily generated from Shippingport Atomic Power (offsite) and 100N (onsite generating organization).

\subsubsection{Source category(s)}

$\square$ Pollution control or waste treatment process

Materials production/recovery effluents

$\triangle$ Facility or equipment operation and maintenance waste

$\bigotimes$ Discarded excess or expired materials

$R \& D / R \& D$ laboratory waste

Spill clean-ups or emergency response actions

$\square$ Analytical laboratory waste

QRemediation/D\&D waste

Other (explain):

1.3.3 Additional notes: This is a general waste category based on physical, chemical, radiological and regulatory factors.

\subsection{WASTE STREAM CHARACTERIZATION}

2.1 Waste stream description (content): This waste stream consists elemental lead solids (lead bricks, shot, gloves, shielding, etc.); however, the lead is primarly comingled with hetergeneous debris and/or the lead is a component of a debris article. The current WSRd making up this waste stream is EPB.

\subsection{Radiological characteristics}

\subsubsection{Mixed Waste type $\square$ High-level $\square$ Transuranic $\bigotimes$ Low-level} 囚Contact-handled $\square$ Remote-handled

2.2.2 Comments on radiological characteristics (e.g., more specific content, treatment concerns caused by radiation, confidence level): Because this waste is a general category based on dangerous waste physical characteristics, the radiological characteristics are expected to vary greatly. However, there is high confidence that the waste is CH-MLLW.

\subsection{Matrix characteristics (physical content)}

2.3.1 Matrix constituent table (comprising at least $1 \%$ of the total volume or mass)

\begin{tabular}{ccc}
$\begin{array}{c}\text { Matrix Parameter } \\
\text { Category Code }\end{array}$ & $\begin{array}{c}\text { Matrix Constituent } \\
\text { Description }\end{array}$ & $\begin{array}{c}\text { Typical } \\
\text { or Range (\%) }\end{array}$ \\
\hline X7210 & Elemental Lead & $\mathbf{3 0 - 5 0 \%}$
\end{tabular}


Overall matrix parameter category code: $\quad \mathbf{X 7 2 1 0}$

Overall matrix description: Elemental Lead

2.3.2 Confidence level for matrix characteristics data in 2.3.1

$\square$ Low $\square^{M e d i u m ~} \square$ High

2.3.3 Comments on matrix characteristics and/or confidence level: Subject waste will undergo matrix characterization verifications as part of the past-practice upgrading program. Once the waste meets all the upgrading requirements, it will be assigned to the proper WSS WSRd associated with waste stream MLLW-05B. If during the upgrading process it is determined that some of the waste does not meet one of the MLLW-05B waste stream WSRds, then the appropriate WSRd will be assigned to the waste, and then the waste will be transferred to the correct waste stream.

\subsection{Regulated contaminant characteristics}

\subsubsection{Wastewater/non-wastewater under RCRA}

$\square$ Wastewater $\bigotimes$ Non-wastewater $\square$ Unknown

2.4.2 Regulated contaminant table including treatment requirements, and UHCs if applicable

\begin{tabular}{|c|c|c|c|c|c|}
\hline $\begin{array}{l}\text { EPA/ } \\
\text { State } \\
\text { Code }\end{array}$ & Waste Description & $\begin{array}{c}\text { LDR } \\
\text { Sub- } \\
\text { category } \\
\end{array}$ & $\begin{array}{c}\text { Concentration } \\
\text { (Typical or } \\
\text { Range)* }\end{array}$ & Basis & $\begin{array}{c}\text { LDR } \\
\text { Concentration Limit or } \\
\text { Technology Code } \\
\end{array}$ \\
\hline D001 & Ignitable $\quad$ Ig & Ignitable Char & $* * *$ & $\begin{array}{l}\text { Treatment } \\
\text { Technology }\end{array}$ & $\begin{array}{l}\text { Alternative Treatment } \\
\text { Stds. For Haz. Debris } \\
\text { (40 CFR 268.45) }\end{array}$ \\
\hline D002 & Corrosive & Corrosive Char & $* * *$ & " & " \\
\hline D004 & TC-Arsenic & NA & *** & $"$ & $"$ \\
\hline D005 & TC-Barium & NA & $* * *$ & $"$ & " \\
\hline D006 & TC-Cadmium & Cadmium Char & $* * *$ & $"$ & $"$ \\
\hline D007 & TC-Chromium & NA & $* * *$ & $"$ & $"$ \\
\hline D008 & TC-Lead & Lead Char & $* * *$ & $"$ & $"$ \\
\hline D009 & TC-Mercury & Low Mercury & $<260 \mathrm{mg} / \mathrm{kg}$ & $"$ & $"$ \\
\hline D010 & TC-Selenium & .NA & $* * *$ & " & " \\
\hline D011 & TC-Silver & NA & $* * *$ & $"$ & $"$ \\
\hline D018 & Benzene & NA & $* * *$ & $"$ & $"$ \\
\hline D019 & Carbon Tetrachlorid & NA & $* * *$ & $"$ & $"$ \\
\hline D022 & Chloroform & NA & $* * *$ & $"$ & $"$ \\
\hline D023 & o-Cresol & NA & $* * *$ & $"$ & $"$ \\
\hline D028 & 1,2-Dichloroethane & NA & $* * *$ & $"$ & $"$ \\
\hline D029 & 1,1-Dichlorethylene & NA & $* * *$ & $"$ & $"$ \\
\hline D035 & Methyl Ethyl Ketone & NA & $* * *$ & $"$ & $n$ \\
\hline D038 & Pyridine & NA & $* * *$ & $"$ & $"$ \\
\hline D039 & Tetracholorethylene & NA & $* \ddot{* *}$ & $"$ & $\because$ \\
\hline D040 & Trichloroethylene & NA & $* * *$ & $"$ & $"$ \\
\hline D043 & Vinyl Chloride & NA & $* * *$ & $"$ & $"$ \\
\hline F001 & $1,1,1$-Trichloroethan & ne Spent solvent & $* * *$ & $"$ & $"$ \\
\hline F002 & Methylene Chloride & Spent solvent & $* * *$ & $"$ & $"$ \\
\hline F003 & Acetone \& Hexone & Spent solvent & $* * *$ & " & $"$ \\
\hline F004 & o-Cresol \& p-Cresol & Spent solvent & $* * *$ & $"$ & $"$ \\
\hline F005 & Methyl Ethyl Ketone & Spent solvnet & $* * *$ & $"$ & $"$ \\
\hline P012 & Arsenic Trioxide & NA & $\star * *$ & $"$ & $"$ \\
\hline
\end{tabular}




$\begin{array}{lllcll}\text { WT01 } & \text { Toxic, EHW } & \text { NA } & * * * & * * * & \text { None (1) } \\ \text { WT02 } & \text { Toxic, DW } & \text { NA } & * * * & * * * & \text { None } \\ \text { WP01 } & \text { Persistant, EHW } & \text { NA } & * * * & * * * & \text { None (1) } \\ \text { WP02 } & \text { Persistant, DW } & \text { NA } & * * * & * * * & \text { None } \\ \text { WSC2 } & \text { Solid Corrosive } & \text { NA } & * * * & * * * & \text { Remove Solid-Acid } \\ & & & & \text { Characteristic }\end{array}$

UHCs Identification not required for D008 radioactive lead solids and hazardous debris when using the alternative treatment standards for hazardous debris.

1) Mixed extremely hazardous wastes may be land-disposed in Washington State in DOE facilities in accordance with RCW 70.105.050(2).

2) The combination of waste codes varies on a per-package basis in accordance with WAC 173-303$070(3)$ and (5).

* If waste isn't consistent in concentration, this may not apply. Describe in 2.4.5.

2.4.2.1 List any waste codes from 2.4.2 for which the stream already meets established LDR treatment standards: It is assumed that all waste in this waste stream will require various levels of treatment prior to disposal

2.4.3 Does this waste stream contain PCBs?

$$
\triangle Y \text { es } \square \text { No } \square \text { Unknown. }
$$

If no, skip to 2.4.4.

2.4.3.1 Is waste stream subject to TSCA regulations for PCBs?

$\square$ Yes $\bigotimes$ No $\square$ Unknown

2.4.3.2 Indicate the $\mathrm{PCB}$ concentration range.

\<50 ppm $\square \geq 50 \mathrm{ppm} \quad \square$ Unknown

2.4.4 What is the confidence level for the regulated contaminant characteristic data?

$\square$ Low 凶Medium $\square$ High

2.4.5 Comments on regulated contaminant characteristics and/or confidence level: Subject waste will undergo characterization verifications as part of the past-practice upgrading program. Once the waste meets all the upgrading requirements, it will be assigned to the proper WSS WSRd associated with waste stream MLLW-05B. If during the upgrading process it is determined that some of the waste does not meet one of the MLLW-05B waste stream WSRds, then the appropriate WSRd will be assigned to the waste, and then the waste will be transferred to the correct waste stream.

2.4.6 Will further characterization be performed? $\quad$ Yes $\square$ No $\square$ Unknown

2.4.6.1 If yes, provide details and schedule: Currently scheduled to go through characterization upgrading beginning in FY2000; however, this is contingent on other Hanford Site priorities and budget constrants.

2.4.6.2 If yes, provide Tri-Party Agreement milestone numbers: No TPA milestone is directly associated with characterization of this waste stream.

\subsection{WASTE STREAM STORAGE, INVENTORY, AND GENERATION}

3.1 Is this waste stream currently stored? $\square$ Yes $\square$ No If no, skip to 3.7.

3.2 Current storage method 


$\begin{array}{lll}\square \text { Container (pad) } & \text { QContainer (covered) } & \square \text { Container (retrievably buried) } \\ \square \text { Tank } & \square \text { Waste pile } & \square \text { Surface impoundment } \\ \square \text { Other (explain): } & \end{array}$

3.3 TSD unit name and building number and (if applicable/available) number of containers/tanks in each: There are approximately 940 packages residing in the CWC.

3.4 Is storage of waste in compliance with applicable State and Federal standards?

$\otimes$ Yes $\square$ No

If no, provide details:

3.5 Applicable Tri-Party Agreement milestones related to storage: No TPA milestone is directly associated with storage of this waste stream.

3.6 Current inventory for this stream

Total LDR volume (cubic meters): 287

Date of inventory values: $9 / 30 / 98$

Comments on waste inventory: Based on inventory residing at the $\mathrm{CWC}$ as reported in the Solid Waste Information Tracking System (SWITS) for WSRd EPB.

3.7 Is this waste stream currently generated; if not, will this waste stream be generated in the next 5 years? $\square$ Yes $\square$ No. If no, skip to 3.11 .

3.8 The current or future generation of this waste is best described as:

$\square$ Routine $\square$ One-time or sporadic

3.9 Estimated generation projection by fiscal year

\begin{tabular}{lllll}
1999 & $\mathrm{~m}^{3} \quad$ (and/or) & $\mathrm{kg}$ & $\mathrm{NA}$ \\
2000 & & & & \\
2001 & & & & \\
2002 & & & & \\
2003 & & & & \\
\hline
\end{tabular}

3.10 Last year's fiscal year 1998 generation projection:

Actual quantity generated in 1998 :

(both in $\mathrm{m}^{3}$ unless otherwise noted)

3.11 Has there ever been any unusual release of this stream to the environment?

If yes, summarize releases and quantities:

\subsection{WASTE STREAM TREATMENT}

4.1 Is this stream currently being treated?

$\square$ Yes $\bigotimes$ No If yes, provide details:

4.2 Planned treatment: Check the appropriate box indicating future plans for treating this waste stream to meet applicable regulations, including LDR treatment standards.

$\square$ No treatment required (skip to 5.0 )
Treating or plan to treat onsite
Treating or plan to treat offsite
Treatment options still being assessed 
4.3. Planned treatment method, facility, extent of treatment capacity available: Since this waste is a past-practice waste stream and must undergo characterization upgrading before treatment, the specific treatment methods, facilities and schedules will not be determined until the appropriate WSS WSRd is assigned.

\subsection{Treatment schedule information: TBD}

4.5 Applicable treatment Tri-Party Agreement milestone numbers (including permitting): Counts toward M-19-00 volume commitments (treatment).

4.6 If treating or planning to treat on site, was or will waste minimization be addressed in developing and/or selecting the treatment method? $\square$ Yes $\square$ No $\square$ Unknown

If yes, please describe:

4.7 Treatability equivalency petitions, rulemaking petitions, and case-by-case exemptions needed for treatment:

\subsection{WASTE STREAM DISPOSAL}

5.1 After treatment, how will the waste stream be disposed of (include description, locations, variances required, etc., as applicable): Subject waste ultimately will be disposed into a RCRA Subtitle-C disposal cell located on the Hanford Site; however, the waste must under go upgrading and treatment prior to disposal.

5.2 Applicable Tri-Party Agreement milestone number(s) for disposal: $\quad$ M-91-13

5.3 Planned disposal dates/time-frame: TBD

\subsection{WASTE MINIMIZATION}

6.I Has a waste minimization assessment been completed for this stream?

$$
\square \text { Yes } \square \text { No } \square \text { NA }
$$

6.2 Explain any waste minimization activities (e.g., process changes) under way or to be implemented for generation of this stream: No new waste was generated during 1998. Therefore a waste assessment for this stream is not necessary and was not conducted during the calendar year.

To the extent practical, all mixed waste is segregated and packaged separately from LLW or TRU wastes. The volume of mixed waste is reduced by in-drum compaction when possible, and where it does not interfere with future treatment activities. To minimize the generation of mixed waste, generators actively seek nondangerous alternatives for the dangerous constituents in their processes. Minimization goals are set annually and tracked quarterly, and waste treatment is used to destroy the hazardous constituents, as allowable.

6.3 Schedule for implementing waste minimization methods: Waste minimization activities are an ongoing activity.

6.4 Waste reduction achieved during the calendar year and projected future reductions (volume or mass): None

6.4.1 Assumptions used in above estimates: 


\section{WASTE STREAM PROFILE SHEET}

\subsection{WASTE STREAM IDENTIFICATION AND SOURCE}

\subsection{Waste stream ID: MLLW-05B}

\subsection{Waste stream name: Waste Specification System Elemental Lead}

\subsection{Waste stream source information}

1.3.1 Stream source and history description. Include how the waste was managed prior to storage, timeframe when waste was placed into storage. Subject waste was generated and put into CWC storage either after the implementation of the Waste Specification System (WSS) or has been upgraded from various past-practice waste streams. The waste was generated by many onsite generating organizations and offsite generators. There was no one primary generator for this waste stream.

\subsubsection{Source category(s)}

$\square$ Pollution control or waste treatment process $\square$ Materials production/recovery effluents

$\triangle$ Facility or equipment operation and maintenance waste

$\bigotimes$ Discarded excess or expired materials

$\mathrm{R} \& \mathrm{D} / \mathrm{R} \& \mathrm{D}$ laboratory waste

Spill clean-ups or emergency response actions Other (explain):

1.3.3 Additional notes: This is a general waste category based on physical, chemical, radiological and regulatory factors.

\subsection{WASTE STREAM CHARACTERIZATION}

2.1 Waste stream description (content): This waste stream consists elemental lead solids (lead bricks, shot, gloves, shielding, etc.); however, the lead is primarly comingled with hetergeneous debris and/or the lead is a component of a debris article. The current WSRds making up this waste stream are 800,801 and 803 .

\subsection{Radiological characteristics}

\subsubsection{Mixed Waste type $\square$ High-level $\square$ Transuranic QLow-level} $\bigotimes$ Contact-handled $\square$ Remote-handled

2.2.2 Comments on radiological characteristics (e.g., more specific content, treatment concerns caused by radiation, confidence level): Since this waste is a general category based on dangerous waste physical characteristics, the radiological characteristics are expected to vary greatly. However, there is high confidence that the waste is CH-MLLW.

2.3 Matrix characteristics (physical content)

2.3.1 Matrix constituent table (comprising at least $1 \%$ of the total volume or mass)

\begin{tabular}{ccc}
$\begin{array}{c}\text { Matrix Parameter } \\
\text { Category Code }\end{array}$ & $\begin{array}{c}\text { Matrix Constituent } \\
\text { Description }\end{array}$ & $\begin{array}{c}\text { Typical } \\
\text { or Range }(\%)\end{array}$ \\
\hline $\mathbf{X 7 2 1 0}$ & Elemental Lead & $\mathbf{3 0 - 5 0 \%}$
\end{tabular}


Overall matrix parameter category code: $\quad \mathbf{X 7 2 1 0}$

Overall matrix description: Elemental Lead

2.3.2 Confidence level for matrix characteristics data in 2.3 .1

$\square$ Low $\square$ Medium $\bigotimes$ High

2.3.3 Comments on matrix characteristics and/or confidence level: This waste stream can consist of many different physical matrix characteristic types since it based on LDR requirements for treatment and disposal of a dangerous waste. There is a high confidence that subject waste stream will not contain physical matrix characteristics that do not meet the waste stream discription.

\subsection{Regulated contaminant characteristics}

\subsubsection{Wastewater/non-wastewater under RCRA}

[Wastewater $\bigotimes$ Non-wastewater

Unknown

2.4.2 Regulated contaminant table including treatment requirements, and UHCs if applicable

\begin{tabular}{|c|c|c|c|c|c|}
\hline $\begin{array}{l}\text { EPA/ } \\
\text { State } \\
\text { Code }\end{array}$ & Waste Description & $\begin{array}{l}\text { LDR } \\
\text { Sub- } \\
\text { category }\end{array}$ & $\begin{array}{l}\text { Concentration } \\
\text { (Typical or } \\
\text { Range) }\end{array}$ & Basis & $\begin{array}{c}\text { LDR } \\
\text { Concentration Limit or } \\
\text { Technology Code }\end{array}$ \\
\hline D004 & TC-Arsenic & NA & $* * *$ & Treatment & Alternative Treatment \\
\hline D005 & TC-Barium & NA & $\star * *$ & Technology & Stds. for Haz. Debris \\
\hline D006 & TC-Cadmium & admium Char & $* * *$ & "1 & (40 CFR 268.45) \\
\hline D007 & TC-Chromium & NA & $* * *$ & $"$ & " \\
\hline D008 & TC-Lead & Lead Char & $* * *$ & " & $"$ \\
\hline D009 & TC-Mercury & Low Mercury & $<260 \mathrm{mg} / \mathrm{kg}$ & $"$ & " \\
\hline D010 & TC-Selenium & NA & $* * *$ & $"$ & $"$ \\
\hline D018 & Benzene & NA & $* * *$ & $"$ & $"$ \\
\hline D030 & 2,4-Dinitrotoluene & NA & $* * *$ & $"$ & $"$ \\
\hline D033 & Hexachlorobutadiene & $\mathbf{N A}$ & *** & " & $"$ \\
\hline D043 & Vinyl Chloride & NA & $* * *$ & "t & $"$ \\
\hline F001 & 1,1,1-Trichloroethane & Spent soivent & $* * *$ & $"$ & $"$ \\
\hline F002 & Methylene Chloride & Spent solvent & $* * *$ & $"$ & $"$ \\
\hline F003 & Acetone \& Hexone & Spent solvent & $* * *$ & "1 & $"$ \\
\hline Fo04 & o-Cresol \& p-Cresol & Spent solvent & $* * *$ & $"$ & $"$ \\
\hline F005 & Methyl Ethyl Ketone & Spent solvent & $* * *$ & 11 & $"$ \\
\hline WT01 & Toxic, EHW & NA & $* * *$ & $* *$ & None (1) \\
\hline WP02 & Persistant, DW & NA & $* * *$ & $* * *$ & None \\
\hline WSC2 & Solid Corrosive & NA & $* * *$ & $* * *$ & $\begin{array}{l}\text { Remove Solid-Acid } \\
\text { Characteristic }\end{array}$ \\
\hline
\end{tabular}

UHCs Identification not required for D008 radioactive lead solids and hazardous debris when using the alternative treatment standards for hazardous debris.

1) Mixed extremely hazardous wastes may be land-disposed in Washington State in DOE facilities in accordance with RCW 70.105.050(2).

2) The combination of waste codes varies on a per-package basis in accordance with WAC 173-303$070(3)$ and (5).

* If waste isn't consistent in concentration, this may not apply. Describe in 2.4.5. 
2.4.2.1 List any waste codes from 2.4.2 for which the stream already meets established LDR treatment standards: It is assumed that all waste in this waste stream will require various levels of treatment prior to disposal.

2.4.3 Does this waste stream contain PCBs? $\triangle$ Yes $\square$ No $\square$ Unknown. If no, skip to 2.4.4.

2.4.3.1 Is waste stream subject to TSCA regulations for PCBs?

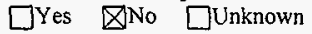

2.4.3.2 Indicate the $\mathrm{PCB}$ concentration range.

$\bigotimes<50 \mathrm{ppm} \quad \square \geq 50 \mathrm{ppm} \quad \square$ Unknown

2.4.4 What is the confidence level for the regulated contaminant characteristic data? $\square$ Low $\bigotimes$ Medium $\square$ High

2.4.5 Comments on regulated contaminant characteristics and/or confidence level: Subject waste has met the rigors of the Waste Specification System for waste storage and treatment.

2.4.6 Will further characterization be performed? $\square$ Yes $\quad$ No $\square$ Unknown

2.4.6.1 If yes, provide details and schedule:

2.4.6.2 If yes, provide Tri-Party Agreement milestone numbers:

\subsection{WASTE STREAM STORAGE, INVENTORY, AND GENERATION}

3.1 Is this waste stream currently stored? $\quad$ Yes $\square$ No $\quad$ If no, skip to 3.7.

3.2 Current storage method

$\begin{array}{ll}\square \text { Container (pad) } & \square \text { Container (covered) } \\ \square \text { Tank } & \square \text { Container (retrievably buried) } \\ \square \text { Other (explain): } & \square \text { Surface impoundment }\end{array}$

3.3 TSD unit name and building number and (if applicable/available) number of containers/tanks in each: There are approximately 105 packages residing in the $\mathrm{CWC}$.

3.4 Is storage of waste in compliance with applicable State and Federal standards?

$\triangle$ Yes $\square$ No

If no, provide details:

3.5 Applicable Tri-Party Agreement milestones related to storage: No TPA milestone is directly associated with storage of this waste stream.

3.6 Current inventory for this stream

Total LDR volume (cubic meters): 56

Date of inventory values: $\quad \mathbf{9 / 3 0 / 9 8}$

Comments on waste inventory: Based on inventory residing at the $\mathrm{CWC}$ as reported in the Solid Waste Information Tracking System (SWITS) for WSRds: 800, 801 and 803.

3.7 Is this waste stream currently generated; if not, will this waste stream be generated in the next 5 years? \Yes $\square$ No. If no, skip to 3.11 . 
3.8 The current or future generation of this waste is best described as:

$\triangle$ Routine $\square$ One-time or sporadic

3.9 Estimated generation projection by fiscal year

$\begin{array}{lllll} & \mathrm{m}^{3} & \text { (and/or) } & \mathrm{kg} & \\ 1999 & 17 & & & \\ 2000 & 22 & & & \\ 2001 & 10 & & & \\ 2002 & 14 & & & \\ 2003 & 10 & & & \end{array}$

3.10 Last year's fiscal year 1998 generation projection: 9

Actual quantity generated in 1998: 20 (both in $\mathrm{m}^{3}$ unless otherwise noted)

3.11 Has there ever been any unusual release of this stream to the environment?

$\square$ Yes $\bigotimes$ No.

If yes, summarize releases and quantities:

\subsection{WASTE STREAM TREATMENT}

4.1 Is this stream currently being treated?

$\square$ Yes $\square$ No If yes, provide details:

4.2 Planned treatment: Check the appropriate box indicating future plans for treating this waste stream to meet applicable regulations, including LDR treatment standards.

$\square$ No treatment required (skip to 5.0 )
Treating or plan to treat onsite
Treating or plan to treat offsite
. Treatment options still being assessed

4.3 Planned treatment method, facility, extent of treatment capacity available: Macroencapsulation per the Treatment Standards for Hazardous Wastes (40 CFR 268.40) is specified treatment technology for elemental lead. Treatment will be performed by means of onsite and offsite commercial treatment contracts, and/or by onsite treatment units. There are no treatment campaigns planned for this waste until FY2001.

4.4 Treatment schedule information: FY1999 ...... $0 \mathrm{~m}^{\wedge} 3$

FY2000 ..... $0 \mathrm{~m}^{\wedge} 3$

FY2001 ..... 58 $\mathrm{m}^{\wedge} 3$

FY2002 ..... $58 \mathrm{~m}^{\wedge} 3$

FY2003 ..... 58 $\mathrm{m}^{\wedge} 3$

FY2004 ..... 58 $\mathrm{m}^{\wedge} 3$

FY2005 …. $58 \mathrm{~m}^{\wedge} 3$

FY2006 ..... 58 $\mathrm{m}^{\wedge} 3$

FY2007 ..... 58 $\mathrm{m}^{\wedge} 3$

FY $2008 \ldots . . . .58 \mathrm{~m}^{\wedge} 3$

FY2009 ..... as generated

4.5 Applicable treatment Tri-Party Agreement milestone numbers (including permitting): Counts toward M-19-00 volume commitments (treatment).

4.6 If treating or planning to treat on site, was or will waste minimization be addressed in developing and/or selecting the treatment method? $\square$ Yes $\square$ No $\square$ Unknown

If yes, please describe: 
4.7 Treatability equivalency petitions, rulemaking petitions, and case-by-case exemptions needed for treatment: TBD

\subsection{WASTE STREAM DISPOSAL}

5.1 After treatment, how will the waste stream be disposed of (include description, locations, variances required, etc., as applicable): Subject waste will be disposed into a RCRA Subtitle-C disposal cell located on the Hanford Site.

5.2 Applicable Tri-Party Agreement milestone number(s) for disposal: M-91-13

5.3 Planned disposal dates/time-frame: Following treatment

\subsection{WASTE MINIMIZATION}

6.1 Has a waste minimization assessment been completed for this stream?

$\square$ Yes QNo $\square$ NA

6.2 Explain any waste minimization activities (e.g., process changes) under way or to be implemented for generation of this stream: The Lead Return on Investment Project recovered lead from 80 drums that were stored at $\mathrm{CWC}$ which were subsequently radiologically decontaminated and shipped out for recycle, saving $\mathbf{1 6 . 8}$ cubic meters of waste.

To the extent practical, all mixed waste is segregated and packaged separately from LLW or TRU wastes. The volume of mixed waste is reduced by in-drum compaction when possible, and where it does not interfere with future treatment activities. To minimize the generation of mixed waste, generators actively seek nondangerous alternatives for the dangerous constituents in their processes. Minimization goals are set annually and tracked quarterly, and waste treatment is used to destroy the hazardous constituents, as allowable.

6.3 Schedule for implementing waste minimization methods: Waste minimization activities are an ongoing activity.

6.4 Waste reduction achieved during the calendar year and projected future reductions (volume or mass): None

6.4.1 Assumptions used in above estimates: NA 


\section{WASTE STREAM PROFILE SHEET}

\subsection{WASTE STREAM IDENTIFICATION AND SOURCE}

\subsection{Waste stream ID: MLLW-06A}

1.2 Waste stream name: Past-Practice Elemental Mercury

1.3 Waste stream source information

1.3.1 Stream source and history description. Include how the waste was managed prior to storage, timeframe when waste was placed into storage. Subject waste was generated and put into CWC storage prior to the implementation of the Waste Specification System (WSS); therefore, it is considered as a past-practice waste. The waste was generated by onsite generating organizations and offsite generators. The primary onsite generating location for this waste stream has been the 325 Building.

\subsubsection{Source category(s)}
Pollution control or waste treatment process
Facility or equipment operation and maintenance waste
D Discarded excess or expired materials
X R\&D/R\&D laboratory waste
$\triangle$ Spill clean-ups or emergency response actions
Materials production/recovery effluents
$\triangle$ Analytical laboratory waste
Remediation/D\&D waste
Source unknown

Other (explain):

1.3.3 Additional notes: This is a general waste category based on physical, chemical, radiological and regulatory factors.

\subsection{WASTE STREAM CHARACTERIZATION}

2.1 Waste stream description (content): This waste stream consists of elemental mercury, amalgamated mercury, and debris articles containing small amounts of elemental mercury in their componentry (mercury switches, thermometers, etc). The current WSRd making up this waste stream is EHG.

\subsection{Radiological characteristics}

2.2.1 Mixed Waste type $\square$ High-level $\square$ Transuranic 凶Low-level \Contact-handled $\square$ Remote-handled

2.2.2 Comments on radiological characteristics (e.g., more specific content, treatment concerns caused by radiation, confidence level): Since this waste is a general category based on dangerous waste physical characteristics, the radiological characteristics are expected to vary greatly. However, there is high confidence that the waste is CH-MLLW.

\subsection{Matrix characteristics (physical content)}

2.3.1 Matrix constituent table (comprising at least $1 \%$ of the total volume or mass)

\begin{tabular}{ccc}
$\begin{array}{c}\text { Matrix Parameter } \\
\text { Category Code }\end{array}$ & $\begin{array}{c}\text { Matrix Constituent } \\
\text { Description }\end{array}$ & $\begin{array}{c}\text { Typical } \\
\text { or Range (\%) }\end{array}$ \\
\hline X7100 & Elemental Mercury & $\mathbf{2 0 - 4 0 \%}$
\end{tabular}


Overall matrix parameter category code: $\mathbf{X 7 1 0 0}$

Overall matrix description: Elemental Mercury

2.3.2 Confidence level for matrix characteristics data in 2.3 .1

$\square$ Low $\triangle$ Medium $\square$ High

2.3.3 Comments on matrix characteristics and/or confidence level: Subject waste will undergo matrix characterization verifications as part of the past-practice upgrading program. Once the waste meets all the upgrading requirements, it will be assigned to the proper WSS WSRd associated with waste stream MLLW-06B. If during the upgrading process it is determined that some of the waste does not meet one of the MLLW-06B waste stream WSRds, then the appropriate WSRd will be assigned to the waste, and then the waste will be transferred to the correct waste stream.

\subsection{Regulated contaminant characteristics}

\subsubsection{Wastewater/non-wastewater under RCRA}

$\square$ Wastewater $\bigotimes$ Non-wastewater $\square$ Unknown.

2.4.2 Regulated contaminant table including treatment requirements, and UHCs if applicable

\begin{tabular}{|c|c|c|c|c|c|}
\hline $\begin{array}{l}\text { EPA/ } \\
\text { State } \\
\text { Code }\end{array}$ & Waste Description & $\begin{array}{c}\text { LDR } \\
\text { Sub- } \\
\text { category }\end{array}$ & $\begin{array}{c}\text { Concentration } \\
\text { (Typical or } \\
\text { Range) } \\
\end{array}$ & Basis & $\begin{array}{c}\text { LDR } \\
\text { Concentration Limit or } \\
\text { Technology Code }\end{array}$ \\
\hline D001 & Ignitable & Ignitable Char & $\pi * \dot{x}$ & $\begin{array}{l}\text { Treatment } \\
\text { Technology }\end{array}$ & $\begin{array}{l}\text { Alternative Treatment } \\
\text { Stds. For Haz. Debris } \\
\text { (40 CFR 268.45) }\end{array}$ \\
\hline D002 & Corrosive & Corrosive Char & $* * *$ & $"$ & $"$ \\
\hline D005 & TC-Barium & NA & $* * *$ & $"$ & " \\
\hline D006 & TC-Cadmium & Cadmium Char & $* * *$ & $"$ & $"$ \\
\hline D007 & TC-Chromium & NA & $* * *$ & " & $"$ \\
\hline D009 & TC-Mercury & Elemental $\mathrm{Hg}$ & $* * * *$ & $"$ & Amalgamation \\
\hline U151 & Mercury & Elemental Hg & $* * *$ & $"$ & " \\
\hline WT01 & Toxic, EHW & NA & $* * *$ & $\star * *$ & None (1) \\
\hline
\end{tabular}

UHCs Identification not required for Dó09 elemental mercury and hazardous debris when using the alternative treatment standards for hazardous debris.

1) Mixed extremely hazardous wastes may be land-disposed in Washington State in DOE facilities in accordance with RCW 70.105.050(2).

2) The combination of waste codes varies on a per-package basis in accordance with WAC 173-303$070(3)$ and (5).

* If waste isn't consistent in concentration, this may not apply. Describe in 2.4.5.

2.4.2.1 List any waste codes from 2.4.2 for which the stream already meets established LDR treatment standards: It is assumed that all waste in this waste stream will require various levels of treatment prior to disposal.

2.4.3 Does this waste stream contain PCBs?

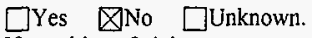

If no, skip to 2.4.4. 
2.4.3.1 Is waste stream subject to TSCA regulations for PCBs?

$\square$ Yes $\square$ No $\square$ Unknown

2.4.3.2 Indicate the PCB concentration range. $\square<50 \mathrm{ppm} \quad \square \geq 50 \mathrm{ppm} \quad \square$ Unknown

2.4.4 What is the confidence level for the regulated contaminant characteristic data?

$\square$ Low $\bigotimes$ Medium $\square$ High

2.4.5 Comments on regulated contaminant characteristics and/or confidence level: Subject waste will undergo characterization verifications as part of the past-practice upgrading program. Once the waste meets all the upgrading requirements, it will be assigned to the proper WSS WSRd associated with waste stream MLLW-06B. If during the upgrading process it is determined that some of the waste does not meet one of the MLLW-06B waste stream WSRds, then the appropriate WSRd will be assigned to the waste, and then the waste will be transferred to the correct waste stream.

\title{
2.4.6 Will further characterization be performed? \Yes $\square$ No $\square$ Unknown
}

2.4.6.1 If yes, provide details and schedule: Currently scheduled to go through characterization upgrading beginning in FY2002; however, this is contingent on other Hanford Site priorities and budget constrants.

2.4.6.2 If yes, provide Tri-Party Agreement milestone numbers: No TPA milestone is directly associated with characterization of this waste stream.

\subsection{WASTE STREAM STORAGE, INVENTORY, AND GENERATION}

3.1 Is this waste stream currently stored? QYes $\square$ No If no, skip to 3.7.

3.2 Current storage method

$\square$ Container (pad)
$\square$ Tank
$\square$ Other (explain):

\author{
QContainer (covered) \\ Waste pile
}

$\square$ Container (retrievably buried)

Surface impoundment

3.3 TSD unit name and building number and (if applicable/available) number of containers/tanks in each: There are approximately 37 packages residing in the $\mathrm{CWC}$.

3.4 is storage of waste in compliance with applicable State and Federal standards?

$$
\begin{aligned}
& \bigotimes \text { Yes } \square \text { No } \\
& \text { If no, provide details: }
\end{aligned}
$$

3.5 Applicable Tri-Party Agreement milestones related to storage: No TPA milestone is directly associated with storage of this waste stream.

3.6 Current inventory for this stream

Total LDR volume (cubic meters): 7

Date of inventory values: $9 / 30 / 98$

Comments on waste inventory: Based on inventory residing at the $\mathrm{CWC}$ as reported in the

Solid Waste Information Tracking System (SWITS) for WSRd EHG.

3.7 is this waste stream currently generated; if not, will this waste stream be generated in the next 5 years? $\square$ Yes $\bigotimes$ No. If no, skip to 3.11.

3.8 The current or future generation of this waste is best described as: 
$\square$ Routine $\square$ One-time or sporadic

3.9 Estimated generation projection by fiscal year

$\begin{array}{llll}1999 & \mathrm{~m}^{3} \text { (and/or) } \mathrm{kg} & \text { NA } \\ 2000 & & & \bigotimes \\ 2001 & & & \\ 2002 & & & \end{array}$

3.10 Last year's fiscal year 1998 generation projection:

Actual quantity generated in 1998: (both in $\mathrm{m}^{3}$ unless otherwise noted)

3.11 Has there ever been any unusual release of this stream to the environment?

$\square$ Yes QNo

If yes, summarize releases and quantities:

\subsection{WASTE STREAM TREATMENT}

4.1 Is this stream currently being treated?

$\square$ Yes $\bigotimes$ No If yes, provide details:

4.2 Planned treatment: Check the appropriate box indicating future plans for treating this waste stream to meet applicable regulations, including LDR treatment standards.

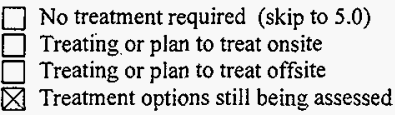

4.3 Planned treatment method, facility, extent of treatment capacity available: Since this waste is a past-practice waste stream and must undergo characterization upgrading before treatment, the specific treatment methods, facilities and schedules will not be determined until the appropriate WSS WSRd is assigned.

4.4 Treatment schedule information: TBD

4.5 Applicable treatment Tri-Party Agreement milestone numbers (including permitting): Counts toward M-19-00 volume commitments (treatment).

4.6 If treating or planning to treat on site, was or will waste minimization be addressed in developing and/or selecting the treatment method? $\square$ Yes $\square$ No $\square$ Unknown

If yes, please describe:

4.7 Treatability equivalency petitions, rulemaking petitions, and case-by-case exemptions needed for treatment:

\subsection{WASTE STREAM DISPOSAL}

5.1 After treatment, how will the waste stream be disposed of (include description, locations, variances required, etc., as applicable): Subject waste ultimately will be disposed into a RCRA Subtitle-C disposal cell located on the Hanford Site; however, the waste must undergo upgrading and treatment prior to disposal. 
DOE/RL-99-01

5.2 Applicable Tri-Party Agreement milestone number(s) for disposal: M-91-13

5.3 Planned disposal dates/time-frame: TBD

\subsection{WASTE MINIMIZATION}

6.1 Has a waste minimization assessment been completed for this stream?

$\square$ Yes $\bigotimes$ No $\square \mathrm{NA}$

6.2 Explain any waste minimization activities (e.g., process changes) under way or to be implemented for generation of this stream: No new waste was generated during 1998. Therefore a waste assessment for this stream is not necessary and was not conducted during the calendar year.

To the extent practical, all mixed waste is segregated and packaged separately from LLW or TRU wastes. The volume of mixed waste is reduced by in-drum compaction when possible, and where it does not interfere with future treatment activities. To minimize the generation of mixed waste, generators actively seek nondangerous alternatives for the dangerous constituents in their processes. Minimization goals are set annually and tracked quarterly, and waste treatment is used to destroy the hazardous constituents, as allowable.

6.3 Schedule for implementing waste minimization methods: Waste minimization activities are an ongoing activity.

6.4 Waste reduction achieved during the calendar year and projected future reductions (volume or mass): None

6.4.1 Assumptions used in above estimates: NA 


\section{WASTE STREAM PROFILE SHEET}

\subsection{WASTE STREAM IDENTIFICATION AND SOURCE}

1.1 Waste stream ID: MLLW-06B

\subsection{Waste stream name: Waste Specification System Elemental Mercury}

1.3 Waste stream source information

1.3.1 Stream source and history description. Include how the waste was managed prior to storage, timeframe when waste was placed into storage. Subject waste was generated and put into CWC storage either after the implementation of the Waste Specification System (WSS) or has been upgraded from various past-practice waste streams. The waste was generated by a few onsite generating organizations and offsite generators. There was no one primary generator for this waste stream.

1.3.2 Source category $(\mathrm{s})$

$\square$ Pollution control or waste treatment process $\square$ Materials production/recovery effluents

叉 Facility or equipment operation and maintenance waste

Discarded excess or expired materials

R\&D/R\&D laboratory waste

Spill clean-ups or emergency response actions Other (explain):

1.3.3 Additional notes: This is a general waste category based on physical, chemical, radiological and regulatory factors.

\subsection{WASTE STREAM CHARACTERIZATION}

2.1 Waste stream description (content): This waste stream consists of elemental mercury, amalgamated mercury, and debris articles containing small amounts of elemental mercury in their componentry (mercury switches, thermometers, etc). The current WSRds making up this waste stream are 810 and 811 .

\subsection{Radiological characteristics}

\subsubsection{Mixed Waste type $\square$ High-level $\square$ Transuranic $\square$ Low-level 区Contact-handled $\square$ Remote-handled}

2.2.2 Comments on radiological characteristics (e.g., more specific content, treatment concerns caused by radiation, confidence level): Since this waste is a general category based on dangerous waste physical characteristics, the radiological characteristics are expected to vary greatly. However, there is high confidence that the waste is CH-MLLW.

\subsection{Matrix characteristics (physical content)}

2.3.1 Matrix constituent table (comprising at least $1 \%$ of the total volume or mass)

\begin{tabular}{ccc}
$\begin{array}{c}\text { Matrix Parameter } \\
\text { Category Code }\end{array}$ & $\begin{array}{c}\text { Matrix Constituent } \\
\text { Description }\end{array}$ & $\begin{array}{c}\text { Typical } \\
\text { or Range }(\%)\end{array}$ \\
\hline X7100 & Elemental Mercury & $\mathbf{3 0 - 5 0 \%}$
\end{tabular}


DOE/RL-99-01

Overall matrix parameter category code: $\quad X 7100$

Overall matrix description: Elemental Mercury

2.3.2 Confidence level for matrix characteristics data in 2.3.1

$\square$ Low $\square$ Medium 区High

2.3.3 Comments on matrix characteristics and/or confidence level: This waste stream can consist of many different physical matrix characteristic types since it based on LDR requirements for treatment and disposal of a dangerous waste. There is a high confidence that subject waste stream will not contain physical matrix characteristics that do not meet the waste stream description.

\subsection{Regulated contaminant characteristics}

2.4.1 Wastewater/non-wastewater under RCRA

$\square$ Wastewater $\bigotimes$ Nori-wastewater $\square$ Unknown

2.4.2 Regulated contaminant table including treatment requirements, and UHCs if applicable

\begin{tabular}{|c|c|c|c|c|c|}
\hline $\begin{array}{l}\text { EPA/ } \\
\text { State } \\
\text { Code } \\
\end{array}$ & Waste Description & $\begin{array}{c}\text { LDR } \\
\text { Sub- } \\
\text { category }\end{array}$ & $\begin{array}{c}\text { Concentration } \\
\text { (Typical or } \\
\text { Range)* }\end{array}$ & Basis & $\begin{array}{c}\text { LDR } \\
\text { Concentration Limit or } \\
\text { Technology Code }\end{array}$ \\
\hline$\overline{\mathrm{D} 002}$ & Corrosive & Corrosive Char & $\mathbf{r} \quad \star \star * x$ & $\begin{array}{l}\text { Treatment } \\
\text { Technology }\end{array}$ & $\begin{array}{l}\text { Alternative Treatment } \\
\text { Stds. For Haz. Debris } \\
\text { (40 CFR 268.45) }\end{array}$ \\
\hline D009 & TC-Mercury & Elemental & $* * *$ & $"$ & Amalgamation \\
\hline U151 & Mercury & Elemental & $* * *$ & $"$ & $"$ \\
\hline WT01 & Toxic, EHW & NA & $* * *$ & $* * *$ & None (1) \\
\hline
\end{tabular}

UHCs Identification not required for D009 elemental mercury and hazardous debris when using the alternative treatment standards for hazardous debris.

1) Mixed extremely hazardous wastes may be land-disposed in Washington State in DOE facilities in accordance with RCW 70.105.050(2).

2) The combination of waste codes varies on a per-package basis in accordance with WAC 173-303070(3) and (5).

* If waste isn't consistent in concentration, this may not apply. Describe in 2.4.5.

2.4.2.1 List any waste codes from 2.4.2 for which the stream already meets established LDR treatment standards: It is assumed that all waste in this waste stream will require various levels of treatment prior to disposal

\subsubsection{Does this waste stream contain PCBs?}

$$
\begin{aligned}
& \square \text { Yes } \square \text { No } \square \text { Unknown. } \\
& \text { If no, skip to } 2.4 .4 \text {. }
\end{aligned}
$$

2.4.3.1 Is waste stream subject to TSCA regulations for PCBs?

$$
\square \text { Yes } \square \text { No } \square \text { Unknown }
$$

2.4.3.2 Indicate the PCB concentration range.

$$
\square<50 \mathrm{ppm} \square \geq 50 \mathrm{ppm} \quad \square \text { Unknown }
$$


2.4.4 What is the confidence level for the regulated contaminant characteristic data?

$\square$ Low $\square$ Medium $\triangle$ High

2.4.5 Comments on regulated contaminant characteristics and/or confidence level: Subject waste has met the rigors of the Waste Specification System for waste storage and treatment.

2.4.6 Will further characterization be performed? $\square$ Yes $\quad$ No $\square$ Unknown

2.4.6.1 If yes, provide details and schedule:

2.4.6.2 If yes, provide Tri-Party Agreement milestone numbers:

\subsection{WASTE STREAM STORAGE, INVENTORY, AND GENERATION}

3.1 Is this waste stream currently stored? $\square$ Yes $\square$ No If no, skip to 3.7.

3.2 Current storage method

$\begin{array}{ll}\square \text { Container (pad) } & \square \text { Container (covered) } \\ \square \text { Tank } & \square \text { Container (retrievably buried) } \\ \square \text { Other (explain): } & \end{array}$

3.3 TSD unit name and building number and (if applicable/available) number of containers/tanks in each: There are approximately 8 packages residing in the $\mathrm{CWC}$.

3.4 Is storage of waste in compliance with applicable State and Federal standards?

$\triangle$ Yes $\square$ No

If no, provide details:

3.5 Applicable Tri-Party Agreement milestones related to storage: No TPA milestone is directly associated with storage of this waste stream.

3.6 Current inventory for this stream

Total LDR volume (cubic meters): 2

Date of inventory values: $\quad 9 / 30 / 98$

Comments on waste inventory: Based on inventory residing at the CWC as reported in the Solid Waste Information Tracking System (SWITS) for WSRds 810 \& 811 .

3.7 Is this waste stream currently generated; if not, will this waste stream be generated in the next 5 years?

QYes $\square$ No. If no, skip to 3.11 .

3.8 The current or future generation of this waste is best described as:

$\bigotimes$ Routine $\square$ One-time or sporadic

3.9 Estimated generation projection by fiscal year

$\begin{array}{lllll} & \mathrm{m}^{3} & \text { (and/or) } & \mathrm{kg} & \\ 1999 & \mathbf{0 . 4} & & & \\ 2000 & \mathbf{0 . 4} & & & \square \\ 2001 & 0.4 & & & \square \\ 2002 & 0.4 & & & \square \\ 2003 & \mathbf{0 . 4} & & & \square\end{array}$

3.10 Last year's fiscal year 1998 generation projection: .008

Actual quantity generated in 1998: .62 (both in $\mathrm{m}^{3}$ unless otherwise noted) 
3.11 Has there ever been any unusual release of this stream to the environment?

$\square$ Yes 区No

If yes, summarize releases and quantities:

\subsection{WASTE STREAM TREATMENT}

4.1 Is this stream currently being treated?

$\square$ Yes $\square$ No If yes, provide details:

4.2 Planned treatment: Check the appropriate box indicating future plans for treating this waste stream to meet applicable regulations, including LDR treatment standards.
No treatment required (skip to 5.0 )
Treating or plan to treat onsite
Treating or plan to treat offsite
\Treatment options still being assessed

4.3 Planned treatment method, facility, extent of treatment capacity available: Mercury amalgamation per the Treatment Standards for Hazardous Wastes (40 CFR268.40) is the specified treatment technology for elemental mercury. Treatment will be performed by means of onsite and offsite commercial treatment contracts, and/or by onsite treatment units. There are no treatment campaigns planned for this waste until FY2002.

$\begin{array}{llll}4.4 \text { Treatment schedule information: } & \text { FY1999 ..... } & 0 \mathrm{~m}^{\wedge} 3 \\ & \text { FY2000 } \ldots . . . & 0 \mathrm{~m}^{\wedge} 3 \\ & \text { FY2001 } \ldots . . & 0 \mathrm{~m}^{\wedge} 3 \\ & \text { FY2002 } \ldots . . . & 2 \mathrm{~m}^{\wedge} 3 \\ & \text { FY2003 } \ldots . . . & 2 \mathrm{~m}^{\wedge} 3 \\ & \text { FY2004 } \ldots . . . & 2 \mathrm{~m}^{\wedge} 3 \\ & \text { FY2005 } \ldots . . . & 2 \mathrm{~m}^{\wedge} 3 \\ & \text { FY2006 } \ldots . . . & 2 \mathrm{~m}^{\wedge} 3 \\ & \text { FY2007 } & \ldots . . & 1 \mathrm{~m}^{\wedge} 3 \\ & \text { FY2008 } & \ldots . . . & 1 \mathrm{~m}^{\wedge} 3 \\ & \text { FY2009 } \ldots . . . & \text { as generated }\end{array}$

4.5 Applicable treatment Tri-Party Agreement milestone numbers (including permitting): Counts toward M-19-00 volume commitments (treatment).

4.6 If treating or planning to treat on site, was or will waste minimization be addressed in developing and/or selecting the treatment method? $\square$ Yes $\square$ No $\square$ Unknown

If yes, please describe:

4.7 Treatability equivalency petitions, rulemaking petitions, and case-by-case exemptions needed for treatment: TBD

\subsection{WASTE STREAM DISPOSAL}

5.1 After treatment, how will the waste stream be disposed of (include description, locations, variances required, etc., as applicable): Subject waste will be disposed into a RCRA Subtitle-C disposal cell located on the Hanford Site.

5.2 Applicable Tri-Party Agreement milestone number(s) for disposal: M-91-13 


\subsection{WASTE MINIMIZATION}

6.1 Has a waste minimization assessment been completed for this stream?

$\square$ Yes $\square$ No $\square$ NA

6.2 Explain any waste minimization activities (e.g., process changes) under way or to be implemented for generation of this stream: To the extent practical, all mixed waste is segregated and packaged separately from $L L W$ or TRU wastes. The volume of mixed waste is reduced by in-drum compaction when possible, and where it does not interfere with future treatment activities. To minimize the generation of mixed waste, generators actively seek nondangerous alternatives for the dangerous constituents in their processes. Minimization goals are set annually and tracked quarteriy, and waste treatment is used to destroy the hazardous constituents, as allowable.

6.3 Schedule for implementing waste minimization methods: Waste minimization activities are an ongoing activity.

6.4 Waste reduction achieved during the calendar year and projected future reductions (volume or mass): None

6.4.I Assumptions used in above estimates: NA 


\section{WASTE STREAM PROFILE SHEET}

\subsection{WASTE STREAM IDENTIFICATION AND SOURCE}

\subsection{Waste stream ID: MLLW-07A}

\subsection{Waste stream name: M-91 Remote Handled Mixed Low-Level Waste (Past-Practice)}

\subsection{Waste stream source information}

1.3.1 Stream source and history description. Include how the waste was managed prior to storage, timeframe when waste was placed into storage. Subject waste was generated and put into CWC storage prior to the implementation of the Waste Specification System (WSS); therefore, it is considered as a past-practice waste. This waste stream is the remote-handled mixed waste generated on the Hanford Facility and offsite excluding the Long-Length Contaminated Equipment waste stream residing in MLLW-01D. Hanford began accumulating RH-LLMW in the CWC buildings during 1987. The waste is awaiting treatment capacity at Hanford or within the DOE Complex. The waste was generated by onsite generating organizations, primarily the SST/DST systems.

\subsubsection{Source category(s)}

$\square$ Pollution control or waste treatment process $\square$ Materials production/recovery effluents

$\bigotimes$ Facility or equipment operation and maintenance waste

Discarded excess or expired materials $\square$ Analytical laboratory waste

$\square$ R\&D/R\&D laboratory waste

Spill clean-ups or emergency response actions $\square$ Source unknown

Other (explain):

1.3.3 Additional notes: This is a general waste category based on radiological and regulatory factors.

\subsection{WASTE STREAM CHARACTERIZATION}

2.1 Waste stream description (content): This waste stream is comprised of remote-handled mixed low-level waste (RH-MLLW) with various chemical (organics, inroganics, metals) and physical (particulates, debris, sludges, etc.) characteristics. Many different regulated constituents could be represented in this waste stream; however, the primary waste type is heterogeneous debris from the SST/DST systems operations. The current WSRd making up this waste stream is HRW.

\subsection{Radiological characteristics}

\subsubsection{Mixed Waste type $\square$ High-level $\square$ Transuranic QLow-level} $\square$ Contact-handled $\bigotimes$ Remote-handled

2.2.2 Comments on radiological characteristics (e.g., more specific content, treatment concerns caused by radiation, confidence level): Remote-handled $(\mathrm{RH})$ waste must be shielded down to contact-handled (CH) levels before it can be accepted into the $C W C$; therefore, $\mathrm{RH}$ waste packages in CWC are actually input into SWITS as CH. To determine if a CWC waste package contains RH waste, the radionuclide, dose rate, physical form and generator information in SWITS is reviewed for clues that might lead a reviewer to believe a waste may be $R H$. If there are any indications the waste maybe RH, then it is assigned to the HRW WSRd.

2.3 Matrix characteristics (physical content) 
2.3.1 Matrix constituent table (comprising at least $1 \%$ of the total volume or mass)

\begin{tabular}{ccc}
$\begin{array}{c}\text { Matrix Parameter } \\
\text { Category Code }\end{array}$ & $\begin{array}{c}\text { Matrix Constituent } \\
\text { Description }\end{array}$ & $\begin{array}{c}\text { Typical } \\
\text { or Range (\%) }\end{array}$ \\
\hline S5000 & Debris & $90 \%$ \\
S4000 & Soil/Gravel & $5 \%$ \\
X7210 & Lead & $\mathbf{2 \%}$ \\
$\mathbf{S 3 2 0 0}$ & Organic Solids & $1 \%$ \\
X6000 & Labpacks & $<1 \%$ \\
X7100 & Mercury & $<1 \%$ \\
\$3100 & Inorganic Solids & $<1 \%$
\end{tabular}

Overall matrix parameter category code: $\mathbf{S 5 0 0 0}$

Overall matrix description: Debris

2.3.2 Confidence level for matrix characteristics data in 2.3.1

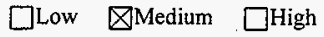

2.3.3 Comments on matrix characteristics and/or confidence level: Subject waste will undergo matrix characterization verifications as part of the past-practice upgrading program. Once the waste meets all the upgrading requirements, it will be assigned to the proper WSS WSRd associated with waste stream MLLW-07B. If during the upgrading process it is determined that some of the waste does not meet one of the MLLW-07B waste stream WSRds, then the appropriate WSRd will be assigned to the waste, and then the waste will be transferred to the correct waste stream.

\subsection{Regulated contaminant characteristics}

2.4.1 Wastewater/non-wastewater under RCRA

$\square$ Wastewater $\triangle$ Non-wastewater $\square$ Unknown

2.4.2 Regulated contaminant table including treatment requirements, and UHCs if applicable

\begin{tabular}{|c|c|c|c|c|c|}
\hline $\begin{array}{l}\text { EPA/ } \\
\text { State } \\
\text { Code } \\
\end{array}$ & Waste Description & $\begin{array}{c}\text { LDR } \\
\text { Sub- } \\
\text { category }\end{array}$ & $\begin{array}{l}\text { Concentration } \\
\text { (Typical or } \\
\text { Range) }\end{array}$ & Basis & $\begin{array}{c}\text { LDR } \\
\text { Concentration Limit or } \\
\text { Technology Code }\end{array}$ \\
\hline D001 & Ignitable & Various & $* * *$ & $\star \star \star$ & Various \\
\hline D002 & Corrosive & Various & $* * *$ & $\star \star *$ & Various \\
\hline D006 & TC-Cadmium & Various & $* * *$ & $* * *$ & Various \\
\hline D007 & TC-Chromium & NA & $* * *$ & $* * *$ & $5.0 \mathrm{mg} / \mathrm{l} \mathrm{TCLP}$ \\
\hline D008 & TC-Lead & Various & $* * \star$ & $* * *$ & Various \\
\hline D011 & TC-Silver & NA & $* * *$ & $* * *$ & $5.0 \mathrm{mg} / \mathrm{TCLP}$ \\
\hline F001 & Spent Solvent & 1,1,1-Trichloroethane & $e^{* * *}$ & *** & $6.0 \mathrm{mg} / \mathrm{kg}$ \\
\hline F002 & Spent Solvent & Methylene Chloride & $\star * *$ & $\star \star \star *$ & $30 \mathrm{mg} / \mathrm{kg}$ \\
\hline F003 & Spent Solvent & Acetone \& Hexone & $* * *$ & *** & $160 \mathrm{mg} / \mathrm{kg}$ \\
\hline F004 & Spent Solvent & o-Cresol \& p-Cresol & $* * *$ & $* * *$ & $5.6 \mathrm{mg} / \mathrm{kg}$ \\
\hline F005 & Spent Solvent & Methyl Ethyl Ketone & $* * *$ & $* \star *$ & $36 \mathrm{mg} / \mathrm{kg}$ \\
\hline WT01 & Toxic, EHW & NA & $* * *$ & *** & None (1) \\
\hline WT02 & Toxic, DW & NA & $* * *$ & *** & None \\
\hline WP01 & Persistant, EHW & NA & $* * *$ & $* * *$ & None (1) \\
\hline WP02 & Persistant, DW & NA & $* * *$ & *** & None \\
\hline
\end{tabular}

UHCs TBD on a per-package basis during waste "up-grading" and/or when the waste is sent for treatment. 
1) Mixed extremely hazardous wastes may be land-disposed in Washington State in DOE facilities in accordance with RCW 70.105.050(2).

2) The combination of waste codes varies on a per-package basis in accordance with WAC 173-303070(3) and (5).

* If waste isn't consistent in concentration, this may not apply. Describe in 2.4.5.

2.4.2.1 List any waste codes from 2.4 .2 for which the stream already meets established LDR treatment standards: It is assumed that all waste in this waste stream will require various levels of treatment prior to disposal

2.4.3 Does this waste stream contain PCBs?

$$
\square \text { Yes } \underset{\text { If no, skip to 2.4.4. }}{\square \text { No }}
$$

2.4.3.1 Is waste stream subject to TSCA regulations for PCBs?

$\square$ Yes $\square$ No $\square$ Unknown

2.4.3.2 Indicate the $\mathrm{PCB}$ concentration range.

$\square<50 \mathrm{ppm} \square 250 \mathrm{ppm} \square$ Unknown

2.4.4 What is the confidence level for the regulated contaminant characteristic data?

$\square$ Low $\nabla$ Medium $\square$ High

2.4.5 Comments on regulated contaminant characteristics and/or confidence level: Subject waste will undergo characterization verifications as part of the past-practice upgrading program. Once the waste meets all the upgrading requirements, it will be assigned to the proper WSS WSRd associated with waste stream MLLW-07B. If during the upgrading process it is determined that some of the waste does not meet one of the MLLW-07B waste stream WSRds, then the appropriate WSRd will be assigned to the waste, and then the waste will be transferred to the correct waste stream.

2.4.6 Will further characterization be performed? $\quad$ Yes $\square$ No $\square$ Unknown

2.4.6.1 If yes, provide details and schedule: TBD

2.4.6.2 If yes, provide Tri-Party Agreement milestone numbers: M-91-10, M-91-11-T01, and M-91-15

\subsection{WASTE STREAM STORAGE, INVENTORY, AND GENERATION}

3.1 Is this waste stream currently stored? $\quad$ Yes $\square$ No If no, skip to 3.7.

3.2 Current storage method

$\begin{array}{ll}\square \text { Container (pad) } & \square \text { Container (covered) } \\ \square \text { Tank } & \square \text { Container (retrievably buried) } \\ \square \text { Other (explain): } & \end{array}$

3.3 TSD unit name and building number and (if applicable/available) number of containers/tanks in each: There are approximately 20 packages residing in the CWC.

3.4 Is storage of waste in compliance with applicable State and Federal standards?

$\triangle$ Yes $\square$ No

If no, provide details: 
DOE/RL-99-01

3.5 Applicable Tri-Party Agreement milestones related to storage: M-91-10, M-91-11-T01, and M-9115

3.6 Current inventory for this stream

Total LDR volume (cubic meters): 38

Date of inventory values: $9 / 30 / 98$

Comments on waste inventory: Based on inventory residing at the $\mathrm{CWC}$ as reported in the Solid Waste Information Tracking System (SWITS) for WSRd HRW.

3.7 Is this waste stream currently generated; if not, will this waste stream be generated in the next 5 years? $\square$ Yes 区No. If no, skip to 3.I1.

3.8 The current or future generation of this waste is best described as:

$\square$ Routine $\square$ One-time or sporadic

3.9 Estimated generation projection by fiscal year

$\begin{array}{lllll}1999 & \mathrm{~m}^{3} \quad \text { (and/or) } & \mathrm{kg} & \mathrm{NA} \\ 2000 & & & & \bigotimes \\ 2001 & & & & \bigotimes \\ 2002 & & & & \bigotimes \\ 2003 & & & \end{array}$

3.10 Last year's fiscal year 1998 generation projection:

Actual quantity generated in 1998: (both in $\mathrm{m}^{3}$ unless otherwise noted)

3.11 Has there ever been any unusual release of this stream to the environment?

$\square$ Yes 区No

If yes, summarize releases and quantities:

\subsection{WASTE STREAM TREATMENT}

4.1 Is this stream currently being treated?

पYes 区No If yes, provide details:

4.2 Planned treatment: Check the appropriate box indicating future plans for treating this waste stream to meet applicable regulations, including LDR treatment standards.

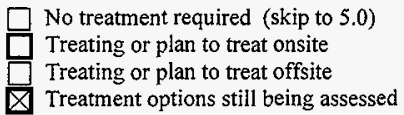

4.3 Planned treatment method, facility, extent of treatment capacity available: Treatment options are being addressed as part of the Project Management Plan specified in TPA Milestone M-91-10.

4.4 Treatment schedule information: TBD

4.5 Applicable treatment Tri-Party Agreement milestone numbers (including permitting): $\mathbf{M - 9 1 - 1 0 ,} \mathbf{M}-$ 91-11-T01, and M-91-15.

4.6 If treating or planning to treat on site, was or will waste minimization be addressed in developing and/or selecting the treatment method? $\square$ Yes $\square$ No $\bigotimes$ Unknown

If yes, please describe: 
4.7 Treatability equivalency petitions, rulemaking petitions, and case-by-case exemptions needed for treatment: TBD

\subsection{WASTE STREAM DISPOSAL}

5.1 After treatment, how will the waste stream be disposed of (include description, locations, variances required, etc., as applicable): Subject waste ultimately will be disposed into a RCRA Subtitle-C disposal cell located on the Hanford Site; however, the waste must undergo upgrading and treatment prior to disposal.

5.2 Applicable Tri-Party Agreement milestone number(s) for disposal: M-91-10, M-91-11-T01, and M91-15

5.3 Planned disposal dates/time-frame: TBD

\subsection{WASTE MINIMIZATION}

6.1 Has a waste minimization assessment been completed for this stream?

$\square$ Yes $\square$ No $\square$ NA

6.2 Explain any waste minimization activities (e.g., process changes) under way or to be implemented for generation of this stream: No new waste was generated during 1998. Therefore a waste assessment for this stream is not necessary and was not conducted during the calendar year.

To the extent practical, all mixed waste is segregated and packaged separately from LLW or TRU wastes. The volume of mixed waste is reduced by in-drum compaction when possible, and where it does not interfere with future treatment activities. To minimize the generation of mixed waste, generators actively seek nondangerous alternatives for the dangerous constituents in their processes. Minimization goals are set annually and tracked quarterly, and waste treatment is used to destroy the hazardous constituents, as allowable.

6.3 Schedule for implementing waste minimization methods: Waste minimization activities are an ongoing activity.

6.4 Waste reduction achieved during the calendar year and projected future reductions (volume or mass): None

6.4.1 Assumptions used in above estimates: NA 


\title{
WASTE STREAM PROFILE SHEET
}

\subsection{WASTE STREAM IDENTIFICATION AND SOURCE}

\author{
1.1 Waste stream ID: MLLW-07B
}

1.2 Waste stream name: System )

\section{M-91 Remote-Handled Mixed Low-Level Waste (Waste Specification}

\subsection{Waste stream source information}

1.3.1 Stream source and history description. Include how the waste was managed prior to storage, timeframe when waste was placed into storage. Subject waste was generated and put into CWC storage either after the implementation of the Waste Specification System (WSS) or has been upgraded from various past-practice waste streams. The waste was generated by onsite generating organizations. There was no one primary onsite generating organization for this waste stream.

\subsubsection{Source category(s)}
$\square$ Pollution control or waste treatment process
Materials production/recovery effluents
$\triangle$ Facility or equipment operation and maintenance waste
Discarded excess or expired materials
$\triangle$ R\&D/R\&D laboratory waste
Spill clean-ups or emergency response actions

Other (explain):

1.3.3 Additional notes: This is a very broad waste stream based on radiological and regulatory factors.

\subsection{WASTE STREAM CHARACTERIZATION}

2.1 Waste stream description (content): This waste stream is comprised of remote-handled mixed low-level waste (RH-MLLW) with various chemical (organics, inorganics, metals) and physical (particulates, debris, sludges, etc.) characteristics. Many different regulated constituents could be represented in this waste stream; however, the primary waste type is heterogeneous debris from the SST/DST systems operations. The current WSRds making up this waste stream are $601,605,606$, 800 , and 801 .

\subsection{Radiological characteristics}

\subsubsection{Mixed Waste type $\square$ High-level $\square$ Transuranic \Low-level} $\square$ Contact-handled $\bigotimes$ Remote-handled

2.2.2 Comments on radiological characteristics (e.g., more specific content, treatment concerns caused by radiation, confidence level): Remote handled (RH) waste must be shielded down to contact-handled (CH) levels before it can be accepted into the $\mathrm{CWC}$; therefore, $\mathrm{RH}$ waste packages in CWC are actually input into SWITS as $\mathrm{CH}$. To determine if a $\mathrm{CWC}$ waste package contains $\mathrm{RH}$ waste, the radionuclide, dose rate, physical form and generator information in SWITS is reviewed for clues that might lead a reviewer to believe a waste may be $\mathrm{RH}$. If there are any indications the waste maybe $\mathrm{RH}$, then it is assigned to this waste stream.

2.3 Matrix characteristics (physical content) 
2.3.1 Matrix constituent table (comprising at least $\mathrm{I} \%$ of the total volume or mass)

\begin{tabular}{ccc} 
Matrix Parameter & Matrix Constituent & Typical \\
Category Code & Description & or Range (\%) \\
\hline S5400 & Heterogeneous Debris & $\mathbf{9 0 - 9 5 \%}$ \\
X7210 & Elemental Lead & $\mathbf{5 - 1 0 \%}$
\end{tabular}

Overall matrix parameter category code: $\mathbf{S 5 4 0 0}$

Overall matrix description: Heterogeneous Debris

2.3.2 Confidence level for matrix characteristics data in 2.3.1

$\square$ Low $\square$ Medium $\bigotimes$ High

2.3.3 Comments on matrix characteristics and/or confidence level: This waste stream can consist of many different physical matrix characteristic types since it based on LDR requirements for treatment and disposal of a dangerous waste. There is a high confidence that subject waste stream will not contain physical matrix characteristics that do not meet the waste stream description.

\subsection{Regulated contaminant characteristics}

\subsubsection{Wastewater/non-wastewater under RCRA}

$\square$ Wastewater 囚Non-wastewater $\square$ Unknown

2.4.2 Regulated contaminant table including treatment requirements, and UHCs if applicable

\begin{tabular}{|c|c|c|c|c|c|}
\hline $\begin{array}{l}\text { EPA/ } \\
\text { State } \\
\text { Code }\end{array}$ & Waste Description & $\begin{array}{c}\text { LDR } \\
\text { Sub- } \\
\text { category }\end{array}$ & $\begin{array}{l}\text { Concentration } \\
\text { (Typical or } \\
\text { Range)* }\end{array}$ & Basis & $\begin{array}{c}\text { LDR } \\
\text { Concentration Limit or } \\
\text { Technology Code }\end{array}$ \\
\hline$\overline{\mathrm{D} 001}$ & Ignitable & Various & $\star \star \star \star$ & $\star \star \star *$ & Various \\
\hline D002 & Corrosive & Various & $* * *$ & $* * *$ & Various \\
\hline D006 & TC-Cadmium & Various & $* * *$ & $* * *$ & Various \\
\hline D007 & TC-Chromium & NA & $\approx * *$ & $* * *$ & $5.0 \mathrm{mg} / \mathrm{l} \mathrm{TCLP}$ \\
\hline D008 & TC-Lead & Various & *** & $* * *$ & Various \\
\hline D011 & TC-Silver & $\mathbf{N A}_{\mathbf{A}}$ & $* * *$ & $* * *$ & $5.0 \mathrm{mg} / \mathrm{I}$ TCLP \\
\hline F001 & Spent Solvent & 1,1,1-Trichloroethan & $n e^{* * *}$ & $\star * *$ & $6.0 \mathrm{mg} / \mathrm{kg}$ \\
\hline F002 & Spent Solvent & Methylene Chloride & $* * *$ & $* * *$ & $30 \mathrm{mg} / \mathrm{kg}$ \\
\hline F003 & Spent Solvent & Acetone \& Hexone & $* * *$ & $* \star *$ & $160 \mathrm{mg} / \mathrm{kg}$ \\
\hline F004 & Spent Solvent & o-Cresol \& p-Cresol & $* * *$ & $* * *$ & $5.6 \mathrm{mg} / \mathrm{kg}$ \\
\hline F005 & Spent Solvent & Methyl Ethyl Ketone & le $* * *$ & $* * *$ & $36 \mathrm{mg} / \mathrm{kg}$ \\
\hline WT01 & Toxic, EHW & NA & $* * *$ & $* * *$ & None (1) \\
\hline WT02 & Toxic, DW & NA & $* * *$ & $* * *$ & None \\
\hline WP01 & Persistant, EHW & NA & $\star * *$ & $* * *$ & None (1) \\
\hline WP02 & Persistant, DW & NA & $\star \star \star \star$ & $\star \star \star *$ & None \\
\hline
\end{tabular}

UHCs TBD on a per-package basis during waste receipt and/or when the waste is sent for treatment (see Section 2.4.5).

1) Mixed extremely hazardous wastes may be land-disposed in Washington State in DOE facilities in accordance with RCW 70.105.050(2).

2) The combination of waste codes varies on a per-package basis in accordance with WAC 173-303$070(3)$ and (5).

* If waste isn't consistent in concentration, this may not apply. Describe in 2.4.5. 
2.4.2.1 List any waste codes from 2.4.2 for which the stream already meets established LDR treatment standards: It is assumed that all waste in this waste stream will require various levels of treatment prior to disposal

2.4.3 Does this waste stream contain PCBs?

$\square$ Yes 区No $\square$ Unknown.

If no, skip to 2.4 .4 .

2.4.3.1 Is waste stream subject to TSCA regulations for PCBs?

$\square$ Yes $\square$ No $\square$ Unknown

2.4.3.2 Indicate the $\mathrm{PCB}$ concentration range.

$\square<50$ ppm $\square>50$ ppm $\square$ Unknown

2.4.4 What is the confidence level for the regulated contaminant characteristic data?

$\square$ Low $\square$ Medium $\bigotimes$ High

2.4.5 Comments on regulated contaminant characteristics and/or confidence level: Subject waste has met the rigors of the Waste Specification System for waste storage and treatment. However, the WSS came into effect in 1995 and was based on the dangerous waste regulations imposed at that time. There has been several changes to the dangerous waste regulations since then that impose additional characterization requirements onto the generator, namely identification of UHCs for all waste designated with a characteristic waste code (i.e., D001 through D043).

2.4.6 Will further characterization be performed? \Yes $\square$ No $\square$ Unknown

2.4.6.1 If yes, provide details and schedule: UHCs will be determined and identified on the corresponding LDR notification/certification at the time subject waste is sent to treatment.

2.4.6.2 If yes, provide Tri-Party Agreement milestone numbers: $\quad$ M-91-10, M-91-11-T01, and M-91-15

\subsection{WASTE STREAM STORAGE, INVENTORY, AND GENERATION}

3.1 Is this waste stream currently stored? $\bigotimes$ Yes $\square$ No If no, skip to 3.7.

3.2 Current storage method

$\square$ Container (pad)
$\square$ Tank
$\square$ Other (explain):

QContainer (covered)

$\square$ Waste pile

$\square$ Container (retrievably buried)

Gurface impoundment

3.3 TSD unit name and building number and (if applicable/available) number of containers/tanks in each: There are approximately 18 packages residing in the $\mathrm{CWC}$.

3.4 Is storage of waste in compliance with applicable State and Federal standards?

$\bigotimes$ Yes $\square$ No

If no, provide details:

3.5 Applicable Tri-Party Agreement milestones related to storage: No TPA milestone is directly associated with storage of this waste stream.

3.6 Current inventory for this stream

Total LDR volume (cubic meters): 23

Date of inventory values: $\quad 9 / 30 / 98$ 
Comments on waste inventory: Based on inventory residing at the $\mathrm{CWC}$ as reported in the Solid Waste Information Tracking System (SWITS) for MLLW with a dose rate exceeding $100 \mathrm{mR}$.

3.7 Is this waste stream currently generated; if not, will this waste stream be generated in the next 5 years? $\triangle Y$ es $\square$ No. If no, skip to 3.11 .

3.8 The current or future generation of this waste is best described as:

$\square$ Routine $\quad$ One-time or sporadic

3.9 Estimated generation projection by fiscal year

$\begin{array}{ccccc} & \mathrm{m}^{3} & \text { (and/or) } & \mathrm{kg} & \\ 1999 & \mathbf{4} 7 & & & \\ 2000 & \mathbf{1 6 0} & & & \square \\ 2001 & 177 & & & \square \\ 2002 & 176 & & & \square \\ 2003 & 136 & & & \square\end{array}$

3.10 Last year's fiscal year 1998 generation projection: 2.2

Actual quantity generated in 1998: 44 (both in $\mathrm{m}^{3}$ unless otherwise noted)

3.11 Has there ever been any unusual release of this stream to the environment?

$\square$ Yes 区No

If yes, summarize releases and quantities:

\subsection{WASTE STREAM TREATMENT}

4.1 Is this stream currently being treated?

$\square$ Yes $\bigotimes$ No If yes, provide details:

4.2 Planned treatment: Check the appropriate box indicating future plans for treating this waste stream to meet applicable regulations, including LDR treatment standards.

$\square$ No treatment required (skip to 5.0)
$\square$ Treating or plan to treat onsite
$\square$ Treating or plan to treat offsite
$\square$ Treatment options still being assessed

4.3 Planned treatment method, facility, extent of treatment capacity available: Treatment options are being addressed as part of the Project Management Plan specified in TPA Milestone M-91-10.

4.4 Treatment schedule information: TBD

4.5 Applicable treatment Tri-Party Agreement milestone numbers (including permitting): $\mathbf{M - 9 1 - 1 0 ,} \mathbf{M -}$ 91-11-T01, and M-91-15.

4.6 If treating or planning to treat on site, was or will waste minimization be addressed in developing and/or selecting the treatment method? $\square$ Yes $\square$ No $\square$ Unknown

If yes, please describe:

4.7 Treatability equivalency petitions, rulemaking petitions, and case-by-case exemptions needed for treatment: TBD

\subsection{WASTE STREAM DISPOSAL}


5.1 After treatment, how will the waste stream be disposed of (include description, locations, variances required, etc., as applicable): Subject waste will be disposed into a RCRA Subtitle-C disposal cell located on the Hanford Site.

5.2 Applicable Tri-Party Agreement milestone number(s) for disposal: M-91-10, M-91-11-T01, and M91-15.

5.3 Plarned disposal dates/time-frame: TBD

\subsection{WASTE MINIMIZATION}

6.1 Has a waste minimization assessment been completed for this stream?

$\square$ Yes 区No $\square$ NA

6.2 Explain any waste minimization activities (e.g., process changes) under way or to be implemented for generation of this stream: To the extent practical, all mixed waste is segregated and packaged separately from LLW or TRU wastes. The volume of mixed waste is reduced by in-drum compaction when possible, and where it does not interfere with future treatment activities. To minimize the generation of mixed waste, generators actively seek nondangerous alternatives for the dangerous constituents in their processes. Minimization goals are set annually and tracked quarterly, and waste treatment is used to destroy the hazardous constituents, as allowable.

6.3 Schedule for implementing waste minimization methods: Waste minimization activities are an ongoing activity.

6.4 Waste reduction achieved during the calendar year and projected future reductions (volume or mass): None

6.4.1 Assumptions used in above estimates: NA 


\section{WASTE STREAM PROFILE SHEET}

\subsection{WASTE STREAM IDENTIFICATION AND SOURCE}

\subsection{Waste stream ID: MLLW-08}

\subsection{Waste stream name: Greater-Than-Class 3 Waste}

1.3 Waste stream source information

1.3.1 Stream source and history description. Include how the waste was managed prior to storage, timeframe when waste was placed into storage. This waste stream consists of Greater-Than-Class 3 waste. The waste is made up of radioisotope thermoelectric generators (RTG). The first receipt into CWC of this waste was in 1980 and the second was in 1983.

\subsubsection{Source category(s)}

Pollution control or waste treatment process $\square$ Materials production/recovery effluents Facility or equipment operation and maintenance waste Discarded excess or expired materials $R \& D / R \& D$ laboratory waste Spill clean-ups or emergency response actions Other (explain): Discarded naval equipment

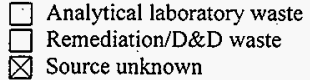

Source unknown

1.3.3 Additional notes:

\section{0 - WASTE STREAM CHARACTERIZATION}

2.1 Waste stream description (content): Greater-than-class 3 waste.

2.2 Radiological characteristics

2.2.1 Mixed Waste type $\square$ High-level $\square$ Transuranic $\bigotimes$ Low-level $\bigotimes$ Contact-handled $\square$ Remote-handled

2.2.2 Comments on radiological characteristics (e.g., more specific content, treatment concerns caused by radiation, confidence level): This waste stream has been packaged to contact-handled levels so it can be stored inside of the $\mathrm{CWC}$.

2.3 Matrix characteristics (physical content)

2.3.1 Matrix constituent table (comprising at least $1 \%$ of the total volume or mass)

\begin{tabular}{ccc}
$\begin{array}{c}\text { Matrix Parameter } \\
\text { Category Code }\end{array}$ & $\begin{array}{c}\text { Matrix Constituent } \\
\text { Description }\end{array}$ & $\begin{array}{c}\text { Typical } \\
\text { or Range (\%) }\end{array}$ \\
\hline $\mathbf{S 5 0 0 0}$ & Debris Waste & $95 \%$ \\
$\mathbf{X 7 2 1 0}$ & Lead Shielding & $\mathbf{5 \%}$
\end{tabular}

Overall matrix parameter category code: $\mathbf{S 5 0 0 0}$ 
Overall matrix description: Debris

2.3.2 Confidence level for matrix characteristics data in 2.3 .1 $\square$ Low $\square$ Medium $\bigotimes$ High

2.3.3 Comments on matrix characteristics and/or confidence level: The existing inventory has a high confidence level for matrix characteristic data.

2.4 Regulated contaminant characteristics

2.4.1 Wastewater/non-wastewater under RCRA

$\square$ Wastewater छNon-wastewater $\square$ Unknown.

2.4.2 Regulated contaminant table including treatment requirements, and UHCs if applicable

\begin{tabular}{lccccc}
$\begin{array}{l}\text { EPA/ } \\
\text { State } \\
\text { Code }\end{array}$ & Waste Description & $\begin{array}{c}\text { LDR } \\
\text { Sub- } \\
\text { category }\end{array}$ & $\begin{array}{c}\text { Concentration } \\
\text { (Typical or } \\
\text { Range)* }\end{array}$ & Basis & $\begin{array}{c}\text { Concentration Limit or } \\
\text { Technology Code }\end{array}$ \\
\hline D008 & Lead & NA & NA & NA & None (1)
\end{tabular}

(1) Lead shielding is a State-only waste when backfilled and is not subject to Federal LDRs.

* If waste isn't consistent in concentration, this may not apply. Describe in 2.4.5.

2.4.2.1 List any waste codes from 2.4.2 for which the stream already meets established LDR treatment standards: State-only D008. The Radioisotope Thermoelectric Generator waste is prohibited from disposal by DOE Orders.

2.4.3 Does this waste stream contain PCBs?

$$
\square_{\text {If no, skip to 2.4.4. }}^{\square} \text { YUnknown. }
$$

2.4.3.1 is waste stream subject to TSCA regulations for PCBs?

$\square$ Yes $\square$ No $\square$ Unknown

2.4.3.2 Indicate the $P C B$ concentration range.

$\square<50 \mathrm{ppm} \quad \square>50 \mathrm{ppm} \quad \square$ Unknown

2.4.4 What is the confidence level for the regulated contaminant characteristic data?

$\square$ Low $\square$ Medium $\otimes$ High

2.4.5 Comments on regulated contaminant characteristics and/or confidence level: None

2.4.6 Will further characterization be performed? $\square$ Yes $\square$ No QUnknown

2.4.6.1 If yes, provide details and schedule: TBD

2.4.6.2 If yes, provide Tri-Party Agreement milestone numbers: M-91-10, M-91-11-T01, and M-91-15

\subsection{WASTE STREAM STORAGE, INVENTORY, AND GENERATION}

3.1 Is this waste stream currently stored? QYes $\square$ No If no, skip to 3.7. 
3.2 Current storage method

$\begin{array}{lll}\square \text { Container (pad) } & \bigotimes \text { Qontainer (covered) } & \square \text { Container (retrievably buried) } \\ \square \text { Tank } & \square \text { Waste pile } & \square \text { Surface impoundment } \\ \square \text { Other (explain): } & \end{array}$

3.3 TSD unit name and building number and (if applicable/available) number of containers/tanks in each: CWC

3.4 Is storage of waste in compliance with applicable State and Federal standards?

$\triangle$ Yes $\square$ No

If no, provide details:

3.5 Applicable Tri-Party Agreement milestones related to storage: M-91-10, M-91-11-T01, and M-9115

3.6 Current inventory for this stream

Total LDR volume (cubic meters): 1

Date of inventory values: $\quad 9 / 30 / 97$

Comments on waste inventory: Based on inventory residing at the $\mathrm{CWC}$ reported in the

Solid Waste Information Tracking System (SWITS).

3.7 Is this waste stream currently generated; if not, will this waste stream be generated in the next 5 years? $\square$ Yes $\bigotimes$ No. If no, skip to 3.11.

3.8 The current or future generation of this waste is best described as:

$\square$ Routine $\square$ One-time or sporadic

3.9 Estimated generation projection by fiscal year

$\begin{array}{llll}1999 & \mathrm{~m}^{3} \quad \text { (and/or) } \mathrm{kg} \\ 2000 & & & \\ 2001 & & \end{array}$

3.10 Last year's fiscal year 1998 generation projection: NA

Actual quantity generated in 1998: (both in $\mathrm{m}^{3}$ unless otherwise noted)

3.11 Has there ever been any unusual release of this stream to the environment?

$\square$ Yes $\bigotimes$ No

If yes, summarize releases and quantities:

\subsection{WASTE STREAM TREATMENT}

4.1 Is this stream currently being treated?

$\square$ Yes 区No If yes, provide details:

4.2 Planned treatment: Check the appropriate box indicating future plans for treating this waste stream to meet applicable regulations, including LDR treatment standards.

$\bigotimes$ No treatment required (skip to 5.0)

$\square$ Treating or plan to treat onsite

$\square$ Treating or plan to treat offsite

$\square$ Treatment options still being assessed 
4.3 Planned treatment method, facility, extent of treatment capacity available:

4.4 Treatment schedule information:

4.5 Applicable treatment Tri-Party Agreement milestone numbers (including permitting):

4.6 If treating or planning to treat on site, was or will waste minimization be addressed in developing and/or selecting the treatment method? $\square$ Yes $\square$ No $\square$ Unknown

If yes, please describe:

4.7 Treatability equivalency petitions, rulemaking petitions, and case-by-case exemptions needed for treatment:

\subsection{WASTE STREAM DISPOSAL}

5.1 After treatment, how will the waste stream be disposed of (include description, locations, variances required, etc., as applicable): Disposal path will be determined as part of TPA milestone M-91-10.

5.2 Applicable Tri-Party Agreement milestone number(s) for disposal: $\quad$ M-91-00

5.3 Planned disposal dates/time-frame: TBD

\subsection{WASTE MINIMIZATION}

6.1 Has a waste minimization assessment been completed for this stream?

$$
\square \text { Yes \No } \square \text { NA }
$$

6.2 Explain any waste minimization activities (e.g., process changes) under way or to be implemented for generation of this stream: No new waste was generated during 1998 . Therefore a waste assessment for this stream is not necessary and was not conducted during the calendar year.

6.3 Schedule for implementing waste minimization methods: NA

6.4 Waste reduction achieved during the calendar year and projected future reductions (volume or mass): NA

6.4.1 Assumptions used in above estimates: NA 


\section{WASTE STREAM PROFILE SHEET}

\subsection{WASTE STREAM IDENTIFICATION AND SOURCE}

1.1 Waste Stream 1D:

1.2 Waste stream name

TRUM-01A

Contact-handled mixed TRU combustible debris

1.3.1 Stream source and history description:

on The waste was generated from the Plutonium Finishing Plant, the PUREX plant, the Critical Mass Laboratory, the Materials Engineering Laboratory, Kerr-McGee, and the REDOX facility. The waste was generated and placed into storage from 1987 to 1998.

1.3.2 Source category(s)

$\square$ Pollution control or waste treatment process

Material production/recovery effluents

$\nabla$ Facility or equipment operation and maintenance waste

$\square$ Discarded excess or expired materials

$\checkmark$ Analytical laboratory waste

$\checkmark$ RD/RD laboratory waste

$\checkmark$ Remediation/DD waste

$\square$ spill clean-ups or emergency response actions

$\square$ Source unknown

$\square$ Other (explain)

1.3.3 Additional notes:

\subsection{WASTE STREAM CHARACTERIZATION}

2.1 Waste stream description (content)

The waste stream consists of plastics, paper/cardboard, filters, rubber, wood, cloth/rags, metals, soil/rocks, chemicais, and glass.

2.2 Radiological characteristics

2.2.1 Mixed waste type

$\square$ High-level

$\nabla$ Transuranic

Low-level

(1) Contact-handled

Remote-handled

2.2.2 Comments on radiological characteristics (e.9., more specific content, treatment concerns caused by radiation, cónfidence level):

Each container of waste has more than $100 \mathrm{nCi} / \mathrm{g}$ of transuranic nuclide activity. Non-transuranic activity is either not reported or reported in trace quantities.

2.3 Matrix characteristics (physical content)

2.3.1 Matrix constituent table (comprising at least $1 \%$ of the total volume or mass)

\begin{tabular}{|c|c|c|}
\hline (1) & 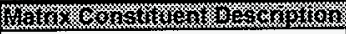 & 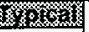 \\
\hline 54200 & Soil/debris. & $2.0 \%$ \\
\hline 55190 & Inorganic debris & $6.0 \%$ \\
\hline$\$ 5390$ & Organic debris & $75.9 \%$ \\
\hline$\$ 5490$ & Heterogeneous debris & $12.7 \%$ \\
\hline U9999 & Unknown/other & $2.0 \%$ \\
\hline
\end{tabular}

Overall matrix parameter category code: 55390 Overall matrix description: Organic debris

2.3.2 Confidence level for matrix characteristics data in 2.3.1 $\square$ low $\square$ medium $\square$ high 
2.3.3 Comments on matrix charaoteristics and/or confidence level:
The Waste Specification Record number for this waste stream is CBDTC, combustible debris - transuranic contact-handled.

\subsection{Regulated contaminant characteristics}

\subsubsection{Wastewater/non-wastewater under RCRA}
$\square$ wastewater
$\checkmark$ non-wastewater
unknown

2.4.2 Regulated contaminant table including treatment requirements, and UHC's if applicable

\begin{tabular}{|c|c|c|c|c|c|}
\hline 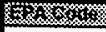 & 特 & 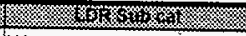 & shes & 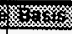 & 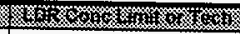 \\
\hline Do01 & Various & NA & 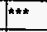 & *nt & Remove characteristic (1) \\
\hline 0002 & Various & NA & $n=$ & $\cdots$ & Remove characteristic (1) \\
\hline Do04 & ARSENIC & NA & $n+\star \pi$ & $* * *$ & Exempt (61 FR 60704) \\
\hline 2005 & BARIUM & NA & $+\infty$ & $+\ldots$ & Exempt (61 FR 60704) \\
\hline 006 & CADMIUM & NA & $* * * *$ & 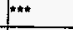 & Exempt (61 FR 60704) \\
\hline $\mathrm{D} 007$ & CHROMIUM & NA & ton* & $n+\cdots$ & Exempt (61 FR 60704) \\
\hline $\mathrm{D} 008$ & LEAD & NA & *A* & $n$ & Exempt (61 FR 60704) \\
\hline poog & MERCURY & NA & $* * *$ & $* * *$ & Exempt (61 FR 60704) \\
\hline bo11 & SILVER & NA & $i^{+ \text {Ant }}$ & treat & Exempt (61 FR 60704) \\
\hline po19 & CARBON TETRACHLORIDE & NA & $\cdots$ & $+\infty$ & Exempt (61 FR 60704) \\
\hline 035 & METHYL ETHYL KETONE & NA & + & 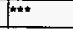 & Exempt (61 FR 60704) \\
\hline Foo1 & Spent Solvent & 1,1,1-TRICHLOROETHANE & $+*$ & $i^{\infty n+\infty}$ & Exempt (61 FR 60704) \\
\hline 5002 & Spent Solvent & TRICHLOROETHYLENE & 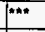 & $F * *$ & Exempt (61 FR 60704) \\
\hline Fo03 & Spent Solvent & ACETONE & $n * k$ & 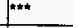 & Exempt (61 FR 60704) \\
\hline F004 & Spent Solvent & CRESOL & 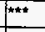 & $\pm * *$ & Exempt (61 FR 60704) \\
\hline Foo5 & Spent Solvent & METHYL ETHYL KETONE & $* * *$ & $+*$ & Exempt (61 FR 60704) \\
\hline WP01 & Persistant, EHW & NA & $+* *$ & $+\infty$ & N/A \\
\hline NPO2 & Persistant, DW & NA & $+\cdots$ & $+n$ & N/A \\
\hline WSC2 & Solid Corrosive & NA & $+* *$ & 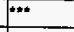 & N/A \\
\hline WTO1 & Toxic, EHW & NA & $\cdots$ & $+n *$ & $\mathrm{~N} / \mathrm{A}$ \\
\hline NTO2 & Toxic, DW & NA & F** & $* \star * \pi$ & N/A \\
\hline
\end{tabular}

UHC's are not applicable to the WIPP facility. (1) Treatment standards are based on the WIPP facility waste acceptance criteria.

2.4.2.1 List any waste codes from 2.4.2 for which the stream already meets established LDR treatment standard Waste is exempt from Federal LDR's when being disposed of at the WIPP facility.

2.4.3 Does this waste stream contain PCBs?
Yes
No
Unknown

2.4.3.1 Is the waste stream subject to TSCA regulations for PCBs?
$\square$ Yès
No
Unknown

2.4.3.2 Indicate the $\mathrm{PCB}$ concentration range
$\square<50 \mathrm{ppm}$
$>=50 \mathrm{ppm}$
Unknown

2.4.4 What is the confidence level for the regulated contaminant characteristic data?
$\square$ low
[.] medium
high 


\subsubsection{Comments on regulated contaminant characteristics} and/or confidence level: The regulated contaminants listed above are applicable to the existing
inventory. The contaminants vary widely in concentration. The
contaminants with the highest concentration are:
Lead and carbon tetrachloride.

2.4.6 Will further characterization be performed $\square$ Yes No Unknown
2.4.6.1 If yes, provide details and schedule:

Additional characterization will be conducted as necessary to meet the waste acceptance criteria for WIPP. Characterization will include process knowledge, and sampling and analysis.

2.4.6.2 If yes, provide Tri-Party Agreement milestone number No milestones are, applicable to characterization of TRU waste.

\subsection{WASTE STREAM STORAGE, INVENTORY, AND GENERATION \\ 3.1 Is the waste stream currently stored? $\quad \square$ Nos \\ 3.2 Current storage method

$\begin{array}{ll}\square \text { Container (pad) } & \square \text { Container (covered) } \\ \square \text { Tank } & \square \text { Waste pile } \\ \square \text { Other (explain) } & \end{array}$ \\ Surface impoundment}


id: $\quad$ TRUM:OIA

3.3 TSD unit name and building number and (if applicable/available) number of containers/tanks in each:

TSD Unit Name

Number of containers

Central Waste Complex

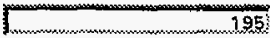

3.4 Is storage of waste in compliance with applicable State and Federal standards?

$\square$ Yes $\square$ No

If no, provide details:

3.6 Current inventory for this stream

Total LDR volume (cubic meters):

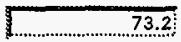

Date of Inventory values:

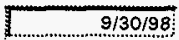

Comments on waste inventory:

The inventory is based on the Solid Waste Information and Tracking System. The waste is packaged in drums and boxes.

3.7 Is the waste stream gurrently generated; and if not, will this waste stream be generated in the next 5 years?
Yes
$\square$ No

3.8 The current or future generation of this waste is best described as:
Droutine
One-time or sporadic

3.9 Estimated generation projection by fiscal year

$\begin{array}{ll}\text { 1999: } & 1.3 \\ \text { 2000: } & 5.4 \\ 2001: & 10.3\end{array}$

Last Year's fiscal year 1998 generation projection:

Actual quantity generated in 1998:
2002:

2003:

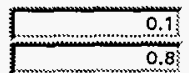

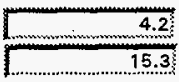

(Both in $\mathrm{m} 3$ unless otherwise noted)

3.11 Has there ever been any unusual selease of this stream to the environment?

$$
\square \text { Yes } \square \text { No }
$$

If yes, summarize releases and quantities:

\subsection{WASTE STREAM TREATMENT}

4.1 Is this stream currently being treated? 
$\square$ Yes $\square$ No If yes, provide details:

4.2 Planned treatment: Check the appropriate box indicating future plans for treating this waste stream to meet applicable regulations, including LDR treatment standards:

$\square$ No treatment required (skip to 5.0 )

$\square$ Treating or plan to treat onsite

$\square$ Treating or plan to treat offsite

$\square$ Treatment options still being assessed

4.3 Planned treatment method, facility, and extent of treatment capacity available:

Drumed waste and boxed waste are planned to be treated at WRAP and the proposed M91 facility, respectively. Treatment will be performed as necessary to meet the applicable waste acceptance criteria at WIPP. The extent of treatment will vary, and may include decontamination, solidification, and Irpackaging ...WRAP has a design capacity of 4725 drums $(983 \mathrm{~m}$ 3). por.year on a single stift basis.

4.4 Treatment schedule The programmatic treatment schedule for mixed and non-mixed TRU information:: waste is from 1999 to 2032 . The schedule is subject to change as it depends on the ability of DOE to accept mixed TRU waste at WIPP and available funding for treatment.

4.5 Applicable treatment Tri-Party Agreement milestone numbers (including permitting):

$M-91-01$

4.6 If treating or planning to treat on site, will waste minimization be addressed in developing and/or selecting the treatment method?

If yes, please describe

4.7 Treatability equivalency petitions, rulemaking petitions, and case-by-case exernptions needed for treatment

None are planned.

\subsection{WASTE STREAM DISPOSAL}

5.1 After treatment, how will the waste stream be disposed of (include description, locations, variances required, etc., as applicable):

Mixed TRU waste is planned to be disposed of at the Waste Isolation Pilot Plant (WIPP), a deep geological repository.

5.2 Applicable TPA milestone number(s) for disposal:

No TPA milestones exist for the disposal of TRU waste. The disposal of TRU waste at WIPP is a national DOE policy, and is required by DOE Order $5820.2 \mathrm{~A}$ (to be replaced by 435.1 ).

5.3 Planned disposal

The planned time frame of mixed and non-mixed TRU waste shipments to WIPP dates/time-trame: is from 2000 to 2032 . The time frame is subject to change depending on DOE's ability to accept waste at WIPP and adequate funding of TRU waste program activities.

\subsection{WASTE MINIMIZATION}

6.1 Has a waste minimization assessment been completed for this stream?
Yes
No
Not applicable

6.2 Explain any waste minimization activities (e.g., process changes) under way or to be implemented for generation of this stream: 
In the next 5 years, only the Plutonium Finishing Plant has forecasted generation of mixed TRU combustible debris. Forecasted quantities are small. Nevertheless, minimization practices include sorting mixed from non-mixed TRU waste and application of best management practices for reducing waste.

Schedule for implementing waste minimization methods:

Because only best management'practices have been identified, which are ongoing, a specific schedule is not applicable.

6.4 Waste reduction achieved during the calendar year and projected future reductions (volume or mass):

Calendar year: $1 . . . . . . . . . . . . . . . .13$

Projected:

of $\ln _{3}$

\subsubsection{Assumptions used in above estimate:}

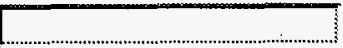




\section{WASTE STREAM PROFILE SHEET}

\subsection{WASTE STREAM IDENTIFICATION AND SOURCE}

1.1 Waste Stream ID:

1.2 Waste stream name

\subsubsection{Stream source and} history description:

\section{TRUM-01B}

Contact-handled mixed TRU composite filters

Toн

The waste was generated from the Plutonium Finishing Plant. The waste was

generated and placed into storage from 1996 to 1997.

1.3.2 Source category(s)
$\square$ Pollution control or waste treatment process
Material production/recovery effluents
$\checkmark$ Facility or equipment operation and maintenance waste
$\square$ Discarded excess or expired materials
$\square$ Analytical laboratory waste
RD/RD laboratory waste
$\square$ Remediation/DD waste
$\square$ spill clean-ups or emergency response actions
Source unknown
$\square$ Other (explain)

1.3.3 Additional notes:

\subsection{WASTE STREAM CHARACTERIZATION}

2.1 Waste stream The waste stream consists of cement, plastics, and chemicais.

description

(content)

2.2 Radiological characteristics

2.2.1 Mixed waste type $\square$ High-level $\square$ Transuranic $\square$ Low-level

( Contact-handled $\square$ Remote-handied

2.2.2 Comments on radiological characteristics (e.g., more specific content, treatment concerns caused by radiation, confidence level):

Each container of waste has more than $100 \mathrm{nCi} / \mathrm{g}$ of transuranic nuclide activity. Non-transuranic activity is either not reported or reported in trace quantities.

2.3 Matrix characteristics (physical content)

2.3.1 Matrix constituent table (comprising at least $1 \%$ of the total volume or mass)

\begin{tabular}{|c|c|c|}
\hline \multicolumn{3}{|c|}{ W } \\
\hline$\$ 3190$ & Inorganic homogeneous solids & $67.4 \%$ \\
\hline$\$ 5390$ & Organic debris & $22.0 \%$ \\
\hline U9999 & Unknown/other & $10.3 \%$ \\
\hline
\end{tabular}

Overall matrix parameter category code: $\$ 5410$

Overall matrix description:

$$
\text { Composite filters }
$$

2.3.2 Confidence level for matrix characteristics data in 2.3.1 $\square$ low $\square$ medium $\square$ high 
2.3.3 Comments on matrix characteristics and/or confidence level:

The Waste Specification Number for this waste stream is CMFTC, composite fịtters - transuranic contact-handled.

2.4 Regulated contaminant characteristics

2.4.1 Wastewater/non-wastewater under RCRA
$\square$ wastewater
$\checkmark$ non-wastewater
$\square$ unknown

2.4.2 Regulated conteminant table including treatment requirements, and UHC's if applicable

\begin{tabular}{|c|c|c|c|c|c|}
\hline 80 & 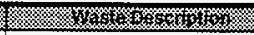 & 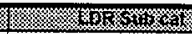 & 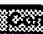 & 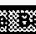 & 6 (6) \\
\hline D004 & ARSENIC & NA & $=$ & \pm+ & Exempt (61 FR 60704) \\
\hline 005 & BARIUM & NA & $* * *$ & $* * *$ & Exempt (61 FR 60704) \\
\hline 0006 & CADMIUM & NA & 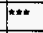 & tank & Exempt (61 FR 60704) \\
\hline 0007 & CHROMIUM & NA & tma* & $+*$ & Exempt (61 FR 60704) \\
\hline 2008 & LEAD & NA & $+*$ & $* * \star *$ & Exempt (61 FR 60704) \\
\hline 5019 & GARBON TETRACHLORIDE & NA & $\neq *$ & t*t* & Exempt (61 FR 60704) \\
\hline WP01 & Persistant, EHW & NA & $\{+\infty$ & 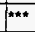 & N/A \\
\hline WSC2 & Solid Corrosive & INA & $* * *$ & $a+k$ & N/A \\
\hline WTO2 & Toxic, DW & INA & $\approx+$ & tante & N/A \\
\hline
\end{tabular}

UHC's are not applicable to the WIPP facility. (1) Treatment standards are based on the WIPP facility waste acceptance criteria.

2.4.2.1 List any waste codes from 2.4.2 for which the stream atready meets established LDR treatment standard Waste is exempt from Federal LDR's when being disposed of at the WIPP facility.

2.4.3 Does this waste stream contain PCBs?
$\square$ Yes
No
Unknown

2.4.3.1 is the waste stream subject to TSCA regulations for PCBs?
$\square$ Yes
$\nabla$ No
Unknown

2.4.3.2 Indicate the PCB concentration range
$\square<50 \mathrm{ppm}$
$>=50 \mathrm{ppm}$
Unknown

2.4.4 What is the confidence level for the regulated contaminant characteristic data?

$\square$ low

medium

high

2.4.5 Comments on regulated contaminant characteristics and/or confidence level: The regulated contaminants listed above are applicable to the existing
inventory. The contaminants vary widely in concentration. The
contaminants with the highest concentration are:
Chromium cadimum, and carbon tetrachloride:
performed $\square$ Yes No

\subsubsection{Will further characterization be performed $\square$ Yes $\square$ No Unknown}

2.4.6.1 If yes, provide details and Additional characterization will be conducted as necessary to meet the schedule: waste acceptance criteria for WIPP. Characterization will include process knowledge, and sampling and analysis.

2.4.6.2 If yes, provide Tri-Party Agreement milestone number No milestones are applicable to characterization of TRU waste.

\subsection{WASTE STREAM STORAGE, INVENTORY, AND GENERATION}


DOE/RL-99-01

3.1 Is the waste stream currently stored?

3.2 Current storage method

$\square$ Container (pad)
$\square$ Tank
$\square$ Other (explain)

If no, skip to 3.7

Container (retrievably buried)

Surface impoundment 
id: TRUM-018

3.3 TSD unit name and building number and (if applicable/available) number of containers/tanks in each:

TSD Unit Name

Central Waste Complex
Number of containers

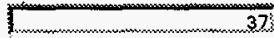

3.4 is storage of waste in compliance with applicable State and Federal standards?

田 Yes

No

If no, provide details:

3.6 Current inventory for this stream

Total LDR volume (cubic meters):

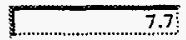

Date of Inventory values:

$9 / 30 / 98$

Comments on waste inventory:

The inventory is based on the Solid Waste Information and Tracking System. The waste is packaged in drums.

3.7

Is the waste stream currently generated; and if not, will-this waste stream be generated in the next 5 years? $\square$ Yes

No

3.8

The current or future generation of this waste is best described as:

Routine

One-time or sporadic

3.9 Estimated generation projection by fiscal year

1999 :

2000:

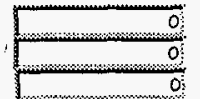

2002:

2003:

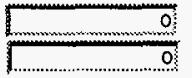

2001:

Last Year's fiscal year 1998 generation projection:

Actual quantity generated in 1998:

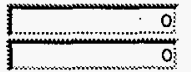

(Both in $\mathrm{m} 3$ unless atherwise noted)

3.11 Has there ever been any unusual release of this stream to the environment?

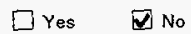

If yes, summarize releases and quantities:

\subsection{WASTE STREAM TREATMENT}

4.1 Is this stream currently being treated? 
Yes No If yes, provide details:

4.2 Planned treatment: Check the appropriate box indicating future plans for treating this waste stream to meet applicable regulations, including LDR treatment standards:
No treatment required (skip to 5.0 )
Treating or plan to treat onsite
Treating or plan to treat offsite
Treatment options still being assessed

4.3 Planned treatment method, facility, and extent of treatment capacity available:

Waste is planned to be treated at WRAP. Treatment will be performed as necessary to meet the applicable waste acceptance criteria at WIPP. The extent of treatment will vary, and may include no treatment, solidification, or repackaging. WRAP has a design capacity of 4725 drums (983 m3) per year on a.single.shift basis

4.4 Treatment schedule information::

The programmatic treatment schedule for mixed and non-mixed TRU waste is from 1999 to 2032 . The schedule is subject to change as it depends on the ability of DOE to accept mixed TRU waste at WIPP and available funding for treatment.

4.5 Applicable treatment Tri-Party Agreement milestone numbers (including permitting): M-91-01

4.6 If treating or planning to treat on site, will waste minimization be addressed in developing and/or selecting the treatment method?

$\square$ No $\square$ Yes $\square$ Unknown

If yes, please describe

4.7 Treatability equivalency petitions, rulemaking petitions, and case-by-case exemptions needed for treatment None are planned.

\subsection{WASTE STREAM DISPOSAL}

5.1 After treatment, how will the waste stream be disposed of (include description, locations, variances required, etc., as applicable):

Mixed TRU waste is planned to be disposed of at the Waste Isolation Pilot Plant (WIPP), a deep geological repository.

5.2 Applicable TPA milestone number(s) for disposal:

No TPA milestones exist for the disposal of TRU waste. The disposal of TRU waste at WIPP is a national DOE policy, and is required by DOE Order $5820.2 A$ (to be replaced by 435.1 ).

5.3 Planned disposal dates/time-trame:

The planned time frame of mixed and non-mixed TRU waste shipments to WIPP is from 2000 to 2032 . The time frame is subject to change deperiding on DOE's ability to accept waste at WIPP and adequate funding of TRU waste Sprogram activities.

\subsection{WASTE MINIMIZATION}

6.1 Has a waste minimization assessment been completed for this stream?
Yes
(7) No
Not applicable

6.2 Explain any waste minimization activities (e.g., process changes) under way or to be implemented for generation of this stream: 
The minimization activities are not applicable because waste has not been forecasted within the next five years (1999 to 2003$)$.

6.3 Schedule for implementing waste minimization methods:

Because only best management practices have been identified, which are ongoing, a specific schedule is not applicable.

6.4 Waste reduction achieved during the calendar year and projected future reductions (volume or mass):
Calendar year:
0
Projected:
0

6.4.1 Assumptions used in above estimate:

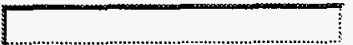




\section{WASTE STREAM PROFILE SHEET}

\subsection{WASTE STREAM IDENTIFICATION AND SOURCE}

1.1 Waste Stream ID: TRUM-01C

1.2 Waste stream name Contact-handled mixed TRU heterogeneous debris

1.3.1 Stream souree and. The waste was generated from the Plutonium Finishing Plant, the PUREX plant, Kerrhistory description: McGee, the Radiochemistry Building, and the Semi-works D\&D. The waste was generated and placed into storage from 1987 to 1998 .

1.3.2 Source category(s)

$\square$ Pollution control or waste treatment process
$\square$ Facility or equipment operation and maintenance waste

$\square$ Discarded excess or expired materials

$\square$ Analytical laboratory waste

$\checkmark$ RD/RD laboratory waste

$\checkmark$ Remediation/DD waste

$\square$ spill clean-ups or emergency response actions

Source unknown

$\square$ other (explain)

1.3.3 Additional notes:

\subsection{WASTE STREAM CHARACTERIZATION}

2.1 Waste stream description (content)

The waste strearn consists of plastics, metals, wood, rubber, paper/cardboard, soil/rocks, chemicals, lead, cloth/rags, glass, and filters.

2.2 Radiological characteristics

2.2.1 Mixed waste type

$[$ High-level

D Transuranic

Low-level

V Contact-handled

Remote-handled

2.2.2 Comments on radiological characteristics (e.g., more specific content, treatment concerns caused by radiation, confidence level):

Each containet of waste has more than $100 \mathrm{nCi} / \mathrm{g}$ of transuranic nuclide activity. Non-transuranic activity is either not reported or reported in trace quantities.

2.3 Matrix characteristics (physical content)

2.3.1 Matrix constituent table (comprising at least $1 \%$ of the total volume or mass)

\begin{tabular}{|c|c|c|}
\hline 1) & (3) & 3. \\
\hline$\$ 3190$ & inorganic homogeneous solids & $1.1 \%$ \\
\hline$\$ 4200$ & Soil/debris & $7.0 \%$ \\
\hline 55190 & Inorganic debris & $27.2 \%$ \\
\hline 55390 & Organic debris & $56.5 \%$ \\
\hline 55490 & Heterogeneous debris & $1.1 \%$ \\
\hline 09999 & Unknown/other & $3.5 \%$ \\
\hline$\times 7290$ & Elemental lead & $3.3 \%$ \\
\hline
\end{tabular}

Overall matrix parameter category code: $\$ 5490$

Overall matrix description:

Heterogeneous debris 
2.3.2 Confidence level for matrix characteristics data in $2.3 .1 \quad \square$ low $\square$ medium $\square$ high

2.3.3 Comments on matrix The Waste Specification Record Number for this waste steam is HTDTC,

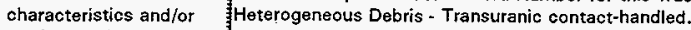

confidence level:

2.4.1 Wastewater/non-wastewater under RCRA

$\square$ wastewater $\square$ non-wastewater $\square$ unknown

2.4.2 Regulated contaminant table including treatment requirements, and UHC's if applicable

\begin{tabular}{|c|c|c|c|c|c|}
\hline 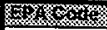 & $\alpha_{1}$ & XY Y Y & 3 & 8 & 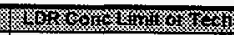 \\
\hline 0001 & Various & NA & 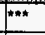 & $* * *$ & Remove characteristic (1) \\
\hline 0002 & Various & NA & $+* *$ & \pm \pm & Remove characteristic (1) \\
\hline 0004 & ARSENIC & NA & 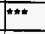 & 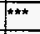 & Exempt (61. FR 60704) \\
\hline Do05 & BARIUM & NA & $f^{n n *}$ & $* * *$ & Exempt (61 FR 60704) \\
\hline 0006 & CADMIUM & NA & $* * *$ & 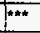 & Exempt (61 FR 60704) \\
\hline poo7 & CHROMLUM & NA & tont* & $\$ \star \star *$ & Exempt (61 FR 60704) \\
\hline 5008 & LEAD & NA & $+n$ & $* * *$ & Exempt (61 FR 60704) \\
\hline 009 & MERCURY & NA & $+n+$ & $x+n$ & Exempt (61 FR 60704) \\
\hline 5010 & SELENIUM & NA & $\because *$ & $+\infty$ & Exempt (61 FR 60704) \\
\hline 5011 & SILVER & NA & $\star \star \star \star *$ & $n+\infty$ & Exempt (6ł FR 60704) \\
\hline po18 & BENZENE & NA & $* * *$ & ten & Exempt (61 FR 60704) \\
\hline 5019 & CARBON TETRACHLORIDE & NA & 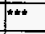 & + & Exempt (61 FR 60704) \\
\hline $\mathrm{p} 030$ & 2,4-DINITROTOLUENE & NA & $m n *$ & $\neq *$ & Exempt (61 FR 60704) \\
\hline 5001 & Spent Solvent & TRICHLOROETHYLENE & $* *$ & $n$ & Exempt (61 FR 60704) \\
\hline F002 & Spent Solvent & TRICHLOROETHENE & $\neq+\infty$ & 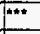 & Exempt (61 FR 60704) \\
\hline Foo3 & Spent Solvent & XYLENE & $* * *$ & men:" & Exempt (61 FR 60704) \\
\hline 7004 & Spent Solvent & CRESOL & $* * *$ & + \pm & Exempt (61 FR 60704) \\
\hline F005 & Spent Solvent & METHYL ETHYL KETONE & 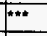 & $* *$ & Exempt (61 FR 60704) \\
\hline WP01 & Persistant, EHW & NA & F"* & 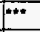 & N/A \\
\hline NP02 & Persistant, DW & NA & 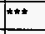 & $+\ldots+\infty$ & $\mathrm{N} / \mathrm{A}$ \\
\hline WSC2 & Solid Corrosive & INA & 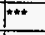 & $+* *$ & $\mathrm{~N} / \mathrm{A}$ \\
\hline NTO1 & Toxic, EHW & NA & $n * k$ & $+* \pi *$ & N/A \\
\hline NT02 & Toxic, DW & INA & $+*$ & $+\ldots$ & N/A \\
\hline
\end{tabular}

UHC's are not applicable to the WIPP facility. (1) Treatment standards are based on the WIPP facility waste acceptance criteria.

2.4.2.1 List any waste codes from 2.4.2 for which the stream already meets es tablished LDR treatment standard Waste is exempt from Federal LDR's when being disposed of at the WIPP facility.

2.4.3 Does this waste stream contain PCBs?
$\square$ Yes
No
Unknown

2.4.3.1 Is the waste stream subject to TSCA regulations for PCBs?
Yes
$\triangle$ No
Unknown

2.4.3.2 Indicate the $\mathrm{PCB}$ concentration range
$\square<50 \mathrm{ppm}$
$\square>=50 \mathrm{ppm}$
Unknown

2.4.4 What is the confidence level for the regulated contaminant characteristic data?
$\square$ low
$\sqrt{\mathrm{B}}$ medium
high 
2.4.5 Comments on regulated contaminant characteristics and/or confidence level:

Lead and carbon tetrachloride

2.4.6 Will further characterization be performed $\bar{\nabla}$ Yes $\square$ No $\quad \square$ Unknown

2.4.6.1 If yes, provide details and schedule:

Additional characterization will be conducted as necessary to meet the waste acceptance criteria for WIPP. Characterization will include process Knowledge, and sampling and analysis.

2.4.6.2 If yes, provide Tri-Party Ágreement milestone number No milestones are applicable to characterization fof TंRU waste.

\subsection{WASTE STREAM STORAGE, INVENTORY, AND GENERATION}

3.1 is the waste stream currently stored? $\square$ Yes $\square$ No

3.2 Current storage method

$\square$ Container (pad)

$\square$ Tank

$\square$ Other (explain)

$$
\text { If no, skip to } 3.7
$$

Container (retrievably buried)

surface impoundment 
id: $\quad$ TRUM-O1C

3.3 TSD unit name and building number and (if applicable/available) number of containers/tanks in each: TSD Unit Name Central Waste Complex Number of containers 300

3.4 is storage of waste in compliance with applicable State and Federal standards?

$\square$ Yes $\square$ No

If no, provide details:

3.6 Current inventory for this stream

Total LDR volume (cubic meters):

Date of Inventory values:

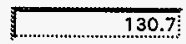

Comments on waste inventory:

$9 / 30 / 98$

The inventory is based on the Solid Waste Information and Tracking System. The waste is packaged in drums and boxes.

3.7

Is the waste stream currently generated; and if not, will this waste stream be generated in the next 5 years? Yes

3.8 The current or future generation of this waste is best described as:
Q Routine
One-time or sporadic

3.9 Estimated generation projection by fiscal year

1999:
2000:
2001:

3.11 Has there ever been any unusual release of this stream to the environment?

$\square$ Yes $\square$ No

If yes, summarize releases and quantities:

\subsection{WASTE STREAM TREATMENT}

4.1 Is this stream currently being treated? 
4.2 Planned treatment: Check the appropriate box indicating future plans for treating this waste stream to meet applicable regulations, including LDR treatment standards:

$\square$ No treatment required (skip to 5.0)

$\square$ Treating or plan to treat onsite

$\square$ Treating or plan to treat offsite

7reatment options still being assessed

4.3 Planned treatment method, facility, and extent of treatment capacity available:

Drumed waste and boxed waste are planned to be treated at WRAP and the proposed M91 facility, respectively. Treatment will be performed as necessary to meet the applicable waste acceptance criteria at WIPP. The extent of treatment will vary, and may include decontamination, solidification, and repackaging. WRAR has a.design capacity of 4725 drums $(983 \mathrm{~m}$ 3).per vear on a single shift basis

4.4. Treatment schedule information::

The programmatic treatment schedule for mixed and non-mixed TRU waste is from 1999 to 2032 . The schedule is subjeot to change as it depends on the ability of DOE to accept mixed TRU waste at WIPP and available funding for treatment.

4.5 Applicable treatment Tri-Party Agreernent milestone numbers (including permitting): M-91-01

4.6 If treating or planning to treat on site, will waste minimization be addressed in developing and/or

selecting the treatment method?

If yes, please describe

No

Unknown

Treatability equivalency petitions, rulemaking petitions, and case-by-case exemptions needed for treatment None are planned.

\subsection{WASTE STREAM DISPOSAL}

5.1 After treatment, how will the waste stream be disposed of (include description, locations, variances required, etc., as applicablel:

Mixed TRU waste is planned to be disposed of at the Waste Isolation Pilot Plant (WIPP), a deop geological repository.

5.2 Applicable TPA milestone number(s) for disposal:

No TPA milestones exist for the disposal of TRU waste. The disposal of TRU waste at WIPP is a national DOE policy, and is required by DOE Order $5820.2 \mathrm{~A}$ (to be replaced by 435.1 ).

5.3 Planned disposal

dates/ time-frame:

The planned time frame of mixed and non-mixed TRU waste shipments to WIPP
is from 2000 to 2032 . The time frame is subject to change depending on
DOE's ability to accept waste at WIPP and adequate funding of TRU waste
program activities.

\subsection{WASTE MINIMIZATION}

6.1 Has a waste minimization assessment been completed for this stream?
Yes
No
Not applicable

6.2 Explain any waste minimization activities (e.g., process changes) under way or to be implemented for generation of this stream: 
In the next 5 years, waste is forecated from the 200 Area Accelerated Deactivation Project, the Plutonium Finishing Plant, the Chemical Engineering Building, Tank Pre-treatment processing, R\&D facilities, and environmental restoration activities. Best management practices will be applied to reduce miscellaneous heterogeneous debris during the deactivation project, the deactivation at the Plutonium Finishing Plant and Chemical Engineering Building, and pretreatment processing of tank waste. Though R\&D activities generate small quantities of waste, best management practices are in-place to minimize waste to the extent possible.

Because only best management practices have been identified, which are ongoing, a specific schedule is not applicable.

6.4 Waste reduction achieved during the calendar yeat and projected future reductions (volume or mass):

Calendar year:

of $\mathrm{m3}$

Projected:

o

6.4.1 Assumptions used in above estimate:

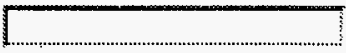




\section{WASTE STREAM PROFILE SHEET}

\subsection{WASTE STREAM IDENTIFICATION AND SOURCE}

1.1 Waste Stream ID:

1.2 Waste stream name

$$
\text { TRUM-01D }
$$

Contact-handled mixed TRU metal debris

1.3.1 Stream source and history description:

The The waste was generated from the PUREX plant, the Plutonium Finishing Plant, KerrMcGee, and the Radiochemistry Building. The waste was generated and placed into storage from 1987 to 1998 .

\subsubsection{Source category(s)}

$\square$ Pollution control or waste treatment process

Material production/recovery effluents

$\square$ Facility or equipment operation and maintenance waste

$\square$ Discardied excess or expired materials

Analytical laboratory waste

RD/RD laboratory waste

Remediation/DD waste

Spill clean-ups or emergency response actions

Other (explain)

1.3.3 Additional notes:

\subsection{WASTE STREAM CHARACTERIZATION}

2.1 Waste stream description (content)

The waste stream consists of metals, plastics, wood, and paper/cardboard.

2.2 Radiological characteristics

2.2. Mixed waste type High-level 4 Transuranic Low-level

(V) Contact-handled Remote-handled

2.2.2 Comments on radiological characteristics le.g., more specific content, treatment concerns caused by radiation, confidence (evel):

Each container of waste has more than $100 \mathrm{nCi} / \mathrm{g}$ of transuranic nuclide activity. Non-transuranic activity is either not reported or reported in trace quantities.

2.3 Matrix characteristics (physical content)

2.3.1 Matrix constituent table (comprising at least $1 \%$ of the total volume or mass)

\begin{tabular}{|c|c|c|}
\hline 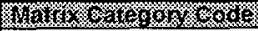 & 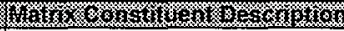 & \% \\
\hline$\$ 5190$ & Inorganic debris & $83.2 \%$ \\
\hline$\$ 5390$ & Organic debris & $16.5 \%$ \\
\hline
\end{tabular}

Overall matrix parameter category code: $\$ 5119$

Overall matrix description:

Metal debris

2.3.2 Confidence level for matrix characteristics data in 2.3.1

$\square$ low $\square$ medium $\square$ high 
2.3.3 Comments on matrix characteristics and/or confidence level:

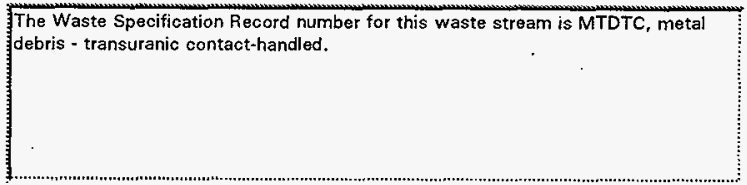

2.4 Regulated contaminant characteristics

2.4.1 Wastewater/non-wastewater under RCRA

$\square$ wastewater $\square$ non-wastewater

unknown

2.4.2 Regulated contaminant table including treatment requirements, and UHC's if applicable

\begin{tabular}{|c|c|c|c|c|c|}
\hline 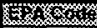 & 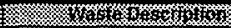 & $x \rightarrow x_{3}$ & \multicolumn{3}{|c|}{ 3 } \\
\hline Do06 & CADMIUM & NA & $\approx * *$ & T** & Exempt (6t FR 60704) \\
\hline 0008 & LEAD & NA & 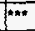 & 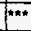 & Exempt (61 FR 60704) \\
\hline 5009 & MERCURY & NA & $\ldots *$ & $+\cdots$ & Exempt (61 FR 60704) \\
\hline NSC2 & Solid Corrosive & NA & tax & t+*: & N/A \\
\hline NTO1 & Toxic, EHW & INA & $+* *$ & $+*+$ & N/A \\
\hline NTO2 & Toxic, DW & INA & F*** & 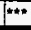 & N/A \\
\hline
\end{tabular}

UHC's are not appliciable to the WIPP facility. (1) Treatment standards are based on the WIPP facility waste acceptance criteria.

2.4.2.1 List any waste codes from 2.4.2 for which the stream already meets established LDR treatment standard Waste is exempt from Federal LDR's when being disposed of at the WIPP facility.

2.4.3 Does this waste stream contain PCBs?
Yes
$\nabla$ No
Unknown

2.4.3.1 Is the waste stream subject to TSCA regulations for PCBs?
$\square$ Yes
$\checkmark$ No
Unknown

2.4.3.2 Indicate the $\mathrm{PCB}$ concentration range
$\square<50 \mathrm{ppm}$
$]>=50 \mathrm{ppm}$
Unknown

2.4.4 What is the confidence level for the regulated contaminant characteristic data?
$\square$ low
$\nabla$ medium
high

2.4.5 Comments on regulated contaminant characteristics and/or confidence level:

The regulated contaminants listod apove are applicable to the existing
inventory. The contaminants vary widely in concentration. The
contaminants with the highest concentration are:

2.4.6 Will further characterization be performed $\square$ Yes $\square$ No $\square$ Unknown

2.4.6.1 If yes, provide details and Additional characterization will be conducted as necessary to meet the schedule: waste acceptance criteria for WIPP. Characterization will include process knowledge, and sampling and analysis.

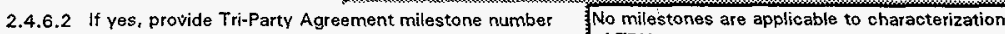
of TRU waste.

\subsection{WASTE STREAM STORAGE, INVENTORY, AND GENERATION}
3.1
is the waste stream currently stored?
(a) Yes
No

3.2 Current storage method

Container (pad)

Container (covered)

Tank

Waste pile

If no, skip to 3.7

Other (explain) 
DOE/RL-99-01 
DOE/RL-99-01

id:

TRUM-01D

3.3 TSD unit name and building number and (if applicable/available) number of containers/tanks in each: TSD Unit Name

Number of containers

Central Waste Complex

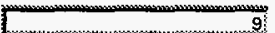

3.4

Is storage of waste in compliance with applicable State and Federal standards?

$\nabla$ Yes $\quad \square$ No

If no, provide details:

3.6 Current inventory for this stream

Total LDR volume (cubic meters):

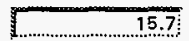

Date of Inventory values:

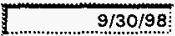

Comments on waste inventory:

The inventory is based on the Solid Waste information and Tracking System. The waste is packaged in drums and boxes.

Is the waste stream currently generated; and if not, will this waste stream be generated in the next 5 years?

$$
\square \text { Yes } \square \text { No }
$$

3.8 The current or future generation of this waste is best described as:
Routine
$\square$ One-time or sporadic

3.9 Estimated generation projection by fiscal year

$\begin{array}{lll}\text { 1999: } & 0.2 \\ 2000: & 2002: \\ 2001: & 2003:\end{array}$

3.10
Last Year's fiscal year 1998 generation projection:

Actual quantity generated in 1998 :

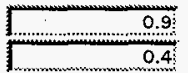

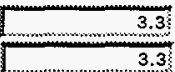

(Both in $\mathrm{m} 3$ unless otherwise noted)

Has there ever been any unusual release of this stream to the environment?

$\square$ Yes No

If yes, summarize releases and quantities:

\subsection{WASTE STREAM TREATMENT}

4.1 Is this stream currently being treated? 
4.2 Plannod treatment: Check the appropriate box indicating future plans for treating this waste stream to meet applicable regulations, including LDR treatment standards:

$\square$ No treatment required (skip to 5.0 )

$\square$ Treating or plan to treat onsite

$\square$ Treating or plan to treat offsite

V Treatment options still being assessed

4.3 Planned treatment method, facility, and extent of treatment capacity available:

Drumed waste and boxed waste are planned to be treated at WRAP and the proposed M91 facility, respectively. Treatment will be performed as necessary to meet the applicable waste acceptance criteria at WIPP. The extent of treatment will vary, and may include decontamination, solidification, and Irepackaging. WRAP has a design capacity of 4725 drums. $(983 \mathrm{~m} 3 \mathrm{~L}$. par year on a single shift basis.

4.4 Treatment schedule The programmatic treatment schedule for mixed and non-mixed TRU information:: waste is from 1999 to 2032 . The schedule is subject to change as it depends on the ability of DOE to accept mixed TRU waste at WIPP and available funding for treatment.

4.5 Applicable treatment Tri-Party Agreement milestone numbers (including permitting): $M-91-01$

4.6 If treating or planning to treat on site, will waste minimization be addressed in developing and/or selecting the treatment method?

If yes, please describe

4.7 Treatability equivalency petitions, rulemaking petitions, and case-by-case exemptions needed for treatment None are planned.

\subsection{WASTE STREAM DISPOSAL}

5.1 After treatment, how will the waste stream be disposed of (include description, locations, variances required, etc., as applicable):

Mixed TRU waste is planned to be disposed of at the Waste Isolation Pilot Plant (WIPP), a deep geological

5.2 Applicable TPA milestone number(s) for disposal:
No TPA milestones exist for the disposal of TRU waste. The disposal of TRU waste at WIPP DOE policy, and is required by DOE Order $5820.2 \mathrm{~A}$ (to be replaced by 435.1 ).

5.3 Planned disposal dates/time-frame:

The planned time frame of mixed and non-mixed TRU waste shipments to WIPP is from 2000 to 2032 . The time frame is subject to change depending on DOE's ability to accept waste at WIPP and adequate funding of TRU waste program activities.

\subsection{WASTE MINIMIZATION}

6.1 Has a waste minimization assessment been completed for this stream?
$\square$ Yes
(v) No
Not applicable

6.2 Explain any waste minimization activities (e.g., process changes) under way or to be implemented for generation of this stream: 
Between 1996 and 1998 , only two drums $10.4164 \mathrm{m3}$ ) were received from the Radiochemistry Building. A volume of $0.6 \mathrm{~m} 3$ was avoided through development of a portable apparatus to clean a spray wand. Without the apparatus, the spray wand, which would have been contaminated with TRU material, would have been generated as solid waste. Future waste are expected from the 200 Area Accelerated Deactivation Project and Environmental Restoration. Waste minimization is not applicable to the 200 Area Accelerated Deactivation Project because the waste is existing loose, excess equipment that needs to be removed and relocated to a safer location and in a safer configuration.

6.3 Schedule for implementing waste minimization methods:

Because waste minimization is not applicable, a waste minimization implementation schedule is not required.

6.4 Waste reduction achieved during the calendar year and projected future reductions (volume or mass):
Calendar year:
ons
Projected:
0

6.4.1 Assumptions used in above estimate:

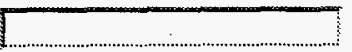




\section{WASTE STREAM PROFILE SHEET}

\subsection{WASTE STREAM IDENTIFICATION AND SOURCE}

1.1 Waste Stream ID:

$$
\text { TRUM-01E }
$$

1.2 Waste stream name Contact-handled mixed TRU non-metal inorganic particulates

1.3.1 Stream source and history description: The waste was generated from the Radiological Calibrations Laboratory, Kerr-
$M c G e e$, and the Plutonium Finishing Plant. The waste was generated from and placed into storage from 1987 to 1988.

1.3.2 Source category(s)

$\square$ Pollution control or waste treatment process $\quad \square$ Material production/recovery effluents

$\checkmark$ Facility or equipment operation and maintenance waste

$\square$ Discarded excess or expired materials

$\square$ Analytical laboratory waste

$\square$ RD/RD laboratory waste

$\square$ Remediation/DD waste

$\square$ Spill clean-ups or emergency response actions

Source unknown

$\square$ Other (explain)

1.3.3 Additional notes:

\subsection{WASTE STREAM CHARACTERIZATION}

2.1 Waste stream description (content)

The waste stream consists of absorbents, metals, soil/rocks, absorbed liquids, lead, filters, concrete, plastics, glass, and rubber.

2.2 Radiological characteristics

2.2.1 Mixed waste type

High-level

Transuranic

Low-level

(V) Contact-handled

$\square$ Remote-handled

2.2.2 Comments on radiological characteristics (e.g., more specific content, treatment concerns caused by radiation, confidence level):

Each container of waste has more than $100 \mathrm{nCi} / \mathrm{g}$ of transuranic nuclide activity. Non-transuranic activity is either not reported or reported in trace quantities.

2.3 Matrix characteristics (physical content)

2.3.1 Matrix constituent table (comprising at least $1 \%$ of the total volume or mass)

\begin{tabular}{|c|c|c|}
\hline 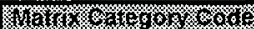 & (6) & Wofom \\
\hline 12290 & Organic Liquids & $3.0 \%$ \\
\hline$\$ 3190$ & Inorganic homogeneous solids & $61.7 \%$ \\
\hline 55190 & Inorganic debris & $25.3 \%$ \\
\hline $55390^{\circ}$ & Organic debris & $3.6 \%$ \\
\hline 55490 & Heterogeneous debris & $2.8 \%$ \\
\hline$\times 7290$ & Elemental lead & $3.0 \%$ \\
\hline
\end{tabular}

Overall matrix parameter category code: 53119

Overall matrix description:

2.3.2 Confidence level for matrix characteristics data in 2.3.1 $\square$ low $\square$ medium $\square$ high 
2.3.3 Comments on matrix. characteristics and/or confidence level:
The Waste Specification Record Number for this waste stream is NMTTC, Nonmetal - transuranic contact-handled.

\subsection{Regulated contaminant characteristics}

2.4.1 Wastewater/non-wastewater under RCRA
$\square$ wastewater
non-wastewater
unknown

2.4.2 Riegulated contaminant table including treatment requirements, and UHC's if applicable

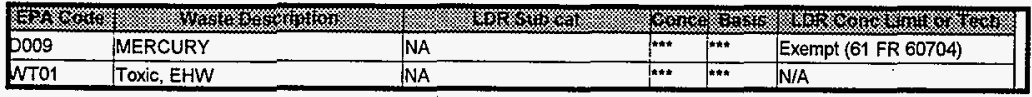

UHC's are not applicable to the WIPP facility. (1) Treatment standards are based on the WIPP facility waste acceptance criteria.

2.4.2.1 List any waste codes from 2.4 .2 for which the stream already meets established LDR treatment standard Waste is exempt from Federal LDR's when being disposed of at the WIPP facility.

2.4.3 Does this waste stream contain PCBs?
$\square$ Yes
No No
Unknown

2.4.3.1 Is the waste stream subject to TSCA regulations for PCBs?
$\square$ Yes
$\checkmark$ No
Unknown

2.4.3.2 Indicate the PCB concentration range
$<50 \mathrm{ppm}$
$>=50 \mathrm{ppm}$
Unknown

2.4.4 What is the confidence level for the regulated contaminant characteristic data?
$\square$ low
$\checkmark$ medium
high

2.4.5 Comments on regulated contaminant characteristics and/or confidence level:

The regulated contamin ants listed above are applicable to the existing
inventory. The contaminants vary widely in concentration. The
contaminants with the highest concentration are:

2.4.6 Will further characterization be performed $\bar{\nabla}$ Yes $\quad \square$ No $\square$ Unknown
2.4.6.1 If yes, provide details and
Additional characterization will be conducted as necessary to meet the schedule: waste acceptance criteria for WIPP. Characterization will to meet the knowledge, and sampling and analysis.

2.4.6.2 If yes, provide Tri-Party Agreement milestone number No milestones are applicable to characterization of TRU waste.

\subsection{WASTE STREAM STORAGE, INVENTORY, AND GENERATION}
3.1
Is the waste stream currently stored?
$\square$ Yes
$\square$ No

\subsection{Current storage method}

$\square$ Container (pad)

Tank

$\square$ Other (explain) $\nabla$ Container (covereo)

$\square$ Waste pile

$$
\text { If no, skip to } 3.7
$$

(4) Container (retrievably buried)

- Surface impoundment 
id: TRUM-O1E

3.3 TSD unit name and building number and (if applicable/available) number of containers/tanks in each:

TSD Unit Name

Central Waste Complex
Number of containers

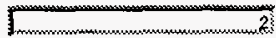

3.4 Is storage of waste in compliance with applicable State and Federal standards?

$\square$ Yes $\square$ No

if no, provide details:

Current inventory for this stream

Total LDR volume (cubic meters):

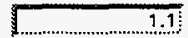

Date of inventory values:

$9 / 30 / 98$

Comments on waste inventory: The inventory is based on the Solid Waste information and Tracking System. The waste is packaged in drums.

3.7

Is the waste stream currently generated; and if not, will this waste stream be generated in the next 5 years?

$$
\square \text { Yes } \square \text { No }
$$

3.8 The current or future generation of this waste is best described as:

$$
\square \text { Routine } \square \text { One-time or sporadic }
$$

Estimated generation projection by fiscal year

1999:
2000:
$2001:$
12.4

Last Year's fiscal year 1998 generation projection:

Actual quantity generated in 1998:
2002:

2003:

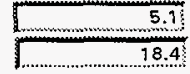

(Both in $\mathrm{m} 3$ unless otherwise noted)

3.11 Has there ever been any unusual release of this stream to the environment?

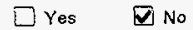

If yes, summarize releases and quantities:

\subsection{WASTE STREAM TREATMENT}

4. 1 Is this stream currently being treated? 
Yes $\square$ No If yes, provide details:

4.2 Planned treatment: Check the appropriate box indicating future plans for treating this waste stream to meet applicable regulations, including LDR treatment standards:

No treatment required (skip to 5.0 )

Treating or plan to treat onsite

$\square$ Treating or plan to treat offsite

$\checkmark$ Treatment options still being assessed

4.3 Planned treatment method, facility, and extent of treatment capacity available:

Waste is planned to be treated at WRAP. Treatment will be performed as necessary to meet the applicable waste acceptance criteria at WIPP. The extent of treatment will van, and may include no Itreatment, solidification, or repackaging. WRAP has a design capacity of 4725 drums $(983 \mathrm{~m} 3)$ per year Ion a single shift.basis.

4.4 Treatment schedule information:

The programmatic treatment schedule for mixed and non-mixed TRU waste is from 1999 to 2032 . The schedule is subject to change as it depends on the ability of DOE to accept mixed TRU waste at WIPP and available funding for treatment.

4.5 Applicable treatment Tri-Party Agreement milestone numbers (including permitting): M-91-01

If treating or planning to treat on site, will waste minimization be addressed in developing and/or selecting the treatment method?

$\square$ No $\square$ Unknown

If yes, please describe

4.7 T.reatability equivalency petitions, rulemaking petitions, and case-by-case exemptions needed for treatment None are planned.

\subsection{WASTE STREAM DISPOSAL}

5.1 After treatment, how. will the waste stream be disposed of (include description, locations, variances required, etc., as applicable):

Mixed TRU waste is planned to bo disposed of at the Waste Isolation Pilot Plant (WIPP), a deep geological repository.

5.2 Applicable TPA milestone number(s) for disposal:

No TPA milestones exist for the disposal of TRU waste. The disposal of TRU waste at WIPP is a national DOE policy, and is required by DOE Order $5820.2 A$ (to be replaced by 435.1 ).

5.3 Planned disposal dates/time-frame:

The planned time frame of mixed and non-mixed TRU waste shipments to WIPP is from 2000 to 2032 . The time frarne is subject to change depending on DOE's ability to accept waste at WIPP and adequate funding of TRU waste program activities.

\subsection{WASTE MINIMIZATION}

6.1 Has a waste minimization assessment been completed for this stream?
Yes
No
Not applicable

6.2 Explain any waste minimization activities (e.g., process changes) under way or to be implemented for generation of this stream: 
Future waste will be solidified residues from the Plutonium Finishing Plant by a cementation process. A fixed quantity of cement is needed to place the residues in a safe configuration for long-term storage. Practices will be implemented to keep cement addition to a minimum without compromising the stabilization of plutonium within the waste matrix.

6.3 Schedule for implementing waste minimization methods:

Because only best management practices have been identified, which are ongoing, a specific schedule is not applicable.

6.4

Waste reduction achieved during the calendar year and projected future reductions (volume or mass):

Calendar year:

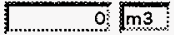

Projected:

0

6.4.1 Assumptions used in above estimate:

A 


\section{WASTE STREAM PROFILE SHEET}

\subsection{WASTE STREAM IDENTIFICATION AND SOURCE}

1.1 Waste Stream ID:

1.2 Waste stream name

\subsubsection{Stream source and} history description:

TRUM-01F
The waste was generated from the Plutonium Finishing Plant, the Radiochemistry
Building, research laboratories, and the Fuels Development Laboratory. The waste
was generated and placed into storage from 1988 to 1995 .

\subsubsection{Source category(s)}

$\square$ Pollution control or waste treatment process $\square$

0 Facility or equipment operation and maintenance waste

$\square$ D. Discarded excess or expired materials RD/RD laboratory waste

Spill clean-ups or emergency response actions Other (explain)

Material production/recovery effluents

$\square$ Analytical laboratory waste

$\square$ Remediation/DD waste

\subsubsection{Additional notes:}

\subsection{WASTE STREAM CHARACTERIZATION}

2.1 Waste stream description (content)

\section{The waste stream consists of lead, rubber, metals, plastics, soil/rocks, ceramic/bricks, lead shielding, cadmium, glass, paper/cardboard, and cloth/r}

2.2 Radiological characteristics

2.2.1 Mixed waste type

High-level $\square$ Transuranic

$\square$ Contact-handled
Low-level

Remote-handled

2.2.2 Comments on radiological characteristics (e.g., more specific content, treatment concerns caused by radiation, confidence level):

Each container of waste has more than $100 \mathrm{nCi} / \mathrm{g}$ of transuranic nuclide activity. Non-transuranic activity is either not reported or reported in trace quantitios.

2.3 Matrix characteristics (physical content)

2.3.1 Matrix constituent table (comprising at least $1 \%$ of the total volume or mass)

\begin{tabular}{|l|l|r|}
\hline Sind & & \\
\hline 54200 & Soil/debris & $4.5 \%$ \\
\hline 55190 & Inorganic debris & $17.2 \%$ \\
\hline 5390 & Organic debris & $24.0 \%$ \\
\hline$X 7290$ & Elemental lead & $54.1 \%$ \\
\hline
\end{tabular}

Overall matrix parameter category code: $\times 7219$

Overall matrix description:

Elemental lead

2.3.2 Confidence level for matrix characteristics data in 2.3.1 $\square$ low $\square$ medium $\square$ high 
2.3.3 Comments on matrix characteristics and/or confidence level:

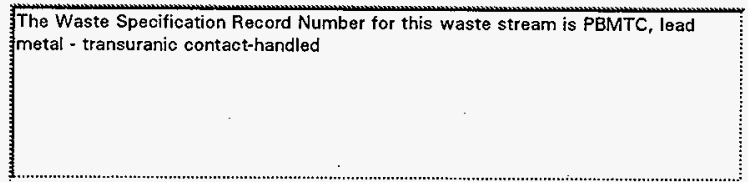

2.4 Regulated contaminant characteristics

2.4.1 Wastewater/non-wastewater under RCRA

$\square$ wastewater $\square$ non-wastewater $\square$ unknown

2.4.2 Regulated contaminant table including treatment requirements, and UHC's if applicable H

\begin{tabular}{|c|c|c|c|c|c|c|}
\hline $\mathrm{p} 002$ & SULFURIC ACID & NA & & 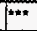 & $\star \star \star * *$ & Remove characteristic (1) \\
\hline 0006 & CADMIUM & NA & & $+* *$ & $+\cdots *$ & Exempt (61 FR 60704) \\
\hline 5007 & LEEAD CHROMATE & NA & & $* n$ & $\hbar \star \star \star k^{\circ}$ & Exempt (61 FR 60704) \\
\hline 000 & LEAD & NA & $\cdot$ & $+*$ & $\cdots * *$ & Exempt (61 FR 60704) \\
\hline Do09 & MERCURY & INA & & 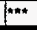 & $n * \pi$ & Exempt (61 FR 60704) \\
\hline
\end{tabular}

UHC's are not applicable to the WIPP facility. (1) Treatment standards are based on the WIPP facility waste acceptance criteria.

2.4.2.1 List any waste codes from 2.4.2 for which the stream already meets established LDR treatment standard Waste is exempt from Federal LDR's when being disposed of at the WIPF facility.

2.4.3 Does this waste stream contain PCBs?
7 Yes
$\checkmark$ No
Unknown

2.4.3.1 Is the waste stream subject to TSCA regulations for PCBs?
$\square$ Yes
$\square$ No
Unknown

2.4.3.2 Indicate the $\mathrm{PCB}$ concentration range
$\square<50$ ppm
$\square>=50 \mathrm{ppm}$
Unknown

2.4.4 What is the confidence level for the regulated contaminant characteristic data?
low
$\nabla$ medium
high

2.4.5 Comments on regulated contaminant characteristics and/or confidence level:

The regulated contaminants listed above are applicable to the existing inventory. The contaminants vary widely in concentration. The contaminants with the highest concentration are:

\section{Lead and cadimum.}

2.4.6 Will further characterization be performed $\square$ Yes $\square$ No $\square$ Unknown

2.4.6.1 If yes, provide details and Additional characterization will be conducted as necessary to meet the schedule:

waste acceptance criteria for WIPP. Characterization will include process knowledge, and sampling and analysis.

2.4.6.2 If yes, provide Tri-Party Agreement milestone number No milestones are applicable to characterization of TRU waste.

\subsection{WASTE STREAM STORAGE, INVENTORY, AND GENERATION}

3.1

Is the waste stream currently stored?

D Yes

3.2

Current storage method

$\square$ Container (pad)

Container (covered)

Tank

Waste pile

If no, skip to 3.7

$\square$ Other (explain) 
id:

TRUM-01F

3.3 TSD unit name and building number and (if applicable/available) number of containers/tanks in each:

TSD Unit Name

Central Waste Complex
Number of containers

3.4 Is storage of waste in compliance with applicable State and Federal standards?

$\square$ Yes $\square$ No

If no, provide details:

3.6 Current inventory for this stream

Total LDR volume (cubic meters):

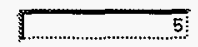

Date of inventory values: 9/30/98:

Comments on waste inventory:

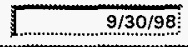

The inventory is based on the Solid Waste Information and Tracking System. The waste is packaged in drums.

Is the waste stream currently generated; and if not, will this waste stream be generated in the next 5 years? $\checkmark$ Yes 7 No

3.8 The current or future generation of this waste is best described as:

$\triangle$ Routine One-time or sporadic

3.9 Estimated generation projection by fiscal year
1999:
2000:

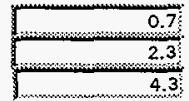
2002:
2003:

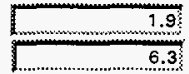
2001:

4.3
Last Year's fiscal year 1998 generation projection:
Actual quantity generated in 1998:

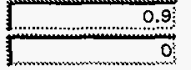
(Both in $\mathrm{m} 3$ unless otherwise noted)

3.10

3.11 Has there ever been any unusual release of this stream to the environment?

$$
\square \text { Yes } \nabla \text { No }
$$

If yes, summarize releases and quantities:

\subsection{WASTE STREAM TREATMENT}

4.1 Is this stream currently being treated? 
$\checkmark$ Yes $\square$ No . If yes, provide details:

4.2 Planned treatment: Check the appropriate box indicating future plans for treating this waste stream to meet applicable regulations, including LDR treatment standards:

No treatment required (skip to 5.0 )

$\square$ Treating or plan to treat onsite

$\square$ Treating or plan to treat offsite

Treatment options still being assessed

4.3 Planned treatment method, facility, and extent of treatment capacity available:

Waste is planned to be treated at WRAP. Treatment will be performed as necessary to meet the applicable waste acceptance criteria at WIPP. The extent of treatment will vary, and may inolude no treatment, solidification, or repackaging. WRAP has a design capacity of 4725 drums (983 m3) per year on a singles shitt.basis.

4.4 Treatment schedule information::

The programmatic treatment schedule for mixed and non-mixed TRU waste is from 1999 to 2032 . The schedule is subject to change as it depends on the ability of DOE to accept mixed TRU waste at WIPP and available funding for treatment.

4.5 Applicable treatment Tri-Party Agreement milestone numbers (including permitting):

M-91-01

If treating or planning to treat on site, will waste minimization be addressed in developing and/or selecting the treatment method?

$\square$ Yes $\square$ No $\square$ Unknown

If yes, please describe

4.7 Treatability equivalency petitions, rulemaking petitions, and case-by-case exemptions needed for treatment None are planned.

\subsection{WASTE STREAM DISPOSAL}

5.1 After treatment, how will the waste stream be disposed of (include description, locations, variances required, etc., as applicable):

(Mixed TRU waste is planned to be disposed of at the Waste Isolation Pilot Plant (WIPP), a deep geological
repository.

5.2 Applicable TPA milestons number(s) for disposal:

No TPA milestones exist for the disposal of TRU waste. The disposal of TRU waste at WIPP is a national DOE policy, and is required by DOE Order $5820.2 A$ (to be replaced by 435.1 ).

\begin{tabular}{|c|c|c|}
\hline 5.3 & $\begin{array}{l}\text { Planned disposal } \\
\text { dates/ time-frame: }\end{array}$ & $\begin{array}{l}\text { The planned time frame of mixed and non-mixed TRU waste shipments to WIPP } \\
\text { is from } 2000 \text { to } 2032 \text {. The time frame is subject to change depending on } \\
\text { DOE's ability to accept waste at WIPP and adequate funding of TRU waste } \\
\text { program activities. }\end{array}$ \\
\hline
\end{tabular}

\subsection{WASTE MINIMIZATION}

6.1 Has a waste minimization assessment been completed for this stream?
$\square$ Yes
(v) No
Not applicable

6.2 Explain any waste minimization activities (e.g., process changes) under way or to be implemented for generation of this stream: 
No waste has been received between 1996 and 1998. Therefore, a waste assessment for this stream was not necessary and was not conducted during the calendar year. Future lead waste has been forecasted from the Pacific Northwest National Laboratory. This waste will result from R\&D activities. Because R\&D waste generations are small, best management practices are applied for minimization of future waste.

6.3 Schedule for implementing waste minimization methods:

Because only best management practices have been identified, which are ongoing, 'a specific schedule is not applicable.

6.4 Waste reduction achieved during the calendar year and projected future reductions (volume or mass):

Calendar year:

$$
\text { m3 }
$$

Projected:

of $m^{3}$

6.4.1 Assumptions used in above estimate:

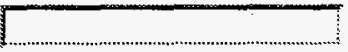




\section{WASTE STREAM PROFILE SHEET}

\subsection{WASTE STREAM IDENTIFICATION AND SOURCE}

1.1 Waste Stream ID: TRUM-01G

1.2 Waste stream name Contact-handled mixed TRU solidified inorganics

1.3.1 Stream source and The waste was generated from the Plutonium Finishing Plant. The waste was history description: generated and placed into storage from 1996 to 1998.

1.3.2 Source category(s)

$\square$ Pollution control or waste treatment process
$\square$ Facility or equipment operation and maintenance waste

$\square$ Discarded excess or expired materials

Material production/recovery effluents

$\square$ RD/RD laboratory waste

$\square$ Analytical laboratory waste

$\square$ Spill clean-ups or emergency response actions

$\square$ Remediation/DD waste

$\square$ Other (explain)

1.3.3 Additional notes:

\subsection{WASTE STREAM CHARACTERIZATION}

2.1 Waste stream description (content)

The waste stream consists of plastics, absorbed liquids, glass,
absorbents, soil/rocks, and rubber.
$\square$ High-level $\quad \square$ Transuranic $\square$ Low-level
$\square$ Contact-handled $\square$ Remote-handled

2.2 Radiological characteristics

2.2.1 Mixed waste type D Contact-handled

Remote-handled

2.2.2 Comments on radiological characteristics (e.g., more specific content, treatment concerns caused by radiation, confidence level):

Each container of waste has more than $100 \mathrm{nCi} / \mathrm{g}$ of transuranic nuclide activity. Non-transuranic activity is either not reported or reported in trace quantities.

2.3 Matrix characteristics (physical content)

2.3.1 Matrix constituent table (comprising at least $1 \%$ of the total volume or mass)

\begin{tabular}{|c|c|c|}
\hline X7a & 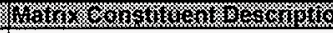 & 1) \\
\hline$\$ 3190$ & Inorganic homogeneous solids & $1.2 \%$ \\
\hline$\$ 4200$ & Soil/debris & $2.3 \%$ \\
\hline$\$ 5190$ & Inorganic debris & $24.5 \%$ \\
\hline 55390 & Organic debris & $58.8 \%$ \\
\hline 09999 & Unknown/other & $13.2 \%$ \\
\hline
\end{tabular}

Overall matrix parameter category code: $\$ 3150$

Overall matrix description: Solidified homogeneous solids

2.3.2 Confidence level for matrix characteristics data in 2.3.1 $\square$ low $\square$ medium $\square$ high 
2.3.3 Comments on matrix characteristics and/or confidence level:
The Waste Specification Record Number for this waste stream is SLITC, solidified liquids - inorganic - transuranic contact-handled. The waste matrix table lists forganic debris as the predominant waste form. In review of the waste records, the organic debris is universal polypropylenes. Even though the material acts as an absorbent, it is categoried as organic debris. These materials range from 26.5 to 53 percent.

害.......

2,4 Regulated contaminant characteristics

2.4.1 Wastewater/nor-wastewater under RCRA

$\square$ wastewater $\square$ non-wastewater unknown

2.4.2 Regulated contaminant table including treatment requirements, and UHC's if applicable K

\begin{tabular}{|c|c|c|c|c|c|}
\hline 6004 & ARSENIC & NA & $1+*$ & $+*$ & Exempt (61 FR 60704) \\
\hline 5007 & DIPOTASSIUM DICHROMATE & NA & +***+* & $+*$ & Exempt (61 FR 60704) \\
\hline 008 & LEAD & NA & $\neq *$ & 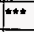 & Exempt (61 FR 60704) \\
\hline 5009 & MERCURY & INA & int & ank & Exempt (61 FR 60704) \\
\hline 2010 & SELENIUM & NA & $\cdots$ & $* * *$ & Exempt (61 FR 60704) \\
\hline Do11 & SILVER & NA & $+* * *$ & 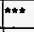 & Exempt (61 FR 60704) \\
\hline NTO2 & Toxic, DW & INA & 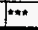 & $n n * *$ & N/A \\
\hline
\end{tabular}

UHC's are not applicable to the WIPP facility. (1) Treatment standards are based on the WIPP facility waste acceptance criteria.

2.4.2.1 List any waste codes from 2.4 .2 for which the stream already meets established LDR treatment standard Waste is exempt from Federal LDR's when being disposed of at the WIPP facility.

2.4.3 Does this waste stream contain PCBs?

$\square$ Yes $\square$ No $\square$ unknown

2.4.3.1 Is the waste stream subject to TSCA regulations for PCBs?
$\square$ Yes
4 No
Unknown

2.4.3.2 Indicate the PCB concentration range

$\square<50 \mathrm{ppm}$

$\square>=50 \mathrm{ppm}$

Unknown

2.4.4 What is the confidence level for the regulated contaminant characteristic data?
$\square$ low
$\square$ medium
high

2.4.5 Comments on regulated contaminant characteristics and/or confidence level:

The regulated contaminants listed above are applicable to the existing
inventory. The contaminants vary widely in concentration. The
contaminants with the highest concentration are:

$$
\text { Silver and mercury. }
$$

2.4.6 Will further characterization be performed $\overline{D e s}$$$
\text { No }
$$

Unknown
Additional characterization will be conducted as necessary to meet the waste acceptance criteria for WIPP. Characterization will include process knowledge, and sampling and analysis. schedule:

2.4.6.2 If yes, provide Tri-Party Agreement milestone number
No milestones are applicable to characterization of TRU waste.

\subsection{WASTE STREAM STORAGE, INVENTORY, AND GENERATION}
3.1
Is the waste stream currently stored?
1] Yes
No
3.2 Current storage method
$\square$ Container (pad)
$\square$ Tank
$\square$ Other (explain)
If no, skip to 3.7
Container (retrievably buried)
Surface impoundment 
TSD Unit Name

Central Waste Complex
Number of containers

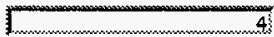

3.4 is storage of waste in compliance with applicable State and Federal standards?

$\triangle$ Yes

No

If no, provide details:

Current inventory for this stream

Total LDR volume (cubic meters):

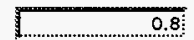

Date of inventory values: $9 / 30 / 98$

Comments on waste inventory: The inventory is based on the Solid Waste Information and Tracking System. The waste is packaged in drums.

3.7 Is the waste stream currently generated; and if not, will this waste stream be generated in the next 5 years?

$$
\square \text { Yes } \square \text { No }
$$

3.8 The current or future generation of this waste is best described as:

Routine

One-time or sporadic

3.9 Estimated generation projection by fiscal year

$\begin{array}{lrl}\text { 1999: } & 0.21 \\ \text { 2000: } & 2002: \\ \text { 2001: } & 0.9 & 2003: \\ & 1.7 & \end{array}$

3.10 Last Year's fiscal year 1998 generation projection:

Actual quantity generated in 1998:

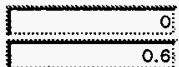

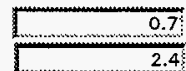

(Both in $\mathrm{m} 3$ unless otherwise noted)

3.11 Has there ever been any unusual release of this stream to the environment?

$\square$ Yes $D$ No

If yes, summarize releases and quantities:

\subsection{WASTE STREAM TREATMENT}

4.1 Is this stream currently being treated? 
4.2 Planned treatment: Check the appropriate box indicating future plans for treating this waste stroam to meet applicable regulations, including LDR treatment standards:

$[$ No treatment required (skip to 5.0 )

$\square$ Treating or plan to treat onsite

Treating or plan to treat offsite

Treatment options still being assessed

4.3 Planned treatment method, facility, and extent of treatment capacity available:

Waste is planned to be treated at WRAP. Treatment will be performed as necessary to meet the Japplicable waste acceptance criteria at WIPP. The extent of treatment will vary, and may include no treatment, solidification, or repackaging. WRAP has a design capacity of 4725 drums $(983 \mathrm{~m} 3)$ per year Oon a single shift pasis.

4.4 Treatment schedule

information::

The programmatic treatment schedule for mixed and non-mixed TRU
waste is from 7999 to 2032 . The schedule is subject to change as it
depends on the ability of DOE to accopt mixed TRU waste at WIPP and
available funding for treatment.

4.5 Applicable treatment T'ri-Party Agreement milestone numbers (including permitting):

M-91+01

If treating or planning to treat on site, will waste minimization be addressed in developing and/or selecting the treatment method?

If yes, please describe

4.7 Treatability equivalency petitions, rulemaking petitions, and case-by-case exemptions needed for treatment None are planned.

\subsection{WASTE STREAM DISPOSAL}

5.1 After treatiment, how will the waste stream be disposed of (include description, locations, variances required, ete., as applicable):

Mixed TRU waste is planned to be disposed of at the Waste isolation Pilot Plant (WIPP), a deep geological repository.

5.2 Applicable TPA milestone number(s) for disposal:

No TPA milestones exist for the disposal of TRU waste. The disposal of TRU waste at WIPP is a national DOE policy, and is required by DOE Order $5820.2 A$ (to be replaced by 435.1 ).

5.3 Planned disposal dates/time-frame:

The planned time frame of mixed and non-mixed TRU waste shipments to WIPP is from 2000 to 2032 . The time frame is subject to change depending on DOE's ability to accept waste at WIPP and adequate funding of TRU waste program activities.

\subsection{WASTE MINIMLZATION}

6.1 Has a waste minimization assessment been completed for this stream?
$\square$ Yes
No
Not applicable

6.2 Explain any waste minimization activities (e.g., process changes) under way or to be implemented for generation of this stream: 
Between 1996 and 1998 , only one drum $(0.2082 \mathrm{~m} 3)$ was received. Future waste generation is forecasted from the Plutonium Finishing Plant and is relatively small. The plant is proceeding in a

deactivation mode. Waste will likely consist of miscellaneous residues as a result of deactivation activities

\subsection{Schedule for implementing waste minimization methods:}

Because only best management practices have been identified, which are ongoing, a specific schedule is not applicable.

6.4 Waste reduction achieved during the calendar year and projected future reductions (volume or mass):

Calendar year: 0

Projected:

O)

6.4.1 Assumptions used in above estimate:

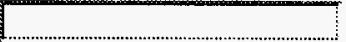




\section{WASTE STREAM PROFILE SHEET}

\subsection{WASTE STREAM IDENTIFICATION AND SOURCE}

1.1 Waste Stream ID: TRUM-0TH

1.2 Waste stream name

Contact-handled mixed TRU solidified organics

1.3.1 Stream source and history description: Thom

The waste was generated from the Plutonium Finishing Plant, the Chemical Engineering Building, and the Fuels Development Laboratory. The waste was generated and placed into storage from 1993 to 1998 .

1.3.2 Source category(s)

$\square$ Pollution control or waste treatment process

Material production/recovery effluents

$\mathbf{V}$ Facility or equipment operation and maintenance waste

$\square$ Discarded excess or expired materials

$\square$ Analytical laboratory waste

$\checkmark$ RD/RD laboratory waste

$\square$ Remediation/DD waste

$\square$ spill clean-ups or emergency response actions

$\square$ Source unknown

$\square$ Other (explain)

1.3.3 Additional notes:

\subsection{WASTE STREAM CHARACTERIZATION}

2.1 Waste stream description (content)

The waste stream consists of plastics, chemicals, absorbents, glass, animal waste, metals, and absorbed organic liquids.

2.2 Radiological characteristics

2.2.1 Mixed waste type High-level Transuranic Low-level D Contact-handled Remote-handled

2.2.2 Comments on radiological characteristics (e.g., more specific content, treatment concerns caused by radiation, confidence level):

Each container of waste has more than $100 \mathrm{nCi} / \mathrm{g}$ of transuranic nuclide activity. Non-transuranic activity is either not reported or reported in trace quantities.

2.3 Matrix characteristics (physical content)

2.3.1 Matrix constituent table (comprising at least $1 \%$ of the total volume or mass)

\begin{tabular}{|c|c|c|}
\hline 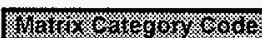 & 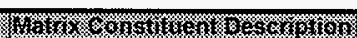 & matis \\
\hline S3190 & Ilnorganic homogeneous solids & $18.8 \%$ \\
\hline$\$ 5190$ & Inorganic debris & $16.5 \%$ \\
\hline$\$ 5390$ & Organic debris & $37.7 \%$ \\
\hline U9999 & Unknown/other & $27.0 \%$ \\
\hline
\end{tabular}

Overall matrix parameter category code: $\$ 3150$

Overall matrix description:

Solidified homogeneous solids

2.3.2 Confidence level for matrix characteristics data in 2.3.1 $\square$ low $\square$ medium $\square$ high 
2.3.3 Comments on matrix characteristics and/or confidence level:
The Waste Specification Record Number for this waste stream is SLOTC,
solidified liquids - organic - transuranic contact-handled. The waste matrix table
lists organic debris as the predominant waste form. In review of the waste
records, the organic debris is universal polypropylenes. Even though the material
acts as an absorbent, it is categoried as organic debris. These materials range
from 26.5 to 53 percent.

2.4 Regulated contaminant characteristics

2.4.1 Wastewater/non-wastewater under RCRA

$\square$ wastewater $\square$ non-wastewater $\square$ unknown

2.4.2 Regulated contaminant table including treatment requirements, and UHC's if applicable

\begin{tabular}{|l|l|l|l|l|l|}
\hline & & & & & \\
\hline
\end{tabular}

UHC's are not applicable to the WIPP facility. (1) Treatment standards are based on the WIPP facility waste acceptance criteria.

2.4.2.1 List any waste codes from 2.4.2 for which the stream already meets established LDR treatment standard Waste is exempt from Federal LDR's when being disposed of at the WIPP facility.

2.4.3 Does this waste stream contain PCBs?
$\square$ Yes
(2) No
Unknown

2.4.3.1 Is the waste stream subject to TSCA regulations for PCBs?
$\square$ Yes
[u No
Unknown

2.4.3.2 Indicate the PCB concentration range
$\square<50 \mathrm{ppm}$
$\square>=50$ ppm
Unknown

2.4.4 What is the confidence level for the regulated contaminant characteristic data?
$\square$ low
D. medium
high

2.4.5 Comments on regulated contaminant characteristics The regulated contaminants listed above are applicable to the existing inventory. The contaminants vary widely in concentration. The and/or confidence level: contaminants with the highest concentration are:

$$
\text { Carbon tetrachloride. }
$$

2.4.6 Will further characterization be performed $D$ Yes 7 No Unknown

2.4.6.1 If yes, provide details and Additional characterization will be conducted as necessary to meet the schedule: waste acceptance criteria for WIPP. Characterization will include process knowledge, and sampling and analysis.

2.4.6.2 If yes, provide Tri-Party Agreement milestone number No milestones are applicable to characterization of TRU waste.

\subsection{WASTE STREAM STORAGE, INVENTORY, AND GENERATION}


DOE/RL-99-01

3.1 Is the waste stream currently stored? $\square$ Yes

$\square$ No

3.2 Current storage method

$\square$ Container (pad) $\square$ container (covered)

$\square$ Waste pile

If no, skip to 3.7

$\square$ Tank

$\square$ Other (explain)

Container (retrievably buried)

Surface impoundment 
3.3 TSD unit name and building number and (if applicable/available) number of containers/tanks in each:

TSD Unit Name

Central Waste Complex
Number of containers

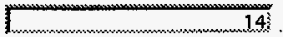

3.4 Is storage of waste in compliance with applicable State and Federal standards?

Yes.

No

If no, provide details:

Current inventory for this stream

Total LDR volume (cubic meters):

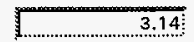

Date of Inventory values:

$9 / 30 / 98$

Comments on waste inventory:

The inventory is based on the Solid Waste Information and Tracking System. The waste is packaged in drums.

Is the waste stream currently generated; and if not, will this waste stream be generated in the next 5 years?

$$
\square \text { Yes } \square \text { No }
$$

3.8 The current or future generation of this waste is best described as:
$\square$ Routine
One-time or sporadic

3.9 Estimated generation projection by fiscal year

\begin{tabular}{l|l} 
1999: & 0.1 \\
$2000:$ & 0.5 \\
$2001:$ & 0.5
\end{tabular}

Last Year's fiscal year 1998 generation projection: Actual quantity generated in 1998:

$\frac{0}{0.9}$

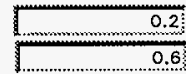

(Both in $\mathrm{m} 3$ unless otherwise noted)

3.11 Has there ever been any unusual release of this stream to the environment?

$\square$ Yes $\sqrt{ }$ No

If yes, summarize releases and quantities:

\subsection{WASTE STREAM TREATMENT}

4.1 Is this stream currently being treated? 
Yes $\square$ No If yes, provide details:

4.2 Planned treatment: Check the appropriate box indicating future plans for treating this waste stream to meet applicable regulations, including LDR treatment standards:

Wo treatment required (skip to 5.0 )

$\square$ Treating or plan to treat onsite

$\square$ Treating or plan to treat offsite

Treatment options still being assessed

4.3 Planned treatment method, facility, and extent of treatment capacity available:

Waste is planned to be treated at WRAP. Treatment will be performed as necossary to meet the applicable waste acceptance criteria at WIPP. The extent of treatment will vary, and may include no treatment, solidification, or repackaging. WRAP has a design capacity of 4725 drums $(983 \mathrm{~m} 3)$ per year on.a.single shift basis.

4.4 Treatment schedule information::

The programmatic treatment schedule for mixed and non-mixed TRU waste is from 1999 to 2032 . The schedule is subject to change as it depends on the ability of DOE to accept mixed TRU waste at WIPP and available funding for treatment.

4.5 Applicable treatment Tri-Party Agreement milestone numbers (including permitting): M-91-01

4.6 If treating or planning to treat on site, will waste minimization be addressed in developing and/or selecting the treatment method?

$\nabla$ No $\square$ Unknown

If yes, please describe

4.7 Treatability equivalency petitions, rulemaking petitions, and case-by-case exemptions needed for treatment

None are planned.

\subsection{WASTE STREAM DISPOSAL}

5.1 After treatment, how will the waste stream be disposed of (include description, locations, variances required, etc., as applicable):

Mixed TRU waste is planned to be disposed of at the Waste Isolation Pilot Plant (WIPP), a deep geological Frepository.

5.2 Applicable TPA milestone number(s) for disposal:

No TPA milestones exist for the disposal of TRU waste. The disposal of TRU waste at WIFP is a national DOE policy, and is required by DOE Order $5820.2 A$ (to be replaced by 435.1 ).

5.3 Planned disposal

The planned time frame of mixed and non-mixed TRU waste shipments to WIPP dates/ time-frame:

is from 2000 to 2032 . The time frame is subject to change depending on DOE's ability to accept waste at WIPP and adequate funding of TRU waste program activities.

\subsection{WASTE MINIMIZATION}

6.1 Has a waste minimization assessment been completed for this stream?
$\square$ Yes
No
Not applicable

6.2 Explain any waste minimization activities (e.g., process changes) under way or to be implemented for generation of this stream: 
A total of $1.25 \mathrm{m3}$ of waste has been received from 1996 and 1998. The generators are the Plutonium Finishing Plant and the Chemical Engineering Building. Small quantities of future waste have been forecasted from the Plutonium Finishing Plant and Environmental Restoration. Waste from the Plutonium Finishing Plant will be the removal of excess residues for safer storage. As such, waste minimization is not applicable. Because environmental restoration activities involve relocation of existing waste to a safer location and in a safer configuration, waste minimization is not applicable. Best management practices will be implemented, however, to minimize future generation to the extent possible.

6.3 Schedule for implementing waste minimization methods:

Because only best management practices have been identified, which are ongoing, a specific schedule is not applicable.

6.4 Waste reduction achieved during the calendar year and projected future reductions (volume or mass):

Calendar year:

Projected:

os $\sqrt{m 3}$

6.4.1 Assumptions used in above estimate:

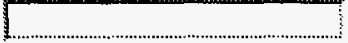




\section{WASTE STREAM PROFILE SHEET}

\subsection{WASTE STREAM IDENTIFICATION AND SOURCE}

1.1 Waste Stream ID:

1.2 Waste stream name

1.3.1 Stream source and history description:

The waste was generated from the Semi-works Decontamination and
Decommissioning Project, and the Plutonium Finishing Plant. The waste was
generated and placed into storage from 1992 to 1998 .

1.3.2 Source category(s)

$\square$ Pollution control or waste treatment process

Material production/recovery effluents

Vacility or equipment operation and maintenance waste

$\square$ Discarded excess or expired materials

$\square$ Analytical laboratory waste

$\square$ RD/RD laboratory waste

$\checkmark$ Remediation/DD waste

$\square$ Spill clean-ups or emergency response actions

Source unknown

Other (explain)

1.3.3 Additional notes:

\subsection{WASTE STREAM CHARACTERIZATION}

2.1. Waste stream description (content)

The waste sțream consists of soil/rocks, wood, metals, plastics, and chemicals.

2.2 Radiological characteristics

2.2.1 Mixed waste type
High-level
(D) Transuranic
Low-level
(1) Contact-handled
Remote-handled

2.2.2 Comments on radiological characteristics (e.g., more specific content, treatment concerns caused by radiation, confidence level):

Each container of waste has more than $100 \mathrm{nCi} / \mathrm{g}$ of transuranic nuclide activity. Non-transuranic activity is either not reported or reported in trace quantities.

2.3 Matrix characteristics (physical content)

2.3.1 Matrix constituent table (comprising at least $1 \%$ of the total volume or mass)

\begin{tabular}{|c|c|c|}
\hline W & 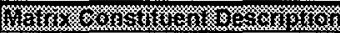 & Wat \\
\hline 54200 & Soil/debris & $68.6 \%$ \\
\hline$\$ 5190$ & Inorganic debris & $7.5 \%$ \\
\hline$\$ 5390$ & Organic debris & $18.7 \%$ \\
\hline U9999 & Unknown/other & $5.3 \%$ \\
\hline
\end{tabular}

Overall matrix parameter category code: 54200

Overall matrix description:

2.3.2 Confidence level for matrix characteristics data in 2.3.1 $\square$ low $\square$ medium $\square$ high 
2.3.3 Comments on matrix characteristics and/or confidence level:

The Waste Specification Record Number for this waste stream is SOITC, soil -
transuranic contact-handled.

2.4 Regulated contaminant characteristics

2.4.1 Wastewater/non-wastewater under RCRA
$\square$ wastewater
$\checkmark$ non-wastewater
unknown

2.4.2 Regulated contaminant table including treatment requirements, and UHC's if applicable

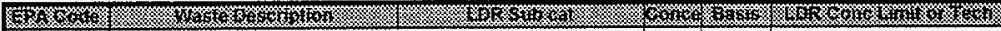

\begin{tabular}{|c|c|c|c|c|c|}
\hline 5007 & CHROMIUM & NA & ankn & $\pi *$ & Exempt (61 FR 60704) \\
\hline 0010 & SELENIUM & NA & $+*+$ & tank & Exempt (61 FR 60704) \\
\hline WTO2 & Toxic, DW & NA & 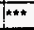 & $m * *$ & N/A \\
\hline
\end{tabular}

UHC's are not applicable to the WIPP facility. (1) Treatment standards are based on the WIPP facility waste acceptance criteria.

2.4.2.1 List any waste codes from 2.4.2 for which the stream al ready meets established LDR treatment standard Waste is exempt from Federal LDR's when being disposed of at the WIPP facility.

2.4.3 Does this waste stream contain PCBs?
Yès
$\checkmark$ No
Unknown

2.4.3.1 Is the waste stream subject to TSCA regulations for PCBs?
$\square$ Yes
$\nabla$ No
$\square$ Unknown

2.4.3.2 Indicate the $\mathrm{PCB}$ concentration range
$<50 \mathrm{ppm}$
$>=50 \mathrm{ppm}$
Unknown

2.4.4 What is the confidence level for the regulated contaminant characteristic data?
$\square$ low
$\square$ medium
high

2.4.5 Comments on regulated contaminant characteristics and/or confidence level:

The regulated contaminants listed above are applicable to the existing inventory. The contaminants vary widely in concentration. The contaminants with the highest concentration are:

\section{Chromium.}

2.4.6 Will further characterization be performed

Vij $\square$ No

Unknown
2.4.6.1 If yes, provide details and schedule:
Additional characterization will be conducted as necessary to meet the waste acceptance criteria for WIPP. Characterization will include process Knowledge, and sampling and analysis.

2.4.6.2 If yes, provide Tri-Party Agreement milestone number No milestones are applicable to characterization of TRU waste.

\subsection{WASTE STREAM STORAGE, INVENTORY, AND GENERATION}
3.1
is the waste stream currently stored?
(V) Yes
3.2 Current storage method
$\square$ Container (pad)
Tank
Other (explain)
Container (covered)
Waste pile
If no, skip to 3.7
Container (retrievably buried)
Surface impoundment 
3.3 TSD unit name and building number and (if applicable/available) number of containers/tanks in each: TSD Unit Name Number of containers Central Waste Complex .

3.4 Is storage of waste in compliance with applicable State and Federal standards?

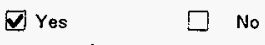

If no, provide details:

3.6 Current inventory for this stream

Total LDR volume (cubic meters):

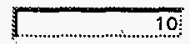

Date of Inventory values: 9/30/98:

Comments on waste inventory: The inventory is based on the Solid Waste Information and Tracking System. The waste is packaged in drums.

3.7 is the waste stream currently generated; and if not, will this waste stream be generated in the next 5 years? $\square$ Yes No

3.8 The current or future generation of this waste is best described as:

$\square$ Routine 7 One-time or sporadic

3.9 Estimated generation projection by fiscal year
1999:
2000:

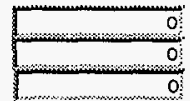
2002:
2001:
2003:

Last Year's fiscal year 1998 generation projection:

Actual quantity generated in 1998:

3.11 Has there ever been any unusual release of this stream to the environment?

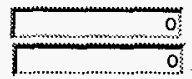

(Both in $\mathrm{m} 3$ unless otherwise noted)

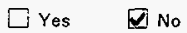

If yes, surnmarize releases and quantities:

\subsection{WASTE STREAM TREATMENT}

4.1 Is this stream currently being treated? 

Yes
No
If yes, provide details:

4.2 Planned treatment: Check the appropriate box indicating future plans for treating this waste stream to meet applicable regulations, including LDR treatment standards:

$\square$ No treatment required (skip to 5.0 )

$\square$ Treating or plan to treat onsite

Treating or plan to treat offsite

$\square$ Treatment options still being assessed

4.3 Planned treatment method, facility, and extent of treatment capacity available:

Waste is planned to be treated at WRAP. Treatment will be performed as necessary to meet the applicable waste acceptance criteria at WIPP. The extent of treatment will vary, and may include no treatment, solidification, or repackaging. WRAP has a design capacity of 4725 drums $(983 \mathrm{~m} 3$ ) per year Ion a single shift basis.

4.4 Treatment schedule information::

The programmatic treatment schedule for mixed and non-mixed TRU waste is from 1999 to 2032 . The schedule is subject to change as it depends on the ability of DOE to accept mixed TRU waste at WIPP and available funding for treatment.

4.5 Applicable treatment Tri-Party Agreement milestone numbers (including permitting): M-91-01

If treating or planning to treat on site, will waste minimization be addressed in developing and/or selecting the treatment method?

If yes, please describe

4.7 Treatability equivalency petitions, rulemaking petitions, and case-by-case exemptions needed for treatment None are planned.

\subsection{WASTE STREAM DISPOSAL}

5.1 After treatment, how will the waste stream be disposed of (include description, locations, variances required, etc., as applicable):

Mixed TRU waste is planned to be disposed of at the Waste Isolation Pilot Plant (WIPP), a deep geological repository.

5.2 Applicable TPA milestone number(s) for disposal:

No TPA milestones exist for the disposal of TRU waste. The disposal of TRU waste at WIPP is a national DOE policy, and is required by DOE Order $5820.2 \mathrm{~A}$ (to be replaced by 435.1 ).

\subsection{Planned disposal}

dates/time-frame:

The planned time frame of mixed and non-mixed TRU waste shipments to WIPP is from 2000 to 2032 . The time frame is subject to change depending on DOE's ability to accept waste at WIPP and adequate funding of TRU waste program activities.

\subsection{WASTE MINIMIZATION}

6.1 Has a waste minimization assessment been completed for this stream?
$Y \in S$
$\nabla$ No
Not applicable

6.2 Explain any waste minimization activities (e.g.. process changes) under way or to be implemented for generation of this stream: 


\section{DOE/RL-99-01}

Only one drum of soil waste was received from 1996 to 1998 . The waste came from the Plutonium Finishing Plant. Future waste containing soils contaminated with TRU elements and hazardous constituents has not been forecasted within the next 5 years (1999 to 2003).

6.3

Schedule for implementing waste minimization methods:

Because only best management practices have been identified, which are ongoing, a specific schedule is not applicable.

6.4 Waste reduction achieved during the calendar year and projected future reductions (volume or mass):
Calendar year:
$0 \sqrt{13}$
Projected:
o $m 3$

6.4.1 Assumptions used in above estimate:

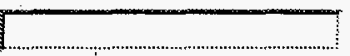




\section{WASTE STREAM PROFILE SHEET}

\subsection{WASTE STREAM IDENTIFICATION AND SOURCE}

1.1 Waste Stream 10:

1.2 Waste stream name

TRUM-OIK

Contact-ha

Contact-handled mixed TRU unknown or mixture

1.2 Waste strea Then

1.3.1 Stream sourće and history description:

The waste was generated from the Radiochemistry Building, the Plutonium Finishing Plant, and the PUREX plant. The waste was generated and placed into storage from 1987 to 1995 .

\subsubsection{Source category(s)}

$\square$ Pollution control or waste treatment process Material production/recovery effluents

$\square$ Facility or equipment operation and maintenance waste

$\square$ Discarded excess or expired materials

7 RD/RD laboratory waste

$\square$ Spill clean-ups or emergency response actions

$\square$ Analytical laboratory waste

Spill clean-ups or

\subsubsection{Additional notes:}

\subsection{WASTE STREAM CHARACTERIZATION}

2.1 Waste stream description (content)

\section{The waste stream consists of miscellaneous/unknown, plastics, metals,} cloth/rags, rubber, glass, paper/cardboard, and chemicals.

2.2 Radiological characteristics

2.2.1 Mixed waste type

$\square$ High-level

V Transuranic

\section{Low-level}

Contact-handled

\section{Remote-handled}

2.2.2 Comments on radiological characteristics (e.g., more specific content, treatment concerns caused by radiation, confidence level):

Each container of waste has more than $100 \mathrm{nCi} / \mathrm{g}$ of transuranic nuclide activity. One container from the Radiochemistry Building in 1995 contains $8.1 \mathrm{Ci}$ of $\mathrm{Sr}-90,8.1 \mathrm{Ci}$ of $\mathrm{Y}-90,1.6 \mathrm{Ci}$ of $\mathrm{Cs}-137$, and $1.5 \mathrm{Ci}$ of Ba-137m. Non-transuranic activity is either not reported or reported in trace quantities.

2.3 Matrix characteristics (physical content)

2.3.1 Matrix constituent table (comprising at least $1 \%$ of the total volume or mass)

\begin{tabular}{|c|c|c|}
\hline (A) & H & \& 20 \\
\hline$\$ 5190$ & Inorganic debris & $11.7 \%$ \\
\hline 55390 & Organic debris & $21.7 \%$ \\
\hline U9999 & Unknown/other & $59.2 \%$ \\
\hline
\end{tabular}

Overall matrix parameter category code: U9999

Overall matrix description: Unknown or mixture of forms

2.3.2 Confidence lovel for matrix characteristics data in 2.3.1 $\square$ low $\square$ medium $\square$ high 
2.3.3 Comments on matrix characteristics and/or confidence level:

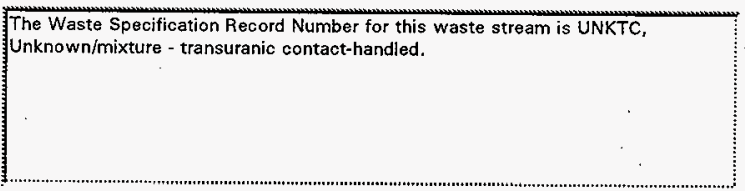

2.4 Regulated contaminant characteristics

2.4.1 Wastewater/non-wastewater under RCRA
wastewater
[V] non-wastewater
$\square$ unknown

2.4.2 Regulated contaminant table including treatment requirements, and UHC's if applicable

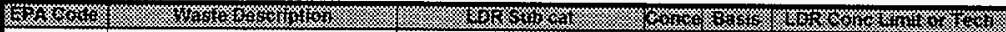

\begin{tabular}{|c|c|c|c|c|c|}
\hline boo7 & LEAD CHROMATE & NA & $* * *$ & $* \star \star *$ & Exempt (61 FR 60704) \\
\hline 0008 & LEAD & NA & \pm+ & $* * *$ & Exempt (61 FR 60704) \\
\hline D009 & MERCURIC OXIDE & NA & $\cdots$ & $\infty$ & Exempt (61 FR 60704) \\
\hline pot11 & SILVER & NA & neth & $+\$$ & Exempt (61 FR 60704) \\
\hline F001 & Spent Solvent & ITRICHLOROETHYLENE & + tin & $* * *$ & Exempt (61 FR 60704) \\
\hline 002 & Spent Solvent & Methylene Chloride & $+\infty$ & $* *$ & Exempt (61 FR 60704) \\
\hline$=003$ & Spent Solvent & Methyl isobutyl ketone & $a * *$ & tatk & Exempt (61 FR 60704) \\
\hline 0004 & Spent Solvent & CRESOL & $+\infty$ & 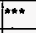 & Exempt (61 FR 60704) \\
\hline Foos & Spent Solvent & METHYL ETHYL KETONE & $+*$ & $a * \star$ & Exempt (61 FR 60704) \\
\hline NPO2 & Persistant, DW & $\mathrm{NA}$ & $n+\hbar$ & $+\infty$ & N/A \\
\hline NSC2 & Solid Corrosive & NA & ank & $k \cdots *$ & N/A \\
\hline WT01 & Toxic, EHW & NA & $+*$ & $n * *$ & N/A \\
\hline NT02 & Toxic, DW & NA & $\approx *$ & $\neq * *$ & N/A \\
\hline
\end{tabular}

UHC's are not applicable to the WIPP facility. (1) Treatment standards are based on the WIPP facility waste acceptance criteria.

2.4.2.1 List any waste codes from 2.4.2 for which the stream already meets established LDR treatment standard Waste is exempt from Federal LDR's when being disposed of at the WIPP facility.

2.4.3 Does this waste stream contain PCBs?
$\square$ Yes
$\checkmark$ No
Unknown

2.4.3.1 Is the waste stream subject to TSCA regulations for PCBs?
$\square$ Yes
$\checkmark$ No
$\square$ Unknown

2.4.3.2 Indicate the $\mathrm{PCB}$ concentration range
$<50 \mathrm{ppm}$
$\square>=50 \mathrm{ppm}$
Unknown

2.4.4 What is the confidence level for the regulated contaminant characteristic data?
$\square$ low
(V) medium
high

2.4.5 Comments on regulated contaminant characteristics and/or confidence level:

The regulated contaminants listed a bove are applicable to the existing inventory. The contaminants vary widely in concentration. The contaminants with the highest concentration are: Lead, mercury, and silver.

2.4.6 Will further characterization be performed $\square$ Yes $\square$ No $\square$ Unknown

2.4.6.1 If yes, provide details and Additional characterization will be conducted as necessary to meet the schedule:

waste acceptance criteria for WIPP. Characterization will include process knowledge, and sampling and analysis.

2.4.6.2 If yes, provide Tri-Party Agreement milestone number No milestones are applicable to characterization H. TRU waste.

\subsection{WASTE STREAM STORAGE, INVENTORY, AND GENERATION}


DOE/RL-99-01

3.1 Is the waste stream currently stored? $\square$ Yes $\square$ No

3.2 Current storage method

$\begin{array}{lll}\square \text { Container (pad) } & \square \text { Container (covered) } & \square \text { Container (retrievably buried) } \\ \square \text { Tank } & \square \text { Waste pile } & \square \text { Surface impoundment }\end{array}$

Other (explain)

Waste pile

Surface impoundment

If no, skip to 3.7 
id: TRUM-01K

3.3 TSD unit name and building number and (if applicable/available) number of containers/tanks in each:

TSD Unit Name

Central Waste Complex
Number of containers

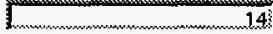

3.4 Is storage of waste in compliance with applicable State and Federal standards?

D Yes No

If no, provide details:

3.5 Applicable Tri-Party Agreement milestones related to storage:

M-91-01

3.6 Current inventory for this stream

Total LDR volume (cubic meters):

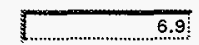

Date of inventory values:

Comments on waste inventory:

$$
9 / 30 / 98
$$

The inventory is based on the Solid Waste Information and Tracking System. The waste is packaged in drums and boxes.

Is the waste stream currently generated; and if not, will this waste stream be generated in the next 5 years? प Yes

No

3.8

The current or future generation of this waste is best described as:

Routine

One-time or sporadic

3.9 Estimated generation projection by fiscal year

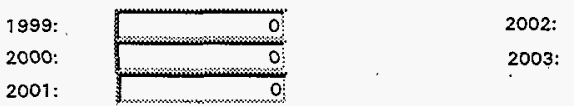

3.10

Last Year's fiscal year 1998 generation projection:

Actual quantity generated in 1998:
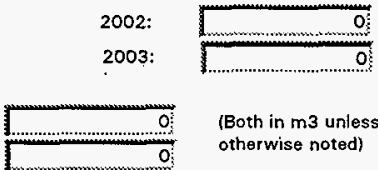

(Both in $\mathrm{m} 3$ unless otherwise noted)

3.11 Has there ever been any unusual release of this stream to the environment?

$\square$ Yes $\square$ No

If yes, summarize releases and quantities:

\subsection{WASTE STREAM TREATMENT}

4.1 is this stream currently being treated? 
$\square$ Yes $\square$ No If yes, provide details:

4.2 Planned treatment: Check the appropriate box indicating future plans for treating this wasto stream to meet applicable regulations, including LDR treatment standards:

$\square$ No treatment required (skip to 5.0 )

$\square$ Treating or plan to treat onsite

$\square$ Treating or plan to treat offsite

$\square$ Treatment options still beting assessed

4.3 Planned treatment method, facility, and extent of treatment capacity available:

Drumed waste and boxed waste are planned to be treated at WRAP and the proposed M91 facility, respectively. Treatment will be performed as necessary to meet the applicable waste acceptance criteria at WIPP. The extent of treatment will vary, and may include decontamination, solidification, and trepackaging. WRAP has a design capacity of 4725 drums (983 m3) por year on a single shift basis.

4.4 Treatment schedule information::

The programmatic treatment schedule for mixed and non-mixed TRU waste is from 1999 to 2032 . The schedule is subject to change as it depends on the ability of DOE to accept mixed TRU waste at WIPP and available funding for treatment.

4.5 Applicable treatment Tri-Party Agreement milestone numbers (including permitting): M-91-01

If treating or planning to treat on site, will waste minimization be addressed in developing and/or selecting the treatment method?

selecting the treatment method? $\square$ Nos $\square$ Unknown

If yes, please describe

4.7 Treatability equivalency petitions, rulemaking petitions, and case-by-case exemptions needed for treatment None are planned.

\subsection{WASTE STREAM DISPOSAL}

5.1 After treatment, how will the waste stream be disposed of (include description, locations, variances required, etc., as applicable):

Mixed TRU waste is planned to be disposed of at the Waste Isolation Pilot Plant (WIPP), a deep geological repository.

5.2 Applicable TPA milestone number(s) for disposal:

No TPA milestones exist for the disposal of TRU waste. The disposal of TRU waste at WIPP is a national DOE policy, and is required by DOE Order $5820.2 \mathrm{~A}$ (to be replaced by 435.1 ).

5.3 Planned disposal The planned time frame of mixed and non-mixed TRU waste shipments to WIPP dates/ time-frame:

is from 2000 to 2032 . The time frame is subject to change depending on DOE's ability to accept waste at WIPP and adequate funding of TRU waste program activities.

\subsection{WASTE MINIMIZATION}

6.1 Has a waste minimization assessment been completed for this stream?
$\square$ Yes
No
Not applicable

6.2 Explain any waste minimization activities (e.g., process changes) under way or to be implemented for generation of this stream: 
Contact-handled mixed TRU waste with unknown physical characteristics or mixture of physical forms has not been forecasted within the next 5 years (1999 to 2003). As such, waste minimization is not applicable to this waste stream.

6.3 Schedule for implementing waste minimization methods:

Because only best management practices have been identified, which are ongoing, a specific schedule is not applicable.

6.4 Waste reduction achieved during the calendar year and projected future reductions (volume or mass):
Calendar year:
o 3
Projected:
o)

6.4.1 Assumptions used in above estimate: 


\section{WASTE STREAM PROFILE SHEET}

\subsection{WASTE STREAM IDENTIFICATION AND SOURCE}

1.1 Waste Stream ID: TRUM-02A

1.2 Waste stream name

1.3.1 Stream source and
history description: Shielded mixed TRU heterogeneous debris The $w$ as The waste was generated from the Radiochemistry Building and the Post Irradiation Test Laboratory. The waste was generated and placed into storage from 1996 to 1998.

1.3.2 Source category(s)

$\square$ Pollution control or waste treatment process

Material production/recovery effluents

$\square$ Facility or equipment operation and maintenance waste

$\square$ Discarded excess or expired materials

$\square$ Analytical laboratory waste

$\checkmark$ RD/RD laboratory waste

$\square$ Spill clean-ups or emergency response actions

$\square$ Remediation/DD waste

$\square$ Other (explain)

1.3,3 Additional notes:

\subsection{WASTE STREAM CHARACTERIZATION} 2.1 Waste stream (content)

The waste stream consists of plastics, metals, lead shielding, steel shielding,
glass, paper/cardboard, and absorbents.

2.2 Radiological characteristios

2.2.1 Mixed waste type

$\square$ High-level

Source unknown

\section{Contact-handled $\mathbf{W}$ Remote-handled}

2.2.2 Comments on radiological characteristics (e.g., more specific content, treatment concerns caused by radiation, confidence level):

Each containet of waste has more than $100 \mathrm{nCi} / \mathrm{g}$ of transuranic nuclide activity. Because this waste stream is shielded waste, the waste typically contains non-transuranic activity. One container from the Post Irradiation Test Facility is reported to contain $0.33 \mathrm{Ci}$ of $\mathrm{Cs}-137$ and $0.31 \mathrm{Ci}$ of Ba-137m. Twenty (20) containers from the Radiochemistry Building, received either on $5 / 7 / 97$ or $9 / 30 / 97$, are reported to contain a total of $4.26 \mathrm{Ci}$ of $\mathrm{Sr}-90,4.26 \mathrm{Cl}$ of $\mathrm{Y}-90,1.70 \mathrm{Ci}$ of $\mathrm{Cs}-137$, and $1.61 \mathrm{Ci}$ of $\mathrm{Ba}-137 \mathrm{~m}$. Two containers from the Radiochemistry Building received on $9 / 30 / 98$ are reported to contain a total of $3.54 \mathrm{Ci}$ of $\mathrm{Sr}-90,3.54 \mathrm{Ci}$ of $\mathrm{Y}-90,2.34 \mathrm{Ci}$ of $\mathrm{Cs}-137$, and $2.21 \mathrm{Ci}$ of $8 \mathrm{a}-137 \mathrm{~m}$.

2.3 Matrix characteristics (physical content)

2.3.1 Matrix constituent table (comprising at least $1 \%$ of the total volume or mass)

\begin{tabular}{|c|c|c|}
\hline \multicolumn{3}{|c|}{ W1 } \\
\hline$\$ 3190$ & lnorganic homogeneous solids & $1.3 \%$ \\
\hline$\$ 5190$ & Inorganic debris & $13.4 \%$ \\
\hline$\$ 5390$ & Organic debris & $63.9 \%$ \\
\hline$\times 7290$ & Elemental lead & $11.2 \%$ \\
\hline$\times 7900$ & Special waste form & $9.3 \%$ \\
\hline
\end{tabular}

Overall matrix parameter category code: $\$ 5490$

Overall matrix description: Heterogeneous debris

2.3.2 Confidence level for matrix characteristics data in 2.3.1 $\square$ low $\square$ medium $\square$ high 
2.3.3 Comments on matrix charaoteristios and/or confidence level:
The Waste Specification Record Number for this waste stream is HTDTS,
Theterogeneous debris - transuranic remote-handled (shielded to contact-handled
(evels for storage).

2.4 Regulated contaminant characteristics

2.4.1 Wastewaterinon-wastewater under RCRA

$\square$ wastewater $\square$ non-wastewater $\square$ unknown

2.4.2 Regulated contaminant table including treatment requirements, and UHC's if applicable

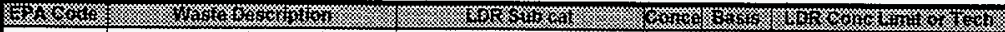

\begin{tabular}{|c|c|c|c|c|c|}
\hline Doo4 & ARSENIC & $\mathrm{NA}$ & $* *$ & $=m$ & Exempt (61 FR 60704) \\
\hline 005 & BARIUM & NA & fait* & $+\infty$ & Exempt (61 FR 60704) \\
\hline 0006 & CADMIUM & NA & $\$ *$ & $\neq * *$ & Exempt (61 FR 60704) \\
\hline 5007 & CHROMIUM & INA & $* *$ & $=* *$ & Exempt (61 FR 60704) \\
\hline Do08 & LEAD & NA & $* \pm$ & $* n$ & Exempt (61 FR 60704) \\
\hline 5009 & MERCURY & NA & 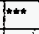 & $+\infty$ & Exempt (61 FR 60704) \\
\hline 2010 & SELENIUM & NA & $+*$ & $=m$ & Exempt (61 FR 60704) \\
\hline 011 & SILVER & NA & $\star \star \star *$ & $* * * *$ & Exempt (61 FR 60704) \\
\hline po18 & BENZENE & NA & $\geqslant * *$ & ;en & Exempt (61 FR 60704) \\
\hline 019 & CARBON TETRACHLORIDE & NA & the* & 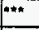 & Exempt (61 FR 60704) \\
\hline 027 & 1,4-DICHLOROBENZENE & NA & $* * *$ & $* *$ & Exempt (61 FR 60704) \\
\hline Do28 & 1,2-DICHLOROETHANE & NA & $* n *$ & n*t & Exempt (61 FR 60704) \\
\hline 5029 & 1,1-DICHLOROETHYLENE & NA & $+* *$ & 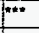 & Exempt (61 FR 60704) \\
\hline $\mathrm{p} 030$ & 2,4-DINITROTOLUENE & NA & 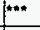 & 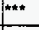 & Exempt (61 FR 60704) \\
\hline po33 & HEXACHLOROBUTADIENE & NA & $+*$ & $m *$ & Remove characteristic (1) \\
\hline po34 & HEXACHLOROETHANE & NA & $m * *$ & $\# *$ & Exempt (61 FR 60704) \\
\hline $\mathrm{p} 036$ & NITROBENZENE & NA & $n=*$ & 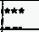 & Exempt (61 FR 60704) \\
\hline 2043 & VINYL CHLORIDE & NA & + & $+n+\infty$ & Exempt (61 FR 60704) \\
\hline F001 & Spent Solvent & TETRACHLOROETHYLENE & $* * *$ & $\neq=$ & Exempt (61 FR 60704) \\
\hline 002 & Spent Solvent & 1,1,1-TRICHLOROETHANE & $+*$ & 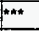 & Exempt (61 FR 60704) \\
\hline 003 & Spent Solvent & ACETONE & $+\infty$ & 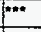 & Exempt (61 FR 60704) \\
\hline F004 & Spent Solvent & CRESOL & $+\ldots+\infty$ & 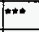 & Exempt (61 FR 60704) \\
\hline F005 & Spent Solvent & METHYL ETHYL KETONE & $\approx *$ & $+* *$ & Exempt (61 FR 60704) \\
\hline NSC2 & Solid Corrosive & INA & a** & $m *$ & $\mathrm{~N} / \mathrm{A}$ \\
\hline
\end{tabular}

UHC's are not applicable to the WIPP facility. (1) Treatment standards are based on the WIPP facility waste acceptance criteria.

2.4.2.1 List any waste codes from 2.4.2 for which the stream already meets established LOR treatment standard Waste is exempt from Federal LDR's when being disposed of at the WIPP facility.

2.4.3 Does this waste stream contain PCBs?
$\square$ Yes
No
Unknown

2.4.3.1 Is the waste stream subject to TSCA regulations for PCBs?
$\square$ Yes
No
Unknown

2.4.3.2 Indicate the $\mathrm{PCB}$ concentration range
$\square<50$ ppm
$\square>=50 \mathrm{ppm}$
Unknown

2.4.4 What is the confidence level for the regulated contaminant characteristic data?
$\square$ low
$\checkmark$ medium
high 
2.4.5 Comments on regulated contaminant characteristics and/or confidence level:
The regulated contaminants listed above are applicable to the existing inventory. The contaminants vary widely in concentration. The contaminants with the highest concentration are:

Lead and silver.

2.4.6 Will further characterization be performed $D$ Yes No Unknown

2.4.6.1 If yes, provide details and schedule:
Additional characterization will be conducted as necessary to meet the waste acceptance criteria for WIPP. Characterization will include process knowledge, and sampling and analysis.

2.4.6.2 If yes; provide Tri-Party Agreement milestone number No milestones are applicable to characterization of TRU waste.

\subsection{WASTE STREAM STORAGE, INVENTORY, AND GENERATION}

3.1 Is the waste stream currently stored?

QYes

3.2 Current storage method $\square$ Container (pad) Tank

Other (explain) $\checkmark$ Container (covered)

$\square$ Waste pile $\square$ No

$$
\text { If no, skip to } \mathbf{3 . 7}
$$

Container (retrievably buried)

$\square$ surface impoundment

(n)


id:

TRUM-02A

3.3 TSD unit name and building number and (if applicable/available) number of containers/tanks in each:

TSD Unit Name

Central Waste Complex
Number of containers

23

3.4 is storage of waste in compliance with applicable State and federal standards?

V) Yes

No

If no, provide details:

3.6 Current inventory for this stream

Total LDR volụme (cubic meters):

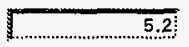

Date of Inventory values:

$$
9 / 30 / 98
$$

Comments on waste inventory:

The inventory is based on the Solid Waste Information and
Tracking System. The waste is packaged in drums.

3.7 Is the waste stream currently generated; and if not, will this waste stream be generated in the next 5 years? Yes $\square$ No

3.8 The current or future generation of this waste is best described as:
Doutine
$\checkmark$ One-time or sporadic

$3.9 \quad$ Estimated generation projection by fiscal year

\begin{tabular}{|c|c|c|c|}
\hline 1999: & 0 & 2002: & 10.5 \\
\hline 2000: & 0 & 2003: & 10.5 \\
\hline 2001: & 0 & & \\
\hline
\end{tabular}

Last Year's fiscal year 1998 generation projection: Actual quantity generated in 1998:

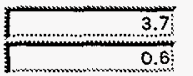

Both in $\mathrm{m} 3$ unless otherwise noted)

3.11 Has there ever been any unusual release of this stream to the environment?

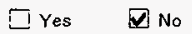

If yes, summarize releases and quantities:

\subsection{WASTE STREAM TREATMENT}

4.1 Is this stream currently being treated? 
$\square$ Yes $\square$ No If yes, provide details:

4.2 Planned treatment: Check the appropriate box indicating future plans for treating this waste stream to meet applicable regulations, including LDR treatment standards:

No treatment required (skip to 5.0 )

Treating or plan to treat onsite

$\square$ Treating or plan to treat offsite

Treatment options still being assessed

4.3 Planned treatment method, facility, and extent of treatment capacity available:

Waste are planned to be treated at the proposed M91 facility as needed to meet the applicable waste acceptance.criteria at WIPP. The extent of treatment will vary, and may include decontamination, solidification, and repackaging. The treatment capacity of the M91 facility has yet to be determined.

4.4 Treatment schedule

information::

The programmatic treatment schedule for mixed and non-mixed TRU waste is from 1999 to 2032 . The schedule is subject to change as it depends on the ability of DOE to accept mixed TRU waste at WIPP and available funding for treatment.

4.5 Applicable treatment.Tri-Party Agreement milestone numbers (including permitting):

$M-91-01$

4.6

If treating or planning to treat on site, will waste minimization be addressed in developing and/or

selecting the treatment method?

$\square$ Yes $\square$ No $\square$ Unknown

If yes, please describe

4.7 Treatability equivalency petitions, rulemaking petitions, and case-by-case exemptions needed for treatment None are planned.

\subsection{WASTE STREAM DISPOSAL}

5.1 After treatment, how will the waste stream be disposed of (include description, locations, variances required, etc., as applicable):

Mixed TRU waste is planned to be disposed of at the Waste Isolation Pilot Plant (WIPP), a deep geological repository,

5.2 Applicable TPA milestone number(s) for disposal:

No TPA milestones exist for the disposal of TRU waste. The disposal of TRU waste at WIPP is a national DOE policy, and is required by DOE Order $5820.2 A$ (to be replaced by 435.1 ).

5.3 Planned disposal

dates/ time-frame:

The planned time frame of mixed and non-mixed TRU waste shipments to WIPP

is from 2000 to 2032 . The time frame is subject to change depending on

DOE's ability to accept waste at WIPP and adequate funding of TRU waste

program activities.

\subsection{WASTE MINIMIZATION}

6.1 Has a waste minimization assessment been completed for this stream?
$\square$ Ves
(V) No
Not applicable

6.2 Explain any waste minimization activities (e.g., process changes) under way or to be implemented for generation of this stream: 
In the past three years, shielded heterogeneous debris has been received from the Radiochemistry Building the the Post Irradiation Test Laboratory. Future waste has been forecasted from the pre-treatment of tank waste. Waste minimization is compromised because shielding must be added to each container to store the waste as contact-handed waste. Nevertheless, best management practices are applied to minimize waste to the extent possible.

6.3 Schedule for implementing waste minimization methods:

Because only best management practices have been identified, which are ongoing, a specific schedule is not applicable.

6.4 Waste reduction achieved during the calendar year and projected future reductions (volume or mass):

Calendar year: $0.1 \mathrm{~m}$

Projected:

$0 \longdiv { m ^ { 3 } }$

6.4.1 Assumptions used in above estimate: 


\section{WASTE STREAM PROFILE SHEET}

\subsection{WASTE STREAM IDENTIFICATION AND SOURCE}

1.1 Waste Stream Ib:

TRUM-O2B

1.2 Waste stream name

Shielded mixed TRU lead waste

1.3.1 Stream source and

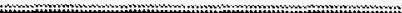
history description:

The waste was generated from the Argonne Nat Labs - East, the Chemical Engineering Building, the Battelle Columbus Labs, and the Post Irradiation Test Laboratory. The waste was generated and placed into storage from 1987 to 1996.

1.3.2 Source category(s)

$\square$ Pollution control or waste treatment process

Material production/recovery effluents

$\square$ Facility or equipment operation and maintenance waste

$\square$ Discarded excess or expired materials

RD/RD laboratory waste

Spill clean-ups or emergency response actions

Other (explain)

1.3.3 Additional notes:

\subsection{WASTE STREAM CHARACTERIZATION}

2.1 Waste stream description (content)

Analytical laboratory waste

Remediation/DD waste

Source unknown

2.2 Radiological characteristics

2.2.1 Mixed waste type The waste stream consists of lead, metals, cement, and lead shielding.

$\nabla$ High-level

$\checkmark$ Transuranic

Low-level

\section{$\square$ Contact-handled $\square$ Remote-handied}

2.2.2 Comments on radiological characteristics (e.g., more specific content, treatment concerns caused by radiation, confidence level):

Each container of waste has more than $100 \mathrm{nCi} / \mathrm{g}$ of transuranic nuclide activity. Because this waste stream is shieided waste, the waste typically contains non-transuranic activity. One container from the Post Irradiation Test Facility in 1996 is reported to contain $118 \mathrm{Ci}$ of Cs-137 and $112 \mathrm{Ci}$ of Ba-137m. Six (6) containers from the Argonne National Labs-East in 1987 are reported to contain a total of $1,800 \mathrm{Cj}$ of unspecified beta-gamma radioactivity. One container of waste from the Chemical Engineering Butlding in 1987 contain $35.4 \mathrm{Ci}$ of unspecified beta-gamma radioactivity.

Matrix characteristics (physical content)

2.3.1 Matrix constituent table (comprising at least $1 \%$ of the total volume or mass)

\begin{tabular}{|l|r|r|}
\hline Yine & & \\
\hline 53190 & Inorganic nomogeneous solids & $9.7 \%$ \\
\hline 55190 & Inorganic debris & $14.5 \%$ \\
\hline$\times 7290$ & Elemental lead & $75.8 \%$ \\
\hline
\end{tabular}

Overall matrix parameter category code: $\mathrm{X7219}$

Overall matrix description:

Elemental lead

2.3.2 Confidence level for matrix characteristics data in 2.3 .1

$\square$ low $\square$ medium $\square$ high


2.3.3 Comments on matrix characteristics and/or confidence level:

The Waste Specification Record number for this waste stream is PBMTS, lead metal - transuranic remote-handled (shielded to contact handled levels for storage).

2.4 Regulated contaminant characteristics

2.4.1 Wastewater/non-wastewater under RCRA
$\square$ wastewater
$\nabla$ non-wastewater
unknown

2.4.2 Regulated contaminant table including treatment requirements, and UHC's if applicable

\begin{tabular}{|c|c|c|c|c|c|}
\hline 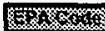 & $8 x_{2}$ & $30 \%$ \% & 3 & 20 & 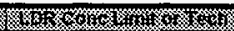 \\
\hline 0006 & CADMIUM & NA & $\because *$ & 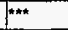 & Exempt (61 FR 60704) \\
\hline 0008 & LEAD & NA & $+\ldots$ & $\$ * \star *$ & Exempt (61 FR 60704) \\
\hline P015 & BERYLLIUM & NA & $\approx *=$ & $\# *$ & Exempt (61 FR 60704) \\
\hline NT01 & Toxic, EHW & NA & F** & $\pi * \star *$ & N/A \\
\hline
\end{tabular}

UHC's are not applicable to the WIPP facility. (1) Treatment standards are based on the WIPP facility waste acceptance criteria.

2.4.2.1 List any waste codes from 2.4.2 for which the stream already meets established LDR treatment standard Waste is exempt from Federal LDR's when being disposed of at the WIPP facility.

2.4.3 Does this waste stream contain PCBs?
$\square$ Yes
$\checkmark$ No
Unknown

2.4.3.1 Is the waste stream subject to TSCA regulations for PCBs?
$\square$ Yes
No
Unknown

2.4.3.2 Indicate the $\mathrm{PCB}$ concentration range
$\square<50 \mathrm{ppm}$
$\square>=50 \mathrm{ppm}$
Unknown

2.4.4 What is the confidence level for the regulated contaminant characteristic data?
$\square$ low
D] medium
high

2.4.5 Comments on regulated contaminant characteristics and/or confidence level:

The regulated contaminants listed above are applicable to the existing
inventory. The contaminants vary widely in concentration. The
contaninants with the highest concentration are:

2.4.6 Will further characterization be performed $\nabla$ Yes $\square$ No
2.4.6.1 If yes, provide details and schedule:
Additional characterization will be conducted as necessary to meet the waste acceptance criteria for WIPP. Characterization will include process knowledge, and sampling and analysis.

2.4.6.2 If yes, provide Tri-Party Agreement milestone number No milestones are applicable to characterization of TRU waste.

\subsection{WASTE STREAM STORAGE, INVENTORY, AND GENERATION}

3.1 Is the waste stream currently stored?

3.2 Current storage method

$\square$ Container (pad)

Tank

Other (explain)
V Yes

No

$$
\text { If no, skip to } 3.7
$$

Container \{retrievably buried)

Container (covered)

Waste pile
Surface impoundment 
3.3 TSD unit name and building number and (if applicable/available) number of containers/tanks in each:

TSD Unit Name

Central Waste Complex
Number of containers

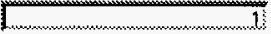

3.4 Is storage of waste in compliance with applicable State and Federal standards?

$\checkmark$ Yes

No

If no, provide details:

Current inventory for this stream

Total LDR volume (cubic meters):

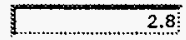

Date of inventory values:

9/30/98:

Comments on waste inventory:

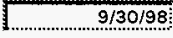

The inventory is based on the Solid Waste Information and Tracking System. The waste is packaged in drums, boxes, and special types of containers.

3.7 Is the waste stream currently generated; and if not, will this waste stream be generated in the next 5 years? $\square$ Yes $\square$ No

3.8. The current of future generation of this waste is best described as:
$\bar{V}$ Routine
One-time or sporadic

3.9 Estimated generation projection by fiscal year

$\begin{array}{lll}\text { 1999: } & 2002: \\ \text { 2000: } & 0.4 \\ 2001: & 0.4 & 2003:\end{array}$

3.10

3.11

Last Year's fiscal year 1998 generation projection:

Actual quantity generated in 1998:

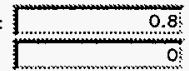

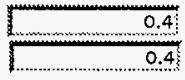

(Both in $\mathrm{m} 3$ unless otherwise noted)

If yes, summarize releases and quantities:

\subsection{WASTE STREAM TREATMENT}

4.1 Is this stream currently being treated? 
$\square$ Yes. $\square$ No If yes, provide details:

4.2 Planned treatment: Check the appropriate box indicating future plans for treating this waste stream to meet applicable regulations, including LDR treatment standards:

$\square$ No treatment required (skip to 5.0 )

$\square$ Treating or plan to treat onsite

Treating or plan to treat offsite

Treatment options still being assessed

4.3 Planned treatment method, facility, and extent of treatment capacity available:

Waste are planned to be treated at the proposed M91 facility as needed to meet the applicable waste acceptance criteria at WIPP. The extent of treatment will vary, and may include decontamination, solidification, and repackaging. The treatment capacity of the M91 facility has yet to be determined.

4.4 Treatment schedule information::

The programmatic treatment schedule for mixed and non-mixed TRU
waste is from 1999 to 2032 . The schedule is subject to change as it
depends on the ability of DOE to accept mixed TRU waste at WIPP and
available funding for treatment.

4.5 Applicable treatment Tri-Party Agreement milestone numbers (including permitting):

4.6 If treating or planning to treat on site, will waste minimization be addressed in developing and/or selecting the treatment method?

selecting the treatment method?

4.7 Treatability equivalency petitions, rulemaking petitions, and case-by-case exemptions needed for treatment None are planned.

\subsection{WASTE STREAM DISPOSAL}

5.1 After treatment, how will the waste stream be disposed of (include description, locations, variances required, etc., as applicable):

Mixed TRU waste is planned to be disposed of at the Waste Isolation Pilot Plant (WIPP), a deep geological
repository.

5.2 Applicable TPA milestone number(s) for disposal:

No TPA milestones exist for the disposal of TRU waste. The disposal of TRU waste at WIPP is a national DOE policy, and is required by DOE Order $5820.2 \mathrm{~A}$ (to be replaced by 435.1 ).
5.3 Planned disposal dates/ time-frame:
The planned time frame of mixed and non-mixed TRU waste shipments to WIPP is from 2000 to 2032 . The time frame is subject to change depending on DOE's ability to accept waste at WIPP and adequate funding of TRU waste program activities.

\subsection{WASTE MINIMIZATION}

6.1 Has a waste minimization assessment been completed for this stream?
$\square$ Yes
(D)
$\square$ Not applicable

6.2 Explain any waste minimization activities (e.g., process changes) under way or to be implemented for generation of this stream: 
Only one drum of waste has been generated between 1996 and 1998 . The drum was from the Post Irradiation Test Facility. Future waste is expected from R\&D activities at the Pacific Northwest National Laboratories. Because $R \& D$ activities generate relatively small volumes of waste, waste minimization, other than best management practices, is not applicable to these activities. Waste has also been forecasted from T Plant. Operations at T Plant involve treating existing wastes so that they can be stored safely. Because no new solid radioactive waste is generated from the process, waste minimization, other than best management practices, is not applicable. Because only best management practices have been identified, which are ongoing, a specific schedule is not applicable.

6.4 Waste reduction achieved during the calendar year and projected future reductions (volume or mass):
Calendar year:
$0 \mathrm{~m}^{3}$
Projected:
$0 \sqrt{\min 3}$

6.4.1 Assumptions used in above estimate: 
DOE/RL-99-01

This page intentionally left blank. 


\section{WASTE STREAM PROFILE SHEET}

\subsection{WASTE STREAM IDENTIFICATION AND SOURCE}

1.1 Waste Stream ID: TRUM-03

1.2 Waste stream name

1.3.1 Stream source and history description:

PFP hydraulic fluids contaminated with PCB's Pom The waste was generated from the Plutonium Finishing Plant. The waste was generated and placed into storage from 1988 to 1989 .

\subsubsection{Source category(s)}

$\square$ Pollution control or waste treatment process

Material production/recovery effluents

$\square$ Facility or equipment operation and maintenance waste

$\square$ Discarded excess or expired materials

Analytical labioratory waste $\mathrm{RD} / \mathrm{RD}$ laboratory waste

Remediation/DD waste

Spill clean-ups or emergency response actions

Source unknown

$\square$ other (explain)

1.3.3 Additional notes:

\subsection{WASTE STREAM CHARACTERIZATION}

2.1 Waste stream The waste stream consists of metals, plastics, wood, lead, glass, concrete, description absosbed organic liquids, cloth/rags, absorbents, and absorbed liquids.

(content)

2.2 Radiological characteristics

2.2.1 Mixed waste type

$$
\begin{gathered}
\text { High-level } \square \text { Transuranic } \square \text { Low-level } \\
\square \text { Contact-handled } \square \text { Remote-handled }
\end{gathered}
$$

2.2.2 Comments on radiological characteristics (e.g., more specific content, treatment concerns caused by radiation, confidence (evel):

Each container of waste has more than $100 \mathrm{nCi} / g$ of transuranic nuclide activity. Non-transuranic activity is either not reported or reported in trace quantities.

2.3 Matrix characteristics (physical content)

\begin{tabular}{|c|c|c|}
\hline W & 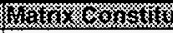 & 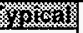 \\
\hline$\$ 5190$ & Inorganic debris & $66.9 \%$ \\
\hline$\$ 5390$ & Organic debris & $26.8 \%$ \\
\hline 19999 & Unknown/other & $1.2 \%$ \\
\hline$\times 7290$ & Elemental lead & $4.2 \%$ \\
\hline
\end{tabular}

2.3.1 Matrix constituent table (comprising at least $1 \%$ of the total volume or mass)

Overall matrix parameter category code: 55490

Overall matrix description: $\quad$ Heterogeneous debris

2.3.2 Confidence level for matrix characteristics data in 2.3.1 $\square$ low $\square$ medium $\square$ high 
2.3.3 Comments on matrix characteristics and/or confidence level:

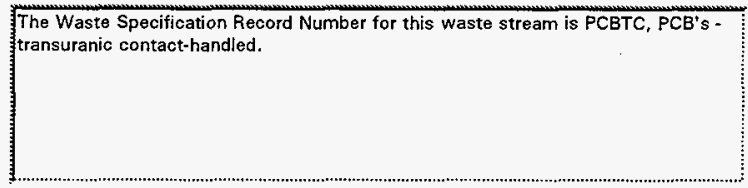

2.4 Regulated contaminant characteristics

2.4.1 Wastewater/non-wastewater under RCRA
wastewater
$\checkmark$ non-wastewater
unknown

2.4.2 Regulated contaminant table including treatment requirements, and UHC's if applicable

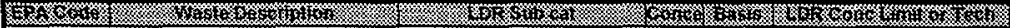

\begin{tabular}{|c|c|c|c|c|c|}
\hline boot & Various & NA & $m *$ & $* \pm *$ & Remove characteristic (1) \\
\hline 5006 & CADMIUM & NA & $\cdots$ & $+*$ & Exempt (61 FR 60704) \\
\hline $\mathrm{b} 008$ & LEAD & NA & math & to*** & Exempt (61 FR 60704) \\
\hline Do09 & MERCURY & INA & $\infty$ & $* *+$ & Exempt (61 FR 60704) \\
\hline WT01 & Toxic, EHW & NA & *at & 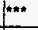 & N/A \\
\hline NTO2 & Toxic, DW & NA & $1+\cdots \star$ & $* * *$ & N/A \\
\hline
\end{tabular}

UHC's are not applicable to the WIPP facility. (1) Treatment standards are based on the WIPP facility waste acceptance criteria.

2.4.2.1 List any waste codes from 2.4.2 for which the stream already meets established LDR treatment standard Waste is exempt from Federal LDR's when being disposed of at the WIPP facility.

2.4.3 Does this waste stream contain PCBs?
$\checkmark$ Yes
No
Unknown

2.4.3.1 Is the waste stream subject to TSCA regulations for PCBs?
$\square$ Yes
No
Unknown

2.4.3.2 Indicate the $\mathrm{PCB}$ concentration range
$\square<50 \mathrm{ppm}$
$>=50 \mathrm{ppm}$
Unknown

2.4.4 What is the confidence level for the regulated contaminant characteristic data?

low

medium

high

2.4.5 Comments on regulated contaminant characteristics and/or confidence lovel:

The regulated contaminants listed above are applicable to the existing inventory. The contaminants vary widely in concentration. The contaminants with the highest concentration are:

$$
\text { PCB's and lead. }
$$

2.4.6 Will further characterization be pertormed $\square$ Yes

No

Unknown

2.4.6.1 If yes, provide details and schedule:

Additional characterization will be conducted as necessary to meet the waste acceptance criteria for WIPP. Characterization will include process knowledge, and sampling and analysis.

2,4.6.2 If yes, provide Tri-Party Agreement milestone number No milestones are applicable to characterization of TRU waste.

\subsection{WASTE STREAM STORAGE, INVENTORY, AND GENERATION}

3.1 is the waste stream currently stored? $\square$ No

3.2 Current storage method

$\begin{array}{ll}\square \text { Container (pad) } & \square \text { Container (covered) } \\ \square \text { Tank } & \square \text { Waste pile } \\ \square \text { Other (explajn) } & \end{array}$

If no, skip to 3.7

Container (retrievably buried)

Surface impoundment 
TSD unit name and building number and (if applicable/available) number of containers/tanks in each: TSD Unit Name

Number of containers

Central Waste Complex

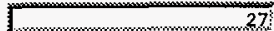

3.4 Is storage of waste in compliance with applicable State and Federal standards?
0 Yes
No

If no, provide details:

Total LDR volume (cubic meters):

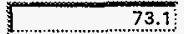

Date of Inventory values: $9 / 30 / 98$

Comments on waste inventory:

The inventory is based on the Solid Waste information and Tracking System. The waste is packaged in drums and boxes.

Is the waste stream currently generated; and if not, will this waste stream be generated in the next 5 years?
7 Yes.
$\mathbf{N o}$

3.8

The current or future generation of this waste is best described as:

3.9

$\square$ Routine

One-time or sporadic

3.9 Estimated generation projection by fiscal year

1999:

2000:

2001:

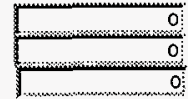

3.10

Last Year's fiscal year 1998 generation projection:

Actual quantity generated in 1998 :

2002:

2003:

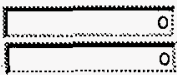

(Both in $\mathrm{m} 3$ unless otherwise noted)

3.11 Has there ever been any unusual release of this stream to the environment?

$\square$ Yes

No

If yes, summarize releases and quantities:

\subsection{WASTE STREAM TREATMENT}

4.1 Is this stream currently being treated? 


\section{DOE/RL-99-01}

$\square$ Yes $\quad \square$ No if yes, provide details:

4.2 Planned treatment: Check the appropriate box indicating future plans for treating this waste stream to meet applicable regulations, including LDR treatment standards:

$\square$ No treatment required (skip to 5.0 )

Treating or plan to treat onsite

Treating or plan to treat offsite

V] Treatment options still being assessed

4.3 Planned treatment method, facility, and extent of treatment capacity available:

The waste is planned to be treated at the proposed M91 facility. Thermal destruction of PCB's are required prior to acceptance of this TRU waste at WIPP.

4.4 Treatment schedule

information::

The programmatic treatment schedule for mixed and non-mixed TRU
waste is from 1999 to 2032 . The schedule is subject to change as it
depends on the ability of DOE to accept mixed TRU waste at WIPP and
available funding for treatment.

4.5 Applicable treatment Tri-Party Agreement milestone numbers (including permitting):

M-91-01

4.6

If treating or planning to treat on site, will waste mininization be addressed in developing and/or

selecting the treatment method?

पYes $\square$ No $\square$ Unknown

If yes, please describe

4.7 Treatability equivalency petitions, rulemaking petitions, and case-by-case exemptions needed for treatment None are planned.

\subsection{WASTE STREAM DISPOSAL}

5.1 After treatment, how will the waste stream be disposed of tinclude description, locations, variances required, etc., as applicable):

Due to the presense of PCB's in the TRU waste, the dispostion of this waste has yet to be determined.

5.2 Applicable TPA miles tone number(s) for disposal:

No TPA milestones exist for the disposal of TRU waste. The disposal of TRU waste at WIPP is a national DOE policy, and is required by DOE Order $5820.2 \mathrm{~A}$ (to be replaced by 435.1 ).

5.3 Planned disposal

dates/time-frame:

The schedule for disposal of TRU waste contaminated with PCB's has yet to be determined.

\subsection{WASTE MINIMIZATION}

6.1 Has a waste minimization assessment been comploted for this stream?
Y.es
No
Not applicable

6.2 Explain any waste minimization activities (e.g., process changes) under way or to be implemented for generation of this stream: 
TRU waste contaminated with PCB's has not been generated since 1989. Additional TRU waste contaminated with PCB's is not expected in the future.

6.3 Schedule for implementing waste minimization methods:

Because only best management practices have been identified, which are ongoing, a specific schedule is not applicable.

6.4 Waste redwction achieved during the calendar year and projected future reductions (volume or mass):

Calendar year:

Projected:

o) $\ln _{3}$

6.4.1 Assumptions used in above estimate:

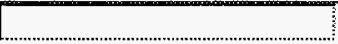


DOE/RL-99-01

This page intentionally left blank. 


\section{WASTE STREAM PROFILE SHEET}

\subsection{WASTE STREAM IDENTIFICATION AND SOURCE}

1.1 Waste Stream ID: TRUM-04A

1.2 Waste stream name

1.3.1 Stream source and history description:

300 Area Revitalization contact-handled mixed TRU heterogeneous debris Thom An This waste is from 300 Area facilities in which the process missions have terminated. The waste has yet to be generated.

1.3.2 Source category(s)
$\square$ Pollution control or waste treatment process
Material production/recovery effluents
$\square$ Facility or equipment operation and maintenance waste
$\square$ Discarded excess or expired materials
$\square$ Analytical laboratory waste
$\square$ RD/RD laboratory waste
$\square$ Remediation/DD waste
spill clean-ups or emergency response actions
Souree unknown
$\square$ Other (explain)

1.3.3 Additional notes:

\subsection{WASTE STREAM CHARACTERIZATION}

Waste stream
description (content)

The waste will consist of heterogeneous debris to include glass, rags, metal,
paper, cardboard, plastics, and rubber.

2.2 Radiological characteristios
2.2.1 Mixed waste type
$\square$ High-level
D Transuranic
Low-level
D. Contact-handled
Remote-handled

2.2.2 Comments on radiological characteristics (e.g., more specific content, treatment concerns caused by radiation, confidence level):

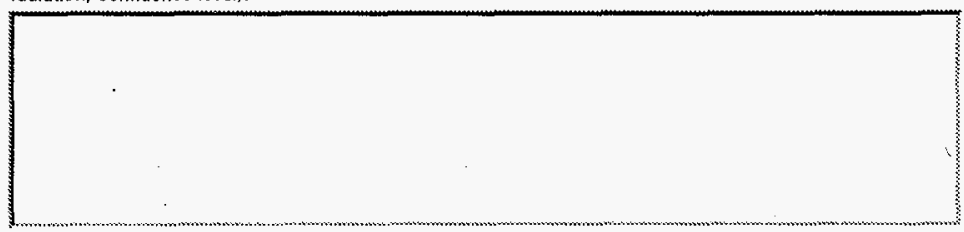

2.3 Matrix characteristics (physical content)

2.3.1 Matrix constituent table (comprising at least $1 \%$ of the total volume or mass)

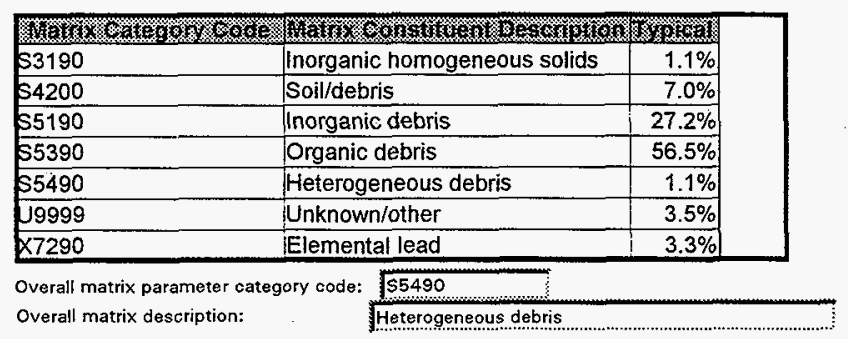


2.3.2 Confidence level for matrix characteristics data in 2.3.1 $\square$ low $\square$ medium $\square$ high

2.3.3 Comments on matrix characteristics and/or confidence level:

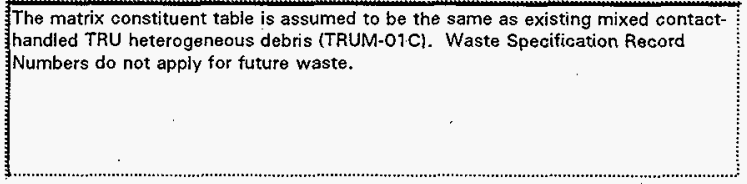

2.4 Regulated contaminant characteristics

2.4.1 Wastewater/non-wastewater under RCRA

$\square$ wastewater $\square$ non-wastewater $\square$ unknown

2.4.2 Regulated contaminant table including treatment requirements, and UHC's if applicable F

\begin{tabular}{l|l|l|l|l|l|} 
TBD & TBD & TBD & TBD & TBD & TBD \\
\hline
\end{tabular}

UHC's are not applicable to the WIPP facility. (1) Treatment standards are based on the WIPP facility waste acceptance criteria.

2.4.2.1 List any waste codes from 2.4.2 for which the stream already meets established LDR treatment standard Waste is exempt from Federal LDR's when being disposed of at the WIPP facility.

2.4.3 Does this waste stream contain PCBs?
$\square$ Yes
$\checkmark$ No
Unknown

2.4.3.1 Is the waste stream subject to TSCA regulations for PCBs?
$\square$ Yes
7 No
Unknown

2.4.3.2 Indicate the PCB concentration range
$\square<50 \mathrm{ppm}$
$>=50 \mathrm{ppm}$
Unknown

2.4.4 What is the confidence level for the regulated contaminant characteristic data?
$\square$ low
D. medium
high

2.4.5 Comments on regulated contaminant characteristics and/or confidence level:

Because the waste has yet to be generated the regulated contaminant characteristics are unknown.

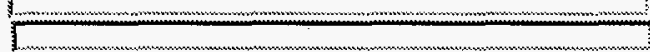

2.4.6 Will further characterization be performed $\square$ Yes $\square$ No $\square$ Unknown

2.4.6.1 If yes, provide details and Additional characterization will be conducted as necessary to meet the schedule: waste acceptance criteria for WIPP. Characterization will include process knowledge, and sampling and analysis.

2.4.6.2 If yes, provide Tri-Party Agreement milestone number No milestones are applicable to characterization of TRU waste.

\subsection{WASTE STREAM STORAGE, INVENTORY, AND GENERATION}
3.1
Is the waste stream currently stored?
G Yes
No

3.2 Current storage method

$\square$ Container (pad)
$\square$ Tank.
$\square$ Other (explain)

Container (covered)

Waste pile

$$
\text { If no, skip to } 3.7
$$

Container (retrievably buried)

Surface impoundment 
DOE/RL-99-01

id:

TRUM.04A

3.3 TSD unit name and building number and (if applicable/available) number of containers/tanks in each:

TSD Unit Name

Central Waste Complex
Number of containers

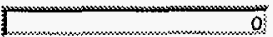

Is storage of waste in compliance with applicable State and Federal standards?

Yes

No

If no, provide details:

3.6 Current inventory for this stream

Total LDR volume (cubic meters):

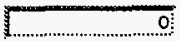

Date of Inventory values:

$9 / 30 / 98$

Comments on waste inventory:

Is the waste stream currently generated; and if not, will this waste stream be generated in the next 5 years? $\nabla$ Yes $\square$ No

The current or future generation of this waste is best described as:

$\square$ Routine

One-time or sporadic

3.9 Estimated gemeration projection by fiscal year

1999:

2000:

2001:

Last Year's fiscal vear 1998 generation projection: Actual quantity generated in 1998 :
2002:

2003:

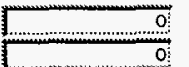

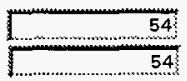

(Both in $\mathrm{m} 3$ unless otherwise noted)

3.11 Has there ever been any unusual release of this stream to the environment?

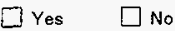

If yes, summarize releases and quantities:

\subsection{WASTE STREAM TREATMENT}


(V) Yes $\square$ No If yes, provide details:

4.2 Planned treatment: Check the appropriate box indicating future plans for treating this waste stream to meet applicable regulations, including LDR treatment standards:

No treatment required (skip to 5.0)

Treating or plan to treat onsite

Treating or plan to treat offsite

Treatment options still being assessed

4.3 Planned treatment method, facility, and extent of treatment capacity available:

Drumed waste and boxed waste are planned to be treated at WRAP and the proposed M91 facility, respectively. Treatment will be performed as necessary to meet the applicable waste acceptance criteria at WIPP. The extent of treatment will vary, and may include decontamination, solidification, and repackaging. WRAP has a design capacity of 4725 drums $(983 \mathrm{~m} 3$ ) per year on a single shift basis.

Treatment schedule

The programmatic treatment schedule for mixed and non-mixed TRU

information: waste is from 1999 to 2032 . The schedule is subject to change as it depends on the ability of DOE to accept mixed TRU waste at WIPP and available funding for treatment.

4.5 Applicable troatment Tri-Party Agreement milestone numbers (including permitting): M-91-01

If treating or planning to treat on site, will waste minimization be addressed in developing and/or selecting the treatment method?

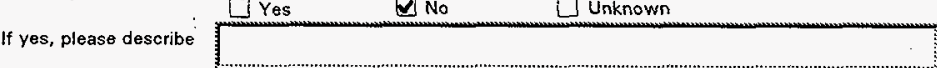

4.7 Treatability equivalency petitions, rulemaking petitions, and case-by-case exemptions needed for treatment None are planned.

\subsection{WASTE STREAM DISPOSAL}

5.1 After treatment, how will the waste stream be disposed of linclude description, locations, variances required, etc., as applicable):

Mixed TRU waste is planned to be disposed of at the Waste Isolation Pilot Plant (WIPP), a deep geological repository.

5.2 Applicable TPA milestone number(s) for disposal:

No TPA milestones exist for the disposal of TRU waste. The disposal of TRU waste at WIPP is a national DOE policy, and is required by DOE Order $5820.2 \mathrm{~A}$ (to be replaced by 435.1 ).

5.3 Planned disposal dates/ time-frame:

The planned time frame of mixed and non-mixed TRU waste shipments to WIPP is from 2000 to 2032. The time frame is subject to change depending on DOE's ability to accept waste at WIPP and adequate funding of TRU waste program activities.

\subsection{WASTE MINIMIZATION}

6.1 Has a waste minimization assessment been completed for this stream?
E Yes
No
Not applicable

6.2 Explain any waste minimization activities (e.g., process changes) under way or to be implemented for generation of this stream: 


The waste consists of so called "step-off pad" waste (clothing, tape, etc) associated with deactivation
operations. These waste materials are associated with working within a radiation protection zone and
therefore best management practices ususally provide the best waste minimization alternatives.

6.3 Schedule for implementing waste minimization methods:

Because only best management practices have been identified, which are ongoing, a specific schedule is not applicable.

6.4 Waste reduction achieved during the calendar year and projected future reductions (volume or mass):
Calendar year:
0 .
Projected:
0 的3

6.4.1 Assumptions used in above estimate:

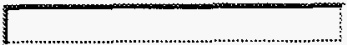




\section{WASTE STREAM PROFILE SHEET}

\subsection{WASTE STREAM IDENTIFICATION AND SOURCE}

1.1 Waste Stream ID:

1.2 Waste stream name

1.3.7 Stream source and history description:

$$
\text { TRUM-04B }
$$

300 Area Revitalization contact-handled mixed TRU inorganic nonmetal debris This waste is from 300 Area facilities in which the process missions have terminated. The waste has yet to be generated.

\subsubsection{Source category(s)}

$\square$ Pollution control or waste treatment process

Material production/recovery effluents

$\square$ Facility or equipment operation and maintenance waste

$\checkmark$ Discarded excess or expired materials

Analytical laboratory waste

RD/RD laboratory waste

$\square$ Remediation/DD waste

Spill clean-ups or emergency response actions

$\square$ Source unknown

$\square$ other (explain)

1.3.3 Additional notes:

\subsection{WASTE STREAM CHARACTERIZATION}

2.1 Waste stream description (content) The waste will consist of heterogeneous debris to include glass, rags, paper,
cardboard, plastics, and rubber.

2.2 Radiological characteristics

2.2.1 Mixed waste type

[.] High-level

Contact-handled

Low-level

Remote-handled

2.2.2 Comments on tadiological characteristics (e.g., more specific content, treatment concerns caused by radiation, confidence level):

2.3 Matrix characteristics (physical content)

2.3.1 Matrix constituent table (comprising at least $1 \%$ of the total volume or mass)

\begin{tabular}{|c|c|c|}
\hline \multicolumn{3}{|c|}{ 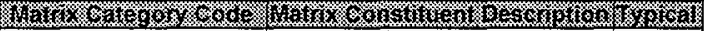 } \\
\hline$\$ 3190$ & Inorganic homogeneous solids & $1.1 \%$ \\
\hline$\$ 4200$ & Soil/debris & $7.0 \%$ \\
\hline$\$ 5190$ & Inorganic debris & $27.2 \%$ \\
\hline$\$ 5390$ & Organic debris & $56.5 \%$ \\
\hline 55490 & Heterogeneous debris & $1.1 \%$ \\
\hline J9999 & Unknown/other & $3.5 \%$ \\
\hline$\times 7290$ & Elemental lead & $3.3 \%$ \\
\hline
\end{tabular}

Overall matrix parameter category code: $\$ 5129$

Overall matrix description:

Inorganic non-metal debris 
2.3.2 Confidence level for matrix characteristics data in 2.3.1 $\square$ low $\square$ medium $\square$ high

2.3.3 Comments on matrix characteristics and/or handled TRU heterogeneous debris (TRUM-01C). Waste Specification Record confidence level:

The matrix constituent table is assumed to be the same as existing mixed contact-
Nandled TRU heterogeneous debris (TRUM-01 C). Waste Specification Record
Numbers do not apply for future waste.

2.4 Regulated contaminant characteristics

2.4.1 Wastewater/non-wastewater under RCRA
$\square$ wastewater
$\square$ non-wastewater
unknown

2.4.2 Regulated contaminant table including treatment requirements, and UHC's if applicable

\begin{tabular}{|c|c|c|c|c|}
\hline 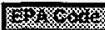 & 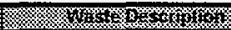 & Y Ins & sons & 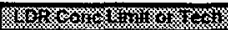 \\
\hline TBD & TBD & TBD & TBD TBD & TBD \\
\hline
\end{tabular}

UHC's are not applicable to the WIPP facility. (1) Treatment standards are based on the WIPP facility waste acceptance criteria.

2.4.2.1 List any waste codes from 2.4.2 for which the stream already meets established LDR treatment standard Waste is exempt from Federal LDR's when being disposed of at the WIPP facility.

2.4.3 Does this waste stream contain PCBs?
Yes
No
Unknown

2.4.3.1 is the waste stream subject to TSCA regulations for PCBs?

$\square$ Yes $\square$ No Unknown

2.4.3.2 Indicate the PCB concentration range
$\square<50 \mathrm{ppm}$
$>=50 \mathrm{ppm}$
Unknown

2.4.4 What is the confidence level for the regulated contaminant characteristic data?
D low
$\checkmark$ medium
high

2.4.5 Comments on regulated contaminant characteristics and/or confidence level:

Because the waste has yet to be generated, the regulated contaminant characteristics are unknown.

2.4.6 Will further characterization be performed $\square$ Yes $\square$ No $\quad \square$ Unknown

2.4.6.1 If yes, provide details and Additional characterization will be conducted as necessary to meet the schedule: waste acceptance criteria for WIPP. Characterization will include process knowledge, and sampling and analysis.

2.4.6.2 If yes, provide Tri-Party Agreement milestone number No milestones are applicable to characterization of TRU waste.

\subsection{WASTE STREAM STORAGE, INVENTORY, AND GENERATION}
3.1
Is the waste stream currently stored?
Yes
DNo

3.2 Current storage method

$\square$ Container (pad)
$\square$ Tank
$\square$ Other (explain)

Container (covered)

Waste pile

$$
\text { If no, skip to } 3.7
$$

Container (retrievably buried)

surface impoundment 
id: $\quad$ TRUM.04B

3.3 TSD unit name and building number and (if applicable/available) number of containers/tanks in each:

TSD Unit Name

Central Waste Complex
Number of containers

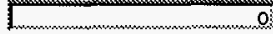

3.4 Is storage of waste in compliance with applicable State and Federal standards?

$\square$ Yes $\square$ No

If no, provide details:

3.5 Applicable Tri-Party Agreement milestones related to storage:
M-91-01

3.6

Current inventory for this stream

Total LDF volume \{cubic meters:

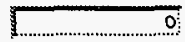

Date of inventory values:

$9 / 30 / 98:$

Comments on waste inventory:
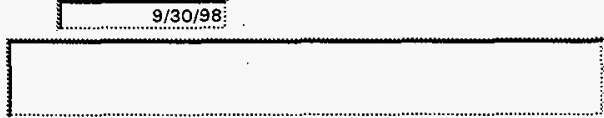

3.7 Is the waste stream currently generated; and if not, will this waste stream be generated in the next 5 years?

$$
\square \text { Yes } \square \text { No }
$$

3.8 The current or future generation of this waste is best described as:
$\square$ Routine
One-time or sporadic

3.9 Estimated generation projection by fiscal year

1999:

2000:

2001:

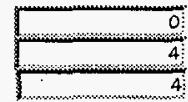

3.10

Last Year's fiscal year 1998 generation projection:

Actual quantity generated in 1998:

3.11 Has there ever been any unusual release of this stream to the environment?

$\square$ Yes $\square$ No

If yes, summarize releases and quantities:

\subsection{WASTE STREAM TREATMENT}

4.1 Is this stream currently being treated?
2002:

2003:

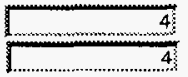

(Both in $\mathrm{m} 3$ unless otherwise noted) 
$\square$ Yes $\square$ No If yes, provide details:

4.2 Planned treatment: Check the appropriate box indicating future plans for treating this waste stream to meet applicable regulations, including LDR treatment standards:

$\square$ No treatment required (skip to 5.0 )

G Treating or plan to treat onsite

$\square$ Treating or plan to treat offsite

V. Treatment options still being assessed

4.3 Planned treatment method, facility, and extent of treatment capacity available:

Drumed waste and boxed waste are planned to be treated at WRAP and the proposed M91 facility, respectively. Treatment will be performed as necessary to meet the applicable waste acceptance criteria at WIPP. The extent of treatment will vary, and may include decontamination, solidification, and Irepackaging, WRAP has a design capacity of 4725 drums (983 m3) per year on a single shift basis.

4.4 Treatment schedule information:

The programmatic treatment schedule for mixed and non-mixed TRU waste is from 1999 to 2032 . The schedule is subject to change as it depends on the ability of DOE to accept mixed TRU waste at WIPP and available funding for treatment.

4.5 Applicable treatment Tri-Party Agreoment milestone numbers (including permitting):

M-91-01

4.6

If treating or planning to treat on site, will waste minimization be addressed in developing and/or selecting the treatment method?

$\square$ Yes $\square$ No Unknown

If yes, please describe

4.7 Treatability equivalency petitions, rulemaking petitions, and case-by-case exemptions needed for treatment

None are planned.

5.0 WASTE STREAM DISPOSAL

5.1 After treatment, how will the waste stream be disposed of (include description, locations, variances required, etc., as applicable):

Mixed TRU waste is planned to be disposed of at the Waste Isolation Pilot Plant (WIPP), a deep geological repository.

5.2 Applicable TPA milestone number(s) for disposal:

No TPA milestones exist for the disposal of TRU waste. The disposal of TRU waste at WIPP is a national DOE policy, and is required by DOE Order $5820.2 \mathrm{~A}$ (to be replaced by 435.1 ).

5.3 Planned disposal dates/ time-frame:

The planned time frame of mixed and non-mixed TRU waste shipments to WIPP
is from 2000 to 2032 . The time frame is subject to change depending on
DOE's ability to accept waste at WIPP and adequate funding of TRU waste
program activities.

\subsection{WASTE MINIMIZATION}

6.7 Has a waste minimization assessment been completed for this stream?
$\square$ Yes
No
Not applicable

6.2 Explain any waste minimization activities (e.g., process changes) under way or to be implemented for generation of this stream: 
The waste is typically residues from gloveboxes as part of deactivation operations. Because the activity involves a relocation of waste material to a safer location and in a safer configuration, waste minimization opportunities that can be identified are usually best management practices. Therefore, best management practices will be applied in the deactivation operations.

6.3

Schedule for implementing waste minimization methods:

Because only best management practices have been identified, which are ongoing, a specific schedule is not applicable.

6.4 Waste reduction achieved during the calendar year and projected future reductions (volume or mass):

Calendar year:

Projected:

0 m3

6.4.1 Assumptions used in above estimate:

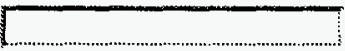




\title{
WASTE STREAM PROFILE SHEET
}

\subsection{WASTE STREAM IDENTIFICATION AND SOURCE}

1.1 Waste Stream iD:

$$
\text { TRUM-04C }
$$

1.2 Waste stream name 300 Area Revitalization contact-handled mixed TRU metal debris

1.3.1 Stream source and history description:

This waste is from 300 Area facilities in which the process missions have terminated. The waste has yet to be generated.

\subsubsection{Source category(s)}

$\square$ Pollution control or waste treatment process

Material production/recovery effluents

Facility or equipment operation and maintenance waste

$\square$ Discarded excess or expired materials

$\square$ Analytical laboratory waste

RD/RD laboratory waste

$\square$ Remediation/DD waste

$\square$ Spill clean-ups or emergency response actions

$\square$ Source unknown

$\square$ Other (explain)

1.3.3 Additional notes:

\subsection{WASTE STREAM CHARACTERIZATION}

\subsection{Waste stream} description (content)

The waste will consist of unsecured equipment and other misc
pieces. The waste will be cut as necessary for packaging.
$\square$ High-level
$\square$ Contact-handled $\square$ Remote-handled

2.2 Radiological characteristics

2.2.1 Mixed waste type

$$
\square \text { Contact-handled }
$$

\author{
Remote-handled
}

2.2.2 Comments on radiological characteristics le.g., more specific content, treatment concerns caused by radiation, confidence level):

\subsection{Matrix characteristics (physical content)}

2.3.1 Matrix constituent table (comprising at least $1 \%$ of the total volume or mass)

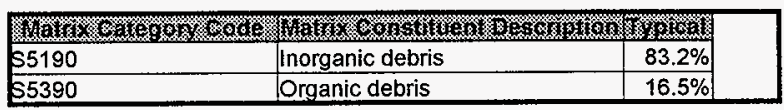

Overall matrix parameter category code: 55119 Overall matrix description:

2.3.2 Confidence level for matrix characteristics data in 2.3.1

$\square$ low $\square$ medium high 
2.3.3 Comments on matrix characteristics and/or confidence level:

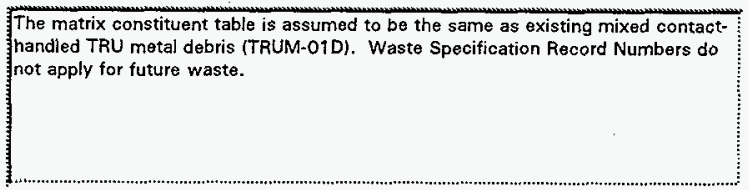

2.4 Regulated contaminant characteristics

2.4.1 Wastewater/non-wastewater under RCRA
$\square$ wastewater
$\checkmark$ non-wastewater
unknown

2.4.2 Regulated contaminant table including treatment requirements, and UHC's if applicable 174)

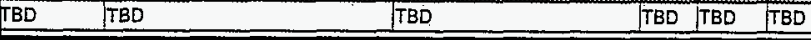

UHC's are not applicable to the WIPP facility. (1) Treatment standards are based on the WIPP facility waste acceptance criteria.

2.4.2.1 List any waste codes from 2.4.2 for which the stream al ready meets established LDR treatment standard Waste is exempt from Federal LDR's when being disposed of at the WIPP facility.

2.4.3 Does this waste stream contain PCBs?
Yes
No
Unknown

2.4.3.1 Is the waste stream subject to TSCA regulations for PCBs?
$\square$ Yes
$\square$ No
Unknown

2.4.3.2 Indicate the $\mathrm{PCB}$ concentration range
$<50 \mathrm{ppm}$
$\square>=50 \mathrm{ppm}$
Unknown

2.4.4 What is the confidence level for the regulated contaminant characteristic data?
$\square$ low
$\nabla$ medium
high

2.4.5 Comments on regulated contaminant characteristics and/or confidence level:

Because the waste has yet to be generated, the regulated contaminant characteristics are unknown.

2.4.6 Will further characterization be performed $\square$ Ves $\square$ No $\square$ Unknown

2.4.6.1 If yes, provide details and Additional characterization will be conducted as necessary to meot the schedule:

waste acceptance criteria for WIPP. Characterization will include process knowledge, and sampling and analysis.

2.4.6.2 If yes, provide Tri-Party Agreement milestone number No milestones are applicable to characterization of TRU waste.

\subsection{WASTE STREAM STORAGE, INVENTORY, AND GENERATION}

3.1 Is the waste stream currently stored? $\square$ Yes

3.2 Current storage method

$\square$ Container (pad)

$[$ Tank

Container (covered)

If no, skip to 3.7

Other (explain)

Surface impoundment 
3.3 TSD unit name and building number and (if applicable/available) number of containers/tanks in each: TSD Unit Name Number of containers Central Waste Complex

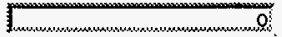

3.4 Is storage of waste in compliance with applicable State and Federal standards?

Yes No

If no, provide details:

M-91-01

3.6 Current inventory for this stream

Total LDR volume (cubic meters):

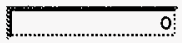

Date of Inventory values:

Comments on waste inventory:

3.7

Is the waste stream currently generated; and if not, will this waste stream be generated in the next 5 years?

$$
\text { Ves } \square \text { No }
$$

The current or future generation of this waste is best described as:

$$
\text { (V) Routine }
$$

One-time or sporadic

3.9

Estimated generation projection by fiscal year

$\begin{array}{ll}\text { 1999: } & \text { - } \\ \text { 2000: } & 42 \\ \text { 2001: } & 42\end{array}$

Last Year's fiscal year 1998 generation projection:

Actual quantity generated in 1998:
2002:

2003:

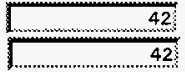

(Both in $\mathrm{m} 3$ unless otherwise noted)

Has there ever been any unusual release of this stream to the environment?

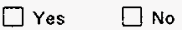

If yes, summarize releases and quantities:

\subsection{WASTE STREAM TREATMENT}

4.1 Is this stream currently being treated? 
$\square$ Yes $\square$ No If yes, provide details:

4.2 Planned treatment: Check the appropriate box indicating future plans for treating this waste stream to meet applicable regulations, including LDR treatment standards:

$\square$ No treatment required (skip to 5.0 )

$\square$ Treating or plan to treat onsite

$\square$ Treating or plan to treat offsite

Treatment options still being assessed

4.3 Planned treatment method, facility, and extent of treatment capacity available:

Drumed waste and boxed waste are planned to be treated at WRAP and the proposed M9 1 facility, respectively. Treatment will be performed as necessary to meet the applicable waste acceptance criteria at WIPP. The extent of treatment will vary, and may include decontamination, solidification, and (repackaging . WRAP has a design capacity of 4725 drums 1983 m3) per year on a single shift basjs.

4.4 Treatment schedule information::

The programmatic treatment schedule for mixed and non-mixed TRU
waste is from 1999 to 2032 . The schedule is subject to change as it
depends on the ability of DOE to accept mixed TRU waste at WIPP and
available funding for treatment.

4.5 Applicable treatment Tri-Party Agreement milestone numbers (including permitting):

I........... If treating or planning to treat on site, will waste minimization be addressed in developing and/or selecting the treatment method?

If yes, please describe

4.7 Treatability equivalency petitions, rulemaking petitions, and case-by-case exemptions needed for treatment None are planned.

\subsection{WASTE STREAM DISPOSAL}

5.1 After treatment, how will the waste stream be disposed of (include description, locations, variances required, etc., as applicable): .

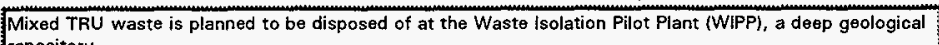
repository.

5.2 Applicable TPA milestone number(s) for disposal:

No TPA milestones exist for the disposal of TRU waste. The disposal of TRU waste at WIPP is a national DOE policy, and is required by DOE Order $5820.2 \mathrm{~A}$ (to be replaced by 435.1 ).

\subsection{WASTE MINIMIZATION}

6.1 Has a waste minimization assessment been completed for this stream?
E Yes
No
Not applicable

6.2 Explain any waste minimization activities (e.g., process changes) under way or to be implemented for generation of this stream: 
The waste is typically excess equipment and piping that is loose (not fixed in-place). Size reduction by cutting large or long pieces will be applied to fit into a container acceptable by Hanford's solid waste acceptance criteria. Because deactivation involves relocating existing excess material to a safe location and in a safer configuration, waste minimization, other than best management practices, are not applicable.

6.3 Schedule for implementing waste minimization methods:

Because only best management practices have been identified, which are ongoing, a specific schedule is not applicable.

6.4 Waste reduction achieved during the calendar year and projected future reductions (volume or mass):

Calendar year:

Projected:

$0 \sqrt{\mathrm{m} 3}$

6.4.I Assumptions used in above estimate:

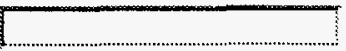


DOE/RL-99-01

This page intentionally left blank. 


\section{WASTE STREAM PROFILE SHEET}

\subsection{WASTE STREAM IDENTIFICATION AND SOURCE}

1.1 Waste Stream ID:

\section{TRUM-05A} Contact-handled TRU single-shell tank long-length equipment

1.2 Waste stream name

1.3.1 Stream source and

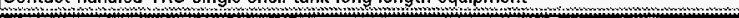
This waste is from future maintenance of underground waste storage tanks at history description: Hanford. The waste has yet to be generated.

1.3.2 Source category(s)
$[$ Pollution control or waste treatment process
Material production/recovery effluents
$\square$ Facility or equipment operation and maintenance waste
Discarded excess or expired materials
$\square$ Analytical laboratory waste
[ RD/RD laboratory waste
$\square$ Remediation/DD waste
Spill clean-ups or emergency response actions
$\square$ other (explain)
$\square$ Source unknown

\subsubsection{Additional notes:}

\subsection{WASTE STREAM CHARACTERIZATION}

2.1 Waste stream description (content)

The waste will consists of pumps, thermocouples, and other excess equipment that is removed from single-shell tanks.

2.2 Radiological characteristics

2.2.1 Mixed waste type

$\Xi$ High-leve

\section{(V) Contact-handled}

2.2.2 Comments on radiological characteristics (e.g., more specific content, treatment concerns caused by radiation, confidence (evel):

2.3 Matrix characteristics (physical content)

2.3.1 Matrix constituent table (comprising at least $1 \%$ of the total volume or mass)

\begin{tabular}{|c|c|c|}
\hline 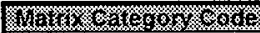 & 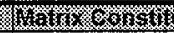 & S \\
\hline 55390 & Organic debris & $61.0 \%$ \\
\hline$\$ 5119$ & Metal debris & $37.0 \%$ \\
\hline$\times 7290$ & Elemental lead & $2.0 \%$ \\
\hline
\end{tabular}

Overall matrix parameter category code: $\$ 5490$

Overall matrix description:

Heterogeneous debris

2.3.2 Confidence level for matrix characteristics data in 2.3.1

$\square$ low $\nabla$ medium

high 
2.3.3 Comments on matrix characteristios and/or confidence level:

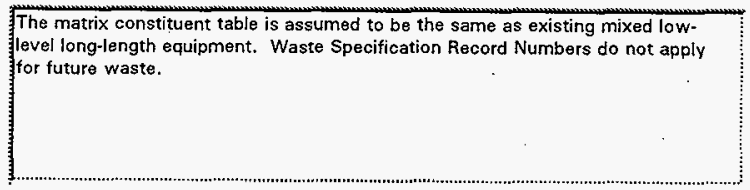

2.4 Regulated contaminant characteristics

2.4.7 Wastewater/non-wastewater under RCRA

$\square$ wastewater $\square$ non-wastewater $\square$ unknown

2.4.2 Regulated contaminant table including treatment requirements, and UHC's if applicable

\begin{tabular}{|c|c|c|c|c|c|}
\hline 258 & Sho & $4 \mathrm{x}$ & (n) & 40 & $x$ \\
\hline 5001 & Spent Solvent & 1,1,1-TRICHLOROETYHANE & that & $n * *$ & Exempt (61 FR 60704) \\
\hline F002 & Spent Solvent & Methylene Chloride & $* * *$ & $x * *$ & Exempt (61 FR 60704) \\
\hline 0003 & Spent Solvent & ACETONE & $=n$ & $\Leftrightarrow *$ & Exempt (61 FR 60704) \\
\hline F004 & Spent Solvent & CRESOL & $+* *$ & $+a *$ & Exempt (61 FR 60704) \\
\hline 5005 & Spent Solvent & METHYL ETHYL KETONE & $\pi \star \star$ & $* *$ & Exempt (61 FR 60704) \\
\hline 5007 & CHROMIUM & INA & 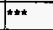 & $* *$ & Exempt (61 FR 60704) \\
\hline 6009 & MERCURY & NA & 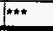 & $x * *$ & Exempt (61 FR 60704) \\
\hline
\end{tabular}

UHC's are not applicable to the WIPP facility. (1) Treatment standards are based on the WIPP facility waste acceptance criteria.

2.4.2.1 List any waste codes from 2.4 .2 for which the stream already meets established LDR treatment standard Waste is exempt from Federal LDR's when being disposed of at the WIPP facility.

2.4.3 Does this waste stream contain PCBs?

$\square$ Yes $\square$ No $\square$ Unknown

2.4.3.1 is the waște stream subject to TSCA regulations for PCBs?
$\square$ Yes
[ No
Unknown

2.4.3.2 Indicate the $\mathrm{PCB}$ concentration range
$\square<50 \mathrm{ppm}$
D $>=50 \mathrm{ppm}$
Unknown

2.4.4 What is the confidence level for the regulated contaminant characteristic data?
$\square$ low
0 medium
high

2.4.5 Comments on regulated contaminant characteristics and/or confidence level:

The contaminants are based on existing mixed low-leve long-length equipment, which is presently stored in Trench 34 of 21 8-W-5.

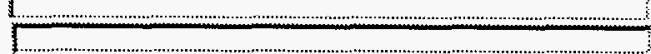

2.4.6 Will further characterization be performed $\square$ Yes $\square$ No $\square$ Unknown

2.4.6.1 If yes, provide details and Additional characterization will be conducted as necessary to meet the schedule: waste acceptance eriteria for WIPP. Characterization will include process knowledge and sampling and analysis.

2.4.6.2 If yes, provide Tri-Party Agreement milestone number No milestones are applicable to characterization fof TRU waste.

\subsection{WASTE STREAM STORAGE, INVENTORY, AND GENERATION}

3.1

Is the waste stream currently stored?

3.2

Current storage method

$\square$ Container (pad)
$\square$ Tank
$\square$ Other (explain)
Yes

0 No

If no, skip to 3.7

Container (covered)

Waste pile
Container (retrievably buried)

Surface impoundment 
3.3 TSD unit name and building number and (if applicable/available) number of containers/tanks in each:

TSD Unit Name Number of containers Central Waste Complex

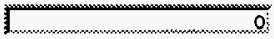

3.4 Is storage of waste in compliance with applicable State and Federal standards?

Yes

No

If no, provide details:

3.5 Applicable Tri-Party Agreement milestones related to storage:

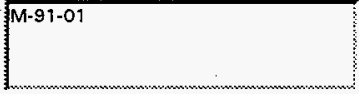

3.6

Current inventory for this stream

Total LDR volume (cubic meters):

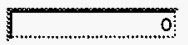

Date of Inventory values:

$9 / 30 / 98$

Comments on waste inventory:

3.7 Is the waste stream currently generated; and if not, will this waste stream be generated in the next 5 years?

$$
\square \text { Yes } \square \text { No }
$$

3.8 The current or future generation of this waste is best described as:

$\square$ Routine $\square$ One-time or sporadic

3.9 Estimated generation projection by fiscal year

$\begin{array}{lll}\text { 1999: } & \text { 2002: } \\ \text { 2000: } & \text { 2003: } \\ \text { 2001: } & \end{array}$

3.10 Last Year's fiscal year 1998 generation projection:

Actual quantity generated in 1998:

|0:

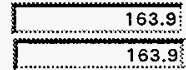

(Both in $\mathrm{m} 3$ unless otherwise noted)

3.11 Has there ever been any unusual release of this stream to the environment?

$\square$ Yes

$\square$ No

If yes, summarize releases and quantities:

\subsection{WASTE STREAM TREATMENT}

4.1. Is this stream currently being treated? 
$\square$ Yes [No If yes, provide details:

4.2 Planned treatment: Check the appropriate box indicating future plans for treating this waste stream to meet applicable regulations, including LDR treatment standards:

$\square$ No treatment required (skip to 5.0 )

$\square$ Treating or plan to treat onsite

$\square$ Treating or plan to treat offsite

$\checkmark$ Treatment options still being assessed

4.3 Planned treatment method, facility, and extent of treatment capacity available:

Waste are planned to be treated at the proposed M91 facility as needed to meet the applicable waste acceptance criteria at WIPP. The extent of treatment will vary, and may include decontamination, solidification, and repackaging. The treatment capacity of the M91 facility has yet to be determined.

Treatment schedule

The programmatic treatment schedule for mixed and non-mixed TRU

information:: waste is from 1999 to 2032 . The schedule is subject to change as it depends on the ability of DOE to accept mixed TRU waste at WIPP and available funding for treatment.

4.5 Applicable treatment Tri-Party Agreement milestone numbers (including permitting):

$M-91-01$

4.6

If treating or planning to treat on site, will waste minimization be addressed in developing and/or selecting the treatment method?

$\square$ Yes $\square$ No $\square$ Unknown

If yes, please describe

4.7 Treatability equivalency petitions, rulemaking petitions, and case-by-case exemptions needed for treatment None are planned.

\subsection{WASTE STREAM DISPOSAL}

5.1 After treatment, how will the waste stream be disposed of (include description, locations, variances required, etc., as applicable):

Mixed TRU waste is planned to be disposed of at the Waste Isolation Pilot Plant (WIPP), a deep geological repository.

5.2 Applicable TPA milestone number(s) for disposal:

No TPA milestones exist for the disposal of TRU waste. The disposal of TRU waste at WIPP is a national DOE policy, and is required by DOE Order $5820.2 \mathrm{~A}$ (to be replaced by 435.1 ).

5.3 Planned disposal

dates/time-frame:

The planned time frame of mixed and non-mixed TRU waste shipments to WIPP is from 2000 to 2032 . The time frame is subject to change depending on DOE's ability to accept waste at WIPP and adequate funding of TRU waste program activities.

\subsection{WASTE MINIMIZATION}

6.1 Has a waste minimization assessment been completed for this stream?
$\square$ Yes
No
Not applicable

6.2 Explain any waste minimization activities (e.g., process changes) under way or to be implemented for generation of this stream: 
The waste is long-length operational equipment removed from the 102-SY tank as part of the TWRS

upgrade project (Project No. 211). Waste minimization is not directly applicable because the project is not

tied to the production of new tank waste. However, best management practices will be applied

throughout the project duration to minimize waste to the extent possible.

6.3 Schedule for implementing waste minimization methods:

Because only best management practices have been identified, which are ongoing, a specific schedule is not applicable.

1 .

6.4 Waste reduction achieved during the calendar year and projected future reductions (volume or mass):
Calendar year:
of $m 3$
Projected:
$0 \longdiv { m 3 }$

6.4.1 Assumptions ised in above estimate: 


\section{WASTE STREAM PROFILE SHEET}

\subsection{WASTE STREAM IDENTIFICATION AND SOURCE}

1.1 Waste Stream ID: TRUM-05B

1.2 Waste stream name

1.3.1 Stream source and history description:

Remote-handled TRU single-shell tank long-Jength equipment

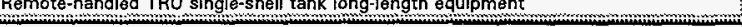

This waste is from future maintenance of underground waste storage tanks at

Hanford. The waste has yet to be generated.

1.3.2 Source category(s)
[ Pollution control or waste treatment process
Material production/recovery effluents
$\square$ Facility or equjpment operation and maintenance waste
$\square$ Discarded excess or expired materials
$\square$ Analytical laboratory waste
$\square$ RD/RD laboratory waste
$\square$ Remediation/DD waste
$\square$ spill clean-ups or emergency response actions
[] Source unknown
$\square$ Other (explain)

1.3.3 Additional notes:

\subsection{WASTE STREAM CHARACTERIZATION}

2.1 Waste stream description (content)

The waste will consist of pumps, thermocouples, and other exces
that is removed from single-shell tanks.
$\square$ High-level
$\square$ Contact-handled $\square$ Remote-handled

2.2 Radiological characteristics

2.2.1 Mixed waste type Contact-handled

\section{$\square$ Remote-handled}

2.2.2 Comments on radiological characteristics (e.g., more specific content, treatment concerns caused by radiation, confidence level):

2.3 Matrix characteristics (physical content)

2.3.1 Matrix constituent table (comprising at least $1 \%$ of the total volume or mass)

\begin{tabular}{|c|c|c|}
\hline $3 \mathrm{X}$ & 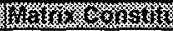 & 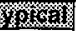 \\
\hline$\$ 5390$ & Organic debris & $61.0 \%$ \\
\hline$\$ 5119$ & Metal debris & $37.0 \%$ \\
\hline$\times 7290$ & Elemental lead & $2.0 \%$ \\
\hline
\end{tabular}

Overall matrix parameter category code: 55490

Overall matrix description:

Heterogeneous debris

2.3.2 Confidence level for matrix characteristics data in 2.3.1 $\square$ low $\square$ medium $\square$ high 
2.3.3 Comments on matrix characteristics and/or confidence level:
The matrix constituent table is assumed to be the same as existing mixed lowlevel long-length equipment. Waste Specification Record Numbers do not apply for future waste.

2.4 Regulated contaminant characteristics

2.4.1 Wastewater/non-wastewater under RCRA
$\square$ wastewater
$\checkmark$ non-wastewater
unknown

2,4.2 Regulated contaminant table including treatment requirements, and UHC's if applicable

\begin{tabular}{|c|c|c|c|c|c|}
\hline 西 & 4 & 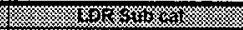 & \multicolumn{2}{|c|}{ 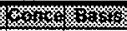 } & 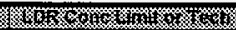 \\
\hline$=001$ & Spent Solvent & 1,1,1-TRICHLOROETHANE & r*m & $=n$ & Exempt (61 FR 60704) \\
\hline 8002 & Spent Solvent & Methylene Chloride & $* * *$ & *** & Exempt (61 FR 60704) \\
\hline 003 & Spent Solvent & ACETONE & $\approx *$ & $+\infty$ & Exempt (61 FR 60704) \\
\hline$F 004$ & Spent Solvent & ICRESOL & $2 * \star \star$ & 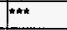 & Exempt (61 FR 60704) \\
\hline 5005 & Spent Solvent & METHYL ETHYL KETONE & $+\cdots$ & $\approx$ & Exempt (61 FR 60704) \\
\hline 0007 & CHROMIUM & NA & ant & $\pi \star \star$ & Exempt (61 FR 60704) \\
\hline 009 & MERCURY & NA & $n * \pi$ & $\star \star * *$ & Exempt (61 FR 60704) \\
\hline
\end{tabular}

UHC's are not applicable to the WIPP facility. (1) Treatment standards are based on the WIPP facility waste acceptance criteria.

2.4.2.1 List any waste codes from 2.4.2 for which the stream already meets established LDR treatment standard Waste is exempt from Federal LDR's when being disposed of at the WIPP facility.

2.4.3 Does this waste stream contain PCBs?
$\square$ Yes
D. No
Unknown

2.4.3.1 Is the waste stream subject to TSCA regulations for PCBs?
$[$ Yes
$\square$ No
Unknown

2.4.3.2 Indicate the PCB concentration range
$\square<50 \mathrm{ppm}$
$\square>=50 \mathrm{ppm}$
Unknown

2.4.4 What is the confidence level for the regulated contaminant characteristic data?
$\square$ low
$\square$ medium
high

2.4.5 Comments on regulated contaminant characteristics and/or confidence level:

The contaminants are based on existing mixed low-leve long-length equipment, which is presently stored in Trench 34 of $218-W-5$.

2.4.6 Will further characterization be performed $\square$ Yes $\square$ No

2.4.6.1 If yes, provide details and schedule:

Additional characterization will be conducted as necessary to meet the waste acceptance criteria for WIPP. Characterization will include process knowledge, and sampling and analysis.

2.4.6.2 If yes, provide Tri-Party Agreement milestone number No milestones are applicable to characterization of TRU waste.

\subsection{WASTE STREAM STORAGE, INVENTORY, AND GENERATION}

3.1

3.2 is the waste stream currently stored?

Current storage method

Contairier (pad)
Tank
Other (explain)

\section{No}

If no, skip to 3.7

Container (covered)

Waste pile
Container (retrievably buried)

Surface impoundment 
id: TRUM-05B

3.3 TSD unit name and building number and (if applicable/available) number of containers/tanks in each:

TSD Unit Name

Central Waste Complex
Number of containers

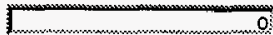

3.4 Is storage of waste in compliance with applicable State and Federal standards?
Yes
No

If no, provide details:

3.6 Current inventory for this stream

Total LDR volume (cubic meters):

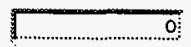

Date of Inventory values:

$9 / 30 / 98$

Comments on waste inventory:

is the waste stream currently generated; and if not, will this waste stream be generated in the next 5 years? $\checkmark$ Yes

$\square$ No

The current or future generation of this waste is best described as:

$\checkmark$ Routine

One-time or sporadic

3.9 Estimated generation projection by fiscal year

$\begin{array}{lll}\text { 1999: } & \text { 2002: } \\ \text { 2000: } & \text { 2003: } \\ \text { 2001: } & 163.9 & \end{array}$

Last Year's fiscal year 1998 generation projection:

Actual quantity generated in 1998:

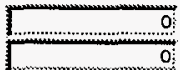

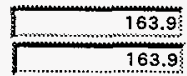

(Both in $\mathrm{m} 3$ unless otherwise noted)

3.11 Has there ever been any unusual release of this stream to the environment?

$\square$ Yes

$\square$ No

If yes, summarize releases and quantities:

\subsection{WASTE STREAM TREATMENT}

4.1

Is this stream currently being treated? 
Q Yes $\square$ No If yes, provide details:

4.2 Planned treatment: Check the appropriate box indicating future plans for treating this waste stream to meet applicable regulations, including LDR treatment standards:

$\square$ No treatment required (skip to 5.0 )

$\square$ Treating or plan to treat onsite

$\square$ Treating or plan to treat offsite

D Treatment options still being assessed

4.3 Planned treatment method, facility, and extent of treatment capacity available:

Waste are planned to be treated at the proposed M91 facility as needed to meet the applicable waste facceptance criteria at WIPP. The extent of treatment will vary, and may include decontamination, solidification, and repackaging. The treatment capacity of the M91 facility has yet to be determined.

4.4 Treatment schedule information:

The programmatic treatment schedule for mixed and non-mixed TRU waste is from 1999 to 2032 . The schedule is subject to change as it depends on the ability of DOE to accept mixed TRU waste at WIPP and available funding for treatment.

4.5 Applicable treatment Tri-Party Agreement milestone numbers (including permitting): M-91-01

4.6 If treating or planning to treat on site, will waste minimization be addressed in developing and/or selecting the treatment method?

If yes, please describe

4.7 Treatability equivalency petitions, rulemaking petitions, and case-by-case exemptions needed for treatment None are planned.

\subsection{WASTE STREAM DISPOSAL}

5.1 After treatment, how will the waste stream be disposed of (include description, locations, variances required, etc., as applicable):

Mixed TRU waste is planned to be disposed of at the Waste Isolation Pilot Plant (WIPP), a desp geological repository.

5.2 Applicable TPA milestone number(s) for disposal:

No TPA milestones exist for the disposal of TRU waste. The disposal of TRU waste at WIPP is a national DOE policy, and is required by DOE Order $5820.2 \mathrm{~A}$ (to be replaced by 435.1 ).

5.3 Planned disposal dates/ time-frame:

The planned time frame of mixed and non-mixed TRU waste shipments to WIPP is from 2000 to 2032 . The time frame is subject to change depending on DOE's ability to accept waste at WIPP and adequate funding of TRU waste program activities.

\subsection{WASTE MINIMIZATION}

6.1 Has a waste minimization assessment been completed for this stream?
L Ves
No
Not applicable

6.2 Explain any waste minimization activities (e.g., process changes) under way or to be implemented for generation of this stream: 
he waste is long-length operational equipment removed from the 102.SY tank as part of the TWRS
upgrade project (Project No. 2111. Shielding will increase the volume; however, this is necessary so that
the waste can be stored safely as contact-handled waste. Waste minimization is not directly applicable
because the project is not tied to the production of new tank waste. However, best management
practices will be applied throughout the project duration to minimize waste to the extent possible.

6.3 Schedule for implementing waste minimization methods:

Because only best management practices have been identified, which are ongoing, a specific schedule is not applicable.

6.4 Waste reduction achieved during the calendar year and projected future reductions (volume or mass):

Calendar year:

o $\mathrm{m}^{3}$

Projected:

o $m 3$

6.4.1 Assumptions used in above estimate:

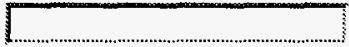




\section{WASTE STREAM PROFILE SHEET}

\subsection{WASTE STREAM IDENTIFICATION AND SOURCE}

1.1 Waste Stream ID:

1.2 Waste stream name

1.3.1 Stream source and history description:

TRUM-O6
Thiscellaneous remote-handled mixed TRU waste will be remote-handled TRU waste from national laboratories and
operations at T-Plant

1.3.2 Source category(s)

$\square$ Pollution control or waste treatment process

Material production/recovery effiuents

$\square$ Facility or equipment operation and maintenance waste

$\square$ Discarded excess or expired materials

$\checkmark$ Analytical laboratory waste

RD/RD laboratory waste

Remediation/DD waste

Spill clean-ups or emergency response actions

Source unknown

$\square$ Other (explain)

1.3.3 Additional notes:

\subsection{WASTE STREAM CHARACTERIZATION}

\subsection{Waste stream} description (content)

The waste will consist of laboratory waste from the Pacific Northwest National
Laboratory, and solidified sludge and lead waste from T Plant

2.2 Radiological characteristics

2.2.1 Mixed waste type

High-level

2 Transuranic

Low-tevel

\section{$\square$ Contact-handled \\ (2) Remote-handled}

2.2.2 Comments on radiological characteristics (e.g., more specific content, treatment concerns caused by radiation, confidence level):

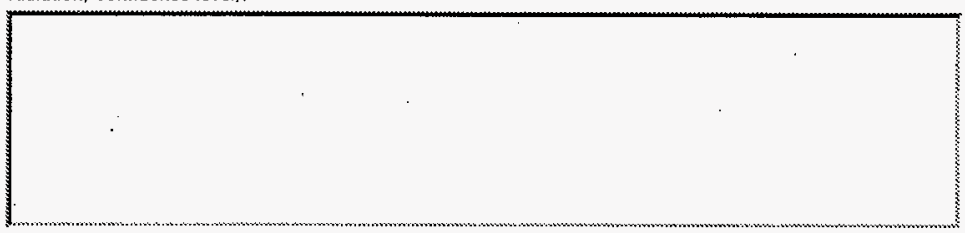

2.3 Matrix characteristics (physical content)

2.3.1 Matrix constituent table (comprising at least $1 \%$ of the total volume or mass)

\begin{tabular}{|c|c|c|}
\hline 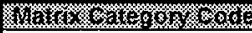 & 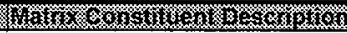 & 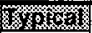 \\
\hline$\$ 5190$ & Inorganic debris & $11.7 \%$ \\
\hline$\$ 5390$ & Organic debris & $21.7 \%$ \\
\hline U9999 & Unknown/other & $59.2 \%$ \\
\hline
\end{tabular}

Overall matrix parameter category code: U9999

Overall matrix description:

$$
\text { Unknown or mixture of forms }
$$

2.3.2 Confidence level for matrix characteristics data in 2.3.1 $\square$ low $\nabla$ medium $\square$ high 
2.3.3 Comments on matrix characteristics and/or confidence level:
The matrix constituent table is assumed to be the same as existing mixed contacthandled other/unknown TRU waste (TRUM-01K). Waste Specification Record Numbers do not apply to future waste.

\subsection{Regulated contaminant characteristics}

2.4.1 Wastewater/non-wastewater under RCRA
$\square$ wastewater
$\square$ non-wastewater
unknown

2.4.2 Regulated contaminant table including treatment requirements, and UHC's if applicable

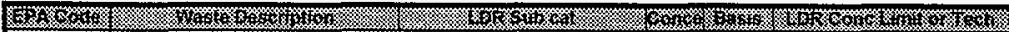

\begin{tabular}{|l|l|l|l|l|l|}
\hline TBD & TBD & TBD & TBD & TBD & TBD \\
\hline
\end{tabular}

UHC's are not applicable to the WIPP facility. (1) Treatment standards are based on the WIPP facility waste acceptance criteria.

2.4.2.1 List any waste codes from 2.4.2 for which the stream already meets established LDR treatment standard Waste is exempt from Federal LDR's when being disposed of at the WIPP facility.

2.4.3 Does this waste stream contain PCBs?
$\square$ Yes
1 No
Unknown

2.4.3.1 is the waste stream subject to TSCA regulations for PCBs?
$\square$ Yes
$\square$ No
Unknown

2.4.3.2 Indicate the $\mathrm{PCB}$ concentration range
$\square<50 \mathrm{ppm}$
$\square>=50 \mathrm{ppm}$
Unknown

2.4.4 What is the confidence level for the regulated contaminant characteristic data?
low
$\nabla$ medium
high

2.4.5 Comments on regulated contaminant characteristics and/or confidence level:

Because the waste has yet to be generated, the regulated contaminant characteristics are unknown.

2.4.6 Will further chatacterization be performed $\square$ Yes $\square$ No

2.4.6.1 If yes, provide details and Additional characterization will be conducted as necessary to meet the schedule: waste acceptance criteria for WIPP. Characterization will include process knowledge, and sampling and analysis.

2.4.6.2 If yes, provide Tri-Party Agreement milestone number No milestones are applicable to characterization of TRU waste.

\subsection{WASTE STREAM STORAGE, INVENTORY, AND GENERATION}
3.1
Is the waste stream currently stored?
$\square$ Yes
No

3.2
$\square$ Container (pad)
$\square$ Tank
Other (explain)

Current storage method

Container (covered)

Waste pile

If no, skip to 3.7

$\square$ Container (retrievably buried) Surface impoundment 
id:

TRUM-06

3.3 TSD unit name and building number and (if applicable/available) number of containers/tanks in each:

TSD Unit Name

Central Waste Complex
Number of containers

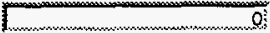

3.4 Is storage of waste in compliance with applicable State and Federal standards?

$\square$ Yes $\square$ No

If no, provide details:

3.5 Applicable Tri-Party Agreement milestones related to storage:

M-91-01

3.6 Current inventory for this stream

Total LDR volume (cubic meters):

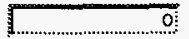

Date of Inventory values:

$9 / 30 / 98$

Comments on waste inventory:

3.7

Is the waste stream currently generated; and if not, will this waste stream be generated in the next 5 years? Yes No

3.8 The current or future generation of this waste is best described as:
$\checkmark$ Routine
One-time or sporadic

3.9

Estimated generation projection by fiscal year

\begin{tabular}{l|l} 
1999: & 2002: \\
2000: & 1.4 \\
2001: & 1.4 \\
$1.2003:$ &
\end{tabular}

2001:

Last Year's fiscal year 1998 generation projection:

Actual quantity generated in 1998:

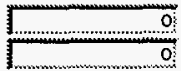

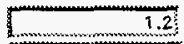

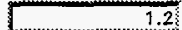

(Both in $\mathrm{m} 3$ unless otherwise noted)

3.11 Has there ever been any unusual release of this stream to the environment?
$\square$ Yes
$\square$ No

If yes, summarize releases and quantities:

\subsection{WASTE STREAM TREATMENT}

4.1 is this stream currently being treated? 
$\square$ Yes $\square$ No if yes, provide details:

4.2 Planned treatment: Check the appropriate box indicating future plans for treating this waste stream to meet applicable regulations, including LDR treatment standards:

[ No treatment required (skip to 5.0 )

[ Treating or plan to treat onsite

L] Treating or plan to treat offsite

Treatment options still being assessed

4.3 Planned treatment method, facility, and extent of treatment capacity available:

Waste are planned to be treated at the proposed M91 facility as needed to meet the applicable waste acceptance criteria at WIPP. The extent of treatment will vary, and may include decontamination, solidification, and repackaging. The treatment capacity of the M91 facility has yet to be determined.

4.4 Treatment schedule

The programmatic treatment schedule for mixed and non-mixed TRU

information:: waste is from 1999 to 2032 . The schedule is subject to change as it depends on the ability of DOE to accept mixed TRU waste at WIPP and available funding for treatment.

4.5 Applicable treatment Tri-Party Agreement milestone numbers (including permitting): M-91-01

4.6 If treating or planning to treat on site, will waste minimization be addressed in developing and/or selecting the treatment method?

If yes, please describe

4.7 Treatability equivalency petitions, rulemaking petitions, and case-by-case exemptions needed for treatment

None are planned.

\subsection{WASTE STREAM DISPOSAL}

5.1 After treatment, how will the waste stream be disposed of include description, locations, variances required, etc., as applicable):

Mixed TRU waste is planned to be disposed of at the Waste Isolation Pilot Plant (WIPP), a deep geological repository.

5.2 Applicable TPA milestone number(s) for disposal:

No TPA milestones exist for the disposal of TRU waste. The disposal of TRU waste at WIPP is a national DOE policy, and is required by DOE Order $5820.2 \mathrm{~A}$ (to be replaced by 435.1 ).

$5.3 \quad$ Planned disposal dates/ time-frame:

The planned time frame of mixed and non-mixed TRU waste shipments to WIPP is from 2000 to 2032 . The time frame is subject to change depending on DOE's ability to accept waste at WIPP and adequate funding of TRU waste program activities.

\subsection{WASTE MINIMIZATION}

6.1 Has a waste minimization assessment been completed for this stream?
Yes
No
$\square$ Not applicable

6.2 Explain any waste minimization activities (e.g., process changes) under way or to be implemented for generation of this stream: 
This waste consists of small quantities of R\&D waste from the Pacific Northwest National Laboratory and solidification operations at T Plant. On account of the small quantities, waste minimization is not applicable to R\&D operations other than application of best management practices. Solidification operations at $T$ Plant typically involve treating existing waste in a manner for safe storage. As such, waste minimization, other than best management practices, are not applicable.

6.3 Schedule for implementing waste minimization methods:

Because only best management practices have been identified, which are ongoing, a specific schedule is not applicable.

6.4 Waste reduction achieved during the calendar year and projected future reductions (volume or mass): Calendar year: o 3 Projected: O) 3

6.4.1 Assumptions used in above estimate:

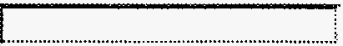


DOE/RL-99-01

This page intentionally left blank. 


\section{WASTE STREAM PROFILE SHEET}

\subsection{WASTE STREAM IDENTIFICATION AND SOURCE}

\subsection{Waste stream ID: DST-1}

\subsection{Waste stream name: Double Shell Tanks}

\subsection{Waste stream source information}

1.3.1 Stream source and history description. Include how the waste was managed prior to storage, timeframe when waste was placed into storage. The DST system contains wastes from current operations (cleanup waste) and past chemical separation processes (legacy waste). The major contributors to the waste stored in the DST system are PUREX, the Plutonium Finishing Plant, $B$ Plant and liquids from the SST system. Smaller amounts of other miscellaneous wastes such as laboratory wastes and wastes from the clean out of facilities in the $100,200,300$, and 400 areas are stored in DSTs. Waste streams are treated with sodium hydroxide and sodium nitrite to minimize tank corrosion and to address compatibility issues. Wastes have been stored in the DST system from 1970 to the present.

\subsubsection{Source category(s)}

$\square$ Pollution control or waste treatment process $\quad$ Materials production/recovery effluents

Facility or equipment operation and maintenance waste

Discarded excess or expired materials $\square$ Analytical laboratory waste

$\bigotimes$ R\&D/R\&D laboratory waste

Spill clean-ups or emergency response actions Other (explain):

1.3.3 Additional notes: None

\subsection{WASTE STREAM CHARACTERIZATION}

2.1 Waste stream description (content): Basic Aqueous Slurry with a layer of settled solids (sludge).

\subsection{Radiological characteristics}

\subsubsection{Mixed Waste type $\bigotimes$ High-level $\square$ Transuranic $\square$ Low-level}

\section{$\square$ Contact-handled $\bigotimes$ Remote-handled}

2.2.2 Comments on radiological characteristics (e.g., more specific content, treatment concerns caused by radiation, confidence level): DST system wastes contain the following major radionuclidies: $3 \mathrm{H}$, $14 \mathrm{C}, 60 \mathrm{Co}, 63 \mathrm{Ni}, 90 \mathrm{Sr}, 90 \mathrm{Y}, 93 \mathrm{Zr}, 93 \mathrm{mNb}, 99 \mathrm{Tc}, 106 \mathrm{Ru}, 113 \mathrm{mCd}, 125 \mathrm{Sb}, 126 \mathrm{Sn}, 129 \mathrm{I}, 134 \mathrm{Cs}, 137 \mathrm{Cs}$, $137 \mathrm{mBa}, 151 \mathrm{Sm}, 152 \mathrm{Eu}, 154 \mathrm{Eu}, 155 \mathrm{Eu}, 234 \mathrm{U}, 235 \mathrm{U}, 238 \mathrm{U}, 238 \mathrm{Pu}, 239 \mathrm{Pu}, 240 \mathrm{Pu}, 241 \mathrm{Am}$, and $241 \mathrm{Pu}$.

2.3 Matrix characteristics (physical content)

2.3.1 Matrix constituent table (comprising at least $1 \%$ of the total volume or mass)

\begin{tabular}{ccc}
$\begin{array}{c}\text { Matrix Parameter } \\
\text { Category Code }\end{array}$ & $\begin{array}{c}\text { Matrix Constituent } \\
\text { Description }\end{array}$ & $\begin{array}{c}\text { Typical } \\
\text { or Range (\%) }\end{array}$ \\
\hline L1220 & Basic Aqueous Slurry & $\mathbf{7 7 \%}$ \\
S9000 & Unknown/Other Solids & $\mathbf{2 3 \%}$
\end{tabular}


Overall matrix parameter category code: $\quad$ L1220 sludge)

Overall matrix description: Basic Aqueous Slurry (precipitated metal salts with layer of

\subsubsection{Confidence level for matrix characteristics data in 2.3.1}

\section{Low $\square$ Medium $\bigotimes$ High}

2.3.3 Comments on matrix characteristics and/or confidence level: The major constituents of DST system wastes are water and sodium salts of aluminate, nitrate, nitrite, phosphate, hydroxide, carbonate, and sulfate. Some calcium and potassium salts are also present. Complexed waste in the DSTs contain sodium salts of chelating agents ethylenediamine-tetraacetic acid and nhydroxyethylenediamine-tetraacetic acid. There may also be detectable concentrations of halogenated and nonhalogenated organic compounds and heavy metals such as lead, chromium and cadmium.

\subsection{Regulated contaminant characteristics}

\subsubsection{Wastewater/non-wastewater under RCRA}

$\square$ Wastewater $\bigotimes$ Non-wastewater $\square$ unknown

2.4.2 Regulated contaminant table including treatment requirements, and UHCs if applicable

\begin{tabular}{|c|c|c|c|c|c|}
\hline $\begin{array}{l}\text { EPA/ } \\
\text { State } \\
\text { Code }\end{array}$ & Waste Description & $\begin{array}{c}\text { LDR } \\
\text { Sub- } \\
\text { category } \\
\end{array}$ & $\begin{array}{l}\text { ncentration } \\
\text { Yypical or } \\
\text { Range) }\end{array}$ & Basis & $\begin{array}{c}\text { LDR } \\
\text { Concentration Limit or } \\
\text { Technology Code }\end{array}$ \\
\hline F001 & 1,1,1 Trichlorethane & Spent solvents & TBD & TBD & $6.0 \mathrm{mg} / \mathrm{kg}$ \\
\hline $\mathrm{F} 002$ & Methylene Chloride & Spent solvents & TBD & TBD & $30 \mathrm{mg} / \mathrm{kg}$ \\
\hline F003 & Acetone & Spent solvents & TBD & TBD & $160 \mathrm{mg} / \mathrm{kg}$ \\
\hline & Methyl Isobutyl Keto & one Spent solvents & TBD & TBD & $33 \mathrm{mg} / \mathrm{kg}$ \\
\hline F004 & Cresols & Spent solvents & TBD & TBD & $5.6 \mathrm{mg} / \mathrm{kg}$ \\
\hline D001 & Ignitability & $\begin{array}{l}\text { Low TOC ignitable } \\
\text { characteristic liquid }\end{array}$ & d NA & TBD & Deactivation (2) \\
\hline D002 & Corrosivity & Radioactive (1) & $>12.5$ & TBD & HLVIT \\
\hline D003 & Reactivity & Reactive Cyanides & TBD & TBD & $\begin{array}{l}\text { Meet Cyanide } \\
\text { conc. std. }\end{array}$ \\
\hline D004 & Arsenic & Radioactive (1) & TBD & TBD & HLVIT \\
\hline D005 & Barium & Radioactive (1) & TBD & TBD & HLVIT \\
\hline D006 & Cadmium & Radioactive (1) & TBD & TBD & HLVIT \\
\hline D007 & Chromium & Radioactive (1) & TBD & TBD & HLVIT \\
\hline D008 & Lead & Radioactive (1) & TBD & TBD & HLVIT \\
\hline D009 & Mercury & Radioactive (1) & TBD & TBD & HLVIT \\
\hline D010 & Selenium & Radioactive (1) & TBD & TBD & HLVIT \\
\hline D011 & Silver & Radioactive (1) & TBD & TBD & HLVIT \\
\hline D018 & Benzene & & TBD & TBD & $10 \mathrm{mg} / \mathrm{kg}(2)$ \\
\hline D019 & Carbon Tetrachlorid & & TBD & TBD & $6.0 \mathrm{mg} / \mathrm{kg}(2)$ \\
\hline D022 & Chloroform & & TBD & TBD & $6.0 \mathrm{mg} / \mathrm{kg}(2)$ \\
\hline D028 & 1,2-Dichloroethane & & TBD & TBD & $6.0 \mathrm{mg} / \mathrm{kg}(2)$ \\
\hline D029 & 1,1-Dichloroethylene & & TBD & TBD & $6.0 \mathrm{mg} / \mathrm{kg}(2)$ \\
\hline D030 & 2,4-Dinitrotoluene & & TBD & TBD & $140 \mathrm{mg} / \mathrm{kg}(2)$ \\
\hline D033 & Hexachlorobutadiene & & TBD & TBD & $5.6 \mathrm{mg} / \mathrm{kg}(2)$ \\
\hline D034 & Hexachloroethane & & TBD & TBD & $30 \mathrm{mg} / \mathrm{kg}(2)$ \\
\hline D035 & Methyl ethly ketone & & TBD & TBD & $36 \mathrm{mg} / \mathrm{kg}(2)$ \\
\hline
\end{tabular}


DOE/RL-99-01

\begin{tabular}{|c|c|c|c|c|}
\hline D036 & Nitrobenzene & TBD & TBD & 14 mg/kg (2) \\
\hline D038 & Pyridine & TBD & TBD & $16 \mathrm{mg} / \mathrm{kg}(2)$ \\
\hline D039 & Tetrachloroethylene & TBD & TBD & $6.0 \mathrm{mg} / \mathrm{kg}(2)$ \\
\hline D040 & Trichloroethylene & TBD & TBD & $6.0 \mathrm{mg} / \mathrm{kg}(2)$ \\
\hline D041 & $2,4,5$-Trichlorophenol & TBD & TBD & $7.4 \mathrm{mg} / \mathrm{kg}(2)$ \\
\hline D043 & Vinyl chloride & TBD & TBD & $6.0 \mathrm{mg} / \mathrm{kg}(2)$ \\
\hline WT01 & $\begin{array}{l}\text { Toxic Dangerous wastes } \\
\text { Extremely hazardous wastes }\end{array}$ & TBD & TBD & None (3) \\
\hline WT02 & $\begin{array}{l}\text { Toxic Dangerous wastes } \\
\text { Dangerous wastes }\end{array}$ & TBD & TBD & None \\
\hline WP01 & $\begin{array}{l}\text { Persistent Dangerous wastes } \\
\text { Extremely hazardous wastes }\end{array}$ & TBD & TBD & None (3) \\
\hline WP02 & Persistent Dangerous wastes & TBD & TBD & None \\
\hline
\end{tabular}

(1) Radioactive high-level wastes generated during the reprocessing of fuel rods.

(2) and meet 40CFR268.48

(3) Mixed extremely hazardous wastes may be land-disposed in Washington State in DOE facilities in accordance with Revised Code of Washington 70.105.050(2)

UHCs See comment below

* If waste isn't consistent in concentration, this may not apply. Describe in 2.4.5.

2.4.2.1 List any waste codes from 2.4.2 for which the stream already meets established LDR treatment standards: None

2.4.3 Does this waste stream contain PCBs?

$\bigotimes$ Yes $\square$ No $\square$ Unknown.

If no, skip to 2.4.4.

2.4.3.1 is waste stream subject to TSCA regulations for PCBs?

$\square$ Yes $\triangle$ No $\square$ Unknown

2.4.3.2 Indicate the PCB concentration range.

$\bigotimes<50 \mathrm{ppm} \square \geq 50 \mathrm{ppm} \square$ Unknown

2.4.4 What is the confidence level for the regulated contaminant characteristic data?

QLow $\square$ Medium $\square$ High

2.4.5 Comments on regulated contaminant characteristics and/or confidence level: The waste codes assigned to DST system waste are based on historical knowledge, and additional waste codes may be added or deleted based on the ongoing characterization program. Refer to Table 3-1 for the estimated mass of chemical components of DST and SST system waste.

Since 1995, LDR requirements have been documented on profile sheets for waste sent to the DST system. On September 25, 1995, waste acceptance criteria for waste entering the DST system specifically required the identification of UHCs. There is no documentation of LDR requirements for waste placed in the SST system and for waste sent to the DST system prior to 1995 . Due to the lack of documentation to the contrary, the position has been taken that waste in the DST system does not meet any of the UHC standards. However, a list is kept of the UHCs that have been documented since 1995. At this time, UHCs relevant to DOE activities at Hanford are considered reasonably expected to be present in the waste.

2.4.6 Will further characterization be performed? \Yes $\square$ No $\square$ Unknown 
Table 3-1. Estimated Mass of Nonradioactive Chemical Components of Single-Shell Tank and Double-Shell Tank Systems Waste.

\begin{tabular}{|c|c|c|c|c|c|c|c|}
\hline \multirow{2}{*}{$\begin{array}{c}\text { Chemical } \\
\text { (metric tons) }\end{array}$} & \multicolumn{4}{|c|}{ Single-shell tank system } & \multicolumn{3}{|c|}{ Double-shell tank system } \\
\hline & Sludge & Salt cake & $\begin{array}{l}\text { Interstitial } \\
\text { liquid }\end{array}$ & Total & Soluble & Insoluble & Total \\
\hline $\mathrm{Ag}^{+}$ & & & & & $3.28 \mathrm{E}-01$ & $1.38 \mathrm{E}+00$ & $1.70 \mathrm{E}+00$ \\
\hline $\mathrm{Al}(\mathrm{OH})_{4}{ }^{-}$ & $6.25 E+02$ & $1.25 \mathrm{E}+03$ & $4.57 \mathrm{E}+02$ & $2.33 \mathrm{E}+03$ & $5.09 \mathrm{E}+03$ & & $5.09 \ddot{\mathrm{E}}+03$ \\
\hline $\mathrm{Al}^{+3(1)}$ & $1.99 \mathrm{E}+03$ & & & $1.99 \overline{\mathrm{E}+03}$ & & $6.78 \overline{\mathrm{E}}+01$ & $6.78 \mathrm{E}+01$ \\
\hline $\mathrm{As}^{+2}$ & & & & & $7.70 \overline{\mathrm{E}-01}$ & $4.98 \mathrm{E}-01$ & $1.27 E+00$ \\
\hline $\mathrm{B}^{+5}$ & & & & & $5.19 \overline{\mathrm{E}-01}$ & $9.94 \mathrm{E} \cdot \overline{01}$ & $1.51 \mathrm{E}+00$ \\
\hline $\mathrm{Ba}^{+2}$ & & & & & $7.91 \mathrm{E}-01$ & $3.09 \mathrm{E}+00$ & $3.88 E+00$ \\
\hline $\mathrm{Be}^{-2}$ & & & & & 8.19 E-02 & $7.61 \mathrm{E}-03$ & $8.95 \mathrm{E}-02$ \\
\hline $\mathrm{Bi}^{+3}$ & $2.61 \mathrm{E}+02$ & & & $2.61 E \div 02$ & $2.26 \mathrm{E}+00$ & & $2.26 \mathrm{E}+00$ \\
\hline $\mathrm{Ca}^{+2}$ & $1.28 \mathrm{E}+02$ & & & $1 . \overline{28 \mathrm{E}+02}$ & $1.03 \mathrm{E}+01$ & $1.15 \overline{\mathrm{E}}+01$ & $2.18 \mathrm{E}+01$ \\
\hline $\mathrm{Cd}^{+2}$ & $3.84 E+00$ & & & $3.84 \mathrm{E}+00$ & $1.67 \mathrm{E}-01$ & $6.01 \mathrm{E}+00$ & $6.18 \mathrm{E}+00$ \\
\hline $\mathrm{Ce}^{+3}$ & $2.35 \mathrm{E}+02$ & & & $2.35 \mathrm{E}+02$ & $2.26 \mathrm{E}-02$ & $3.04 \mathrm{E}+00$ & $3.07 E+02$ \\
\hline $\mathrm{Cl}^{-}$ & $4.00 E+01$ & & & $4.00 \mathrm{E}+01$ & $2.73 \mathrm{E}+02$ & $1.49 E+00$ & $2.74 \mathrm{E}+02$ \\
\hline $\mathrm{CO}_{3}^{-2}$ & $1.15 \mathrm{E}+03$ & $4.13 \mathrm{E}+02$ & $3.96 \mathrm{E}+0 \mathrm{~J}$ & $1.61 \mathrm{E}+03$ & $1.92 \mathrm{E}+03$ & $5.83 \mathrm{E}+0 \mathrm{I}$ & $1.98 E+03$ \\
\hline $\mathrm{Cr}^{+5}$ & $8.63 \mathrm{E}+01$ & & & $8.63 \mathrm{E}+01$ & & $3.41 \mathrm{E}+01$ & $3.41 \mathrm{E}+01$ \\
\hline $\mathrm{CrO}_{4}^{-2}$ & & & $2.14 \bar{E}+01$ & $2.14 \mathrm{E}+01$ & $1.20 \mathrm{E}+02$ & & $1.20 \mathrm{E}+02$ \\
\hline $\mathrm{Cu}^{+2}$ & & & & & $1.77 \mathrm{E}-01$ & $7.46 \mathrm{E}-01$ & $9.23 \overrightarrow{\mathrm{E}}-01$ \\
\hline $\bar{F}$ & $8.00 \mathrm{E}+02$ & & $5.00 \mathrm{E}+01$ & $8.05 E \div 02$ & $3.52 \mathrm{E}+02$ & $1.91 \mathrm{E}+01$ & $3.71 \mathrm{E}+02$ \\
\hline $\mathrm{Fe}(\mathrm{CN})_{6}^{-4}$ & $3.22 \mathrm{E}+02$ & & & $3.22 \mathrm{E}+02$ & & & \\
\hline $\mathrm{Fe}^{+3}$ & $6 . \overline{27} \mathrm{E}+02$ & & & $6.27 E+02$ & $8.09 \overline{E+00}$ & $1.42 \mathrm{E}+02$ & $1.50 \mathrm{E}+02$ \\
\hline $\mathrm{Hg}^{+}$ & $9.00 \mathrm{E}-01$ & & & $9.00 \mathrm{E}-01$ & $5.84 \mathrm{E}-02$ & & $5.84 \mathrm{E}-02$ \\
\hline $\mathrm{K}^{*}$ & & & & & $5.46 \mathrm{E}+02$ & $2.02 \mathrm{E}+01$ & $5.66 \mathrm{E}+02$ \\
\hline $\mathrm{La}^{+}$ & & & & & $2.19 \mathrm{E}-0 \mathrm{I}$ & $2.10 \overrightarrow{\mathrm{E}}+0 \mathrm{l}$ & $2.12 \mathrm{E}+01$ \\
\hline $\mathrm{Li}^{+2}$ & & & & & $5.77 \mathrm{E}-03$ & $2 . 4 6 \longdiv { \mathrm { E } - 0 2 }$ & $3.04 \mathrm{E}-\overline{02}$ \\
\hline $\mathrm{Mg}^{+2}$ & & & & & $9.65 \mathrm{E}-0 \mathrm{I}$ & $1.10 \mathrm{E}+0 \mathrm{I}$ & $1.20 \mathrm{E}+0 \mathrm{I}$ \\
\hline $\mathrm{Mn}^{-4}$ & $1.20 \mathrm{E}+02$ & & & $1.20 \mathrm{E}+02$ & $7.69 E+00$ & $1.80 \mathrm{E}+01$ & $2.57 \mathrm{E}+01$ \\
\hline $\mathrm{Mo}^{+6}$ & & & & & $4.87 \mathrm{E}+00$ & $8.01 \mathrm{E}-01$ & $5.67 \mathrm{E}+00$ \\
\hline $\mathrm{Na}^{+}$ & $1.58 \mathrm{E}+04$ & $3.39 \mathrm{E}+04$ & $2.30 \mathrm{E} \div 03$ & $5.48 \mathrm{E}+04$ & $1.40 \mathrm{E}+04$ & $2.30 \mathrm{E}+02$ & $1.43 \mathrm{E}+04$ \\
\hline $\mathrm{Ni}^{+2}$ & $1.78 \mathrm{E}+02$ & & & $1.78 \overline{E+02}$ & $4.07 E+00$ & $6.57 \mathrm{E}+00$ & $1.06 \mathrm{E}+01$ \\
\hline $\mathrm{NO}_{2}^{-}$ & $2.00 \mathrm{E}+03$ & $1.53 \mathrm{E}+03$ & $1.27 \mathrm{E}+03$ & $4.80 \bar{E}+03$ & $4.80 \mathrm{E}+03$ & $8.42 \mathrm{E}+00$ & $4.81 E+03$ \\
\hline $\mathrm{NO}_{3}^{-}$ & $1.48 \mathrm{E}+04$ & $8.03 E+04$ & $1.71 \mathrm{E}+03$ & $9.68 \mathrm{E}+04$ & $1.03 \mathrm{E}+04$ & $3.91 \mathrm{E}+01$ & $1.03 E+04$ \\
\hline $\mathrm{OH}^{-}$ & $4.22 \mathrm{E}+03$ & $8.51 \mathrm{E}+02$ & $3.15 \mathrm{E}+02$ & $5.39 \mathrm{E}+03$ & $2.33 \mathrm{E}+03$ & $1.23 \mathrm{E}+02$ & $2 . \overline{45 \mathrm{E}+03}$ \\
\hline $\mathrm{Pb}^{+4}$ & & & & & $1.96 \mathrm{E}+00$ & $3.28 \mathrm{E}+00$ & $5.24 E+00$ \\
\hline $\mathrm{PO}_{4}{ }^{-5}$ & $3.89 \mathrm{E}+03$ & $6.4 \overline{\mathrm{E}}+02$ & $8.58 \mathrm{E}+01$ & $4.62 \mathrm{E}+03$ & $3.29 \mathrm{E}+02$ & $2.16 \mathrm{E}+01$ & $3.51 E+02$ \\
\hline $\mathrm{SiO}_{4}{ }^{-2}$ & $1.21 \mathrm{E}+03$ & & & $1.21 \mathrm{E}+03$ & $1.53 \mathrm{E}+01$ & $2.14 \mathrm{E}+02$ & $2.29 E+02$ \\
\hline $\mathrm{SO}_{4}^{-2}$ & $5.01 \mathrm{E}+02$ & $1.15 \mathrm{E}+03$ & & $1.65 \mathrm{E}+03$ & $3.86 \mathrm{E}+02$ & $6.68 \mathrm{E}+00$ & $3.93 \mathrm{E}+02$ \\
\hline $\mathrm{Sr}^{-2}$ & $3.60 \mathrm{E}+\overline{01}$ & & & $3.60 \overline{\mathrm{E}+01}$ & & & \\
\hline$T O C^{(2)}$ & & & $2.00 E+02$ & $2.00 \mathrm{E}+02$ & $1.26 \mathrm{E}+03$ & $6.84 \mathrm{E}+01$ & $1.33 E+03$ \\
\hline $\mathrm{UO}_{2}{ }^{+2}$ & & & & & $3.54 \mathrm{E}+00$ & $2.68 \mathrm{E}+01$ & $3.03 \mathrm{E}+01$ \\
\hline $\mathrm{V}^{73}$ & & & & & $6.20 \mathrm{E}-02$ & $1.88 \mathrm{E}-01$ & $2.50 \mathrm{E}-0 \mathrm{l}$ \\
\hline$W^{74}$ & $1.44 \bar{E} \div 01$ & & & $1.44 \mathrm{E}+01$ & $7.47 \mathrm{E}-01$ & & $7.47 \mathrm{E}-01$ \\
\hline $\mathrm{Zn}^{72}$ & & & & & $3.59 E+00$ & $9.45 \mathrm{E}-01$ & $4.54 \mathrm{E}+00$ \\
\hline $\mathrm{Zr}^{+4}$ & $2.46 E+02$ & & & $2.46 \bar{E}+02$ & $4.48 \mathrm{E}-01$ & $2.77 \mathrm{E}+02$ & $2 . \overline{77} \mathrm{E}+02$ \\
\hline Total w/o $\mathrm{H}_{2} \mathrm{O}$ & $4.93 \mathrm{E}+04$ & $1.23 \mathrm{E}+05$ & $6.40 \mathrm{E}+04$ & $1.79 \overline{\mathrm{E} \div 05}$ & $4.18 E \div 04$ & $1.45 \mathrm{E}+03$ & $4 . \overline{32} \mathrm{E}+04$ \\
\hline $\mathrm{H}_{2} \mathrm{O}$ & $2.62 E+04$ & $1.40 \mathrm{E}+04$ & $5.16 \mathrm{E}+03$ & $4.54 \mathrm{E}+04$ & $8.59 \mathrm{E}+04$ & & $8.95 \mathrm{E}+04$ \\
\hline Total & $7.55 \mathrm{E}+04$ & $1.37 \mathrm{E}+05$ & $1.16 \mathrm{E}+04$ & $2.24 \mathrm{E}+05$ & $1.31 \bar{E}+05$ & $1.45 \mathrm{E}+03$ & $1.33 \mathrm{E}+05$ \\
\hline
\end{tabular}


2.4.6.1 If yes, provide details and schedule: The DSTs will continue to be characterized on the schedule developed yearly through TPA Milestone, M-44-00

2.4.6.2 If yes, provide Tri-Party Agreement milestone numbers: M-44-00

\subsection{WASTE STREAM STORAGE, INVENTORY, AND GENERATION}

3.1 Is this waste stream currently stored? $\otimes Y$ es $\square$ No If no, skip to 3.7.

3.2 Current storage method

$\begin{array}{ll}\square \text { Container (pad) } & \square \text { Container (covered) } \quad \square \text { Container (retrievably buried) } \\ \square \text { Tank } & \square \text { Waste pile }\end{array}$

3.3 TSD unit name and building number and (if applicable/available) number of containers/tanks in each:

TSD S-2-3, Double Shell Tanks. 200 East Area: AN Farm - 7 Tanks, AP Farm - 8 Tanks, AW

Farm - 6 Tanks, AY Farm - 2 Tanks, AZ Farm - 2 Tanks. 200-West Area: SY Farm - 3 Tanks.

There is a total of 28 tanks.

3.4 Is storage of waste in compliance with applicable State and Federal standards?

$\triangle$ Yes $\square$ Nio

If no, provide details:

3.5 Applicable Tri-Party Agreement milestones related to storage: $\mathbf{M - 3 2 ,} \mathbf{M - 4 3 ,} \mathbf{M - 4 6}$

3.6 Current inventory for this stream

Total LDR volume (cubic meters): 70,976 (Reference: FDH 1999)

Date of inventory values: $12 / 98$

Comments on waste inventory: None

3.7 Is this waste stream currently generated; if not, will this waste stream be generated in the next 5 years?

$\triangle$ Yes $\square$ No. If no, skip to 3.11 .

3.8 The current or future generation of this waste is best described as:

$\bigotimes$ Routine $\square$ One-time or sporadic

3.9 Estimated generation projection by fiscal year

\begin{tabular}{lrlll} 
& \multicolumn{1}{c}{$\mathrm{m}^{3}$} & (and/or) & $\mathrm{kg}$ & \\
1999 & $\mathbf{6 , 8 9 0}$ & & \\
2000 & $\mathbf{7 , 4 2 0}$ & & \\
2001 & $\mathbf{1 0 , 2 0 0}$ & & \\
2002 & $\mathbf{9 , 6 0 0}$ & & \\
2003 & $\mathbf{6 , 1 1 0}$ & & \\
\hline
\end{tabular}

3.10 Last year's fiscal year 1998 generation projection: 14,900

Actual quantity generated in 1998: 930 (both in $\mathrm{m}^{3}$ unless otherwise noted)

3.11 Has there ever been any unusual release of this stream to the environment?

$\square$ Yes $\bigotimes$ No

If yes, summarize releases and quantities:

\subsection{WASTE STREAM TREATMENT}


4.1 Is this stream currently being treated?

$\square$ Yes $\bigotimes$ No If yes, provide details: *Note: DST system wastes are not currently being treated for LDR standards.

4.2 Planned treatment: Check the appropriate box indicating future plans for treating this waste stream to meet applicable regulations, including LDR treatment standards.

$\square$ No treatment required (skip to 5.0 )
Treating or plan to treat onsite
$\square$ Treating or plan to treat offsite
$\square$ Treatment options still being assessed

4.3 Planned treatment method, facility, extent of treatment capacity available: DST system wastes will be retrieved, pretreated, and solidified for disposal. The wastes may be vitrified in a process that will destroy or extract organic and cyanide constituents to below treatment standards, neutralize or deactivate dangerous waste and extremely hazardous waste and immobilize toxic metals.

4.4 Treatment schedule information: Per TPA milestone M-50-00:

Start construction of HLW pretreatment facility - 6/30/2001

Start hot operations of HLW pretreatment facility - 6/30/2008

Complete pretreatment processing of Hanford tank waste $-12 / 31 / 2028$

Per TPA milestone M-61-00:

Start of construction of Phase I low-activity waste (LAW) pretreatment and immobilization facilityTBD

Start hot operations of two contractor owned, contractor operated, Phase I LAW pretreatment and immobilization facilities - 12/31/2003

Initiate negotiations on Phase II LAW pretreatment and immobilization Milestone - 12/31/2004

The contract with BNFL Inc. (Contract No. DE-AC06-96RL13308), for Phase I treatment of Hanford tank waste includes the following target schedule. The schedule is planned to be definitized prior to the end of Part B-1 of the contract.

Initiate Construction (first pour of structural concrete)

Initiate Pretreatment Hot Start

Initiate HLW Hot Start

Hot Start LAW Treatment Services

Completion of Phase I Treatment Services
July 2001

April 2006

February 2007

January 2008

February 2018

4.5 Applicable treatment Tri-Party Agreement milestone numbers (including permitting): $\mathbf{M}-\mathbf{5 0 - 0 0}$, M-51-00, M-60-00, and M-61-00. There is a TPA change request for these milestones, M-62-98-01, and proposed changes are ongoing between DOE and Ecology as of 4/5/99.

4.6 If treating or planning to treat on site, was or will waste minimization be addressed in developing and/or selecting the treatment method? $\square$ Yes $\square$ No $\quad$ Uunknown If yes, please describe:

4.7 Treatability equivalency petitions, rulemaking petitions, and case-by-case exemptions needed for treatment: Any required will be applied for in accordance with the procedures detailed in $40 \mathrm{CFR}$ 268, as applicable, the Tri-Party Agreement.

\subsection{WASTE STREAM DISPOSAL}

5.1 After treatment, how will the waste stream be disposed of (include description, locations, variances required, etc., as applicable): In accordance with current plans, the low-activity waste fraction will 
be disposed of onsite in a retrievable form. The vitrified HLW fraction will be stored on site until the Geologic Repository Program is available to receive wastes for disposal.

5.2 Applicable Tri-Party Agreement milestone number(s) for disposal: $\quad$ M-90-00

5.3 Planned disposal dates/time-frame: Completion dates for this milestone will be determined when the treatment methods are finalized.

\subsection{WASTE MINIMIZATION}

6.1 Has a waste minimization assessment been completed for this stream?

$$
\triangle Y \text { Yes } \square \text { No } \square \text { NA }
$$

6.2 Explain any waste minimization activities (e.g., process changes) under way or to be implemented for generation of this stream: Some of the waste sent to the DST system is reduced at the generating location through pretreatment and recycling of streams. Waste is also minimized by treatment at the 242-A Evaporator. The frequency and volumes of flush solutions has also been minimized.

Low-level mixed effluent transferred to the DST system was examined. The low-level mixed effluents that enter the DST system come in contact with existing wastes and all DST system waste must therefore be managed as HLW. Options to reduce effluents managed as HLW are as follows:

- Recyclable phase transition gel

- Filtration

- Carbon adsorption

- Ion exchange

- Reverse osmosis

- Ultrafiltration

- Pulse dryer/evaporation

6.3 Schedule for implementing waste minimization methods: These activities are already underway.

6.4 Waste reduction achieved during the calendar year and projected future reductions (volume or mass): Unknown

6.4.1 Assumptions used in above estimates: None 
DOE/RL-99-01

This page intentionally left blank. 


\title{
WASTE STREAM PROFILE SHEET
}

\subsection{WASTE STREAM IDENTIFICATION AND SOURCE}

\author{
1.1 Waste stream ID; SST-1
}

\subsection{Waste stream name: Single Shell Tanks}

\subsection{Waste stream source information}

1.3.1 Stream source and history description. Include how the waste was managed prior to storage, timeframe when waste was placed into storage. The SST system waste consists of radioactive and chemically hazardous waste generated as a byproduct of processing spent nuclear fuel to recover plutonium, uranium, and neptunium. A variety of analytical, decladding, and separation processes and associated sitewide operations make up the waste which has been placed into the SST system. Four major operations, the bismuth phosphate process, the reduction-oxidation process, the PUREX process, and the tributyl phosphate process make up the majority of the waste in the SST system. Small amounts of waste from research and development programs, facility and equipment decontamination, lab activities, and the Plutonium Finishing Plant is also stored in the SST system. The aqueous waste was made alkaline before storage. Waste was placed in the SST system between 1944 and 1980. Additions to the SST system were stopped in 1980, except for the addition of water for cooling purposes.

\subsubsection{Source category(s)}

$\square$ Pollution control or waste treatment process $\bigotimes$ Materials production/recovery effluents

Facility or equipment operation and maintenance waste

$\square$ Discarded excess or expired materials

Х R\&D/R\&D laboratory waste

$\square$ Spill clean-ups or emergency response actions Other (explain):

\subsubsection{Additional notes: None}

\subsection{WASTE STREAM CHARACTERIZATION}

2.1 Waste stream description (content): Basic Aqueous Slurry with layers of saltcake and sludge. Sludge is defined as solids (i.e., hydrous metal oxides) precipitated from the neutralization of acid wastes. Saltcake is defined as the various salts formed from the evaporation of water.

\subsection{Radiological characteristics}

\subsubsection{Mixed Waste type QHigh-level $\square$ Transuranic $\square$ Low-level}

$\square$ Contact-handled $\otimes$ Remote-handled

2.2.2 Comments on radiological characteristics (e.g., more specific content, treatment concerns caused by radiation, confidence level): SST system wastes contain the following major radionuclides: $3 \mathrm{H}, 14 \mathrm{C}$, $90 \mathrm{Sr}, 90 \mathrm{Y}, 129 \mathrm{I}, 137 \mathrm{Cs}, 137 \mathrm{mBa}, 151 \mathrm{Sm}, 238 \mathrm{Pu}, 239 \mathrm{Pu}, 240 \mathrm{Pu}, 241 \mathrm{Pu}, 241 \mathrm{Am}$, and $242 \mathrm{Am}$.

\subsection{Matrix characteristics (physical content)}

2.3.1 Matrix constituent table (comprising at least $\mathrm{I} \%$ of the total volume or mass) 
DOE/RL-99-01

\begin{tabular}{ccc}
$\begin{array}{c}\text { Matrix Parameter } \\
\text { Category Code }\end{array}$ & $\begin{array}{c}\text { Matrix Constituent } \\
\text { Description }\end{array}$ & $\begin{array}{c}\text { Typical } \\
\text { or Range }(\%)\end{array}$ \\
\hline$\quad$ L1220 & Basic Aqueous Slurry & $\mathbf{2 \%}$ \\
S9000 & Unknown/Other Solids & $\mathbf{9 8 \%}$
\end{tabular}

Overall matrix parameter category code: $\mathbf{S} 9000$ slurry).

Overall matrix description: Unknown/Other Solids (Saltcake and sludge with basic aqueous

\subsubsection{Confidence level for matrix characteristics data in 2.3 .1 \\ DLow 口Medium 区High}

\subsubsection{Comments on matrix characteristics and/or confidence level: None}

\subsection{Regulated contaminant characteristics}

2.4.1 Wastewater/non-wastewater under RCRA

$\square$ Wastewater $\bigotimes$ Non-wastewater $\square$ Unknown

2.4.2 Regulated contaminant table including treatment requirements, and UHCs if applicable

\begin{tabular}{|c|c|c|c|c|c|}
\hline $\begin{array}{l}\text { EPA/ } \\
\text { State } \\
\text { Code }\end{array}$ & Waste Description & $\begin{array}{cr}\text { LDR } & \text { Con } \\
\text { Sub- } & (\mathrm{Ty} \\
\text { category } & \mathrm{R} \\
\end{array}$ & $\begin{array}{l}\text { ncentration } \\
\text { rypical or } \\
\text { Range)* }\end{array}$ & Basis & $\begin{array}{c}\text { LDR } \\
\text { Concentration Limit or } \\
\text { Technology Code }\end{array}$ \\
\hline F001 & 1,1,1 Trichlorethane & Spent solvents & TBD & TBD & $6.0 \mathrm{mg} / \mathrm{kg}$ \\
\hline $\mathrm{F} 002$ & Methylene Chloride & Spent solvents & TBD & TBD & $30 \mathrm{mg} / \mathrm{kg}$ \\
\hline $\mathrm{F} 003$ & Acetone & Spent solvents & TBD & TBD & $160 \mathrm{mg} / \mathrm{kg}$ \\
\hline & Methyl Isobutyl Keto & ne Spent solvents & TBD & TBD & $33 \mathrm{mg} / \mathrm{kg}$ \\
\hline F004 & Cresols & Spent solvents & TBD & TBD & $5.6 \mathrm{mg} / \mathrm{kg}$ \\
\hline F005 & Methyl Ethyl Ketone & Spent solvents & TBD & TBD & $36 \mathrm{mg} / \mathrm{kg}$ \\
\hline D001 & Ignitability & $\begin{array}{l}\text { Low TOC ignitable } \\
\text { characteristic liquid }\end{array}$ & $\begin{array}{l}\text { e NA } \\
\text { id }\end{array}$ & TBD & Deactivation (2) \\
\hline D002 & Corrosivity & Radioactive (1) & $>12.5$ & TBD & HLVIT \\
\hline D003 & Reactivity & Reactive Cyanides & TBD & TBD & $\begin{array}{l}\text { Meet Cyanide } \\
\text { conc. std. }\end{array}$ \\
\hline D004 & Arsenic & Radioactive (1) & TBD & TBD & HLVIT \\
\hline D005 & Barium & Radioactive (1) & TBD & TBD & HLVIT \\
\hline D006 & Cadmium & Radioactive (1) & TBD & TBD & HLVIT \\
\hline D007 & Chromium & Radioactive (1) & TBD & TBD & HLVIT \\
\hline D008 & Lead & Radioactive (1) & TBD & TBD & HLVIT \\
\hline 0009 & Mercury & Radioactive (1) & TBD & TBD & HLVIT \\
\hline D010 & Selenium & Radioactive (1) & TBD & TBD & HLVIT \\
\hline D011 & Silver & Radioactive (1) & TBD & TBD & HLVIT \\
\hline D018 & Benzene & & TBD & TBD & $10 \mathrm{mg} / \mathrm{kg}(2)$ \\
\hline D019 & \multicolumn{2}{|l|}{ Carbon Tetrachloride } & TBD & TBD & $6.0 \mathrm{mg} / \mathrm{kg}(2)$ \\
\hline D022 & \multicolumn{2}{|l|}{ Chloroform } & TBD & TBD & $6.0 \mathrm{mg} / \mathrm{kg}(2)$ \\
\hline D028 & \multicolumn{2}{|l|}{ 1,2-Dichloroethane } & TBD & TBD & $6.0 \mathrm{mg} / \mathrm{kg} \mathrm{(2)}$ \\
\hline D029 & \multicolumn{2}{|l|}{ 1-Dichlorothylene } & TBD & TBD & $6.0 \mathrm{mg} / \mathrm{kg}(2)$ \\
\hline D030 & \multicolumn{2}{|l|}{ 2,4-Dinitrotoluene } & TBD & TBD & $140 \mathrm{mg} / \mathrm{kg}(2)$ \\
\hline D033 & \multicolumn{2}{|l|}{ Hexachlorobutadiene } & TBD & TBD & $5.6 \mathrm{mg} / \mathrm{kg}(2)$ \\
\hline D034 & \multicolumn{2}{|l|}{ Hexachloroethane } & TBD & TBD & $30 \mathrm{mg} / \mathrm{kg}(2)$ \\
\hline D035 & \multicolumn{2}{|l|}{ Methyl ethly ketone } & TBD & TBD & $36 \mathrm{mg} / \mathrm{kg} \mathrm{(2)}$ \\
\hline D036 & \multicolumn{2}{|l|}{ Nitrobenzene } & TBD & TBD & $14 \mathrm{mg} / \mathrm{kg}(2)$ \\
\hline D038 & \multicolumn{2}{|l|}{ Pyridine } & TBD & TBD & $16 \mathrm{mg} / \mathrm{kg}(2)$ \\
\hline
\end{tabular}




\begin{tabular}{|c|c|c|c|c|}
\hline D039 & Tetrachloroethylene & TBD & TBD & $6.0 \mathrm{mg} / \mathrm{kg}(2)$ \\
\hline D040 & Trichloroethylene & TBD & TBD & $6.0 \mathrm{mg} / \mathrm{kg}(2)$ \\
\hline D041 & 2,4,5-Trichlorophenol & TBD & TBD & $7.4 \mathrm{mg} / \mathrm{kg}(2)$ \\
\hline D043 & Vinyl chloride & TBD & TBD & $6.0 \mathrm{mg} / \mathrm{kg} \mathrm{(2)}$ \\
\hline WT01 & $\begin{array}{l}\text { Toxic Dangerous wastes } \\
\text { Extremely hazardous wastes }\end{array}$ & TBD & TBD & None (3) \\
\hline WT02 & $\begin{array}{l}\text { Toxic Dangerous wastes } \\
\text { Dangerous wastes }\end{array}$ & TBD & TBD & None \\
\hline WP01 & $\begin{array}{l}\text { Persistent Dangerous wastes } \\
\text { Extremely hazardous wastes }\end{array}$ & TBD & TBD & None (3) \\
\hline
\end{tabular}

(1) Radioactive high-level wastes generated during the reprocessing of fuel rods.

(2) and meet 40 CFR268.48

(3) Mixed extremely hazardous wastes may be land-disposed in Washington State in DOE facilities in accordance with Revised Code of Washington 70.105.050(2)

\section{UHCs See comment below}

* If waste isn't consistent in concentration, this may not apply. Describe in 2:4.5. .

2.4.2.1 List any waste codes from 2.4.2 for which the stream already meets established LDR treatment standards: None

2.4.3 Does this waste stream contain PCBs?

$$
\begin{aligned}
& \square \text { Yes } \square \text { No } \square \text { Unknown. } \\
& \text { If no, skip to 2.4.4. }
\end{aligned}
$$

2.4.3.1 Is waste stream subject to TSCA regulations for PCBs?

$$
\square \text { Yes } \square \text { No } \square \text { Unknown }
$$

2.4.3.2 Indicate the PCB concentration range.

$$
\square<50 \mathrm{ppm} \square>50 \mathrm{ppm} \text { 冈Unknown }
$$

2.4.4 What is the confidence level for the regulated contaminant characteristic data? \Low $\square$ Medium $\square$ High

2.4.5 Comments on regulated contaminant characteristics and/or confidence level: The wastes in the SSTs continue to be sampled, analyzed, and characterized. Refer to Table 3-1 for the estimated mass of chemical components of DST and SST system waste.

Waste was sent to the SST system prior to the enactment of LDR requirements, so pertinent LDR requirements were not documented. When SST system waste is transferred to the DST system, known $L D R$ requirements are documented on profile sheets based on the Part $A$, Form 3 Permit Application for the SST system. Typically, no UHCs are identified because there is little or no analytical information on the concentrations of UHCs. At this time, all UHCs are considered reasonably expected to be present in the waste.

2.4.6 Will further characterization be performed? $\quad$ Yes $\square$ No $\square$ Unknown

2:4.6.1 If yes, provide details and schedule: The waste stored in the SSTs will be characterized prior to treatment.

2.4.6.2 If yes, provide Tri-Party Agreement milestone numbers: TBD 


\subsection{WASTE STREAM STORAGE, INVENTORY, AND GENERATION}

3.1 Is this waste stream currently stored? YYes $\square$ No If no, skip to 3.7.

3.2 Current storage method

$\begin{array}{ll}\square \text { Container (pad) } & \square \text { Container (covered) } \\ \square \text { Tank } & \square \text { Container (retrievably buried) } \\ \square \text { Other (explain): } & \square \text { Surface impoundment }\end{array}$

3.3 TSD unit name and building number and (if applicable/available) number of containers/tanks in each: TSD S-2-4, Single Shell Tanks. 200 East Area: A Farm - 6 tanks, AX Farm - 4 tanks, B Farm - 16 tanks, BX Farm - 12 tanks, BY Farm - 12 tanks, C Farm - 16 tanks. 200-West Area: S Farm - 12 tanks, SX Farm - 15 tanks, T Farm - 16 tanks, TX Farm - 18 tanks, TY Farm - 6 tanks, U Farm -16 tanks. The tanks range from $210 \mathrm{~m} 3$ to $3,800 \mathrm{~m} 3$ in capacity, and there are 149 tanks in total.

3.4 Is storage of waste in compliance with applicable State and Federal standards?

$\square$ Yes $\bigotimes$ No

If no, provide details: SST wastes are stored in tanks that do not have secondary containment and do not have an integrity assessment. As such, SSTs will be closed pursuant to TPA Milestone M45, Complete Closure of all Single Shell Tank Farms, rather than upgraded.

3.5 Applicable Tri-Party Agreement milestones related to storage: M-41, M-45. M-41 will be replaced with a consent decree in May 1999.

3.6 Current inventory for this stream

Total LDR volume (cubic meters): $\quad 133,600$

Date of inventory values: $12 / 98$

Comments on waste inventory: None

3.7 Is this waste stream currently generated; if not, will this waste stream be generated in the next 5 years? $\square$ Yes $\bigotimes$ No. If no, skip to 3.11.

3.8 The current or future generation of this waste is best described as:

$\square$ Routine $\square$ One-time or sporadic

3.9 Estimated generation projection by fiscal year

$\begin{array}{lllll}1999 & \mathrm{~m}^{3} \quad \text { (and/or) } \mathrm{kg} & \\ 2000 & & & & \\ 2001 & & & & \\ 2002 & & & & \\ 2003 & & & \end{array}$

3.10 Last year's fiscal year 1998 generation projection: NA

Actual quantity generated in 1998: 0 (both in $\mathrm{m}^{3}$ unless otherwise noted)

3.11 Has there ever been any unusual release of this stream to the environment?

$\triangle$ Yes $\square N_{0}$

If yes, summarize releases and quantities: See Table 3-2

\subsection{WASTE STREAM TREATMENT}

4.1 Is this stream currently being treated? 
Table 3-2. Hanford Facility Single-Shell Tank System Releases. ${ }^{a}$ (2 sheets)

\begin{tabular}{|c|c|c|c|c|c|}
\hline Tank & Volume $\left(\mathrm{m}^{3}\right)$ & Leak reported & Tank & Volume $\left(\mathrm{m}^{3}\right)$ & Leak reported \\
\hline $241-A-103$ & 21 & 1987 & 241-SX-107 & $<19$ & 1964 \\
\hline $241-A-104$ & 2 to 10 & 1975 & $241-S X+108$ & 9 to 133 & 1962 \\
\hline $241-A-105$ & 38 to 1048 & 1963 & $241-S X-109^{\circ}$ & 38 & 1965.1996 \\
\hline $241-A X-102$ & 11 & 1988 & $241-S X-110$ & 21 & 1976 \\
\hline $241-\mathrm{AX}-104^{\mathrm{b}}$ & - & $19 \overline{77}$ & $241-\mathrm{SX}-111^{5}$ & 2 to 8 & 1974 \\
\hline $241-\mathrm{B}-101^{\circ}$ & - & 1974 & $241-S X-112^{C}$ & 114 & 1969 \\
\hline $241-B-103^{6}$ & - & 1978 & 241-SX-113 & 57 & 1962 \\
\hline $241-\mathrm{B}-105^{6}$ & -- & 1978 & $24 !-5 X-114^{b}$ & -- & 1972 \\
\hline $241-B-107$ & 30 & 1980 & $241-S X-115$ & 189 & 196 \\
\hline 241-B-110 & 38 & 1981 & $241-T-101$ & 28 & 1992 \\
\hline $241-B-111^{6}$ & $\because$ & 1978 & $241-\mathrm{T}-103$ & $<4$ & 1974 \\
\hline 241-BI12 & 8 & 1978 & $241-T-106$ & 435 & 1973 \\
\hline 241-B-201 & 5 & 1980 & $241-T-107^{6}$ & $=$ & 1984 \\
\hline $241-\bar{B}-203$ & 1 & 1983 & $241-\Upsilon-108$ & $<4$ & 1974 \\
\hline 241-B-204 & 2 & 1984 & $241-T-109$ & $<4$ & 1974 \\
\hline $241-8 X-101^{\circ}$ & -- & 1972 & $241-T-111$ & $<\dot{4}$ & 1979.1994 \\
\hline $241-\mathrm{BX}-102$ & 265 & 1971 & $241-\mathrm{TX}-105^{\circ}$ & $\because$ & 1977 \\
\hline $241-B \mathrm{X}-108$ & 10 & 1974 & $241-1 X-107$ & 10 & 1984 \\
\hline $241-\mathrm{BX}-110^{6}$ & -- & 1976 & $241-\mathrm{TX} \cdot 110^{6}$ & -- & 1977 \\
\hline $241-\mathrm{BX}-111^{\mathrm{b}}$ & -- & 1984 & $241-\mathrm{TX}-113^{\circ}$ & $*$ & 1974 \\
\hline 241-BY-103 & $<19$ & 1973 & $241-\mathrm{TX}-114^{6}$ & - & 1974 \\
\hline $241-\mathrm{BY}-105^{\circ}$ & -- & 1984 & $241-\mathrm{TX}-115^{6}$ & -- & 1977 \\
\hline $241-B Y-106^{\circ}$ & - & 1984 & $241-\mathrm{TX}-116^{6}$ & $-\cdots$ & 1977 \\
\hline $241-B Y-107$ & 57 & 1984 & $241-\mathrm{TX}-117^{b}$ & -- & 1977 \\
\hline 241-BY-108 & $<19$ & 1972 & 241-TY-101 & $<\overline{4}$ & 1973 \\
\hline $241 \times C-101$ & 76 & 1980 & 241-TY-103 & 11 & 1973 \\
\hline $241-C-110$ & 8 & 1984 & $241-\mathrm{TY}-104$ & 5 & 1981 \\
\hline $241-C-111$ & 21 & 1968 & 241-TY-105 & 133 & 1960 \\
\hline $241-C-201$ & 2 & 1988 & $241-$ TY-106 & 76 & 1959 \\
\hline $241-C-202$ & 2 & 1988 & $241-U-101$ & 114 & 1959 \\
\hline $241-C-203$ & 2 & 1984 & $241-U-104$ & 208 & 1961 \\
\hline $241 \cdot C-204$ & 1 & 1988 & $241-\mathrm{U}-110$ & 19 to 3 ! & 1975 \\
\hline $241-S \cdot 104$ & 91 & 1968 & $241-U-112$ & 32 & 1980 \\
\hline 24l-SX-104 & 23 & 1988 & Total & 2862 to 4022 & \\
\hline
\end{tabular}

${ }^{a}$ After some tanks were declared to be leaking, water may have been added to aid evaporative cooling. Some of this water is thought to have not evaporated. but leaked into the ground. As of October 1990, estimates of the volume leaked ranged from 190 to 3000 cubic meters. The volumes provided and the date of initial release are being continually evaluated and refined and may be revised for improved accuracy. In addition, documents show that from 1946 to $1966,456.700$ cubic meters $(120,661,000$ gallons) of liquid waste were intentionally discharged from SSTs at the Hanford Facility directly to the ground on the 200 Area plateau (WHC 1991c). Most of this waste was discharged from 1946 to 1958 as a result of the early plutonium and uranium recover processes conducted in the 221-B Facility (B Plant), 221-T Facility (T Plant), and 221-U Facility (U Plant). In addition, from 1960 to 1966 laboratory waste from the 300 Area and equipment decontamination waste from the 200 West Area were routed through SSTs before discharge to the ground. No waste has been intentionally discharged to the ground from SSTs since 1966, and no waste has ever been discharged directly to the ground from the newer DST system.

'Individual release volumes for these tanks have not been determined. The total volume is estimated to be 570 cubic meters. 


\section{Table 3-2. Hanford Facility Single-Shell Tank System Releases. ${ }^{a}$ (2 sheets)}

The leah volume and curie release estinutes on $\$ X-10 \$ . \$ X-109$. $\$ X-111$, and $\$ X-112$ hate been re-cratuated using a

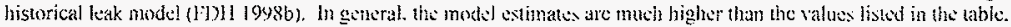
both for volume and curies released. The values listed in the wble do not retlect this revisol estinate becane. "In particular. it is worth smphasizing that this report was never metant to be a definitive update for the leak baseline at the Hanford Sitc. It was rather meant wo be an atcmpt to vien the issus of leak inventories with a new and ditherem methotology," (This quete is from the first page of the referenced report). Therefore an uncertainy analy sis to determine the applicathiliz of this methudology is cumenty in progress.

\section{References:}

FDH, 1999, Waste Tank Summary Report for Month Ending December 31, 1998, HNF-EP-0182-129, prepared by Lockheed Martin Hanford Corporation for Fluor Daniel Hanford Company, Richland, Washington.

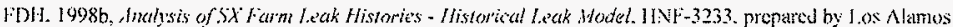
¿ational Laboratory to Fluor Danicl Hanterd Company. Richland. Washinglon. 
$\square$ Yes \No If yes, provide details: *Note: SST system wastes are not currently being treated for LDR standards.

4.2 Planned treatment: Check the appropriate box indicating future plans for treating this waste stream to meet applicable regulations, including LDR treatment standards.

$\square$ No treatment required (skip to 5.0)

$\square$ Treating or plan to treat onsite

Treating or plan to treat offsite

Treatment options still being assessed

4.3 Planned treatment method, facility, extent of treatment capacity available: Wastes in the SST system will undergo retrieval, treatment, and disposal per the DST system waste disposal plan. This may include pretreatment, and vitrification, which will destroy or extract organic and cyanide constituents to below treatment standards, neutralize or deactivate dangerous waste and extremely hazardous waste, and immobilize toxic metals.

4.4 Treatment schedule information: The SST waste will be treated and disposed of as DST waste, per TPA Milestones.

4.5 Applicable treatment Tri-Party Agreement milestone numbers (including permitting): $\quad \mathbf{M}-50-00, \mathbf{M}-$ 60-00, and M-61-00

4.6 If treating or planning to treat on site, was or will waste minimization be addressed in developing and/or selecting the treatment method? $\square$ Yes $\square$ No $\quad$ Unknown

If yes, please describe:

4.7 Treatability equivalency petitions, rulemaking petitions, and case-by-case exemptions needed for treatment: Any required will be applied for in accordance with the procedures detailed in the TriParty Agreement.

\subsection{WASTE STREAM DISPOSAL}

5.1 After treatment, how will the waste stream be disposed of (include description, locations, variances required, etc., as applicable): In accordance with current plans, the low-activity waste fraction will be disposed of onsite in a retrievable form. The vitrified HLW fraction will be stored on site until the Geologic Repository Program is available to receive wastes for disposal.

5.2 Applicable Tri-Party Agreement milestone number(s) for disposal: M-90-00

5.3 Planned disposal dates/time-frame: Completion of the milestone is to be determined when Privatization Plans are finalized.

\subsection{WASTE MINIMIZATION}

6.1 Has a waste minimization assessment been completed for this stream?

$\square$ Yes $\square$ No $\square$ NA

6.2 Explain any waste minimization activities (e.g., process changes) under way or to be implemented for generation of this stream: This stream is no longer being generated.

6.3 Schedule for implementing waste minimization methods: NA

6.4 Waste reduction achieved during the calendar year and projected future reductions (volume or mass): NA 
DOE/RL-99-01

6.4.1 Assumptions used in above estimates: NA 


\section{WASTE STREAM PROFILE SHEET}

\subsection{WASTE STREAM IDENTIFICATION AND SOURCE}

1.1 Waste stream ID: PUREX-1

1.2 Waste stream name: PUREX Containment Building

1.3 Waste stream source information

1.3.1 Stream source and history description. Include how the waste was managed prior to storage, timeframe when waste was placed into storage. Concrete rubble from the E-Cell canyon floor was placed in a metal box during the floor renovation. The waste was generated in September, 1989.

1.3.2 Source category(s)

$\square$ Pollution control or waste treatment process

Materials production/recovery effluents

$\triangle$ Facility or equipment operation and maintenance waste
Discarded excess or expired materials
$R \& D / R \& D$ laboratory waste
Spill clean-ups or emergency response actions

Other (explain):

1.3.3 Additional notes: None

\subsection{WASTE STREAM CHARACTERIZATION}

2.1 Waste stream description (content): Trace chromium as a corrosion product in concrete debris.

2.2 Radiological characteristics

2.2.1 Mixed Waste type $\square$ High-level \Transuranic 囚Low-level

$\square$ Contact-handled 凶Remote-handled

2.2.2 Comments on radiological characteristics (e.g., more specific content, treatment concerns caused by radiation, confidence level): Approximately $500 \mathbf{R a d} / \mathbf{h r}$.

2.3 Matrix characteristics (physical content)

2.3.1 Matrix constituent table (comprising at least $1 \%$ of the total volume or mass)

\begin{tabular}{ccc}
$\begin{array}{c}\text { Matrix Parameter } \\
\text { Category Code }\end{array}$ & $\begin{array}{c}\text { Matrix Constituent } \\
\text { Description }\end{array}$ & $\begin{array}{c}\text { Typical } \\
\text { or Range (\%) }\end{array}$ \\
\hline \$5121 & Concrete Debris & 99.9
\end{tabular}

Overall matrix parameter category code: $\quad$ S5121

Overall matrix description: Concrete Debris 
2.3.2 Confidence level for matrix characteristics data in 2.3.1 $\square$ Low $\square$ Medium 邓High

2.3.3 Comments on matrix characteristics and/or confidence level: None

2.4 Regulated contaminant characteristics

2.4.1 Wastewater/non-wastewater under RCRA

$\square$ Wastewater $\bigotimes$ Non-wastewater $\square$ Unknown

2.4.2 Regulated contaminant table including treatment requirements, and UHCs if applicable

\begin{tabular}{|c|c|c|c|c|c|}
\hline $\begin{array}{l}\text { EPA/ } \\
\text { State } \\
\text { Code }\end{array}$ & Waste Description & $\begin{array}{l}\text { LDR } \\
\text { Sub- } \\
\text { category }\end{array}$ & $\begin{array}{l}\text { Concentration } \\
\text { (Typical or } \\
\text { Range)* }\end{array}$ & Basis & $\begin{array}{l}\text { LDR } \\
\text { Concentration Limit or } \\
\text { Technology Code }\end{array}$ \\
\hline$\overline{\mathrm{D} 007}$ & Chromium & Chromium & $\sim 1000 \mathrm{ppm}$ & Analytical Results & Debris standard \\
\hline
\end{tabular}

UHCs Not applicable to this waste

* If waste isn't consistent in concentration, this may not apply. Describe in 2.4 .5 .

2.4.2.1 List any waste codes from 2.4.2 for which the stream already meets established LDR treatment standards: None

2.4.3 Does this waste stream contain PCBs?

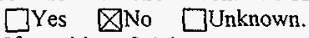

If no, skip to 2.4.4.

2.4.3.1 Is waste stream subject to TSCA regulations for PCBs?

$\square$ Yes $\square$ No $\square$ Unknown

2.4.3.2 Indicate the PCB concentration range.

$\square<50 \mathrm{ppm} \quad \square>50 \mathrm{ppm} \quad \square$ Unknown

2.4.4 What is the confidence level for the regulated contaminant characteristic data?

$\square$ Low $\square$ Medium $\bigotimes$ High

2.4.5 Comments on regulated contaminant characteristics and/or confidence level: Based on laboratory analysis.

2.4.6 Will further characterization be performed? $\square$ Yes $\square$ No $\square$ Unknown

2.4.6.I If yes, provide details and schedule:

2.4.6.2 If yes, provide Tri-Party Agreement milestone numbers:

\subsection{WASTE STREAM STORAGE, INVENTORY, AND GENERATION}

3.1 Is this waste stream currently stored? XYes $\square$ No If no, skip to 3.7.

3.2 Current storage method

$\square$ Container (pad) $\square$ Container (covered) $\square$ Container (retrievably burię)




\section{$\square$ Tank (explain):- $\square$ Waste pile $\square$ Surface impoundment}

3.3 TSD unit name and building number and (if applicable/available) number of containers/tanks in each: 202A PUREX facility, F-Cell canyon floor, single metal box.

3.4 Is storage of waste in compliance with applicable State and Federal standards?

叉Yes $\square$ No

If no, provide details:

3.5 Applicable Tri-Party Agreement milestones related to storage: None

3.6 Current inventory for this stream

Total LDR volume (cubic meters): 1.

Date of inventory values: $\mathbf{9 / 3 0 / 9 8}$

Comments on waste inventory: None

3.7 Is this waste stream currently generated; if not, will this waste stream be generated in the next 5 years? $\square$ Yes $\bigotimes$ No. If no, skip to 3.11.

3.8 The current or future generation of this waste is best described as:

$\square$ Routine $\square$ One-time or sporadic

3.9 Estimated generation projection by fiscal year

$\begin{array}{lllll}1999 & \mathrm{~m}^{3} \quad \text { (and/or) } & \mathrm{kg} & \\ 2000 & & & & \text { МA } \\ 2001 & & & & \\ 2002 & & & & \\ 2003 & & & & \end{array}$

3.10 Last year's fiscal year 1998 generation projection: NA

Actual quantity generated in 1998: 0 (both in $\mathrm{m}^{3}$ unless otherwise noted)

3.11 Has there ever been any unusual release of this stream to the environment?

$\square$ Yes 冈No

If yes, summarize releases and quantities:

\subsection{WASTE STREAM TREATMENT}

4.1 Is this stream currently being treated?

$\square$ Yes $\bigotimes$ No if yes, provide details:

4.2 Planned treatment: Check the appropriate box indicating future plans for treating this waste stream to meet applicable regulations, including LDR treatment standards.

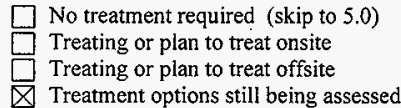

4.3 Planned treatment method, facility, extent of treatment capacity available: None

\subsection{Treatment schedule information: None}


DOE/RL-99-01

4.5 Applicable treatment Tri-Party Agreement milestone numbers (including permitting): None

4.6 If treating or planning to treat on site, was or will waste minimization be addressed in developing and/or selecting the treatment method? $\square$ Yes $\square$ No UUnknown

If yes, please describe:

4.7 Treatability equivalency petitions, rulemaking petitions, and case-by-case exemptions needed for treatment: Unknown

\subsection{WASTE STREAM DISPOSAL}

5.1 After treatment, how will the waste stream be disposed of (include description, locations, variances required, etc., as applicable): Unknown

5.2 Applicable Tri-Party Agreement milestone number(s) for disposal: None

5.3 Planned disposal dates/time-frame: Unknown

\subsection{WASTE MINIMIZATION}

6.1 Has a waste minimization assessment been completed for this stream?

$\square$ Yes $\square$ No $\quad$ NA

6.2 Explain any waste minimization activities (e.g., process changes) under way or to be implemented for generation of this stream: No waste was generated in 1998, nor forecast for outyears. Therefore, a waste minimization assessment is not required.

6.3 Schedule for implementing waste minimization methods: None

6.4 Waste reduction achieved during the calendar year and projected future reductions (volume or mass): NA

6.4.1 Assumptions used in above estimates: NA 


\section{WASTE STREAM PROFILE SHEET}

\subsection{WASTE STREAM IDENTIFICATION AND SOURCE}

1.1 Waste stream ID: PUREX-2

1.2 Waste stream name: PUREX Storage Tunnels

1.3 Waste stream source information

1.3.1 Stream source and history description. Include how the waste was managed prior to storage, timeframe when waste was placed into storage. The bulk of the waste is failed equipment from the PUREX facility. However, waste from other Hanford Facility locations, including 324 and 327 research and development laboratories, has been placed in the tunnels. Failed rail cars are also in the tunnels.

\subsubsection{Source category (s)}
Discarded excess or expired materials
$\triangle R \& D / R \& D$ laboratory waste
Spill clean-ups or emergency response actions
$\square$ Pollution control or waste treatment process $\square$
Other (explain):
Materials production/recovery effluents
$\square$ Analytical laboratory waste
Remediation/D\&D waste
Source unknown

1.3.3 Additional notes: None

\subsection{WASTE STREAM CHARACTERIZATION}

2.1 Waste stream description (content): Varies from very large equipment vessels with lead counterweights to very fine powder in canisters.

\subsection{Radiological characteristics}

\subsubsection{Mixed Waste type $\square$ High-level \Transuranic $\square$ Low-level} $\square$ Contact-handled $\bigotimes$ Remote-handled

2.2.2 Comments on radiological characteristics (e.g., more specific content, treatment concerns caused by radiation, confidence level): Varies from medium $(\sim 1 \mathrm{rad} / \mathrm{hr})$ to very high $(>1,000 \mathrm{rad} / \mathrm{hr})$.

2.3 Matrix characteristics (physical content)

2.3.1 Matrix constituent table (comprising at least $1 \%$ of the total volume or mass)

\begin{tabular}{|c|c|c|}
\hline $\begin{array}{l}\text { Matrix Parameter } \\
\text { Category Code }\end{array}$ & $\begin{array}{l}\text { Matrix Constituent } \\
\text { Description } \\
\end{array}$ & $\begin{array}{c}\text { Typical } \\
\text { or Range (\%) }\end{array}$ \\
\hline S5111 & Stainless steel and/or iron & 99.9 \\
\hline$S 5112$ or $\$ 5113$ & $\begin{array}{l}\text { Stainless steel and/or iron with } \\
\text { metallic lead and/or cadmium }\end{array}$ & $1-99.9$ \\
\hline $\begin{array}{l}X 7211 \text { or } X 7220 \\
\times 7100\end{array}$ & $\begin{array}{l}\text { Lead or cadmium, separated } \\
\text { Mercury in stainless steel } \\
\text { containers }\end{array}$ & $\begin{array}{l}1-99.9 \\
100\end{array}$ \\
\hline S3117 & Vitrified test wastes from & $90-99.9$ \\
\hline
\end{tabular}


R\&D Labs

Overall matrix parameter category code: NA, see below

Overall matrix description: NA, see below

2.3.2 Confidence level for matrix characteristics data in 2.3.1

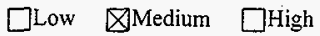

2.3.3 Comments on matrix characteristics and/or confidence level: Several different waste types are included in this stream. Therefore, no overall code was assigned. The matrix constituent table considers each waste type separately, so the percentages add to over 100.

2.4 Regulated contaminant characteristics

2.4.1 Wastewater/non-wastewater under RCRA

$\square$ Wastewater $\bigotimes$ Non-wastewater $\square$ Unknown

2.4.2 Regulated contaminant table including treatment requirements, and UHCs if applicable

\begin{tabular}{|c|c|c|c|c|c|}
\hline $\begin{array}{l}\text { EPA/ } \\
\text { State } \\
\text { Code }\end{array}$ & Waste Description & $\begin{array}{r}\text { LDR } \\
\text { Sub- } \\
\text { category }\end{array}$ & $\begin{array}{c}\text { Concentration } \\
\text { (Typical or } \\
\text { Range) }\end{array}$ & Basis & $\begin{array}{c}\text { LDR } \\
\text { Concentration Limit or } \\
\text { Technology Code }\end{array}$ \\
\hline D005 & Barium & Barium & 100-1000s ppm & Analytical/proc. know. & v. $100 \mathrm{mg} / \mathrm{L} \mathrm{TCLP}$ \\
\hline D006 & ium & Cadmium & & know. & L TCLP \\
\hline 0007 & & Chro & $5-1000 s$ & know. & $g / L$ TCLP \\
\hline 008 & & Rad. & Solids & Pro & RO \\
\hline D009 & & High $\mathbf{H}$ & organic & Pro & \\
\hline D010 & um & Selenium & & edge & $5.7 \mathrm{mg} / \mathrm{L}$ TCLP \\
\hline D011 & Silv & Silver & 5 - 1000's ppm & Pro & $5.0 \mathrm{mg} / \mathrm{L}$ TCLP \\
\hline D001 & Oxidizer & Low TOC & & Process knowledge & $\begin{array}{l}\text { DEACT \& meet } \\
40 \text { CFR } 268.48\end{array}$ \\
\hline WT02 & Toxic (mineral oil) & & & Process knowledge & None \\
\hline
\end{tabular}

UHCs must be determined for the PUREX Tunnels waste.

* If waste isn't consistent in concentration, this may not apply. Describe in 2.4.5.

2.4.2.I List any waste codes from 2.4 .2 for which the stream already meets established LDR treatment standards: None

2.4.3 Does this waste stream contain PCBs?

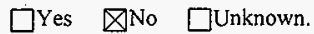

If no, skip to 2.4.4.

2.4.3.1 Is waste stream subject to TSCA regulations for PCBs?

$\square$ Yes $\square$ No $\square$ Unknown

2.4.3.2 Indicate the $P C B$ concentration range.

$\square<50 \mathrm{ppm} \quad \square<50 \mathrm{ppm} \quad \square$ Unknown

2.4.4 What is the confidence level for the regulated contaminant characteristic data?

$\square$ Low $\otimes$ Medium $\square$ High 
2.4.5 Comments on regulated contaminant characteristics and/or confidence level: Confidence varies depending on waste item. Contaminants vary with different containers/equipment. (Not all of the waste would have all waste codes). D001 nitrate residue is from nitric acid.

2.4.6 Will further characterization be performed? $\square$ Yes $₫$ No $\square$ Unknown

2.4.6.1 If yes, provide details and schedule: NA

2.4.6.2 If yes, provide Tri-Party Agreement milestone numbers: NA

\subsection{WASTE STREAM STORAGE, INVENTORY, AND GENERATION}

3.1 Is this waste stream currently stored? 囚Yes $\square$ No If no, skip to 3.7.

\subsection{Current storage method}

$\square$ Container (pad)
$\square$ Tank
$\begin{aligned} & \square \text { Container (covered) } \\ & \text { Wather (explain): }\end{aligned} \quad \begin{aligned} & \text { Container (retrievably buried) } \\ & \square \text { Surface impoundment }\end{aligned}$

3.3 TSD unit name and building number and (if applicable/available) number of containers/tanks in each: PUREX \#1 Storage Tunnel (218-E-14) 8 rail cars

PUREX \#2 Storage Tunnel (218-E-15) 28 rail cars ( 40 positions available)

3.4 Is storage of waste in compliance with applicable State and Federal standards?

$\bigotimes$ Yes $\square$ No

If no, provide details:

3.5 Applicable Tri-Party Agreement milestones related to storage: None

3.6 Current inventory for this stream

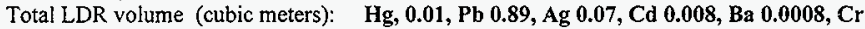

0.0001 , Mineral Oil 0.007. Total volume including equipment, containers, etc, is estimated at 2,800 m3.

Date of inventory values: Current as of $12 / 98$

Comments on waste inventory: Estimated

3.7 Is this waste stream currently generated; if not, will this waste stream be generated in the next 5 years?

QYes $\square$ No. If no, skip to 3.11 .

3.8 The current or future generation of this waste is best described as:

$\square$ Routine 冈One-time or sporadic

3.9 Estimated generation projection by fiscal year

$\begin{array}{lllll} & \mathrm{m}^{3} & \text { (and/or) } & \mathrm{kg} & \\ 1999 & 400 & & & \\ 2000 & & & & \\ 2001 & & & & \\ 2002 & & & & \\ 2003 & & & \end{array}$

3.10 Last year's fiscal year 1998 generation projection: NA Actual quantity generated in 1998: 0 (both in $\mathrm{m}^{3}$ unless otherwise noted) 
3.11 Has there ever been any unusual release of this stream to the environment?

$\square$ Yes 囚No

If yes, summarize releases and quantities:

\subsection{WASTE STREAM TREATMENT}

4.1 Is this stream currently being treated?

$\square$ Yes 区No If yes, provide details:

4.2 Planned treatment: Check the appropriate box indicating future plans for treating this waste stream to meet applicable regulations, including LDR treatment standards.

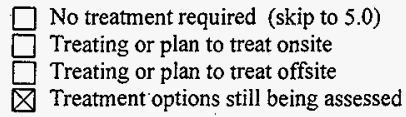

4.3 Planned treatment method, facility, extent of treatment capacity available: NA

4.4 Treatment schedule information: NA

4.5 Applicable treatment Tri-Party Agreement milestone numbers (including permitting): NA

4.6 If treating or planning to treat on site, was or will waste minimization be addressed in developing and/or selecting the treatment method? $\square$ Yes $\square$ No QUnknown

If yes, please describe:

4.7 Treatability equivalency petitions, rulemaking petitions, and case-by-case exemptions needed for treatment: NA

\subsection{WASTE STREAM DISPOSAL}

5.1 After treatment, how will the waste stream be disposed of (include description, locations, variances required, etc., as applicable): Unknown, however the most likely disposal location is the LLBG Subtitle-C or LLBG LLW trenches.

5.2 Applicable Tri-Party Agreement milestone number(s) for disposal: NA

5.3 Planned disposal dates/time-frame: NA

\subsection{WASTE MINIMIZATION}

6.1 Has a waste minimization assessment been completed for this stream?

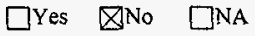

6.2 Explain any waste minimization activities (e.g., process changes) under way or to be implemented for generation of this stream: No new waste was generated during 1998. Therefore a waste assessment for this stream is not necessary and was not conducted during the calendar year.

6.3 Schedule for implementing waste minimization methods: NA 


\section{DOE/RL-99-01}

6.4 Waste reduction achieved during the calendar year and projected future reductions (volume or mass): NA

6.4.1 Assumptions used in above estimates: NA 
DOE/RL-99-01

This page intentionally left blank. 


\section{WASTE STREAM PROFILE SHEET}

\subsection{WASTE STREAM IDENTIFICATION AND SOURCE}

1.1 Waste stream ID: 324 REC

1.2 Waste stream name: 324 Building Radiochemical Engineering Cell

1.3 Waste stream source information

1.3.1 Stream source and history description. Include how the waste was managed prior to storage, timeframe when waste was placed into storage. A variety of high-activity radioactive wastes containing regulated quantities of predominantly toxic heavy metals, generated during research and development activities ongoing since the mid-1960s and the processing of 324 Building High-Level Vault (HLV) waste.

\subsubsection{Source category(s)}

$\square$ Pollution control or waste treatment process
$\square$ Macility or equipment operation and maintenance waste
$\square$ Discarded excess or expired materials
$\begin{aligned} & \text { R\&D/R\&D laboratory waste } \\ & \square \text { Spill clean-ups or emergency response actions }\end{aligned}$
$\square$ Analytical laboratory waste
Semediation/D\&D waste

1.3.3 Additional notes: The 324 Building Radiochemical Engineering Cell (REC) waste stream includes the B-Cell dispersible material, the B-Cell tank heels, the REC pipe trench sludge, and HLV tank filters.

\subsection{WASTE STREAM CHARACTERIZATION}

2.1 Waste stream description (content): Most of the materials now in the Radiochemical Engineering Cells (REC) accumulated during research activities from 1965 to 1987, except for solid residues from the treatment of HLV tank waste. Over the 20+ years of these engineering demonstrations, equipment (such as tools, manipulator boots, and construction materials) were dropped, and liquids (such as feed materials and samples) leaked onto the floor. In addition, particulate materials (essentially dust) and filters introduced with normal air flow into the cell became contaminated. This waste also includes elemental lead, used as shielding and counterbalances.

\subsection{Radiological characteristics}

\subsubsection{Mixed Waste type $\square$ High-level $\bigotimes$ Transuranic $\square$ Low-level}

$\square$ Contact-handled $\bigotimes$ Remote-handled

2.2.2 Comments on radiological characteristics (e.g., more specific content, treatment concerns caused by radiation, confidence level): This waste consists of approximately $3,855 \mathrm{kCi}$ of primarily $\mathrm{Sr}$ and $\mathrm{Cs}$.

\subsection{Matrix characteristics (physical content)}

2.3.1 Matrix constituent table (comprising at least $1 \%$ of the total volume or mass)

Matrix Parameter

Matrix Constituent

Typical

Category Code

Description or Range (\%) 
Overall matrix parameter category code: $\quad \mathbf{S 5 1 1 0}$

Overall matrix description: Metal debris

2.3.2 Confidence level for matrix characteristics data in 2.3.1

$\square$ Low $\square$ Medium $\triangle$ High

2.3.3 Comments on matrix characteristics and/or confidence level: None

2.4 Regulated contaminant characteristics

2.4.1 Wastewater/non-wastewater under RCRA

$\square$ Wastewater $\bigotimes$ Non-wastewater $\square$ Unknown

2.4.2 Regulated contaminant table including treatment requirements, and UHCs if applicable

\begin{tabular}{|c|c|c|c|c|c|}
\hline $\begin{array}{l}\text { EPA/ } \\
\text { State } \\
\text { Code }\end{array}$ & Waste Description & $\begin{array}{c}\text { LDR } \\
\text { Sub- } \\
\text { category }\end{array}$ & $\begin{array}{c}\text { Concentration } \\
\text { (Typical or } \\
\text { Range)* }\end{array}$ & $\begin{array}{r}\text { L } \\
\text { Concentrati } \\
\text { Technol }\end{array}$ & $\begin{array}{l}\text { DR } \\
\text { tion Limit or } \\
\text { logy Code }\end{array}$ \\
\hline D005 & Barium & Barium & $420 \mathrm{ppm}$ & Sample Analysis & (1) \\
\hline D006 & Cadmium & Cadmium & $1.0 \mathrm{ppm}$ & Sample Analysis ( 1 container) & (1) \\
\hline D007 & Chromium & Chromium & $6.3 \mathrm{ppm}$ & Sample Analysis (1 container) & (1) \\
\hline D008 & Lead & Rad. Lead Solids & & Sample Analysis. ( 5 containers) & MACRO \\
\hline D008 & Lead & Lead & & Process Knowledge & (1) \\
\hline
\end{tabular}

UHCs must be determined for this waste.

(1) Debris standards in 40 CFR 268.45

* If waste isn't consistent in concentration, this may not apply. Describe in 2.4.5.

2.4.2.1 List any waste codes from 2.4 .2 for which the stream already meets established LDR treatment standards: None

2.4.3 Does this waste stream contain PCBs?

$\square$ Yes $\square$ No $\square$ Unknown.
If no, skip to 2.4.4.

2.4.3.1 Is waste stream subject to TSCA regulations for PCBs?

$\square$ Yes $\square$ No $\square$ Unknown

2.4.3.2 Indicate the PCB concentration range.

$\square<50 \mathrm{ppm} \quad \square<50 \mathrm{ppm} \quad \square$ Unknown

2.4.4 What is the confidence level for the regulated contaminant characteristic data?

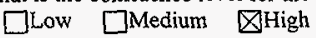

2.4.5 Comments on regulated contaminant characteristics and/or confidence level: Only metal filters are regulated for $\mathrm{Ba}, \mathrm{Cd}, \mathrm{Cr}$, and $\mathrm{Pb}$. The other wastes are regulated for $\mathrm{Cd}, \mathrm{Cr}$, and $\mathrm{Pb}$ only.

2.4.6 Will further characterization be performed? $\square$ Yes $\quad$ No $\square$ Unknown 
2.4.6.1 If yes, provide details and schedule:

2.4.6.2 If yes, provide Tri-Party Agreement milestone numbers:

\subsection{WASTE STREAM STORAGE, INVENTORY, AND GENERATION}

3.1 Is this waste stream currently stored? $\bigotimes$ Yes $\square$ No If no, skip to 3.7.

3.2 Current storage method

$\begin{array}{lll}\square \text { Container (pad) } & \square \text { Container (covered) } & \square \text { Container (retrievably buried) } \\ \bigotimes \text { Tank } & \square \text { Waste pile } & \square \text { Surface impoundment }\end{array}$

3.3 TSD unit name and building number and (if applicable/available) number of containers/tanks in each: 324 Facility REC

3.4 Is storage of waste in compliance with applicable State and Federal standards?

$\triangle$ Yes. $\square$ No

If no, provide details: Storage compliance is not believed to be an issue. The 324 Facility REC is not a TSD unit.

3.5 Applicable Tri-Party Agreement milestones related to storage: $\quad$ M-89-00

3.6 Current inventory for this stream

Total LDR volume (cubic meters): approx. 2.66

Date of inventory values: $12 / 30 / 98$

Comments on waste inventory: This waste has not been containerized for transport.

3.7 Is this waste stream currently generated; if not, will this waste stream be generated in the next 5 years?

$\square$ Yes 区No. If no, skip to 3.11.

3.8 The current or future generation of this waste is best described as:

$\square$ Routine $\bigotimes$ One-time or sporadic

3.9 Estimated generation projection by fiscal year

$\begin{array}{lllll} & \mathrm{m}^{3} \quad \text { (and/or) } & \mathrm{kg} & \\ 1999 & & & \\ 2000 & & & & \bigotimes \\ 2001 & & & & \\ 2002 & & & & \\ 2003 & & & & \end{array}$

3.10 Last year's fiscal year 1998 generation projection: NA Actual quantity generated in 1998: 0 (both in $\mathrm{m}^{3}$ unless otherwise noted)

3.11 Has there ever been any unusual release of this stream to the environment?

$\square$ Yes $\otimes$ No

If yes, summarize releases and quantities:

\subsection{WASTE STREAM TREATMENT}

4.1 Is this stream currently being treated? 
$\square$ Yes $\bigotimes$ No If yes, provide details:

4.2 Planned treatment: Check the appropriate box indicating future plans for treating this waste stream to meet applicable regulations, including LDR treatment standards.

$\square$ No treatment required (skip to 5.0)
$\square$ Treating or plan to treat onsite
$\square$ Treating or plan to treat offsite
Treatment options still being assessed

4.3 Planned treatment method, facility, extent of treatment capacity available: Milestone M-89-02 provides for removal of all REC B-Cell mixed waste and equipment by May 31, 1999. The 324 Facility will not treat this waste prior to transfer to permitted storage. Waste will be moved from the 324 Facility REC to the PUREX Storage Tunnels.

\subsection{Treatment schedule information: NA}

4.5 Applicable treatment Tri-Party Agreement milestone numbers (including permitting): NA

4.6 If treating or planning to treat on site, was or will waste minimization be addressed in developing and/or selecting the treatment method? $\square$ Yes $\square$ No \Unknown

If yes, please describe: Treatment method unknown.

4.7 Treatability equivalency petitions, rulemaking petitions, and case-by-case exemptions needed for treatment: None known at this time.

\subsection{WASTE STREAM DISPOSAL}

5.1 After treatment, how will the waste stream be disposed of (include description, locations, variances required, etc., as applicable): Some 324 mixed waste will be shipped to the PUREX tunnels and the CWC for interim storage. Waste will be treated and disposed in accordance with the proposed treatment scenarios for these units. Disposal will be in the LLBG Subtitle-C or LLBG unlined trenches depending on regulatory status of the waste after treatment.

5.2 Applicable Tri-Party Agreement milestone number(s) for disposal: None

5.3 Planned disposal dates/time-frame: None

\subsection{WASTE MINIMIZATION}

6.1 Has a waste minimization assessment been completed for this stream?

$\square$ Yes $\bigotimes$ No $\square$ NA

6.2 Explain any waste minimization activities (e.g., process changes) under way or to be implemented for generation of this stream: Waste reduction has been accomplished through waste segregation. Waste volume has been reduced by approximately 35 percent during cleanout of the REC. A waste compactor in the shielded airlock at the 324 REC will be installed.

6.3 Schedule for implementing waste minimization methods: There is no schedule for additional minimization.

6.4 Waste reduction achieved during the calendar year and projected future reductions (volume or mass): None

6.4.1 Assumptions used in above estimates: NA 


\section{WASTE STREAM PROFILE SHEET}

\subsection{WASTE STREAM IDENTIFICATION AND SOURCE}

1.1 Waste stream ID: $\quad$ B PLANT-1

1.2 Waste stream name: B Plant Cell 4 Waste

1.3 Waste stream source information

1.3.1 Stream source and history description. Include how the waste was managed prior to storage, timeframe when waste was placed into storage. WESF hot cell maintenance waste (i.e., manipulator boots, light bulbs, HEPA filters, misc. debris). Containerized in drums. Drums placed in storage from 1988 to 1997.

1.3.2 Source category(s)

$\square$ Pollution control or waste treatment process

Materials production/recovery effluents

Facility or equipment operation and maintenance waste

Discarded excess or expired materials

R\&D/R\&D laboratory waste

Spill clean-ups or emergency response actions

Other (explain):

1.3.3 Additional notes: None

\subsection{WASTE STREAM CHARACTERIZATION}

2.1 Waste stream description (content): Radioactive lead solder from incandescent light bulbs, other miscellaneous radioactive maintenance waste.

\subsection{Radiological characteristics}

2.2.1 Mixed Waste type $\square$ High-level $\square$ Transuranic छLow-level $\square$ Contact-handled $\bigotimes$ Remote-handled

2.2.2 Comments on radiological characteristics (e.g., more specific content, treatment concerns caused by radiation, confidence level): High personnel dose potential, remote handled. Range from $200 \mathrm{mR}$ to $500 \mathrm{R}$ at $30 \mathrm{~cm}$. Confidence high.

2.3 Matrix characteristics (physical content)

2.3.1 Matrix constituent table (comprising at least $1 \%$ of the total volume or mass)

\begin{tabular}{ccc}
$\begin{array}{c}\text { Matrix Parameter } \\
\text { Category Code }\end{array}$ & $\begin{array}{c}\text { Matrix Constituent } \\
\text { Description }\end{array}$ & $\begin{array}{c}\text { Typical } \\
\text { or Range (\%) }\end{array}$ \\
\hline S5110 & Inorganic Debris & $>99$ \\
X7210 & Elemental Lead & $<1$
\end{tabular}

Overall matrix parameter category code: $\mathbf{S 5 1 1 0}$ 
Overall matrix description: Inorganic Debris

2.3.2 Confidence level for matrix characteristics data in 2.3.1

$\square$ Low $\square$ Medium $\otimes$ High

2.3.3 Comments on matrix characteristics and/or confidence level: Lead component represents $<1 \%$ of the entire waste matrix as it is mixed with other miscellaneous non-hazardous radioactive materials in the drum due to packaging constraints in WESF. The lead component is lead solder from contaminated light bulbs. However, due to the packaging constraints, if a drum contains lead in any proportions, the entire drum is managed appropriately for the lead component.

2.4 Regulated contaminant characteristics

2.4.1 Wastewaterinon-wastewater under RCRA

$\square$ Wastewater $\triangle$ Non-wastewater $\square$ Unknown

2.4.2 Regulated contaminant table including treatment requirements, and UHCs if applicable

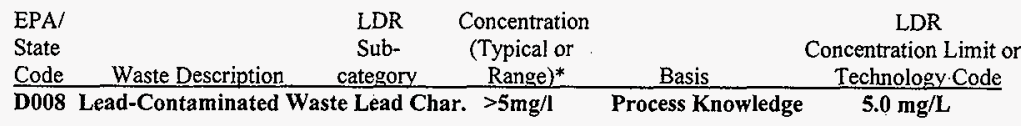

UHCs must be determined.

* If waste isn't consistent in concentration, this may not apply. Describe in 2.4.5.

2.4.2.1 List any waste codes from 2.4.2 for which the stream already meets established LDR treatment standards: None

2.4.3 Does this waste stream contain PCBs?

$\square$ Yes $\bigotimes$ No $\square$ Unknown.

If no, skip to 2.4.4.

2.4.3.1 Is waste stream subject to TSCA regulations for PCBs?

$\square$ Yes $\square$ No $\square$ Unknown

2.4.3.2 Indicate the $\mathrm{PCB}$ concentration range.

$\square<50 \mathrm{ppm} \square \geq 50 \mathrm{ppm} \square$ Unknown

2.4.4 What is the confidence level for the regulated contaminant characteristic data?

$\square$ Low $\square$ Medium 囚High

2.4.5 Comments on regulated contaminant characteristics and/or confidence level: None

2.4.6 Will further characterization be performed? $\square$ Yes $\bigotimes$ No $\square$ Unknown

2.4.6.1 If yes, provide details and schedule: NA

2.4.6.2 If yes, provide Tri-Party Agreement milestone numbers: NA

3.0 WASTE STREAM STORAGE, INVENTORY, AND GENERATION 
3.1 Is this waste stream currently stored? $\square$ Yes $\square$ No $\quad$ If no, skip to 3.7 .

3.2 Current storage method

Plant.

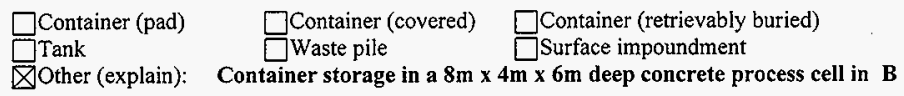

3.3 TSD unit name and building number and (if applicable/available) number of containers/tanks in each: B Plant Complex, Cell 4. 7 drums mixed waste, 36 drums highly radioactive LLW (not mixed waste, reported here for information only).

3.4 is storage of waste in compliance with applicable State and Federal standards?

区Yes $\square$ No

If no, provide details:

3.5 Applicable Tri-Party Agreement milestones related to storage: $\mathbf{M - 8 2 - 8 0}$

3.6 Current inventory for this stream

Total LDR volume (cubic meters): 1.4 (7 drums of mixed waste)

Date of inventory values: $12 / 98$

Comments on waste inventory: NA

3.7 Is this waste stream currently generated; if not, will this waste stream be generated in the next 5 years? $\square$ Yes $\bigotimes$ No. If no, skip to 3.11 .

3.8 The current or future generation of this waste is best described as:

$\square$ Routine $\square$ One-time or sporadic

3.9 Estimated generation projection by fiscal year

$\begin{array}{llll}1999 & \mathrm{~m}^{3} \quad \text { (and/or) } \mathrm{kg} & \\ 2000 & & & \end{array} 2$

3.10 Last year's fiscal year 1998 generation projection: NA

Actual quantity generated in 1998: 0 (both in $\mathrm{m}^{3}$ unless otherwise noted)

3.11 Has there ever been any unusual release of this stream to the environment?

$\square$ Yes $\square$ No

If yes, summarize releases and quantities:

\subsection{WASTE STREAM TREATMENT}

4.1 Is this stream currently being treated?

$\square$ Yes $\bigotimes$ No If yes, provide details:

4.2 Planned treatment: Check the appropriate box indicating future plans for treating this waste stream to meet applicable regulations, including LDR treatment standards.

No treatment required (skip to 5.0) 
DOE/RL-99-0 1

$\square$ Treating or plan to treat onsite

$\square$ Treating or plan to treat offsite

$\triangle$ Treatment options still being assessed

4.3 Planned treatment method, facility, extent of treatment capacity available: TBD

4.4 Treatment schedule information: TBD

4.5 Applicable treatment Tri-Party Agreement milestone numbers (including permitting): $\mathbf{M - 2 0 - 2 1 A}$, "Submit B Plant Pre-Closure Plan"

4.6 If treating or planning to treat on site, was or will waste minimization be addressed in developing and/or selecting the treatment method?. $\square$ Yes $\square$ No $\square$ Unknown

If yes, please describe:

4.7 Treatability equivalency petitions, rulemaking petitions, and case-by-case exemptions needed for treatment: NA

\subsection{WASTE STREAM DISPOSAL}

5.1 After treatment, how will the waste stream be disposed of (include description, locations, variances required, etc., as applicable): Waste will be disposed of in the LLBG Subtitle-C or LLBG LLW trenches depending on the treatment performed.

5.2 Applicable Tri-Party Agreement milestone number(s) for disposal: NA

5.3 Planned disposal dates/time-frame: NA

\subsection{WASTE MINIMIZATION}

6.1. Has a waste minimization assessment been completed for this stream?

$\square$ Yes $\square$ No $\square$ NA

6.2 Explain any waste minimization activities (e.g., process changes) under way or to be implemented for generation of this stream: No additional waste planned to be received in Cell 4

6.3 Schedule for implementing waste minimization methods: NA

6.4 Waste reduction achieved during the calendar year and projected future reductions (volume or mass): NA

6.4.1 Assumptions used in above estimates: NA 


\section{WASTE STREAM PROFILE SHEET}

\subsection{WASTE STREAM IDENTIFICATION AND SOURCE}

1.1 Waste stream ID: B PLANT-2

1.2 Waste stream name: B Plant Containment Building Storage

1.3 Waste stream source information

1.3.1 Stream source and history description. Include how the waste was managed prior to storage, timeframe when waste was placed into storage. Stream consists of failed equipment (e.g., process jumpers, pumps, etc.) used in the 221-B canyon. Waste has been, and will continue to be generated until September 1998 and stored in the B Plant Complex.

1.3.2 Source category(s)

$\square$ Pollution control or waste treatment process

$\square$ Facility or equipment operation and maintenance waste

$\square$ Discarded excess or expired materials

$\square$ R\&D/R\&D laboratory waste

$\square$ Spill clean-ups or emergency response actions

$\triangle$ Other (explain): Facility Deactivation

Materials production/recovery effluents

$\square$ Analytical laboratory waste

Remediation/D\&D waste

Source unknown

\subsubsection{Additional notes: None}

\subsection{WASTE STREAM CHARACTERIZATION}

2.1 Waste stream description (content): Contaminated debris/equipment derived from the processing of "F" listed wastes for the recovery of strontium and cesium. Also contains elemental lead used for counterbalances and shielding.

2.2 Radiological characteristics

2.2.1 Mixed Waste type $\square$ High-level $\square$ Transuranic $\bigotimes$ Low-level $\square$ Contact-handled $\triangle$ Remote-handled

2.2.2 Comments on radiological characteristics (e.g., more specific content, treatment concerns caused by radiation, confidence level): Highly radioactive, remote handled. Confidence high.

2.3 Matrix characteristics (physical content)

2.3.1 Matrix constituent table (comprising at least $1 \%$ of the total volume or mass)

\begin{tabular}{clc}
$\begin{array}{c}\text { Matrix Parameter } \\
\text { Category Code }\end{array}$ & $\begin{array}{c}\text { Matrix Constituent } \\
\text { Description }\end{array}$ & $\begin{array}{c}\text { Typical } \\
\text { or Range }(\%)\end{array}$ \\
\hline $\mathbf{S 5 1 1 0}$ & Inorganic Debris & 99 \\
$\mathbf{X 7 2 1 0}$ & Elemental Lead & 1
\end{tabular}

Overall matrix parameter category code: $\mathbf{S 5 1 0 0}$ 
Overall matrix description: Inorganic Debris

2.3.2 Confidence level for matrix characteristics data in 2.3.1

Jow 区Medium $\square$ High

2.3.3 Comments on matrix characteristics and/or confidence level: Waste inventories are currently maintained by estimates of mass. An exact determination of constituent percentages would require investigation of all drawings. This effort is currently cost and time prohibitive. Percentages are based on engineering estimates.

2.4 Regulated contaminant characteristics

2.4.1 Wastewater/non-wastewater under RCRA

$\square$ Wastewater \Non-wastewater $\square$ Unknown

2.4.2 Regulated contaminant table including treatment requirements, and UHCs if applicable

\begin{tabular}{|c|c|c|c|c|c|}
\hline $\begin{array}{l}\text { EPA/ } \\
\text { State } \\
\text { Code }\end{array}$ & Waste Description & $\begin{array}{l}\text { LDR } \\
\text { Sub- } \\
\text { category }\end{array}$ & $\begin{array}{c}\text { Concentration } \\
\text { (Typical or } \\
\text { Range)* }\end{array}$ & $\begin{array}{r}\text { Conce } \\
\text { Tec }\end{array}$ & $\begin{array}{l}\text { LDR } \\
\text { tration Limit or } \\
\text { nology Code }\end{array}$ \\
\hline F001 & 1,1,1-Trichloroethane & (1) & Unknown & Process Knowledge & (2) \\
\hline $\mathrm{F} 002$ & Methylene Chloride & (1) & Unknown & Process Knowledge & (2) \\
\hline F003 & Acetone, Hexone & (1) & Unknown & Process Knowledge & (2) \\
\hline $\mathrm{F} 004$ & o-Cresol, p-Cresol & (1) & Unknown & Process Knowledge & (2) \\
\hline F005 & Methyl Ethyl Ketone & (1) & Unknown & Process Knowledge & (2) \\
\hline
\end{tabular}

UHCs are not applicable to this waste unless waste is determined to be corrosive.

(1) F001 through F005 Solvent Wastes

(2) Debris standards in 40 CFR 268.48

* If waste isn't consistent in concentration, this may not apply. Describe in 2.4.5.

2.4.2.1 List any waste codes from 2.4 .2 for which the stream already meets established LDR treatment standards: Unknown. Listed waste codes are identified as the waste stored in this unit is derived from the processing of " $F$ " listed waste.

2.4.3 Does this waste stream contain PCBs?

$$
\underset{\text { If no, skip to } 2.4 .4 \text {. }}{\square \text { Yes } \square \text { Unknown. }}
$$

2.4.3.1 Is waste stream subject to TSCA regulations for PCBs?

$$
\square \text { Yes } \square \text { No } \square \text { Unknown }
$$

2.4.3.2 Indicate the $\mathrm{PCB}$ concentration range.

$$
\square<50 \text { ppm } \square>50 \text { ppm } \square \text { Unknown }
$$

2.4.4 What is the confidence level for the regulated contaminant characteristic data?

$$
\square \text { Low } \bigotimes \text { Medium } \square \text { High }
$$

2.4.5 Comments on regulated contaminant characteristics and/or confidence level: Potential exists for other waste characteristies to exist such as corrosivity. However, until each individual component in storage is evaluated for additional characteristics, an assumption has been made that it is unlikely additional waste codes will be required.

2.4.6 Will further characterization be performed? $\square$ Yes $\quad$ No $\square$ Unknown 
2.4.6.1 If yes, provide details and schedule: NA

2.4.6.2 If yes, provide Tri-Party Agreement milestone numbers: NA

\subsection{WASTE STREAM STORAGE, INVENTORY, AND GENERATION}

3.1 Is this waste stream currently stored? $\bigotimes$ Yes $\square$ No If no, skip to 3.7.

3.2 Current storage method

$\begin{array}{lcl}\square \text { Container (pad) } & \square \text { Container (covered) } & \square \text { Container (retrievably buried) } \\ \square \text { Tank } & \square \text { Waste pile } & \square \text { Surface impoundment } \\ \square \text { Other (explain): } & \text { Containment Building } & \end{array}$

3.3 TSD unit name and building number and (if applicable/available) number of containers/tanks in each: B Plant Complex, 221-B Canyon. (Further details in the B-Plant pre-closure work plan, DOE/RL98-12).

3.4 Is storage of waste in compliance with applicable State and Federal standards?

$\triangle$ Yes $\square$ No

If no, provide details:

\subsection{Applicable Tri-Party Agreement milestones related to storage: M-82-00 Agreement Package}

\subsection{Current inventory for this stream}

Total LDR volume (cubic meters): Unavailable

Date of inventory values: 1998

Comments on waste inventory: Quantity estimated at 294,000 kg. This includes an estimated $500 \mathrm{~kg}$ generated in 1998. A more detailed determination of waste volume would require extensive item identification and specific drawing information. At this time, obtaining this information is cost and schedule prohibitive.

3.7 Is this waste stream currently generated; if not, will this waste stream be generated in the next 5 years? $\square$ Yes \No.' If no, skip to 3.11 .

3.8 The current or future generation of this waste is best described as:

$\square$ Routine $\square$ One-time or sporadic

3.9 Estimated generation projection by fiscal year

$\begin{array}{llll}1999 & \mathrm{~m}^{3} \quad \text { (and/or) } \mathrm{kg} & \\ 2000 & & & \end{array}$

3.10 Last year's fiscal year 1998 generation projection: $97,000 \mathrm{~kg}$ Actual quantity generated in 1998: $500 \mathrm{~kg}$ (this is an estimate, but does not include equipment left in place). (both in $\mathrm{m}^{3}$ unless otherwise noted)

3.11 Has there ever been any unusual release of this stream to the environment?

$\square$ Yes $\square$ No

If yes, summarize releases and quantities: 


\subsection{WASTE STREAM TREATMENT}

4.1 Is this stream currently being treated?

$\square$ Yes $\square$ No If yes, provide details:

4.2 Planned treatment: Check the appropriate box indicating future plans for treating this waste stream to meet applicable regulations, including LDR treatment standards.

$\square$ No treatment required (skip to 5.0 )
Treating or plan to treat onsite
$\square$ Treating or plan to treat offsite
Treatment options still being assessed

4.3 Planned treatment method, facility, extent of treatment capacity available: Unknown

4.4 Treatment schedule information: Unknown

4.5 Applicable treatment Tri-Party Agreement milestone numbers (including permitting): $\quad$ M-20-21A

4.6 If treating or planning to treat on site, was or will waste minimization be addressed in developing and/or selecting the treatment method? $\square$ Yes $\square$ No $\quad$ Uunknown

If yes, please describe:

4.7 Treatability equivalency petitions, rulemaking petitions, and case-by-case exemptions needed for treatment: NA

\subsection{WASTE STREAM DISPOSAL}

5.1 After treatment, how will the waste stream be disposed of (include description, locations, variances required, etc., as applicable): Waste will be disposed of in the LLBG Subtitle-C or LLBG LLW trenches depending on the treatment performed.

5.2 Applicable Tri-Party Agreement milestone number(s) for disposal: NA

5.3 Planned disposal dates/time-frame: NA

\subsection{WASTE MINIMIZATION}

6.1 Has a waste minimization assessment been completed for this stream?

$\square$ Yes \No $\square$ NA

6.2 Explain any waste minimization activities (e.g., process changes) under way or to be implemented for generation of this stream: B Plant minimizes as much as practical the use of new equipment in the 221-B canyon.

6.3 Schedule for implementing waste minimization methods: NA

6.4 Waste reduction achieved during the calendar year and projected future reductions (volume or mass): NA

6.4.1 Assumptions used in above estimates: NA 


\section{WASTE STREAM PROFILE SHEET}

\subsection{WASTE STREAM IDENTIFICATION AND SOURCE}

1.1 Waste stream ID: T-Drag-off

1.2 Waste stream name: T Plant Complex Drag-off Box

1.3 Waste stream source information

1.3.1 Stream source and history description. Include how the waste was managed prior to storage, timeframe when waste was placed into storage. Primarily miscellaneous pieces of equipment accumulated over a period of years that have not been completely characterized.

\subsubsection{Source category(s)}

Pollution control or waste treatment process

Materials production/recovery effluents

$\square$ Facility or equipment operation and maintenance waste

$\square$ Discarded excess or expired materials

R\&D/R\&D laboratory waste

Spill clean-ups or emergency response actions

Other (explain):

\subsubsection{Additional notes: None}

\subsection{WASTE STREAM CHARACTERIZATION}

2.1 Waste stream description (content): Miscellaneous pieces of equipment that have not been completely characterized.

\subsection{Radiological characteristics}

\subsubsection{Mixed Waste type $\square$ High-level $\square$ Transuranic \Low-level} \Contact-handled $\square$ Remote-handled

2.2.2 Comments on radiological characteristics (e.g., more specific content, treatment concerns caused by radiation, confidence level): Radionuclides that may be present include $\mathrm{C}-14, \mathrm{Na}-22, \mathrm{Co}-60, \mathrm{Sr}-90$, Zr-95, Tc-99, Cs-137, Eu-152, Eu-154, Eu-155, Np-237, Pu-239, and Am-241. There is low confidence in the radiological characterization since the waste equipment is old and there is limited information on use of the equipment.

\subsection{Matrix characteristics (physical content)}

2.3.1 Matrix constituent table (comprising at least $1 \%$ of the total volume or mass)

\begin{tabular}{ccc}
$\begin{array}{c}\text { Matrix Parameter } \\
\text { Category Code }\end{array}$ & $\begin{array}{c}\text { Matrix Constituent } \\
\text { Description }\end{array}$ & $\begin{array}{c}\text { Typical } \\
\text { or Range (\%) }\end{array}$ \\
\hline S5111 & Metal debris without lead or cadmium & $\mathbf{5 0 \%}$ \\
$\mathbf{S 5 3 3 0}$ & Paper/cloth debris & $\mathbf{2 0 \%}$ \\
$\mathbf{S 5 3 1 2}$ & HOC plastic debris & $29 \%$ \\
$\mathbf{S 5 1 2 2}$ & Glass debris & $1 \%$
\end{tabular}


Overall matrix parameter category code: S5111

Overall matrix description: Metal debris without lead or cadmium

2.3.2 Confidence level for matrix characteristics data in 2.3.1

$\square$ Low $\bigotimes$ Medium $\square$ High

2.3.3 Comments on matrix characteristics and/or confidence level: The matrix characteristics were based on material removed from the drag-off box in the past. Material removed in the future may not have the same characteristics.

2.4 Regulated contaminant characteristics

2.4.1 Wastewater/non-wastewater under RCRA

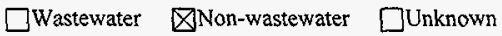

2.4.2 Regulated contaminant table including treatment requirements, and UHCs if applicable

\begin{tabular}{|c|c|c|c|c|c|}
\hline $\begin{array}{l}\text { EPA/ } \\
\text { State } \\
\text { Code } \\
\end{array}$ & Waste Description & $\begin{array}{l}\text { LDR } \\
\text { Sub- } \\
\text { category }\end{array}$ & $\begin{array}{c}\text { Concentration } \\
\text { (Typical or } \\
\text { Range) }\end{array}$ & Basis & $\begin{array}{l}\text { LDR } \\
\text { Concentration Limit or } \\
\text { Technology Code }\end{array}$ \\
\hline F001 & 1,1,1 Trichloroethane & (1) & $0.002 \%$ & Process Knowledge & $\begin{array}{ll} & (2)\end{array}$ \\
\hline F002 & Methylene Chloride & (1) & $0.002 \%$ & Process knowledge & (2) \\
\hline F003 & Acetone & (1) & $0.002 \%$ & Process knowledge & (2) \\
\hline F004 & Cresol/Cresylic Acid & (1) & $0.002 \%$ & Process knowledge & (2) \\
\hline F005 & Methyl Ethyl Ketone & (1) & $0.002 \%$ & Process Knowledge & (2) \\
\hline
\end{tabular}

UHCs are not applicable to this waste.

(1) F001 through F005 Solvent Waste

(2) Debris standards in 40 CFR 268.45

* If waste isn't consistent in concentration, this may not apply. Describe in 2.4.5.

2.4.2.1 List any waste codes from 2.4.2 for which the stream already meets established LDR treatment standards: None, if debris treatment standards are used.

2.4.3 Does this waste stream contain PCBs?

$\square Y e s \square$ Qu Qunnown.

If no, skip to 2.4.4.

2.4.3.1 Is waste stream subject to TSCA regulations for PCBs?

$\square$ Yes $\square$ No $\bigotimes U$ Unknown

2.4.3.2 Indicate the $\mathrm{PCB}$ concentration range.

$\square<50 \mathrm{ppm} \square \geq 50 \mathrm{ppm}$ \Unknown

2.4.4 What is the confidence level for the regulated contaminant characteristic data?

凶Low $\square$ Medium $\square$ High

2.4.5 Comments on regulated contaminant characteristics and/or confidence level: A standard or default estimate of "listed" constituents was used. The waste probably does not contain any of the regulated contaminants, but since the waste may have come into contact with the contaminants in the past, the waste must be considered to contain a minimal percentage of the contaminants.

2.4.6 Will further characterization be performed? $\quad$ Yes $\square$ No $\square$ Unknown 
2.4.6.1 If yes, provide details and schedule: If possible, LLW will be segregated from mixed waste. Funding has not been identified to continue segregation.

2.4.6.2 If yes, provide Tri-Party Agreement milestone numbers: $\mathrm{NA}$

\subsection{WASTE STREAM STORAGE, INVENTORY, AND GENERATION}

3.1 Is this waste stream currently stored? QYes $\square$ No If no, skip to 3.7.

3.2 Current storage method

$\begin{array}{lc}\square \text { Container (pad) } & \square \text { Container (covered) } \\ \square \text { Tank } & \square \text { Container (retrievably buried) } \\ \text { WOther (explain): } & \text { Container is stored within a "containment building." }\end{array}$

3.3 TSD unit name and building number and (if applicable/available) number of containers/tanks in each: T Plant Complex, Building 221-T, one container

3.4 Is storage of waste in compliance with applicable State and Federal standards?

$\triangle$ Yes $\square$ No

If no, provide details:

3.5 Applicable Tri-Party Agreement milestones related to storage: NA

3.6 Current inventory for this stream

Total LDR volume (cubic meters): Approximately $\mathbf{1 0 . 0}$

Date of inventory values: $1 / 29 / 98$

Comments on waste inventory: NA

3.7 Is this waste stream currently generated; if not, will this waste stream be generated in the next 5 years?

$\square$ Yes $\bigotimes$ No. If no, skip to 3.11 .

3.8 The current or future generation of this waste is best described as:

$\square$ Routine $\bigotimes$ One-time or sporadic

3.9 Estimated generation projection by fiscal year

$\begin{array}{llll}1999 & \mathrm{~m}^{3} \quad \text { (and/or) } & \mathrm{kg} & \\ 2000 & & & \end{array}$

3.10 Last year's fiscal year 1998 generation projection: NA Actual quantity generated in 1998: 0 (both in $\mathrm{m}^{3}$ unless otherwise noted)

3.11 Has there ever been any unusual release of this stream to the environment?

$\square$ Yes $\square$ No

If yes, summarize releases and quantities:

\subsection{WASTE STREAM TREATMENT}

4.1 Is this stream currently being treated?

$\square$ Yes $\bigotimes$ No If yes, provide details: 
4.2 Planned treatment: Check the appropriate box indicating future plans for treating this waste stream to meet applicable regulations, including LDR treatment standards.

$\square$ No treatment required (skip to 5.0 )
Treating or plan to treat onsite
$\square$ Treating or plan to treat offsite
$\square$ Treatment options still being assessed

4.3 Planned treatment method, facility, extent of treatment capacity available: The waste may have to be treated before disposal, but treatment has not been planned.

4.4 Treatment schedule information: NA

4.5 Applicable treatment Tri-Party Agreement milestone numbers (including permitting): NA

4.6 If treating or planning to treat on site, was or will waste minimization be addressed in developing and/or selecting the treatment method? $\square$ Yes $\square$ No QUnknown

If yes, please describe:

4.7 Treatability equivalency petitions, rulemaking petitions, and case-by-case exemptions needed for treatment: NA

\subsection{WASTE STREAM DISPOSAL}

5.1 After treatment, how will the waste stream be disposed of (include description, locations, variances required, etc., as applicable): Will be disposed of into the LLBG Subtitle-C or LLBG LLW trenches depending on the type of treatment performed.

5.2 Applicable Tri-Party Agreement milestone number(s) for disposal: None

5.3 Planned disposal dates/time-frame: Not yet planned.

\subsection{WASTE MINIMIZATION}

6.1. Has a waste minimization assessment been completed for this stream?

QYes $\square$ No $\square$ NA

6.2 Explain any waste minimization activities (e.g., process changes) under way or to be implemented for generation of this stream: The waste is to be sorted to separate low-level waste from mixed waste. This will reduce the quantity of mixed waste that must be treated and save money.

6.3 Schedule for implementing waste minimization methods: There is no schedule for continuing or completing this work.

6.4 Waste reduction achieved during the calendar year and projected future reductions (volume or mass): $13.6 \mathrm{~m} 3$.

6.4.1 Assumptions. used in above estimates: NA 


\section{WASTE STREAM PROFILE SHEET}

\subsection{WASTE STREAM IDENTIFICATION AND SOURCE}

1.1 Waste stream ID: T-Condenser

1.2 Waste stream name: T Plant Complex EC-1 Condenser

1.3 Waste stream source information

1.3.1 Stream source and history description. Include how the waste was managed prior to storage, timeframe when waste was placed into storage. OId condenser from the 242-A Evaporator. The condenser was received at T Plant in 1995.

1.3.2 Source category(s)

冈 Pollution control or waste treatment process

Materials production/recovery effluents

Facility or equipment operation and maintenance waste

Discarded excess or expired materials

R\&D/R\&D laboratory waste

$\square$ Spill clean-ups or emergency response actions

$\square$ Other (explain):

\subsubsection{Additional notes: None}

\subsection{WASTE STREAM CHARACTERIZATION}

2.1 Waste stream description (content): Big piece of steel equipment contaminated with listed mixed waste.

2.2 Radiological characteristics

2.2.1 Mixed Waste type $\square$ High-level $\square$ Transuranic $\bigotimes$ Low-level 区Contact-handled $\square$ Remote-handled

2.2.2 Comments on radiological characteristics (e.g., more specific content, treatment concerns caused by radiation, confidence level): The dose rate is $12.0 \mathrm{mRem}$.

2.3 Matrix characteristics (physical content)

2.3.1 Matrix constituent table (comprising at least $1 \%$ of the total volume or mass)

\begin{tabular}{ccc}
$\begin{array}{c}\text { Matrix Parameter } \\
\text { Category Code }\end{array}$ & $\begin{array}{c}\text { Matrix Constituent } \\
\text { Description }\end{array}$ & $\begin{array}{c}\text { Typical } \\
\text { or Range (\%) }\end{array}$ \\
\hline S5111 & Metal debris without lead or cadmium & 100
\end{tabular}

Overall matrix parameter category code: $\mathbf{S 5 1 1 1}$

Overall matrix description: Metal debris without lead or cadmium 
2.3.2 Confidence level for matrix characteristics data in 2.3.1

$\square$ Low $\square$ Medium 囚High

2.3.3 Comments on matrix characteristics and/or confidence level:

2.4 Regulated contaminant characteristics

2.4.1 Wastewater/non-wastewater under RCRA $\square$ Wastewater 区Non-wastewater $\square$ Unknown

2.4.2 Regulated contaminant table including treatment requirements; and UHCs if applicable

\begin{tabular}{llcccc}
$\begin{array}{l}\text { EPA/ } \\
\text { State } \\
\text { Code }\end{array}$ & \multicolumn{1}{c}{ Waste Description } & $\begin{array}{c}\text { LDR } \\
\text { Sub- } \\
\text { category }\end{array}$ & $\begin{array}{c}\text { Concentration } \\
\text { (Typical or } \\
\text { Range)* }\end{array}$ & Basis & $\begin{array}{c}\text { LDR } \\
\text { Concentration Limit or } \\
\text { Technology Code }\end{array}$ \\
\hline F001 & 1,1,1 Trichloroethane & $(1)$ & Unknown & Process Knowledge & $(2)$ \\
F002 & Methylene Chloride & $(1)$ & Unknown & Process Knowledge & $(2)$ \\
F003 & Acetone & $(1)$ & Unknown & Process Knowledge & $(2)$ \\
F003 & Methyl Isobutyl Ketone & $(1)$ & Unknown & Process Knowledge & $(2)$ \\
F004 & Cresol/Cresylic Acid & $(1)$ & Unknown & Process Knowledge & (2) \\
F005 & Methyl Ethyl Ketone & $(1)$ & Unknown & Process Knowledge & $(2)$
\end{tabular}

UHCs are not applicable to this waste.

(1) F001 through F005 spent solvents

(2) Debris standards in 40 CFR 288.45

* If waste isn't consistent in concentration, this may not apply. Describe in 2.4.5.

2.4.2.1 List any waste codes from 2.4 .2 for which the stream already meets established LDR treatment standards: None

2.4.3 Does this waste stream contain PCBs?

$\square$ Yes 区No $\square$ Unknown.

If no, skip to 2.4.4.

2.4.3.1 Is waste stream subject to TSCA regulations for PCBs?

$\square$ Yes $\square$ No $\square$ Unknown

2.4.3.2 Indicate the $\mathrm{PCB}$ concentration range.

$\square<50 \mathrm{ppm} \square \geq 50 \mathrm{ppm} \square$ Unknown

2.4.4 What is the confidence level for the regulated contaminant characteristic data?

$\square$ Low $\$ Medium $\square$ High

2.4.5 Comments on regulated contaminant characteristics and/or confidence level: None

2.4.6 Will further characterization be performed? $\square$ Yes $\square$ No $\quad$ Unknown

2.4.6.1 If yes, provide details and schedule: No funding identified.

2.4.6.2 If yes, provide Tri-Party Agreement milestone numbers: NA

\subsection{WASTE STREAM STORAGE, INVENTORY, AND GENERATION}

3.1 Is this waste stream currently stored? $\bigotimes Y$ es $\square$ No If no, skip to 3.7. 
3.2 Current storage method

$\bigotimes$ Container (pad)
$\square$ Tank
$\square$ Other (explain):

Container (covered)

Waste pile $\square$ Container (retrievably buried)

Surface impoundment

3.3 TSD unit name and building number and (if applicable/available) number of containers/tanks in each:

\section{T Plant Complex}

3.4 Is storage of waste in compliance with applicable State and Federal standards?

$\triangle$ Yes $\square$ No

If no, provide details:

3.5 Applicable Tri-Party Agreement milestones related to storage: NA

3.6 Current inventory for this stream

Total LDR volume (cubic meters): 32.11

Date of inventory values: $1 / 28 / 99$

Comments on waste inventory: NA

3.7 Is this waste stream currently generated; if not, will this waste stream be generated in the next 5 years?

$\square Y$ Yes $\bigotimes$ No. If no, skip to 3.11 .

3.8 The current or future generation of this waste is best described as:

$\square$ Routine OOne-time or sporadic

3.9 Estimated generation projection by fiscal year

$\begin{array}{ccccc}1999 & \mathrm{~m}^{3} & \text { (and/or) } & \mathrm{kg} & \text { NA } \\ 2000 & & & & \\ 2001 & & & & \\ 2002 & & & & \\ 2003 & & & & \end{array}$

3.10 Last year's fiscal year 1998 generation projection: NA

Actual quantity generated in 1998: 0 (both in $\mathrm{m}^{3}$ unless otherwise noted)

3.11 Has there ever been any unusual release of this stream to the environment?

$\square$ Yes 区No

If yes, summarize releases and quantities:

\subsection{WASTE STREAM TREATMENT}

4.1 Is this stream currently being treated?

$\square$ Yes $\square$ No If yes, provide details:

4.2 Planned treatment: Check the appropriate box indicating future plans for treating this waste stream to meet applicable regulations, including LDR treatment standards.

$\square$ No treatment required (skip to 5.0)

Treating or plan to treat onsite

$\square$ Treating or plan to treat offsite

Treatment options still being assessed 
4.3 Planned treatment method, facility, extent of treatment capacity available: Treatment in accordance with alternative debris standards is likely.

\subsection{Treatment schedule information: NA}

4.5 Applicable treatment Tri-Party Agreement milestone numbers (including pernitting): NA

4.6 If treating or planning to treat on site, was or will waste minimization be addressed in developing and/or selecting the treatment method? $\square$ Yes $\square$ No $\square$ Unknown

If yes, please describe:

4.7 Treatability equivalency petitions, rulemaking petitions, and case-by-case exemptions needed for treatment: NA

\subsection{WASTE STREAM DISPOSAL}

5.1 After treatment, how will the waste stream be disposed of (include description, locations, variances required, etc., as applicable): Disposal to the LLBG Subtitle-C or LLBG LLW trenches depending on the type of treatment performed.

5.2 Applicable Tri-Party Agreement milestone number(s) for disposai: None

5.3 Planned disposal dates/time-frame: Not yet planned.

\subsection{WASTE MINIMIZATION}

6.1 Has a waste minimization assessment been completed for this stream? $\square$ Yes $\square$ No $\square$ NA

6.2 Explain any waste minimization activities (e.g.; process changes) under way or to be implemented for -generation of this stream: NA

6.3 Schedule for implementing waste minimization methods: NA

6.4 Waste reduction achieved during the calendar year and projected future reductions (volume or mass): NA

6.4.1 Assumptions used in above estimates: NA 


\section{WASTE STREAM PROFILE SHEET}

\subsection{WASTE STREAM IDENTIFICATION AND SOURCE}

1.1 Waste stream ID: T-Tank

\subsection{Waste stream name: T Plant Complex Tank Trailer Waste}

1.3 Waste stream source information

1.3.1 Stream source and history description. Include how the waste was managed prior to storage, timeframe when waste was placed into storage. The tank trailer (approx. 5,000 gal capacity) contains liquid and sludge from the 222-S Laboratory complex, 219-S tank, and B Plant. The tank trailer is used to transfer waste to the DST system. The tank was emptied as far as the DST system equipment was able. In July 1998, approximately $3,783 \mathrm{~kg}$ (approx. 1,000 gal.) of liquid waste from the $B$ Plant Complex was added to the tank trailer. The trailer is staged at $T$ Plant awaiting approval to send to 204-AR (transfer to DST system).

\subsubsection{Source category(s)}

$\square$ Pollution control or waste treatment process $\square$ Materials production/recovery effluents $\square$ Facility or equipment operation and maintenance waste

Discarded excess or expired materials

$\square$ R\&D/R\&D laboratory waste

Spill clean-ups or emergency response actions Other (explain):

1.3.3 Additional notes: None

\subsection{WASTE STREAM CHARACTERIZATION}

2.1 Waste stream description (content):

2.2 Radiological characteristics

2.2.1 Mixed Waste type $\square$ High-level . $\square$ Transuranic $\bigotimes$ Low-level \Contact-handled $\square$ Remote-handled

2.2.2 Comments on radiological characteristics (e.g., more specific content, treatment concerns caused by radiation, confidence level): $\quad$ Sr-90 - 6.33e-4 Ci; Cs-137 - 6.75e-4; total beta/gamma - $2.58 \mathrm{e}-3$

2.3 Matrix characteristics (physical content)

2.3.1 Matrix constituent table (comprising at least $1 \%$ of the total volume or mass)

\begin{tabular}{ccc}
$\begin{array}{c}\text { Matrix Parameter } \\
\text { Category Code }\end{array}$ & $\begin{array}{c}\text { Matrix Constituent } \\
\text { Description }\end{array}$ & $\begin{array}{c}\text { Typical } \\
\text { or Range }(\%)\end{array}$ \\
\hline L1120 & $\begin{array}{l}\text { Basic wastewater, or } \\
\text { L1220 }\end{array}$ & 100
\end{tabular}


Overall matrix parameter category code: $\mathbf{L 1}$

Overall matrix description: Aqueous Liquids/Slurries

2.3.2 Confidence level for matrix characteristics data in 2.3.1

$\square$ Low $\square$ Medium 区High

2.3.3 Comments on matrix characteristics and/or confidence level: Tank trailer is relatively new, history of waste is well known.

2.4 Regulated contaminant characteristics

2.4.1 Wastewater/non-wastewater under RCRA 冈Wastewater $\square$ Non-wastewater

$\square$ Unknown

2.4.2 Regulated contaminant table including treatment requirements, and UHCs if applicable

\begin{tabular}{|c|c|c|c|c|c|}
\hline $\begin{array}{l}\text { EPA/ } \\
\text { State } \\
\text { Code }\end{array}$ & Waste Description & $\begin{array}{l}\text { LDR } \\
\text { Sub- } \\
\text { category } \\
\end{array}$ & $\begin{array}{c}\text { Concentration } \\
\text { (Typical or } \\
\text { Range)* }\end{array}$ & Basis & $\begin{array}{c}\text { LDR } \\
\text { Concentration Limit or } \\
\text { Technology Code }\end{array}$ \\
\hline F001 & 1,1,1 Trichloroethane & (1) & $0.00001 \%$ & Process Knowledge & $.0054 \mathrm{mg} / \mathrm{L}$ \\
\hline $\mathrm{F} 002$ & Methylene Chloride & (1) & $0.00001 \%$ & Process Knowledge & $.0089 \mathrm{mg} / \mathrm{L}$ \\
\hline F003 & Acetone & (1) & $0.00001 \%$ & Process Knowledge & $.28 \mathrm{mg} / \mathrm{L}$ \\
\hline F003 & Methyl Isobutyl Ketone & (1) & $0.00001 \%$ & Process Knowledge & $.14 \mathrm{mg} / \mathrm{L}$ \\
\hline F004 & Cresol/Cresylic Acid & (1) & $0.00001 \%$ & Process Knowledge & $.88 \mathrm{mg} / \mathrm{L}$ \\
\hline F005 & Methyl Ethyl Ketone & (1) & $0.00001 \%$ & Process Knowledge & $.28 \mathrm{mg} / \mathrm{L}$ \\
\hline D002 & \multirow{2}{*}{\multicolumn{2}{|c|}{ Non-CWA Char. Waste }} & pH 13.6 & Analysis & DEACT and meet 268.48 \\
\hline D007 & & & $39.6 \mathrm{mg} / \mathrm{L}$ & Analysis & $5 \mathrm{mg} / \mathrm{L}$ \\
\hline
\end{tabular}

UHCs have not been determined for this waste stream

(1) F001 through F005 spent solvents.

* If waste isn't consistent in concentration, this may not apply. Describe in 2.4.5.

2.4.2.1 List any waste codes from 2.4.2 for which the stream already meets established LDR treatment standards: Waste probably meets treatment standards as generated for F001 through F005.

2.4.3 Does this waste stream contain PCBs?
$\square$ Yes $\triangle$ No $\square$ Unknown.
If no, skip to 2.4.4.

2.4.3.1 Is waste stream subject to TSCA regulations for PCBs?
$\square$ Yes $\square$ No $\square$ Unknown

2.4.3.2 Indicate the $\mathrm{PCB}$ concentration range.

$\square<50 \mathrm{ppm} \square>50 \mathrm{ppm} \square$ Unknown

2.4.4 What is the confidence level for the regulated contaminant characteristic data?

$\square$ Low $\square$ Medium $\triangle$ High

2.4.5 Comments on regulated contaminant characteristics and/or confidence level: The regulated contaminant concentrations for "listed" wastes are estimates. The waste may contain little or none of these contaminants. Wastewater treatability group status must be verified.

2.4.6 Will further characterization be performed? $\square$ Yes $\bigotimes$ No $\square$ Unknown

2.4.6.1 If yes, provide details and schedule: TBD 
DOE/RL-99-01

2.4.6.2 If yes, provide Tri-Party Agreement milestone numbers: None

\subsection{WASTE STREAM STORAGE, INVENTORY, AND GENERATION}

3.1 Is this waste stream currently stored? $\bigotimes Y$ es $\square$ No If no, skip to 3.7.

3.2 Current storage method

\begin{tabular}{|c|c|c|}
\hline 冈Container (pad) & $\begin{array}{l}\square \text { Container (covered) } \\
\square \text { Waste pile }\end{array}$ & $\square$ Container (retrievably buried) \\
\hline $\begin{array}{l}\square \text { Tank } \\
\text { Other (explain): }\end{array}$ & & \\
\hline
\end{tabular}

3.3 TSD unit name and building number and (if applicable/available) number of containers/tanks in each: T Plant Complex

3.4 Is storage of waste in compliance with applicable State and Federal standards?

$\bigotimes$ Yes $\square$ No

If no, provide details:

3.5 Applicable Tri-Party Agreement milestones related to storage: NA

3.6 Current inventory for this stream

Total LDR volume (cubic meters): Approximately 4.54 cubic meter $(4540 \mathrm{~kg})$

Date of inventory values: $12 / 98$

Comments on waste inventory: This waste was generated at $222 \mathrm{~S}$ Laboratory Complex and B Plant Complex. The trailer will remain in storage at the $T$ Plant Complex pending approval to transfer the waste to 204-AR.

3.7 Is this waste stream currently generated; if not, will this waste stream be generated in the next 5 years?

$\square$ Yes $\square$ No. If no, skip to 3.11 .

3.8 The current or future generation of this waste is best described as:

$\square$ Routine $\square$ One-time or sporadic

3.9 Estimated generation projection by fiscal year

$\begin{array}{lllll}1999 & \mathrm{~m}^{3} \quad \text { (and/or) } & \mathrm{kg} & \\ 2000 & & & & \\ 2001 & & & & \\ 2002 & & & & \\ 2003 & & & & \end{array}$

3.10 Last year's fiscal year 1998 generation projection: NA

Actual quantity generated in 1998: 0 (both in $\mathrm{m}^{3}$ unless otherwise noted)

3.11 Has there ever been any unusual release of this stream to the environment?

$\square$ Yes $\bigotimes$ No

If yes, summarize releases and quantities:

\subsection{WASTE STREAM TREATMENT}

4.1 Is this stream currently being treated?

$\square$ Yes $\bigotimes$ No If yes, provide details: 
4.2 Planned treatment: Check the appropriate box indicating future plans for treating this waste stream to meet applicable regulations, including LDR treatment standards.

$\square$ No treatment required (skip to 5.0)

Treating or plan to treat onsite

Treating or plan to treat offsite

Treatment options still being assessed

4.3 Planned treatment method, facility, extent of treatment capacity available: Waste will be added to the DST system and it will be treated with DST waste.

\subsection{Treatment schedule information: TBD}

4.5 Applicable treatment Tri-Party Agreement milestone numbers (including permitting): NA

4.6 If treating or planning to treat on site, was or will waste minimization be addressed in developing and/or selecting the treatment method? $\square$ Yes $\square$ No Wunknown

If yes, please describe:

4.7 Treatability equivalency petitions, rulemaking petitions, and case-by-case exemptions needed for treatment: NA

\subsection{WASTE STREAM DISPOSAL}

5.1 After treatment, how will the waste stream be disposed of (include description, locations, variances required, etc., as applicable): Currently awaiting certification that the waste is PCB-free. Once certified, waste will be sent the DST system. Refer to DST System treatment plans for treatment and disposal details.

5.2 Applicable Tri-Party Agreement milestone number(s) for disposal: None

5.3 Planned disposal dates/time-frame: TBD

\subsection{WASTE MINIMIZATION}

6.1 Has a waste minimization assessment been completed for this stream? $\square$ Yes 区No $\square$ NA

6.2 Explain any waste minimization activities (e.g., process changes) under way or to be implemented for generation of this stream: Recommended use of a tank trailer for processing 222-S Laboratory Complex waste has been curtailed due to the installation of a dedicated line from the 222-S Laboratory Complex to the DST system. An opportunity assessment is in progress to study liquid waste generation in the laboratory. Once emptied, the trailer will be used for transfer of approximately 25,000 gallons of liquid waste from $T$ Plant to the DST system.

6.3 Schedule for implementing waste minimization methods: NA

6.4 Waste reduction achieved during the calendar year and projected future reductions (volume or mass): It is estimated that the waste reduction is approximately 2000 gal per year. This is based on avoiding two 1000 gal flushes that would have been required if transfers were made by using the tanker instead of the dedicated line.

6.4.1 Assumptions used in above estimates: NA 


\section{WASTE STREAM PROFILE SHEET}

\subsection{WASTE STREAM IDENTIFICATION AND SOURCE}

1,1 Waste stream ID: WESF

1.2 Waste stream name: Cesium and Strontium Capsules

1.3 Waste stream source information

1.3.1 Stream source and history description. Include how the waste was managed prior to storage, timeframe when waste was placed into storage. Cesium and Strontium were removed from Tank Farm waste, separated and purified at B Plant and converted to dry salt for storage at WESF. The cesium and strontium capsules were declared waste in 1997 with the certification for a Part A, Form 3 permit application.

\subsubsection{Source category(s)}
Pollution control or waste treatment process
Facility or equipment operation and maintenan
$\square$ Facility or equipment operation and main
$\square$ R\&D/R\&D laboratory waste
$\square$ Spill clean-ups or emergency response actions
Analytical laboratory waste
Remediation/D\&D waste
$\triangle$ Other (explain): Reclaimed materials from DST or SST wastes

Materials production/recovery effluents

1.3.3 Additional notes:

\subsection{WASTE STREAM CHARACTERIZATION}

2.1 Waste stream description (content): The stream consists of 1335 cesium capsules and 601 strontium capsules. The capsules are double encapsulated and stored in pool celis at WESF.

\subsection{Radiological characteristics}

\subsubsection{Mixed Waste type $\square$ High-level $\square$ Transuranic 凶Low-level} $\square$ Contact-handled $\bigotimes$ Remote-handled

2.2.2 Comments on radiological characteristics (e.g., more specific content, treatment concerns caused by radiation, confidence level): The salt contents consist of purified cesium and strontium salts in the form of cesium chloride and strontium fluoride. The curie content of each capsule varies depending on when it was reclaimed and the amount of impurities it contains. With the daughter products included, there are 95.6 mega curies of Cesium and 42 mega curies of Strontium.

\subsection{Matrix characteristics (physical content)}

2.3.1 Matrix constituent table (comprising at least $1 \%$ of the total volume or mass)

\begin{tabular}{ccc}
$\begin{array}{c}\text { Matrix Parameter } \\
\text { Category Code }\end{array}$ & $\begin{array}{c}\text { Matrix Constituent } \\
\text { Description }\end{array}$ & $\begin{array}{c}\text { Typical } \\
\text { or Range (\%) }\end{array}$ \\
\hline S3140 & Salt Waste & 100
\end{tabular}


DOE/RL-99-01

Overall matrix parameter category code: $\$ 3140$

Overall matrix description: Inorganic Homogeneous Solids - Salt Waste

2.3.2 Confidence level for matrix characteristics data in 2.3 .1

$\square$ Low $\square$ Medium \High

2.3.3 Comments on matrix characteristics and/or confidence level:

2.4 Regulated contaminant characteristics

2.4.1 Wastewater/non-wastewater under RCRA

$\square$ Wastewater $\square$ Non-wastewater $\square$ Unknown

2.4.2 Regulated contaminant table including treatment requirements, and UHCs if applicable

\begin{tabular}{|c|c|c|c|c|c|}
\hline $\begin{array}{l}\text { EPA/ } \\
\text { State } \\
\text { Code }\end{array}$ & Waste Description & $\begin{array}{r}\text { LDR } \\
\text { Sub- } \\
\text { category } \\
\end{array}$ & $\begin{array}{c}\begin{array}{c}\text { Concentration } \\
\text { (Typical or } \\
\text { Range) }\end{array} \\
\end{array}$ & Basis & $\begin{array}{c}\text { LDR } \\
\text { Concentration Limit or } \\
\text { Technology Code }\end{array}$ \\
\hline \multicolumn{6}{|c|}{ Cesium Capsules } \\
\hline D005 & TC-Barium & NA & $0.55-0.94 \%$ & (1) & $100 \mathrm{mg} / \mathrm{l} \mathrm{TCLP}$ \\
\hline D006 & TC-Cadmium & Cadmium Cha & r. $\mathbf{0 . 0 2} \%$ & (1) & $1.0 \mathrm{mg} / \mathrm{l} \mathrm{TCLP}$ \\
\hline D007 & TC-Chromium & NA & $0.02-1.4 \%$ & (1) & $5.0 \mathrm{mg} / 1 \mathrm{TCLP}$ \\
\hline D008 & TC-Lead & Lead Char. & $.14-1.4 \%$ & (1) & $5.0 \mathrm{mg} / \mathrm{l}$ TCLP \\
\hline D0011 & TC-Silver & NA & NA & (1) & $5.0 \mathrm{mg} / 1 \mathrm{TCLP}$ \\
\hline WT02 & Toxic, DW & NA & & & None \\
\hline
\end{tabular}

Strontium Capsules

\begin{tabular}{llcccc} 
D005 & TC-Barium & \multicolumn{1}{c}{ NA } & $0.1-2 \%$ & $(1)$ & $100 \mathrm{mg} / \mathrm{I}$ TCLP \\
D006 & TC-Cadmium & Cadmium Char. $<0.1 \%$ & $(1)$ & $1.0 \mathrm{mg} / \mathrm{l}$ TCLP \\
D007 & TC-Chromium & NA & $<0.2 \%$ & $(1)$ & $5.0 \mathrm{mg} /$ TCLP \\
D008 & TC-Lead & Lead Char. & $<0.2 \%$ & $(1)$ & $5.0 \mathrm{mg} / \mathrm{l}$ TCLP \\
D0011 & TC-Silver & NA & Unknown & $(1)$ & $5.0 \mathrm{mg} / 1$ TCLP \\
WT02 & Toxic, DW & NA & & & None
\end{tabular}

(1) Process knowledge (flowsheets and history)

* If waste isn't consistent in concentration, this may not apply. Describe in 2.4.5.

2.4.2.1 List any waste codes from 2.4.2 for which the stream already meets established LDR treatment standards: None

2.4.3 Does this waste stream contain PCBs?

$\square$ Yes $\bigotimes$ No $\square$ Unknown.

If no, skip to 2.4.4.

2.4.3.1 Is waste stream subject to TSCA regulations for PCBs?

$\square$ Yes $\square$ No $\square$ Unknown

2.4.3.2 Indicate the $\mathrm{PCB}$ concentration range.

$\square<50 \mathrm{ppm} \quad \square \geq 50 \mathrm{ppm} \quad \square$ Unknown

2.4.4 What is the confidence level for the regulated contaminant characteristic data?

$\square$ Low $\square$ Medium छHigh

2.4.5 Comments on regulated contaminant characteristics and/or confidence level: 
2.4.6 Will further characterization be performed? $\square$ Yes $\quad$ No $\square$ Unknown

2.4.6.1 If yes, provide details and schedule:

2.4.6.2 If yes, provide Tri-Party Agreement milestone numbers:

\subsection{WASTE STREAM STORAGE, INVENTORY, AND GENERATION}

3.1 Is this waste stream currently stored? $\quad$ Yes $\square$ No If no, skip to 3.7.

3.2 Current storage method

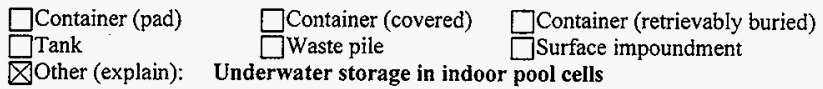

3.3 TSD unit name and building number and (if applicable/available) number of containers/tanks in each: Waste Encapsulation and Storage Facility, 225-B Building, 200-E Area. There are 9 pool cells containing 1335 Cs-137 capsules and 201 Sr-90 capsules.

3.4 Is storage of waste in compliance with applicable State and Federal standards?

$\triangle$ Yes $\square$ No

If no, provide details:

3.5 Applicable Tri-Party Agreement milestones related to storage: None

3.6 Current inventory for this stream

Total LDR volume (cubic meters): 2.0

Date of inventory values: $12 / 31 / 98$

Comments on waste inventory:

3.7 Is this waste stream currently generated; if not, will this waste stream be generated in the next 5 years? $\square$ Yes 冈No. If no, skip to 3.11 .

3.8 The current or future generation of this waste is best described as:

$\square$ Routine $\square$ One-time or sporadic

3.9 Estimated generation projection by fiscal year

$\begin{array}{lllll}1999 & \mathrm{~m}^{3} & \text { (and/or) } & \mathrm{kg} & \\ 2000 & & & & \\ 2001 & & & & \\ 2002 & & & & \\ 2003 & & & & \end{array}$

3.10 Last year's fiscal year 1998 generation projection: NA (not in report)

Actual quantity generated in 1998: 0 (both in $\mathrm{m}^{3}$ unless otherwise noted)

3.11 Has there ever been any unusual release of this stream to the environment?

$\square$ Yes $\otimes$ No

If yes, summarize releases and quantities:

\subsection{WASTE STREAM TREATMENT}


4.1 Is this stream currently being treated?

$\square$ Yes $\bigotimes$ No If yes, provide details:

4.2 Planned treatment: Check the appropriate box indicating future plans for treating this waste stream to meet applicable regulations, including LDR treatment standards.

No treatment required (skip to 5.0 )

$\square$ Treating or plan to treat onsite

Treating or plan to treat offsite

\reatment options still being assessed

4.3 Planned treatment method, facility, extent of treatment capacity available: TBD

4.4 Treatment schedule information: Capsules are expected to be stored at the WESF until 2017.

4.5 Applicable treatment Tri-Party Agreement milestone numbers (including permitting): None

4.6 If treating or planning to treat on site, was or will waste minimization be addressed in developing and/or selecting the treatment method? $\square$ Yes $\square$ No $\bigotimes$ Unknown

If yes, please describe:

4.7 Treatability equivalency petitions, rulemaking petitions, and case-by-case exemptions needed for treatment: TBD

\subsection{WASTE STREAM DISPOSAL}

5.1 After treatment, how will the waste stream be disposed of (include description, locations, variances required, etc., as applicable): Blended with tank farm waste.

5.2 Applicable Tri-Party Agreement milestone number(s) for disposal: None

\subsection{Planned disposal dates/time-frame: 2013-2017}

\subsection{WASTE MINIMIZATION}

6.1 Has a waste minimization assessment been completed for this stream?

$\square$ Yes $\square$ No $\quad \mathrm{NA}$

6.2 Explain any waste minimization activities (e.g., process changes) under way or to be implemented for generation of this stream: Capsules are no longer heing generated.

6.3 Schedule for implementing waste minimization methods: NA

6.4 Waste reduction achieved during the calendar year and projected future reductions (volume or mass): NA

6.4.1 Assumptions used in above estimates: NA 


\subsection{ADDITIONAL WASTE STREAM INFORMATION}

The information in this chapter augments and supports the information in the Waste Stream Profile Sheets in Chapter 3. The format and included subsections vary by waste stream, although generation, storage, and treatment are discussed for all streams.

\subsection{THE HANFORD FACILITY'S SOLID LOW-LEVEL MIXED WASTE}

This section covers waste streams MLLW-01 through MLLW-08.

\subsubsection{MLLW Summary}

MLLW contains concentrations of both low-level radioactive materials and hazardous chemicals. The radioactive component is identified by DOE Order 5820.2A (DOE 1988), and the hazardous component is identified by RCRA or the State Hazardous Waste Management Act. This waste category pertains only to solid waste meeting the nonwastewater treatability group as defined by the federal LDR requirements.

The Hanford Facility's solid MLLW is generated by numerous onsite generator activities and offsite generators authorized by the DOE to ship waste to the Hanford Facility. Currently, the waste is put into aboveground buildings and modules at the CWC located in the 200 West Area. The CWC is operated under interim status until its incorporation into the Hanford Facility RCRA permit. The waste received at the CWC is generated by ongoing operations (e.g., facility stabilization, waste management, SST and DST system operations, etc.) and research and development activities (e.g., SST waste sampling and analysis). Offsite waste has come primarily from DOE research facilities and other DOE processing sites. The characteristics of the MLLW received at the CWC vary greatly; ranging from "derived-from" debris waste to concentrated discarded chemicals ("P" and " $U$ " coded waste). The MLLW contains a variety of contaminated materials, including personal protective equipment, air filters, cleaning solutions, engine oils and grease, spent or unused chemicals, paint residues, soils, construction and building materials, water-treatment secondaries, and decommissioning plant equipment.

As of September 30, 1998, approximately 8990 cubic meters of MLLW are stored at the CWC and 181 cubic meters are stored in Trench 34 of LLBG 218-W-5. Of this volume, approximately 1540 cubic meters currently meet federal and state LDRs and approximately 7630 cubic meters require treatment before land disposal. During the next 5 years, approximately 5470 cubic meters of MLLW are forecast to be generated by both onsite generating organizations and offsite generators. 'Of this volume, approximately 2680 cubic meters would be received meeting federal and state LDRs and approximately 2290 cubic meters would require treatment before land disposal. The MLLW is divided into eight basic categories based on the waste's physical, chemical, and radiological characteristics, and LDR treatment 
dispositions. These categories, titled MLLW-01 through MLLW-08, are summarized in Table 4-1.

Table 4-1. Waste Stream Summaries.

\begin{tabular}{|c|c|c|c|c|c|c|}
\hline $\begin{array}{l}\text { Hanford Site waste } \\
\text { category }\end{array}$ & Description & $\begin{array}{l}\text { Inventory } \\
\text { FY } 1997 \\
\left(\mathrm{~m}^{3}\right)\end{array}$ & \begin{tabular}{|c|} 
Waste \\
receipts \\
FY $1998\left(\mathrm{~m}^{3}\right)$ \\
\end{tabular} & $\begin{array}{c}\text { Inventory } \\
\text { FY } 1998 \\
\left(\mathrm{~m}^{3}\right) \\
\end{array}$ & $\begin{array}{c}5 \text {-yr forecast } \\
\text { generation } \\
\left(\mathrm{m}^{3}\right)\end{array}$ & $\begin{array}{c}\begin{array}{c}\text { Subtotals } \\
\left(\mathrm{m}^{3}\right)\end{array} \\
\end{array}$ \\
\hline RL-MLLW-01A & Backlog soils & 224 & 0 & 224 & 0 & 224 \\
\hline$\overline{R L-M L L W-01 B}$ & $\begin{array}{l}\text { General PP LDR-compliant } \\
\text { Waste }\end{array}$ & 114 & 0 & 114 & 0 & 114 \\
\hline RL-MLLW-01C & $\begin{array}{l}\text { General WSS LDR-compliant } \\
\text { Waste }\end{array}$ & 1004 & 15 & 1019 & 2684 & 3703 \\
\hline RL-MLLW-01D & Trench 34 WSS stored inventory & 181 & 0 & 181 & 0 & 181 \\
\hline RL-MLLW-02A & $\begin{array}{l}\text { General PP inorganic solids and } \\
\text { labpacks }\end{array}$ & 229 & 0 & 229 & 0 & 229 \\
\hline RL-MLLW-02B & $\begin{array}{l}\text { General WSS inorganic solids } \\
\text { and Labpacks }\end{array}$ & 2784 & 22 & 2806 & 165 & 2971 \\
\hline RL-MLLW-03A & $\begin{array}{l}\text { General PP organic solids and } \\
\text { labpacks }\end{array}$ & 275 & 0 & 275 & 0 & 275 \\
\hline$\overline{R L-M L L W-03 B}$ & $\begin{array}{l}\text { General WSS organic solids and } \\
\text { labpacks }\end{array}$ & 200 & 71 & 271 & 278 & 549 \\
\hline RL-MLLW-04A & PP hazardous debris & 2401 & 0 & 2401 & 0 & 2401 \\
\hline RL-MLLW-04B & WSS hazardous debris & $9 \overline{973}$ & 265 & 1238 & 2264 & 3502 \\
\hline RL-MLLW-05A & PP elemental lead & 286 & 0 & 286 & 0 & 286 \\
\hline $\mathrm{RL}-\mathrm{MLLW}-05 \mathrm{~B}$ & WSS elemental lead & 35 & 21 & 56 & 73 & 129 \\
\hline RL-MLLW-06A & PP elemental mercury & 7 & 0 & 7 & 0 & 7 \\
\hline RL-MLLW-06B & WSS elemental mercury & 1 & 1 & 2 & 1 & 2 \\
\hline RL-MLLW-07A & PP RH-MLLW & 38 & 0 & 38 & 0 & 38 \\
\hline RL-MLLW-07B & WSS RH-MLLW & 21 & 2 & 23 & I & 24 \\
\hline RL-MLLW-08 & GTC3 (PP) & 1 & 0 & 1 & 0 & 1 \\
\hline \multicolumn{2}{|c|}{ FY Fiscal vear } & 8773 & 397 & 9170 & 5466 & 14636 \\
\hline \multicolumn{2}{|c|}{$\begin{array}{ll}\text { FY } & \text { Fiscal year } \\
\text { LDR } & \text { Land disposal restrictions } \\
\text { O/C } & \text { Organic/carbonaceous }\end{array}$} & \multicolumn{3}{|c|}{ PP Past practice } & & \\
\hline
\end{tabular}

Waste generation information and records are maintained in the computerized Solid Waste Information and Tracking System database (SWITS), and in the TSD unit operating record. The TSD unit operating record contains the official quality records for the CWC. SWITS readily tracks and summarizes waste inventories and physical, chemical, and radiological information.

\subsubsection{MLLW Generation}

During FY 1998, most MLLW shipped to the CWC was generated in small quantities by routine plant operation and maintenance activities. Generation rates and the type of waste. generated vary greatly. The following 18 locations for onsite generation activity at the Hanford Facility and 3 offsite generators sent waste to the CWC during FY 1998. 
- Onsite Bechtel Hanford, Inc., Dyncorp, the Fast Flux Test Facility, 324 Building, Plutonium Finishing Plant, 200 Area Liquid Effluent Treatment Facility, the CWC, 327 Building, B Plant, Pacific Northwest National Laboratory (includes 305B), K Basins, DST system, SST system, T Plant, 222-S Laboratory, Waste Sampling and Characterization Facility, and well-drilling services

- Offsite Quanterra, Knolls Atomic Power-Laboratory, and Puget Sound Naval Shipyards.

The overall volume of mixed waste generated and received by the CWC during FY 1998 was 397 cubic meters (see Table 4-1). Approximately 65 percent were debris waste. The DST and SST systems were the largest MLLW generation location during the period; the 222-S Laboratory was second. Approximately 2 cubic meters of MLLW were received from offsite generators during FY 1998.

Approximately 16 cubic meters of PCB-contaminated LLW and MLLW were generated during the period. The PCB-contaminated waste was generated by maintenance and periodic flushing of PCB hydraulic systems, failure of transformers and capacitors, and removal of PCB ballasts from light fixtures located in radioactively contaminated areas. The waste is packaged and shipped to the CWC for storage. Future generation of PCB-contaminated waste is expected to vary. Hanford Facility cleanup efforts may identify PCB-contaminated areas that will require cleanup and packaging.

4.1.2.1 MLLW-01 - LDR-Compliant Solids. This waste stream comprises various substreams that either currently meet the LDRs in the regulations, are anticipated to meet the LDRs, or are disposable through specific regulatory action (i.e., contained-in determination, variance, delisting, etc.). The following four profile sheets make up this category.

A) Backlog Soils. Backlog soils consist of 224 cubic meters of dirt, sand, gravel, and rocks excavated from various SST and DST system locations on the Hanford Facility. The waste was part of the Backlog Waste Program and was originally designated with listed waste codes F001 through F005 as being derived from SST and DST system waste. A contained-in determination was obtained from Ecology in 1997 for the waste, which is scheduled for disposal in the LLW portion of the LLBG during FY 1999.

B) General Past-Practice LDR-Compliant Waste. This stream contains 114 cubic meters of past-practice waste anticipated to meet LDRs without additional treatment. Two past-practice WSRds comprise this waste stream: SOW (state only waste) and TFS (tank farms soils).

SOW. Approximately 69 cubic meters of existing waste are designated with state-only dangerous waste codes that are not subject to any LDRs (e.g., WT02, WP02). Once the waste has been verified, the portion of waste that qualifies will be disposed of in the LLBG (Subtitle-C portion). The activity is scheduled for FY 2001. 
TFS. This stream consists of 45 cubic meters of dirt, sand, gravel, and rocks excavated from SST and DST system farms at the Hanford Facility. This waste was generated after the Backlog Waste Program was completed, but came from the same sites as the backlog soils. The waste is designated with listed waste codes F001 through F005 as being derived from SST and DST system waste. The waste will be sampled to verify that the hazardous constituents are below the specified LDR limits. This waste would be disposed of in the appropriate Subtitle-C disposal cell located on the Hanford Site. Disposal currently is scheduled for FY 2001.

C) General Waste Specification System LDR-Compliant Waste. This stream contains approximately 3700 cubic meters (1019 existing and 2684 forecast) of waste received under the WSS. This waste is anticipated to meet LDRs without additional treatment. Currently, four WSS WSRds make up this waste stream: 502 (only waste received from the 200 Area Liquid Effluent Treatment Facility), 903, 930, and 931. The chemical (organics, inorganics, metals) and physical characteristics (debris, particulates, soils, etc.) are very broad for this waste stream. The major contributors to this stream include: SST and DST systems macroencapsulated long-length contaminated equipment (LLCE) items ( 2630 cubic meters), stabilized liquids from the closure of the 183-H Solar Basins (935 cubic meters), and the LDR-compliant secondary solids from 200 Area Liquid Effluent Treatment Facility's thin film dryer (130 cubic meters). This waste is scheduled to be disposed of in the LLBG (Subtitle-C portion) beginning in FY 2000.

D) Trench 34 Stored Inventory. Beginning in FY 1997, Trench 34 of the 218-W-5 LLBG has been used to store MLLW. Waste stored in Trench 34 is LDR compliant and typically large bulky packages that would be difficult to manage in the CWC. This stream includes 10 macroencapsulated LLCE items from the SST/DST systems (95 cubic meters), 22 "ArrowPacs," which are macroencapsulated packages of hazardous debris (64 cubic meters), and 1 macroencapsulated nuclear reactor core basket from the U.S. Navy ( 22 cubic meters). This waste will remain in place when Trench 34 transfers from a storage operation to a disposal operation (currently scheduled to begin in FY 2000); then the waste will be declared disposed of.

4.1.2.2 MLLW-02 - Inorganic Solids. This waste stream consists of inorganic-based waste contaminated with heavy metals. The waste requires treatment to meet all applicable federal and state LDRs. This waste stream contains the following two profile sheets: "General Past-Practice Inorganic Solids \& Labpacks," and "General Waste Specification System Inorganic Solids \& Labpacks."

A) General Past-Practice Inorganic Solids \& Labpacks. This stream consists of 229 cubic meters of inorganic sludge, salt, soil, resins, and aqueous solutions (non-bulk) contaminated with RCRA-listed heavy metals and/or having certain ignitable or corrosive characteristics. This waste was received into the CWC before the WSS was implemented. The waste is generated from many different sources and contains a variety of inorganic contaminants. The primary contributors to this waste stream are Argonne National Laboratory, an offsite generator ( 37 cubic meters), and unverified waste from the 183-H Solar Basin closure, an onsite generator (22 cubic meters).

The common characteristic of this waste stream is that non-thermal treatment technologies (e.g., stabilization and/or deactivation) are either the specified treatment or the best demonstrated 
available technology (BDAT). Because this waste requires upgrading to meet the WSS requirements before treatment, its actual treatment and disposal would take place under waste stream MLLW-02B.

This waste stream also contains the waste associated with past-practice WSRds BAT (lead-acid/cadmium batteries) and ENA (elemental sodium). The treatment path forward has not been determined for this waste, but non-thermal treatment technologies are expected to be used.

B) General Waste Specification System Inorganic Solids \& Labpacks. This stream consists of approximately 2970 cubic meters (2806 existing and 165 forecasted) of inorganic sludge, salt, soil, resins, and aqueous solutions (non-bulk) contaminated with RCRA-listed heavy metals and/or having certain ignitable or corrosive characteristics. This waste was received into the CWC under the requirements of the WSS. The waste is generated from many different sources and contains a variety of inorganic contaminants. The primary generators include sludge and particulates from the 183-H Solar Basin closure (2640 cubic meters), and salt solids containing toxic-characteristic metals from the 200 Area Liquid Effluent Treatment Facility thinfilm dryer ( 50 cubic meters).

The common characteristic of this waste stream is that non-thermal treatment technologies (e.g., stabilization and/or deactivation) are either the specified treatment or the BDAT. Treatment of this waste is scheduled to begin in FY 2001; the waste will be disposed of in the LLBG (Subtitle-C portion) after treatment.

This waste stream also contains the waste associated with WSRds 802 (lead-acid batteries), 820 (water-reactive metals, sodium), and 830 (cadmium-containing batteries). The treatment path forward for these subcomponents of the waste stream has not yet been determined because no treatment capacity is currently available for the waste.

4.1.2.3 MLLW-03 - Organic Solids. These waste stream profile sheets cover various organicand inorganic-based waste types mainly contaminated with organic waste constituents. This stream contains the following two profile sheets: "General Past-Practice Organic Solids \& Labpacks," and "General Waste Specification System Organic Solids \& Labpacks."

A) General Past-Practice Organic Solids \& Labpacks. This stream consists of 275 cubic meters of sludge, salt, particulates, soil, resins, and non-bulk liquids contaminated mainly with organic constituents including PCBs. This waste was received into the CWC before the WSS was implemented. The waste is generated from many different sources and contains a variety of organic contaminates. The PFP onsite generating location ( 45 cubic meters) and Battelle Columbus, an offsite generator ( 26 cubic meters), are the primary contributors to this waste stream.

The common characteristic of this waste stream is that thermal treatment technologies (e.g., combustion, incineration) are either the specified treatment or the BDAT. Because this waste requires upgrading to meet the WSS requirements before treatment, its actual treatment and disposal would take place under waste stream MLLW-03B. 
B) General Waste Specification System Organic Solids \& Labpacks. This stream consists of 550 cubic meters ( 270 existing and 280 forecast) of sludge, salt, particulates, soil, resins, and non-bulk liquids contaminated mainly with organic constituents, including PCBs. This waste was received into the CWC under the requirements of the WSS. The waste is generated from many different sources and contains a variety of organic contaminants. The primary contributors to this waste stream are Argonne National Laboratory, an offsite generator ( 77 cubic meters), and PFP, an onsite generating location (21 cubic meters).

The common characteristic of this waste stream is that thermal treatment technologies (e.g., combustion, incineration) are either the specified treatment or the BDAT. Treatment of this waste is scheduled to begin in FY 2001; this waste will be disposed of in the LLBG (Subtitle-C portion) after treatment.

4.1.2.4 MLLW-04 - Debris. These waste stream profile sheets cover waste meeting the definition of hazardous debris. This category contains the following two profile sheets: PastPractice Hazardous Debris and Waste Specification System Hazardous Debris.

A) Past-Practice Hazardous Debris. This waste stream profile sheet covers past-practice waste meeting the definition of hazardous debris. Currently, 2400 cubic meters of this waste are in CWC storage. This waste was received into the CWC before the WSS was implemented, and was generated from many different sources. The primary onsite generating locations for this waste stream have been TWRS ( $\sim 1700$ cubic meters) and PFP (130 cubic meters).

From evaluations performed on the hazardous debris inventory in the $\mathrm{CWC}$, the waste contains approximately 90 percent organic/carbonaceous $(\mathrm{O} / \mathrm{C})$ constituents (e.g., plastics, rubber, wood, paper, rags, etc.) and 10 percent non-O/C constituents (e.g., metals, concrete, asbestos). Because Hanford Site practices have not segregated $\mathrm{O} / \mathrm{C}$ constituents from non- $\mathrm{O} / \mathrm{C}$ constituents, the entire debris waste inventory is essentially $\mathrm{O} / \mathrm{C}$ waste as defined in WAC 173-303-040. Because this waste requires upgrading to meet the WSS requirements before treatment, its actual treatment and disposal would take place under waste stream MLLW-04B.

B) Waste Specification System Hazardous Debris. This stream consists of 3480 cubic meters (1535 existing and 1945 forecast) of waste meeting the definition of hazardous debris. This waste was received into the CWC under the requirements of the WSS, and was generated from many different sources. The primary onsite generating locations for this waste stream have been TWRS ( 700 cubic meters) and the 222-S Laboratory (206 cubic meters).

From evaluations performed on the hazardous debris inventory in the CWC, the waste contains approximately 90 percent organic/carbonaceous $(\mathrm{O} / \mathrm{C}$ ) constituents (e.g., plastics, rubber, wood, paper, rags, etc.) and 10 percent non- $\mathrm{O} / \mathrm{C}$ constituents (e.g., metals, concrete, asbestos). Because Hanford Facility practices have not segregated $\mathrm{O} / \mathrm{C}$ constituents from non$\mathrm{O} / \mathrm{C}$ constituents, the entire debris waste inventory is essentially $\mathrm{O} / \mathrm{C}$ waste as defined in WAC 173-303-040. The WAC specifies incineration as the minimum management method for treating $\mathrm{O} / \mathrm{C}$ waste (173-303-140(4)(d)(i)). Because non-O/C constituents are normally technically difficult to manage in many thermal treatment units, they are sorted out before thermal treatment and sent for treatment using non-thermal technologies (e.g., immobilization). 
At present, limited capabilities and capacity are available to treat this waste using thermal technologies. To move forward with treating this waste, a certification was submitted to Ecology in accordance with the requirements of WAC 173-303-140(4)(d)(iii). This certification allows debris waste to be treated via immobilization, in accordance with the "Alternative Treatment Standards for Hazardous Debris" (40 CFR 268.45). Some debris macroencapsulation has already been performed, and additional treatment ( 560 cubic meters) is scheduled for FY 1999 at the ATG facility in Richland, Washington. Once Allied Technology Group treatment is available the certificate is anticipated to be invalid.

4.1.2.5 RL-MLLW-05 - Elemental Lead. This waste stream consists of radioactive lead solids and/or debris waste containing radioactive lead solids as defined in 40 CFR 268.40, "Treatment Standards for Hazardous Wastes." This waste stream contains the following two profile sheets: "Past-Practice Elemental Lead" and "Waste Specification System Elemental Lead."

A) Past-Practice Elemental Lead. This waste stream profile sheet covers past-practice waste containing radioactive lead solids. The physical forms of lead include bricks, sheets, pipe, shot, composites (e.g., lead-lined steel doors, blankets, shield walls) and lead-containing articles (light bulbs, printed circuit boards). A significant amount of debris-type material packaged with the elemental lead may not need to be managed as dangerous waste once the lead has been segregated from it. Currently, 286 cubic meters of this waste are in CWC storage. This waste was received into the CWC before the WSS was implemented from many different sources. The primary contributors to this waste stream have been Shippingport Atomic Power Laboratory (offsite) and $100 \mathrm{~N}$ at the Hanford Facility. Because this waste requires upgrading to meet the WSS requirements before treatment, its actual treatment and disposal would take place under waste stream MLLW-05B.

B) Waste Specification System Elemental Lead. This waste stream profile sheet covers WSS waste containing radioactive lead solids. The physical forms of lead include bricks, sheets, pipe, shot, composites (e.g., lead-lined steel doors, blankets, shield walls) and lead-containing articles (light bulbs, printed circuit boards). A significant amount of debris-type material packaged with the elemental lead may not need to be managed as dangerous waste once the lead has been segregated from it. Currently, 56 cubic meters of this waste are in CWC storage and another 73 cubic meters are forecast. This waste was received into the CWC under the requirements of the WSS from many different sources. The primary contributors to this waste stream have been B Plant and 300 Area facilities at the Hanford Facility. The primary path for this waste stream is the specified RCRA treatment, macroencapsulation, in accordance with 40 CFR 268.40. Some of the lead may be recycled through decontamination efforts. Treatment of this stream is planned to begin in FY 2001 at the ATG facility in Richland, Washington.

4.1.2.6 MLLW-06 - Elemental Mercury. This waste stream consists of elemental mercury contaminated with radioactive material as defined in 40 CFR 268.40, "Treatment Standards for Hazardous Wastes." This waste stream contains the following two profile sheets: "Past-Practice Elemental Mercury" and "Waste Specification System Elemental Mercury."

A) Past-Practice Elemental Mercury. This waste stream profile sheet covers past-practice waste containing elemental mercury contaminated with radioactive materials. The physical forms include liquid mercury in labpacks and some amalgamated mercury. A significant amount 
of debris-type material packaged along with the mercury may not need to be managed as dangerous waste once the mercury has been segregated from it. Currently, 7 cubic meters of this waste are in CWC storage. This waste was received into the CWC before the WSS was implemented from many different onsite sources. The primary contributors to this waste stream have been various 300 Area facilities and the PUREX Facility at the Hanford Facility. Because this waste requires upgrading to meet the WSS requirements before treatment, its actual treatment and disposal would take place under waste stream MLLW-06B.

B) Waste Specification System Elemental Mercury: This waste stream profile sheet covers WSS waste containing elemental mercury contaminated with radioactive materials. The physical forms include liquid mercury in labpacks and some amalgamated mercury. A significant amount of debris-type material packaged along with the mercury may not need to be managed as dangerous waste once the mercury has been segregated from it. Currently, 2 cubic meters of this waste are in CWC storage and 1 additional cubic meter is forecast. This waste was received into the CWC under the requirements of the WSS, and was generated by six onsite generating locations. The primary contributor was the PUREX facility. The treatment path for this waste stream is the RCRA-specified treatment of amalgamation, followed by stabilization as applicable. Treatment of this waste is scheduled to begin in FY 2001.

\subsubsection{MLLW-07 - Remote Handled MLLW. This waste stream consists of MLLW in} which the waste package surface dose rate exceeds $200 \mathrm{mR} / \mathrm{hr}$, or the waste inside the package is known to or expected to exceed $200 \mathrm{mR} / \mathrm{hr}$. The waste stream is broad based and comprises various types of debris, inorganic solids, organic solids, and elemental lead. Because of health, safety, and/or physical facility constraints associated with treating this waste, it will remain in storage until treatment technologies and capacity can accommodate the waste. Tri-Party Agreement M-91 milestones address the path forward for this waste stream. This waste stream contains the following two profile sheets: "Past-Practice RH-MLLW" and "Waste Specification System RH-MLLW."

A) Past-Practice RH-MLLW. This waste stream covers past-practice waste where with a package surface dose rate exceeds $200 \mathrm{mR} / \mathrm{hr}$ and/or the waste inside the package exceeds, or is suspected to exceed, $200 \mathrm{mR} / \mathrm{hr}$. This waste was generated and put into CWC storage before the WSS was implemented; therefore, it is considered a past-practice waste. The Hanford Facility began accumulating RH-MLLW in the CWC buildings in 1987. Currently, approximately 38 cubic meters of this waste is stored in the CWC. The waste was generated from various . onsite sources. The primary contributor to this waste stream has been SST/DST system operations. Because this waste requires upgrading to meet the WSS requirements before treatment, its actual treatment and disposal would take place under waste stream MLLW-07B.

B) Waste Specification System RH-MLLW. This waste stream addiresses waste accepted under the WSS requirements that is considered remote-handled (i.e., the package surface dose rate exceeds $200 \mathrm{mR} / \mathrm{hr}$ and/or the waste inside the package exceeds, or is suspected to exceed, $200 \mathrm{mR} / \mathrm{hr}$ ). The waste contains various chemical (organics, inorganics, metals) and physical (particulate, debris, sludges, etc.) characteristics. Many different regulated constituents could be represented in this waste stream; however, the primary waste type is heterogeneous debris from the SST/DST systems operations. Currently, 43 cubic meters of this waste are in CWC storage, and 1 additional cubic meter is forecast. The current WSRds making up this waste stream are 
$601,605,606,800$ and 80 ; the waste has been generated by various sources. The primary onsite generating location has been the 241S Tank Farm. The treatment path for this waste is being determined following the project management plan being formulated under the jurisdiction of the Tri-Party Agreement M-91 milestone series.

4.1.2.8 MLLW-08 - GTC3 MLLW. This waste stream is MLLW that exceeds the Hanford Site Solid Waste Acceptance Criteria (FDH 1998) for Class 3 radioactive material limits. Disposal is currently prohibited by DOE orders. Currently 1.4 cubic meters of this waste, consisting of two radioisotope thermoelectric generators received in the early 1980 s from the U.S. Navy are in CWC storage as LLW. When the waste is backfilled, this waste will be designated as State-only mixed waste because of the lead shielding components in the generators (D008). Waste stream disposition is pending changes to DOE policy affecting GTC3 waste. The disposition path forward is covered under Tri-Party Agreement M-91 milestones. This waste is not prohibited from disposal under WAC 173-303 and may be land disposed of after resolution of DOE policy issues regarding GTC3 waste.

\subsubsection{MLLW Characterization}

From the Sampling and Analysis Plan for Mixed Waste Treatment (Josephson 1996), the Hanford Facility's solid mixed waste is divided chronologically into two categories: newly generated waste and unverified waste (past-practice waste). Waste accepted before the WSS (Kirkpatrick and Oswald 1995) was implemented is called unverified mixed waste. It spans the period between 1987 when MLLW was first put into storage through February 1995. Waste accepted after implementation of the WSS is called newly generated waste. These waste characterization categories are summarized in Sections -4.1.3.1 and 4.1.3.2.

4.1.3.1 Newly Generated Waste Characterization and Designation. This section discusses waste designation and characterization activities performed by the waste generator and the TSD unit receiving the waste.

Before any waste is accepted at the CWC, it must be characterized and packaged as described in the Hanford Site Solid Waste Acceptance Criteria (FDH 1998). These criteria require that the waste generator characterize each individual container of waste with sufficient accuracy to permit proper segregation, storage, treatment, certification, shipment, and, if applicable, disposal. The characterization shall ensure that, on generation and after processing, the actual physical, chemical, and radiological characteristics are recorded and known during all stages of the waste management process.

Waste is designated based on the information provided by the generator. Waste designation is performed by the waste analysis organization as part of a waste acceptance evaluation in accordance with the Hanford Site Solid Waste Acceptance Criteria, and recorded in the SWITS database. The SWITS database includes Washington State and RCRA waste codes resulting from designations based on process knowledge and testing. Each waste stream shipped from a generator to a Hanford Facility TSD must pass a formal compliance assessment before shipment, in accordance with the implementing procedures of DOE Order 5820.2A. 
The dangerous waste designation of each waste package is determined at its point of generation based on process knowledge of the waste placed in the container. The waste is sampled and analyzed if sufficient process knowledge is unavailable.

- Process Knowledge. The waste characterization is typically determined by the waste generator based on documented knowledge of the process generating the waste. Process knowledge is backed by acceptable evidence that relates the characterization to a definite process generating the waste.

Process knowledge has been used to characterize PCB-contaminated LLW currently in storage. Equipment containing PCBs, such as hydraulic systems, transformers, capacitors, and fluorescent light ballasts have been clearly identified. These systems are managed in accordance with 40 CFR 761; waste is immediately handled and packaged as PCB LLW material.

- Testing. If process knowledge is not sufficient to fully characterize a waste package, the generator must augment the characterization with sampling and analysis. Testing is normally required only for characteristic waste constituents (i.e., D00l through D043). The level of analytical data quality is determined by the receiving TSD unit's waste acceptance criteria. The generator also uses sampling and analysis to determine whether its "listed" waste constituents are below a concentration-based treatment standard, thus enabling storage in CWC ready for Subtitle-C disposal.

Hydraulic systems and transformers have been sampled to determine PCB concentrations. Any waste resulting from the management of these systems is designated based on the concentration of PCBs in the source system. Light ballasts are designated based on data from the manufacturers.

Acceptance of newly generated waste requires the generator to complete and sign an LDR notification/certification before the CWC can receive the waste. Acceptance of waste shipped from locations south of the Hanford Facility's 200 Area normally requires that a uniform hazardous waste manifest be prepared for the shipment. Waste generated inside the 200 Area normally is accepted by the onsite waste-transfer protocol as specified by the Hanford Facility's transportation requirements.

An integral component in the waste designation process is the use of WSRds as described in the Hanford Facility's WSS. The WSRds functionally categorize waste being received from various sources into streams requiring similar management. WSRds specify the waste's general radiological description; hazardous constituents; allowable waste codes; recommended packaging, storage, and treatment requirements, and any special handling and/or storage instructions that apply. The WSRds are not location specific and are used for waste from many sources.

4.1.3.2 Unverified Waste Characterization and Designation. This section covers waste designation and characterization activities performed by the CWC TSD unit on unverified waste in storage. 
Unverified MLLW is the principal sampling and analysis problem confronting the CWC TSD unit. Unverified MLLW receipts were governed by individual acceptance documents called storage disposal approval records (SDAR). The SDARs conveyed acceptance criteria to meet safe handling and storage requirements; they do not meet treatment and disposal requirements. Furthermore, because the unverified waste has been in storage for many years, changes in the regulations (e.g., UHCs, State LDRs, etc.) may have made the original waste designation incomplete or obsolete.

In 1996, to address issues of unverified waste, a plan for sampling and analyzing mixed waste treatment was implemented. This plan expands on the WSS by using sorting algorithm software to assign special unverified WSRds to this waste. Once an unverified WSRd is assigned to a waste package, all packages with the same unverified WSRd are scheduled for characterization verification. (See Table 4-2 for the current characterization verification schedule). Characterization verification, "upgrading," consists of reviewing the original characterization records, performing real-time radiography on a specified quantity of packages, sampling and analyzing the waste as needed, then accepting the packages under the newly generated waste acceptance protocol (the WSS). Once the waste has been accepted, the unverified WSRd is converted to the applicable WSS WSRd. Characterization verification began in FY 1997; it will continue until all the unverified waste meets the WSS acceptance criteria.

During FY 1998, 13,630 past-practice MLLW packages were upgraded. Of these, 12,361 were successfully upgraded to WSS WSRds and 1,269 were deferred to a more appropriate pastpractice WSRd. Table 4-3 lists the past-practice WSRds that were upgraded in FY 1998 and the resulting WSRds.

4.1.3.3 Uncertainty of Waste Characterization or Designation. When the waste was first put into storage, the waste characterization and designation were appropriate and compliant for storage purposes. However, because of changes in designation regulations and acceptance rigor, unverified waste will require characterization verification before it can be sent to the proper treatment and/or disposal unit. While waiting for the characterization verifications, the unverified waste does not pose a health or environmental threat.

\subsubsection{MLLW Storage}

This section describes the storage units used to store waste streams MLLW-01 through MLLW-08.

4.1.4.1 Description of Storage Units and Capacities. The waste resides in the CWC located in the 200 West Area. The CWC is an interim-status TSD storage unit and will be incorporated into the Hanford Facility RCRA Permit in 1999. It consists of the following storage buildings and modules. 
Table 4-2. Past-Practice Waste Characterization Verification Schedule.

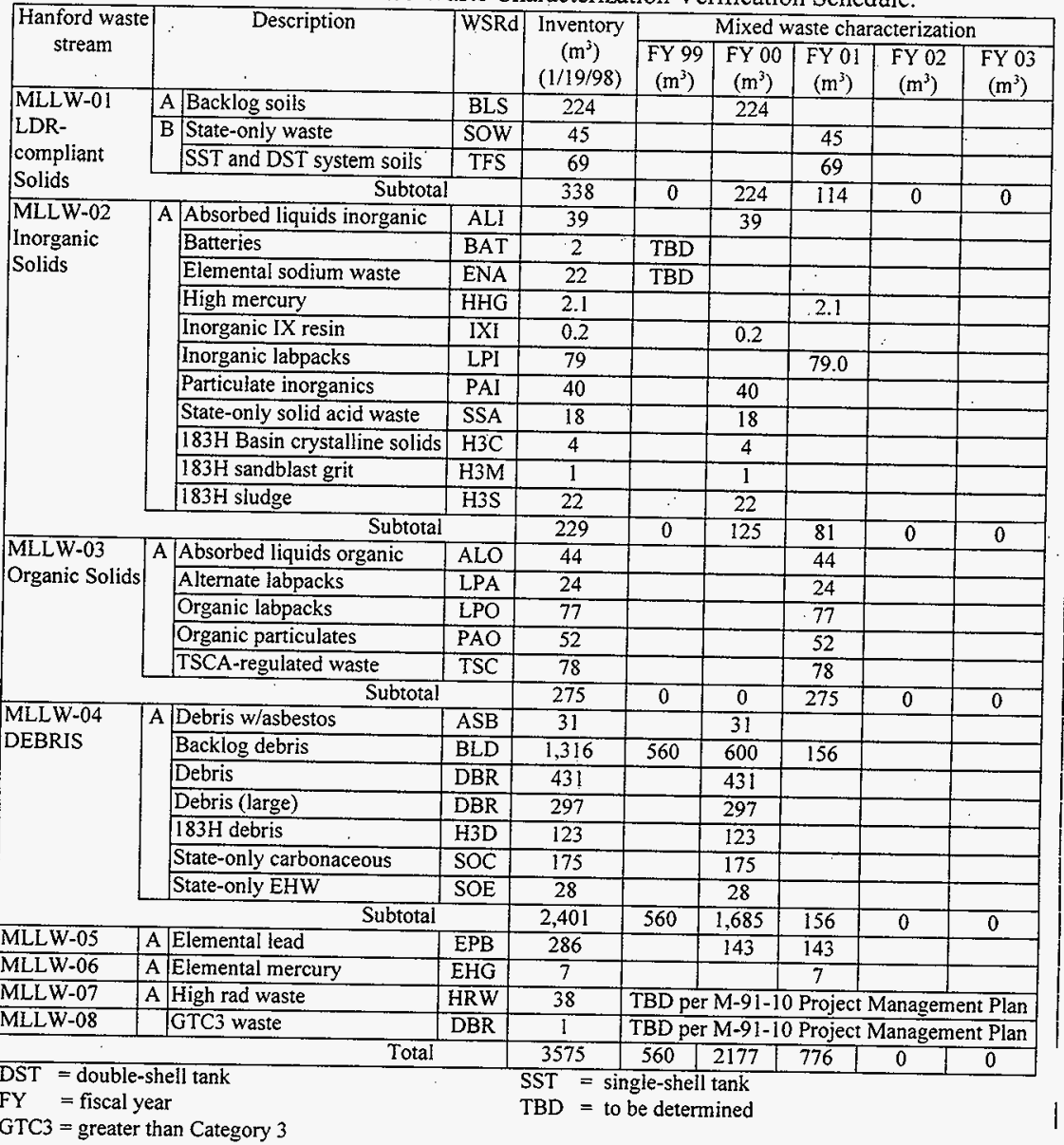


Table 4-3. FY1998 Characterization Verification Activity

\begin{tabular}{|c|c|c|c|c|c|c|c|c|c|c|c|}
\hline \multirow{3}{*}{$\begin{array}{c}\text { Upgraded or } \\
\text { reassigned WSRd }\end{array}$} & \multirow{3}{*}{$\begin{array}{c}\text { Number of } \\
\text { packages }\end{array}$} & \multicolumn{10}{|c|}{\begin{tabular}{|l|} 
Initial WSRd assignment $\mathrm{w} /$ number of packages \\
\end{tabular}} \\
\hline & & $\overline{\mathrm{LPA}}$ & ALI & UUU & PAI & ASB & $\mathrm{HSL}$ & $\mathrm{H3} \mathrm{C}$ & $\mathrm{H} 3 \mathrm{~S}$ & $\mathrm{JH} 3 \mathrm{G}$ & H3D \\
\hline & & 318 & 257 & 408 & 178 & 266 & 2705 & 4370 & 4707 & 198 & 223 \\
\hline 100 & 3 & & & & $\overline{3}$ & & & & & & \\
\hline 101 & 1 & & & & 1 & & & & & & \\
\hline 311 & 12 & & & & 12 & & & & & & \\
\hline 400 & 23 & 23 & & & & & & & & & \\
\hline 401 & $\frac{3}{3}$ & 3 & & & & & & & & & \\
\hline 402 & 7 & 7 & & & & & & & & & \\
\hline 404 & 4 & 4 & & & & & & & & & \\
\hline 406 & 0 & & & & & & & & & & \\
\hline 431 & 0 & 7 & & & & & & & & & \\
\hline 500 & 9 & & 9 & & & & & & & & \\
\hline 501 & 45 & & 45 & & & & & & & & \\
\hline 503 & 1 & & 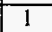 & & & & & & & & \\
\hline 505 & 9230 & & & & & & & 4369 & 4616 & 198 & 47 \\
\hline 506 & 2 & & 2 & & & & & & & & \\
\hline 507 & 2 & & 1 & & 1 & & & & & & \\
\hline 522 & 1 & & 1 & & & & & & & & \\
\hline $53 \mathrm{~A}$ & 5 & & 5 & & & & & & & & \\
\hline 601 & 6 & & 6 & & & & & & & & \\
\hline 621 & 1 & & 1 & & & & & & & & \\
\hline 701 & 317 & & 2 & 315 & & & & & & & \\
\hline 820 & 1 & & 1 & & & & & & & & \\
\hline 930 & 2688 & & & & & & 2688 & & & & \\
\hline ALO & 180 & 4 & 154 & 22 & & & & & & & \\
\hline ASB & 65 & & & & & 65 & & & & & \\
\hline BLD & 146 & & & & & 146 & & & & & \\
\hline BLS & 8 & & & & & 8 & & & & & \\
\hline DBR & 52 & 2 & & 15 & 14 & 21 & & & & & \\
\hline EPB & 9 & & & & & 9 & & & & & \\
\hline ENA & 7 & & & 7 & & & & & & & \\
\hline $\mathrm{H} 3 \mathrm{D}$ & 193 & & & & & & 17 & & & & 176 \\
\hline $\mathrm{H} 3 \mathrm{C}$ & 4 & 1 & & 2 & & & & 1 & & & \\
\hline $\mathrm{H} 3 \mathrm{~L}$ & 12 & & & 12 & & & & & & & \\
\hline $\mathrm{H} 3 \mathrm{M}$ & 0 & 5 & & & & & & & & & \\
\hline $\mathrm{H} 3 \mathrm{~S}$ & 91 & & & & & & & & 91 & & \\
\hline $\mathrm{HRW}$ & 6 & & & & 6 & & & & & & \\
\hline LPA & 1 & & & 1 & & & & & & & \\
\hline LPI & 66 & 53 & 13 & & & & & & & & \\
\hline LPO & 244 & 220 & \begin{tabular}{|l|}
16 \\
\end{tabular} & 8 & & & & & & & \\
\hline PAI & 33 & 1 & & 17 & 15 & & & & & & \\
\hline PAO & 68 & & & 5 & 63 & & & & & & \\
\hline SOW & 2 & & & 1 & & 1 & & & & & \\
\hline SSA & 16 & & & 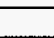 & & 16 & & & & & \\
\hline TCS & 2 & & & & 2 & & & & & & \\
\hline TFS & 64 & & & 3 & 61 & & & & & & \\
\hline
\end{tabular}

WSRd $=$ Waste Specification Record 
2402W through WL Series MW Storage Buildings. This complex consists of 12 individual storage buildings designed to store contact-handled MLLW, TRUM waste, and PCB waste. Each building has 372 square meters of floor space that can hold approximately 100055 -gallon drum equivalents of waste. These buildings provide a combined storage capacity of 12,000 drum equivalents. These 12 buildings ( $2402 \mathrm{~W}$ series) were built between 1988 and 1990.

2403WA-WC Series MW Storage Buildings. This complex consists of three individual storage buildings designed to store contact-handled MLEW and TRUM waste. (TSCA-regulated PCB waste would require additional secondary containment if placed in the buildings.) Each building has 3160 square meters of floor space that can hold approximately 11,600 drum. equivalents. These buildings provide a combined storage capacity of 34,800 drum equivalents. They became operational during 1991.

2403WD MW Storage Building. This storage building was designed to store contacthandled MLLW, TRUM waste, and PCB waste. (TSCA-regulated PCB waste would require additional secondary containment if placed in the buildings.) The $2403 \mathrm{WD}$ building has 5135 square meters of floor space that can hold approximately 17,500 drum equivalents. The building became operational during 1991 .

2404WA-WC Series MW Storage Buildings. This complex consists of three individual storage buildings designed to store contact-handled MLLW, TRUM waste, and PCB waste. Each building has 2000 square meters of floor space that can hold approximately 4600 drum equivalents. These buildings provide a combined storage capacity of 13,800 drum equivalents. They became operational during 1997.

Flammable Mixed-Waste Storage Modules (FS-01-FS-24). This complex consists of 27 individual modules designed to store flammable LLW, TRU waste, mixed LLW, TRU-mixed waste, and PCBs with flash points below $38^{\circ} \mathrm{C}$. Each unit can hold approximately $22 \mathrm{drum}$ equivalents for a total capacity of 528 drum equivalents. The modules are small preengineered buildings with 16.3 square meters of floor space each.

Alkali Metal Waste Storage Modules (AMW-01-AMW-04). This complex consists of four individual modules designed to store contaminated alkali metal (sodium, lithium., etc.) waste. Each unit can hold approximately 21 drum equivalents for a total capacity of 84 drum equivalents. The modules are small preengineered buildings with 16.3 square meters of floor space each.

Waste Unloading and Staging Area. This pad is 836 cubic meters in area and can hold approximately 2500 drums stacked two high. This pad is not intended for long-term storage.

LLBGs (Subtitle-C Portion) (218-W-5 T31 and T34). This complex consists of two individual RCRA-compliant disposal trenches currently used for storage only. Containerized treated MLLW is placed into Trench 34 awaiting the start of disposal operations (scheduled for FY 2000). Each trench has approximately 2300 square meters of RCRA-compliant storage area and can effectively hold approximately 5000 drum equivalents in the storage mode. These storage units became operational during 1997 and eventually will transition to disposal. 
4.1.4.2 CWC Storage Capacity and Existing Stored Volume. The CWC currently has a design capacity of approximately 80,000 drum equivalents (approximately 16,800 cubic meters) of long-term (i.e., inside building storage) storage capacity for mixed, PCB, and TRU waste. The effective operational capacity is approximately 80 percent of the design capacity or 64,000 drum equivalents. The amount of waste currently stored in the CWC is approximately 50,300 drum equivalents $(10,460$ cubic meters). As of September 30,1998, this includes 8,645 cubic meters of MLLW, 255 cubic meters of MLLW with PCBs, 1165 cubic meters of TRUM waste, 79 cubic meters of TRUM waste with PCBs, and 228 cubic meters of LLW. This means that the CWC is at 75 to 80 percent of its operational capacity, and 60 to 65 percent of its design capacity.

Except for low-flash-point modules, which are procured as needed, no plans have been made to build additional long-term storage buildings. The current mixed waste treatment activities and plans would maintain the stored waste volume below the CWC capacity. This situation is contingent on no major increase to current waste forecasts, no change to the LLCE item direct-disposal baseline, and no treatment-program funding shortfalls. The capacity of the $\mathrm{CWC}$ to store mixed waste will be evaluated periodically. If changes to the current programmatic baseline affect the long-term storage capacity needs at the $\mathrm{CWC}$, these will be addressed through the DOE.

\subsubsection{MLLW Treatment}

Sections 4.1.5.1 through 4.1.5.8 describe the treatment activities for waste streams MLLW-01 through MLLW-08.

4.1.5.1 FY 1998 and First Quarter FY 1999 Treatment Activities. During FY 1998 and the first quarter of FY 1999, the following low-level mixed waste treatment activities took place:

Backlog Soils Disposal. Before 1993, the cleanup of various contaminated areas in the SST and DST system generated 260 cubic meters of soil in drums and boxes. The waste was originally designated with listed waste codes F001 through F005 because it was associated with (derived from) the SST and DST system waste. The DOE obtained a "contained-in" determination from Ecology in February 1997 to allow disposal of the waste into the LLBGs (LLW portion). During FY 1997, approximately 30 cubic meters of this waste were disposed of; another 224 cubic meters were scheduled for FY 1998; however, funding for the activity was redirected to the assist in dispositioning the Argonne National Laboratory bulging waste containers at the CWC. Disposal of the remaining backlog soils is pushed out to FY 1999. The remaining 30 cubic meters have TSCA-driven concerns about $\mathrm{PCB}$ contamination that must be addressed before disposal can take place. This waste is part of the MLLW-01A waste stream. The waste volume that has been disposed of is not included in the inventory.

Idaho National Environmental Engineering Laboratory/Bettis Flyash. The Idaho National Environmental Engineering Laboratory (INEEL) sent 0.9 cubic meter of flyash and inorganic non-incinerables during September 1997. The original waste was generated by Bettis Atomic Power Laboratory and was incinerated at INEEL's Waste Experimental Reduction 
Facility (WERF). The waste was received by the T Plant facility where it was treated using stabilization technology. Treatment was completed during the third quarter of FY1998; and the treated waste residues were returned to Bettis Atomic Power Laboratory during August 1998.

4.1.5.2 Planned Treatment Activities. To meet regulatory and consent agreements, a significant amount of the waste currently being stored and newly generated waste will need to be treated and/or disposed of during the next 8 years. The Tri-Party Agreement has several milestones influencing MLLW treatment and disposal. The milestones specifying MLLW disposition volumes and schedule are summarized in the following paragraphs.

M-19-00 For CH-MLLW, this milestone requires that the cumulative treatment and/or disposal volume to be at least 246 cubic meters by the end of FY 2000. It must be 822 cubic meters by the end of FY 2001 and 1644 cubic meters by September 30, 2002.

M-19-01 This milestone requires that treatment of CH-MLLW begin on or before September 30, 1999.

M-91-12 This milestone requires that thermal treatment for CH-MLLW begin by December 2000. At least 600 cubic meters of CH-MLLW must be provided for thermal treatment by December 2005.

M-91-13 This milestone requires disposal of CH-MLLW to begin by June 2001.

M-91-14 This milestone requires that acquisition of facilities be completed and treatment of RH and large-container CH-MLLW initiated by June 2008.

To meet these commitments, the Hanford Facility is following a dynamic program to obtain treatment and/or disposal pathways for MLLW. With the exception of MLLW-08 (GTC3), treatment and disposal pathways have been or are being determined for each waste stream. The Hanford Facility's MLLW baseline disposition map' (see Figure 4-1) depicts these implementing pathways. Table 4-4 shows the current disposition schedule and associated waste volumes.

4.1.5.3 Treatment Projects/Programs. The following summarizes the various treatment projects and programs being pursued at the Hanford Facility to disposition waste streams RL-MLLW-01 through RL-MLLW-07. A disposition path forward has not been established for the RL-MLLW-08 waste stream because the Hanford Site Solid Waste Acceptance Criteria (FDH 1998) prohibit disposing of GTC-C waste, which includes most GTC-3 waste.

\footnotetext{
'The LLMW baseline disposition map covers all LLMW that is forecasted for the life cycle of the Environmental Management function at the Hanford Facility (i.e., through FY 2046).
} 


\section{Hanford MLLW Baseline Disposition Map - Waste Stream Version}

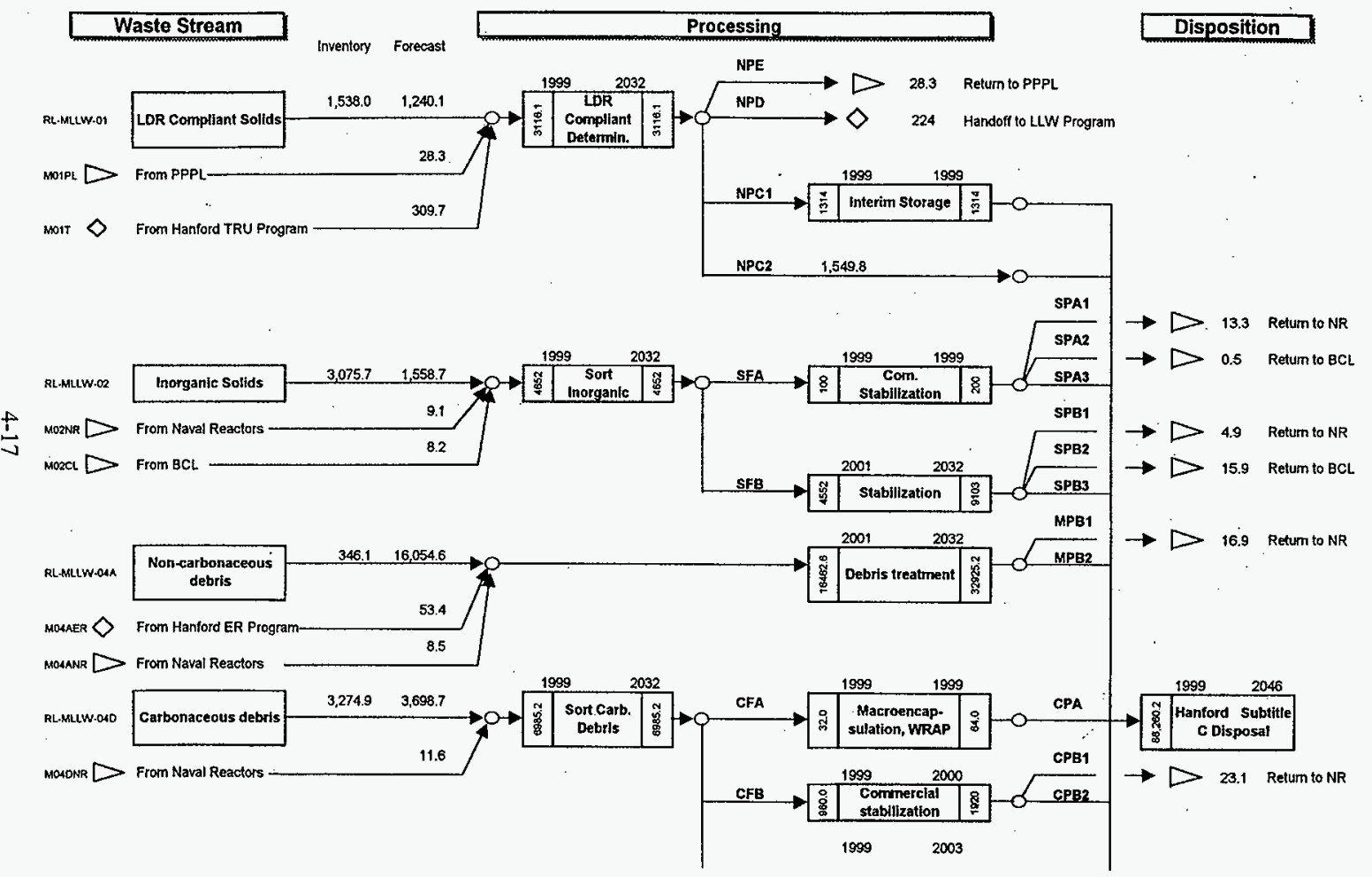

F. M. Coony 3/4/99 9:46 AM 


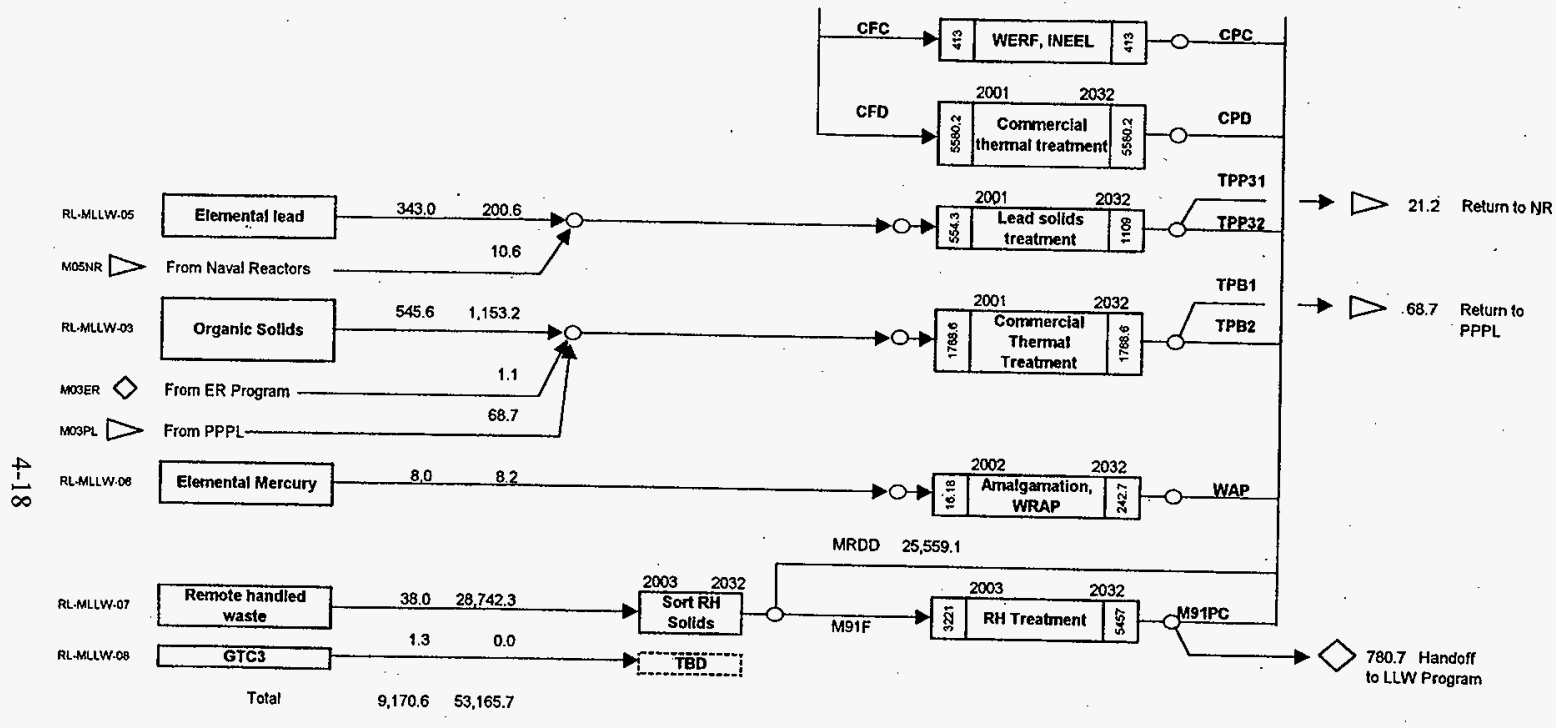


Figure 4-1. Hanford MLLW Baseline Disposition Map. (3 sheets)

Key:

The numbers on the left refer to waste streams as used in this report.

Numbers above boxed indicate the years for the start and end of processes.

Numbers inside boxes on the left and right vertically represent cubic meters of waste at the beginning and end of processes. Numbers above flowchart arrows represent cubic meters of waste in inventory, projected to be generated, or projected for disposal.

Inventory is as of September 30, 1998.

Generation refers to generation in the years 1999 to 2046.

Codes before and after process boxes (e.g., SFA) generally have the following meaning:

The first part of the code denotes the process:

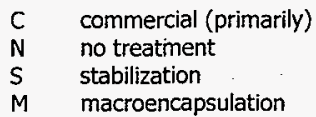

The next part of the code is either $F$ for feed or $P$ for product.

The last part of the code (optional) is sequential (e.g., A, B, C or 1, 2,3).

BCL
ER
GTC3
INEEL
LDR compliant
determin
LLW
MLLW
NR
PPPL
RH
TRU
WERF
WRAP

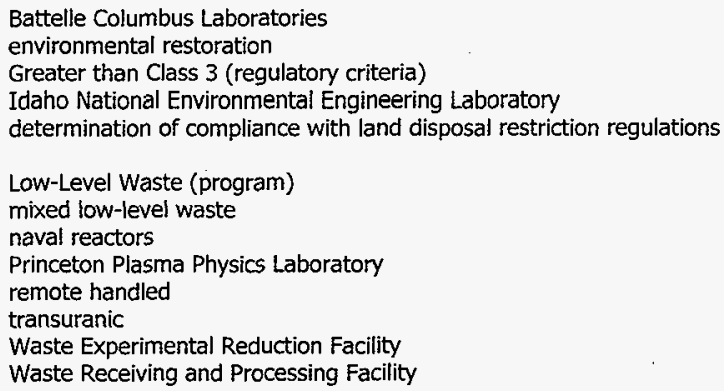


Table 4-4. Waste Treatment Schedule and Volume. (3 sheets)

\begin{tabular}{|c|c|c|c|c|c|c|c|c|c|c|c|}
\hline \multirow{3}{*}{\begin{tabular}{|c|}
$\begin{array}{c}\text { Hanford } \\
\text { waste } \\
\text { stream ID }\end{array}$ \\
MLLW-01
\end{tabular}} & \multirow{2}{*}{$\begin{array}{c}\text { Waste } \\
\text { stream title }\end{array}$} & \multirow{2}{*}{\multicolumn{2}{|c|}{ Description }} & \multirow[t]{2}{*}{ Associated WSRds } & \multicolumn{7}{|c|}{ MLLW treatment and/or disposal volumes } \\
\hline & & & & & $\begin{array}{c}\text { FY 1997 } \\
\left(\mathrm{m}^{3}\right)\end{array}$ & \begin{tabular}{|c|} 
FY 1998 \\
$\left(\mathrm{~m}^{3}\right)$
\end{tabular} & $\begin{array}{c}\begin{array}{c}\text { FY } 1999 \\
\left(\mathrm{~m}^{3}\right)\end{array} \\
\end{array}$ & $\begin{array}{c}\begin{array}{c}F Y 2000 \\
\left(\mathrm{~m}^{3}\right)\end{array} \\
\end{array}$ & $\begin{array}{c}\text { FY 2001 } \\
\left(\mathrm{m}^{3}\right)\end{array}$ & $\begin{array}{c}\mathrm{FY} 2002 \\
\left(\mathrm{~m}^{3}\right)\end{array}$ & $\begin{array}{c}\text { FY 2003 } \\
\left(\mathrm{m}^{3}\right)\end{array}$ \\
\hline & \begin{tabular}{|l|} 
LDR- \\
compliant \\
solids
\end{tabular} & $\mathrm{A}$ & Backlog soils & BLS & 54 & \begin{tabular}{l|l}
0 \\
0
\end{tabular} & 224 & & & & \\
\hline & & $\overline{\mathrm{B}}$ & $\begin{array}{l}\text { General PP LDR- } \\
\text { compliant waste } \\
\end{array}$ & SOW, TFS & 196 & & & & 114 & & \\
\hline & & C & $\begin{array}{l}\text { General WSS LDR- } \\
\text { compliant waste }\end{array}$ & $\begin{array}{l}502(200 \mathrm{LEF}), 903, \\
930,931\end{array}$ & & & & 1132 & 706 & 704 & 142 \\
\hline & & $\mathrm{D}$ & $\begin{array}{l}\text { Trench } 34 \text { stored } \\
\text { inventory (WSS) }\end{array}$ & (various) & & & & 181 & & & \\
\hline & & & & Subtotal $=$ & 250 & 0 & 224 & 1313 & 820 & 704 & 142 \\
\hline MLLW-02 & $\begin{array}{l}\text { Inorganic } \\
\text { solids }\end{array}$ & A & $\begin{array}{l}\text { General PP } \\
\text { inorganic solids \& } \\
\text { labpacks }\end{array}$ & $\begin{array}{l}\text { ALI, BAT, ENA, } \\
\text { HHG, IXI, LPI, } \\
\text { PAI, SSA, H3C, } \\
\text { H3M, H3S } \\
\end{array}$ & & & & & & & \\
\hline & & B & \begin{tabular}{|l|} 
General WSS \\
inorganic solids \& \\
labpacks
\end{tabular} & $\begin{array}{l}420,42 \mathrm{I}, 422,425, \\
426,428,429 \\
44 \mathrm{~A}, 500(183 \mathrm{H}), \\
501-0 \& 1,503-0 \\
\& 1,504-0,505 \\
\text { (except 505-3), } \\
521,523,524,525, \\
802,812,820,821, \\
82 \mathrm{~A}, 830,900, \\
901,902,904, \\
90 \mathrm{~A}, \\
\end{array}$ & 3 & 1 & & & 431 & 431 & 431 \\
\hline MLLW-03 & $\begin{array}{l}\text { Organic } \\
\text { solids }\end{array}$ & $\mathrm{A}$ & $\begin{array}{l}\text { General PP organic } \\
\text { solids \& labpacks }\end{array}$ & $\begin{array}{r}\text { Subtotal }= \\
\text { ALO, LPA, } \overline{\mathrm{LPO}} \\
\mathrm{PAO}, \mathrm{TSC}\end{array}$ & 3 & 1 & 0 & 0 & 431 & 431 & $43 !$ \\
\hline
\end{tabular}


Table 4-4. Waste Treatment Schedule and Volume. (3 sheets)

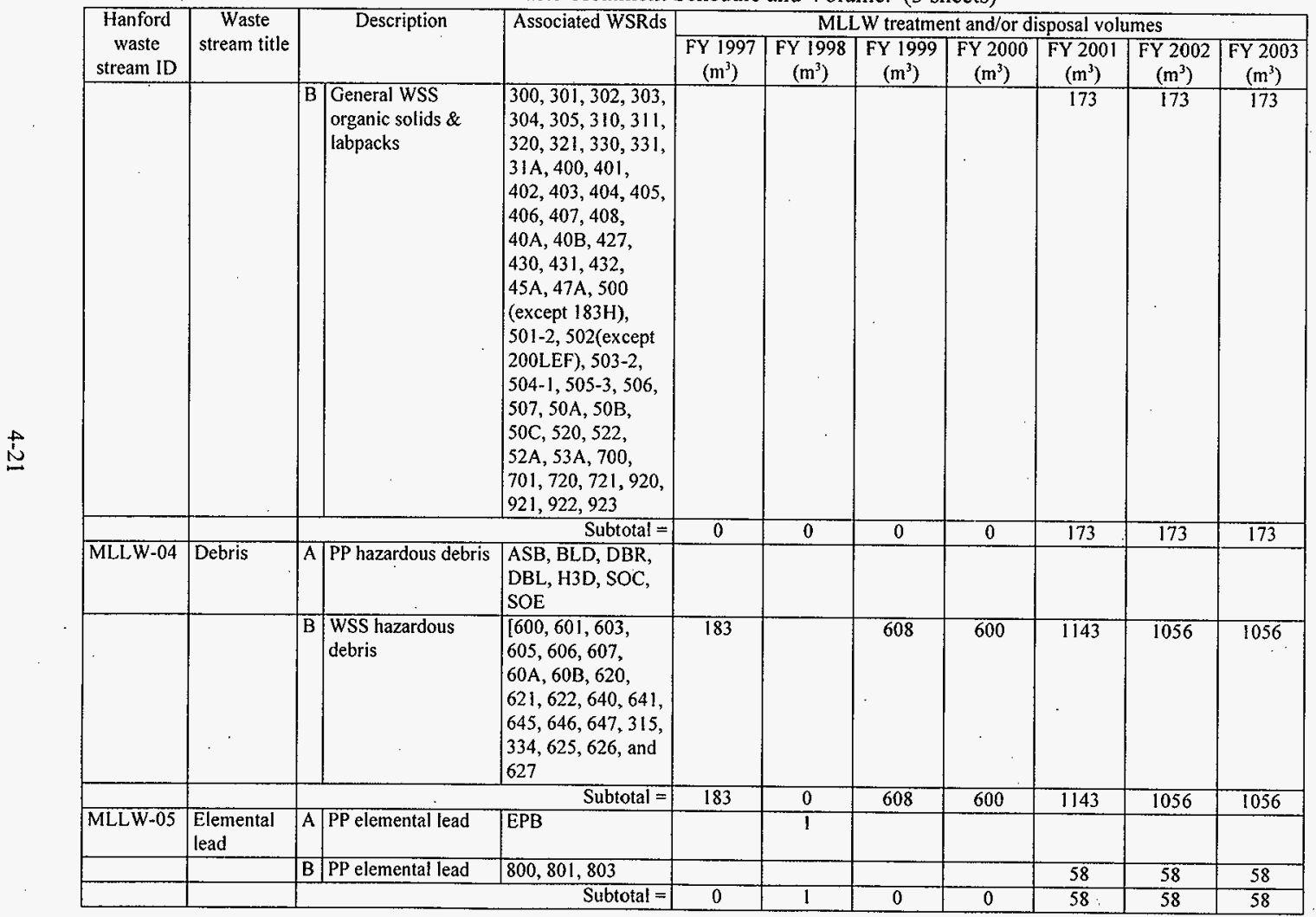


Table 4-4. Waste Treatment Schedule and Volume. (3 sheets)

\begin{tabular}{|c|c|c|c|c|c|c|c|c|c|c|c|}
\hline \multirow{2}{*}{$\begin{array}{c}\text { Hanford } \\
\text { waste } \\
\text { stream ID }\end{array}$} & \multirow{2}{*}{$\begin{array}{c}\text { Waste } \\
\text { stream title }\end{array}$} & \multirow{2}{*}{\multicolumn{2}{|c|}{ Description }} & \multirow[t]{2}{*}{ Associated WSRds } & \multicolumn{7}{|c|}{ MLLW treatment and/or disposal volumes } \\
\hline & & & & & $\begin{array}{c}\text { FY } 1997 \\
\left(\mathrm{~m}^{3}\right)\end{array}$ & $\begin{array}{c}\text { FY } 1998 \\
\left(\mathrm{~m}^{3}\right)\end{array}$ & $\begin{array}{c}\text { FY } 1999 \\
\left(\mathrm{~m}^{3}\right)\end{array}$ & $\begin{array}{c}\begin{array}{c}\text { FY } 2000 \\
\left(\mathrm{~m}^{3}\right)\end{array} \\
\end{array}$ & $\begin{array}{c}\text { FY } 2001 \\
\left(\mathrm{~m}^{3}\right)\end{array}$ & $\begin{array}{c}\text { FY 2002 } \\
\left(\mathrm{m}^{3}\right)\end{array}$ & $\begin{array}{c}\text { FY } 2003 \\
\left(\mathrm{~m}^{3}\right)\end{array}$ \\
\hline MLLW-06 & $\begin{array}{l}\text { Elemental } \\
\text { mercury }\end{array}$ & $\bar{A}$ & $\begin{array}{l}\text { PP elemental } \\
\text { mercury }\end{array}$ & $\overline{\mathrm{EHG}}$ & & & & & & & \\
\hline & & B & $\begin{array}{l}\text { WSS elemental } \\
\text { mercury }\end{array}$ & 810,811 & & & & & 2 & 2 & 2 \\
\hline & & & & Subtotal $=$ & 0 & 0 & 0 & 0 & 2 & 2 & $\overline{2}$ \\
\hline MLLW-07 & $\begin{array}{l}\text { Remote- } \\
\text { handled - } \\
\text { MLLW }\end{array}$ & $\mathrm{A}$ & PP RH-MLLW & HRW & & & & & & & \\
\hline & & $\mathrm{B}$ & WSS RH-MLLW & (various) & & & & & & & \\
\hline & & & & Subtotal $=$ & $\overline{0}$ & 0 & 0 & 0 & 0 & 0 & 0 \\
\hline MLLW-08 & GTC3 (PP) & & & DBR (RTGs only) & & & & & & & \\
\hline & & & & Total $=$ & 436 & 2 & 832 & 1913 & 2627 & 2424 & 1862 \\
\hline $\begin{array}{l}\text { MLLW } \\
\text { PP } \\
\text { RH }\end{array}$ & $\begin{array}{l}\text { mixed low-le } \\
\text { past practice } \\
\text { remote handl }\end{array}$ & & waste & & $\begin{array}{l}\text { WSRd } \\
\text { WSS }\end{array}$ & $\begin{array}{l}=\text { Waste } \\
=\text { Waste }\end{array}$ & $\begin{array}{l}\text { Specificatio } \\
\text { Specificatic }\end{array}$ & $\begin{array}{l}\text { n Record } \\
\text { n System }\end{array}$ & & & \\
\hline
\end{tabular}


Waste Receiving and Processing Facility Module 1 (WRAP-1): The WRAP-1 facility is located in the 200 West Area. It became operational in March 1997 with the startup of its nondestructive evaluation and nondestructive analysis (NDE/NDA) line. LLW processing and verification began in September 1998. TRUM waste processing is scheduled to begin in FY 2000. WRAP-1 will accept contact-handied TRUM waste and MLLW in both box and drum forms. Capabilities are limited to NDE/NDA of boxes. Capabilities for drummed waste include NDE/NDA, segregation, decontamination, characterization, verification, and treatment. Operations other than NDE/NDA are performed in the TRU waste and MLLW restricted-waste gloveboxes. MLLW treatment capabilities include neutralization, stabilization, amalgamation, macroencapsulation, and controlled reaction with water; however, the capacity is limited and the activity currently is not funded.

Thermal Treatment Program. The thermal treatment program consists of two private and two DOE-complex facilities. Each waste package requiring thermal treatment (streams MLLW-03B, and MLLW-04B when O/C waste debris requires thermal treatment) will be evaluated and assigned to the best alternative. The private facilities are the ATG Richland thermal treatment facility and the DSSI incinerator at Oak Ridge, Tennessee. The DOE complex facilities are the WERF incinerator at the INEEL and the incinerator at Oak Ridge National Laboratory operated under the TSCA. Currently only the ATG and WERF facilities are used in the baseline dispositions.

ATG Richland Thermal Treatment Facility. The ATG Richland thermal treatment facility will be able to treat mixed waste and alpha-contaminated PCB waste in solid, absorbed liquid, and laboratory packed-liquid forms. Incoming waste will be segregated into two fractions. One will require thermal treatment; the other may be treated by less expensive lowtemperature methods. For the thermal treatment fraction, ATG will thermally destroy organic hazardous constituents and radionuclides in a grouted final form as needed to stabilize any metals.

A contract is in place with the ATG facility to begin thermal treatment of Hanford Facility waste during FY 2001. At this point, it is anticipated that the State-only LDR for organic/carbonaceous waste will become applicable to disposal activities once again. The contract is for a 5-year base period with five 1-year options. The contract with ATG will allow thermal treatment of up to 717 cubic meters of waste during each base-period year (FY 2001through FY 2005) and up to 310 cubic meters during each option year. Currently, ATG is going through a joint permitting process (i.e., RCRA and TSCA) with permit issuance scheduled for spring 1999.

WERF Incinerator. The WERF incinerator at the INEEL can treat combustible mixed waste in solid or absorbed liquid forms. The WERF incinerator does not accept PCB waste. The WERF incinerator has limited capability for segregating waste into combustible and noncombustible fractions. Combustible fractions are packed into incinerable boxes $(0.6$ cubic foot) and mechanically fed into a controlled-air incinerator. Noncombustible waste and treatment residues are returned to the generator. The WERF incinerator currently is operating and can process up to 518 cubic meters of waste annually. The Hanford Facility is scheduled to have 16 cubic meters of waste incinerated at the WERF facility during FY 1999 (O/C debris), with additional volumes (up to 100 cubic meters per year) during FY 2000 through FY 2003). 
Non-Thermal Treatment Program. The non-thermal treatment program was initiated after the WRAP Module 2A project was terminated in December 1994. The program is scoped to disposition waste that was originally assigned to WRAP $2 \mathrm{~A}$. The program consists of three components: commercial treatment, onsite treatment, and direct disposal. Waste that does not require thermal treatment will be evaluated container by container and assigned to the best disposition path.

Commercial Treatment. This component will procure treatment services to meet RCRA and WAC requirements. Targeted technologies include macroencapsulation for debris and elemental lead waste and stabilization for particulates, soil, and sludge. The waste may be treated at the vendor's site under the vendor's RCRA permit, or by the vendor at the Hanford Facility under the Hanford Facility RCRA permit. Contracts will be placed for individual streams so the best treatment process and location can be selected for each stream. Waste streams designated for commercial treatment are generally those with large volumes that would require substantial capital investment or new facilities to treat effectively. Treatment will begin no later than September 1999, as required by Tri-Party Agreement milestone M-19-01.

A commercial contract has been awarded to the ATG in Richland, Washington, to treat up to 560 cubic meters of inorganic solids (MLLW-02B) and/or 1760 cubic meters of debris (MLLW-04B). Treatment is scheduled to begin in June 1999 with the treatment of 560 cubic meters of $\mathrm{O} / \mathrm{C}$ debris. Treatment will be by macroencapsulation; therefore, the Hanford Facility contractors will be using the $\mathrm{O} / \mathrm{C}$ waste exemption that was certified to the WDOE during December 1998. Treatment under this contract could be extended through FY 2001 if the contract options are picked up. Currently, ATG is going through a joint permitting process (i.e., RCRA and TSCA) with permit issuance scheduled for spring 1999.

Onsite Treatment. This component will use existing small-scale macroencapsulation, neutralization, and stabilization capabilities at the T Plant complex to treat specialty waste streams. This component is separate from amalgamation, neutralization, and stabilization treatment at WRAP. Waste streams designated for onsite treatment are generally those with small volumes that would not require substantial capital investment or new facilities for effective treatment or those that require significant pretreatment characterization and sorting (e.g., labpacks). This component is currently operating.

Direct Disposal. This component seeks to meet LDR treatment standards without additional treatment, as recommended by the report from RL's direct disposal team (RL 1995). Typically, direct disposal efforts use analytical data to demonstrate that waste meets LDR treatment standards or pursues regulatory relief of LDR treatment standards when technically advisable. Waste streams designated for direct disposal are those where existing analytical data or process knowledge indicate that the concentration of hazardous constituents is small or those where some type of treatment already has been performed. Direct disposal waste volumes are included in waste streams MLLW-01A, B, and C.

Milestone M-91 Program. This program is the successor to the M-33 milestone discussions and will develop treatment capability, where applicable, for remote-handled and large-size TRU waste, TRUM waste, MLLW, and GTC 3 waste. Treatment alternatives will be evaluated by developing separate project management plans for TRU and TRUM waste (M-91-03) and for MLLW and GTC3 waste (M-91-10). For each waste type, the preferred 
alternative developed in the project management plan will be constructed or procured. Capability to process TRU and TRUM waste will be available no later than June 2005. Capability to treat MLLW and GTC3 waste will be available no later than June 2008.

\subsubsection{MLLW Strategic Plan Objective}

Accelerated treatment plans and scoping studies also have been pursued by DOE-HQ, $\mathrm{RL}$, and the Facility's contractors to meet the "10-year plan" objectives laid out by the DOE Office of Environmental Restoration and Waste Management during 1996 and 1997. This objective has been carried forward into the Strategic Plan for the Hanford Facility's PHMC. The goal and planning guidance is to treat and/or directly dispose of all CH-MLLW that has been in storage for more than 1 year by FY 2008. This objective is commonly called "getting-whole" and would set the stage for treating and disposing of waste in the same year in which it is generated.

\subsubsection{MLLW Waste Reduction}

All plants and processes that generate waste shipped to the CWC must have a waste minimization program and waste specification summaries in place.

\subsubsection{MLLW Treatability Variances, Equivalency Petitions, Rulemaking Petitions, and Case-by-Case Exemptions}

If treatability variances, equivalency petitions, rulemaking petitions, or case-by-case exemptions are required because of delays in the development of treatment, storage, or disposal capacity or the demonstrated need for using alternative treatment technologies, they will be applied for in accordance with the procedures detailed in the Tri-Party Agreement and/or regulations.

The following list summarizes approved variances, alternative treatments, and containedin determinations.

- A treatment equivalency petition has been approved by EPA and Ecology in accordance with 40 CFR 268.42(b) for treating the formic acid in the 183-H Solar Basin waste. The treatment equivalency allows for nonthermal "stabilization" treatment in lieu of thermal "combustion" treatment.

- In February 1997, Ecology granted a "contained-in" determination for 828 drums of backlog waste soil from the SST and DST systems. This determination allowed waste codes F001 through F005 to be removed from these drums. State-only dangerous waste codes are not required for the PCBs contained in the waste; however, all soil containing PCBs with concentrations higher than 50 parts per million still must be managed in accordance with the TSCA. This determination was the result of reviewing analytical data from soil samples. 
- In March 1997, Ecology granted DOE an exemption to the O/C State-only LDR for 880 drums of Hanford Facility debris mixed waste. The exemption allowed the use of debris "macroencapsulation" treatment in lieu of thermal "incineration" treatment. The macroencapsulation treatment was performed during FY 1997.

- In December 1998, RL submitted a certification for the state-only LDR for O/C waste to Ecology. The certification was submitted in accordance with the requirements of WAC 173-303-140(4)(d)(iii). By using this exemption, the Hanford Facility contractors can pursue other treatment technologies that are appropriate for the waste; primarily immobilization, in accordance with the Alternative Treatment Standards for Hazardous Debris (40 CFR 268.45). Ecology will periodically assess thermal treatment capacity for MLLW O/C waste. When Ecology deems that significant capacity does exist and $\mathrm{RL}$ cannot demonstrate otherwise, the certification will be invalid and the Hanford Facility will be required to use thermal destruction of $\mathrm{O} / \mathrm{C}$ debris or pursue exemptions on a waste-stream basis.

\subsection{TRUM WASTE SUMMARY}

Transuranic mixed waste contains concentrations of both transuranic radioactive materials and hazardous chemicals, including PCBs. The radioactive component is identified by DOE Order 5820.2A. This waste category pertains only to solid waste meeting the nonwastewater treatability group as defined by federal LDR requirements.

Although DOE established a policy for segregating radioactive waste with long-lived radionuclides in 1970, it was not until RCRA was adopted at the Hanford Facility in 1987. that TRU waste containing RCRA-defined hazardous waste constituents was managed as mixed TRU waste. With the adoption of RCRA, mixed TRU waste has been placed in storage units operating under interim status. The storage units will be incorporated into the Hanford Facility RCRA permit. Most mixed TRU waste is placed at the CWC. Waste is presently generated from onsite operations and research facilities. Offsite waste has not been received since 1989, pending the startup of WIPP.

Mixed TRU waste streams are organized by radiological and physical characteristics. Mixed TRU waste contains TRU radioactivity greater than $100 \mathrm{nCi} / \mathrm{g}$. In most of the waste streams, non-transuranic radioactivity either is not present or is present in a trace amount. Some mixed TRU waste does contain significant non-TRU radioactivity. This waste usually is shielded so that it can be stored at CWC. Most of the mixed TRU waste is debris.

The hazardous characteristics of mixed TRU waste are highly varied. Some of the waste is designated with waste codes F001 through F005 because of the "derived-from" rule. Otherwise, the waste is designated with many of the organic or metal hazardous characteristic constituents.

As of September 30,1998, 336 cubic meters of mixed TRU waste are stored at the CWC. Because mixed TRU waste is to be disposed of at the WIPP facility outside Washington State, compliance to state-only LDRs will not apply. In addition, the WIPP facility has been exempted from federal LDRs (61 FR 60704). Any treatment performed on mixed TRU will be to meet transportation requirements and WIPP facility waste acceptance requirements. Of the mixed 
TRU stored at CWC, 73 cubic meters contain PCBs. Waste containing PCBs is not acceptable for disposal at WIPP. A small amount of mixed TRU waste contains material carrying D001 and D003 codes. Waste with these codes is not acceptable at WIPP. However, this waste will be recharacterized as part of the TRU characterization process. The waste will be treated, if necessary, so that it no longer exhibits ignitable or reactive characteristics.

A relatively large volume of TRU waste (over 14,800 cubic meters) is located in the 200 Area burial grounds. By DOE policy, this waste has been situated in a manner that permits future retrieval and disposal in a deep geological repository. An unknown fraction of this waste would be mixed TRU waste if the waste were designated today. If the waste is retrieved and relocated outside the burial grounds, it will be classified as newly generated waste under RCRA. The waste will be characterized. If it contains hazardous constituents, the waste will be designated as mixed TRU waste.

The waste streams are summarized in Table 4-5. Waste generation information and records are maintained in the SWITS database and in the TSD units. The original purpose of the SWITS system is to account for the volume and the radioactivity level of waste stored or buried on the Hanford Facility. The system also maintains data on packaging and physical and chemical characteristics. Waste characteristics are provided by the waste generators.

Last year, existing mixed TRU waste streams were divided by radiological handling and by the presence of PCBs. This year, the waste streams are further divided by physical characteristics. This division results in 13 existing waste streams. The selected physical characteristics are those used by WIPP. Dividing the waste streams in this manner provides WIPP with needed information on the long-term production of volatile compounds within the waste containers.

Six future waste streams are presented (TRUM-04, -05 , and -06 categories). They are presented as separate waste streams because of the scope and magnitude of the projects that will produce the waste. The forecast waste generation from these projects depends highly on future funding and actual waste designation. Forecast volumes, therefore, are subject to change at any time.

Table 4-5. Mixed TRU Waste Streams.

\begin{tabular}{|l|l|r|r|r|}
\hline Waste stream & \multicolumn{1}{|c|}{ Description } & $\begin{array}{c}\text { Inventory, FY } \\
1998\left(\mathrm{~m}^{3}\right)\end{array}$ & $\begin{array}{c}5 \text {-yr forecast } \\
\text { generation }\end{array}$ & Total \\
\hline TRUM-01A & $\begin{array}{l}\text { Contact-handled mixed TRU } \\
\text { combustible debris }\end{array}$ & 73 & 35 & 108 \\
\hline TRUM-01B & $\begin{array}{l}\text { Contact-handled mixed TRU } \\
\text { composite filters }\end{array}$ & 8 & 0 & 8 \\
\hline TRUM-01C & $\begin{array}{l}\text { Contact-handled mixed TRU } \\
\text { heterogeneous debris }\end{array}$ & 131 & 88 & 219 \\
\hline TRUM-01D & $\begin{array}{l}\text { Contact-handled mixed TRU } \\
\text { metal debris }\end{array}$ & 16 & 16 & 32 \\
\hline TRUM-01E & $\begin{array}{l}\text { Contact-handled mixed TRU } \\
\text { non-metal inorganic } \\
\text { particulates }\end{array}$ & 1 & 45 & 46 \\
\hline
\end{tabular}


DOE/RL-99-01

Table 4-5. Mixed TRU Waste Streams.

\begin{tabular}{|c|c|c|c|c|}
\hline Waste stream & Description & $\begin{array}{c}\text { Inventory, FY } \\
1998\left(\mathrm{~m}^{3}\right)\end{array}$ & $\begin{array}{l}5 \text {-yr forecast } \\
\text { generation }\end{array}$ & Total \\
\hline TRUM-01F & $\begin{array}{l}\text { Contact-handled mixed TRU } \\
\text { lead waste }\end{array}$ & 5 & 15 & 20 \\
\hline TRUM-01G & $\begin{array}{l}\text { Contact-handled mixed TRU } \\
\text { solidified inorganics }\end{array}$ & 1 & 6 & 7 \\
\hline TRUM-01H & $\begin{array}{l}\text { Contact-handled mixed TRU } \\
\text { solidified organics }\end{array}$ & 3 & 2 & 5 \\
\hline TRUM-01J & $\begin{array}{l}\text { Contact-handled mixed TRU } \\
\text { soils }\end{array}$ & 10 & 0 & 10 \\
\hline TRUM-0IK & $\begin{array}{l}\text { Contact-handled mixed TRU } \\
\text { unknown or mixture }\end{array}$ & 7 & 0 & \\
\hline TRUM-02A & $\begin{array}{l}\text { Shielded mixed TRU } \\
\text { heterogeneous debris }\end{array}$ & 5 & 22 & 27 \\
\hline TRUM-02B & $\begin{array}{l}\text { Shielded mixed TRU lead } \\
\text { waste }\end{array}$ & 3 & 0 & 3 \\
\hline TRUM-03 & $\begin{array}{l}\text { PFP hydraulic fluids } \\
\text { contaminated with PCB's }\end{array}$ & 73 & 0 & 73 \\
\hline TRUM-04A & $\begin{array}{l}300 \text { Area revitalization contact- } \\
\text { handled mixed TRU } \\
\text { heterogeneous debris }\end{array}$ & 0 & 216 & 216 \\
\hline TRUM-04B & \begin{tabular}{|l|}
00 Area Revitalization \\
contact-handled mixed TRU \\
inorganic nonmetal debris
\end{tabular} & 0 & 16 & 16 \\
\hline TRUM-04C & $\begin{array}{l}300 \text { Area Revitalization } \\
\text { contact-handled mixed TRU } \\
\text { metal debris }\end{array}$ & 0 & 168 & 168 \\
\hline TRUM-05A & $\begin{array}{l}\text { Contact-handled TRU single- } \\
\text { shell tank long-length } \\
\text { equipment }\end{array}$ & 0 & 492 & 492 \\
\hline TRUM-0SB & $\begin{array}{l}\text { Remote-handled TRU single- } \\
\text { shell tank long-length } \\
\text { equipment }\end{array}$ & 0 & 492 & 492 \\
\hline TRUM-06 & $\begin{array}{l}\text { Miscellaneous remote-handled } \\
\text { mixed TRU waste }\end{array}$ & 0 & 5 & 5 \\
\hline Total & & 336 & 1,618 & 1,954 \\
\hline $\begin{array}{ll}\mathrm{FY} & =\text { fise } \\
\mathrm{PCB} & =\text { pol }\end{array}$ & & suranic & & \\
\hline
\end{tabular}

\subsubsection{Mixed TRU Waste Generation}

The volumes of waste generated in FY 1998 are presented by waste stream in Table 4-6. Waste generation was small and was much lower than forecast. Original forecast volumes for FY 1998 include solidified residues from a cementation unit at PFP. During FY 1998, the discovery was made that the cementation unit needed to be permitted at a TSD unit. The permit process had postponed the production of solidified waste from the PFP. 
Table 4-6. Mixed TRU Waste Generation.

\begin{tabular}{|l|l|r|}
\hline \multicolumn{1}{|c|}{ Description } & $\begin{array}{c}\text { FY 1998 } \\
\text { Seneration }\left(\mathrm{m}^{3}\right)\end{array}$ \\
\hline TRUM-01A & Contact-handled mixed TRU combustible debris & 0.83 \\
\hline TRUM-01B & Contact-handled mixed TRU composite filters & 0.00 \\
\hline TRUM-01C & Contact-handled mixed TRU heterogeneous debris & 0.83 \\
\hline TRUM-01D & Contact-handled mixed TRU metal debris & 0.42 \\
\hline TRUM-01E & Contact-handled mixed TRU non-metal inorganic particulates & 0.00 \\
\hline TRUM-01F & Contact-handled mixed TRU lead waste & 0.00 \\
\hline TRUM-01G & Contact-handled mixed TRU solidified inorganics & 0.62 \\
\hline TRUM-01H & Contact-handled mixed TRU solidified organics & 0.86 \\
\hline TRUM-01J & Contact-handled mixed TRU soils & 0.21 \\
\hline TRUM-01K & Contact-handled mixed TRU unknown or mixture & 0.00 \\
\hline TRUM-02A & Shielded mixed TRU heterogeneous debris & 0.64 \\
\hline TRUM-02B & Shielded mixed TRU lead waste & 0.00 \\
\hline TRUM-03 & PFP hydraulic fluids contaminated with PCB's & 0.00 \\
\hline Total & & 4.41 \\
\hline FY = fiscal year & TRUM = transuranic \\
PCB = polychlorinated biphenyl & Transic mixed & \\
\hline
\end{tabular}

The total inventory of TRU mixed waste decreased from last year's LDR report. Table 4-7 explains the decrease in inventory. To simplify accounting for the changes, waste streams TRUM-01A through TRUM-01K and waste streams TRUM-02A and TRUM-02B have been combined. The inventory decreased because 16 cubic meters of waste were generated in 1987. This waste is located in the burial grounds. Also, the description of the waste streams in the TRUM-02 series was changed from remote-handled waste to shielded waste. Some contacthandled waste at CWC is actually remote-handled waste, but the waste is shielded to permit safe storage at CWC. Shielded waste is considered remote-handled waste by WIPP waste acceptance criteria. A review of existing waste records found that 7.5 cubic meters received through FY 1997 were TRUM waste that had been shielded to contact-handled levels.

Table 4-7. Inventory Changes.

\begin{tabular}{|l|l|r|r|r|r|}
\hline \multicolumn{1}{|c|}{ Stream IDs } & \multicolumn{1}{|c|}{ Description } & $\begin{array}{l}\text { Inventory, } \\
\text { end of FY } \\
1997\end{array}$ & $\begin{array}{l}\text { Revision/ } \\
\text { correction } \\
(+/-)\end{array}$ & $\begin{array}{c}\text { Additions, } \\
\text { FY 1998 }\end{array}$ & $\begin{array}{l}\text { Inventory, } \\
\text { end of FY } \\
1998\end{array}$ \\
\hline $\begin{array}{l}\text { TRUM-01A through } \\
\text { TRUM-01K }\end{array}$ & $\begin{array}{l}\text { Contact-handied mixed } \\
\text { TRU waste streams }\end{array}$ & 274 & -23.5 & 3.8 & 254.3 \\
\hline $\begin{array}{l}\text { TRUM-02A and } \\
\text { TRUM-02B }\end{array}$ & $\begin{array}{l}\text { Shielded mixed TRU } \\
\text { waste streams }\end{array}$ & 0 & 7.5 & 0.6 & 8.1 \\
\hline TRUM-03 & $\begin{array}{l}\text { Contact-handled mixed } \\
\text { TRU with PCB's }\end{array}$ & 73 & 0 & 0 & 73.1 \\
\hline Total & & 347 & -16 & 4.4 & 335.5 \\
\hline $\begin{array}{l}\text { FY = fiscal year } \\
\text { PCB = polychlorinated biphenyl }\end{array}$
\end{tabular}


Chapter 3 presents information on each waste stream. Regardless of the designated waste stream, the TRUM waste streams are managed in the same manner. As stated earlier, TRUM waste disposed of at WIPP is exempt from federal LDRs. Nevertheless, the waste will be processed to meet the WIPP waste acceptance criteria. Additional characterization will be performed, which will include a review of the generators' records and sampling and analysis. The present acceptance criteria at WIPP require sampling and analysis of each container for volatile gases before shipment to WIPP. The waste will be treated as needed to meet the waste acceptance criteria. Contact-handled waste in drums will be examined, opened, and repackaged at the WRAP facility. If found, free liquids will be solidified. Shielded waste, remote-handled waste, and waste in boxes will be processed in accordance with provisions established with the project management plans for Milestone M-91. The project management plan will define the need for remote container opening, repackaging, size reduction, decontamination, and repackaging.

One waste stream, TRUM-03, CH with PCBs, consists of hydraulic fluids and crushed fluorescent light tubes with ballasts. The WIPP waste acceptance criteria prohibit placing PCBcontaminated waste at WIPP. RL plans to evaluate options that will lead to disposal of this waste stream. One possibility is using a coordinated approach through implementation of the Mega Rule (40 CFR 761), which became effective on August 28, 1998. This rule provides relief for disposal of some PCB waste. Under this rule, PCBs are added to the RCRA permits for storage and treatment, and disposal becomes a possibility based on risk analysis.

\subsubsection{TRUM Characterization}

TRU and TRUM waste will require WIPP facility certification for shipment to and disposal at the WIPP facility. WIPP facility certification is independent from the waste characterization and designation activity that authorizes waste receipt at the CWC.

4.2.2.1 TRUM Waste Characterization and Designation for Storage. TRUM waste is accepted for storage by implementation of the WSS (Kirkpatrick and Oswald 1995). This section covers waste designation and characterization activities performed by the onsite organization or offsite generators and by the storage or treatment unit receiving the waste.

Before any waste is accepted at the CWC, it is characterized and packaged as described in the Hanford Site Solid Waste Acceptance Criteria (FDH 1998). These criteria require that the generator of the waste characterize each individual container with sufficient accuracy to permit proper segregation, storage, treatment, certification, shipment, and, if applicable, disposal. The characterization ensures that, on generation and after processing, the actual physical, chemical, and radiological characteristics are recorded and known during all stages of the waste management process.

Waste is designated based on the information provided by the generator. The waste analysis organization assigns the waste designation as part of a waste acceptance evaluation in accordance with the Hanford Site Solid Waste Acceptance Criteria (FDH 1998). The designation is recorded in the SWITS database, which includes Washington State and RCRA waste codes. resulting from designations based on process knowledge and sample analysis. Each waste stream shipped from a generator to a Hanford Facility TSD is required to pass a formal compliance 
assessment before shipment in accordance with the implementing procedures of DOE Order 5820.2A.

The dangerous waste designation of each waste package is determined at its point of generation based on process knowledge of the waste placed in the container. The package is sampled and analyzed if sufficient process knowledge is unavailable.

Process Knowledge. The waste characterization is typically determined by the waste generator based on documented knowledge of the process generating the waste (i.e., process knowledge). Process knowledge is backed by acceptable evidence that relates the characterization to a definite process that generated the waste.

Process knowledge has been used to characterize PCB-contaminated TRU waste currently in storage. Equipment containing PCBs, such as hydraulic systems, transformers, capacitors, and fluorescent light ballasts, have been identified clearly. These systems are managed in accordance with 40 CFR 761; waste is immediately handled and packaged as PCB TRU material.

Testing. If process knowledge is not sufficient to fully characterize a waste package, the generator must augment the characterization with sampling and analysis. This is normally required only for characteristic waste constituents (i.e., D001 through D043). The quality level of analytical data is determined by the receiving TSD unit's waste acceptance criteria.

Hydraulic systems and transformers have been sampled to determine PCB concentrations. Any waste resulting from the management of these systems is designated based on the concentration of PCBs in the source system. Light ballasts are designated based on data from the manufacturers.

Acceptance of newly generated waste requires the generator to complete and sign an LDR notification/certification before the $\mathrm{CWC}$ can receive the waste because storage of the TRU waste must be in compliance with record keeping requirements. Acceptance of waste shipped from locations south of the Hanford Facility's 200 Area normally requires completion of a uniform hazardous waste manifest for the shipment. Waste generated inside the 200 Area at the Facility normally is shipped by the onsite waste-transfer protocol as specified by the Hanford Facility's transportation requirements.

An integral component in the waste designation process is the use of WSRds as described in the Hanford Facility's WSS. WSRds functionally categorize waste received from various generators into streams requiring similar management. WSRds specify the waste's general radiological description; hazardous constituents; allowable waste codes; recommended packaging, storage, and treatment requirements; and any special handling and/or storage instructions applicable to the waste. WSRds are not location specific, and therefore are used for waste from many sources.

4.2.2.2 WIPP Certification. TRU waste certification from WIPP involves proving that the TRU waste sent to Carisbad, New Mexico, meets the WIPP waste acceptance criteria without opening the containers. The certification process is primarily a quality assurance exercise with a focus on complying with the Transuranic Waste Characterization Quality Assurance Program Plan (CAO 1996) for the WIPP. The process also will require complying with Hanford Facility- 
Plan (CAO 1996) for the WIPP. The process also will require complying with Hanford Facilityspecific and waste-specific certification documents are not yet written. When completed, the certification documents will comply with CAO (1996).

In addition to the base certification documents, a number of items must be completed before the TRU Waste Certification process can be approved. These items have not been written, but are expected to include the following:

- Certification of acceptable knowledge of the waste inside the containers

- Hanford Facility procedures that refiect the requirements of the quality assurance program plan and WIPP waste acceptance criteria

- Demonstrations to confirm that personnel, laboratories, and equipment performing NDA and RCRA and head space gas sampling comply with CAO (1996)

- A sampling plan and a transportation packaging plan

- Data interface with WIPP

- Carlsbad Area Office certification audits.

Once the audits have been successfully completed, the Carlsbad Area Office will approve the certification process at the Facility with annual audits to ensure compliance.

4.2.2.3 Uncertainty of Waste Characterization and Designation. When the waste was placed in storage, the waste characterization and designation was appropriate and compliant for storage purposes. However, changes in designation regulations, acceptance criteria, and WIPP certification have made that characterization obsolete. Additional characterization will be required before the waste can be sent to the WIPP facility.

\subsubsection{TRUM Storage}

This section describes the storage units used to store waste streams TRUM-01A through TRUM-03.

4.2.3.1 Description of Storage Units and Capacities. Each waste stream is stored in one or more of the buildings located at the $\mathrm{CWC}$, an interim-status RCRA storage unit under the Hanford Facility RCRA Permit. The waste profile forms indicate the number of containers located in each building as of September, 1998. Containers are subject to relocation at a different building within the CWC, or at WRAP, at any time. The CWC consists of many storage buildings and modules. These are briefly described in the following paragraphs.

2402W through 2402WL Series Mixed Waste Storage Buildings. This complex consists of 13 individual storage buildings designed to store contact-handled MLLW and TRUM and PCB waste. Each building has 372 square meters of floor space that can hold approximately 
100055 -gal-drum equivalents of waste. These buildings provide a combined storage capacity of 13,000 drum equivalents. The $112402 \mathrm{~W}$ series buildings were built between 1988 and 1990 .

2403WA - WC Series MW Storage Buildings. This complex consists of three individual storage buildings designed to store contact-handled MLLW, and TRUM and PCB waste. (TSCA-regulated PCB waste would require additional secondary containment if placed into the buildings.) TSCA PCBs are not allowed in these buildings. Each building has 3160 square meters of floor space that can hold approximately 11,600 drum equivalents. These buildings, with a combined storage capacity of 34,800 dram equivalents, became operational during 1991.

2403WD MW Storage Building. This storage building is designed to store contacthandled MLLW and TRUM and PCB waste. (TSCA-regulated PCB waste would require. additional secondary containment if placed in the buildings.) TSCA PCBs are not allowed in this building. The $2403 \mathrm{WD}$ Building contains 5135 square meters of floor space that can hold approximately 17,500 drum equivalents. This building became operational during 1991 .

2404WA - WC Series MW Storage Buildings. This complex consists of three individual storage buildings designed to store contact-handled MLLW and TRUM and PCB waste. Each building has 2000 square meters of floor space that can hold approximately 4600 drum equivalents. These buildings, with a combined storage capacity of 13,800 drum equivalents, became operational during 1997.

Flammable Mixed-Waste Storage Modules (FS-01-FS-24). This complex consists of 24 individual modules designed to store flammable LLW, TRU waste, MLLW, and TRUM and PCB waste with flash points below $38^{\circ} \mathrm{C}$. Each unit can hold approximately 22 drum equivalents for a total capacity of 528 drum equivalents. The modules are small preengineered buildings, each with 16.3 square meters of floor space.

Waste Unloading and Staging Area. This pad is 836 square meters in area and can hold approximately 2500 drums stacked two high. This pad is not intended for long-term storage.

\subsubsection{CWC Storage Capacity and Existing Stored Volume. See Section 4.1.4.2.}

\subsubsection{TRUM Treatment and WIPP Certification}

4.2.4.1 FY 1997 Treatment Activities. The glove-box side of WRAP was initiated in FY 1998

4.2.4.2 Planned Treatment Activities. To meet consent agreements, TRU waste will need to be prepared, shipped, and disposed of at the WIPP facility. The Tri-Party Agreement has several milestones influencing TRUM waste disposition, the ones specifying TRUM waste disposition are as follows:

- M-91-01. Complete acquisition of facilities necessary for storage and treatment or processing before disposal of all Hanford Facility post-1970 TRU/TRUM by December 2000. 
- M-91-02. Initiate processing of CH-TRUM waste at WRAP-1 by December 1998.

- M-91-03. Submit Hanford Facility TRU/TRUM waste project management plan to Ecology by June 2000.

- M-91-04. Complete construction of small-container.CH-TRUM waste retrieval facilities and initiate retrieval from the 200 Area burial grounds by September 2000.

- M-91-05-T01. Complete a TRU/TRUM waste retrieval and processing facility engineering study or functional design criteria study and submit it to Ecology by December 2002.

- M-91-06-T01. Award necessary contracts for processing remote-handled and largesize TRUM waste packages by September 2003.

- M-91-07. Complete Project W-113 for post-1970 CH-TRUM waste retrieval by September 2004.

- M-91-08-T01. Complete construction and initiate hot operations of processing facility for remote-handled and large-size TRUM waste by June 2005.

The DOE is actively pursuing these compliance agreements for the Hanford Facility. WRAP-1 began its LLW activities and funding has been obtained to begin the TRUM waste certification program. The Hanford Facility's TRUM waste baseline disposition map (Figure 4-2) depicts the established path forward for treatment and disposal, and Table 4-8 shows the current disposition schedule and associated waste volumes. (Figure 4-2 shows TRU waste, not included elsewhere in this report, as well as TRUM waste.)

4.2.4.3 Accelerated and Alternative Treatment. The TRUM disposition program will begin with two parts. The first is to initiate the TRUM Waste WIPP facility certification program by putting in place the onsite certification, quality assurance, and operations procedures for WIPP facility certification. The second is to perform a technology and trade-off study for the various retrieval approaches to be used on the W-113 Project (Suspect TRU Retrieval). Savings from other waste management programs and activities were redirected to or approved for this program.

\subsubsection{TRUM Treatability Variances, Equivalency Petitions, Rulemaking Petitions, and Case-by-Case Exemptions}

The Hanford Facility and the DOE complex have limited capacity for preparing TRUM waste for WIPP disposal. Therefore, the TRUM waste must be stored. The WIPP facility is scheduled to begin accepting certified TRU in 1999 . However, only nonmixed TRU waste will be accepted during the initial operations because the WIPP facility has not yet received a RCRA permit to receive and dispose of TRUM waste.

If additional time extensions are required because of delays in developing WIPP certification capacity for TRUM waste, they will be applied for in accordance with the 
procedures detailed in the Tri-Party Agreement and regulations. Because waste destined for the WIPP facility is not subject to federal and state-only LDRs, variances and other petitions are not anticipated.

Table 4-8. TRUM Disposition Schedule and Waste Volume.

\begin{tabular}{|l|l|c|c|c|c|c|c|}
\hline \multirow{2}{*}{$\begin{array}{c}\text { Waste stream } \\
\text { ID }\end{array}$} & \multirow{2}{*}{ Waste stream title } & $\begin{array}{c}\text { FY } \\
1997 \\
\left(\mathrm{~m}^{3}\right)\end{array}$ & $\begin{array}{c}\text { FY } \\
1998 \\
\left(\mathrm{~m}^{3}\right)\end{array}$ & $\begin{array}{c}\text { FY } \\
1999 \\
\left(\mathrm{~m}^{3}\right)\end{array}$ & $\begin{array}{c}\text { FY } \\
2000 \\
\left(\mathrm{~m}^{3}\right)\end{array}$ & $\begin{array}{c}\text { FY } \\
2001 \\
\left(\mathrm{~m}^{3}\right)\end{array}$ & $\begin{array}{c}\text { FY } \\
2002 \\
\left(\mathrm{~m}^{3}\right)\end{array}$ \\
\hline RL-TRUM-01 & $\begin{array}{l}\text { Generalized } \\
\text { CH-TRUM }\end{array}$ & 0 & 0 & 298 & 648 & 583 & 459 \\
\hline RL-TRUM-02 & $\begin{array}{l}\text { Generalized } \\
\text { RH-TRUM }\end{array}$ & 0 & 0 & 0 & 0 & 0 & 0 \\
\hline RL-TRUM-03 & $\begin{array}{l}\text { CH/RH-TRUM with } \\
\text { PCBs }\end{array}$ & 0 & 0 & 0 & 0 & 0 & 0 \\
\hline & Total & 0 & 0 & 298 & 648 & 583 & 459 \\
\hline
\end{tabular}

'Dispositioned waste volumes include TRU and TRUM waste. No determination has been made on how much of each waste type will be sent or when it will be sent.

$\mathrm{CH}$ contact handled

FY fiscal year

PCB polychlorinated bipheny!

$\mathrm{RH}$ remote handled
TRU transuranic

TRUM transuranic mixed

WIPP Waste Isolation Pilot Plant

\subsection{DOUBLE-SHELL TANK SYSTEM WASTE}

The DST system consists of 28 DSTs with a total capacity of 118,000 cubic meters. The waste stored in these tanks is alkaline liquids and solids generated during the past production of nuclear materials. Waste has been received from operations in the 100,200,300, and 400 Areas of the Hanford Facility. The DST system waste consists of legacy and cleanup waste. Legacy waste has been generated from the PUREX process, B Plant operations, PFP operations, research and development programs, and laboratories. Liquid supernatant and interstitial liquids from the SST system are stored in the DST system. Cleanup waste came from the decontamination and decommissioning of plants and equipment.

\subsubsection{DST System Generation}

The DST system contains legacy waste from past chemical separations processes and cleanup waste from current operations. The major legacy waste contributors were the PUREX process, B plant operations, PFP operations, and SST waste from the bismuth phosphate separations, uranium recovery, and reduction-oxidation extraction processes. Cleanup waste comes from the cleanup of various Hanford Facility locations. 
Hanford TRU Baseline Disposition Map

\section{Waste Stream}

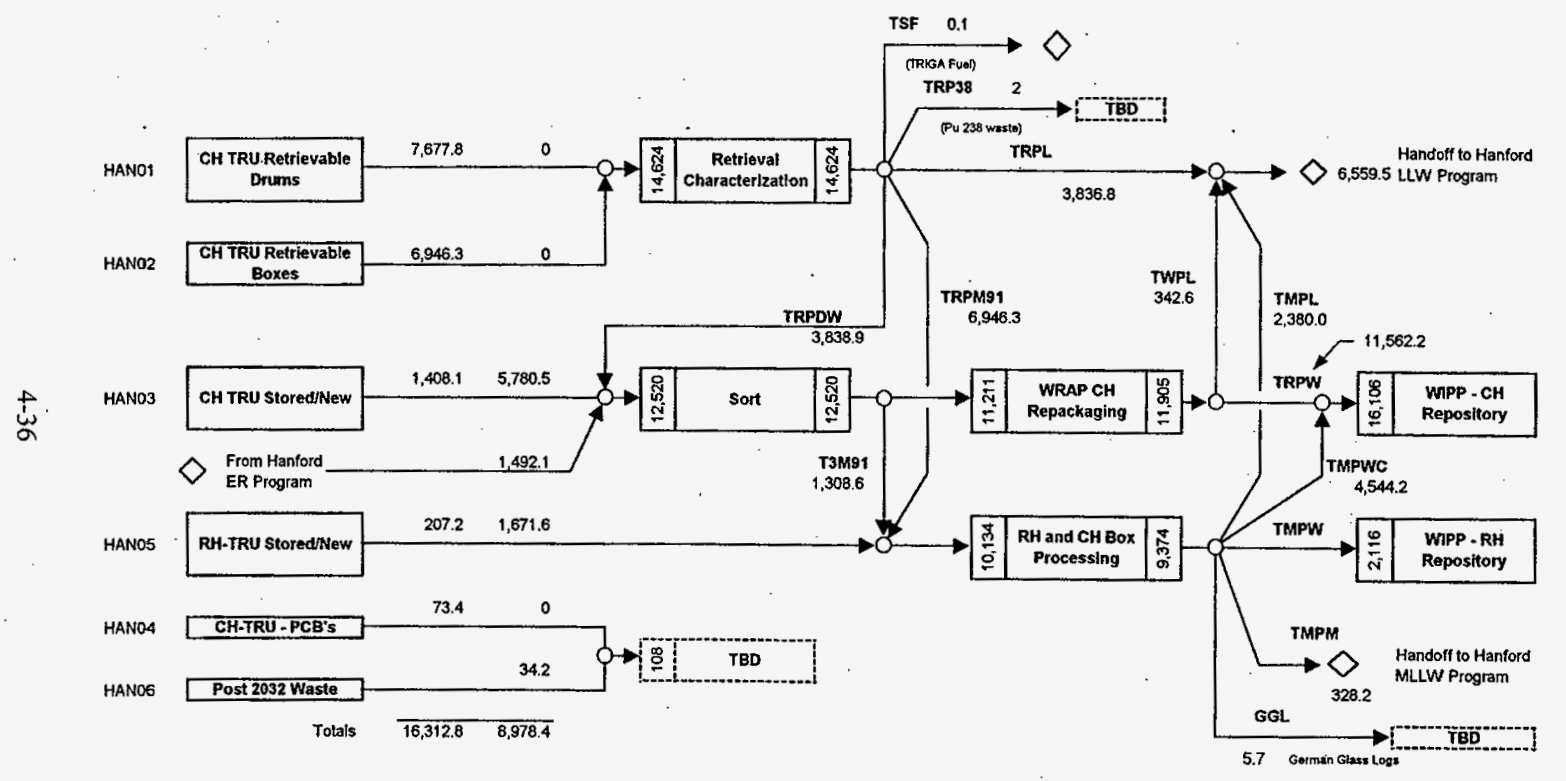


Figure 4-2. Hanford Transuranic Baseline Disposition Map. (2 sheets)

Key:

The numbers on the left refer to waste stream codes not used elsewhere in this report.

Numbers inside the boxes on the left and right vertically represent cubic meters of waste at the beginning and end of processes. Numbers above flowchart arrows represent cubic meters of waste in inventory, projected to be generated, or projected for disposal.

Inventory is as of September 30, 1998.

Generation refers to the years 1999 to 2046.

Codes before and after process boxes (e.g. TSF) have the following meanings.

$\begin{array}{ll}\text { CH } & \text { contact handled } \\ \text { GGL } & \text { German glass logs } \\ \text { LLW } & \text { low-level waste } \\ \text { M91 } & \text { Tri-Party Agreement Milestone M-91 } \\ \text { MLLW } & \text { mixed low-level waste } \\ \text { PCB } & \text { polychlorinated biphenyl } \\ \text { RH } & \text { remote handled } \\ \text { T3M91 } & \text { TRU M91 Feed } \\ \text { TBD } & \text { to be determined } \\ \text { TMPL } & \text { TRU M91 product low-level waste } \\ \text { TMPM } & \text { TRU M91 product mixed low-level waste } \\ \text { TMPW } & \text { TRU Product WRAP (remote-handled implied) } \\ \text { TMPWC } & \text { TRU M91 Product mixed low-level waste (contact handied) } \\ \text { TRP38 } & \text { TRU Plutonium-238 } \\ \text { TRPDW } & \text { TRU Retrieval product drum to WRAP } \\ \text { TRPL } & \text { TRU Retrieval product low-level waste } \\ \text { TRPM91 } & \text { TRU Retrieval product to M91 } \\ \text { TRPW } & \text { TRU WRAP product } \\ \text { TRU } & \text { transuranic } \\ \text { TSF. } & \text { TRU Spent fuel } \\ \text { TWPL } & \text { TRU WRAP product low-level waste } \\ \text { WIPP } & \text { Waste Isolation Pilot Plant } \\ \text { WRAP } & \text { Waste Receiving and Processing }\end{array}$


The PUREX plant, operating from 1956 to 1992, received irradiated fuel from the Hanford Facility reactors. The fuel was dissolved in nitric acid, and processed through several solvent extraction steps to separate the plutonium, uranium, and neptunium from other fission products. The PUREX process waste consists of three major types of waste; aging waste from the first decontamination cycle, process condensate, and ammonia scrubber feed. The aging waste contains most of the fission products in nitric acid. When the stream was determined to be waste, it was treated with sugar to destroy most of the nitric acid, then with sodium hydroxide and sodium nitrite to meet DST system storage specifications. Condensing vapors from the PUREX uranium-nitric acid product and recycle streams generated process condensate. The ammonia scrubber feed was generated when water was sprayed to adsorb ammonia gas generated by the decladding and metathesis reaction from the dissolver off gas stream.

B Plant recovered cesium and strontium from legacy waste. A B Plant fractionation process separated high-heat-producing isotopes from the waste. The strontium was separated by an extraction process using complexing agents (e.g., ethylene-diaminetetraacetic acid, n-hydroxyethylethylenediamine-tetraacetic acid, and citrate) to prevent transition metal extraction. The cesium was extracted and purified by ion exchange. The cesium and strontium were converted to fluoride and chloride salts, which were encapsulated in the Waste Encapsulation and Storage Facility (WESF), part of the B Plant Complex. Sodium hydroxide or sodium carbonate was added to the waste to create an alkaline solution and minimize tank corrosion.

The PFP, which operated from 1949 to the present, converted plutonium in solution to plutonium metal. The process consisted of precipitation, solvent exchange, and ion exchange, producing waste high in metallic nitrates. The current waste stream is a low-salt stream from operating the building systems and stabilization operations.

The products of bismuth phosphate separations, uranium recovery, and reduction-oxidation are part of the SST system waste, which was transferred to the DST system during the interim stabilization of the SST system.

Cleanup waste originated from Hanford Facility locations including, but not limited to, PUREX, B Plant, T Plant, the 222-S Laboratory, the 340 Facility, and the 242-A Evaporator. Cleanup waste from these units varies in composition but consists primarily of dilute inorganic aqueous material. Activities generating this waste include vessel and pipe decontamination, waste concentration in the 242-A Evaporator, and other miscellaneous decommissioning activities. The waste stream includes wastewater, flush water, and liquids generated from analytical laboratories.

See Table 4-9 for an historical summary of DST system waste generation by various Hanford Facility locations. Further historical information on SST and DST systems is given in WHC (1990b). 
Table 4-9. Waste Generation for Various Locations and Programs (cubic meters).

\begin{tabular}{|l|c|c|c|c|c|c|c|c|c|c|c|c|}
\hline FY & $\begin{array}{c}\text { B } \\
\text { Plant }\end{array}$ & PUREX & $\begin{array}{c}\text { Tank } \\
\text { farms }\end{array}$ & $\begin{array}{c}\text { SST to } \\
\text { DST } \\
\text { pumping }\end{array}$ & $\begin{array}{c}\text { UO } \\
\text { Plant }\end{array}$ & $\begin{array}{c}\text { PFP } \\
\text { Plant }\end{array}$ & $\begin{array}{c}\text { S Plant } \\
\text { (labora- } \\
\text { tories) }\end{array}$ & $\begin{array}{c}100 \\
\text { Area }\end{array}$ & $\begin{array}{c}300 \\
\text { Area }\end{array}$ & $\begin{array}{c}400 \\
\text { Area }\end{array}$ & Total \\
\hline 1990 & 2393 & 6882 & 1226 & 0 & 0 & 53 & 151 & 121 & 193 & 136 & 0 & 11,155 \\
\hline 1991 & 1317 & 984 & 776 & 859 & 0 & 0 & 140 & 170 & 0 & 208 & 0 & 4454 \\
\hline 1992 & 435 & 363 & 155 & 859 & 0 & 136 & 250 & 106 & 0 & 132 & 30 & 2065 \\
\hline 1993 & 511 & 291 & 144 & 458 & 0 & 19 & 257 & 38 & 0 & 87 & 45 & $1532^{2}$ \\
\hline 1994 & 53 & 276 & 140 & 140 & 0 & 26 & 76 & 76 & 0 & 110 & 42 & 1635 \\
\hline 1995 & 129 & 1154 & 836 & 360 & 0 & 0 & 83 & 83 & 0 & 220 & 0 & 3225 \\
\hline 1996 & 359 & 621 & 1196 & 500 & 0 & 15 & 91 & 106 & 0 & 197 & 0 & 2797 \\
\hline 1997 & 292 & 0 & 72 & 617 & 0 & 0 & 64 & 27 & 0 & 98 & 0 & 1170 \\
\hline 1998 & 140 & 0 & 322 & 367 & 0 & 0 & 0 & 41 & 0 & 57 & 0 & 927 \\
\hline
\end{tabular}

Note: All generation quantities include the volume of any flush water.

'In addition to the waste categories in the table, in 1993, approximately 1336 cubic meters of water was added to DSTs. This water was used to test the upgraded 242-A Evaporator components before restart.

DST double-shell tank SST single-shell tank

PFP Plutonium Finishing Plant $\quad \mathrm{UO}_{3} \quad$ Uranium Oxide (Plant)

PUREX Plutonium-Uranium Extraction (Plant)

\subsubsection{DST System Storage}

4.3.2.1 Storage Facilities. The 200 Areas contain six DST system farms. Each farm consists of a steel tank in a secondary steel liner inside a reinforced concrete tank buried underground. These tanks are operating under interim status for the storage of HLW. Table 4-10 lists the location of each farm and the number of tanks in that farm.

Table 4-10. DST System Summary.

\begin{tabular}{|l|l|c|}
\hline \multicolumn{1}{|c|}{ Tank farm } & \multicolumn{1}{c|}{ Location } & Number of tanks \\
\hline AN & 200 East Area & 7 \\
\hline AP & 200 East Area & 8 \\
\hline AW & 200 East Area & 6 \\
\hline AY & 200 East Area & 2 \\
\hline AZ & 200 East Area & 2 \\
\hline SY & 200 West Area & 3 \\
\hline
\end{tabular}

4.3.2.2 Storage Capacity. The DST system farms contain 24 DSTs that hold 4300 cubic meters each and 4 DSTs that hold 3700 cubic meters each. The total capacity of the DST system is 118,000 cubic meters. As of December 1998, the system contained 70,976 cubic meters of waste. The DST system was expected to be out of space by 1998 . However, with at least one evaporator run per year and waste minimization, the DST system now is predicted to have room for more waste until after the year 2004. See Figure 4-3 for a current summary of DST system space. This estimate is based on the evaporator continuing to have at least one campaign per year and waste minimization efforts continuing. 


\subsubsection{DST System Characterization}

As described in Chapter 1, several processes contributed to DST system waste. In addition, waste management practices have mingled the types of waste in the system. Therefore, a detailed, quantified characterization of the tanks' contents based strictiy on process knowledge is not possible. Stratification and segregation have occurred in the tanks as solids settled out. The consistency of the waste ranges from liquid supernatant to thick sludge to crust formed as a top layer. The DST system waste is described qualitatively based on historical data and sample analysis.

DST system waste can be categorized into several types, each with a specific history and character.

4.3.3.1 Double-Shell Slurry Feed and Double-Shell Slurry Waste. Double-shell slurry feed (DSSF) is generated by concentrating the dilute waste streams generated by the operating plants to conserve storage space. The DSSF waste has been evaporated to the sodium aluminate phase boundary, so it contains no aluminate solids. Double-shell slurry is a more concentrated waste form produced by evaporating DSSF past the aluminate boundary. Double-shell slurry contains aluminate solids and has a much higher viscosity than DSSF.

4.3.3.2 Neutralized Current Acid Waste. The neutralized current acid waste, also known as PUREX aging waste, consists of water, aluminum hydroxide, sodium nitrate, sodium hydroxide, sodium fluoride, cadmium nitrate, sodium nitrite, and most radionuclides from irradiated fuel. Before 1989, process samples analyzed in the laboratory were recycled to the process system. This practice may have allowed chemicals added to the samples for analysis to enter the aging waste stream. However, sample analysis has never confirmed the presence of these chemicals.

4.3.3.3 Neutralized Cladding Removal Solids Waste. Cladding removal waste resuited from dissolving the zircaloy cladding on the irradiated nuclear fuel. Neutralizing this waste precipitated most of the zirconium, creating slurry.

4.3.3.4 Plutonium Finishing Plant Waste. The PFP waste originated from the conversion of plutonium nitrate to oxide or metal and includes TRU laboratory waste and high-salt solvent extraction waste.

4.3.3.5 Complexant Concentrate Waste. The complexant concentrate waste results from the concentration of waste containing a large amount of organic complexing agents. The organic complexing compounds were introduced to the waste during strontium recovery at B Plant. 


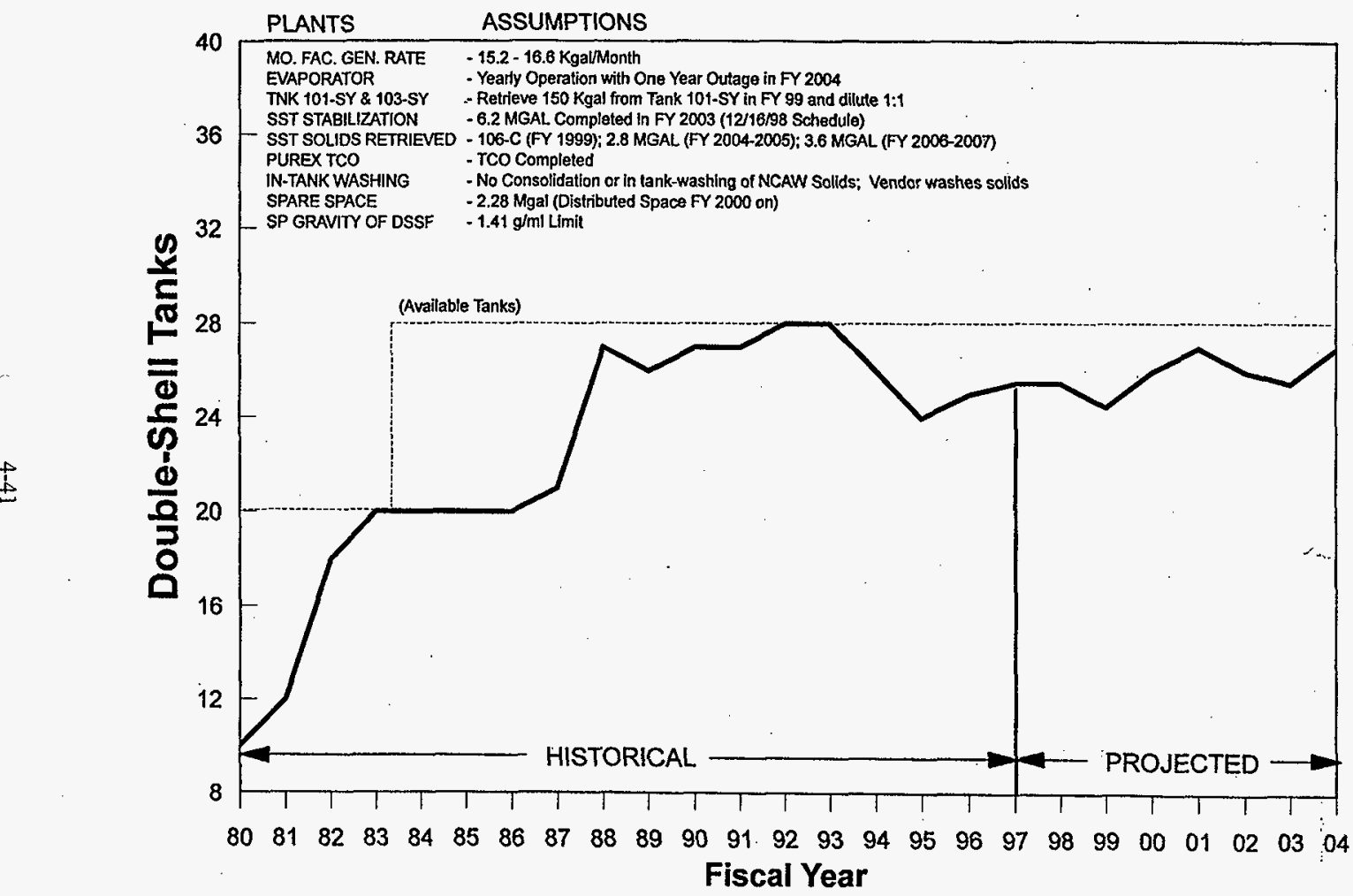


4.3.3.6 Basis for Waste Designation. In accordance with Tri-Party Agreement

Milestone M-44-00, the data-quality-objective process will be used to establish the data needs necessary to determine the waste designation and LDR status of the waste. As part of the LDR status, the process will determine which underlying hazardous constituents must be quantified based on knowledge supplied by the Hanford Facility generating locations at the point of generation.

The waste codes assigned to waste stored in the DST system are based on historical knowledge of waste received into the system and the characteristics the waste displays in storage. Waste codes assigned to waste in storage are a subset of the waste codes identified on the DST system Part A Form 3 Permit application. Additional waste codes can be added or deleted based on the ongoing characterization program. The codes are meant to encompass the entire DST system. Sampling and analysis of the DST system waste is under way and will continue based on the priorities determined using systems engineering.

Since 1995, waste designation and LDR information on waste shipped to the DST system has been documented on "profile sheets" that are different from those included in Chapter 3. The information required in these sheets is specified in the DST waste analysis plan. DST system acceptance criteria specificaliy require that all LDR requirements be identified. LDR requirements for waste in the DST system before 1995 are not documented.

\subsubsection{DST System Treatment}

The DST system waste will be treated and disposed of using processes and facilities adopted by the Tri-Party Agreement. Currently the plan is to separate the DST system waste into LLW and HLW/TRU fractions. This waste then will be solidified and stored in long-term storage in TSD units. Figure 4-4 is a flowchart summarizing current treatment plans.

The current Tri-Party Agreement plan is to separate the DST system waste into LLW and HLW/TRU fractions, so the bulk of the radionuclides are in the HLW. The HLW stream will be treated to further reduce its volume and increase radionuclide loading if necessary. The LLW will have enough radionuclides removed to meet the U.S. Nuclear Regulatory Commission's "incidental waste" classification and the DOE's as low as reasonably achievable (ALARA) policy.

Further work is expected to determine what fraction of DST system waste is subject to the specified technology, high-level waste vitrification, after pretreatment. Regardless of what fraction of the DST system waste is subject to high-level waste vitrification, current federal LDR requirements impose sampling and analysis requirements on the treated waste before disposal.

In separate onsite TSD units, both the LLW and HLW fractions will be vitrified. This process destroys or extracts organic constituents and cyanide to below treatment standards, neutralizes or deactivates dangerous waste and extremely hazardous waste, and immobilizes toxic metals. The LLW fraction will be disposed of near the surface on the Hanford Facility. The vitrified $\mathrm{HLW}$ stream will be stored on site until the geologic repository is available to receive the waste for disposal. 


\section{Hanford Tank Waste Remediation System}

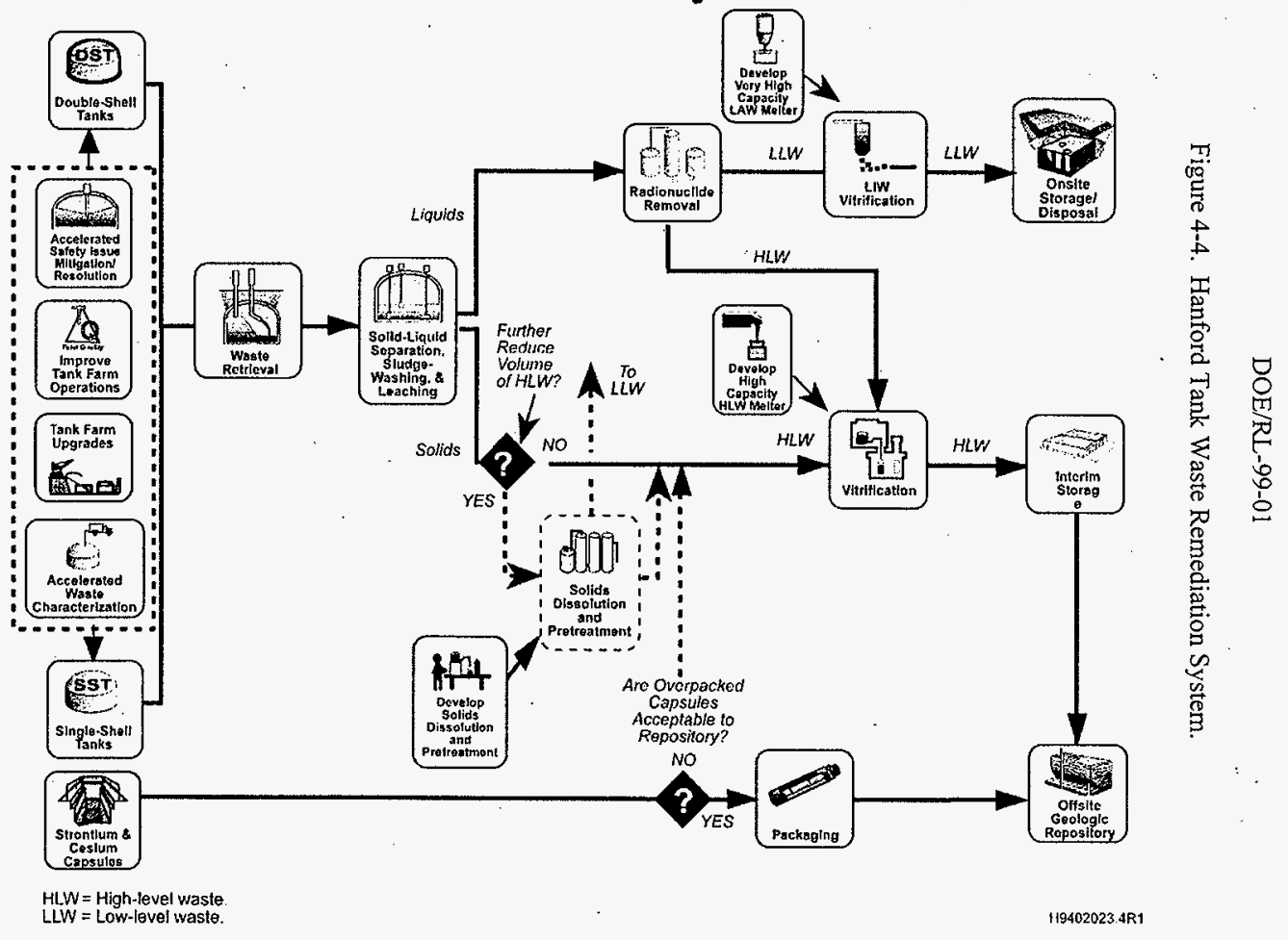


DOE's current plan is to award private contracts ${ }^{1}$ for the SST and DST system treatment facilities with a two-phase contract. The first phase will be a Proof-of-Concept-Commercial Demonstration Phase, which consists of design, permitting, and construction and operation of a TSD unit to pretreat and vitrify waste. During 1998, DOE authorized BNFL, Inc. (BNFL) to proceed with their contract to provide waste treatment and immobilization services using privatized facilities-a contractor-developed, -financed, -constructed, -owned, -operated, and deactivated waste treatment and immobilization system for Hanford tank waste. During Phase I, BNFL will develop and document the approach for demonstrating compliance with the RCRA land disposal restrictions for LAW and collect characterization and demonstration-scale treated waste product data to support the compliance demonstration. Phase II will be the full-scale production phase. Treatment of DST system waste is on a schedule based primarily on Tri-Party Agreement Milestones M-50-00 (HLW pretreatment), M-60-00 (LLW vitrification), and M-51-00 (HLW vitrification). Because of budget limitations, accelerating treatment beyond these milestone dates is not realistic.

\subsubsection{DST System Treatability Variances, Equivalency Petitions, Rulemaking Petitions, and Case-by-Case Exemptions}

Because the DST system waste is a listed waste, further regulatory action is anticipated so the waste can be disposed of at the geologic repository. Because current regulations impose sampling and analysis requirements on treated waste, regulatory action may be necessary to reduce or eliminate this requirement.

\subsection{SINGLE-SHELL TANK SYSTEM WASTE}

The SST system consists of 149 tanks located in 12 tank farms with 2 to 18 tanks each, in the 200 Areas. The amount of waste contained in the tanks varies from 5 to 95 percent of each tank's capacity; the consistency of the waste varies from pumpable liquid to sludge to hard salt cake. These tanks have held chemically hazardous and radioactive waste generated as a byproduct of processing spent nuclear fuel to recover plutonium, uranium, and neptunium since 1944.

\subsubsection{SST System Generation}

Waste has been generated through a variety of analytical, decladding, and separation processes and associated Facility-wide operations. Four major chemical processing operations; the bismuth phosphate, reduction-oxidation, PUREX, and tributyl phosphate processes, produced waste stored in the SST system. The bismuth phosphate, reduction-oxidation, and PUREX processes were specifically designed for plutonium recovery. The initial bismuth phosphate chemical separation process produced large volumes of dilute, low-heat waste. The tributyl phosphate solvent extraction process was designed to recover the relatively large amounts of uranium that remained in waste from the bismuth phosphate process. The bismuth phosphate

'Offsite and private contractors are subject to the same LDRs as government contractors. 
process was superseded by the reduction-oxidation process, which was superseded by the PUREX process.

The bismuth phosphate process, which was used from 1943 to 1957 , was used at B Plant and $\mathrm{T}$ Plant to separate plutonium from uranium in irradiated fuel by coprecipitation with bismuth phosphate from a uranyl nitrate solution. The plutonium was further separated from fission products by successive precipitation cycles using bismuth phosphate and lanthanum fluoride. Waste containing uranium, acid, and many of the fission products was neutralized and stored in the SST system.

The bismuth phosphate metal waste initially was stored in separate tanks, however, the metal waste was reprocessed to recover the uranium and the supernatant was scavenged and disposed of to the cribs, leaving very little of the original waste. .

The uranium recovery process operated in U Plant from 1952 to 1958 and in the PUREX Plant from 1956 to 1958 . In this process, waste was sluiced from the tank, dissolved in nitric acid, and run through a solvent extraction process using tributyl phosphate in a kerosene-like solvent. The U Plant process recovered uranium from bismuth phosphate metal waste and produced waste consisting of fission products and residual plutonium. The PUREX process recovered uranium, plutonium, and neptunium in addition to separating the fission products. The waste was all neutral or alkaline before being stored in the SST system.

A significant increase in the volume of waste resulted from the uranium recovery process in U Plant. The process efficiently recovered uranium from the bismuth phosphate metal waste; however, it generated about 2 liters of waste for every liter of bismuth phosphate metal waste processed. This increase in waste volume was the rationale for the ferrocyanide scavenging campaign. The volume of waste in the tanks had to be reduced, and the ferrocyanide scavenging decontaminated the waste sufficiently to enable disposal to the cribs.

The reduction-oxidation process conducted from 1951 to 1967 in the 202-S Plant used a continuous solvent extraction process to remove plutonium and uranium from dissolved fuel in a hexone solvent. The slightly acidic waste stream contained the fission products and large quantities of aluminum nitrate. This waste was neutralized and stored in the SST system.

\subsubsection{SST System Storage}

The 200 Areas contain 12 single-shell tank farms. Each tank farm consists of 4 to 18 underground, reinforced concrete steel-lined tanks. Table 4-11 lists the location of each farm and the number of tanks in that farm.

The Hanford Facility contains 149 SSTs. Of these, 133 are 22.9 meters in diameter with nominal capacities between 2,000 and 3,800 cubic meters. Sixteen are 6.1 meters in diameter with capacities of 210 cubic meters. Currently, the system contains 133,600 cubic meters of waste. The SST system has not accepted waste since 1980 . The only material added to the SST system has been water to tank $241 \mathrm{C}-106$ to control evaporative cooling. 
Table 4-11. SST System Summary.

\begin{tabular}{|l|c|c|}
\hline \multicolumn{1}{|c|}{ Tank farm } & Location & Number of tanks \\
\hline $\mathrm{A}$ & 200 East Area & 6 \\
\hline$\overline{\mathrm{AX}}$ & 200 East Area & 4 \\
\hline$\overline{\mathrm{B}}$ & 200 East Area & 16 \\
\hline $\mathrm{BX}$ & 200 East Area & 12 \\
\hline $\mathrm{BY}$ & 200 East Area & 12 \\
\hline $\mathrm{C}$ & 200 East Area & 16 \\
\hline $\mathrm{S}$ & 200 West Area & 12 \\
\hline $\mathrm{SX}$ & 200 West Area & 15 \\
\hline $\mathrm{T}$ & 200 West Area & 16 \\
\hline $\mathrm{TX}$ & 200 West Area & 18 \\
\hline$\overline{T Y}:$ & 200 West Area & 6 \\
\hline $\mathrm{U}$ & 200 West Area & 16 \\
\hline
\end{tabular}

\subsubsection{SST System Characterization}

The SST system received waste from five chemical processes: bismuth phosphate, reduction-oxidation, PUREX, and tributyl phosphate, and B Plant waste fractionation. This waste is found in three forms in the SST system: sludge, salt cake, and supernatant. Both the salt cake and the sludge contain interstitial liquids.

The sludge consists of the solids (hydrous metal oxides, iron, and aluminum) precipitated during the neutralization of acid waste before transfer to the SST system. Sludge types vary greatly in their physical properties. Salt cake contains salts, primarily sodium nitrate, formed by the water evaporating from the waste. Damp salt cake is a jelly-like material; dried salt cake is a hard, abrasive, brittle material that may have formed as large single crystals. The salt cake porosity ranges from 10 to 50 percent. Liquid exists as supernatant and interstitial fluid (WHC 1990c).

The SST system waste is made up of primarily sodium hydroxide; sodium salts of nitrate, nitrite, carbonate, aluminate, and phosphate; and hydrous oxides of iron and aluminum.

A relatively small amount of solvents such a tributyl phosphate and normal paraffin hydrocarbon was added to the SST system waste during fuel reprocessing. Water-soluble compelling agents and carboxylic acids from the B Plant waste fractionation process also were added.

Land disposal restriction requirements for the waste placed in the SST system were not documented and the waste currently in the system was placed in the tanks before LDR requirements were enacted. When waste is transferred to the DST system, known LDR requirements are documented in a profile sheet. Because limited analytical information is available, underlying hazardous constituents typically are not identified. 


\subsubsection{SST System Treatment}

The waste in the SST system will undergo retrieval and disposal in accordance with the DST system treatment plan. The Tri-Party Agreement specifies that SST system waste will be treated and disposed of using the DST system pretreatment and disposal facilities and that tank 241-C-106 will be the first to have its contents retrieved from storage. Closure options, which will identify the level of retrieval necessary, will be documented in a comprehensive tank waste remediation system supplemental environmental impact statement.

See the DST system treatment summary (Section 3.4.3) for details of the treatment methods for this waste stream.

The schedule for treating SST system waste is based primarily on Tri-Party Agreement Milestones M-50-00 (pretreatment), M-60-00 (LLW vitrification), and M-51-00 (HLW vitrification). Because of budget limitations, accelerating treatment beyond these milestone dates is not realistic.

Further work is expected to determine what fraction of SST system waste is subject to the specified technology, high-level vitrification, after pretreatment. Regardless of what fraction of the SST system waste is subject to high-level vitrification, current federal LDR requirements impose sampling and analysis requirements on the treated waste before disposal.

\subsubsection{SST System Treatability Variances, Equivalency Petitions, Rulemaking Petitions, and Case-by-Case Exemptions}

Because the SST system waste is a listed waste, further regulatory action is anticipated so the waste can be disposed of at the geologic repository. Because current regulations impose sampling and analysis requirements on treated waste, regulatory action may be necessary to reduce or eliminate this requirement.

\subsection{PUREX CONTAINMENT BUILDING}

This section covers waste stream PUREX-1. The PUREX containment building is permitted under interim status as a waste management unit within the PUREX Plant TSD unit. It is permitted for the storage of solid mixed waste containing arsenic, barium, cadmium, chromium, lead, mercury, selenium, and silver.

\subsubsection{PUREX Containment Building Generation}

The waste stored in Position F17 in F-Cell consists of concrete debris collected from the floor of E-Cell. The concrete material was collected during the replacement of Tank E3. Approximately 1.0 cubic meter of debris, weighing approximately 4100 kilograms, is contained in a 1.2 meter $\times 1.8$ meter $\times 0.6$ meter carbon steel scrap hopper. The debris contains regulated quantities of chromium. 


\subsubsection{PUREX Containment Building Storage}

The PUREX containment building (202A building) is a portion of the plant with concrete floor, walls, and ceiling up to 1.8 meters thick. Waste in the containment building is -protected from external environmental forces such as wind, rain, and flooding.

The shielded area of the PUREX 202A building (thousands of cubic meters) is permitted for storage of dangerous waste. There are no plans to store more waste in the containment building.

\subsubsection{PUREX Containment Building Characterization}

The waste stored in Position F17 in F-Cell consists of concrete debris collected from the floor of E-Cell. The concrete material was retrieved from the floor when Tank E3 was replaced. Sample analyses were conducted to characterize the E-Cell floor solids. Regulated quantities of chromium were detected.

\subsubsection{PUREX Containment Building Treatment}

Currently, no plans exist to treat the chromium-contaminated concrete solids from the E-Cell floor. The waste will remain in place through the surveillance and maintenance phase and will be disposed of during closure. A treatment schedule for this waste has not been established. The material stored in the containment building will be addressed as part of PUREX Plant closure.

\subsection{PUREX STORAGE TUNNELS WASTE}

This section covers waste stream PUREX-2. The PUREX Storage Tunnels 1 and 2 both contain elemental lead. The PUREX Storage Tunnel 2 contains silver (mostly as silver nitrate), elemental mercury, cadmium, and chromium. The lead is in jumper counterweights and equipment weights and shielding. The silver is in discarded silver reactors. The mercury is sealed inside thermowells that are an integral part of the irradiated fuel dissolvers. The cadmium is present as elemental cadmium attached to equipment for neutron shielding. The chromium is a corrosion byproduct from a failed stainless steel process concentrator. (See Table 4-12.)

\subsubsection{PUREX Storage Tunnels Generation}

In June 1996, barium, absorbed mineral oil, and more cadmium, chromium, and lead were added to the PUREX Storage Tunnel 2 from the 324 and 325 Buildings. Barium is present as dispersible particulate contaminated with process materials from the 324 Building process. Cadmium is present as dispersible particulate and dried melter feed residue removed from process equipment. Chromium is present as dispersible particulate and dried meiter feed residue. Lead is present as dispersible particulate, dried melter feed residue, and liquid metal seal material. The mineral oil is contained within an absorbent material. 
Table 4-12. Plutonium Uranium Extraction Plant Storage Tunnels Inventories.

\begin{tabular}{|l|c|c|c|c|c|c|c|c|}
\hline $\begin{array}{c}\text { Date } \\
\text { transferred } \\
\text { to tunnels }\end{array}$ & $\begin{array}{c}\text { Tunnel } \\
\text { number }\end{array}$ & $\begin{array}{c}\text { Mass }(\mathrm{kg}) \\
\text { of lead } \\
\text { transferred }\end{array}$ & $\begin{array}{c}\text { Mass (kg) } \\
\text { of mercury } \\
\text { transferred }\end{array}$ & $\begin{array}{c}\text { Mass (kg) } \\
\text { of silver } \\
\text { nitrate } \\
\text { transferred }\end{array}$ & $\begin{array}{c}\text { Mass (kg) } \\
\text { of } \\
\text { cadmium } \\
\text { transferred }\end{array}$ & $\begin{array}{c}\text { Mass (kg) } \\
\text { of } \\
\text { chromium } \\
\text { transferred }\end{array}$ & $\begin{array}{c}\text { Mass (kg) } \\
\text { of mineral } \\
\text { oil } \\
\text { transferred }\end{array}$ & $\begin{array}{c}\text { Mass (kg) } \\
\text { of barium } \\
\text { transferred }\end{array}$ \\
\hline $06-60$ & 1 & 115 & - & - & - & - & - & - \\
\hline $12-24-60$ & 1 & 115 & - & - & - & - & - & - \\
\hline $02-26-71$ & 2 & - & - & 625 & - & - & - & - \\
\hline $12-22-71$ & 2 & - & 45 & - & - & - & - & - \\
\hline $09-30-72$ & 2 & - & 45 & - & - & - & - & - \\
\hline $01-18-86$ & 2 & - & 40 & - & 43 & - & - & - \\
\hline $11-18-87$ & 2 & 2540 & - & - & - & - & - & - \\
\hline $05-13-88$ & 2 & 230 & - & 115 & 13 & - & - & - \\
\hline $01-27-95$ & 2 & - & - & - & - & 8 & - & - \\
\hline $02-08-95$ & 2 & 1930 & - & - & - & - & - & - \\
\hline $03-11-96$ & 2 & 3232 & - & - & 2 & - & - & - \\
\hline $04-26-96$ & 2 & 1802 & - & - & 10.5 & 1.0 & 8.5 & - \\
\hline $06-12-96$ & 2 & 0.001 & - & - & 0.001 & 0.002 & - & 0.004 \\
\hline & Total & 9964 & 130 & 740 & 68.5 & 9 & 8.5 & 3 \\
\hline
\end{tabular}

Note: $9964 \mathrm{~kg}$ of lead have a volume of $0.89 \mathrm{~m}^{3}$.

$130 \mathrm{~kg}$ of mercury have a volume of about $0.01 \mathrm{~m}^{3}$ at $23^{\circ} \mathrm{C}$.

$740 \mathrm{~kg}$ of silver nitrate have a volume of $0.07 \mathrm{~m}^{3}$.

$68.5 \mathrm{~kg}$ of cadmium have a volume of $7.88 \times 10^{-3} \mathrm{~m}^{3}$.

$9 \mathrm{~kg}$ of chromium have a volume of $1.26 \times 10^{-3} \mathrm{~m}^{3}$

$8.5 \mathrm{~kg}$ of mineral oil have a volume of $7.4 \times 10^{-3} \mathrm{~m}^{3}$.

$3 \mathrm{~kg}$ of barium have a volume of $8.29 \times 10^{-4} \mathrm{~m}^{3}$.

As Hanford Facility cleanup continues, other equipment and materials containing these and other waste types may be added to the tunnels.

Silver in the form of silver salts deposited on unglazed ceramic packing was contained within the discarded silver reactors stored in Tunnel 2 . Three silver reactors were used to remove radioactive iodine from the offgas streams of the irradiated reactor fuel dissolvers in the PUREX process. The silver reactor vessel contained two beds of packing. The packing was coated initially with 114 kilograms of silver nitrate used for iodine retention. Nozzles on the top of the reactor were provided to allow flushing and/or regeneration of the packing with silver nitrate solution as the need arose.

Experience showed that after extended use, the silver reactors lost efficiency. This loss in efficiency normally occurred when about one-half of the silver nitrate on the packing had been converted to silver iodide. Other competing reactions such as reduction of silver nitrate to metallic silver and formation of silver chloride also occurred and affected silver reactor efficiency. To counteract this, the silver reactor was regenerated with fresh silver nitrate periodically. Therefore, the packing of the discarded silver reactor contained a mixture of silver nitrate, silver halides, and silver fines.

Elemental mercury waste was generated when dissolvers in the PUREX process failed or became obsolete and were discarded. The mercury was sealed inside thermowells, which were an integral part of reactor fuel dissolvers used at the PUREX Plant. Each dissolver had two thermowells. Each thermowell consisted of a 2.9 meter length of stainless steel pipe with an extension welded to the downside end. The lower end butted against the outer surface of the 
internal slotted bar screen that separates the undissolved fuel elements from the outer solution chamber of the annular dissolver. The mercury transferred heat from the dissolver interior to the temperature sensor mounted within the thermowell. This mercury remains in the thermowells of discarded dissolvers. In preparation for storage, the thermowells were sealed with a stainless steel nozzle plug. In storage, the discarded dissolvers rest in an inclined position in a cradle on a rail car. Secondary containment is provided by the dissolver vessel itself.

As of December 1997, three dissolvers have been discarded, one each in 1971, 1972, and 1986. The first two dissolvers contain 45 kilograms of elemental mercury each; the third contains 38 kilograms. All three dissolvers are stored on rail cars in PUREX Storage Tunnel 2 (RL 1990).

Cadmium may be present in the PUREX Storage Tunnel 2 as elemental cadmium attached to equipment for neutron shielding. The presence of cadmium is determined by process knowledge and the design of equipment that was used during PUREX operation and is known to possibly contain cadmium metal.

Chromium is present in the PUREX Storage Tunnel 2 as a corrosion byproduct of the stainless steel from a failed process concentrator. This concentrator was evaluated for reuse in 1986 and was determined to be unacceptable because of incompatibilities and a potential short service life. The concentrator was inspected and found to contain silicate solids with high levels of chromium.

In the 324 Building waste, lead is present in dispersible debris, dried melter feed residue, and liquid metal seal material from the 324 Building process. The dispersible debris waste consists of dirt, dust, process residue, equipment, and tools collected from the 324 Building B-Cell floor. This debris has been contaminated with process feed solutions that contained heavy metals. The residual dried melter feed was removed from process equipment after water evaporation from the melter feed slurry used in the repository program for the Federal Republic of Germany. The liquid metal seal, used as a seal material in a glass melter, is inherently hazardous. Barium also is present in the dispersible debris. Cadmium is present in the dispersible debris and liquid metal seal; chromium is present in the dispersible debris and residual dried melter feed. Mineral oil also is present in an absorbent material.

\subsubsection{PUREX Storage Tunnels Storage}

The PUREX Storage Tunnels are a RCRA-permitted mixed-waste storage unit. The two tunnels are connected to the PUREX Plant and, combined, provide storage space for 48 rail cars. The PUREX Storage Tunnels provide long-term storage for process equipment removed from the PUREX Plant and other onsite sources. Equipment transfers into the PUREX Storage Tunnels are made as needed. Radioactively contaminated equipment is loaded on rail cars and remotely transferred into the PUREX Storage Tunnels. Rail cars act as both transport and storage platforms for equipment placed in the tunnels.

The tunnels are weather-tight structures covered by 2.4 meters of earth. This design protects the stored equipment from exposure to natural elements, provides external radiation shielding from the radioactive equipment stored in the tunnels, and protects the environment. 
Tunnel I (218-E-14) was completed in 1956 as part of the PUREX Plant construction project and holds eight rail cars. Tunnel I was filled to capacity (approximately 600 cubic meters of waste) in 1965 and then secured. No elemental mercury waste is stored in Tunnel 1.

Tunnel 2 (218-E-15) was an expansion project constructed in 1964. This tunnel is considerably longer than Tunnel 1, providing storage space for 40 rail cars. Each rail car can hold 497 cubic meters of waste. To date, 28 rail cars containing 2204 cubic meters of discarded equipment and associated waste have been placed in the tunnel, filling 70 percent of the storage area. Sufficient storage capacity remains for all waste projected to be generated. A more complete description of the PUREX Storage Tunnels is available in PUREX Storage Tunnels Dangerous Waste Permit Application, Rev. 2 (RL 1990). The PUREX Storage Tunnels are a final-status TSD unit included in the Hanford Facility RCRA Permit.

The capacity of the storage tunnels is not expected to be reached.

\subsubsection{PUREX Storage Tunnels Characterization}

This section covers the available waste characterization information. Information based on process knowledge and testing is provided along with the waste designations and their bases, the uncertainty of the designations, and the schedule for further analysis. Not all waste in storage has been evaluated for UHCs. A UHC evaluation is expected for this waste in the future.

4.6.3.1 Process Knowledge. The amount of lead generated was identified by reviewing fabrication and design drawings for each piece of equipment placed in storage to determine if the lead weight, counterweight, or shielding was specifically detailed.

The silver salts quantity was estimated by knowing the amount of silver nitrate placed on the bedding and the regeneration history of the silver reactors. For accountability, the total silver content was considered as silver nitrate, the salt that exhibits both ignitability and toxicity characteristic leaching procedure (TCLP) toxicity. Silver nitrate is identified as a DOT oxidizer in 49 CFR 172.101 .

The mercury waste was characterized based on fabrication and installation specifications. The quantity of mercury present in each dissolver was documented on the fabrication drawings. None of the mercury will evaporate because the thermowells are sealed.

The quantity of cadmium was estimated from the dimensions of the cadmium metal sheets attached to the equipment. The quantity of chromium was estimated from knowledge of silicate solids that have high levels of chromium and are contained within the failed concentrator sampled during PUREX operation. The quantities of barium, lead, cadmium, mineral oil, and chromium from the 324 Building waste were estimated from process knowledge. 
4.6.3.2 Testing. Sampling and chemical analysis were not performed on waste associated with the radioactive discarded equipment placed in the PUREX Storage Tunnels. The quantity of waste in storage was determined from process knowledge and equipment design. Provisions for sampling the bedding were not provided in the design of the silver reactor vessels. Therefore, the reactors were not sampled and analyzed for silver salts before being placed in storage.

Sampling and chemical analysis were not performed on mercury associated with the dissolvers. The need for sample analyses will be evaluated during planning for closure of the PUREX Plant, including the storage tunnels.

\subsubsection{PUREX Storage Tunnels Treatment}

No definite agreed-on plans have been made for treating the tunnel waste. However, conceptually, if the waste must be removed from the PUREX storage tunnels, the elemental lead would be removed; from the process equipment to reduce the volume to be treated where feasible. The elemental lead and the silver salts located in the silver reactors could be treated by encapsulating the material in a cementitious grout that immobilizes the lead and silver. No planned treatment concept has been developed for the cadmium, chromium, barium, and selenium associated with the process equipment stored in the tunnels. The EPA-required treatment for elemental mercury is amalgamation. Therefore, the treatment of choice would be the current approach of adding zinc powder to create an amalgam. An alternative treatment would be to mineralize the elemental mercury (creating mercury sulfide). Alternative technologies to this process have not been studied. This will be done as part of closure activities for the PUREX Storage Tunnels, as necessary.

A schedule for treating this waste has not been established. Waste from the tunnels will be handled along with the similar materials currently in the PUREX canyon when the PUREX facility is decontaminated and decommissioned. PUREX decontamination and decommissioning, along with treatment of the PUREX Storage Tunnel waste, is contingent on completing the Facility-wide land-use plan, the Facility-wide decontamination and decommissioning priority schedule, and the environmental impact statement, and resolving public comments on those documents. A basis for the treatment plan for the waste associated with the PUREX Storage Tunnels will be developed after these items are complete.

Currently no capacity exists to treat the waste in the PUREX Storage Tunnels. To treat this waste, capability to handle and ship large containers containing highly radioactive materials will have to be developed or a treatment unit will have to be located near the tunnels. The treatment unit will have to be capable of remotely handling and reducing the size of highly contaminated large and small pieces of equipment and segregating the dangerous waste portions. 


\subsection{RADIOCHEMICAL ENGINEERING CELLS WASTE}

This section covers the $324 \mathrm{REC}$ waste stream. The $324 \mathrm{REC}$ is located in the 324 Building in the 300 Area. It consists of four hot cells (A, B, C, and D) located around a central airlock and a pipe trench located directly underneath the airlock. It has been used in numerous DOE-sponsored research and development programs since the mid-1960s. The major activities that have influenced the generation of mixed waste include the following:

- The Waste Solidification Engineering Prototypes Program (completed in 1972)

- The development of treatment technologies via the Nuclear Waste Vitrification Project for waste from reprocessing spent nuclear fuel (1979)

- A pilot-scale radioactive liquid-fed ceramic melter testing program in conjunction with the Federal Republic of Germany (1984 through 1987).

A closure plan for this location has been prepared and approved by Ecology (March 1998).

\subsubsection{REC Generation}

Most of the materials now in the REC accumulated during research activities from 1965 to 1987. The exception is solid residue from the treatment of high-level vault (HLV) tank waste. Over the 20+ years these engineering demonstrations were conducted, equipment (tools, manipulator boots, and construction materials) was dropped and liquids (feed materials and samples) leaked onto the floor. In addition, dust introduced with normal air flow into the cell became contaminated.

Operational protocols in the REC were based on the radioactive properties of these materials. The materials were secured within the cell where they did not interfere with engineering operations. Because of funding constraints and technical difficulties and safety issues associated with consolidating and/or retrieving, packaging, and/or transporting the waste materials, they were left in place. Cleanout of the hot cells to eliminate the unacceptable radiological hazards associated with the dispersible material in the B-Cell began in 1988 with completion estimated by 2000 .

No generation of additional waste, other than used HEPA filters, is anticipated. Current waste types contained in the REC and their estimated volumes are as follow.

- Approximately 2.3 cubic meters of tools, equipment, and pieces of metal dropped on the floor during operations; dust and particulates contaminated with feed solution; tank heels; and sludge.

- Approximately 0.36 cubic meter of filters loaded with solid residues resulting from treatment of the HLV tank waste. 


\subsubsection{REC Storage}

The $324 \mathrm{REC}$ does not receive waste from other sources. It stores only waste that was generated from REC operations.

The 324 REC is not a RCRA-permitted TSD unit. The 324 Building was constructed to strict nuclear standards to ensure that it would safely house operations involving highly radioactive materials and prevent releases to the environment. The storage capacity for mixed waste in the available hot cell section is estimated to be 15 cubic meters. This estimate is based on the inventory in storage ( 9.17 cubic meters) and the consideration that no future significant generation of waste at this facility is anticipated. No issues of waste storage capacity are associated with this facility.

\subsubsection{REC Characterization}

Dispersible material in B-Cell was sampled and analyzed in 1995 by Pacific Northwest National Laboratory. Based on the analytical results, the dispersible floor debris was designated as a characteristic waste for cadmium (D006), lead (D008), and chromium (D007). B-Cell tank heels and airlock pipe trench sludge are expected to have the same waste designation as the B-Cell dispersible material. The filters that were used in the HLV tank waste processing are designated as follows per analytical results and process knowledge:

$\begin{array}{ll}\text { Strontium filters } & \mathrm{Cr}, \mathrm{Cd} \\ \text { Metal filters } & \mathrm{Pb}, \mathrm{Ba}, \mathrm{Cr}, \mathrm{Cd} \\ \text { TRU filters } & \mathrm{Cr}\end{array}$

\subsubsection{REC Treatment}

Waste currently stored in the 324 REC unit is not being treated and is not expected to be treated. Milestone M-89-02 provides for removal of all REC B-Cell mixed waste and equipment by May 31, 1999. Some REC mixed waste will be shipped to the PUREX Storage Tunnels and the CWC for storage. Waste will be treated in accordance with the proposed treatment scenarios for the waste managed in these TSD units.

\subsection{B PLANT CELL 4 WASTE}

This section covers waste stream B Plant-1. Mixed waste and low-level waste generated in the WESF hot cells are stored in 208 liter drums in the B Plant Cell 4 container storage area, as allowed in the B Plant Complex Part A Form 3 Permit Application. Currently the area holds 7 rums of mixed waste and 36 drums of highly radioactive low-level waste (not mixed waste). The sole hazardous constituent in the mixed waste drums is lead solder on incandescent lamps from the hot cells. Because of space constraints in the hot cell, the lamps were packed in drums with other low-level waste, so the entire drum is managed as mixed waste. 


\subsubsection{B Plant Cell 4 Generation}

Since 1988, waste generated in the WESF hot cells has been packaged into 208 liter drums and transferred to B Plant for storage in Cell 4 . When lights in the hot cells were replaced, the old ones were packaged into the drums along with the other waste. These transfers were performed frequently until 1991. Cleanout activities in the WESF hot cells generated eight additional waste drums that were transferred to Cell 4 in 1997. Agreements with Ecology reached during transition negotiations under the Tri-Party Agreement allow this process to continue through facility deactivation, although- no additional transfers to Cell 4 from WESF occurred during 1998.

\subsubsection{B Plant Cell 4 Storage}

Cell 4 is located in the 221B B Plant canyon building. Its physical dimensions are approximately 8 meters by 4 meters by 6 meters deep. It stores the waste drums on a wooden platform that can hold 59 drums. Additional platforms can be constructed and mounted on top of the existing platform to create additional storage capacity. A maximum of 245 drums or 51 cubic meters can be stored in Cell 4 in this configuration. Cell 4 is isolated from the canyon deck by concrete cover blocks.

Seven drums (1.4 cubic meters) of mixed waste are currently stored in Cell $4 ; 36$ drums of low-level waste also are stored in the area. This leaves about 16 empty spaces on the existing platform. Although the agreement reached with Ecology during Tri-Party Agreement transition negotiations allowed further additions to Cell 4 from WESF, no additions were made in 1998.

\subsubsection{B Plant Cell 4 Characterization}

No sampling and analysis of the waste in Cell 4 has been performed. Because of the high radiation levels of this waste and the high degree of process knowledge about it, sample analysis is not considered necessary to obtain an accurate characterization and waste designation.

Based on multiple sample results for waste matrices with lead solder, including similar incandescent bulbs, these bulbs would likely yield an extract containing greater than 5.0 milligrams/liter of lead when exposed to a leachate. The amount of lead solder on the incandescent lamps from the WESF hot cell was provided by the vendor who supplies the light bulbs. An inventory of the waste is prepared as the drum is packaged in the hot cell.

\subsubsection{B Plant Cell 4 Treatment}

Treatment has not been planned or scheduled for this waste. Current plans call for the waste disposition to be addressed during closure of the B Plant complex. According to 40 CFR 268, the required treatment for radioactive lead solids is macroencapsulation. 


\subsection{B PLANT CONTAINMENT BUILDING WASTE}

This section covers waste stream B Plant-2. The B Plant containment building consists of the $221 \mathrm{~B}$ canyon area and the process cells in the canyon. The waste stored on the canyon deck and in the process cells, primarily discarded process equipment and jumpers, is considered to be stored in a containment building.

\subsubsection{B Plant Containment Building Generation}

The lead in the B Plant containment building consists of material used as shielding or counterweights for process equipment or jumpers in the $221 \mathrm{~B}$ canyon. Approximately 293,447 kilograms of contaminated debris, equipment, and lead counterweights are stored in the containment building as waste. The contaminated debris and equipment remain from processing listed waste and are designated with waste codes F001 through F005.

As part of decoupling WESF from B Plant to complete the deactivation, tank 100 was replaced and placed on the $B$ Plant canyon deck for storage. Hazardous substances and dangerous waste stored in the B Plant containment building are identified in the hazardous substance and dangerous waste inventory.

\subsubsection{B Plant Containment Building Storage}

The B Plant canyon is approximately 260 meters long by 21 meters wide by 22 meters high. It is a concrete structure with walls several feet thick. Waste stored in the B Plant containment building is protected from the elements. Waste stored in the process cells is further protected by large concrete cover blocks that enclose the cells and form the canyon deck. The full capacity of the containment building is 35,000 cubic meters.

Accurate estimates of the waste volume currently stored in the containment building are not available. However, an inventory of the waste in the process cells and on the canyon deck was developed for the $1998 \mathrm{LDR}$ report and was estimated at 293,447 kilograms. An estimated amount of $500 \mathrm{~kg}$ was generated in 1998 , bringing the estimated inventory to $294,000 \mathrm{~kg}$. This waste is made up of 99 percent contaminated debris and equipment and 1 percent lead. No free liquids are stored in the containment building. The estimate does not include equipment abandoned in place.

\subsubsection{B Plant Containment Building Characterization}

The amount of lead in each process jumper and the amount of shielding for each piece of process equipment are known from design drawings. Historical process flow diagrams and operating knowledge provide a basis for determining what types of waste were processed using this equipment. No sampling and analysis of the waste has been performed and none will be performed during facility deactivation. Process knowledge will be used to adequately characterize the waste that will remain in the containment building during deactivation. 
Some of the waste has been designated as TCLP toxic for lead (D008) because of the lead used as a component of the equipment. In addition, waste codes F001 through F005 have been applied to equipment that was used to manage or process listed SST and DST system waste. Heavy metals waste codes (D004 through D011) also may apply in some instances because of potential waste residue on process equipment. However, applicability of these characteristic codes has not been determined.

\subsubsection{B Plant Containment Building Treatment}

Treatment has not been planned or scheduled for this waste. Current plans call for its disposition to be addressed during closure of the B Plant complex. The specified LDR treatment for radioactive elemental lead solids is macroencapsulation. Elemental lead waste may be treated using alternative treatment standards for hazardous debris.

\subsection{T PLANT COMPLEX DRAG-OFF BOX}

\subsubsection{Drag-Off Box Generation}

No detailed information is available on the generation of waste contained in the drag-off box. Much of the waste in the box appears to be equipment from the T Plant complex. However, some of the items were generated at other onsite locations, such as the PUREX complex, and sent to the $T$ Plant complex for repair or decontamination. Few historical records are known to exist for the materials in the box.

\subsubsection{Drag-Off Box Storage}

The drag-off box is located in the 221-T Building at the T Plant complex. No plans have been made to move the materials in the box or the box itself. However, if the box or materials must be moved, they can be stored at various locations at the T Plant complex as long as the storage areas are posted and controlled appropriately and the materials are packaged adequately. Permitting does not limit the storage of the materials to a single location.

No significant increases to the waste quantity stored in the 221-T Building or the T Plant complex are planned. The quantity of waste material stored at the $T$ Plant complex varies from day to day, but does not increase significantly over time. Therefore, it is not expected that the $T$ Plant complex storage capacity will be reached.

\subsubsection{Drag-Off Box Characterization}

The waste items in the drag-off box were being sorted based on limited process knowledge. Items determined to be mixed low-level waste were segregated from items determined to be low-level waste. Sorting was discontinued because of funding cuts. Funding to complete this process has not been identified. 


\subsubsection{Drag-Off Box Treatment}

The mixed waste items from the box are expected to require treatment before disposal. However, treatment has not been funded, therefore no plans have been made to treat the items. Treatment methods, capacity, and alternative technologies have not been investigated. Treatment capacity is unknown because a treatment method has not been identified. The items may be treated at the T Plant complex depending on the specified treatment method and funding.

\subsection{T PLANT COMPLEX TANK TRAILER WASTE}

\subsubsection{Tank Trailer Waste Generation}

The waste in the tank trailer came from the 219S tank at the 222-S Laboratory complex in the 200 West Area and B Plant in the 200 East Area. The waste was derived from analytical laboratory and B Plant operations. The tank trailer was removed from service because the possible presence of PCBs was a concern. Most of the waste in the tank trailer was transferred to the DST system. What remains is a heel of about 757 kilograms that could not be removed. The trailer was sent to the T Plant complex because the 222-S Laboratory complex was not permitted for storing this type of container. The T Plant complex received the trailer on March 6,1997 . In July 1998, 3783 kilograms of liquid waste from 225-B in the B Plant complex was added to the heel in the trailer. The trailer remains in storage pending approval to transfer the waste to the DST system.

\subsubsection{Tank Trailer Waste Storage}

The tank trailer is located in an outside area at the T Plant complex. The trailer may be stored at various locations at the T Plant complex as long as the storage area is posted and controlled appropriately. Permits do not limit the storage of the trailer to a single location.

Significant increases in the quantity of waste stored at the T Plant complex are not planned. The quantity of waste stored at the $T$ Plant complex does not increase significantly over time. Therefore, it is not expected that the storage capacity of the T Plant complex will be reached.

\subsubsection{Tank Trailer Waste Characterization}

The 5,000-gallon tank trailer contains 4540 kilograms (about 4.54 cubic meters) of waste liquid and sludge. Characterization of the waste was based on process knowledge and analytical results. Therefore, the quantities of listed waste under codes F001 through F005 may be smaller than those reported. The waste contains chromium at a concentration of 39.6 milligrams per liter and has a $\mathrm{pH}$ of 13.6 . 


\subsubsection{Tank Trailer Waste Treatment}

The waste will be transferred to the DST system. This would allow the trailer to be an empty container under RCRA for reuse. The waste will then be treated and disposed of as DST system waste.

\subsection{T PLANT COMPLEX EC-1 CONDENSER}

\subsubsection{EC-1 Condenser Generation}

The EC-1 condenser was used at the 242-A Evaporator as part of the process to reduce the quantity of underground liquid waste at the Hanford Facility. The condenser was removed from service and received at the T Plant complex on July 6, 1995.

\subsubsection{EC-1 Condenser Storage}

The EC-1 condenser is stored on a railroad flatcar on a track in the 2706-T Building yard. The EC-1 condenser may be stored at various locations at T Plant complex as long as the storage area is posted and controlled appropriately. Permits do not limit the storage of the condenser to a single location.

Significant increases to the amount of waste stored at the T Plant complex are not planned. The quantity of waste stored at the $T$ Plant complex varies from day to day, but does not increase significantly over time. Therefore, it is not expected that the storage capacity of the T Plant complex will be reached.

\subsubsection{EC-1 Condenser Characterization}

The EC-1 condenser is designated as mixed waste because of contact with the DST and SST system waste, which carries waste codes F001 through F005. The designation is based on process knowledge.

\subsubsection{EC-1 Condenser Treatment}

Treatment of the EC-1 Condenser is a low priority compared to other treatment projects. No plans have been made to treat the condenser. Currently, treatment capacity is not a concern and no alternative technologies are being developed to treat the condenser. 


\subsection{CESIUM AND STRONTIUM CAPSULES}

\subsubsection{Cesium and Strontium Capsule Waste Generation}

Cesium and strontium were received from B Plant as purified cesium carbonate and strontium nitrate. They were converted to dry salts as cesium chloride and strontium fluoride and encapsulated. The encapsulation consists of a capsule inside a capsule to provide redundant protection in case of a capsule failure.

\subsubsection{Cesium and Strontium Capsule Waste Storage}

The WESF facility houses 1335 cesium capsules and 601 strontium capsules. All the strontium capsules are stored in the pool cells and all but 14 cesium capsules are stored in the pool cells. These 14 capsules currently are stored in $\mathrm{G}$ cell and will be transferred to the pool cell area following an uitrasonic scan.

The TSD portion of the WESF facility consists of 8 pool cells, a transfer aisle, and the process cells $A$ through $\mathrm{G}$. Only the $\mathrm{F}$ and $\mathrm{G}$ cells are currently used. The remaining cells are not planned to be used until the capsules are cut up and blended back into Tank Farm waste beginning in 2013 .

There are no plans to add any additional inventory to WESF.

\subsubsection{Cesium and Strontium Capsule Waste Characterization}

Analyses have not been performed to determine the salt content of each individual capsule. A controlled database is maintained that documents the salt content in each capsule by curie content and salt weight. Process logs are maintained that document the process specifications and purity content of each process run. This information is documented in the interim status plan for WESF and is considered to be adequate waste characterization data.

\subsubsection{Cesium and Strontium Capsule Waste Treatment}

Current plans are to cut up the capsules and dissolve the salts at either the Waste Encapsulation and Storage Facility or the future waste vitrification plant for the DST and SST system waste. The capsules then will be blended into the tank waste beginning in 2013 for subsequent vitrification. Alternatively, the capsules could be packaged and sent directly to a future offsite geologic repository. (The alternative disposal methods are shown in Figure 4-4). 


\subsection{REEERENCES}

\subsection{APPLICABLE DOCUMENTS}

Adler, J. G., 1998, B-Plant Complex Preclosure Work Plan, DOE/RL-98-12, U.S. Department of Energy, Richland Operations Office, Richland, Washington.

CAO, 1996, Transuranic Waste Characterization Quality Assurance Program Plan, CAO-94-1010, U.S. Department of Energy, Carlsbad Area Office, Carlsbad, New Mexico.

DOE, 1988, Radioactive Waste Management, DOE Order 5820.2A, U.S. Department of Energy, Washington, D.C.

DOE, 1990, National Report on Prohibited Wastes and Treatment Options, U.S. Department of Energy, Washington, D.C.

DOE, 1995, DOE/LLW-271, U.S. Department of Energy, Washington, D.C.

Ecology, 1993, Treatment by Generators, Technical Information Memorandum 86-3, Hazardous Waste and Toxics Reduction Program, Technical Assistance and Policy Sections, Washington State Department of Ecology, Olympia, Washington.

Ecology, 1998, Hanford Facility Resource Conservation and Recovery Act Permit, Revision 4A, EPA/State ID \#: WA7890008967, Washington State Department of Ecology, Kennewick, Washington.

Ecology, EPA, and DOE, 1989, Hanford Federal Facility Agreement and Consent Order, as amended, Washington State Department of Ecology, U.S. Environmental Protection Agency, and U.S. Department of Energy, Olympia, Washington.

EPA, 1986, Test Methods for the Evaluation of Solid Waste: Physical/Chemical Methods, SW-846, 3rd ed., U.S. Environmental Protection Agency, Washington, D.C.

FDH, 1998, Hanford Site Solid Waste Acceptance Criteria, HNF-EP-0063, Rev. 5, Fluor Daniel Hanford, Inc., Richland, Washington.

FDH, 1999, Waste Tank Summary Report for Month Ending December 31, 1998, HNF-EP-0182-129, Prepared by Lockheed Martin Hanford Company for Fluor Daniel Hanford Company, Richland, Washington.

Josephson, W. S., 1996, Sampling and Analysis Plan for Mixed Waste Treatment, WHC-SD-WM-TP-442, Westinghouse Hanford Company, Richland, Washington. 
Kirkpatrick, K. L., and B. L. Oswald, 1995, Waste Specification System, WHC-EP-0846, Westinghouse Hanford Company, Richland, Washington.

RL, 1990, PUREX Storage Tunnels Dangerous Waste Permit Application, DOE/RL-90-24,

Rev. 2, U.S. Department of Energy, Richland Operations Office, Richland, Washington.

RL, 1995, Direct Disposal Team Report-Options for Treating and Disposing of Current and

Future Mixed Waste Streams on the Hanford Facility, DOE/RL-95-35, Rev. 0,

U.S. Department of Energy, Richland Operations Office, Richland, Washington.

RL, 1998, Hanford Site Waste Minimization and Pollution Prevention Awareness Program Plan, DOE/RL-91-31, Rev. 4, U.S. Department of Energy, Richland Operations Office, Richland, Washington.

RL, 1998, Return on Investment (ROI) Proposal Preparation Guide, DOE/RL-97-12, Rev. 2, U.S. Department of Energy, Richland Operations Office, Richland, Washington.

WHC, 1989, Characterization Plan for Stored Contact Handled Transuranic Waste, WHC-EP-0223, Westinghouse Hanford Company, Richland, Washington.

WHC, 1990a, Hanford Site LDR RMW Stream Data Package, WHC-MR-0224, Westinghouse Hanford Company, Richland, Washington.

WHC, 1990b, A History of the 200 Area Tank Farms, WHC-MR-0132, Westinghouse Hanford Company, Richland, Washington.

WHC, 1990c, Single-Shell Tank Waste Retrieval Study, WHC-EP-0352, Westinghouse Hanford Company, Richland, Washington.

\subsection{CODE OF FEDERAL REGULATIONS AND FEDERAL REGISTER}

40 CFR 268, "Land Disposal Restrictions," Title 40, Code of Federal Regulations, Part 268, as amended, U.S. Environmental Protection Agency, Washington, D.C.

40 CFR 761, "Polychlorinated Biphenyls (PCB) Manufacturing, Processing, Distribution in Commerce, and Use Prohibitions," Title 40, Code of Federal Regulations, Part 761, as amended, U.S. Environmental Protection Agency, Washington, D.C.

55 FR 22520, 1990, "Land Disposal Restrictions for Third-Third Scheduled Wastes; Final Rule," Federal Register, Vol. 55, pp. 22627, (June 1, 1990). 
58 FR 29860, 1993, "Land Disposal Restriction for Ignitable and Corrosive Characteristic Wastes Whose Treatment Standards Were Vacated," Federal Register, Vol. 58, pp. 29860, (May 24, 1993).

59 FR 47982, 1994, "Universal Treatment Standards," Federal Register, Vol. 59, pp. 47982.

61 FR 15660, 1996, "Land Disposal Restrictions Phase III-Decharacterized Wastewaters, Carbonate Waste, and Spent Potliners," Federal Register, Vol. 61, pp. 15660.

61 FR 15666, 1996, "Land Disposal Restrictions Phase III-Decharacterized Wastewaters, Carbonate Waste, and Spent Potliners," Federal Register, Vol. 61, pp. 15666.

61 FR 33680, 1996, "Land Disposal Restrictions Phase III-Decharacterized Wastewaters, Carbonate Waste, and Spent Potliners," Federal Register, Vol. 61, pp. 33680.

61 FR 35419, 1996, "Land Disposal Restrictions Phase III-Decharacterized Wastewaters, Carbonate Waste, and Spent Potliners," Federal Register, Vol. 61, pp. 35419.

61 FR 43924, 1996, "Emergency Revision of the Land Disposal Restrictions (LDR) Phase III Treatment Standards for Listed Hazardous Waste from Carbonate Production, Final Rule," Federal Register, Vol. 61, pp. 43924.

61 FR 60704, 1996, "Termination of Review of Every Petition to EPA for a No-Migration Determination for the Waste Isolation Pilot Plant (WIPP) Under the Resource Conservation and Recovery Act," Federal Register, Vol. 61, pp. 60704.

62 FR 7502, 1997, "Land Disposal Restrictions: Corrections of Tables; Treatment Standards for Hazardous Wastes and Universal Treatment Standards," Federal Register, Vol. 62, pp. 7502.

62 FR 25998, 1997, "Land Disposal Restrictions Phase IV: Treatment Standards for Wood Preserving Wastes, Paperwork Reduction and Streamlining, Exemptions from RCRA for Certain Processed Materials; and Miscellaneous Hazardous Waste Provisions; Final Rule," Federal Register, Vol. 62, pp. 25998.

62 FR 64504, 1997, "Clarification of Standards for Hazardous Waste Land Disposal Restriction Treatment Variances," Federal Register, Vol. 62, pp. 64504.

63 FR 28556, 1998, "Land Disposal Restrictions Phase IV: Final Rule Promulgating Treatment Standards for Metal Wastes and Mineral Processing Wastes; Mineral Processing Secondary Materials and Bevill Exclusion Issues; Treatment Standards for Hazardous Soils, and Exclusion of Recycled Wood Preserving Wastewaters," Federal Register, Vol. 63, pp. 28556. 
63 FR 65874, 1998, "Hazardous Remediation Waste Management Requirements (HWIRmedia)," Federal Register, Vol. 63, pp. 65874

\subsection{FEDERAL AND STATE ACTS}

Atomic Energy Act of 1954, as amended, 42 USC 2011.

Comprehensive Environmental Response, Compensation, and Liability Act of 1980, as amended, 42 USC 9601 et seq.

Federal Facility Compliance Act of 1992, as amended, 42 USC 6901, et seq.

Resource Conservation and Recovery Act of 1976, as amended, 42 USC 6901, et seq.

State of Washington Hazardous Waste Management Act of 1976, as administered through the Washington State Department of Ecology's "Dangerous Waste Regulations," WAC 173-303.

Toxic Substances Control Act of 1976, 15 USC 2601, et seq.

\subsection{WASHINGTON STATE REGULATIONS}

WAC-173-303, "Dangerous Waste Regulations," Washington Administrative Code, as amended. (WAC 173-303-140 covers land disposal restrictions.) 


\section{DISTRIBUTION}

Number of Copies

$\underline{\text { OFFSITE }}$

17

Washington State Department

of Ecology

P. O. Box 47600

Olympia, Washington $98504-7600$

M.A. Wilson (2)

Washington State Department

of Ecology

Kennewick, Washington 99336

M. N. Jaraysi (5) B5-18

U.S. Environmental Protection Agency 1200 Sixth Ave.

Seattle, Washington 98101

D. Ingemansen

D. Bartus

U.S. Environmental Protection Agency

Richland, Washington 99352

D. R. Sherwood (2) B5-01

U.S. Department of Energy-Headquarters Washington. D.C.

R. A. Martinez, EM-38

P. Bubar, EM-35

Lockheed Martin Idaho Technologies Company P. O. Box 1625 MS 3875

Idaho Falls, ID 83415-3875

J. Townsend

Distr-1 


\section{DISTRIBUTION (cont)}

Nez Perce Tribe

Environmental Restoration and

Waste Management Program

P.O. Box 365

Lapwai, Idaho 83540

D. Powaukee, Manager

Confederated Tribes of the Umatilla Indian Reservation P.O. Box 638

Pendleton, Oregon 97801-0638

J. R. Wilkinson

DNR-SSRP

Yakama Indian Nation

Environmental Restoration and

Waste Management Program

P.O. Box 151

Toppenish, Washington 98948

R. Jim, Manager

Number of Copies

ONSITE

19

U.S. Department of Energy,

Richland Operations Office

T. L. Aldridge K8-50

D. T. Evans $\quad$ S2-47

R. F. Guercia $\$ 7-55$

J. E. Kinzer - S7-50

J.S. Klug $\quad$ S7-55

R. N. Krekel A5-15

L. E. Rogers R3-79

G. L. Sinton (10) S7-55

M. L. Talbot A7-75

Public Reading Room H2-53 
DOE/RL-99-01

\section{DISTRIBUTION (cont)}

Bechtel Hanford. Incorporated

J. W. Badden

H9-03

R. J. Landon

$\mathrm{H} 0-02$

G. S. Robinson

$\mathrm{H} 0-02$

Document and

Information Services

H0-09

4

Pacific Northwest National Laboratory

M. W. McCoy

P7-79

W. A. Ross

K7-94

H. T. Tilden II

P7-79

Technical Files

K1-11

Fluor Daniel Hanford Company and Project Hanford Management Subcontractors

W. D. Adair

H6-21

R. W. Bailey

S4-49

D. G. Black (25)

H6-20

J. E. Bramson

T5-54

D. J. Carrell

R1-51

F. J. Carvo

N1-41

F. M. Coony

H6-06

L. I. Covey

S6-51

R. H. Engelmann

H6-26

B. G. Erlandson

R1-51

C. R. Haas

T3-28

G. D. Hendricks

R3-56

J. S. Hertzel

H8-67

S. M. Joyce

H6-06

O. S. Kramer

G3-27

G. J. LeBaron

S6-15

S. S. Lowe

H6-06

K. M. McDonald

H6-06

A. G. Miskho

H6-23

D. E. Nester (5)

H6-06

D. H. Nichols

H6-06

J. L. Pennock

S6-81

M. M. Pereira

S6-81 
DISTRIBUTION (cont).

P. A. Powell (2)

K. M. Quigley

E. D. Robbins

F. M. Simmons

J. O. Skolrud

J. N. Strode

W. E. Toebe

A. G. Weiner

B. D. Williamson

J. A. Winterhalder

WMH Legal

EDMC (7)

Central Files

DPC

Hanford Facility

Operating Record
R1-51

H6-06

S6-71

S6-81

H6-20

R2-11

H6-23

H6-21

B3-15

H6-21

H6-10

H6-08

A3-88

A3-94

H6-08 